\title{
Evaluation of KE Basin Sludge Radioisotopics and Volumes in Support of the Sludge Water System Safety Analysis
}

Division: SNF

P. D. Rittmann

Fluor Federal Services

D. J. Strom

Pacific Northwest National Laboratory

Date Published

August 2002

Prepared for the U.S. Department of Energy

Assistant Secretary for Environmental Management

Project Hanford Management Contractor for the

U.S. Department of Energy under Contract DE-AC06-96RL13200

Fluor Hanford

P. O. Box 1000

Richland, Washington

Aanis Aarckel $\frac{8-22-02}{\text { Date }}$

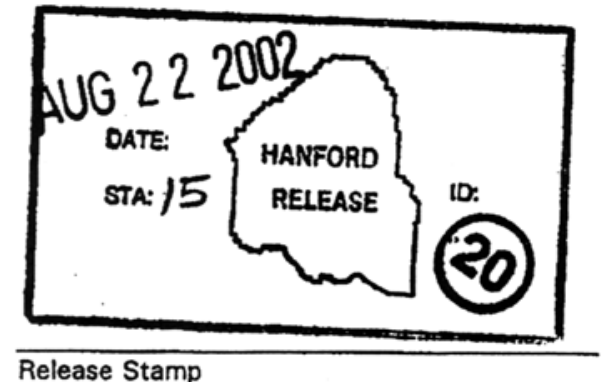


SNF-10293

Revision 0

TRADEMARK DISCLAIMER

Reference herein to any specific commercial product, process,

or service by trade name, trademark, manufacturer, or

otherwise, does not necessarily constitute or imply its

endorsement, recommendation, or favoring by the United

States Govemment or any agency thereof or its contractors or subcontractors.

This report has been reproduced from the best available copy.

Printed in the United States of America

Total Pages: 324 


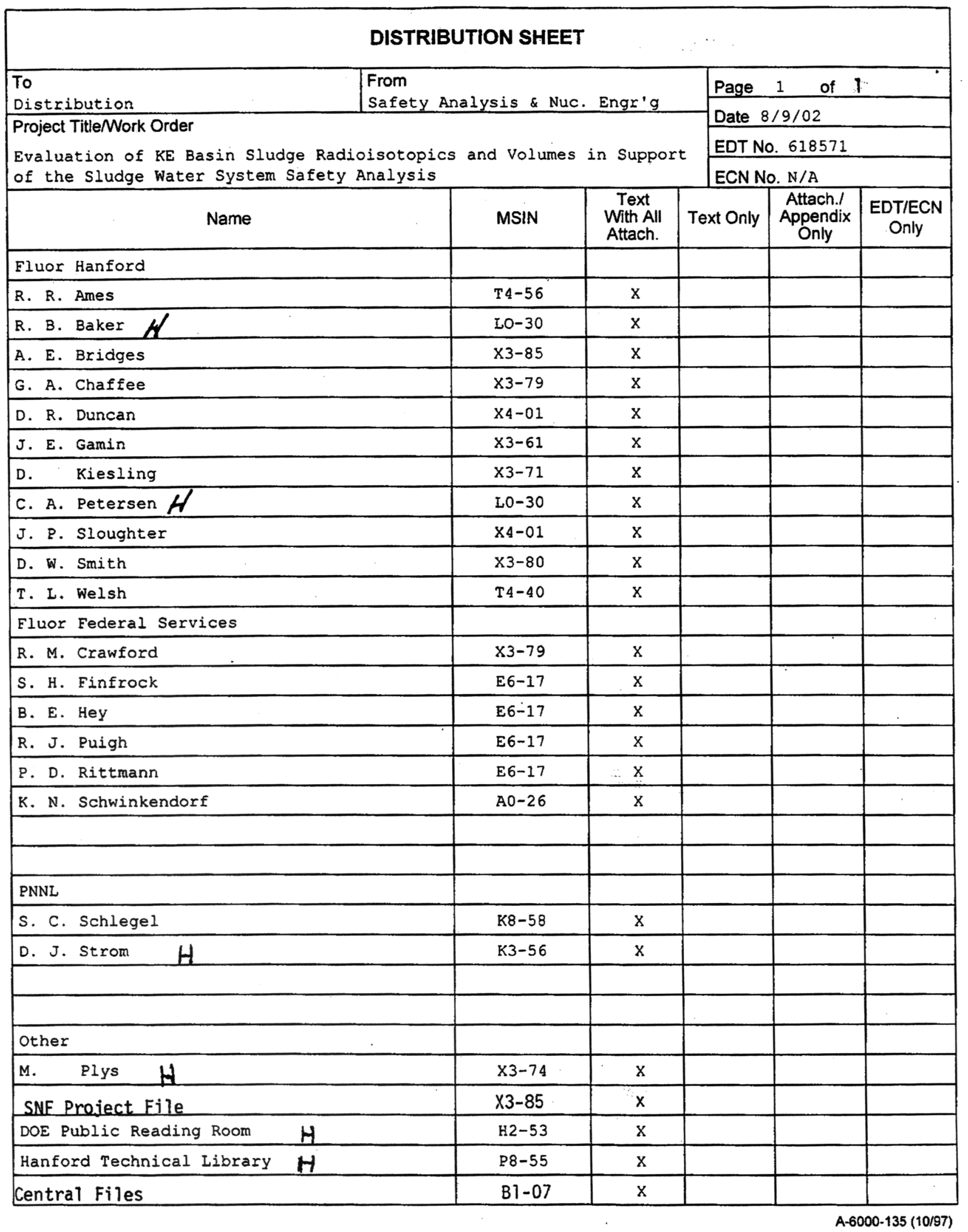




\begin{tabular}{|c|c|}
\hline 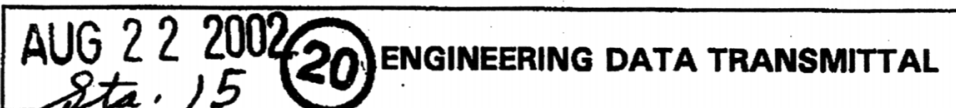 & $\begin{array}{ll}\text { Pagec } 1 \text { of } \frac{1}{1} \\
\text { 1. EDT } \\
618571\end{array}$ \\
\hline
\end{tabular}

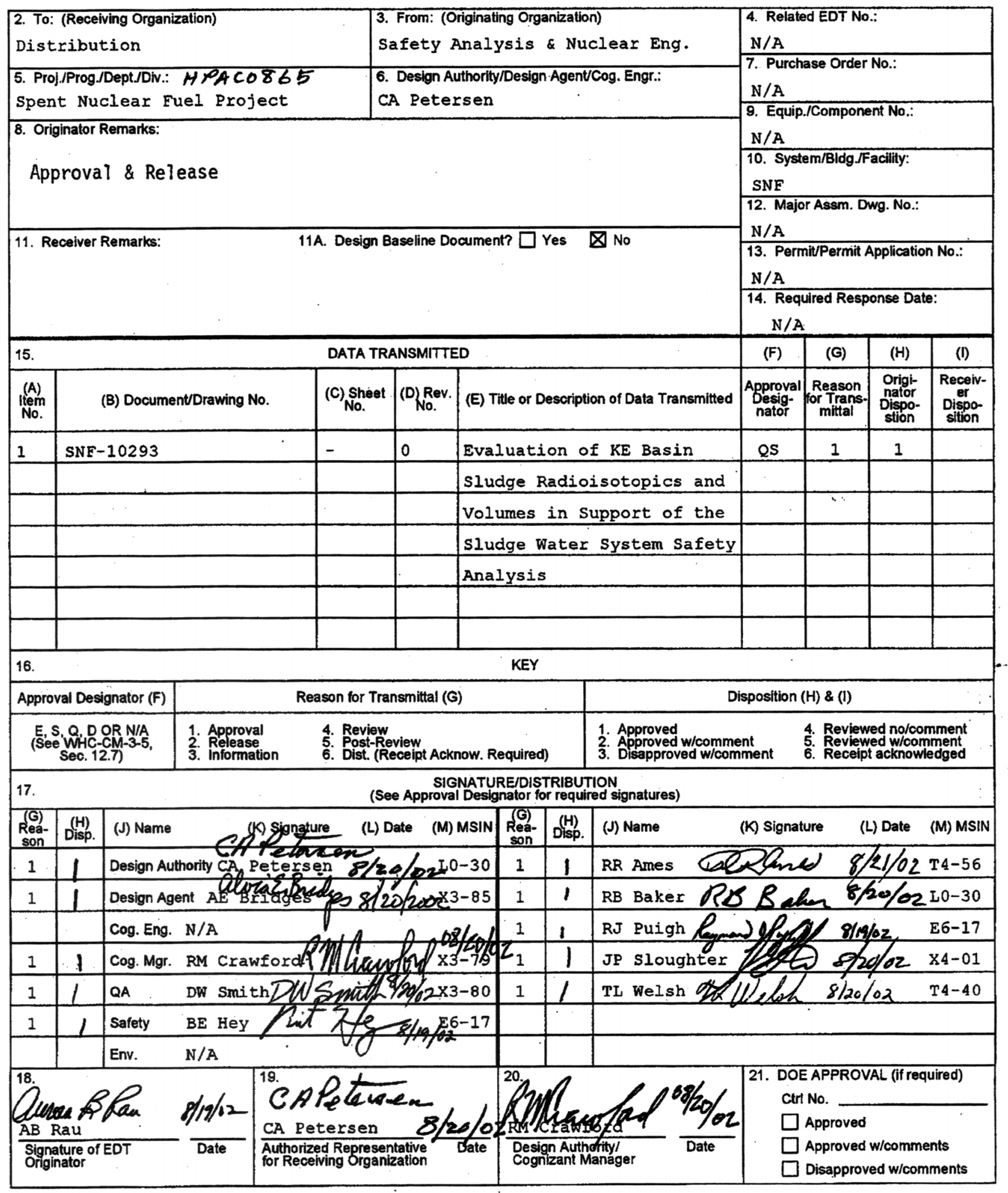


SNF-10293

Revision 0

PNNL-13936

\section{Evaluation of KE Basin Sludge Radioisotopics and Volumes in Support of the Sludge Water System Safety Analysis}

Prepared for the U.S. Department of Energy

Assistant Secretary for Environmental Management

Project Hanford Management Contractor for the

U.S. Department of Energy under Contract DE-AC06-96RL13200

Fluor Hanford

P.O. Box 1000

Richland, Washington 
SNF-10293, Rev. 0

\title{
EVALUATION OF KE BASIN SLUDGE RADIOISOTOPICS AND VOLUMES IN SUPPORT OF THE SLUDGE WATER SYSTEM SAFETY ANALYSIS
}

Key Words: dose factor, heat, monte carlo, sludge retrieval, sludge storage container, stochastic, spent fuel sludge

\begin{abstract}
Stochastic estimates have been generated for the inventories in sludge containers that will be filled in $\mathrm{K}$ East Basin. The containers hold $2 \mathrm{~m}^{3}$ of sludge. Additional volumes $\left(0.5 \mathrm{~m}^{3}\right.$ and $\left.12 \mathrm{~m}^{3}\right)$ were also investigated. The quantities estimated include heat output, inhalation dose factor, and the concentrations of uranium, plutonium, and 13 radionuclides. Floor and canister sludge data collected from 1994 to 2000 has been applied to the canister locations from 1994. The floor and canister sludge from each fuel storage cubicle in a key are retrieved and placed in the containers. The keys are retrieved in any order, i.e., randomly selected retrieval sequences. Alternate probability density functions were tried and the results compared with the base case. Most differences with container concentrations for the base case are less than $10 \%$. Using a single retrieval sequence was found to increase the bounding container concentrations by as much as $20 \%$ for the six sequences that were tested.
\end{abstract}


SNF-10293, REV 0

\section{CONTENTS}

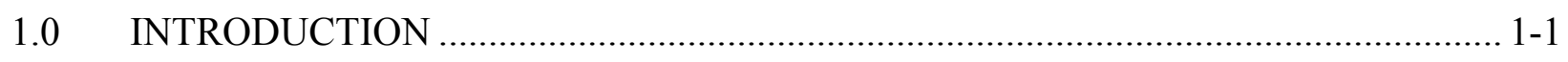

2.0 INTRODUCTION TO THE STOCHASTIC METHODS USED ............................... 2-1

3.0 DATA USED IN THE STOCHASTIC MODEL …............................................... $3-1$

3.1 FLOOR SLUDGE DEPTH..................................................................... 3-1

3.2 FLOOR SLUDGE ISOTOPIC CONCENTRATION ...................................... 3-3

3.2.1 Imputed Values for Floor Sludge....................................................... 3-4

3.2.2 Stochastic Model for Floor Sludge Concentration................................ 3-18

3.3 CANISTER SLUDGE DEPTH ........................................................ 3-22

3.3.1 Canister Sludge Depth Data ........................................................ 3-23

3.3.2 Stochastic Model for Canister Sludge Depth................................... 3-32

3.4 CANISTER SLUDGE ISOTOPIC CONCENTRATION .............................. 3-35

3.4.1 Data and Imputed Values for Canister Sludge.................................... 3-36

3.4.2 Comparisons of Imputed Values with Detection Thresholds ................ 3-44

3.4.3 Canister Sludge Concentration Estimates ....................................... 3-45

3.4.4 Comparisons with HNF-8760 ................................................. 3-52

3.4.5 Stochastic Model for Canister Sludge Concentration ........................... 3-62

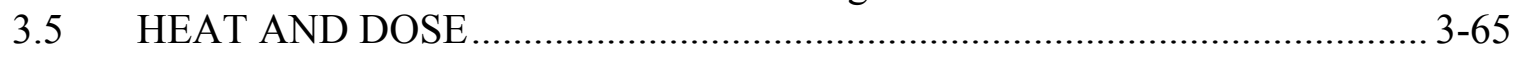

URANIUM METAL ............................................................................... 3-67

3.7 PLUTONIUM WEIGHT PERCENT ....................................................... 3-67

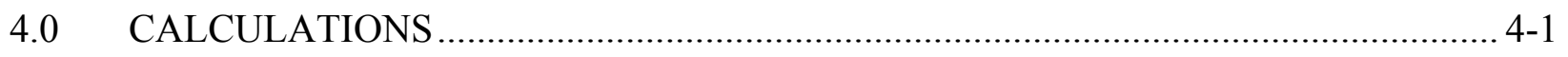

4.1 STOCHASTIC METHOD ............................................................................ 4-1

4.2 PRESERVATION OF MEAN VALUES ..................................................... 4-9

4.2.1 Floor Sludge Input Data ............................................................ 4-10

4.2.2 Canister Sludge Input Data ....................................................... 4-12

4.2.3 Container Results ............................................................. $4-17$

5.0 STOCHASTIC RESULTS FOR THREE CONTAINER VOLUMES ......................... 5-1

5.1 CANISTER-TO-TOTAL VOLUME RATIO.............................................. 5-1

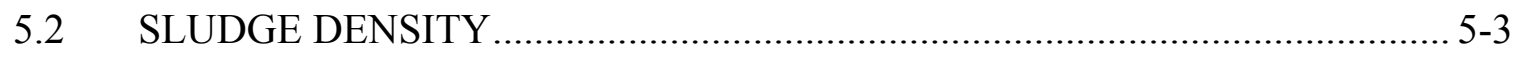

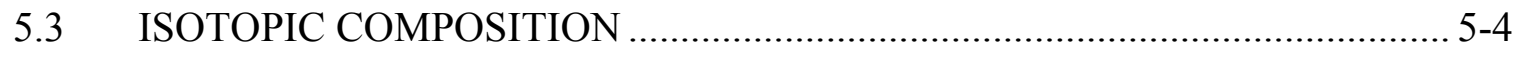

5.4 HEAT GENERATION RATE ................................................................ 5-18

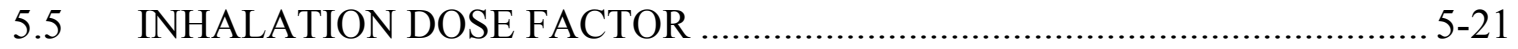

5.6 URANIUM CONCENTRATION …....................................................... 5-22

5.7 PLUTONIUM WEIGHT PERCENT .......................................................... 5-24

5.8 ALTERNATIVES TO THE BASE CASE ................................................. 5-26

5.8.1 Floor Sludge Volume .............................................................. 5-26

5.8.2 Floor Sludge Isotopic Composition ................................................ 5-27

5.8.3 Canister Sludge Depth .......................................................... 5-30

5.8.4 Canister Sludge Isotopic Composition............................................... 5-33

5.8.5 Reduced Variability Factors ........................................................ 5-37

5.8.6 One Retrieval Sequence ........................................................... 5-38 


\section{SNF-10293, REV 0}

5.8.7 Tally All Containers.

6.0 SOFTWARE VERIFICATION AND VALIDATION ........................................ 6-1

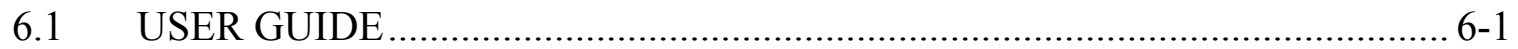

6.2 METHOD OF CALCULATION ............................................................... 6-19

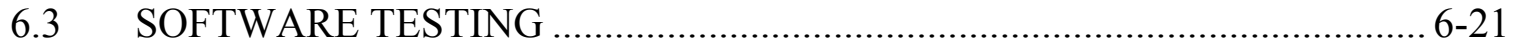

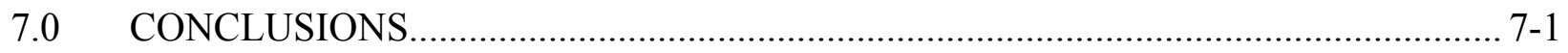

8.0 ACKNOWLEDGMENTS ........................................................................... 8

9.0 REFERENCES …...................................................................................... 9-1

\section{APPENDICES}

A K EAST BASIN FLOOR SLUDGE DEPTH ...................................................... A-i

B EAST BASIN KEY LOCATIONS FROM 1994 ................................................ B-i

C SUMMARY OF DAMAGE CONDITION CATEGORIES ..................................... C-i

D CANISTER SLUDGE DEPTH MEASUREMENTS ........................................... D-i

E COMPARISON OF THE CANISTER SLUDGE SAMPLES WITH RADNUC2A

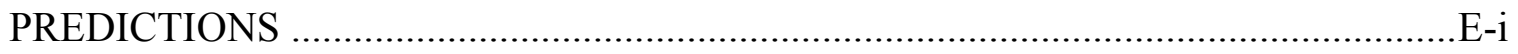

F LISTING OF THE DATA FILES, PDF-10.DAT AND PDF-10.HDR .........................

G LISTING OF THE PROGRAM, PDF-10.PAS ................................................ G-i

H PEER REVIEW AND APPROVAL ................................................................ H-i 


\section{SNF-10293, REV 0}

\section{FIGURES}

Figure 1-1. General Sequence of the Stochastic Calculations. ............................................... 1-2

Figure 2-1. Plot of the Sum of Two Uniform Distributions. ...........................................2-3

Figure 3-1. ${ }^{90}$ Sr Data and Imputed Values from Uranium Measurements in Floor Sludge......... 3-7

Figure 3-2. U.icp Data and Imputation from U.las Measurements in Floor Sludge...................3-8

Figure 3-3. ${ }^{134}$ Cs Data and Imputed Values from ${ }^{137}$ Cs Measurements in Floor Sludge............ 3-9

Figure 3-4. ${ }^{154} \mathrm{Eu}$ Data and Imputed Values from ${ }^{241}$ Am Measurements in Floor Sludge........... 3-9

Figure 3-5. ${ }^{155} \mathrm{Eu}$ Data and Imputed Values from ${ }^{241} \mathrm{Am}$ Measurements in Floor Sludge......... 3-10

Figure 3-6. ${ }^{237} \mathrm{~Np}$ Data and Imputed Values from ${ }^{239+240} \mathrm{Pu}$ Measurements in Floor

Sludge. 3-10

Figure 3-7. ${ }^{238} \mathrm{Pu}$ Data and Imputed Values from ${ }^{239+240} \mathrm{Pu}$ Measurements in Floor Sludge..... 3-11

Figure 3-8. ${ }^{99} \mathrm{Tc}$ Imputations from ${ }^{239+240} \mathrm{Pu}$ Measured Values in Floor Sludge. .................... 3-13

Figure 3-9. ${ }^{152} \mathrm{Eu}$ Imputations from ${ }^{154} \mathrm{Eu}$ Data and Imputed Values in Floor Sludge. ............. 3-14

Figure 3-10. ${ }^{241} \mathrm{Pu}$ Imputations from ${ }^{238} \mathrm{Pu}$ Data and Imputed Values in Floor Sludge............ 3-14

Figure 3-11. Cumulative Probability Distribution Function for Floor Sludge Density............ 3-21

Figure 3-13. Cumulative Probability Distribution for Canisters with Good or Fair Fuel.........3-35

Figure 3-14. ${ }^{60} \mathrm{Co}$ Data and Imputations from ${ }^{154} \mathrm{Eu}$ Data for Canister Sludge. ...................... 3-38

Figure 3-15. ${ }^{90} \mathrm{Sr}$ Data and Imputations from Uranium Data for Canister Sludge...................3-38

Figure 3-16. ${ }^{99} \mathrm{Tc}$ Data and Imputations from Uranium Data for Canister Sludge................. 3-39

Figure 3-17. ${ }^{134} \mathrm{Cs}$ Data and Imputations from ${ }^{137} \mathrm{Cs}$ Data for Canister Sludge. .................... 3-39

Figure 3-18. ${ }^{155} \mathrm{Eu}$ Data and Imputations from ${ }^{154} \mathrm{Eu}$ Data for Canister Sludge.................... 3-40

Figure 3-19. ${ }^{237} \mathrm{~Np}$ Data and Imputations from Uranium Data for Canister Sludge................ 3-40

Figure 3-20. ${ }^{238} \mathrm{Pu}$ Data and Imputations from ${ }^{239+240} \mathrm{Pu}$ Data for Canister Sludge................. 3-41

Figure 3-21. ${ }^{241} \mathrm{Am}$ Data and Imputations from ${ }^{239+240} \mathrm{Pu}$ Data for Canister Sludge. ............... 3-41

Figure 3-22. ${ }^{152} \mathrm{Eu}$ Imputations from ${ }^{154} \mathrm{Eu}$ Data for Canister Sludge.................................... 3-42 
SNF-10293, REV 0

Figure 3-23. ${ }^{241} \mathrm{Pu}$ Imputations from ${ }^{238} \mathrm{Pu}$ Data and Imputations for Canister Sludge. ........... 3-43

Figure 3-24. Uranium Concentration Versus Canister Sludge Density............................... 3-63

Figure 3-25. Specific Activity of Pu-239 and Pu-240 Mixtures in Canister Sludge. .............. 3-69

Figure 4-1. Stochastic Calculation: Floor Sludge Amounts per Key..................................... 4-2

Figure 4-2. Stochastic Calculation: Canister Sludge Volume per Key................................. 4-3

Figure 4-3. Stochastic Calculation: Canister Sludge Amounts per Key................................. 4-4

Figure 4-4. Stochastic Calculation: Total Sludge Amount per Key. .................................... 4-5

Figure 4-5. Stochastic Calculation: Generating a Retrieval Sequence. ................................. 4-6

Figure 4-6. Stochastic Calculation: Filling Sludge Containers........................................... 4-7

Figure 4-7. Stochastic Calculation: Tallying Container Concentrations. .............................. 4-8

Figure 4-8. Stochastic Result for Floor Sludge Density.............................................. 4-10

Figure 4-9. Stochastic Result for Canister Sludge Depth (Good Fuel)................................. 4-14

Figure 4-10. Stochastic Result for Canister Sludge Depth (Fair Fuel)............................... 4-15

Figure 4-11. Stochastic Result for Canister Sludge Depth (Poor and Bad Fuel)....................4-16

Figure 4-12. Stochastic Result for Canister Sludge Density........................................... 4-16

Figure 4-13. Stochastic Result for Canister Sludge Uranium Concentration. ......................... 4-17

Figure 4-14. Stochastic Result for Sludge Total Volume in All Keys.................................. 4-19

Figure 5-1. Stochastic Result for the Canister-to-Total Volume Ratio. ................................ 5-2

Figure 5-2. Stochastic Result for the Sludge Density.................................................. 5-3

Figure 5-3. Stochastic Result for the Co-60 Concentration............................................. 5-5

Figure 5-4. Stochastic Result for the Sr-90 Concentration. ................................................. 5-6

Figure 5-5. Stochastic Result for the Tc-99 Concentration. ........................................... 5-7

Figure 5-6. Stochastic Result for the Cs-134 Concentration. .................................................. 5-8

Figure 5-7. Stochastic Result for the Cs-137 Concentration. ............................................... 5-9

Figure 5-8. Stochastic Result for the Eu-152 Concentration. .......................................... 5-10 
SNF-10293, REV 0

Figure 5-9. Stochastic Result for the Eu-154 Concentration. ......................................... 5-11

Figure 5-10. Stochastic Result for the Eu-155 Concentration. ......................................... 5-12

Figure 5-11. Stochastic Result for the Np-237 Concentration........................................5-13

Figure 5-12. Stochastic Result for the Pu-238 Concentration. ............................................ 5-14

Figure 5-13. Stochastic Result for the $\mathrm{Pu}-239 / 240$ Concentration. ...................................... 5-15

Figure 5-14. Stochastic Result for the Pu-241 Concentration. ............................................ 5-16

Figure 5-15. Stochastic Result for the Am-241 Concentration......................................... 5-17

Figure 5-16. Stochastic Result for Heat Generation per Unit Volume.................................. 5-18

Figure 5-17. Stochastic Result for Heat Generation per Unit Mass of Sludge...................... 5-19

Figure 5-18. Stochastic Result for Heat Generation per Unit Mass of Uranium................... 5-20

Figure 5-19. Stochastic Result for Inhalation Dose per Unit Volume................................. 5-21

Figure 5-20. Stochastic Result for Inhalation Dose per Unit Mass of Sludge...................... 5-22

Figure 5-21. Stochastic Result for Total Uranium Concentration. .................................... 5-23

Figure 5-22. Stochastic Result for Uranium Metal Concentration. .................................... 5-24

Figure 5-23. Stochastic Result for Plutonium Weight Percent........................................... 5-25

Figure 5-24. Stochastic Result for Total Sludge Volume with Independent Key Volumes.....5-26

Figure 5-25. Stochastic Result Using Reduced Variability for Floor Sludge Density............ 5-27

Figure 5-26. Stochastic Result for Lognormal Floor Sludge Uranium Concentration............ 5-28

Figure 5-27. Stochastic Result for Lognormal Floor Sludge Density. ................................ 5-29

Figure 5-28. Stochastic Result for Continuous Canister Sludge Depth (Good Fuel)..............5-31

Figure 5-29. Stochastic Result for Continuous Canister Sludge Depth (Fair Fuel)................. 5-32

Figure 5-30. Stochastic Result for Continuous Canister Sludge Depth (Poor and Bad

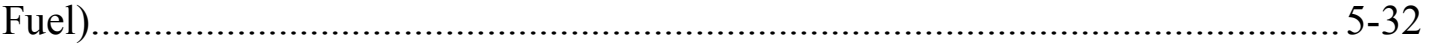

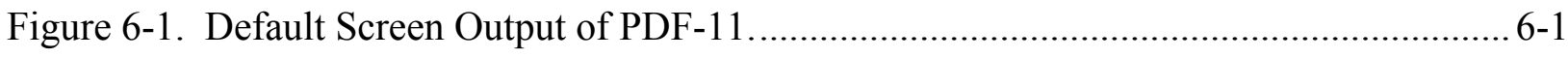

Figure 6-2. Cumulative Total Sludge Volume per Key .................................................. 6-2

Figure 6-3. First Block of Output from PDF-11 (Input Data). ........................................... 6-4 
SNF-10293, REV 0

Figure 6-4. Second Block of Output from PDF-11 (Input Data)................................................. 6-5

Figure 6-5. Third Block of Output from PDF-11 (Input Data).................................................. 6-6

Figure 6-6. Fourth Block of Output from PDF-11 (Input Data) ................................................. 6-7

Figure 6-7. Fifth Block of Output from PDF-11 (Input Data).................................................... 6-9

Figure 6-8. Sixth Block of Output from PDF-11 (Input Data)............................................... 6-10

Figure 6-9. Number of Containers Tallied During a PDF-11 Simulation. ............................... 6-11

Figure 6-10. Two Dimension Tally of Key Positions on the Retrieval List............................ 6-12

Figure 6-11. Two Dimension Tally of Canister Sludge Total Uranium Versus Density. ........ 6-13

Figure 6-12. Tally for the Pu-239/240 Specific Activity...................................................... 6-14

Figure 6-13. Canister Sludge Depth Table Output from PDF-11_......................................... 6-15

Figure 6-14. Summary Table for Various Input Distributions in the Output from PDF-11 ..... 6-16

Figure 6-15. Example Container Distribution Table in the Output from PDF-11.................... 6-18

Figure 6-16. Summary of Container Inventories in the Output from PDF-11 ......................... 6-19

Figure 6-17. Statistical Variation in the Key Position Index................................................. 6-22

Figure 6-18. Statistical Variation in the Total Floor Sludge Counts. ....................................... 6-22 
SNF-10293, REV 0

\section{TABLES}

Table 1-1. Stochastic Results for $0.5 \mathrm{~m}^{3}$ Sludge Containers. ................................................. 1-5

Table 1-2. Stochastic Results for $2 \mathrm{~m}^{3}$ Sludge Containers. ....................................................... 1-6

Table 1-3. Stochastic Results for $12 \mathrm{~m}^{3}$ Sludge Containers. ................................................... 1-7

Table 2-1. Example of the Sum of Two Uniform Distributions..................................................2-2

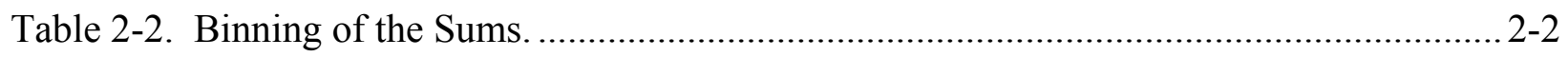

Table 3-1. Average Floor Sludge Volume by Key ................................................................. 3-2

Table 3-2. Floor Sample Data Correlations: Slope and $\mathrm{R}^{2}$.................................................. 3-6

Table 3-3. Correlations Using Radnuc2A Sludge Estimates: Slope and $\mathrm{R}^{2}$........................... 3-12

Table 3-4. Floor Sample Data and Imputed Values............................................................... 3-15

Table 3-5. Floor Sample Data, Cs-134 to Eu-155 ……………………………………........ 3-16

Table 3-6. Floor Sample Data, Np-237 to Am-241 ………………………………............ 3-17

Table 3-7. Variability Factor for the Floor Sample Data...................................................... 3-20

Table 3-8. Summary of Canister Sludge Depths -- Bad Fuel ................................................. 3-24

Table 3-9. Summary of Canister Sludge Depths -- Fair Fuel ................................................. 3-26

Table 3-10. Summary of Canister Sludge Depths -- Good Fuel.............................................. 3-28

Table 3-11. Calculation of Total Canister Sludge Volumes (Liters)......................................... 3-29

Table 3-12. Number of Canisters of Each Type by Key......................................................... 3-29

Table 3-13. Average Canister Sludge Volume by Key.......................................................... 3-31

Table 3-14. Variability Factors for Canister Sludge Depth..................................................... 3-32

Table 3-15. Canister Sample Data Correlations: Slope and $\mathrm{R}^{2}$............................................3-37

Table 3-16. Canister Sample Data with Imputed Values.......................................................... 3-43

Table 3-17. Nuclides in Canister Sludge Reported as Less-thans............................................. 3-45

Table 3-18. Isotope Concentration in Canister Sludge from Radnuc2A: Co-60 to Cs-134 ..... 3-45 
SNF-10293, REV 0

Table 3-19. Isotope Concentration in Canister Sludge from Radnuc2A: Cs-137 to $\mathrm{Np}-237$ $3-48$

Table 3-20. Isotope Concentration in Canister Sludge from Radnuc2A: Pu-238 to Am-241.....

Table 3-21. Nuclide Half Lives and 3-year Decay Factors. $3-54$

Table 3-22. Canister Sludge Volume and Uranium Mass by Key..... $3-55$

Table 3-23. K East Basin Canister Sludge Concentrations. $3-57$

Table 3-24. Summary of KE Basin Canisters Sampled for Sludge in April 1996 that Contained Fuel Elements. $3-60$

Table 3-25. Summary of KE Basin Canisters Sampled for Sludge in 1999 Consolidated Sampling Campaign.

Table 3-26. Summary Table of Estimated Fuel Damage by Category in Barrels Sampled for Sludge KE Basin.

Table 3-27. Variability Factor for the Canister Sample Data. $3-64$

Table 3-28. Heat Generation Rate and Inhalation Dose Factors. 3-66

Table 3-29. Range of Uranium Metal Mass Fractions. $3-67$

Table 3-30. Specific Activity of the Plutonium Isotopes. $3-68$

Table 3-31. Continuous Probability Distribution for Pu-239/240 Specific Activity. 3-69

Table 4-1. Overview of Random Events to Empty K East Basin. 4-9

Table 4-2. Floor Sludge Comparisons -- Samples vs Stochastic. 4-11

Table 4-3. Canister Sludge Comparisons -- Hand Calculated Sums versus Stochastic. 4-13

Table 4-4. Container Sludge Data Mean Values. 4-18

Table 4-5. Stochastic Results for the Total Sludge Volume $\left(\mathrm{m}^{3}\right)$. 4-20

Table 5-1. Stochastic Results for the Canister-to-Total Sludge Volume Ratio. $5-2$

Table 5-2. Stochastic Results for the Sludge Density (kg/L) 5-4

Table 5-3. Stochastic Results for the Co-60 Concentration $\left(\mathrm{Ci} / \mathrm{m}^{3}\right)$. 5-5

Table 5-4. Stochastic Results for the Sr-90 Concentration $\left(\mathrm{Ci} / \mathrm{m}^{3}\right)$. $5-6$

Table 5-5. Stochastic Results for the Tc-99 Concentration $\left(\mathrm{Ci} / \mathrm{m}^{3}\right)$. 5-7 
SNF-10293, REV 0

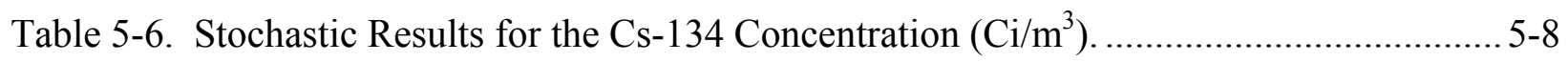

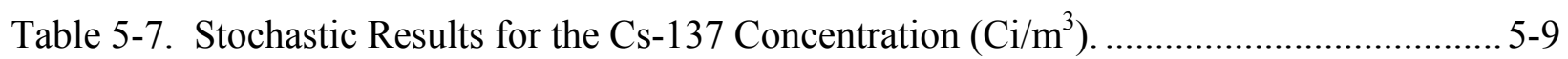

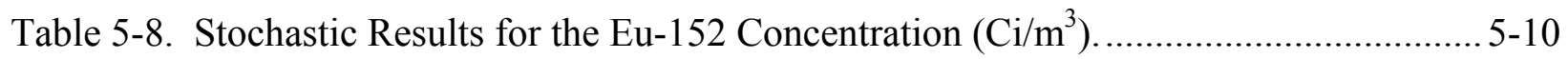

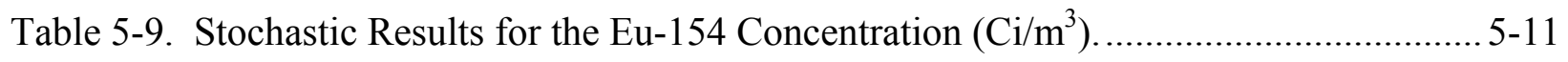

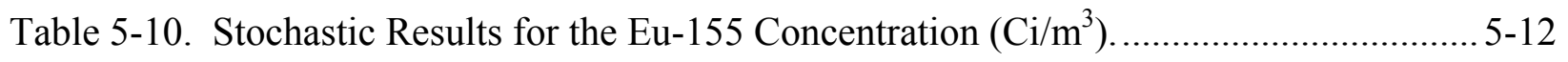

Table 5-11. Stochastic Results for the Np-237 Concentration $\left(\mathrm{Ci} / \mathrm{m}^{3}\right)$. ............................. 5-13

Table 5-12. Stochastic Results for the $\mathrm{Pu}-238$ Concentration $\left(\mathrm{Ci} / \mathrm{m}^{3}\right)$............................... 5-14

Table 5-13. Stochastic Results for the $\mathrm{Pu}-239 / 240$ Concentration $\left(\mathrm{Ci} / \mathrm{m}^{3}\right)$......................... 5-15

Table 5-14. Stochastic Results for the $\mathrm{Pu}-241$ Concentration $\left(\mathrm{Ci} / \mathrm{m}^{3}\right)$................................ 5-16

Table 5-15. Stochastic Results for the Am-241 Concentration $\left(\mathrm{Ci} / \mathrm{m}^{3}\right)$............................. 5-17

Table 5-16. Stochastic Results for Heat Generation Rate per Unit Volume (W/m³ $)$............5-18

Table 5-17. Stochastic Results for Heat Generation per Unit Mass of Sludge (W/MT)......... 5-19

Table 5-18. Stochastic Results for Heat Generation per Unit Mass of Uranium (W/MTU)

Table 5-19. Stochastic Results for Inhalation Dose per Unit Volume (rem/ml). .................. 5-21

Table 5-20. Stochastic Results for Inhalation Dose per Unit Mass of Sludge (rem/g)........... 5-22

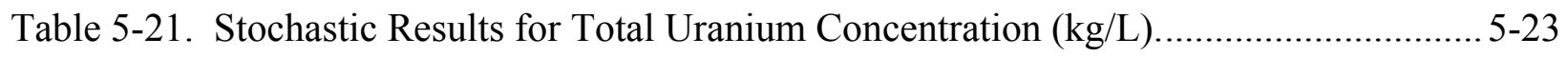

Table 5-22. Stochastic Results for Uranium Metal Concentration....................................... 5-24

Table 5-23. Stochastic Results for Plutonium Weight Fraction. ......................................... 5-25

Table 5-24. Percent Differences: Doubled Imputed Values Versus Base Case. ....................5-30

Table 5-25. Comparison Ratios: Canister Sludge Data Versus HNF-8760........................... 5-35

Table 5-26. One Retrieval Sequence Comparisons with Base Case..................................... 5-38 
SNF-10293, REV 0

\section{TERMS}

CDF

DOE

EPA

FFS

$\mathrm{FH}$

KE

MT

MTU

PDF

PNNL

SNF cumulative probability distribution function

U.S. Department of Energy

U.S. Environmental Protection Agency

Fluor Federal Services

Fluor Hanford, Inc.

K East

metric ton

metric ton of uranium

probability density function

Pacific Northwest National Laboratory

Spent Nuclear Fuel 
SNF-10293, REV 0

\section{DISTRIBUTION}

\section{$\underline{\text { Onsite }}$}

U.S. Department of Energy

$\underline{\text { Richland Operations Office }}$

DOE Public Reading Room

$\mathrm{H} 2-53$

1

$\underline{\text { Pacific Northwest National Laboratory }}$

Hanford Technical Library

P8-55

2

Lockheed Martin Services, Inc.

Central Files

B1-07

Document Processing Center

A3-94

Distr.-1 
SNF-10293, REV 0

This page intentionally left blank.

Distr.-2 
SNF-10293, REV 0

Distr.-3 
SNF-10293, REV 0

\subsection{INTRODUCTION}

The goal of this report is to estimate heat output, potential health risks, and bounding concentrations of uranium, plutonium, and a series of radionuclides in sludge containers that will be filled in $\mathrm{K}$ East Basin. The present project uses a stochastic modeling technique in which values for the volumes of floor and canister sludge, as well as the concentrations of the materials of interest are allowed to vary. The variation is constrained by the available data. The sequence in which the fuel keys are retrieved is allowed to vary. The computed results are distributions showing the probability of finding containers with particular values for the items that are tracked. For this analysis, the tracked items for a given sludge container (i.e. volume) are the sludge mass, the total uranium mass, uranium metal mass, plutonium mass, the activity of 13 isotopes, the heat generation rate, and the inhalation dose. The average values of the output distributions are readily verified with hand calculations. The bounding values are chosen from the cumulative probability curve to be exceeded in less than $5 \%$ of the containers.

The first step is to define the basic calculation of the amounts of each item of interest in a filled sludge container using available sludge data. Normally, the average values are used for this calculation. The next step in the stochastic analysis replaces each of the data averages with a function that represents the data. This function has the same mean as the data. In addition, it has a similar shape and standard deviation. The function is referred to as probability density or probability distribution function (PDF). A probability density function is defined as a real function over some range whose integral over any sub-range is the probability that a random variable has values in the sub-range. Section 3.0 presents both the data and the PDFs used to represent the data. In a stochastic calculation, values are randomly selected from the PDFs, plugged into the same formulas that were used for the basic calculation, and the results are tallied in a histogram. The final histograms can be presented as PDFs, where the bin width is the minimum sub-range, or as cumulative functions, which are the sums of the histogram bins from the lowest value tallied to the value of interest.

The stochastic analysis combines information about floor and canister sludge to generate a spectrum of sludge compositions in the larger sludge volumes associated with sludge retrieval and storage. A simplified flow diagram for the stochastic calculations is shown in Figure 1-1. The four boxes on the left side are calculated for each key using the PDFs derived from the input data. In this table, the word "composition" refers to the 20 items listed in the large box in the middle. Note that at this point these are total amounts associated with a key rather than concentrations. Once the sludge composition has been calculated for all 52 keys, the keys are retrieved in a random order, sludge containers are filled, and the resulting concentrations in the containers is calculated and tallied.

Several assumptions have been made to carry out these calculations. Many of these have been tested by repeating the stochastic analysis using slightly different assumptions to quantify their impact. For example, it is assumed that the canisters are retrieved by key and that the keys are taken in any order. An alternate calculation uses specific key retrieval sequences to quantify the effect. Other assumptions define the calculation in a way that could not be tested. For example, it is assumed that the floor sludge in the immediate vicinity of the canister is retrieved along with 
the canister sludge. The retrieval process is taken to be $100 \%$ efficient, in that all the estimated canister sludge as well as all of the estimated floor sludge is retrieved.

Note that the impacts of retrieving pit sludge or floor sludge outside the cubicles with fuel has not been analyzed.

Figure 1-1. General Sequence of the Stochastic Calculations.

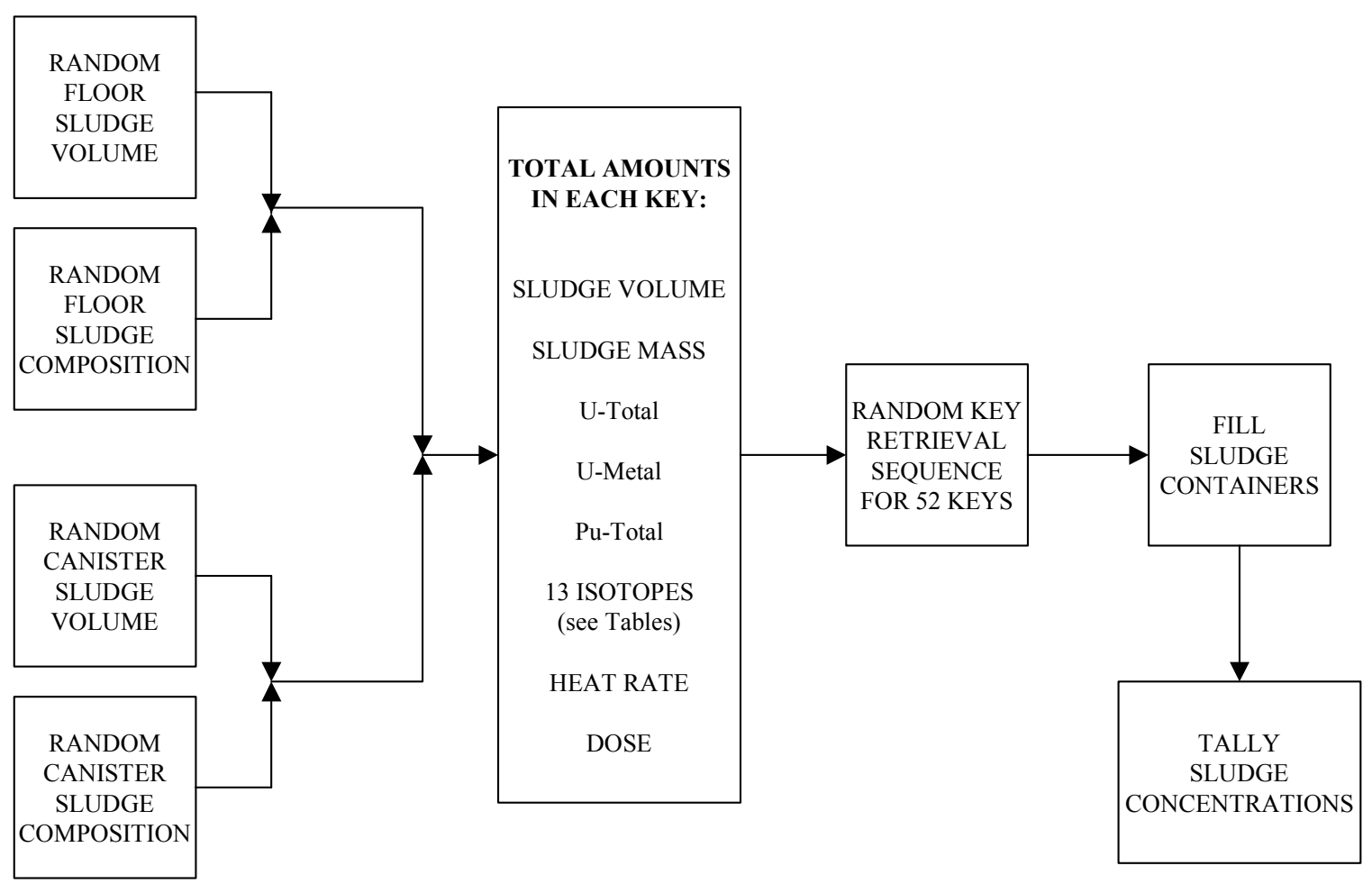

The selection of the data used in the stochastic analysis involved several assumptions. These are presented in greater detail in Section 3.0. An underlying assumption is that the sludge measurements taken during the years 1994 through 2000 provide a consistent source of information to evaluate sludge loading in the larger sludge volumes of interest during sludge retrieval and storage. Currently any of the 3,668 fueled canisters in K East Basin can be moved for operational reasons to any of the over 5,000 storage positions in the basin. These movements are tracked for accountability only. No records are maintained to track canisters by fuel condition or canister type. Hence, while the current positions of specific fuel canisters are known, the corresponding tracking of fuel condition in the K East Basin was not maintained. Note also that the movement of screen bottomed canisters causes some of the canister sludge to fall to the floor and be commingled with the floor sludge. This decreases the volume of canister sludge and increases the mean value for floor sludge concentration from the 1994 through 2000 sampling activity. However, when the floor and canister sludge are both retrieved and added to the same container, the effect of these changes is diminished. Therefore, the 1994/2000 data is assumed adequate for estimates of sludge inventories during sludge removal. 
The data used in the stochastic analysis is summarized below.

1. The 1994 locations of the fuel in each key as found in a spreadsheet are used. The key locations are from private communication with Karen Morris, K Basins Operations, 1995 "K East Basin Storage Map", MAC Version 3.1, Rev. 163, Updated 1/11/94, Report 5/02/94, 124 pages.

2. Floor sludge depth measurements taken in 1994 are used together with the interpolations to estimate floor sludge depths at non-measured locations in $\mathrm{K}$ East. This data is presented in WHC-SD-WM-ANAL-037 (Meling 1994). It is used in the stochastic analysis to calculate the volume of floor sludge associated with each key.

3. Canister sludge depth measurements taken from 1995 to 2000 are used to estimate canister sludge volumes for each key. This data is presented in WHC-SD-SNF-TI-034 (Pitner 1996b).

4. Floor and canister sludge concentration measurements for sludge density, uranium concentration, and various radionuclide concentrations collected from 1994 to 2000 and presented in SNF-7765 Appendix D (FH 2001) are used.

5. Calculated values for various radionuclide concentrations in the fuel and sludge (HNF-8760, Schwinkendorf 2002).

Another assumption is that the amount of canister and floor sludge has not increased since the depth measurements were taken. This assumption has been more fully discussed in HNF-8118 (Baker 2001), Section 2.0. It is concluded that because of the control of basin water conditions and lack of significant environmental additions such as airborne dust, the 1994 through 2000 fuel condition estimates and sludge volume estimates are still valid.

An additional assumption is that the canister barrels selected for the measurements in 1994 are representative of all canister barrels in K East Basin. If additional measurements had been taken, the mean values would not have changed appreciably, nor would the likelihood of particular depth measurements.

PDFs created to represent this data preserve the data mean and may increase the standard deviation a small amount. This is done through the use of uniform distributions with a mean value of 1.0. Random values are selected from these distributions and multiplied by the data value. The mean is preserved and the standard deviation increases a small amount.

The size of the smallest sludge volumes used in the stochastic analysis is the volume of sludge associated with individual keys in K East Basin. A key refers to the group of fuel assemblies discharged from a Hanford Site reactor on a particular day. Although the sludge samples typically have much smaller volumes than are found in the keys, the sample data is applied to the entire volume of sludge associated with a key.

The stochastic results after emptying K East Basin 10 million times are shown in Tables 1-1, 1-2, and 1-3. Three sludge container volumes are used in the stochastic analysis even though there is 
just one storage container size. The $0.5 \mathrm{~m}^{3}$ volume shown in Table 1-1 corresponds to the maximum that may be added to a sludge storage container in 24 hours. The $2.0 \mathrm{~m}^{3}$ volume shown in Table 1-2 is the maximum that will be placed in a storage container. The $12 \mathrm{~m}^{3}$ volume shown in Table 1-3 corresponds to 6 storage containers placed at T-Plant. In this analysis, "container" refers to these three sludge volumes rather than an engineered device for storing sludge.

The stochastic model of container filling adds sludge from randomly ordered keys until the desired volume $\left(0.5,2.0\right.$, or $\left.12 \mathrm{~m}^{3}\right)$ is obtained. Any excess is added to the next container until all the keys are used up. Any partial container volume left over after all the keys are used is discarded to avoid introducing a bias to the full container results. An exception is made for containers that are more than $90 \%$ full. At this level, the container is essentially full. The $90 \%$ criteria is an assumption that was tested and found to have little effect on the final results.

For the largest volume $\left(12 \mathrm{~m}^{3}\right)$, the randomly generated volume of all the keys may not fill the container. However, the smallest total sludge volume $\left(11.3 \mathrm{~m}^{3}\right)$ is greater than $90 \%$ of the container volume, so in all cases there will be at least one container generated.

When a container is filled during the stochastic analysis, several quantities of interest are tallied. The mean, standard deviation, $95^{\text {th }}$ and $99^{\text {th }}$ percentile values for each distribution are listed in Tables 1-1, 1-2, and 1-3. These results generally show the total amounts. The concentrations may be calculated by dividing the amount shown by the container volume. Dividing one of the rows by another row should not be done. As an example, consider dividing the heat generation rate by the uranium mass for comparison with the row labeled "Heat Generation Rate, W/MTU" on the tables. The mean is almost the same, while the other values are quite different from the values shown in the table. The reason for the differences is that both the numerator (heat generation rate) and the denominator (mass of uranium) are distributions. Dividing one distribution by another creates a third distribution, the characteristics of which are not obvious from the table. For this reason, the concentrations that involve division by another distribution have been tallied separately. The special tallies are (1) canister-to-total volume ratio, (2) plutonium weight percent, (3) heat generation rate per metric ton of sludge, (4) heat generation rate per metric ton of uranium, and (5) inhalation dose per unit mass of sludge.

The five special tallies were carried out based on potential future uses for these results. For example, the plutonium weight percent (i.e., the fraction of the sludge mass that is plutonium) is useful for determining various quantities for accountability purposes. 
SNF-10293, REV 0

Table 1-1. Stochastic Results for $0.5 \mathrm{~m}^{3}$ Sludge Containers.

\begin{tabular}{|c|c|c|c|c|}
\hline Quantity & Mean & $9^{\text {th }}$ Percentile & $99^{\text {th }}$ Percentile & $\begin{array}{l}\text { Standard } \\
\text { Deviation }\end{array}$ \\
\hline Canister-to-Total Volume Ratio & $19.6 \%$ & $37.1 \%$ & $45.2 \%$ & $10.7 \%$ \\
\hline Sludge Density, kg/L & 1.39 & 1.78 & 2.28 & 0.23 \\
\hline kg Total Uranium & 112 & 290 & 432 & 90 \\
\hline kg Uranium Metal & 8.39 & 16.0 & 21.3 & 4.11 \\
\hline Plutonium Weight Percent & $0.0366 \%$ & $0.0823 \%$ & $0.1097 \%$ & $0.0240 \%$ \\
\hline Curies Co-60 & $4.68 \mathrm{E}-01$ & $1.09 \mathrm{E}+00$ & $1.57 \mathrm{E}+00$ & $3.06 \mathrm{E}-01$ \\
\hline Curies Sr-90 & $4.95 \mathrm{E}+02$ & $1.31 \mathrm{E}+03$ & $1.86 \mathrm{E}+03$ & $4.13 \mathrm{E}+02$ \\
\hline Curies Tc-99 & $1.56 \mathrm{E}-01$ & 3.93E-01 & $5.63 \mathrm{E}-01$ & $1.22 \mathrm{E}-01$ \\
\hline Curies Cs-134 & $6.08 \mathrm{E}-01$ & $1.94 \mathrm{E}+00$ & $5.48 \mathrm{E}+00$ & $1.16 \mathrm{E}+00$ \\
\hline Curies Cs-137 & $6.67 \mathrm{E}+02$ & $1.72 \mathrm{E}+03$ & $2.48 \mathrm{E}+03$ & $5.45 \mathrm{E}+02$ \\
\hline Curies Eu-152 & 4.70E-02 & $1.28 \mathrm{E}-01$ & $1.92 \mathrm{E}-01$ & 4.19E-02 \\
\hline Curies Eu-154 & $5.45 \mathrm{E}+00$ & $1.51 \mathrm{E}+01$ & $2.34 \mathrm{E}+01$ & $5.06 \mathrm{E}+00$ \\
\hline Curies Eu-155 & $1.08 \mathrm{E}+00$ & $2.85 \mathrm{E}+00$ & $4.63 \mathrm{E}+00$ & $9.50 \mathrm{E}-01$ \\
\hline Curies Np-237 & $3.22 \mathrm{E}-03$ & $8.19 \mathrm{E}-03$ & $1.18 \mathrm{E}-02$ & $2.55 \mathrm{E}-03$ \\
\hline Curies $\mathrm{Pu}-238$ & $6.44 \mathrm{E}+00$ & $1.76 \mathrm{E}+01$ & $2.61 \mathrm{E}+01$ & $5.70 \mathrm{E}+00$ \\
\hline Curies $\mathrm{Pu}-239 \& \mathrm{Pu}-240$ & $2.23 \mathrm{E}+01$ & $5.62 \mathrm{E}+01$ & $8.04 \mathrm{E}+01$ & $1.74 \mathrm{E}+01$ \\
\hline Curies Pu-241 & $3.57 \mathrm{E}+02$ & $9.71 \mathrm{E}+02$ & $1.46 \mathrm{E}+03$ & $3.16 \mathrm{E}+02$ \\
\hline Curies Am-241 & $2.36 \mathrm{E}+01$ & $6.29 \mathrm{E}+01$ & $9.11 \mathrm{E}+01$ & $2.00 \mathrm{E}+01$ \\
\hline Heat Generation Rate, W & 8.30 & 21.6 & 31.0 & 6.81 \\
\hline Heat Generation Rate, W/MT & 11.3 & 26.5 & 35.9 & 8.0 \\
\hline Heat Generation Rate, W/MTU & 74.1 & 100 & 107 & 22.4 \\
\hline Inhalation Dose Factor, rem/ml & 51,310 & 133,800 & 193,900 & 42,250 \\
\hline Inhalation Dose Factor, rem/g & 34,980 & 83,550 & 113,900 & 25,160 \\
\hline
\end{tabular}


SNF-10293, REV 0

Table 1-2. Stochastic Results for $2 \mathrm{~m}^{3}$ Sludge Containers.

\begin{tabular}{|c|c|c|c|c|}
\hline Quantity & Mean & 95 $^{\text {th }}$ Percentile & 99 ${ }^{\text {th }}$ Percentile & $\begin{array}{l}\text { Standard } \\
\text { Deviation }\end{array}$ \\
\hline Canister-to-Total Volume Ratio & $19.6 \%$ & $31.3 \%$ & $36.4 \%$ & $7.2 \%$ \\
\hline Sludge Density, kg/L & 1.39 & 1.65 & 1.89 & 0.15 \\
\hline kg Total Uranium & 445 & 877 & 1,188 & 234 \\
\hline kg Uranium Metal & 33.5 & 52.0 & 64.1 & 10.6 \\
\hline Plutonium Weight Percent & $0.0377 \%$ & $0.0656 \%$ & $0.0799 \%$ & $0.0157 \%$ \\
\hline Curies Co-60 & $1.87 \mathrm{E}+00$ & $3.35 \mathrm{E}+00$ & $4.48 \mathrm{E}+00$ & 7.89E-01 \\
\hline Curies Sr-90 & $1.97 \mathrm{E}+03$ & $3.88 \mathrm{E}+03$ & $4.98 \mathrm{E}+03$ & $1.03 \mathrm{E}+03$ \\
\hline Curies Tc-99 & $6.20 \mathrm{E}-01$ & $1.18 \mathrm{E}+00$ & $1.50 \mathrm{E}+00$ & $3.03 \mathrm{E}-01$ \\
\hline Curies Cs-134 & $2.43 \mathrm{E}+00$ & $7.00 \mathrm{E}+00$ & $1.36 \mathrm{E}+01$ & $2.63 \mathrm{E}+00$ \\
\hline Curies Cs-137 & $2.66 \mathrm{E}+03$ & $5.15 \mathrm{E}+03$ & $6.59 \mathrm{E}+03$ & $1.36 \mathrm{E}+03$ \\
\hline Curies Eu-152 & $1.87 \mathrm{E}-01$ & $3.79 \mathrm{E}-01$ & $4.96 \mathrm{E}-01$ & $1.03 \mathrm{E}-01$ \\
\hline Curies Eu-154 & $2.17 \mathrm{E}+01$ & $4.46 \mathrm{E}+01$ & $5.95 \mathrm{E}+01$ & $1.23 \mathrm{E}+01$ \\
\hline Curies Eu-155 & $4.31 \mathrm{E}+00$ & $8.79 \mathrm{E}+00$ & $1.30 \mathrm{E}+01$ & $2.43 \mathrm{E}+00$ \\
\hline Curies Np-237 & $1.29 \mathrm{E}-02$ & $2.45 \mathrm{E}-02$ & $3.12 \mathrm{E}-02$ & $6.33 \mathrm{E}-03$ \\
\hline Curies Pu-238 & $2.57 \mathrm{E}+01$ & $5.17 \mathrm{E}+01$ & $6.70 \mathrm{E}+01$ & $1.40 \mathrm{E}+01$ \\
\hline Curies $\mathrm{Pu}-239 \& \mathrm{Pu}-240$ & $8.89 \mathrm{E}+01$ & $1.68 \mathrm{E}+02$ & $2.14 \mathrm{E}+02$ & $4.33 \mathrm{E}+01$ \\
\hline Curies Pu-241 & $1.43 \mathrm{E}+03$ & $2.87 \mathrm{E}+03$ & $3.75 \mathrm{E}+03$ & $7.79 \mathrm{E}+02$ \\
\hline Curies Am-241 & $9.42 \mathrm{E}+01$ & $1.86 \mathrm{E}+02$ & $2.38 \mathrm{E}+02$ & $4.96 \mathrm{E}+01$ \\
\hline Heat Generation Rate, W & 33.1 & 64.4 & 82.3 & 17.0 \\
\hline Heat Generation Rate, W/MT & 11.6 & 20.9 & 25.8 & 5.2 \\
\hline Heat Generation Rate, W/MTU & 75.4 & 94.4 & 99.1 & 14.8 \\
\hline Inhalation Dose Factor, rem $/ \mathrm{ml}$ & 51,170 & 99,480 & 127,100 & 26,180 \\
\hline Inhalation Dose Factor, rem/g & 36,100 & 65,530 & 80,730 & 16,400 \\
\hline
\end{tabular}


Table 1-3. Stochastic Results for $12 \mathrm{~m}^{3}$ Sludge Containers.

\begin{tabular}{|c|c|c|c|c|}
\hline Quantity & Mean & $9^{\text {th }}$ Percentile & $99^{\text {th }}$ Percentile & $\begin{array}{l}\text { Standard } \\
\text { Deviation }\end{array}$ \\
\hline Canister-to-Total Volume Ratio & $19.7 \%$ & $25.3 \%$ & $27.7 \%$ & $3.2 \%$ \\
\hline Sludge Density, kg/L & 1.39 & 1.50 & 1.59 & 0.06 \\
\hline kg Total Uranium & 2,683 & 3,798 & 4,445 & 616 \\
\hline kg Uranium Metal & 202 & 250 & 277 & 28 \\
\hline Plutonium Weight Percent & $0.0386 \%$ & $0.0496 \%$ & $0.0547 \%$ & $0.0064 \%$ \\
\hline Curies Co-60 & $1.12 \mathrm{E}+01$ & $1.50 \mathrm{E}+01$ & $1.71 \mathrm{E}+01$ & $2.06 \mathrm{E}+00$ \\
\hline Curies Sr-90 & $1.19 \mathrm{E}+04$ & $1.64 \mathrm{E}+04$ & $1.86 \mathrm{E}+04$ & $2.55 \mathrm{E}+03$ \\
\hline Curies Tc-99 & $3.74 \mathrm{E}+00$ & $5.03 \mathrm{E}+00$ & $5.66 \mathrm{E}+00$ & $7.38 \mathrm{E}-01$ \\
\hline Curies Cs-134 & $1.46 \mathrm{E}+01$ & $2.56 \mathrm{E}+01$ & $3.41 \mathrm{E}+01$ & $5.82 \mathrm{E}+00$ \\
\hline Curies Cs-137 & $1.61 \mathrm{E}+04$ & $2.19 \mathrm{E}+04$ & $2.47 \mathrm{E}+04$ & $3.33 \mathrm{E}+03$ \\
\hline Curies Eu-152 & $1.13 \mathrm{E}+00$ & $1.57 \mathrm{E}+00$ & $1.78 \mathrm{E}+00$ & $2.48 \mathrm{E}-01$ \\
\hline Curies Eu-154 & $1.31 \mathrm{E}+02$ & $1.83 \mathrm{E}+02$ & $2.09 \mathrm{E}+02$ & $2.93 \mathrm{E}+01$ \\
\hline Curies Eu-155 & $2.59 \mathrm{E}+01$ & $3.77 \mathrm{E}+01$ & $4.48 \mathrm{E}+01$ & $6.32 \mathrm{E}+00$ \\
\hline Curies Np-237 & 7.76E-02 & $1.05 \mathrm{E}-01$ & $1.18 \mathrm{E}-01$ & $1.54 \mathrm{E}-02$ \\
\hline Curies Pu-238 & $1.55 \mathrm{E}+02$ & $2.13 \mathrm{E}+02$ & $2.42 \mathrm{E}+02$ & $3.33 \mathrm{E}+01$ \\
\hline Curies $\mathrm{Pu}-239 \& \mathrm{Pu}-240$ & $5.36 \mathrm{E}+02$ & $7.21 \mathrm{E}+02$ & $8.10 \mathrm{E}+02$ & $1.06 \mathrm{E}+02$ \\
\hline Curies Pu-241 & $8.61 \mathrm{E}+03$ & $1.19 \mathrm{E}+04$ & $1.35 \mathrm{E}+04$ & $1.86 \mathrm{E}+03$ \\
\hline Curies Am-241 & $5.68 \mathrm{E}+02$ & $7.76 \mathrm{E}+02$ & $8.76 \mathrm{E}+02$ & $1.18 \mathrm{E}+02$ \\
\hline Heat Generation Rate, W & 200 & 273 & 308 & 42 \\
\hline Heat Generation Rate, W/MT & 11.9 & 15.6 & 17.3 & 2.1 \\
\hline Heat Generation Rate, W/MTU & 75.1 & 85.1 & 87.9 & 7.3 \\
\hline Inhalation Dose Factor, rem $/ \mathrm{ml}$ & 51,490 & 69,820 & 78,740 & 10,490 \\
\hline Inhalation Dose Factor, rem/g & 36,960 & 48,280 & 53,510 & 6,582 \\
\hline
\end{tabular}

Several additional cases were run to investigate the influence of particular modeling assumptions. The results from these alternate cases are summarized below. All differences mentioned below are comparisons with the base case. Detailed discussion is presented in Section 5.8.

1. The $90 \%$ full criterion for containers was compared to no criteria, i.e., all partly filled containers are tallied. If the average concentrations of the partly filled containers are based on the container volume, the concentrations are biased toward lower values. For the 2.0 and $12 \mathrm{~m}^{3}$ containers the mean value decreased $6 \%$ and $39 \%$ while the $95^{\text {th }}$ percentile decreased $4 \%$ and $21 \%$. If the average concentrations of the partly filled containers are based on the sludge volume, the concentrations are biased toward higher values. The mean values increase less than $2 \%$. However, for the 2.0 and $12 \mathrm{~m}^{3}$ containers the $95^{\text {th }}$ percentile increased $3 \%$ and $26 \%$. 
2. The imputed values for floor sludge were increased by a factor of 2 . The resulting container inventories for nuclides with many imputed values increased significantly. The Tc-99 inventory increased $18 \%$, the $\mathrm{Np}-237$ increased $14 \%$, the $\mathrm{Pu}-241$ increased $12 \%$, the Eu-152 increased 10\%, and the Cs-134 increased 7\%. Overall, the inhalation dose increased $2 \%$ and the heat generation rate increased less than $1 \%$ for all container sizes investigated.

3. Values for the canister sludge depth in the two canister barrels with off scale readings were tried that spanned the reasonable range of values, 12 inches to 20 inches. The stochastic results increased less than $2 \%$ for the maximum depth.

4. One retrieval sequence was used rather than any possible sequence. The worst of the 6 sequences tried retrieved the keys in reverse chronological order (youngest first). The resulting container inventories increased by varying amounts. The mean and $95^{\text {th }}$ percentile for the $0.5 \mathrm{~m}^{3}$ volume increased by about $1 \%$ and $3 \%$. The mean and $95^{\text {th }}$ percentile for the $2 \mathrm{~m}^{3}$ volume increased by about $5 \%$ and $19 \%$. The mean and $95^{\text {th }}$ percentile for the $12 \mathrm{~m}^{3}$ volume increased by about $16 \%$ and $12 \%$.

5. Canister sludge sample measurements were used rather than the concentration estimates from HNF-8760. The sludge data gave much lower results for all items tracked except the Tc-99 inventory, which increased by a factor of 7 .

Various modifications to the PDFs for the data were tested and found to decrease the results by a small amount (less than 10\% in all cases). These alternate PDFs include reduced variability factors and continuous cumulative distributions.

The sensitivity analyses performed indicate the base analysis performed was found to be generally robust (insensitive to minor assumption changes) and conservative. The canister sludge sample data for Tc-99 was significantly larger than used for the base case stochastic analysis. Though the magnitude of the activity of this radioisotope is comparatively small, applications specifically addressing Tc-99 should evaluate the difference noted here both for validity of the sample data and the validity of the reactor inventory and decay model. Also noted in this sensitivity analysis was a moderate increase (up to $+19 \%$ for the $12 \mathrm{~m}^{3}$ case) when a single key retrieval sequence is used. 
SNF-10293, REV 0

\subsection{INTRODUCTION TO THE STOCHASTIC METHODS USED}

Once the basic calculation has been defined, certain parameters in the calculation are replaced with a range of values and the associated likelihood of occurring. The replacement is referred to as a probability density function.

Numerous PDFs exist and are used in stochastic analyses. The simplest is the uniform PDF, in which all numbers between the minimum and the maximum are equally likely. The mean value of a uniform distribution is the midpoint of the range. The standard deviation of a uniform distribution is the range divided by the square root of 12, i.e., 3.464. For example, a uniform distribution that ranges from 1 to 3 has a mean value of 2 and a standard deviation of 0.577 . The uniform PDF is used extensively in this report to represent certain data ranges and to represent the expected variability of the data.

When the stochastic calculation is carried out, randomly selected numbers are chosen from the PDFs. These randomly chosen values are used to calculate a new result for container inventory. This process of picking numbers from the PDF is repeated and additional results are found. The results are tallied during this process to obtain the resulting probability distribution function. The tallying operation divides the observed range of results into equal sized bins. Each bin holds the count of how many computed results lie within the range of values spanned by the bin. When enough cases have been run, the outcome of the stochastic analysis is a probability distribution for the result. This histogram of counts is converted to a histogram of probabilities by dividing the number of counts in each bin by the total. The final PDF is a histogram showing the fraction of results that are in each bin.

To illustrate this process with an example, consider the sum of two uniform distributions. The first PDF (PDF A) ranges from 1 to 3 . It has a mean of 2.0, a standard deviation of 0.577 , and a relative standard deviation of $0.577 / 2.0=0.289$. The second PDF (PDF B) ranges from 3.5 to 10.5. It has a mean of 7.0, a standard deviation of 2.02, and a relative standard deviation of $2.02 / 7.0=0.289$. The sum of the two distributions ranges from 4.5 to 13.5. The expected mean is the sum of the means, or 9.0. The expected standard deviation is the square root of the sum of the squares, or 2.10, as shown below. The relative standard deviation is $2.10 / 9.0=0.234$, which is smaller than either of the PDFs that were summed.

$$
\text { Expected Standard Deviation }=\sqrt{0.577^{2}+2.02^{2}}=2.10
$$

To expedite the calculation points will not be taken at random. Instead, 5 evenly spaced values will be taken from each PDF, giving 25 resulting sums. The values and sums are shown in Table 2-1. The mean of the 25 sums is 9.00, while the standard deviation is 2.02 . These agree with the expected values. When uniform PDFs with the same relative standard deviation are added, the sum has a smaller relative standard deviation. 
Table 2-1. Example of the Sum of Two Uniform Distributions.

\begin{tabular}{|c|c||c|c|c|}
\hline $\begin{array}{c}\text { Values from } \\
\text { PDF A }\end{array}$ & $\begin{array}{c}\text { Values from } \\
\text { PDF B }\end{array}$ & \multicolumn{3}{|c|}{ Sums of the Distributions } \\
\hline 1.2 & 4.2 & 5.4 & 11.4 & 10.8 \\
\hline 1.6 & 5.6 & 6.8 & 6.2 & 12.2 \\
\hline 2.0 & 7 & 8.2 & 7.6 & 7 \\
\hline 2.4 & 8.4 & 9.6 & 9 & 8.4 \\
\hline 2.8 & 9.8 & 11 & 10.4 & 9.8 \\
\hline & & 5.8 & 11.8 & 11.2 \\
\hline & & 7.2 & 6.6 & 12.6 \\
\hline & & 8.6 & 8 & \\
\hline & & 10 & 9.4 & \\
\hline
\end{tabular}

The mean of the 25 sums is 9.00 .

The standard deviation of the sums is 2.02 .

The next step is to put the list of sums from Table 2-1 into appropriately sized bins. The bins are shown in Table 2-2, along with the number of sums that were placed in that bin. The final column shows the probability per bin. This column is computed from the number of counts in a bin divided by the total number of counts (25). A graph of the binned products is shown in Figure 2-1. Note that it is not a uniform distribution.

Table 2-2. Binning of the Sums.

\begin{tabular}{|c|c||c|c|}
\hline Bin Low & Bin High & $\begin{array}{c}\text { Counts } \\
\text { per Bin }\end{array}$ & $\begin{array}{c}\text { Probability } \\
\text { per Bin }\end{array}$ \\
\hline 4.5 & 6.3 & 3 & 0.12 \\
\hline 6.3 & 8.1 & 6 & 0.24 \\
\hline 8.1 & 9.9 & 7 & 0.28 \\
\hline 9.9 & 11.7 & 6 & 0.24 \\
\hline 11.7 & 13.5 & 3 & 0.12 \\
\hline \hline & Total & 25 & 1.00 \\
\hline
\end{tabular}


Figure 2-1. Plot of the Sum of Two Uniform Distributions.

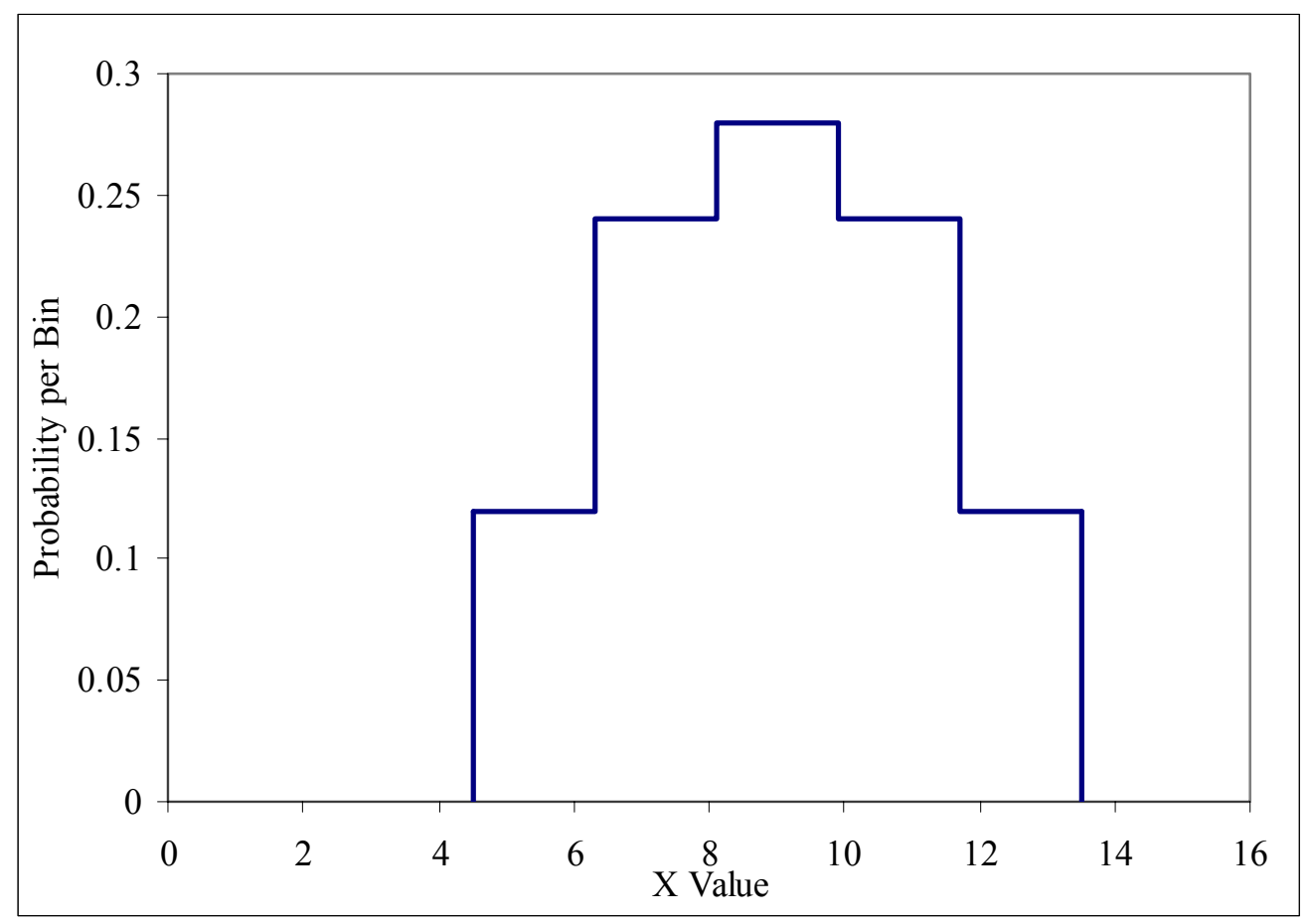

The mean value of the histogram is 9.00 . The standard deviation for the histogram is calculated using the equation below. The standard deviation for the histogram is 2.16 , which differs from the expected value by $3 \%$. (This difference becomes smaller as the number of sample points and tally bins increases.)

$$
\text { Standard Deviation }=\left\{\frac{\sum_{\mathrm{K}=1}^{\mathrm{N}} \operatorname{Count}(\mathrm{K})[\operatorname{Mid}(\mathrm{K})-\mathrm{Mean}]^{2}}{\sum_{\mathrm{K}=1}^{\mathrm{N}} \operatorname{Count}(\mathrm{K})}\right\}^{1 / 2}
$$

Where

$$
\begin{aligned}
\text { Count }(\mathrm{K}) & =\text { number of values in the Kth bin } \\
\mathrm{K} & =\text { index to a particular tally bin } \\
\text { Mean } & =\text { mean value of the parameter being tallied } \\
\operatorname{Mid}(\mathrm{K}) & =\text { midpoint of the Kth bin, i.e., Mid }=(\text { Low }+ \text { High }) / 2 \\
\mathrm{~N} & =\text { number of bins in the histogram }
\end{aligned}
$$

A uniform distribution over the same range as the sum (4.5 to 13.5) would have the same mean, but a larger standard deviation, namely, 2.60. Thus the sum of uniform PDFs is no longer a uniform PDF. Values near the mean are more likely, while values near the bounds are less likely. 
If the first uniform distributions had been divided by the second, the expected mean would be 2.0/7.0 $=0.286$. However, according to the simulated stochastic method described above, the mean is 0.312 . This small difference can be attributed to the effect of division. Multiplication, addition, and subtraction of PDFs preserve the mean, but division introduces a departure from the mean in the final distribution.

The PDF is often used to represent data. The PDF may originate in some curve-fitting regression analysis showing that the data can be represented in a normal or lognormal, or other shape. A simpler method (especially with limited numbers of data values) is to use the measured values themselves as the distribution. An enhancement used in this report is to include the variability in the data with uniform distributions at each data point. The mean value of the uniform distribution is 1.0. The PDF is calculated by first selecting one of the data values and then generating a random value from a uniform distribution whose mean value is 1.0. The value from the uniform distribution is multiplied by the selected data value. This approach preserves the mean value and increases the standard deviation a small amount as illustrated in the stochastic results shown in Section 4.2.

Associated with a PDF is a cumulative distribution function, or CDF. The cumulative distribution function is the integral of the PDF. It gives the probability that the random sampling from the PDF is less than a particular number. The CDF can also be used in the calculation of randomly generated values from a PDF. The random number generator selects a number between 0 and 1. This number is the cumulative probability. The graph of the CDF is used to find the corresponding parameter value. 
SNF-10293, REV 0

\subsection{DATA USED IN THE STOCHASTIC MODEL}

In this section, the data as well as the method used to represent that data in the stochastic model are presented. Later, in the stochastic model results, the tallied distributions for the input parameters are given and compared with the data, where possible. These comparisons help verify that the stochastic calculations have implemented the model correctly. A final consideration in each section is the selection of alternate models for comparison with the chosen method for representing the data. The alternate models test whether the assumed PDF introduces significant bias on the results.

An essential objective in representing the various quantities of interest is capturing the potential variability of each quantity. The PDFs are created so that mean values are preserved, thus the mean value of the stochastic results can be estimated by hand calculations that use the mean values of the input quantities. To be conservative, the variance of the distribution function chosen to represent a parameter is larger than the measured variance. The larger variance increases the spread of the container results and thus increases the bounding $\left(95^{\text {th }}\right.$ percentile) values.

\subsection{FLOOR SLUDGE DEPTH}

Floor sludge depths were measured using a high-resolution, underwater video camera and a stainless steel ruler (Meling 1994). The ruler was inserted into the sludge and the markings on the scale were read using the video camera. The floor sludge depth was measured at 234 locations scattered around K East Basin. This averages to 1 out of every 18 cubicles in the basin.

The depth of the floor sludge at thousands of other locations was imputed using 2-dimensional data fitting software. The purpose was to estimate the total floor sludge present in the K East Basin at that time. Floor areas outside the cubicles as well as the remote pits were included. Various techniques were applied to the data and a conservative approach was selected. The results of these estimates of floor sludge depth that are used in this report are shown in Appendix A. The appendix only shows the depths in cubicles. Floor sludge outside the cubicles or in the adjacent pits is not within the scope of these calculations. Asterisks mark locations that had actual measurements taken.

The previous volume calculation includes the floor areas that are outside the cubicles as well as the presence of empty fuel canisters that displace floor sludge. The estimated total volume of floor sludge was $21,650 \mathrm{~L}\left(21.65 \mathrm{~m}^{3}\right.$ ) with an uncertainty of $\pm 20 \%$ (Tables 4 and 6 of WHC-SD-SNF-TI-006 [Baker 1995]). Note that this volume includes sludge from floor areas outside of cubicles with fuel as well as pit sludge.

For the stochastic analysis, the floor sludge volume associated with each key was needed. This was calculated using the map of key locations (Appendix B) together with the map of floor sludge depths (Appendix A). The calculations were carried out in a spreadsheet containing both the sludge depth map and the key identifier map. The spreadsheet uses a table that gives the floor depth of a selected key identifier in those cells where the key is located. The table has 
zeroes everywhere else. The summation of all the cells in the table gives the total floor sludge depth for that key. The total depth is then multiplied by the area of the cubical that is not under the canister $\left(627 \mathrm{~cm}^{2}\right)$ to give the total volume of floor sludge associated with that key.

The empty cubicle is about $10.625 \mathrm{in}$. by $19.325 \mathrm{in}$. and is modeled using an area of $1,307 \mathrm{~cm}^{2}$. One canister barrel has an outer diameter of about $8.19 \mathrm{in}$. and is modeled using a floor area of $340 \mathrm{~cm}^{2}$. Thus, the floor area in cubicles with a fuel storage canister is $1307 \mathrm{~cm}^{2}-2\left(340 \mathrm{~cm}^{2}\right)=$ $627 \mathrm{~cm}^{2}$.

Table 3-1 shows the calculated floor sludge volume by key. Also shown in Table 3-1 is the number of cubicles with fuel. The total number of cubicles in K East Basin is 3,668. The total volume of floor sludge in cubicles with fueled canisters is $11,700 \mathrm{~L}$. Note that this volume is calculated from the best-estimate of floor sludge depth in each cubicle that has fuel.

Table 3-1. Average Floor Sludge Volume by Key. (2 sheets)

\begin{tabular}{|c|c|c|}
\hline KeyNumber & $\begin{array}{c}\text { Number of } \\
\text { Cubicles }\end{array}$ & $\begin{array}{c}\text { Floor Sludge } \\
\text { Volume (L) }\end{array}$ \\
\hline 9621 & 3 & 3.1 \\
\hline 10001 & 2 & 5.6 \\
\hline 10201 & 11 & 41 \\
\hline 10259 & 66 & 152 \\
\hline 10294 & 120 & 293 \\
\hline 10350 & 2 & 8.4 \\
\hline 10385 & 111 & 295 \\
\hline 10456 & 84 & 280 \\
\hline 10576 & 12 & 51 \\
\hline 10602 & 85 & 284 \\
\hline 10679 & 86 & 203 \\
\hline 10742 & 66 & 233 \\
\hline 10764 & 10 & 8.0 \\
\hline 10770 & 60 & 253 \\
\hline 10832 & 167 & 535 \\
\hline 10982 & 80 & 280 \\
\hline 11036 & 41 & 163 \\
\hline 11109 & 80 & 289 \\
\hline 11183 & 158 & 471 \\
\hline 11372 & 97 & 279 \\
\hline 11449 & 92 & 285 \\
\hline 11540 & 42 & 147 \\
\hline 11733 & 2 & 6.8 \\
\hline & & \\
\hline & 2 & 2 \\
\hline
\end{tabular}

\begin{tabular}{|c|c|c|}
\hline $\begin{array}{c}\text { Key } \\
\text { Number }\end{array}$ & $\begin{array}{c}\text { Number of } \\
\text { Cubicles }\end{array}$ & $\begin{array}{l}\text { Floor Sludge } \\
\text { Volume (L) }\end{array}$ \\
\hline 12127 & 95 & 272 \\
\hline 12201 & 70 & 180 \\
\hline 12240 & 3 & 5.7 \\
\hline 12285 & 199 & 765 \\
\hline 12480 & 168 & 566 \\
\hline 12565 & 122 & 514 \\
\hline 12639 & 142 & 495 \\
\hline 12852 & 8 & 24 \\
\hline 13015 & 3 & 4.0 \\
\hline 13649 & 16 & 49 \\
\hline 13685 & 8 & 29 \\
\hline 14065 & 1 & 1.1 \\
\hline 14355 & 30 & 105 \\
\hline 14356 & 55 & 190 \\
\hline 14722 & 1 & 1.0 \\
\hline 15204 & 313 & 721 \\
\hline 15240 & 2 & 1.8 \\
\hline 15316 & 65 & 201 \\
\hline 15399 & 2 & 7.0 \\
\hline 15444 & 1 & 5.3 \\
\hline 15451 & 30 & 113 \\
\hline 15455 & 5 & 28 \\
\hline 15457 & 2 & 11 \\
\hline
\end{tabular}


SNF-10293, REV 0

Table 3-1. Average Floor Sludge Volume by Key. (2 sheets)

\begin{tabular}{|c|c|c|}
\hline KeyNumber & $\begin{array}{c}\text { Number of } \\
\text { Cubicles }\end{array}$ & $\begin{array}{c}\text { Floor Sludge } \\
\text { Volume (L) }\end{array}$ \\
\hline 11806 & 86 & 230 \\
\hline 11897 & 120 & 420 \\
\hline 11979 & 119 & 384 \\
\hline
\end{tabular}

\begin{tabular}{|c|c|c|}
\hline $\begin{array}{c}\text { Key } \\
\text { Number }\end{array}$ & $\begin{array}{c}\text { Number of } \\
\text { Cubicles }\end{array}$ & $\begin{array}{c}\text { Floor Sludge } \\
\text { Volume (L) }\end{array}$ \\
\hline 15458 & 425 & 1,498 \\
\hline 15460 & 61 & 185 \\
\hline 15461 & 39 & 136 \\
\hline
\end{tabular}

- Notes:

- The floor sludge volumes are best-estimates based on the measured floor sludge depths.

- The total number of canisters is 3,668. The total average volume of floor sludge is $11,700 \mathrm{~L}$.

- The floor sludge volumes were calculated using an exposed floor area of $627 \mathrm{~cm}^{2}$.

Table 6 of WHC-SD-SNF-TI-006 (Baker 1995) gives the estimated uncertainty of the floor sludge total volume as $\pm 20 \%$. This estimate of the overall uncertainty in the floor sludge volume is used as the basis for the stochastic representation of floor sludge volume. The PDF for floor sludge volume is assumed to be a uniform distribution that ranges from $80 \%$ to $120 \%$ of the values shown in Table 3-1. This range was interpreted to apply equally to all the keys at the same time. The stochastic analysis generates a random number from 0.8 to 1.2 and multiplies it by the mean floor sludge volume of every key, i.e., every row in Table 3-1. In this way, the total floor sludge volume follows a uniform distribution that ranges from $80 \%$ to $120 \%$ of the nominal value.

One alternative to the above PDF is to give each key a different random factor. With each key given a different random factor, the resulting total follows something closer to a normal distribution. This feature of uniform distributions was illustrated in Section 2.0. When uniform distributions are added, the result has a roughly normal shape.

Calculations using this alternative PDF were performed to evaluate its effect on the computed results. The uniform distribution applied to each key ranged from 0.19 to 1.81 . This range was selected to give a standard deviation in the total floor sludge volume that is $10 \%$ of the total, namely, $1.17 \mathrm{~m}^{3}$. The estimated uncertainty of $\pm 20 \%$ corresponds to two standard deviations. The results from this alternate case are discussed in Section 5.8.

\subsection{FLOOR SLUDGE ISOTOPIC CONCENTRATION}

Floor sludge samples have been taken at several locations around the basin and adjacent pits. These have been analyzed to measure density, uranium concentration, and activity concentration for several radionuclides. The data for 23 floor sludge samples is listed in Appendix D of SNF-7765. Many of the entries in the table are not available. These could be entered as zeroes in the stochastic analysis. However, this would bias the results toward lower values. Hence, it was decided to estimate appropriate data values to fill in the blanks. 
The methods for calculating total plutonium, uranium metal content, heat generation rate, and inhalation dose are discussed in later sections.

\subsubsection{Imputed Values for Floor Sludge}

Data that are reported as "less than" some value are termed censored data or left-censored data. Data sets that have blank or missing data values also occur, for a variety of reasons. Statisticians and scientists have developed an array of tools to handle censored and missing data. The word "impute" has come into use in the last decade to mean creating a number to put in place of a missing or less-than value. Imputation is a form of prediction, and imputation tools include interpolation, extrapolation, and predictions based on trends in similar data. A value created by imputation is called an imputed value.

Imputation is needed for the K-East Basin sludge because values are censored or missing for many single isotopes results and sometimes for all measurements for a given isotope. In the case of floor sludge, all samples need values for Tc-99, Eu-152, and Pu-241. Measurements for Tc-99 and Eu-152 concentrations are listed in the analytical results for canister sludge and are therefore desired for floor sludge. Estimates for $\mathrm{Pu}-241$ are needed because it is expected to contribute roughly $10 \%$ to the inhalation dose factor.

Two possible approaches to imputation were examined. These two approaches can be generally described as "within analyte" or "across analyte" imputations. Within-analyte approaches presume that, in a rectangular data matrix in which the columns represent analytes such as uranium or ${ }^{241} \mathrm{Am}$, one can impute values to empty cells by using the non-empty values in that column. Across-analyte approaches assume that one analyte can be predicted by another analyte. In the context of the present work, this means that one radionuclide can serve as an indicator for another. For example, HNF-SD-1201 and WHC-SD-1182 (Makenas et al. 1996a; 1997; Appendix $\mathrm{N}$ of each document) have used the across-analyte approach to study the relationship between ${ }^{239+240} \mathrm{Pu}$ and ${ }^{154} \mathrm{Eu}$ in both KE canister and floor sludge.

The within-analyte approach postulates that a distribution, such as the normal or lognormal, could describe the data. If one can assume that the distribution is left-censored, that is, the censored, less-than values are at the bottom end of the distribution, then a widely-used and welldocumented collection of techniques is available (Pan and Connett 2001; Barry and Brattin 1998; Mitchell et. al. 1997; PNL-10653, Gilbert 1995; SARE-9247, Atwood et. al. 1991; Strom 1986). However, the condition of left-censoring does not hold for the "missing", and one does not have any idea where the "not measured" values fall in the distribution. As a result, the within-analyte method was tried and abandoned, and those results are not presented here.

The across-analyte approach was tried by assuming, say, that ${ }^{238} \mathrm{Pu}$ could be predicted by ${ }^{239} \mathrm{Pu}$, that is, that they were likely to occur in about the same ratio in any given sample. One can express this by saying that one analyte is proportional to another. This assumption was borne out by examining graphs of the concentration of one analyte plotted versus another, such as is shown in Figure 3-1 through Figure 3-7. The graphs show the measured values as diamonds. A linear regression that was forced to pass through the origin is also shown. It is a line that ranges from the smallest measured value to the largest. The imputed values are shown in these figures as 
squares. These are computed from measured values for materials on the horizontal axis multiplied by the slope of the regression line. For later use in an alternate case, the location of the imputed values if they are doubled is also shown as asterisks in these figures.

Linear regression has been used to establish the general correlation between isotopes. This is not a completely valid application of regression analysis for the reasons listed below.

1. The values on the horizontal axis are not known exactly. This requirement is implicit in linear regression analysis.

2. The actual relationship between the two nuclides being correlated is not linear in all cases. Other factors, such as fuel burnup, decay time since discharge, and solubility in water make a linear correlation unlikely.

For these reasons, the regression analysis was not carried any further. For example, no estimates of the confidence level of the slope, or estimated variance in the predicted values are evaluated. The regression analysis is simply used to establish the general relationship between the two nuclides with the highest $\mathrm{R}^{2}$ value. The formulas (regression analysis forced through the origin) used for the slope of the line and the $\mathrm{R}^{2}$ parameter are shown below. An additional consideration is that one of the nuclides should have a complete set of measurements. For the floor sludge samples, the nuclides that were measured for each sample are Co-60, Cs-137, Pu-239/240, and Am-241. The uranium concentration was measured for all but one sample, M13 avg.

$$
\begin{gathered}
\text { Imputed } \mathrm{Y}_{\mathrm{J}}=\text { Slope } \cdot \mathrm{X}_{\mathrm{J}} \\
\text { Slope }=\frac{\sum_{\mathrm{K}} \mathrm{X}_{\mathrm{K}} \mathrm{Y}_{\mathrm{K}}}{\sum_{\mathrm{K}} \mathrm{X}_{\mathrm{K}}^{2}} \quad \mathrm{R}^{2}=\frac{\left(\sum_{\mathrm{K}} \mathrm{X}_{\mathrm{K}} \mathrm{Y}_{\mathrm{K}}\right)^{2}}{\left(\sum_{\mathrm{K}} \mathrm{X}_{\mathrm{K}}^{2}\right)\left(\sum_{\mathrm{K}} \mathrm{Y}_{\mathrm{K}}^{2}\right)}
\end{gathered}
$$

Where:

$$
\begin{aligned}
& \mathrm{J}=\text { index to those samples that have measurements for } \mathrm{X} \text { but none for } \mathrm{Y} \\
& \mathrm{K}=\text { index to those samples that have measurements for both isotopes } \\
& \mathrm{X}_{\mathrm{K}}=\text { concentration of an isotope in the Kth sample. This isotope is used } \\
& \text { to estimate values for another isotope. } \\
& \mathrm{Y}_{\mathrm{K}}=\text { concentration of an isotope in the Kth sample. This isotope has } \\
& \text { missing values that will be imputed from the measured values for } X \text {. } \\
& \text { Slope }=\text { slope of the line with the smallest sum of squared differences with } \\
& \text { the } \mathrm{Y}_{\mathrm{K}} \text {. The line passes through the origin. } \\
& \mathrm{R}^{2}=\text { parameter that indicates the proportion of the variability in the } \\
& \text { dependent variable }(\mathrm{Y}) \text { that is explained by the independent variable } \\
& \text { (X). The } \mathrm{R}^{2} \text { value ranges from } 0 \text { to } 1 \text {. }
\end{aligned}
$$

The slopes and $\mathrm{R}^{2}$ values are listed in Table 3-2. The correlations chosen for later imputation are marked with a heavy border in the table. The vertical columns are headed by the nuclides with 
measurements for all 23 samples. The rows are all the other nuclides with at least a few measurements. If $\mathrm{R}^{2}$ is close to 1.00 then the correlation is considered reasonable for the purposes of the stochastic analysis.

Table 3-2. Floor Sample Data Correlations: Slope and $\mathrm{R}^{2}$.

\begin{tabular}{|c|c|c|c|c|c|}
\hline \multirow[b]{2}{*}{ Y-Axis } & \multicolumn{5}{|c|}{ X-AXIS (ISOTOPES MEASURED FOR EVERY SAMPLE) } \\
\hline & Co-60 & Cs-137 & Pu-239/240 & Am-241 & Uranium \\
\hline \multirow[t]{2}{*}{ Co-60 } & 1 & $1.130 \mathrm{E}-03$ & $6.113 \mathrm{E}-02$ & $5.822 \mathrm{E}-02$ & $9.425 \mathrm{E}-06$ \\
\hline & 1 & 0.138 & 0.663 & 0.596 & 0.382 \\
\hline \multirow[t]{2}{*}{ Sr-90 } & $9.108 \mathrm{E}+01$ & $5.145 \mathrm{E}-01$ & $1.144 \mathrm{E}+01$ & $1.314 \mathrm{E}+01$ & $2.961 \mathrm{E}-03$ \\
\hline & 0.195 & 0.695 & 0.551 & 0.721 & 0.919 \\
\hline \multirow[t]{2}{*}{ Cs-134 } & $6.937 \mathrm{E}-02$ & 4.463E-04 & $5.866 \mathrm{E}-03$ & $6.521 \mathrm{E}-03$ & $6.212 \mathrm{E}-07$ \\
\hline & 0.489 & 0.979 & 0.820 & 0.742 & 0.605 \\
\hline \multirow[t]{2}{*}{ Cs-137 } & $1.220 \mathrm{E}+02$ & 1 & $1.778 \mathrm{E}+01$ & $1.613 \mathrm{E}+01$ & $3.706 \mathrm{E}-03$ \\
\hline & 0.138 & 1 & 0.520 & 0.424 & 0.559 \\
\hline \multirow[t]{2}{*}{ Eu-154 } & $1.633 \mathrm{E}+00$ & $3.312 \mathrm{E}-03$ & $1.384 \mathrm{E}-01$ & $1.526 \mathrm{E}-01$ & $2.717 \mathrm{E}-05$ \\
\hline & 0.613 & 0.279 & 0.802 & 0.966 & 0.794 \\
\hline \multirow[t]{2}{*}{ Eu-155 } & $7.286 \mathrm{E}-01$ & $4.487 \mathrm{E}-03$ & 7.353E-02 & 7.504E-02 & $1.450 \mathrm{E}-05$ \\
\hline & 0.590 & 0.518 & 0.829 & 0.982 & 0.831 \\
\hline \multirow[t]{2}{*}{ Np-237 } & $2.077 \mathrm{E}-03$ & $9.676 \mathrm{E}-06$ & $1.410 \mathrm{E}-04$ & $1.233 \mathrm{E}-04$ & $2.503 \mathrm{E}-08$ \\
\hline & 0.958 & 0.395 & 0.885 & 0.773 & 0.541 \\
\hline \multirow[t]{2}{*}{ Pu-238 } & $2.330 \mathrm{E}+00$ & $5.021 \mathrm{E}-03$ & $1.955 \mathrm{E}-01$ & $1.783 \mathrm{E}-01$ & $2.600 \mathrm{E}-05$ \\
\hline & 0.533 & 0.325 & 0.777 & 0.632 & 0.644 \\
\hline \multirow[t]{2}{*}{$\mathrm{Pu}-239 / 240$} & $1.085 \mathrm{E}+01$ & $2.923 \mathrm{E}-02$ & $\bar{~} 1$ & $9.423 \mathrm{E}-01$ & $1.603 \mathrm{E}-04$ \\
\hline & 0.663 & 0.520 & 1 & 0.880 & 0.690 \\
\hline \multirow[t]{2}{*}{ Am-241 } & $1.024 \mathrm{E}+01$ & $2.629 \mathrm{E}-02$ & 9.342E-01 & 1 & $1.840 \mathrm{E}-04$ \\
\hline & 0.596 & 0.424 & 0.880 & 1 & 0.866 \\
\hline \multirow[t]{2}{*}{ Uranium } & $4.050 \mathrm{E}+04$ & $1.508 \mathrm{E}+02$ & $4.301 \mathrm{E}+03$ & $4.704 \mathrm{E}+03$ & 1 \\
\hline & 0.382 & 0.559 & 0.690 & 0.866 & 1 \\
\hline
\end{tabular}

The upper number is the slope of the regression line (intercept is zero) while the lower number is the $\mathrm{R}^{2}$ value of this regression. Numbers with a heavy border were chosen to impute floor sludge concentrations for sample results that are not available in SNF-7765.

In Table 3-2 the possible correlations for Sr-90 are Cs-137, Am-241, and uranium. Because uranium has the largest $\mathrm{R}^{2}$, it was chosen to impute missing values for Sr-90. Similar logic was followed for Cs-134, Eu-154, Eu-155, and Pu-238. In the Np-237 row, the best fit (i.e. largest $\mathrm{R}^{2}$ ) is with Co-60. However, Co-60 is an activation product rather than a fission product. Hence, it was decided to use $\mathrm{Pu}-239 / 240$ to predict values for $\mathrm{Np}-237$. 
In Figure 3-1 through Figure 3-7 the chosen correlations are graphed. The measured values are plotted as diamonds, while the imputed values are plotted as squares. The regression line only extends from the smallest measured value to the largest. In Figure 3-1, the imputed values for Sr-90 are small, and thus have little effect, even if they are doubled. The uranium concentrations need special consideration. First, the values shown for uranium concentration (i.e., U.mean) are the average of two separate measurements, called U.icp (i.e., inductively coupled plasma atomic emission spectrometry, or ICP-AES) and U.las (i.e., laser fluorimetry or phosphorimetry). The ICP values are about 1.20 times greater in virtually all cases, as shown in Figure 3-2. Using this observation, a value was imputed to the missing U.icp for O-09. Then the U.mean value was replaced with the average of the imputed U.icp and the measured U.las. The resulting U.mean is thus about $10 \%$ greater than the U.las value that had been used.

Figure 3-1. ${ }^{90}$ Sr Data and Imputed Values from Uranium Measurements in Floor Sludge.

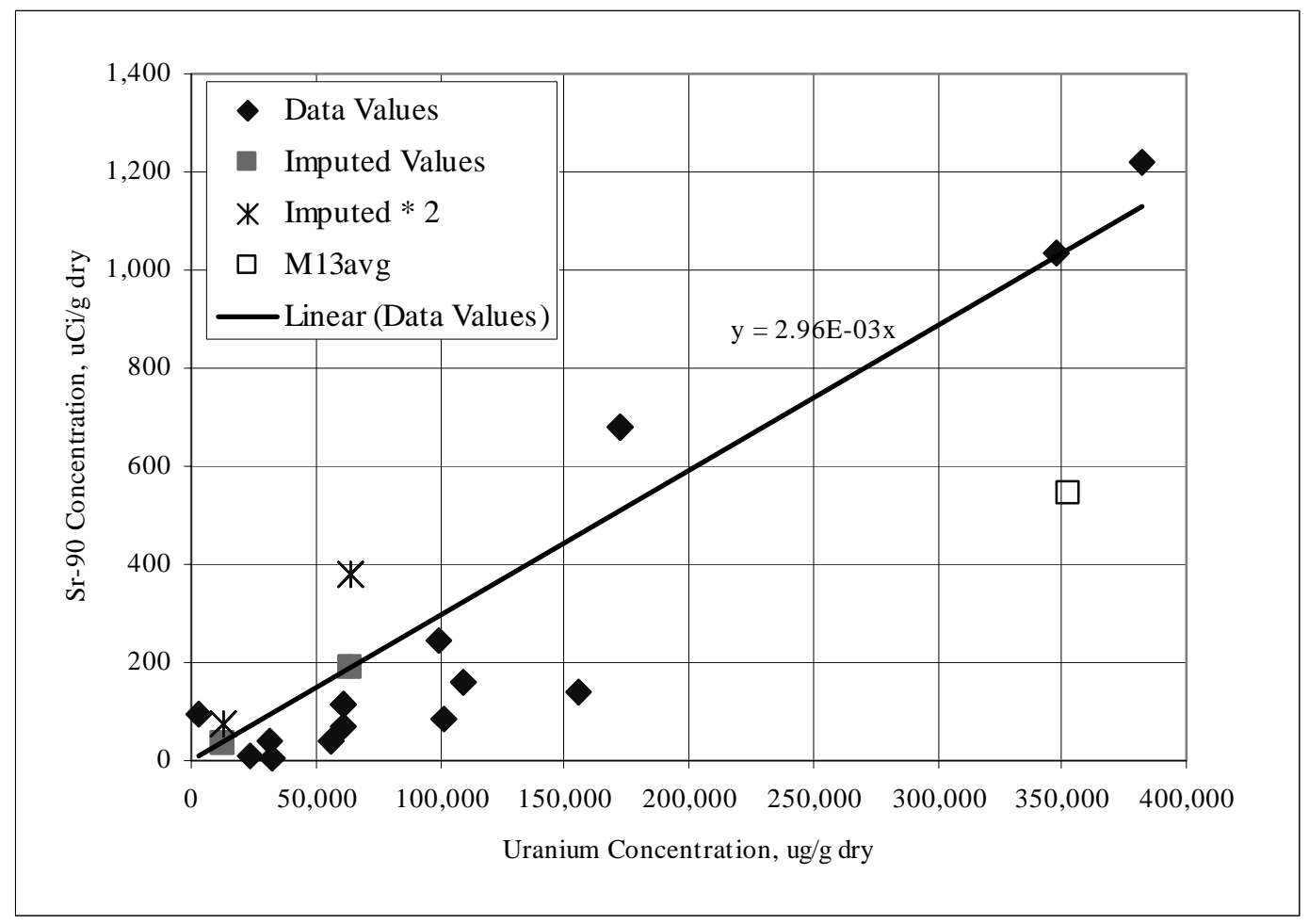

This new value for O-09 was used in the regression of Sr-90 against uranium (Figure 3-1) in all cases except one, sample M13avg, for which there were no uranium measurements. In this case the U.mean was imputed from all the other Sr-90-Umean data pairs. The linear regression has a slope of $310 \mathrm{~g} \mathrm{U}$ per Ci Sr-90. Thus, the measured Sr-90 concentration of $544 \mu \mathrm{Ci} / \mathrm{g}$ can be used to impute the uranium concentration of $353,000 \mu \mathrm{g} / \mathrm{g}$. In Figure 3-1, the Sr-90 measurement and imputed U.mean for sample M13avg are shown separately from the other imputations.

Data and imputations for Cs-134 are shown in Figure 3-3. There are more imputations than data for Cs-134. The imputations define the range of possible concentrations as well. 


\section{SNF-10293, REV 0}

Data and imputations for Eu-154 and Eu-155 are shown in Figure 3-4 and Figure 3-5. Both nuclides are fairly complete. The imputed values are at the low end of the range of concentrations.

Data and imputations for Np-237 are shown in Figure 3-6. There are more imputations than data for Np-237. The imputations are mainly at the low concentrations. The largest concentrations of Np-237 are actually measured. Note that the trend line extends only from the lowest measured value to the largest.

Data and imputations for Pu-238 are shown in Figure 3-7. Most of the points are measured values. The imputed values are spread throughout the range of concentrations.

Figure 3-2. U.icp Data and Imputation from U.las Measurements in Floor Sludge.

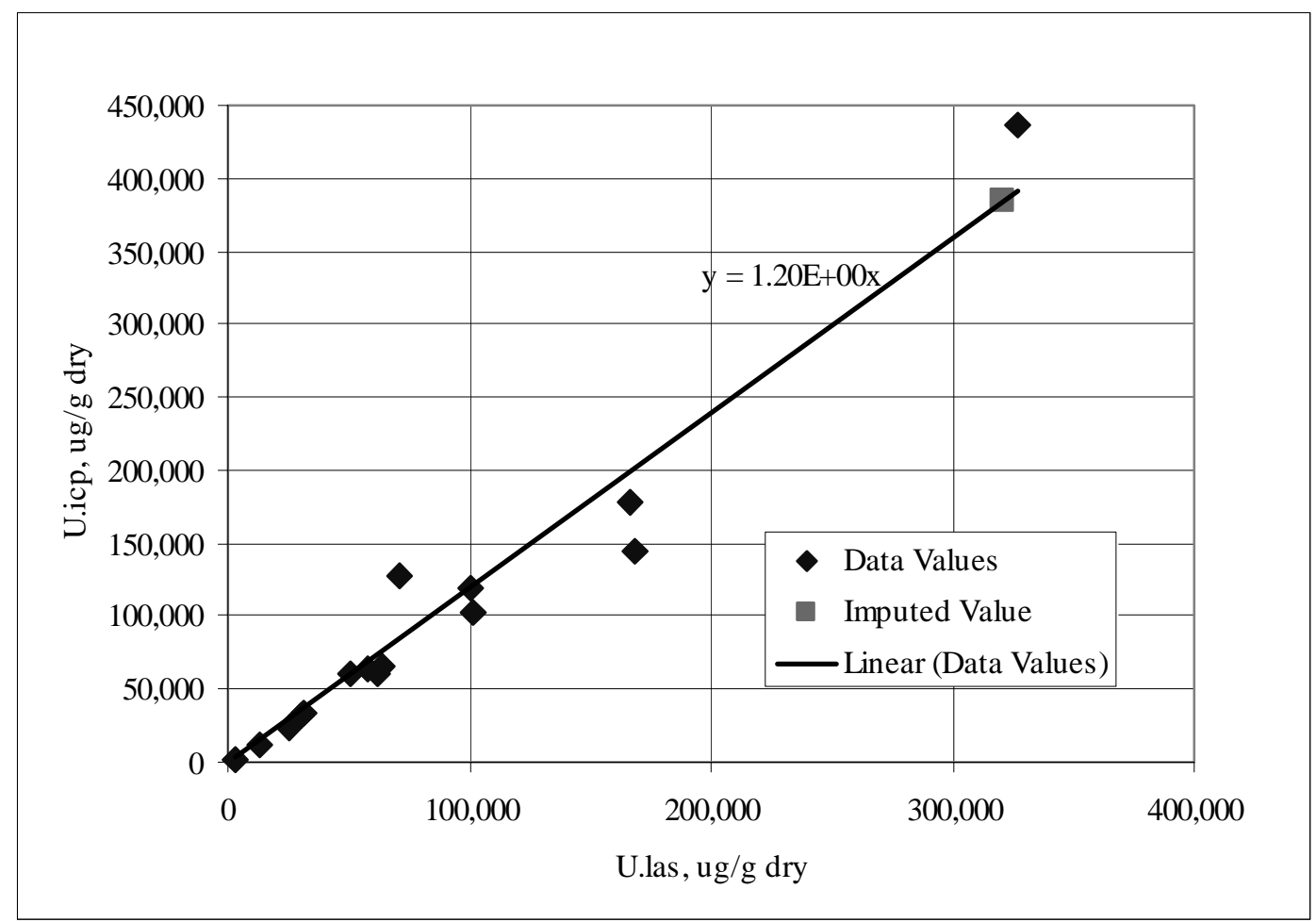


SNF-10293, REV 0

Figure 3-3. ${ }^{134} \mathrm{Cs}$ Data and Imputed Values from ${ }^{137} \mathrm{Cs}$ Measurements in Floor Sludge.

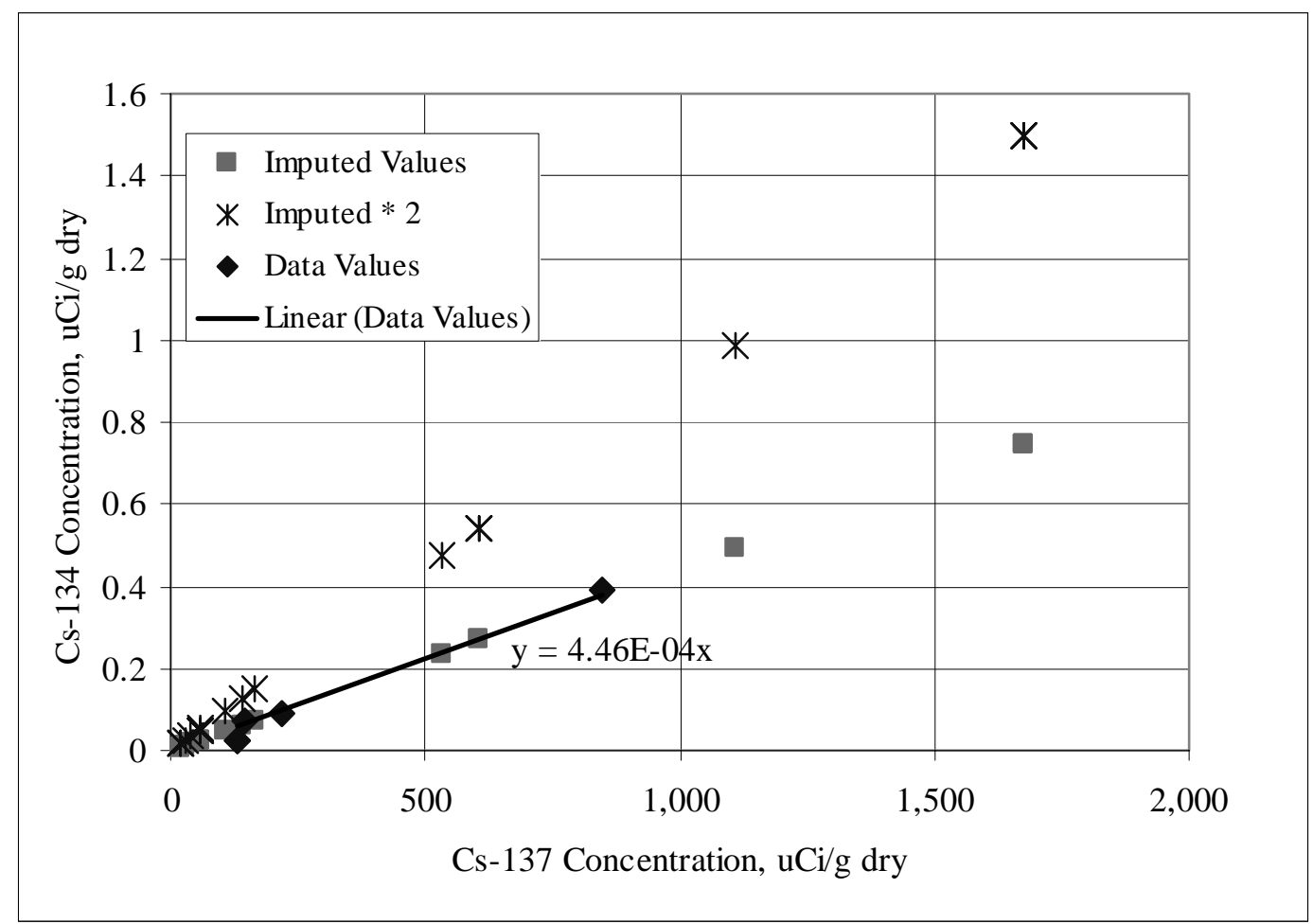

Figure 3-4. ${ }^{154} \mathrm{Eu}$ Data and Imputed Values from ${ }^{241} \mathrm{Am}$ Measurements in Floor Sludge.

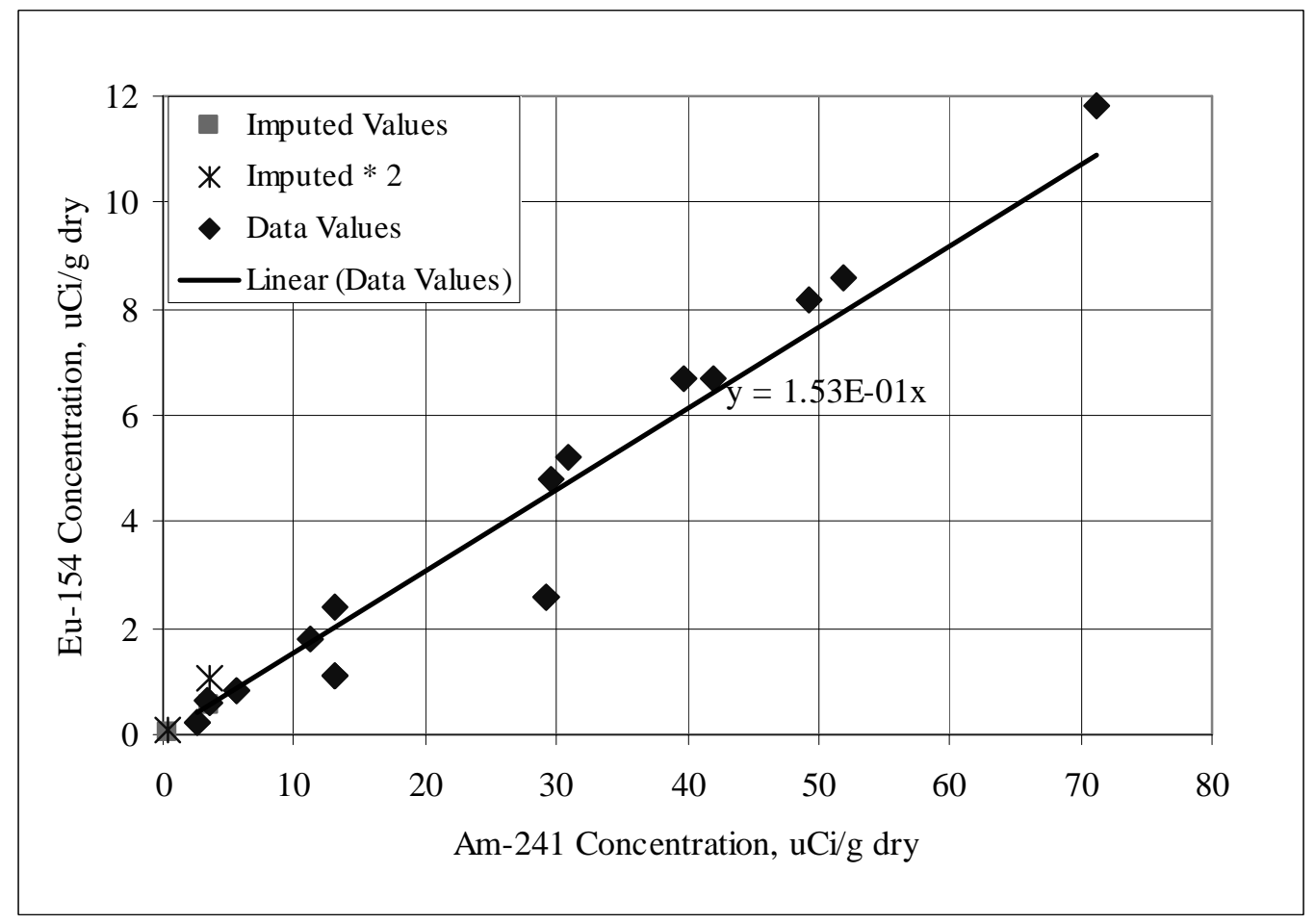


Figure 3-5. ${ }^{155}$ Eu Data and Imputed Values from ${ }^{241} \mathrm{Am}$ Measurements in Floor Sludge.

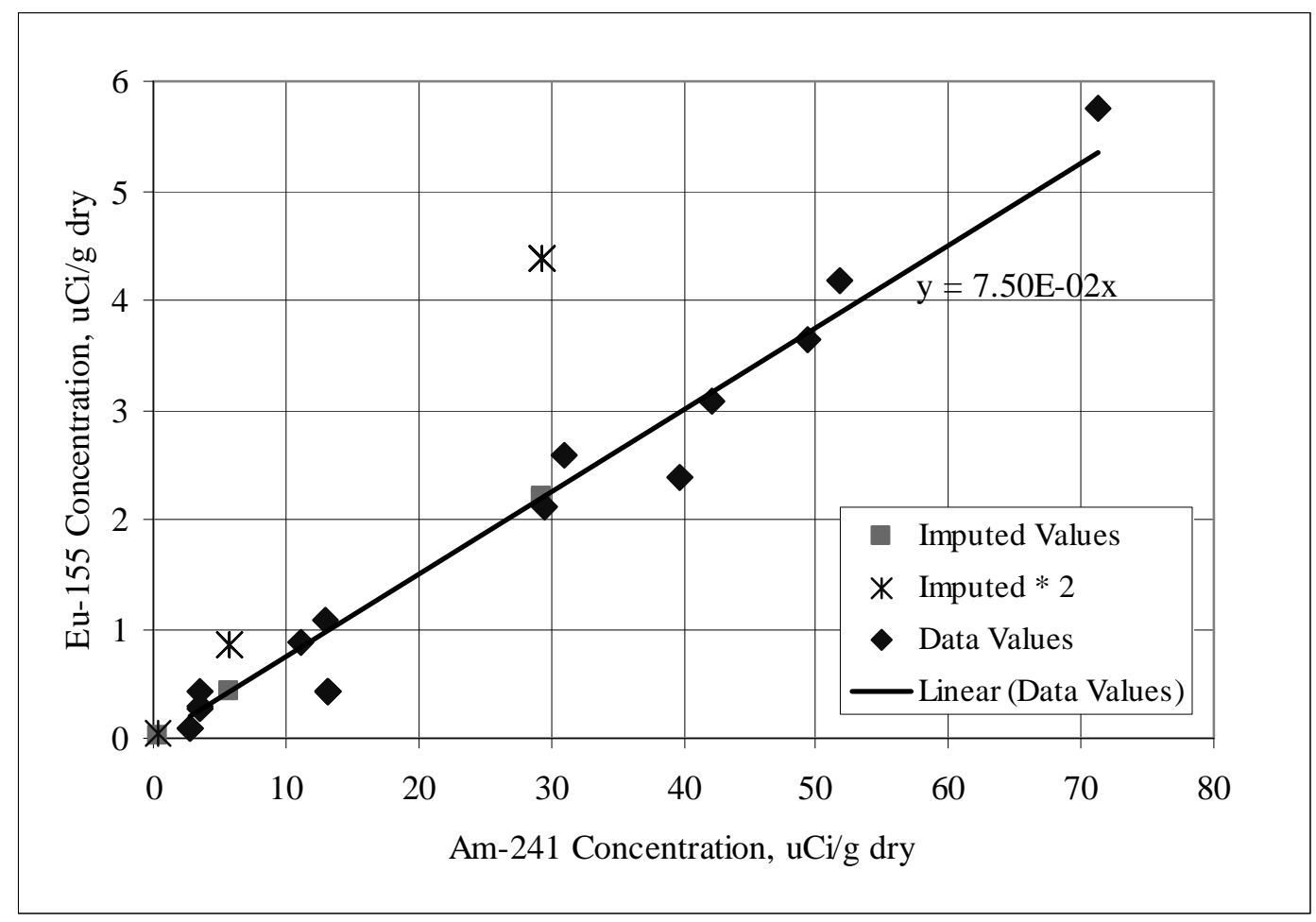

Figure 3-6. ${ }^{237} \mathrm{~Np}$ Data and Imputed Values from ${ }^{239+240} \mathrm{Pu}$ Measurements in Floor Sludge.

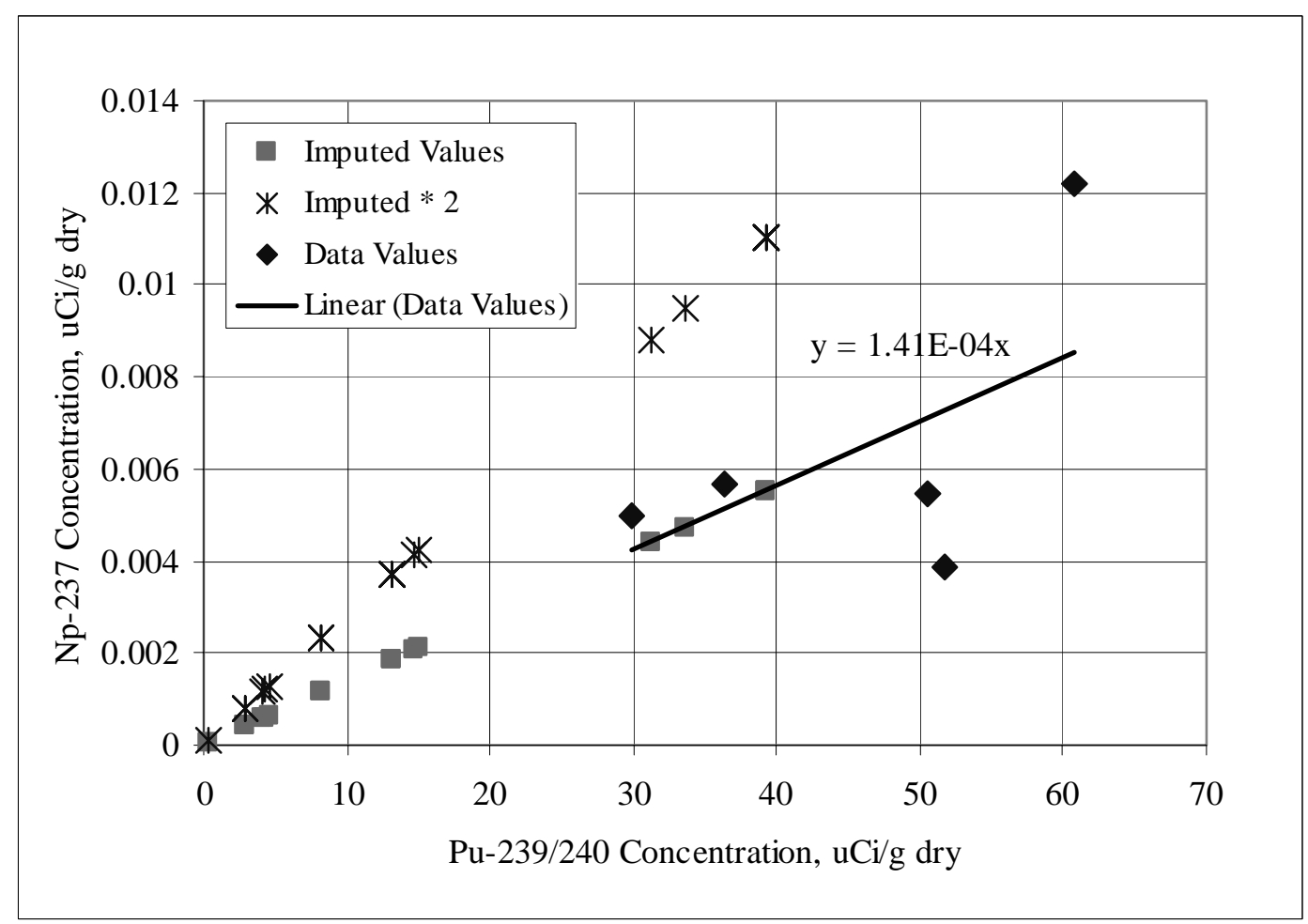


Figure 3-7. ${ }^{238} \mathrm{Pu}$ Data and Imputed Values from ${ }^{239+240} \mathrm{Pu}$ Measurements in Floor Sludge.

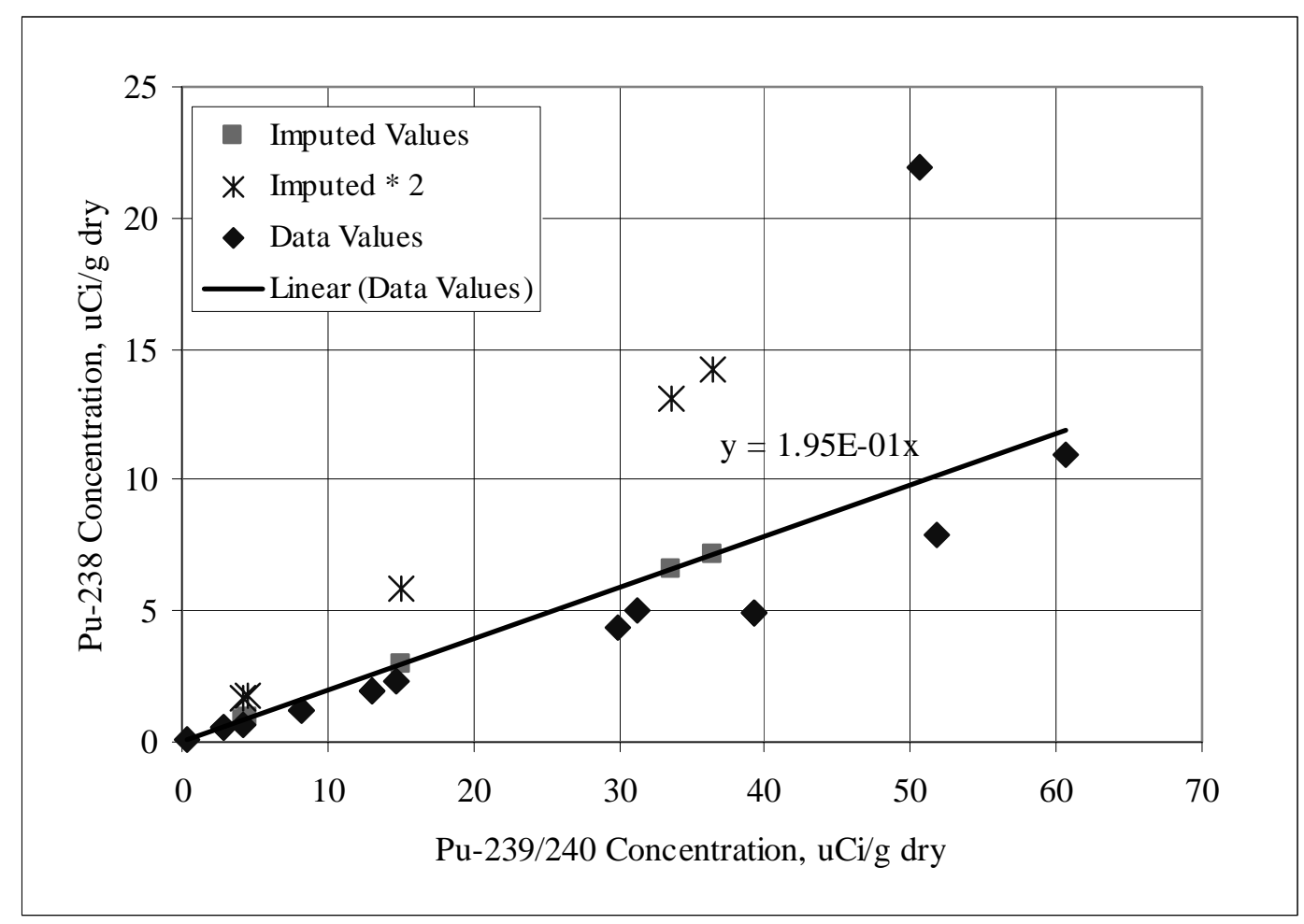

Three radionuclides of interest for the stochastic analysis (Tc-99, Eu-152, and Pu-241) have no measurements in floor sludge. The imputations for these nuclides were therefore based on the average radionuclide inventories in K East Basin canister sludge generated in HNF-8760 (Schwinkendorf 2002).

An approach similar to the previous imputations is followed using the radionuclide concentrations estimated in HNF-8760 for each key. In the formulas used for Table 3-2, the slope is calculated using the concentrations from HNF-8760. The sum is over all 52 keys. This slope is multiplied by the measured concentrations to calculate the imputed values for isotopes without measurements, namely Tc-99, Eu-152, and Pu-241. The slopes and $\mathrm{R}^{2}$ values that could be used to predict these concentrations are listed in Table 3-3. As before, the regression analysis simply establishes the general relationship between the two nuclides. The radionuclide with the highest $\mathrm{R}^{2}$ is chosen for the imputations. The uncertainties are significant and involve factors such as decay time and burnup in addition to the statistical uncertainty. Because doubling the imputed values provides an upper bound, the stochastic calculation was repeated using imputed values that are doubled. This alternate to the base case evaluates the effect of increased imputed values on the final results.

The correlations chosen to impute missing values are marked with heavy borders in Table 3-3. For Tc-99 the chosen predictor is Pu-239/240. For Eu-152 the chosen predictor is Eu-154. For $\mathrm{Pu}-241$, the chosen predictor is $\mathrm{Pu}-238$. 
SNF-10293, REV 0

Table 3-3. Correlations Using Radnuc2A Sludge Estimates: Slope and $\mathrm{R}^{2}$.

\begin{tabular}{|c|c|c|c|c|c|c|}
\hline \multirow{2}{*}{$\begin{array}{l}\text { Nuclide } \\
\text { (X Axis) }\end{array}$} & \multicolumn{2}{|c|}{ Tc-99 Imputations } & \multicolumn{2}{|c|}{ Eu-152 Imputations } & \multicolumn{2}{|c|}{ Pu-241 Imputations } \\
\hline & Slope & $\mathbf{R}^{2}$ & Slope & $\mathbf{R}^{2}$ & Slope & $\mathbf{R}^{2}$ \\
\hline Co-60 & $6.716 \mathrm{E}-01$ & 0.786 & $2.462 \mathrm{E}-01$ & 0.834 & $1.834 \mathrm{E}+03$ & 0.808 \\
\hline Sr-90 & $2.970 \mathrm{E}-04$ & 0.993 & $1.043 \mathrm{E}-04$ & 0.965 & $7.823 \mathrm{E}-01$ & 0.948 \\
\hline Cs-134 & $6.953 \mathrm{E}-02$ & 0.365 & $2.822 \mathrm{E}-02$ & 0.475 & $2.091 \mathrm{E}+02$ & 0.455 \\
\hline Cs-137 & $2.217 \mathrm{E}-04$ & 0.994 & $7.815 \mathrm{E}-05$ & 0.975 & 5.873E-01 & 0.961 \\
\hline Eu-154 & $2.145 \mathrm{E}-02$ & 0.895 & 7.992E-03 & 0.981 & $6.068 \mathrm{E}+01$ & 0.987 \\
\hline Eu-155 & $1.077 \mathrm{E}-01$ & 0.690 & $3.909 \mathrm{E}-02$ & 0.717 & $2.895 \mathrm{E}+02$ & 0.687 \\
\hline Np-237 & $4.774 \mathrm{E}+01$ & 1.000 & $1.667 \mathrm{E}+01$ & 0.963 & $1.255 \mathrm{E}+05$ & 0.951 \\
\hline $\mathrm{Pu}-238$ & $2.051 \mathrm{E}-02$ & 0.956 & $7.388 \mathrm{E}-03$ & 0.979 & $5.615 \mathrm{E}+01$ & 0.987 \\
\hline $\mathrm{Pu}-239 / 240$ & $6.985 \mathrm{E}-03$ & 1.000 & $2.433 \mathrm{E}-03$ & 0.957 & $1.828 \mathrm{E}+01$ & 0.943 \\
\hline Am-241 & $6.203 \mathrm{E}-03$ & 0.964 & 2.192E-03 & 0.950 & $1.665 \mathrm{E}+01$ & 0.956 \\
\hline
\end{tabular}

- These slopes and $\mathrm{R}^{2}$ values were calculated from the radionuclide concentrations estimated for each key in HNF-8760.

- The linear regressions have an intercept of zero. The regressions are by key with no weighting for the expected mass of sludge in that key.

- Numbers with a heavy border were chosen to impute floor sludge concentrations for radionuclides that are not available in SNF-7765.

The results are plotted in Figure 3-8 through Figure 3-10. The graphs show the Radnuc2A concentrations as diamonds. Note that the concentrations that are plotted are in units of curies per metric ton of uranium. A linear regression that was forced to pass through the origin is also shown. It is a line that ranges from the smallest concentration to the largest. The imputed values are shown in these figures as squares. These are computed from measured values for materials on the horizontal axis multiplied by the slope of the regression line. To obtain units of Ci/MTU, the floor sludge concentrations have been divided by the uranium concentration. For later use in an alternate case, the location of the imputed values if they are doubled is also shown as asterisks.

The imputed concentrations for Tc-99 are shown in Figure 3-8. Three of the imputed concentrations are larger than any Radnuc2A result.

The imputed concentrations for Eu-152 are shown in Figure 3-9. The Radnuc2A numbers are clearly on a curve rather than a straight line. From the graph, the imputed values appear nonconservative. However, the doubled values are larger than the Radnuc2A values. The alternate case in which the imputed values are doubled will still be bounding.

The imputed concentrations for Pu-241 are shown in Figure 3-10. The imputed values are spread throughout the range of Radnuc2A concentrations. 
Figure 3-8. ${ }^{99} \mathrm{Tc}$ Imputations from ${ }^{239+240} \mathrm{Pu}$ Measured Values in Floor Sludge.

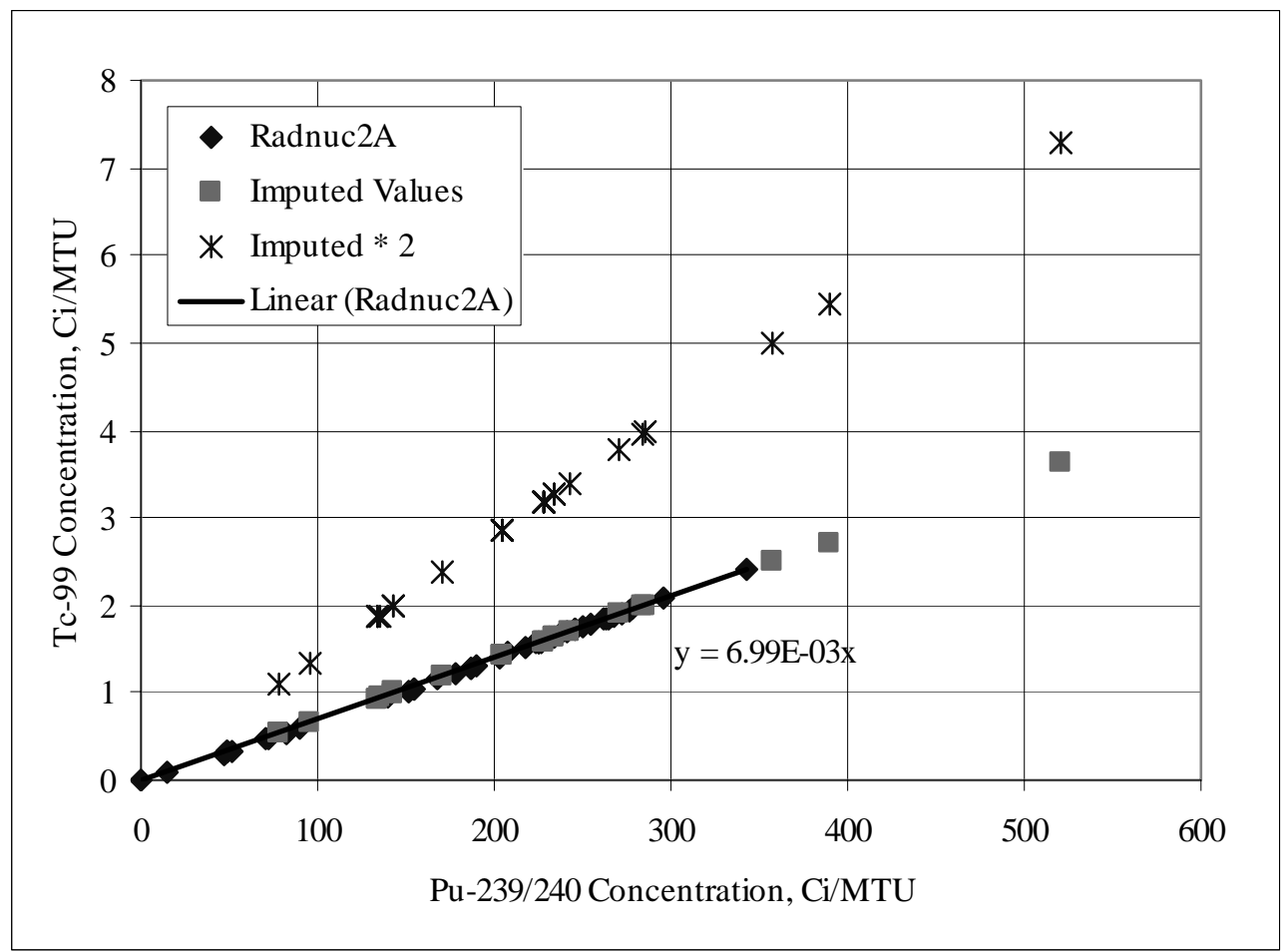

Figure 3-9. ${ }^{152} \mathrm{Eu}$ Imputations from ${ }^{154} \mathrm{Eu}$ Data and Imputed Values in Floor Sludge.

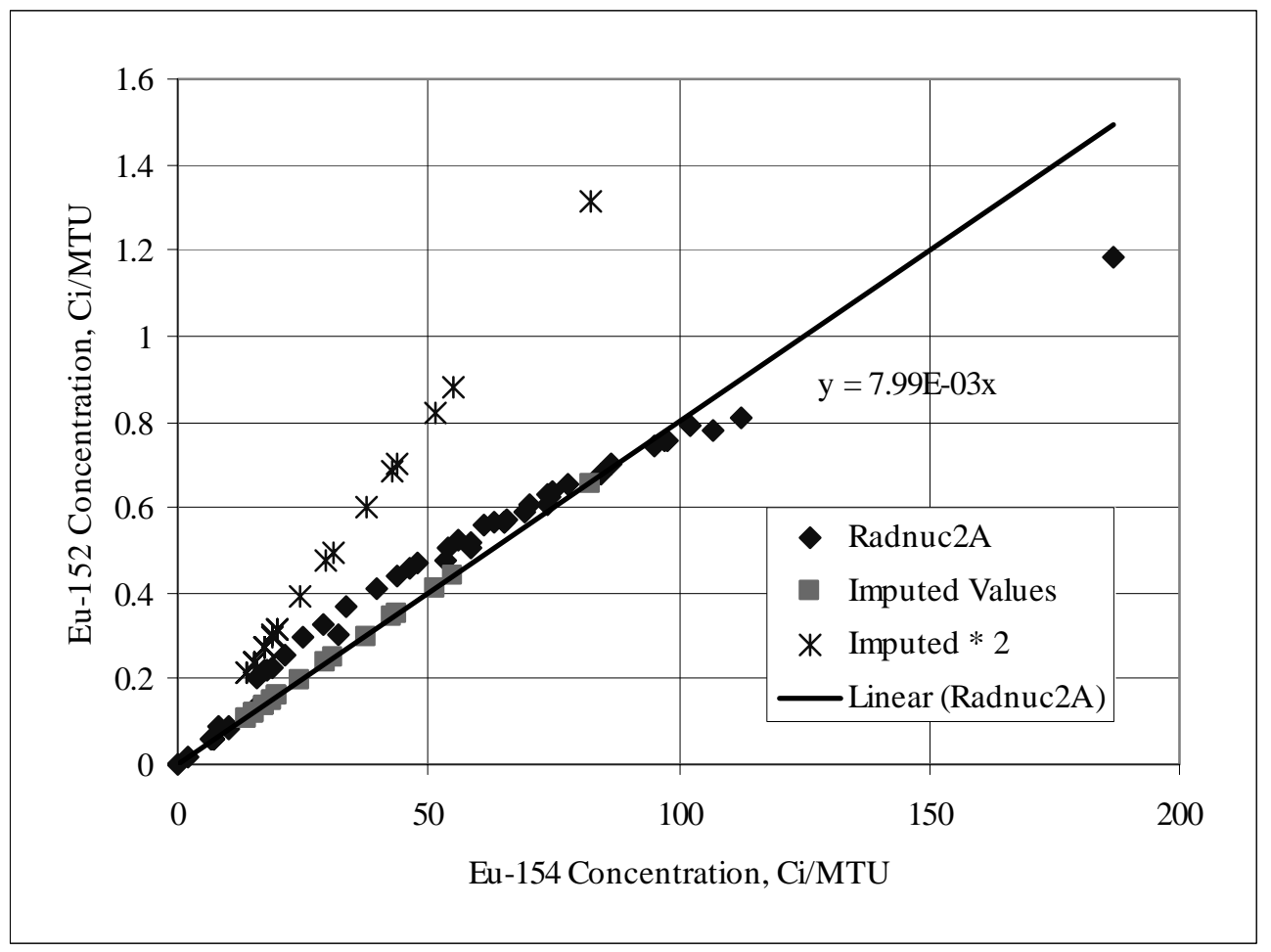


SNF-10293, REV 0

Figure 3-10. ${ }^{241} \mathrm{Pu}$ Imputations from ${ }^{238} \mathrm{Pu}$ Data and Imputed Values in Floor Sludge.

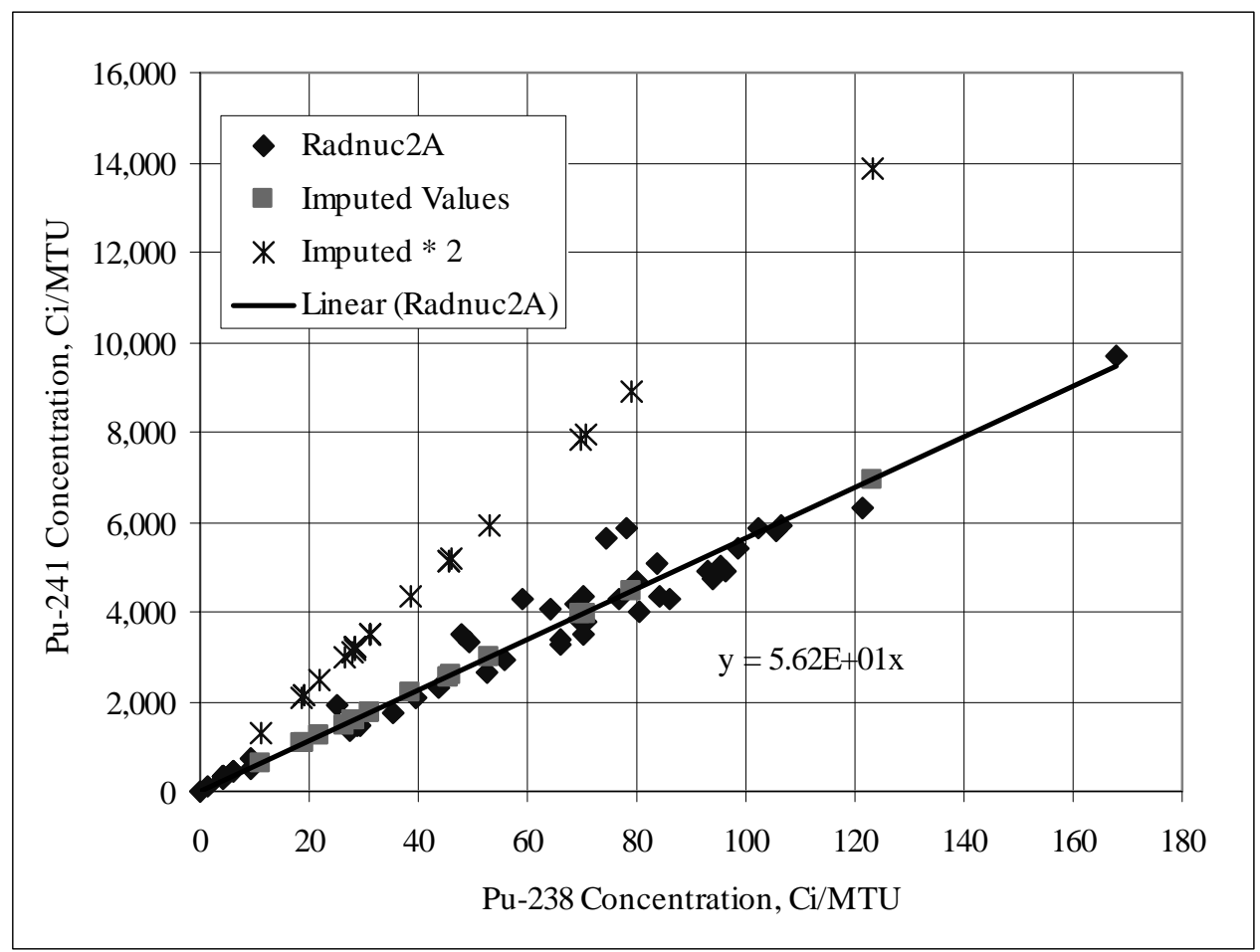

For the stochastic analysis, the preferred units for concentration are the activity or mass per unit volume of settled sludge (wet). This preference follows from the sludge depth measurements and the calculated sludge volumes being in terms of wet sludge. Imputation was done in the $\mu \mathrm{Ci} / \mathrm{g}$ (dry) data domain, and then transformed to $\mu \mathrm{Ci} / \mathrm{mL}$ settled sludge by multiplying each value by the Solids Fraction [g dry/g settled] and the Settled Density [g settled $/ \mathrm{mL}$ settled sludge]. Values for these quantities are listed in Appendix D of SNF-7765.

A complete listing of the floor sludge concentrations is given in Table 3-4, Table 3-5, and Table 3-6. The values imputed for the stochastic analysis are marked with asterisks $(*)$. Also shown in the table are the mean and standard deviations as well as the geometric mean and geometric standard deviation. The geometric mean is the exponential of the mean of the logarithms of the data, while the geometric standard deviation is the exponential of the standard deviation of the logarithms. These summary statistics include the influence of the imputed values and thus may differ from the mean values given in SNF-7765. The mean values are used in later calculations to verify the stochastic calculation. Note that the averages do not include contributions from the pits or floor areas outside of cubicles that have fuel. 
SNF-10293, REV 0

Table 3-4. Floor Sample Data and Imputed Values.

\begin{tabular}{|c|c|c|c|c|c|}
\hline $\begin{array}{l}\text { Sample } \\
\text { Number }\end{array}$ & $\begin{array}{c}\text { Sludge Density } \\
(\mathrm{kg} / \mathrm{L})\end{array}$ & $\begin{array}{l}\text { Uranium } \\
(\mathrm{kg} / \mathrm{L})\end{array}$ & $\begin{array}{c}\text { Co-60 } \\
(\mu \mathrm{Ci} / \mathbf{m L})\end{array}$ & $\begin{array}{c}\mathrm{Sr}-90 \\
(\mu \mathrm{Ci} / \mathrm{mL})\end{array}$ & $\begin{array}{c}\text { Tc-99 } \\
(\mu \mathrm{Ci} / \mathrm{mL})\end{array}$ \\
\hline L-01 & 1.15 & $1.28 \mathrm{E}-01$ & $6.26 \mathrm{E}-01$ & $4.08 \mathrm{E}+02$ & $* 7.00 \mathrm{E}-02$ \\
\hline A-02 & 1.20 & $2.03 \mathrm{E}-02$ & 8.24E-01 & $1.41 \mathrm{E}+01$ & $* 3.84 \mathrm{E}-02$ \\
\hline B-03 & 1.04 & $1.96 \mathrm{E}-02$ & $6.80 \mathrm{E}-01$ & $1.75 \mathrm{E}+01$ & * 5.34E-02 \\
\hline C-04 & 1.15 & 2.12E-02 & $6.62 \mathrm{E}-01$ & $5.22 \mathrm{E}+01$ & $* 7.70 \mathrm{E}-02$ \\
\hline $\mathrm{N}-05$ & 1.11 & $3.79 \mathrm{E}-02$ & $1.05 \mathrm{E}+00$ & $5.57 \mathrm{E}+01$ & $* 7.55 \mathrm{E}-02$ \\
\hline J-06 & 1.25 & $2.83 \mathrm{E}-02$ & 7.49E-01 & $3.19 \mathrm{E}+01$ & $* 4.79 \mathrm{E}-02$ \\
\hline H-08 & 1.21 & $1.76 \mathrm{E}-03$ & $1.16 \mathrm{E}-01$ & $6.04 \mathrm{E}+01$ & $* 1.76 \mathrm{E}-03$ \\
\hline O-09 & 2.28 & $* 6.86 \mathrm{E}-01$ & $2.08 \mathrm{E}+00$ & $2.02 \mathrm{E}+03$ & $* 4.57 \mathrm{E}-01$ \\
\hline F10 & 1.35 & $1.88 \mathrm{E}-02$ & $2.74 \mathrm{E}+00$ & $6.26 \mathrm{E}+00$ & $* 2.24 \mathrm{E}-02$ \\
\hline E11 & 1.14 & $2.00 \mathrm{E}-02$ & $5.86 \mathrm{E}-01$ & $1.66 \mathrm{E}+01$ & * 5.00E-02 \\
\hline K12 & 1.40 & $2.02 \mathrm{E}-02$ & $2.04 \mathrm{E}+00$ & $3.99 \mathrm{E}+00$ & $* 2.01 \mathrm{E}-02$ \\
\hline I15 & 1.30 & $3.71 \mathrm{E}-02$ & $1.96 \mathrm{E}+00$ & $4.51 \mathrm{E}+01$ & $* 3.49 \mathrm{E}-02$ \\
\hline M13avg & 1.43 & $* 6.29 \mathrm{E}-02$ & $7.41 \mathrm{E}-01$ & $1.92 \mathrm{E}+02$ & $* 1.25 \mathrm{E}-01$ \\
\hline KC-4 (1) & 1.24 & $6.85 \mathrm{E}-02$ & 4.30E-01 & $2.69 \mathrm{E}+02$ & $* 1.09 \mathrm{E}-01$ \\
\hline $\mathrm{KC}-4$ (2) & 1.24 & $6.85 \mathrm{E}-02$ & 4.30E-01 & $2.69 \mathrm{E}+02$ & $* 1.09 \mathrm{E}-01$ \\
\hline KC-4 (3) & 1.24 & $6.85 \mathrm{E}-02$ & 4.30E-01 & $2.69 \mathrm{E}+02$ & $* 1.09 \mathrm{E}-01$ \\
\hline KC-5 (1) & 1.19 & $2.68 \mathrm{E}-02$ & $4.57 \mathrm{E}-01$ & $* 7.93 \mathrm{E}+01$ & * 3.82E-02 \\
\hline KC-5 (2) & 1.19 & $2.68 \mathrm{E}-02$ & 4.57E-01 & $* 7.93 \mathrm{E}+01$ & * $3.82 \mathrm{E}-02$ \\
\hline $\mathrm{KC}-5(3)$ & 1.19 & $2.68 \mathrm{E}-02$ & $4.57 \mathrm{E}-01$ & $* 7.93 \mathrm{E}+01$ & $* 3.82 \mathrm{E}-02$ \\
\hline FE-1 (1) & 1.21 & $1.04 \mathrm{E}-02$ & 4.22E-01 & $* 3.09 \mathrm{E}+01$ & $* 1.70 \mathrm{E}-02$ \\
\hline FE-1 (2) & 1.21 & $1.04 \mathrm{E}-02$ & $4.22 \mathrm{E}-01$ & $* 3.09 \mathrm{E}+01$ & $* 1.70 \mathrm{E}-02$ \\
\hline FE-7 (1) & 1.24 & $3.20 \mathrm{E}-02$ & $1.79 \mathrm{E}-01$ & $5.95 \mathrm{E}+01$ & $* 3.00 \mathrm{E}-02$ \\
\hline FE-7 (2) & 1.24 & $3.20 \mathrm{E}-02$ & $1.79 \mathrm{E}-01$ & $5.95 \mathrm{E}+01$ & $* 3.00 \mathrm{E}-02$ \\
\hline Mean & 1.27 & 0.0641 & 8.14E-01 & $1.80 \mathrm{E}+02$ & $6.99 \mathrm{E}-02$ \\
\hline Std Dev & 0.237 & 0.138 & 7.00E-01 & 4.14E+02 & $9.10 \mathrm{E}-02$ \\
\hline Geo Mean & 1.22 & 0.0308 & $6.04 \mathrm{E}-01$ & $6.14 \mathrm{E}+01$ & 4.39E-02 \\
\hline Geo Std Dev & 1.84 & 2.96 & 2.20 & 4.08 & 2.82 \\
\hline
\end{tabular}

- Values shown without asterisks are listed in SNF-7765 Appendix D. Values marked with asterisks are imputed from other isotope amounts. The uranium imputation for M13avg is the Sr-90 concentration times 3.10E-04. The Sr-90 imputations are the uranium concentration times 2,960. Note that the slopes shown in Table 3-2 have uranium concentration in micrograms per gram; hence there is a factor of 1.0E6 difference in the slope.

- Values for Tc-99 were created by multiplying the Pu-239/240 concentration by 0.00699 . This factor was obtained from the canister sludge radioactivity estimates described in HNF-8760. 
Table 3-5. Floor Sample Data, Cs-134 to Eu-155.

\begin{tabular}{|c|c|c|c|c|c|}
\hline $\begin{array}{l}\text { Sample } \\
\text { Number }\end{array}$ & $\begin{array}{c}\text { Cs-134 } \\
(\mu \mathrm{Ci} / \mathrm{mL})\end{array}$ & $\begin{array}{c}\text { Cs-137 } \\
(\mu \mathrm{Ci} / \mathbf{m L})\end{array}$ & $\begin{array}{c}\text { Eu-152 } \\
(\mu \mathrm{Ci} / \mathrm{mL})\end{array}$ & $\begin{array}{c}\text { Eu-154 } \\
(\mu \mathrm{Ci} / \mathrm{mL})\end{array}$ & $\begin{array}{c}\text { Eu-155 } \\
(\mu \mathrm{Ci} / \mathrm{mL})\end{array}$ \\
\hline L-01 & $* 7.94 \mathrm{E}-02$ & $1.78 \mathrm{E}+02$ & $* 3.16 \mathrm{E}-02$ & $3.95 \mathrm{E}+00$ & $1.93 \mathrm{E}+00$ \\
\hline A-02 & $* 9.32 \mathrm{E}-03$ & $2.09 \mathrm{E}+01$ & $* 6.97 \mathrm{E}-03$ & $8.72 \mathrm{E}-01$ & 3.94E-01 \\
\hline B-03 & $* 7.81 \mathrm{E}-03$ & $1.75 \mathrm{E}+01$ & $* 8.62 \mathrm{E}-03$ & $1.08 \mathrm{E}+00$ & $5.27 \mathrm{E}-01$ \\
\hline C-04 & $* 1.58 \mathrm{E}-02$ & $3.55 \mathrm{E}+01$ & $* 1.39 \mathrm{E}-02$ & $1.74 \mathrm{E}+00$ & 7.72E-01 \\
\hline $\mathrm{N}-05$ & $3.13 \mathrm{E}-02$ & $7.52 \mathrm{E}+01$ & $* 1.33 \mathrm{E}-02$ & $1.67 \mathrm{E}+00$ & 7.33E-01 \\
\hline J-06 & $* 1.22 \mathrm{E}-02$ & $2.74 \mathrm{E}+01$ & $* 6.73 \mathrm{E}-03$ & $8.42 \mathrm{E}-01$ & 4.05E-01 \\
\hline $\mathrm{H}-08$ & 4.57E-02 & $8.98 \mathrm{E}+01$ & $* 2.62 \mathrm{E}-04$ & $* 3.28 \mathrm{E}-02$ & $* 1.61 \mathrm{E}-02$ \\
\hline O-09 & $* 9.61 \mathrm{E}-01$ & $2.15 \mathrm{E}+03$ & $* 1.04 \mathrm{E}-01$ & $1.30 \mathrm{E}+01$ & $5.99 \mathrm{E}+00$ \\
\hline F10 & $* 1.33 \mathrm{E}-02$ & $2.97 \mathrm{E}+01$ & $* 3.68 \mathrm{E}-03$ & 4.60E-01 & $2.34 \mathrm{E}-01$ \\
\hline E11 & $* 9.52 \mathrm{E}-03$ & $2.13 \mathrm{E}+01$ & $* 8.22 \mathrm{E}-03$ & $1.03 \mathrm{E}+00$ & $5.09 \mathrm{E}-01$ \\
\hline $\mathrm{K} 12$ & $* 7.73 \mathrm{E}-03$ & $1.73 \mathrm{E}+01$ & $* 3.21 \mathrm{E}-03$ & 4.01E-01 & 2.67E-01 \\
\hline $\mathrm{I} 15$ & $* 2.98 \mathrm{E}-02$ & $6.69 \mathrm{E}+01$ & $* 5.06 \mathrm{E}-03$ & $* 6.33 \mathrm{E}-01$ & 3.24E-01 \\
\hline M13avg & $1.39 \mathrm{E}-01$ & $2.99 \mathrm{E}+02$ & $* 1.88 \mathrm{E}-02$ & $2.36 \mathrm{E}+00$ & $8.43 \mathrm{E}-01$ \\
\hline $\mathrm{KC}-4(1)$ & $* 2.97 \mathrm{E}-01$ & $6.66 \mathrm{E}+02$ & $* 8.24 \mathrm{E}-03$ & $1.03 \mathrm{E}+00$ & $* 8.72 \mathrm{E}-01$ \\
\hline $\mathrm{KC}-4(2)$ & $* 2.97 \mathrm{E}-01$ & $6.66 \mathrm{E}+02$ & $* 8.24 \mathrm{E}-03$ & $1.03 \mathrm{E}+00$ & $* 8.72 \mathrm{E}-01$ \\
\hline KC-4 (3) & $* 2.97 \mathrm{E}-01$ & $6.66 \mathrm{E}+02$ & $* 8.24 \mathrm{E}-03$ & $1.03 \mathrm{E}+00$ & $* 8.72 \mathrm{E}-01$ \\
\hline $\mathrm{KC}-5(1)$ & $1.09 \mathrm{E}-02$ & $5.51 \mathrm{E}+01$ & $* 3.71 \mathrm{E}-03$ & 4.64E-01 & $1.79 \mathrm{E}-01$ \\
\hline $\mathrm{KC}-5(2)$ & $1.09 \mathrm{E}-02$ & $5.51 \mathrm{E}+01$ & $* 3.71 \mathrm{E}-03$ & 4.64E-01 & 1.79E-01 \\
\hline $\mathrm{KC}-5$ (3) & $1.09 \mathrm{E}-02$ & $5.51 \mathrm{E}+01$ & $* 3.71 \mathrm{E}-03$ & 4.64E-01 & $1.79 \mathrm{E}-01$ \\
\hline FE-1 (1) & $* 6.57 \mathrm{E}-03$ & $1.47 \mathrm{E}+01$ & $* 1.65 \mathrm{E}-03$ & $2.06 \mathrm{E}-01$ & 7.30E-02 \\
\hline FE-1 (2) & $* 6.57 \mathrm{E}-03$ & $1.47 \mathrm{E}+01$ & $* 1.65 \mathrm{E}-03$ & $2.06 \mathrm{E}-01$ & $7.30 \mathrm{E}-02$ \\
\hline FE-7 (1) & $* 1.41 \mathrm{E}-01$ & $3.16 \mathrm{E}+02$ & $* 3.48 \mathrm{E}-03$ & $4.35 \mathrm{E}-01$ & $2.23 \mathrm{E}-01$ \\
\hline FE-7 (2) & $* 1.41 \mathrm{E}-01$ & $3.16 \mathrm{E}+02$ & $* 3.48 \mathrm{E}-03$ & 4.35E-01 & $2.23 \mathrm{E}-01$ \\
\hline Mean & $1.12 \mathrm{E}-01$ & $2.55 \mathrm{E}+02$ & $1.18 \mathrm{E}-02$ & $1.47 \mathrm{E}+00$ & $7.26 \mathrm{E}-01$ \\
\hline Std Dev & $2.10 \mathrm{E}-01$ & $4.68 \mathrm{E}+02$ & 2.12E-02 & $2.65 \mathrm{E}+00$ & $1.22 \mathrm{E}+00$ \\
\hline Geo Mean & $3.41 \mathrm{E}-02$ & $8.46 \mathrm{E}+01$ & $5.91 \mathrm{E}-03$ & 7.39E-01 & $3.64 \mathrm{E}-01$ \\
\hline Geo Std Dev & 4.62 & 4.35 & 3.16 & 3.16 & 3.35 \\
\hline
\end{tabular}

- Values shown are listed in SNF-7765 Appendix D. Values marked with asterisks are imputed from other isotope amounts. The Cs-134 imputations are the Cs-137 concentration times 4.46E-04. The Eu-154 imputations are the Am-241 concentration times 0.153. The Eu-155 imputations are the Am-241 concentration times 0.0750 .

- Values for Eu-152 were created by multiplying the Eu-154 concentration by 0.00799 . This factor was obtained from the canister sludge radioactivity estimates described in HNF-8760. 
SNF-10293, REV 0

Table 3-6. Floor Sample Data, Np-237 to Am-241.

\begin{tabular}{|c|c|c|c|c|c|}
\hline $\begin{array}{l}\text { Sample } \\
\text { Number }\end{array}$ & $\begin{array}{c}\text { Np-237 } \\
(\mu \mathrm{Ci} / \mathbf{m L})\end{array}$ & $\begin{array}{c}\text { Pu-238 } \\
(\mu \mathrm{Ci} / \mathrm{mL})\end{array}$ & $\begin{array}{c}\mathrm{Pu}-239 / 240 \\
(\mu \mathrm{Ci} / \mathrm{mL})\end{array}$ & $\begin{array}{c}\text { Pu-241 } \\
(\mu \mathrm{Ci} / \mathrm{mL})\end{array}$ & $\begin{array}{c}\text { Am-241 } \\
(\mu \mathrm{Ci} / \mathrm{mL})\end{array}$ \\
\hline L-01 & $1.67 \mathrm{E}-03$ & $1.45 \mathrm{E}+00$ & $1.00 \mathrm{E}+01$ & $* 8.15 \mathrm{E}+01$ & $2.38 \mathrm{E}+01$ \\
\hline A-02 & $* 7.75 \mathrm{E}-04$ & $* 1.07 \mathrm{E}+00$ & $5.50 \mathrm{E}+00$ & $* 6.03 \mathrm{E}+01$ & $4.76 \mathrm{E}+00$ \\
\hline B-03 & $1.54 \mathrm{E}-03$ & $1.38 \mathrm{E}+00$ & $7.64 \mathrm{E}+00$ & $* 7.77 \mathrm{E}+01$ & $6.52 \mathrm{E}+00$ \\
\hline $\mathrm{C}-04$ & 8.19E-04 & $1.68 \mathrm{E}+00$ & $1.10 \mathrm{E}+01$ & $* 9.41 \mathrm{E}+01$ & $1.05 \mathrm{E}+01$ \\
\hline $\mathrm{N}-05$ & $* 1.52 \mathrm{E}-03$ & $1.75 \mathrm{E}+00$ & $1.08 \mathrm{E}+01$ & $* 9.83 \mathrm{E}+01$ & $1.02 \mathrm{E}+01$ \\
\hline J-06 & $* 9.66 \mathrm{E}-04$ & $1.10 \mathrm{E}+00$ & $6.85 \mathrm{E}+00$ & $* 6.15 \mathrm{E}+01$ & $5.20 \mathrm{E}+00$ \\
\hline H-08 & $* 3.55 \mathrm{E}-05$ & $3.87 \mathrm{E}-02$ & $2.52 \mathrm{E}-01$ & $* 2.17 \mathrm{E}+00$ & $2.15 \mathrm{E}-01$ \\
\hline O-09 & $* 9.22 \mathrm{E}-03$ & $* 1.28 \mathrm{E}+01$ & $6.54 \mathrm{E}+01$ & $* 7.18 \mathrm{E}+02$ & $8.18 \mathrm{E}+01$ \\
\hline F10 & $* 4.53 \mathrm{E}-04$ & 5.32E-01 & $3.21 \mathrm{E}+00$ & $* 2.98 \mathrm{E}+01$ & $2.75 \mathrm{E}+00$ \\
\hline E11 & $1.11 \mathrm{E}-03$ & $* 1.40 \mathrm{E}+00$ & $7.16 \mathrm{E}+00$ & $* 7.85 \mathrm{E}+01$ & $6.09 \mathrm{E}+00$ \\
\hline K12 & $* 4.05 \mathrm{E}-04$ & $* 5.61 \mathrm{E}-01$ & $2.87 \mathrm{E}+00$ & $* 3.15 \mathrm{E}+01$ & $2.17 \mathrm{E}+00$ \\
\hline $\mathrm{I} 15$ & $* 7.05 \mathrm{E}-04$ & $* 9.78 \mathrm{E}-01$ & $5.00 \mathrm{E}+00$ & $* 5.49 \mathrm{E}+01$ & $4.15 \mathrm{E}+00$ \\
\hline M13avg & $1.93 \mathrm{E}-03$ & $7.75 \mathrm{E}+00$ & $1.79 \mathrm{E}+01$ & $* 4.35 \mathrm{E}+02$ & $1.40 \mathrm{E}+01$ \\
\hline $\mathrm{KC}-4(1)$ & $* 2.20 \mathrm{E}-03$ & $1.95 \mathrm{E}+00$ & $1.56 \mathrm{E}+01$ & $* 1.09 \mathrm{E}+02$ & $1.16 \mathrm{E}+01$ \\
\hline KC-4 (2) & $* 2.20 \mathrm{E}-03$ & $1.95 \mathrm{E}+00$ & $1.56 \mathrm{E}+01$ & $* 1.09 \mathrm{E}+02$ & $1.16 \mathrm{E}+01$ \\
\hline $\mathrm{KC}-4$ (3) & $* 2.20 \mathrm{E}-03$ & $1.95 \mathrm{E}+00$ & $1.56 \mathrm{E}+01$ & $* 1.09 \mathrm{E}+02$ & $1.16 \mathrm{E}+01$ \\
\hline KC-5 (1) & $* 7.71 \mathrm{E}-04$ & 8.31E-01 & $5.47 \mathrm{E}+00$ & $* 4.67 \mathrm{E}+01$ & $5.47 \mathrm{E}+00$ \\
\hline $\mathrm{KC}-5(2)$ & $* 7.71 \mathrm{E}-04$ & $8.31 \mathrm{E}-01$ & $5.47 \mathrm{E}+00$ & $* 4.67 \mathrm{E}+01$ & $5.47 \mathrm{E}+00$ \\
\hline $\mathrm{KC}-5$ (3) & $* 7.71 \mathrm{E}-04$ & 8.31E-01 & $5.47 \mathrm{E}+00$ & $* 4.67 \mathrm{E}+01$ & $5.47 \mathrm{E}+00$ \\
\hline FE-1 (1) & $* 3.43 \mathrm{E}-04$ & $4.74 \mathrm{E}-01$ & $2.43 \mathrm{E}+00$ & $* 2.66 \mathrm{E}+01$ & $2.29 \mathrm{E}+00$ \\
\hline FE-1 (2) & $* 3.43 \mathrm{E}-04$ & 4.74E-01 & $2.43 \mathrm{E}+00$ & $* 2.66 \mathrm{E}+01$ & $2.29 \mathrm{E}+00$ \\
\hline FE-7 (1) & $* 6.05 \mathrm{E}-04$ & $6.16 \mathrm{E}-01$ & $4.29 \mathrm{E}+00$ & $* 3.46 \mathrm{E}+01$ & $2.98 \mathrm{E}+00$ \\
\hline FE-7 (2) & $* 6.05 \mathrm{E}-04$ & $6.16 \mathrm{E}-01$ & $4.29 \mathrm{E}+00$ & $* 3.46 \mathrm{E}+01$ & $2.98 \mathrm{E}+00$ \\
\hline Mean & $1.39 \mathrm{E}-03$ & $1.87 \mathrm{E}+00$ & $1.00 \mathrm{E}+01$ & $1.05 \mathrm{E}+02$ & $1.02 \mathrm{E}+01$ \\
\hline Std Dev & $1.83 \mathrm{E}-03$ & $2.81 \mathrm{E}+00$ & $1.30 \mathrm{E}+01$ & $1.58 \mathrm{E}+02$ & $1.65 \mathrm{E}+01$ \\
\hline Geo Mean & 8.74E-04 & $1.06 \mathrm{E}+00$ & $6.28 \mathrm{E}+00$ & $5.96 \mathrm{E}+01$ & $5.63 \mathrm{E}+00$ \\
\hline Geo Std Dev & 2.80 & 2.96 & 2.82 & 2.96 & 3.03 \\
\hline
\end{tabular}

- Values shown are listed in SNF-7765 Appendix D. Values marked with asterisks are imputed from other isotope amounts. The Np-237 imputations are the Pu-239/240 concentration times 1.41E-04. The Pu-238 imputations are the Pu-239/240 concentration times 0.195.

- Values for Pu-241 were created by multiplying the Pu-238 concentration by 56.2. This factor was obtained from the canister sludge radioactivity estimates described in HNF-8760. 
SNF-10293, REV 0

\subsubsection{Stochastic Model for Floor Sludge Concentration}

The stochastic analysis for the floor sludge concentration is based on the 23 samples that have been analyzed. Because there are several isotopes and the concentration of these isotopes is to some extent correlated because of their common origin in a reactor, the stochastic model must include these correlations. The method chosen to preserve existing correlations is to randomly select one of the floor sludge samples to represent all of the floor sludge associated with a key. In addition, a randomly generated factor is applied to every item of interest. The factor is generated from a uniform distribution with a mean of 1.0. Thus, for each key, the concentrations of the 13 isotopes, the uranium (total) concentration, and sludge density are chosen with two random numbers. The first random number selects the sample; the other generates a scale factor from a uniform probability distribution.

Note that the floor sludge wet density will always be greater than $1.0 \mathrm{~kg} / \mathrm{L}$, the density of water. For this reason, the floor sludge density is calculated differently than the other items. Before multiplying by the scale factor, $1.0 \mathrm{~kg} / \mathrm{L}$ is subtracted from the floor sludge density. Then the $1.0 \mathrm{~kg} / \mathrm{L}$ is added. This prevents the floor sludge density from becoming less than $1.0 \mathrm{~kg} / \mathrm{L}$.

In the stochastic analysis, the general method of choosing a sample to represent properties of a key disassociates a measurement from the key it was actually taken from. This is justified by the large number of cubicles per key $(3668 / 52=70.5$ cubicles per key on the average). If specific measurements are anchored to the cubicles from which the samples were taken, the overall effect on the stochastic analysis is expected to be small. For example, consider sample O-09, which has the highest $U$ content. This sample was taken between damaged fuel canisters, and at a location with very deep floor sludge $(19.2 \mathrm{~cm})$. For this cubicle, the floor sludge is very similar to canister sludge. Its location (in 1996) was cubicle 6070, which is one of 55 cubicles with fuel from key 14356. The base case stochastic analysis assigns a concentration randomly selected from the 23 measured or imputed values. Consider the effect when one of the 55 cubicles is assigned the measured value while the other 54 cubicles assigned a concentration randomly selected from the 23 samples. Because one cubicle is always at a higher concentration, the concentration distributions for this key will shift to slightly higher values. This shift is increased if the observed floor sludge depths in this key are used as weighting factors because sample O-09 came from a deeper than average layer of floor sludge. However, if this process is repeated for all the floor sludge samples, the overall effect on the stochastic analysis compared to the base case (which ignores sample location information) should be small. The keys with higher than average distributions will be offset by keys with lower than average distributions when the two are added together.

Consider the effect of using the information measured for one cubicle in all the adjacent cubicles. This alternate case is complicated by the fact that some of the adjacent cubicles may lie in a different key. But again, the effect on the base case should he small because the fixed cubicles will have an average near the mean. In addition, there are more than 3,600 cubicles that will still be assigned concentrations as they were in the base case. No alternate stochastic analyses were run with selected cubicles at measured concentrations while the rest are chosen from a probability density function. 
The next question is what to use as the range for the scale factor in the base case. This factor is intended to assure that the variability of the data used in the stochastic analysis is greater than or equal to the variability calculated from the characterization sample data. A 99\% confidence interval for the mean can be calculated for each analyte using the following formula where $\mathrm{N}$ is the number of observations used in determining the mean (23) and $\sigma$ is the standard deviation of the 23 measurements.

$$
\text { Mean } \pm \frac{2.576 \sigma}{\sqrt{\mathrm{N}}}
$$

The right side of this formula, $\frac{2.576 \sigma}{\sqrt{\mathrm{N}}}$, can be used to determine the range for the scale factor.

For the analytes of interest, the upper bound, relative to the mean value, was determined by dividing the value calculated from the right side of the formula by the mean value, as shown in the equation below. This upper bound, relative to the mean, was then used to determine the range for the variability factor. The calculated upper bounds are listed in Table 3-7. The average of the calculated upper bounds is 1.85 . It is concluded that the variability factor for the floor sludge concentration will range from 0.2 to 1.8 to represent the spread of the data and imputed values.

$$
\text { Bound }=1.0 \pm \frac{2.576 \sigma}{\operatorname{Mean} \sqrt{\mathrm{N}}}
$$

Table 3-7. Variability Factor for the Floor Sample Data.

\begin{tabular}{|c|c|}
\hline Measured Quantity & $\begin{array}{c}\text { Upper Bound for } \\
\text { Variability Factor }\end{array}$ \\
\hline Density & 1.47 \\
\hline Uranium & 2.16 \\
\hline Co-60 & 1.46 \\
\hline Sr-90 & 2.23 \\
\hline Tc-99 & 1.70 \\
\hline Cs-134 & 2.00 \\
\hline Cs-137 & 1.99 \\
\hline Eu-152 & 1.46 \\
\hline
\end{tabular}

\begin{tabular}{|c|c|}
\hline Measured Quantity & $\begin{array}{c}\text { Upper Bound for } \\
\text { Variability Factor }\end{array}$ \\
\hline Eu-154 & 1.97 \\
\hline Eu-155 & 1.97 \\
\hline $\mathrm{Np}-237$ & 1.91 \\
\hline $\mathrm{Pu}-238$ & 1.71 \\
\hline $\mathrm{Pu}-239 / 240$ & 1.81 \\
\hline $\mathrm{Pu}-241$ & 1.70 \\
\hline Am-241 & 1.81 \\
\hline Average & $\mathbf{1 . 8 5}$ \\
\hline
\end{tabular}

- The upper bound on the variability factor is calculated from the $99 \%$ confidence level of the mean and the mean for each material.

- The "Average" is the mean for the values shown in the table.

Four alternate cases for this assumed PDF are (1) reducing the variability factor to a much smaller value, namely, 0.9 to 1.1 , (2) using a piecewise continuous function to be the PDF for floor sludge materials, (3) using a lognormal function to represent the data, and (4) doubling the imputed values. 
The first alternative is readily tested using a different value for the range of variability. The expected outcome is smaller variability in the concentrations in the filled sludge containers because of the reduced variability in the sludge concentrations. The mean values should be the same, but the $95^{\text {th }}$ percentile value should be smaller.

The second alternative takes a different approach to representing the discrete data values. The data values are sorted to produce a cumulative distribution, which is a curve showing the probability of finding a concentration less than a particular value. The cumulative probability function is created by plotting $(\mathrm{k}-0.5) / \mathrm{N}$ versus sludge depth, where $\mathrm{k}$ is the index to the sorted depths $(\mathrm{k}=1,2, \ldots, \mathrm{N})$ and $\mathrm{N}$ is the number of samples $(\mathrm{N}=23)$. For the smallest and largest depths, probabilities of 0 and 1 , respectively, are also plotted. The plotted points are simply connected with straight lines. These assignments preserve the mean values. An example is shown in Figure 3-11 of the cumulative probability function for floor sludge density. This plot is typical of floor sludge cumulative distributions. Most samples are at the low end of the observed range, and one or two are at larger values.

Figure 3-11. Cumulative Probability Distribution Function for Floor Sludge Density.

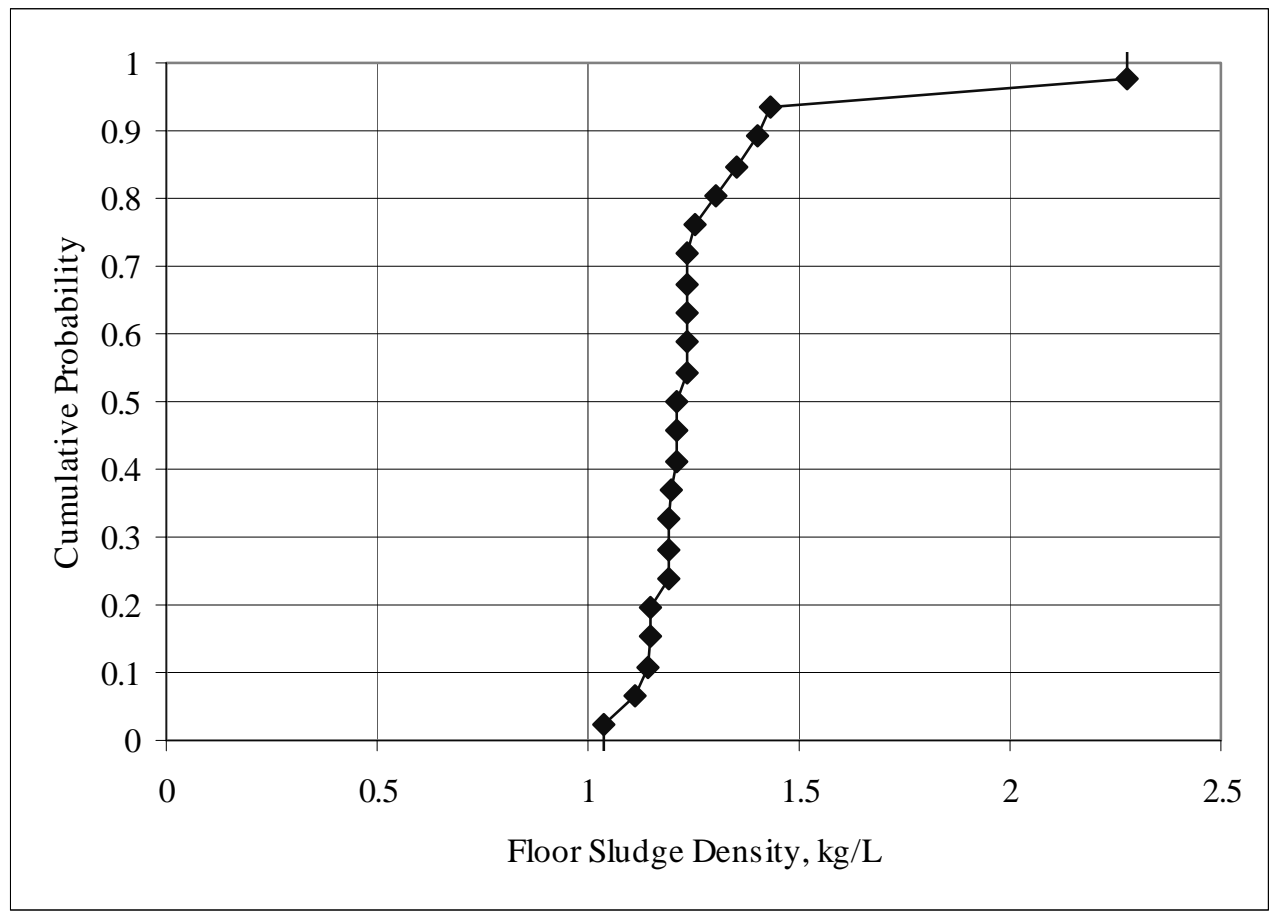

In the second alternative, the isotopic concentrations are calculated by generating one random number and finding the corresponding cumulative probability from the distributions for each material of interest. In this way, the isotopes are completely correlated. The expected outcome is again smaller concentrations in the filled sludge containers because of the reduced variability. The mean values should be no different, but the maximum and minimum must be closer. Thus the $95^{\text {th }}$ percentile result is expected to be smaller. 
In the third alternative, the geometric mean and geometric standard deviation of the data is used in a lognormal function to represent the floor sludge characterization data. The cumulative probability distribution is calculated using the formula shown below (from 26.2.23 in Abramowitz and Stegun 1964).

$$
\begin{aligned}
& \mathrm{x}=\mathrm{GM} \cdot \operatorname{Exp}(-\mathrm{f} \cdot \operatorname{Ln} \mathrm{GS}) \\
& \mathrm{f}=\mathrm{t}-\frac{\mathrm{C}_{\mathrm{A}}+\left(\mathrm{C}_{\mathrm{B}}+\mathrm{C}_{\mathrm{C}} \mathrm{t}\right) \mathrm{t}}{1+\left[\mathrm{D}_{\mathrm{A}}+\left(\mathrm{D}_{\mathrm{B}}+\mathrm{D}_{\mathrm{C}} \mathrm{t}\right) \mathrm{t}\right] \mathrm{t}} \quad \text { and } \quad \mathrm{t}=\sqrt{-2 \cdot \operatorname{Ln}(\mathrm{P})} \\
& \mathrm{C}_{\mathrm{A}}=2.515517 \quad \mathrm{D}_{\mathrm{A}}=1.432788 \\
& \mathrm{C}_{\mathrm{B}}=0.802853 \quad \mathrm{D}_{\mathrm{B}}=0.189269 \\
& \mathrm{C}_{\mathrm{C}}=0.010328 \quad \mathrm{D}_{\mathrm{C}}=0.001308
\end{aligned}
$$

where

$\mathrm{GM}=$ geometric mean value of the floor sludge data

$\mathrm{GS}=$ geometric standard deviation of the floor sludge data

$\mathrm{P}=$ cumulative probability for which the concentrations must be calculated ( $\mathrm{P}$ is a randomly chosen value between 0 and 1.)

Actually, this approximation is only valid for $\mathrm{P}<0.5$. When $\mathrm{P}>0.5$, the value of $\mathrm{P}$ is subtracted from 1 to get a number less than 0.5. This number is used in the above formula for $\mathrm{f}$. Then the sign of $\mathrm{f}$ is reversed and it is used in the equation for $\mathrm{x}$. This approach represents a cumulative lognormal density function over its entire range with an accuracy of better than one part in a thousand $(0.1 \%)$.

Using lognormal distributions to represent floor sludge data is expected to shift the mean values to slightly higher or lower values. This shift is expected because the lognormal distributions are tied to the data through the geometric mean and geometric standard deviation. In addition, the maximum values should be larger, because the lognormal distribution has a long tail near the upper limit.

In the fourth alternative, the values marked with asterisks in Tables 3-4, 3-5, and 3-6 are doubled. The only exception is the uranium concentration in sample O-09 was not changed because this imputation is a minor $(10 \%)$ adjustment to a measured value. The mean values double for Tc-99, Eu-152, and Pu-241, since these are entirely imputed. For Cs-134 and Np-237 the mean almost doubles ( $90 \%$ and $78 \%$ increase), because most of the values are imputed. The mean value for Pu-238 increases 39\%, while the mean value for Eu-155 increases 18\%. The other mean values increase less than $10 \%$.

\subsection{CANISTER SLUDGE DEPTH}

Each KE Basin canister contains from zero to fourteen spent fuel assemblies in two adjacent barrels. The spent fuel assemblies can range in condition from no apparent degradation to severe 
degradation (pieces of cladding and fuel are all that is evident). A video survey of all visible fuel canisters in the KE Basin was conducted in 1994 (WHC-SD-SNF-TI-012 [Pitner 1995]) and further data presented in 1996 (WHC-SD-SNF-TI-018 [Pitner 1996a]). In that survey, an assessment of fuel damage (good, fair, poor, and very bad) in the open canister barrels was made. Appendix B provides a map of K East Basin showing the assessed fuel condition and key number for each canister location in 1994. Note that 110 canisters (3\% of the total) did not have their fuel condition assessed by observation. The condition of these canisters was assumed to be in the same relative proportions as the $97 \%$ that were assessed. Note that the four categories differ from some other assessments. For a discussion of the fuel condition terminology, see Appendix C.

\subsubsection{Canister Sludge Depth Data}

Canister sludge depth measurements were performed in 1995 in selected canister barrels as preparation for the canister sludge sampling (WHC-SD-SNF-TI-034 [Pitner 1996b]). In a few cases, both sides of one canister were measured. Most measurements were for only one of the two barrels at any location.

The basic calculations for canister sludge volume were carried out by A. L. Pitner in WHC-SD-SNF-TI-034 and HNF-5362 (Pitner 1999b). The number of canisters is multiplied by the expected sludge depth in that type of canister and converted to a volume through multiplication by an assumed area. The sludge depth measurements are summarized in WHC-SD-SNF-TI-034. A more detailed listing of the measurements is provided in Appendix D. Additional data from 1999/2000 has been included.

The canister sludge depth measurements shown in Appendix D in a few instances gave negative values. This is interpreted to mean that the ultrasound probe was "seeing" the concrete floor through the screened holes in the bottom of the canisters. When such readings were subtracted from the assumed distance to the bottom of the canister, negative numbers are produced. None of the "bad" fuel canister barrels has negative readings. Three canister barrels containing "fair" fuel have negative values. These are at cubicle locations $1858 \mathrm{E}, 4359 \mathrm{~W}$, and $5608 \mathrm{E}$. Note that the first pair of numbers is the column position while the second pair of numbers is the row position. The last letter (E or W) indicates which of the two canister barrels was measured. Four of the "good" canister barrels have negative depth measurements. These are at locations 2244E, $2244 \mathrm{~W}, 2248 \mathrm{~W}$, and 4661E.

There are three ways that these negative results can be used. The first is to use the negative results without modification. The second is to suppose that the negative result indicates the measurement of a depth through the holes in the bottom of the canister that is below the canister. If the mean depth is less than zero, then the values are all increased by the absolute value of the mean to force the mean to become zero. This method was used in WHC-SD-SNF-TI-034. The third method is to zero all negative values. Each of these methods has been used in the calculations of mean depth. The differences in the results are small. For this reason, the second method has been chosen for consistency with the previous calculations. 
It should be noted that the canister sludge depth measurements in Appendix D are not evenly spread over the canister barrels measured. Some barrels have many measurements while others have few, or just one. It is assumed that no weighting is needed to account for the variable number of measured locations within a canister barrel.

The calculated mean and standard deviation for each sampled canister barrel is shown in Tables 3-8, 3-9, and 3-10. These numbers use the second method for dealing with negative values described above. Any mean value that is less than zero had all of the data adjusted upward equally so that the mean becomes zero. The mean and standard deviations reported in WHC-SD-SNF-TI-034 are also shown in Tables 3-8, 3-9, and 3-10 for comparison. The few differences are noted with asterisks. Cells with "na" indicate measured locations that were added after the measurements documented in WHC-SD-SNF-TI-034 were carried out. The "Overall" mean and standard deviation were computed from the mean values shown in the table.

Canister sludge depths for bad fuel are summarized in Table 3-8. Two measurements (5043W and $6070 \mathrm{~W}$ ) were off scale because the ultrasonic probe was buried in the sludge. The barrel length is 28 inches and the probe is 12 inches from the bottom of the barrel. Thus, the off scale depths lie somewhere between 12 and 28 inches. Because video camera images permit one to see into the barrel several inches, the off scale depths lie between 12 inches and 20 inches. To proceed with the stochastic analysis, it is assumed that the canister sludge depth in these two cases is 17 inches and 18 inches. Whether the stochastic calculations are sensitive to the assumed depth will be determined by running two alternate cases, one with depths of 12 and 13 inches, and the other with depths of 19 and 20 inches.

Table 3-8. Summary of Canister Sludge Depths -- Bad Fuel. (2 sheets)

\begin{tabular}{|c|c|c|c|c|}
\hline \multirow{2}{*}{$\begin{array}{c}\text { Canister Barrel } \\
\text { Location }\end{array}$} & \multicolumn{2}{|c|}{ Present Calculation } & \multicolumn{2}{c|}{ from WHC-SD-SNF-TI-034 } \\
\cline { 2 - 5 } & Mean & $\begin{array}{c}\text { Standard } \\
\text { Deviation }\end{array}$ & Mean & $\begin{array}{c}\text { Standard } \\
\text { Deviation }\end{array}$ \\
\hline $0455 \mathrm{~W}$ & 1.97 & 0.76 & $\mathrm{Na}$ & na \\
\hline $0627 \mathrm{~W}$ & 1.00 & 0.59 & 1.00 & 0.59 \\
\hline $0668 \mathrm{E}$ & 3.23 & 1.37 & 3.23 & 1.37 \\
\hline $0668 \mathrm{~W}$ & 1.81 & 0.98 & $\mathrm{Na}$ & na \\
\hline $1236 \mathrm{~W}$ & 4.36 & 0.31 & $* 4.61 *$ & $* 4.36 *$ \\
\hline $1243 \mathrm{~W}$ & 2.27 & 0.39 & 2.27 & 0.39 \\
\hline $1267 \mathrm{E}$ & 4.77 & 2.41 & 4.77 & 2.41 \\
\hline $2024 \mathrm{E}$ & 1.17 & 1.01 & $\mathrm{Na}$ & na \\
\hline $2031 \mathrm{~W}$ & 1.44 & 1.28 & $\mathrm{Na}$ & na \\
\hline $2211 \mathrm{~W}$ & 4.02 & 1.92 & 4.02 & 1.92 \\
\hline $2214 \mathrm{E}$ & 2.26 & 1.11 & 2.26 & 1.11 \\
\hline $2229 \mathrm{E}$ & 3.31 & 1.75 & 3.31 & 1.75 \\
\hline $2350 \mathrm{E}$ & 3.44 & 1.23 & 3.44 & 1.23 \\
\hline $2355 \mathrm{~W}$ & 1.68 & 0.59 & 1.68 & 0.59 \\
\hline $2540 \mathrm{E}$ & 1.16 & 0.59 & 1.16 & 0.59 \\
\hline
\end{tabular}


SNF-10293, REV 0

Table 3-8. Summary of Canister Sludge Depths -- Bad Fuel. (2 sheets)

\begin{tabular}{|c|c|c|c|c|}
\hline \multirow[b]{2}{*}{$\begin{array}{c}\text { Canister Barrel } \\
\text { Location }\end{array}$} & \multicolumn{2}{|c|}{ Present Calculation } & \multicolumn{2}{|c|}{ from WHC-SD-SNF-TI-034 } \\
\hline & Mean & $\begin{array}{l}\text { Standard } \\
\text { Deviation }\end{array}$ & Mean & $\begin{array}{l}\text { Standard } \\
\text { Deviation }\end{array}$ \\
\hline $2722 \mathrm{E}$ & 1.80 & 0.59 & 1.80 & 0.59 \\
\hline $2756 \mathrm{E}$ & 1.36 & 0.71 & 1.36 & 0.71 \\
\hline $3128 \mathrm{~W}$ & 3.34 & 2.26 & 3.34 & 2.26 \\
\hline $3971 \mathrm{~W}$ & 1.24 & 0.36 & 1.24 & 0.36 \\
\hline $4143 \mathrm{~W}$ & 2.29 & 1.70 & 2.29 & 1.70 \\
\hline $4564 \mathrm{E}$ & 2.01 & 0.38 & $\mathrm{Na}$ & na \\
\hline $4564 \mathrm{~W}$ & 5.93 & 3.35 & $\mathrm{Na}$ & na \\
\hline $4569 \mathrm{E}$ & 3.42 & --- & 3.42 & --- \\
\hline $4569 \mathrm{~W}$ & 10.59 & --- & 10.59 & --- \\
\hline $4571 \mathrm{E}$ & 7.41 & 1.73 & $\mathrm{Na}$ & na \\
\hline $4850 \mathrm{~W}$ & 2.10 & 0.93 & $\mathrm{Na}$ & na \\
\hline ? 5043W ? & 17.00 & --- & 12.5 & -- \\
\hline $5055 \mathrm{~W}$ & 5.66 & 2.01 & 5.66 & 2.01 \\
\hline $5427 \mathrm{~W}$ & 3.24 & 3.05 & 3.24 & 3.05 \\
\hline $5445 \mathrm{E}$ & 7.52 & 3.40 & 7.52 & 3.40 \\
\hline $5465 \mathrm{E}$ & 6.84 & --- & 6.84 & --- \\
\hline $5465 \mathrm{~W}$ & 8.55 & 1.99 & 8.55 & $* 1.87 *$ \\
\hline ? 6070W ? & 18.00 & --- & $\mathrm{Na}$ & na \\
\hline $6071 \mathrm{~W}$ & 8.74 & 2.12 & 8.74 & 2.12 \\
\hline Overall & $4.56 \mathrm{in.}$ & $4.14 \mathrm{in.}$ & $5.32 \mathrm{in.}$ & Not given \\
\hline
\end{tabular}

- All measured values have units of inches.

- The "na" means the values have been added later based on internal memos and logbook notations.

- The "---" indicates there was one measurement in the barrel. Hence, a standard deviation could not be calculated.

- Asterisks indicate numbers in WHC-SD-SNF-TI-034 that differ from the present calculation.

- The line labeled "Overall" shows the mean and standard deviation of the above 34 depths. The mean value from WHC-SD-SNF-TI-034 is for aluminum canisters only.

- The question marks identify two locations where the canister sludge depth was greater than 12 inches. Video camera observations suggest the sludge depth is less than 20 inches. The values shown have been assumed for the base case.

The sludge depths for bad fuel were originally divided into bad fuel located in aluminum canisters and bad fuel located in stainless steel canisters. The mean depth in the aluminum canisters was 5.32 in., while the mean depth in stainless steel canisters was 1.88 in. A later evaluation (HNF-5362) noted that some aluminum canisters were misidentified as stainless steel 
based on the amount of corrosion evident on the canister. In addition, the number of stainless steel canisters with bad fuel is listed as zero. Thus, all canisters with bad fuel are aluminum, and all the depth measurements should be averaged together. Nevertheless, the original depth for aluminum canisters (5.32 in.) was used for all bad canisters. The present calculation uses the measured depths for all bad fuel canisters and assumes they are all made of aluminum. Thus, the mean shown in the last line of Table 3-8 is more than one inch smaller than the mean used in WHC-SD-SNF-TI-034 and HNF-5362. The data for fuel designated as "bad" is assumed to represent fuel designated as "poor".

Canister sludge depths for fair fuel are summarized in Table 3-9. The sludge depths for fair fuel are separated into aluminum and stainless steel canisters. It has been assumed that the canister types shown in WHC-SD-SNF-TI-034 are correct. These assignments lead to the averages shown in Table 3-9. Because the key summaries do not distinguish between canister types, a weighted average was constructed to calculate the depth of sludge in canisters with fair fuel. The weighted mean was calculated using the formulas shown below. There are $2 \times 1,256=2,512$ aluminum canister barrels and $2 \times 330=660$ stainless steel canister barrels containing fair fuel.

$$
\text { Mean }=\frac{\mathrm{N}_{\mathrm{AL}} \mathrm{M}_{\mathrm{AL}}+\mathrm{N}_{\mathrm{SS}} \mathrm{M}_{\mathrm{SS}}}{\mathrm{N}_{\mathrm{AL}}+\mathrm{N}_{\mathrm{SS}}}
$$

Where:

$$
\begin{aligned}
\mathrm{N}_{\mathrm{AL}} \& \mathrm{~N}_{\mathrm{SS}}=\quad \begin{array}{l}
\text { number of aluminum and stainless steel canister barrels }(2,512 \\
\text { and } 660)
\end{array} \\
\mathrm{M}_{\mathrm{AL}} \& \mathrm{M}_{\mathrm{SS}}=\begin{array}{l}
\text { mean values for aluminum and stainless steel canister sludge } \\
\text { measurements }(1.74 \text { in. and } 1.12 \text { in. })
\end{array} \\
\text { Mean }=\begin{array}{l}
\text { weighted mean for any canister containing fuel in fair condition } \\
(1.61 \text { in. })
\end{array}
\end{aligned}
$$

Using the weighted mean formula ensures that the canister sludge volume calculated from the canister counts would agree with the volume calculated for individual keys and summed. For fuel in fair condition, the weighted mean canister sludge depth is $1.61 \mathrm{in}$.

Table 3-9. Summary of Canister Sludge Depths -- Fair Fuel. (2 sheets)

\begin{tabular}{|c|c|c|c|c|}
\hline \multirow{2}{*}{$\begin{array}{c}\text { Canister Barrel } \\
\text { Location }\end{array}$} & Mean & $\begin{array}{c}\text { Standard } \\
\text { Deviation }\end{array}$ & \multicolumn{2}{c|}{ From WHC-SD-SNF-TI-034 } \\
\cline { 2 - 5 } Mean & $\begin{array}{c}\text { Standard } \\
\text { Deviation }\end{array}$ \\
\hline \hline Stainless Steel Canisters & \multicolumn{5}{|l}{} \\
\hline $0418 \mathrm{E}$ & 0.66 & 0.32 & 0.66 & 0.32 \\
\hline $3125 \mathrm{~W}$ & 1.42 & 0.19 & na & na \\
\hline$-4359 \mathrm{~W}-$ & 0.28 & 0.17 & 0.28 & 0.17 \\
\hline $5238 \mathrm{~W}$ & 1.24 & 1.12 & 1.24 & 1.10 \\
\hline
\end{tabular}


SNF-10293, REV 0

Table 3-9. Summary of Canister Sludge Depths -- Fair Fuel. (2 sheets)

\begin{tabular}{|c|c|c|c|c|}
\hline \multirow[b]{2}{*}{$\begin{array}{c}\text { Canister Barrel } \\
\text { Location }\end{array}$} & \multicolumn{2}{|c|}{ Present Calculation } & \multicolumn{2}{|c|}{ From WHC-SD-SNF-TI-034 } \\
\hline & Mean & $\begin{array}{l}\text { Standard } \\
\text { Deviation }\end{array}$ & Mean & $\begin{array}{l}\text { Standard } \\
\text { Deviation }\end{array}$ \\
\hline$-5608 \mathrm{E}-$ & 0.00 & 0.15 & 0.00 & 0.15 \\
\hline $5618 \mathrm{E}$ & 0.85 & 0.15 & 0.85 & 0.15 \\
\hline $5618 \mathrm{~W}$ & 1.53 & 0.33 & 1.53 & 0.33 \\
\hline $6057 \mathrm{E}$ & 1.91 & 1.40 & 1.91 & 1.40 \\
\hline $6413 \mathrm{E}$ & 1.46 & 0.31 & 1.46 & 0.31 \\
\hline $6413 W$ & 1.83 & 0.27 & 1.83 & 0.27 \\
\hline Overall & $1.12 \mathrm{in.}$ & 0.645 in. & 1.15 & not given \\
\hline \multicolumn{5}{|c|}{ Aluminum Canisters } \\
\hline $0450 \mathrm{E}$ & 2.93 & 1.34 & na & na \\
\hline$-1858 \mathrm{E}-$ & 0.81 & 1.03 & 0.81 & 1.03 \\
\hline $2210 \mathrm{E}$ & 1.06 & 0.51 & 1.06 & 0.51 \\
\hline $2353 \mathrm{E}$ & 1.79 & 0.97 & 1.79 & 0.97 \\
\hline $2711 \mathrm{E}$ & 1.70 & 1.44 & 1.71 & 1.44 \\
\hline $2905 \mathrm{E}$ & 1.65 & 0.54 & na & na \\
\hline $3727 \mathrm{~W}$ & 3.02 & 2.03 & 3.02 & 2.03 \\
\hline $4138 \mathrm{~W}$ & 3.32 & 0.78 & 3.32 & 0.78 \\
\hline $4168 \mathrm{E}$ & 0.38 & 0.15 & $* 0.39 *$ & 0.15 \\
\hline $4168 \mathrm{~W}$ & 0.43 & 0.12 & 0.44 & 0.12 \\
\hline $4620 \mathrm{~W}$ & 2.67 & 0.90 & 2.67 & 0.90 \\
\hline $4627 \mathrm{~W}$ & 1.31 & 0.66 & 1.31 & 0.66 \\
\hline $4865 \mathrm{~W}$ & 2.05 & 0.79 & 2.05 & $* 0.76 *$ \\
\hline $4869 \mathrm{E}$ & 2.59 & 0.74 & na & na \\
\hline $5069 \mathrm{E}$ & 2.74 & 1.50 & 2.74 & 1.50 \\
\hline $5254 \mathrm{E}$ & 1.32 & 0.50 & 1.32 & 0.50 \\
\hline $5427 \mathrm{E}$ & 1.58 & 0.48 & 1.58 & 0.48 \\
\hline $6069 \mathrm{~W}$ & 0.07 & na & 0.07 & --- \\
\hline Overall & $1.74 \mathrm{in.}$ & $0.980 \mathrm{in.}$ & $1.63 \mathrm{in.}$ & not given \\
\hline
\end{tabular}

- All measured values have units of inches.

- Cubicle locations marked with dashes (1858E, 4359W, and 5608E) have negative depth readings. For cubicle 5608E, the mean was also negative. The substitution of zero depth is described in the text.

- The "na" means the values have been added later based on internal memos and logbook notations.

- Asterisks indicate numbers in WHC-SD-SNF-TI-034 that differ from the present calculation.

- The "Overall" mean and standard deviation were computed from the canister barrel mean depths shown above. 
Canister sludge depths for good fuel are listed in Table 3-10. While good fuel is found in both stainless steel and aluminum canisters, the depth measurements were only taken in stainless steel canisters. The measurements for stainless steel canisters are assumed to apply to aluminum canisters. The same mean value is used on both types. It should also be noted that more canisters contain good fuel than contain any other kind. However, the number of good canisters that were measured is the smallest.

Table 3-10. Summary of Canister Sludge Depths -- Good Fuel.

\begin{tabular}{|c|c|c|c|c|}
\hline \multirow{2}{*}{$\begin{array}{c}\text { Canister Barrel } \\
\text { Location }\end{array}$} & \multicolumn{2}{|c|}{ Present Calculation } & \multicolumn{2}{c|}{ from WHC-SD-SNF-TI-034 } \\
\cline { 2 - 5 } & Mean & $\begin{array}{c}\text { Standard } \\
\text { Deviation }\end{array}$ & $\begin{array}{c}\text { Standard } \\
\text { Deviation }\end{array}$ \\
\hline $1845 \mathrm{E}$ & 2.33 & 0.35 & 2.33 & 0.35 \\
\hline$-2244 \mathrm{E}-$ & 0.0 & 0.26 & 0.00 & 0.26 \\
\hline$-2244 \mathrm{~W}-$ & 0.0 & 0.16 & 0.00 & 0.16 \\
\hline$-2248 \mathrm{~W}-$ & 0.0 & 0.08 & 0.00 & 0.08 \\
\hline$-4661 \mathrm{E}-$ & 0.0 & 0.20 & 0.00 & 0.20 \\
\hline \hline Overall & 0.465 in. & 1.04 in. & 0.47 in. & not given \\
\hline
\end{tabular}

- All measured values have units of inches.

- The cubicle locations marked with dashes have negative mean values. These have been changed to zero, as discussed in the text.

- The above overall mean values are used for both aluminum and stainless steel canisters.

The total canister sludge volume is calculated in Table 3-11 using the same method as WHC-SD-SNF-TI-034; namely, the depth is multiplied by an assumed area to give the sludge volume in one canister. The "good" and "fair" canisters generally have 7 fuel assemblies per canister barrel and therefore use the conversion factor $0.620 \mathrm{~L} / \mathrm{inch}$. The "poor" and "bad" canisters generally have 6 fuel assemblies per canister barrel and therefore use the conversion factor $0.770 \mathrm{~L} / \mathrm{inch}$. These depth-to-volume conversions are from HNF-5362 Section 4.0, and use $329 \mathrm{~cm}^{2}$ as the surface area of the bottom of one canister barrel. This corresponds to an inside diameter of $8.057 \mathrm{inch}$. The average area displaced by a fuel assembly is $29.6 \mathrm{~cm}^{2}$, which corresponds to a diameter of $2.415 \mathrm{inch}$, assuming there is no sludge in the annulus between the inner assembly and the outer assembly. The total canister sludge volume is summarized in Table 3-11. The values shown differ from HNF-5362. The difference is small (3\%) and largely because of the change in the mean depth for poor and bad fuel canisters.

The canister sludge volume will also be calculated using information about the distribution of the various fuel categories by key. The number of canisters of each kind (good, fair, poor, or bad) for each key is shown in Table 3-12. In 1995 there were 1,065 empty canisters in K East Basin (WHC-SD-SNF-TI-006 Appendix A). These have not been included in the stochastic analysis. The small amount of sludge that was present in the empty canisters can be regarded as floor sludge. Because this sludge is located outside cubicles with fuel, it is not included in the stochastic analysis. 
SNF-10293, REV 0

Table 3-11. Calculation of Total Canister Sludge Volumes (Liters).

\begin{tabular}{|c|c|c|c|c|}
\hline $\begin{array}{c}\text { Fuel } \\
\text { Category }\end{array}$ & Canister Type & $\begin{array}{c}\text { Number of } \\
\text { Canisters }\end{array}$ & $\begin{array}{c}\text { Recomputed } \\
\text { Mean }\end{array}$ & $\begin{array}{c}\text { Previous Mean } \\
\text { (HNF-5362) }\end{array}$ \\
\hline \multirow{2}{*}{ Good } & Stainless Steel & 686 & $198 \mathrm{~L}$ & $200 \mathrm{~L}$ \\
\hline & Aluminum & 1,186 & $342 \mathrm{~L}$ & $346 \mathrm{~L}$ \\
\hline \multirow{2}{*}{ Fair } & Stainless Steel & 330 & $229 \mathrm{~L}$ & $235 \mathrm{~L}$ \\
\hline & Aluminum & 1,256 & $1,359 \mathrm{~L}$ & $1,269 \mathrm{~L}$ \\
\hline Poor & Aluminum & 154 & $540 \mathrm{~L}$ & $631 \mathrm{~L}$ \\
\hline $\mathrm{Bad}$ & Aluminum & 56 & $197 \mathrm{~L}$ & $229 \mathrm{~L}$ \\
\hline \multicolumn{2}{|c|}{ Totals: } & 3,668 & $2,864 \mathrm{~L}$ & $2,910 \mathrm{~L}$ \\
\hline
\end{tabular}

- Canister sludge volumes are calculated from the mean sludge depths shown previously using the depth-to-volume conversion factors of $0.620 \mathrm{~L} / \mathrm{in}$. for good/fair fuel and 0.770 L/in. for poor/bad fuel (WHC-SD-SNF-TI-034 [Pitner 1996b]).

- Empty canisters that were present in 1995 are not listed because they are no longer in K East Basin (HNF-8118). The sludge in these canisters was emptied onto the floor.

The total canister counts by fuel condition and key number are listed in Table 3-12. The total canister counts shown in Table 3-12 (last line) are the same as shown in Table 3-11. In the stochastic analysis the volume of sludge for fair fuel is computed using the weighted average because of the absence of information about whether the canisters are aluminum or stainless steel.

Table 3-13 shows the calculated canister sludge volume by key. Also shown is the number of cubicles with fuel. The total number of cubicles with fuel is 3,668 . The total average volume of canister sludge in $\mathrm{K}$ East Basin is 2,860 L. 
SNF-10293, REV 0

Table 3-12. Number of Canisters of Each Type by Key. (2 sheets)

\begin{tabular}{|c|c|c|c|c|c|}
\hline Key ID & Good & Fair & Poor & Bad & Total \\
\hline 9621 & 0 & 3 & 0 & 0 & 3 \\
\hline 10001 & 1 & 1 & 0 & 0 & 2 \\
\hline 10201 & 0 & 0 & 2 & 9 & 11 \\
\hline 10259 & 20 & 43 & 2 & 1 & 66 \\
\hline 10294 & 23 & 78 & 10 & 9 & 120 \\
\hline 10350 & 0 & 0 & 0 & 2 & 2 \\
\hline 10385 & 23 & 77 & 9 & 2 & 111 \\
\hline 10456 & 23 & 53 & 5 & 3 & 84 \\
\hline 10576 & 7 & 5 & 0 & 0 & 12 \\
\hline 10602 & 18 & 52 & 10 & 5 & 85 \\
\hline 10679 & 21 & 50 & 11 & 4 & 86 \\
\hline 10742 & 17 & 39 & 9 & 1 & 66 \\
\hline 10764 & 9 & 1 & 0 & 0 & 10 \\
\hline 10770 & 17 & 35 & 6 & 2 & 60 \\
\hline 10832 & 38 & 109 & 19 & 1 & 167 \\
\hline 10982 & 11 & 65 & 3 & 1 & 80 \\
\hline 11036 & 5 & 35 & 1 & 0 & 41 \\
\hline 11109 & 12 & 62 & 6 & 0 & 80 \\
\hline 11183 & 55 & 95 & 8 & 0 & 158 \\
\hline 11372 & 6 & 81 & 9 & 1 & 97 \\
\hline 11449 & 20 & 65 & 7 & 0 & 92 \\
\hline 11540 & 15 & 27 & 0 & 0 & 42 \\
\hline 11733 & 1 & 1 & 0 & 0 & 2 \\
\hline 11806 & 28 & 55 & 3 & 0 & 86 \\
\hline 11897 & 43 & 72 & 5 & 0 & 120 \\
\hline 11979 & 49 & 65 & 5 & 0 & 119 \\
\hline 12127 & 43 & 52 & 0 & 0 & 95 \\
\hline 12201 & 32 & 36 & 2 & 0 & 70 \\
\hline 12240 & 3 & 0 & 0 & 0 & 3 \\
\hline 12285 & 108 & 87 & 3 & 1 & 199 \\
\hline 12480 & 93 & 73 & 2 & 0 & 168 \\
\hline 12565 & 84 & 37 & 1 & 0 & 122 \\
\hline 12639 & 95 & 45 & 2 & 0 & 142 \\
\hline 12852 & 5 & 3 & 0 & 0 & 8 \\
\hline 13015 & 1 & 2 & 0 & 0 & 3 \\
\hline
\end{tabular}


SNF-10293, REV 0

Table 3-12. Number of Canisters of Each Type by Key. (2 sheets)

\begin{tabular}{|c|c|c|c|c|c|}
\hline Key ID & Good & Fair & Poor & Bad & Total \\
\hline 13649 & 6 & 10 & 0 & 0 & 16 \\
\hline 13685 & 7 & 1 & 0 & 0 & 8 \\
\hline 14065 & 1 & 0 & 0 & 0 & 1 \\
\hline 14355 & 12 & 16 & 2 & 0 & 30 \\
\hline 14356 & 8 & 21 & 12 & 14 & 55 \\
\hline 14722 & 1 & 0 & 0 & 0 & 1 \\
\hline 15204 & 297 & 16 & 0 & 0 & 313 \\
\hline 15240 & 2 & 0 & 0 & 0 & 2 \\
\hline 15316 & 61 & 4 & 0 & 0 & 65 \\
\hline 15399 & 0 & 2 & 0 & 0 & 2 \\
\hline 15444 & 1 & 0 & 0 & 0 & 1 \\
\hline 15451 & 28 & 2 & 0 & 0 & 30 \\
\hline 15455 & 5 & 0 & 0 & 0 & 5 \\
\hline 15457 & 2 & 0 & 0 & 0 & 2 \\
\hline 15458 & 415 & 10 & 0 & 0 & 425 \\
\hline 15460 & 61 & 0 & 0 & 0 & 61 \\
\hline 15461 & 39 & 0 & 0 & 0 & 39 \\
\hline \hline Totals & 1,872 & 1,586 & 154 & 56 & 3,668 \\
\hline & & & & & \\
\hline
\end{tabular}


SNF-10293, REV 0

Table 3-13. Average Canister Sludge Volume by Key.

\begin{tabular}{|c|c|c|c|c|c|}
\hline $\begin{array}{c}\text { Key } \\
\text { Number }\end{array}$ & $\begin{array}{c}\text { Number of } \\
\text { Cubicles }\end{array}$ & $\begin{array}{c}\text { Average } \\
\text { Canister Sludge } \\
\text { Volume (L) }\end{array}$ & $\begin{array}{c}\text { Key } \\
\text { Number }\end{array}$ & $\begin{array}{c}\text { Number of } \\
\text { Cubicles }\end{array}$ & $\begin{array}{c}\text { Average } \\
\text { Canister Sludge } \\
\text { Volume (L) }\end{array}$ \\
\hline 9621 & 3 & 3.0 & 12127 & 95 & 64 \\
\hline 10001 & 2 & 1.3 & 12201 & 70 & 52 \\
\hline 10201 & 11 & 39 & 12240 & 3 & 0.9 \\
\hline 10259 & 66 & 59 & 12285 & 199 & 132 \\
\hline 10294 & 120 & 151 & 12480 & 168 & 107 \\
\hline 10350 & 2 & 7.0 & 12565 & 122 & 65 \\
\hline 10385 & 111 & 122 & 12639 & 142 & 79 \\
\hline 10456 & 84 & 88 & 12852 & 8 & 4.4 \\
\hline 10576 & 12 & 7 & 13015 & 3 & 2.3 \\
\hline 10602 & 85 & 110 & 13649 & 16 & 12 \\
\hline 10679 & 86 & 109 & 13685 & 8 & 3.0 \\
\hline 10742 & 66 & 79 & 14065 & 1 & 0.3 \\
\hline 10764 & 10 & 3.6 & 14355 & 30 & 26 \\
\hline 10770 & 60 & 68 & 14356 & 55 & 115 \\
\hline 10832 & 167 & 190 & 14722 & 1 & 0.3 \\
\hline 10982 & 80 & 82 & 15204 & 313 & 102 \\
\hline 11036 & 41 & 40 & 15240 & 2 & 0.6 \\
\hline 11109 & 80 & 87 & 15316 & 65 & 22 \\
\hline 11183 & 158 & 139 & 15399 & 2 & 2.0 \\
\hline 11372 & 97 & 118 & 15444 & 1 & 0.3 \\
\hline 11449 & 92 & 95 & 15451 & 30 & 10 \\
\hline 11540 & 42 & 31 & 15455 & 5 & 1.4 \\
\hline 11733 & 2 & 1.3 & 15457 & 2 & 0.6 \\
\hline 11806 & 86 & 74 & 15458 & 425 & 130 \\
\hline 11897 & 120 & 102 & 15460 & 61 & 18 \\
\hline 11979 & 119 & 97 & 15461 & 39 & 11 \\
\hline
\end{tabular}

- The total number of canisters is 3,668. The total average volume of canister sludge is $2,860 \mathrm{~L}$.

- The average canister sludge volumes were calculated using average canister sludge depths of 0.465 inch for good fuel, 1.61 inch for fair fuel, and 4.56 inch for poor and bad fuel.

\subsubsection{Stochastic Model for Canister Sludge Depth}

The stochastic model to represent canister sludge depth uses the values shown in Tables 3-8, 3-9, and 3-10. A random number is generated for each fuel type (good, fair-SS, fair-Al, poor, and bad) and a measured value is randomly selected from the appropriate table. In addition, a 
random factor from a uniform distribution with a mean value of 1.0 is generated for each fuel type.

Each key has canisters in one or more types of fuel conditions as shown in Table 3-12. Thus, for each key, there will be up to 5 pairs of random numbers generated to represent the canister sludge depths in one key. The depths for fair fuel are weighted and combined into one value, as discussed above.

The range used for the variability factor is based on the $99 \%$ confidence interval for the mean as discussed in Section 3.2.2. Table 3-14 shows the upper bound computed using this indicator. Note that the value for good fuel is much larger than the values for the other types of fuel. This is probably because of the small number of measured values (5). Ignoring the upper bound for the good fuel condition, the remaining bounds are all less than 1.5. Because one variability factor is used for all fuel types, its range was selected to be 0.5 to 1.5 .

Table 3-14. Variability Factors for Canister Sludge Depth.

\begin{tabular}{|c|c|}
\hline Fuel Condition & Upper Bound \\
\hline Bad & 1.40 \\
\hline Poor & 1.40 \\
\hline Fair-AL & 1.34 \\
\hline Fair-SS & 1.47 \\
\hline Good & 3.58 \\
\hline
\end{tabular}

The upper bound on the variability factor is calculated from the $99 \%$ confidence interval of the mean depth for each type of fuel.

The variation in canister sludge depth in one key is increased by using one random depth to represent all of the canisters of a given type. If the individual canisters had been assigned random values, the sum would have less variation as discussed in Section 2.0.

Four alternate cases for the base case PDFs described above are (1) reducing the variability factor to a much smaller value, namely, 0.9 to $1.1,(2)$ using a piecewise continuous function to be the PDF for canister sludge depths, (3) using the smallest possible off scale depth, and (4) using the largest possible off scale result.

The first alternative is readily tested using a different value for the range of variability. The expected outcome is smaller variability in the concentrations in the filled sludge containers because of the reduced variability. The mean values should be the same, but the $95^{\text {th }}$ percentile value should be smaller.

The second alternative represents the data for each fuel condition as a cumulative distribution, which is a curve showing the probability of finding a concentration less than a particular value. In the second alternative, the expected outcome is again smaller concentrations in the filled sludge containers because of the reduced variability. The mean values should be no different, but the maximum and minimum must be closer. Thus the $95^{\text {th }}$ percentile result is expected to be smaller. 
In the second alternative PDF, the continuous cumulative probability distribution for canisters with bad and poor fuel is shown in Figure 3-12. The smaller depths are more likely because the cumulative probability curve rises rapidly at small depths. The curve is used in the stochastic analysis by randomly selecting a number between 0 and 1 . All values between 0 and 1 (cumulative probabilities) are equally likely. From the CDF in Figure 3-12, the corresponding sludge depth can be determined. Note that 34 of the 420 canister barrels containing poor and bad fuel were sampled ( $8 \%$ ). These poor/bad fuel canisters provide just $26 \%$ of the total canister sludge volume from Table 3-11.

Figure 3-12. Cumulative Probability Distribution for Canisters with Bad or Poor Fuel.

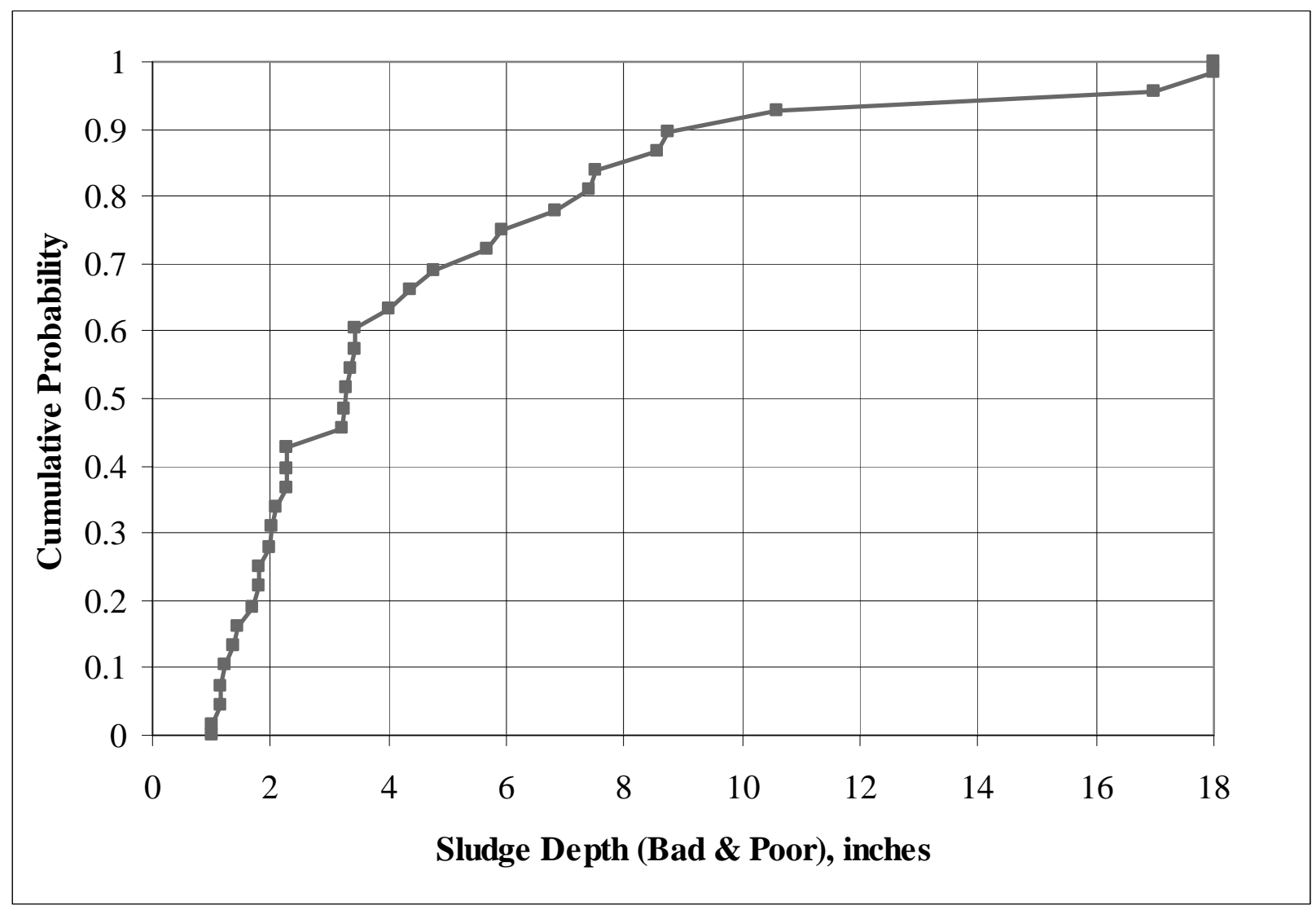

In the second alternative PDF, the continuous cumulative probability distribution for sludge depth in canisters with fair fuel is shown in Figure 3-13. In the stochastic analysis, the canisters with fair fuel are evaluated using two distributions, one for aluminum canisters and one for stainless steel. Two random numbers are generated to calculate two values for canister sludge depth. These are combined using a weighting based on the number of aluminum and stainless steel canisters as described earlier. Note that 10 of the 660 stainless steel canister barrels were sampled $(1.5 \%)$ and 18 of the 2,718 aluminum canister barrels were sampled $(0.66 \%)$. The fair fuel canisters contain about 55\% of the total volume of canister sludge (see Table 3-11). 
The continuous cumulative probability distribution for canisters with good fuel is also shown in Figure 3-13. The figure shows that $70 \%$ of canisters with good fuel have no sludge and $10 \%$ have the maximum depth, $2.33 \mathrm{in}$. The other $20 \%$ are assumed to be evenly distributed between 0 and $2.33 \mathrm{in}$. The reason for the simple function is the dearth of data for canisters with good fuel. Only 5 of the 3,744 canister barrels with good fuel were examined $(0.13 \%)$. It is assumed that this limited data set nonetheless represents the actual sludge depths found in good fuel canisters. The good fuel canisters contain about $19 \%$ of the total volume of canister sludge as shown in Table 3-11.

The stochastic calculation of canister sludge volume begins by randomly selecting four depths, one for each of the canister types (good, fair, poor, and bad). The depth-to-volume conversion factors from Table 3-11 are used along with the canister counts in Table 3-12 to calculate the total volume of canister sludge in one key. This is repeated for all the keys.

Figure 3-13. Cumulative Probability Distribution for Canisters with Good or Fair Fuel.

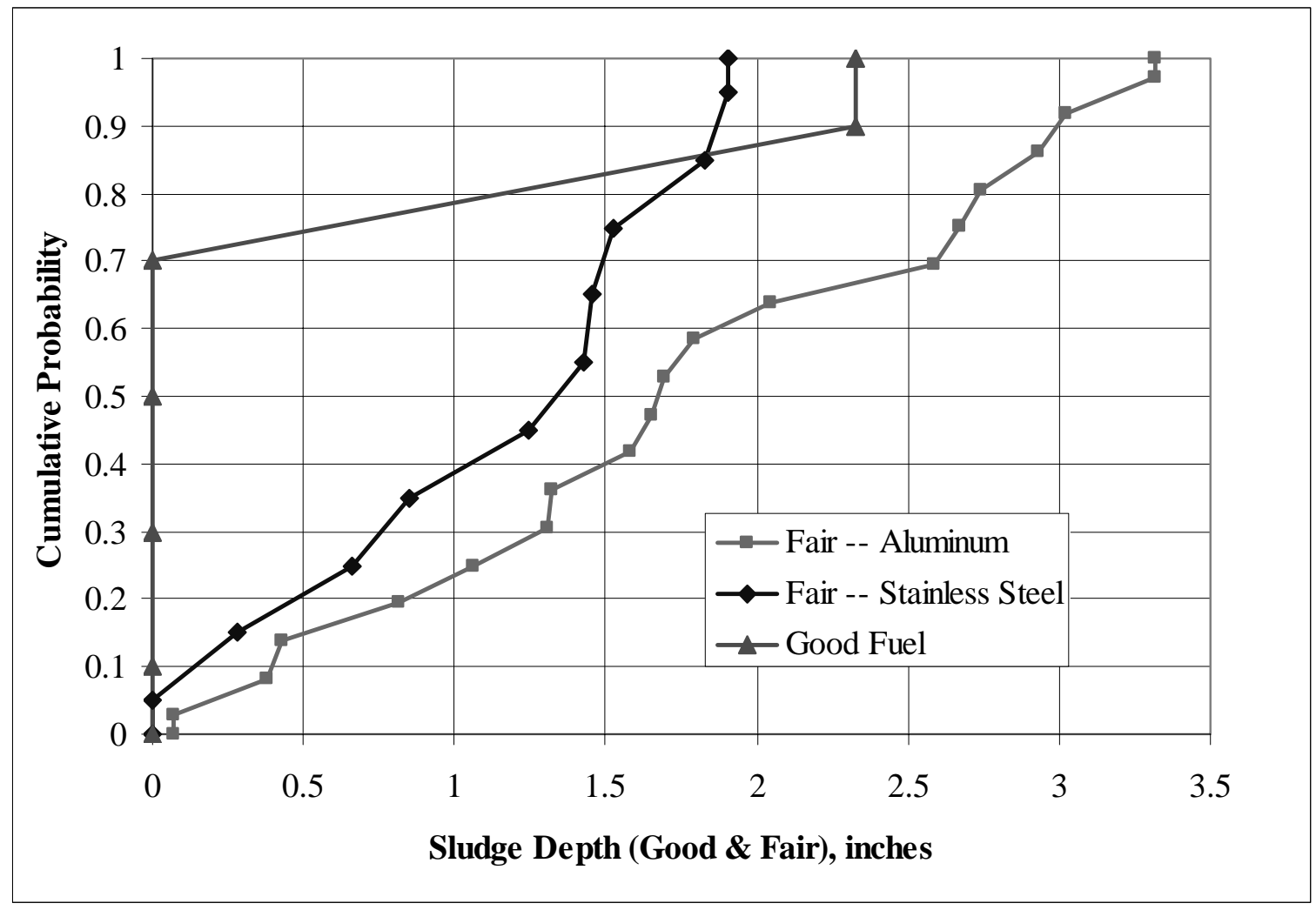

The third and fourth alternatives to the base case are to test the effect of the assumed depth for the two off scale canister barrels (see Table 3-8). For the third alternative the depths are 12 and 13 inches. For the fourth alternative the depths are 19 and 20 inches. 


\subsection{CANISTER SLUDGE ISOTOPIC CONCENTRATION}

Canister sludge samples from K East Basin have been analyzed to measure density, uranium concentration, and activity concentration for several radionuclides. The data for 8 canister sludge samples from K East are listed in Appendix D of SNF-7765. Many of the entries in the table are not available. If this data were to be used in the stochastic analysis, a measured value would be needed for every analyte in every sample. Using a value of zero would bias the stochastic results toward lower values. The canister sludge sample data is used in an alternate case and in comparisons with the Radnuc2A estimates. Therefore, appropriate data values were imputed to fill in the blanks.

The methods for calculating total plutonium, uranium metal content, heat generation rate, and inhalation dose are discussed in later sections.

\subsubsection{Data and Imputed Values for Canister Sludge}

A linear regression approach identical to that used for floor sludge concentrations has been used to impute canister sludge concentrations. Measurements of the Cs-137, Eu-154, Pu-239/240, and the uranium concentrations were obtained for each of the 8 samples. Across-analyte imputations have been performed for Co-60, Sr-90, Tc-99, Eu-155, Np-237, Pu-238, and Am-241, for which there are some measurements. The imputation for Cs-134, which has only one measurement in canister sludge, is based on the floor sludge data. The imputation for Eu-152 and Pu-241, for which there are no measurements, is based on estimates from HNF-8760.

As with floor sludge, the linear regression is carried out to indicate the general correlation between two isotopes. The limitations of the regression method are significant. The slope and $\mathrm{R}^{2}$ value for each correlation is listed in Table 3-15. The correlations chosen for later imputation are marked. The vertical columns are headed by the nuclides with measurements for all 23 samples. The rows are all the other nuclides with at least a few measurements. If $\mathrm{R}^{2}$ is close to 1.00 then the correlation is considered reasonable for the purposes of the stochastic analysis. In Table 3-15 the possible correlations for Co-60 are Cs-137 and Eu-154. Because Eu-154 has the largest $\mathrm{R}^{2}$, it was chosen to impute missing values for Co-60. Similar logic was followed for Sr-90, Tc-99, Eu-155, Np-237, Pu-238, and Am-241.

Graphs are provided in Figure 3-14 through Figure 3-21. Note that the trend line extends only from the lowest measured value to the largest. The measured values are plotted as diamonds. The imputed values are plotted as squares. The asterisks plotted in the figures are twice the imputed values. These doubled values were used as one of the alternatives to the base case stochastic analysis.

For Cs-134 there is only one measurement. Comparing Table 3-15 with Table 3-2 leads to the conclusion that the floor sludge slopes are generally larger than those for the canister sludge. Therefore, the correlation between Cs-134 and Cs-137 in floor sludge (slope=4.46E-04) will be used for canister sludge. The data value and imputations for Cs-134 are shown in Figure 3-17. 
Table 3-15. Canister Sample Data Correlations: Slope and $\mathrm{R}^{2}$.

\begin{tabular}{|c|c|c|c|c|}
\hline \multirow[b]{2}{*}{ Y-Axis } & \multicolumn{4}{|c|}{ X-Axis (Isotopes Measured for Every Sample) } \\
\hline & Cs-137 & Eu-154 & Pu-239/240 & Uranium \\
\hline \multirow[t]{2}{*}{ Co-60 } & $8.673 \mathrm{E}-04$ & $6.513 \mathrm{E}-02$ & $6.227 \mathrm{E}-03$ & $1.184 \mathrm{E}-06$ \\
\hline & 0.869 & 0.897 & 0.690 & 0.554 \\
\hline \multirow[t]{2}{*}{ Sr-90 } & $1.413 \mathrm{E}+00$ & $1.055 \mathrm{E}+02$ & $1.331 \mathrm{E}+01$ & $2.362 \mathrm{E}-03$ \\
\hline & 0.745 & 0.801 & 0.905 & 0.992 \\
\hline \multirow[t]{2}{*}{ Tc-99 } & $1.474 \mathrm{E}-02$ & 9.324E-01 & $1.018 \mathrm{E}-01$ & $1.396 \mathrm{E}-05$ \\
\hline & 0.619 & 0.416 & 0.419 & 0.293 \\
\hline \multirow[t]{2}{*}{ Cs-137 } & $1.000 \mathrm{E}+00$ & 7.077E+01 & $7.219 \mathrm{E}+00$ & $1.192 \mathrm{E}-03$ \\
\hline & 1.000 & 0.942 & 0.875 & 0.761 \\
\hline \multirow[t]{2}{*}{ Eu-154 } & $1.330 \mathrm{E}-02$ & $1.000 \mathrm{E}+00$ & $1.016 \mathrm{E}-01$ & $1.691 \mathrm{E}-05$ \\
\hline & 0.942 & 1.000 & 0.922 & 0.815 \\
\hline \multirow[t]{2}{*}{ Eu-155 } & $6.880 \mathrm{E}-03$ & $5.284 \mathrm{E}-01$ & $5.370 \mathrm{E}-02$ & $1.113 \mathrm{E}-05$ \\
\hline & 0.894 & 0.965 & 0.839 & 0.800 \\
\hline \multirow[t]{2}{*}{ Np-237 } & $1.237 \mathrm{E}-05$ & $8.107 \mathrm{E}-04$ & $1.039 \mathrm{E}-04$ & $1.781 \mathrm{E}-08$ \\
\hline & 0.748 & 0.731 & 0.844 & 0.914 \\
\hline \multirow[t]{2}{*}{ Pu-238 } & $1.826 \mathrm{E}-02$ & $1.365 \mathrm{E}+00$ & $1.501 \mathrm{E}-01$ & $2.538 \mathrm{E}-05$ \\
\hline & 0.861 & 0.901 & 0.929 & 0.788 \\
\hline \multirow[t]{2}{*}{$\mathrm{Pu}-239 / 240$} & $1.212 \mathrm{E}-01$ & $9.073 \mathrm{E}+00$ & $1.000 \mathrm{E}+00$ & $1.707 \mathrm{E}-04$ \\
\hline & 0.121 & 9.073 & 1.000 & 0.930 \\
\hline \multirow[t]{2}{*}{ Am-241 } & $9.644 \mathrm{E}-02$ & $7.252 \mathrm{E}+00$ & $8.068 \mathrm{E}-01$ & $1.374 \mathrm{E}-04$ \\
\hline & 0.856 & 0.906 & 0.957 & 0.824 \\
\hline \multirow[t]{2}{*}{ Uranium } & $6.382 \mathrm{E}+02$ & $4.819 \mathrm{E}+04$ & $5.447 \mathrm{E}+03$ & 1 \\
\hline & 0.761 & 0.815 & 0.930 & 1 \\
\hline
\end{tabular}

The upper number is the slope of the regression line (intercept is zero) while the lower number is the $R^{2}$ value of this regression.

Numbers with a heavy border were chosen to impute floor sludge concentrations for sample results that are not available in SNF-7765. For Tc-99 a regression with a non-zero intercept was used to impute values from uranium. 
Figure 3-14. ${ }^{60} \mathrm{Co}$ Data and Imputations from ${ }^{154} \mathrm{Eu}$ Data for Canister Sludge.

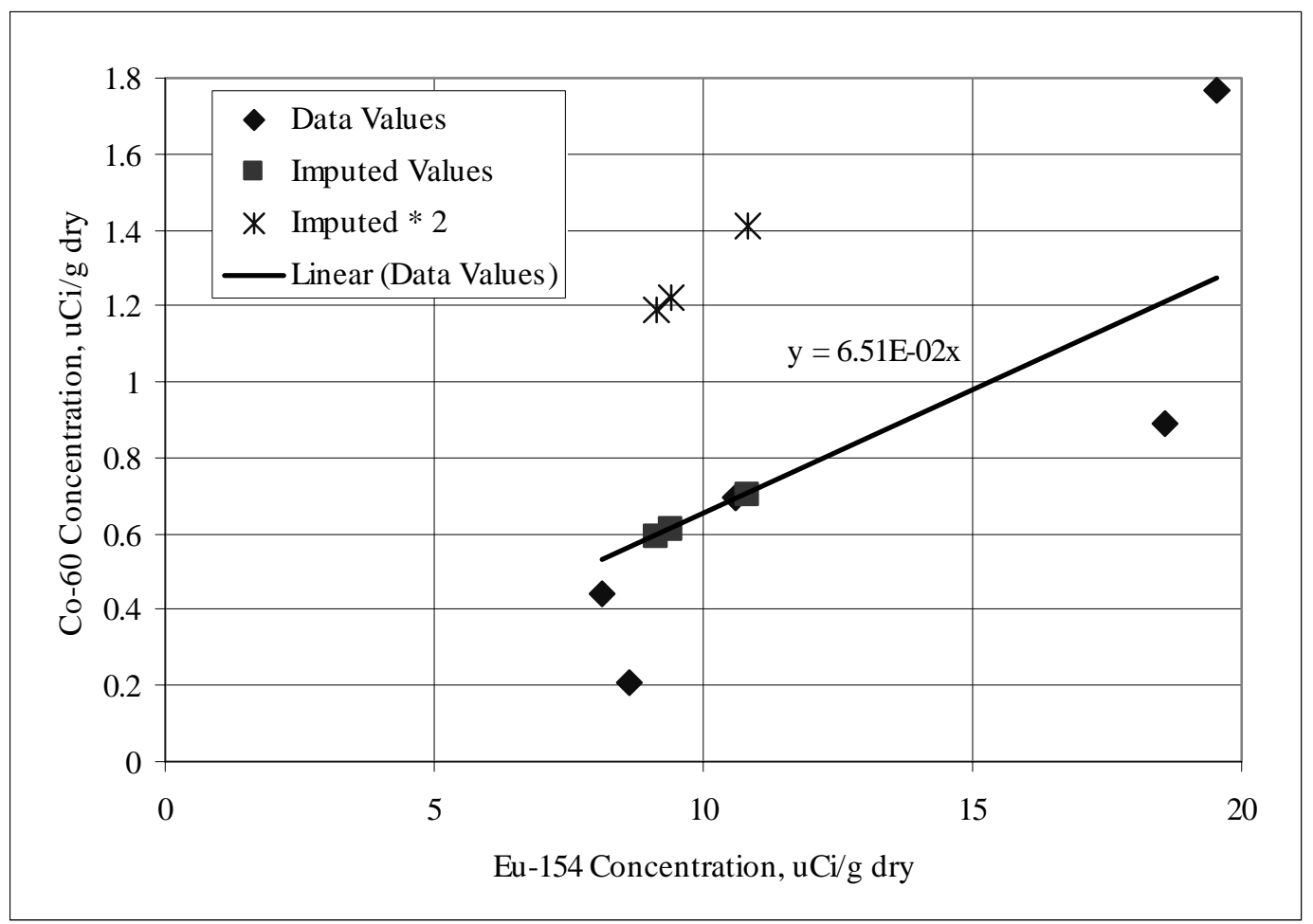

Figure 3-15. ${ }^{90} \mathrm{Sr}$ Data and Imputations from Uranium Data for Canister Sludge.

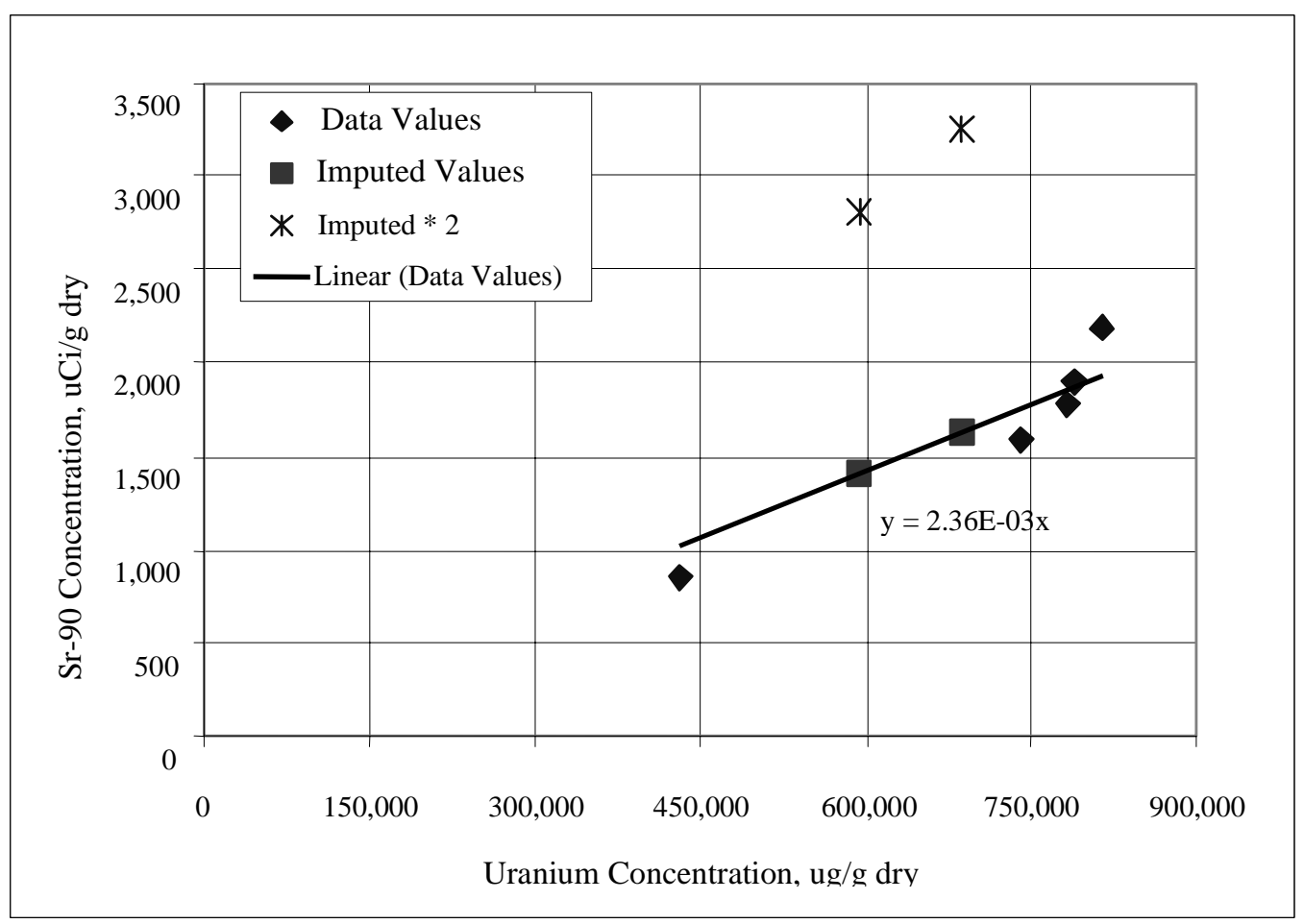


Figure 3-16. ${ }^{99} \mathrm{Tc}$ Data and Imputations from Uranium Data for Canister Sludge.

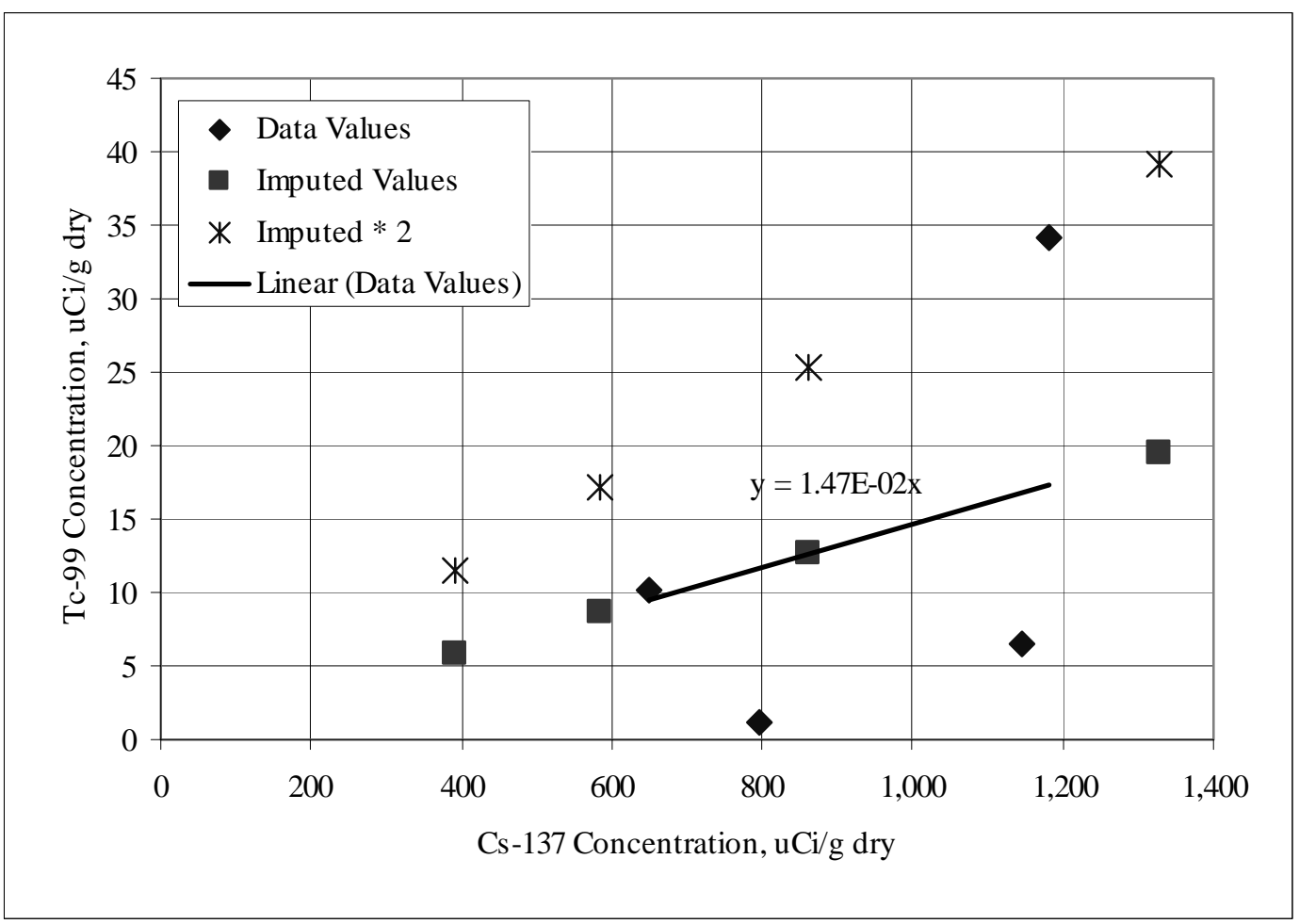

Figure 3-17. ${ }^{134} \mathrm{Cs}$ Data and Imputations from ${ }^{137} \mathrm{Cs}$ Data for Canister Sludge.

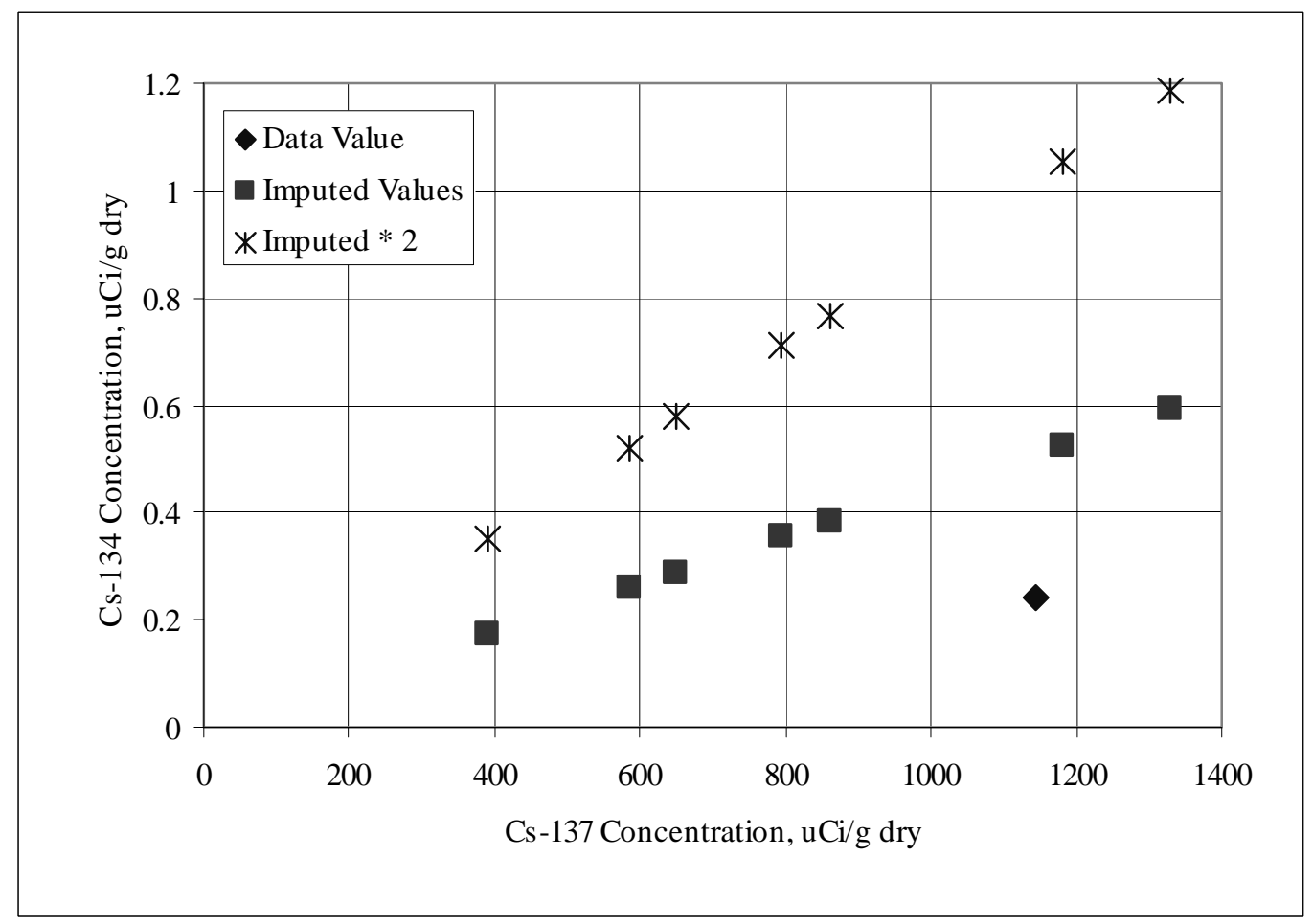


Figure 3-18. ${ }^{155} \mathrm{Eu}$ Data and Imputations from ${ }^{154} \mathrm{Eu}$ Data for Canister Sludge.

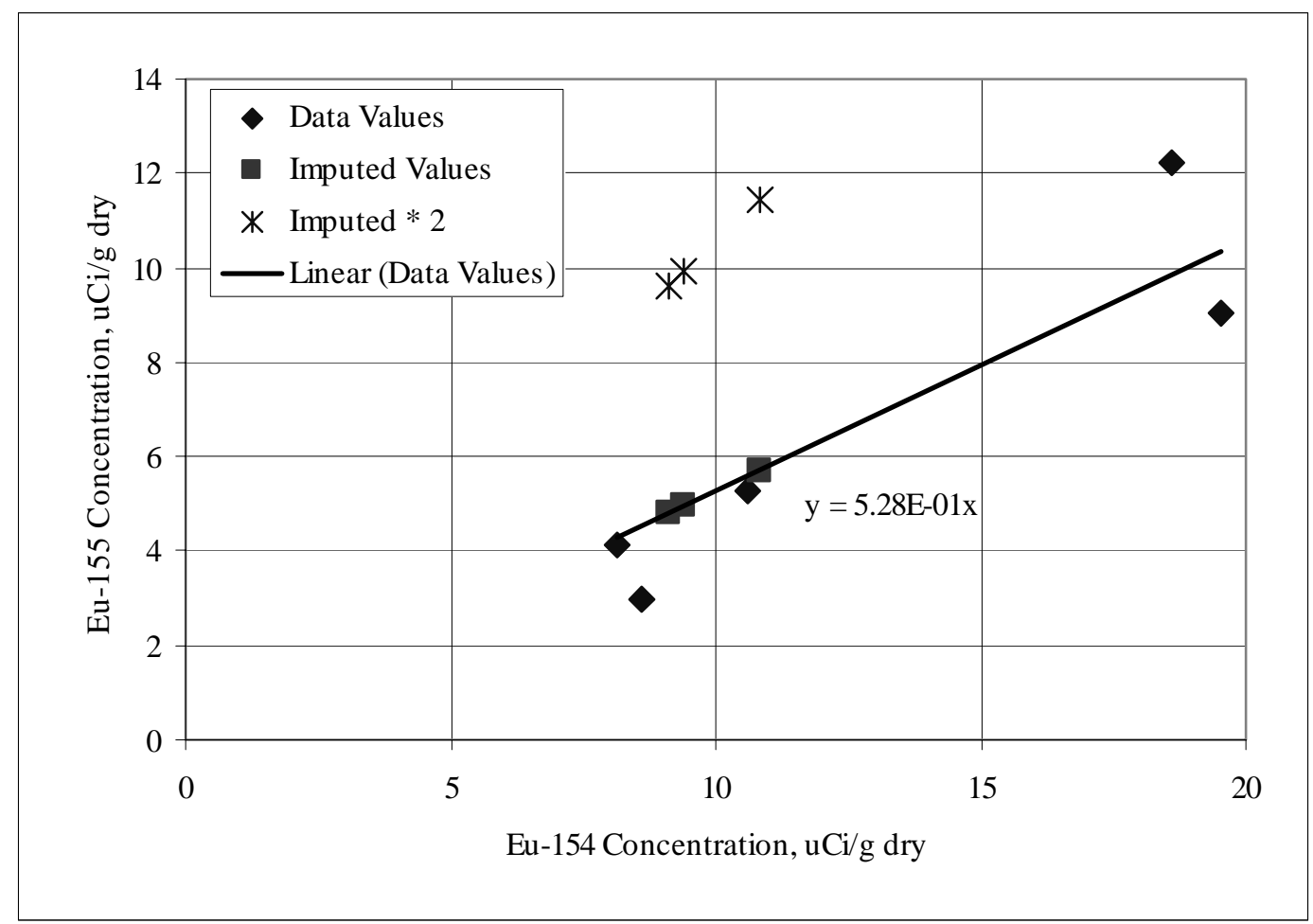

Figure 3-19. ${ }^{237} \mathrm{~Np}$ Data and Imputations from Uranium Data for Canister Sludge.

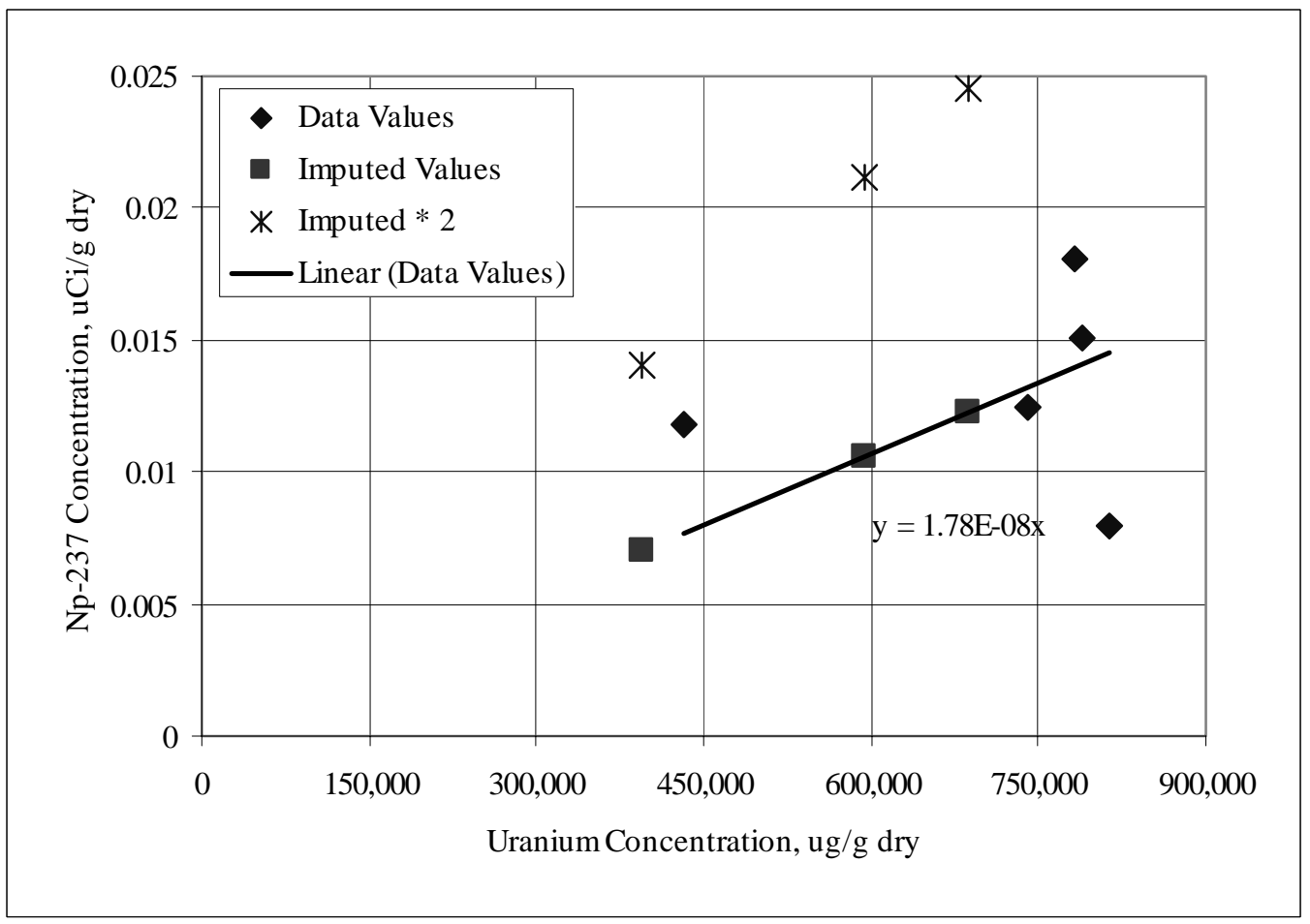


Figure 3-20. ${ }^{238} \mathrm{Pu}$ Data and Imputations from ${ }^{239+240} \mathrm{Pu}$ Data for Canister Sludge.

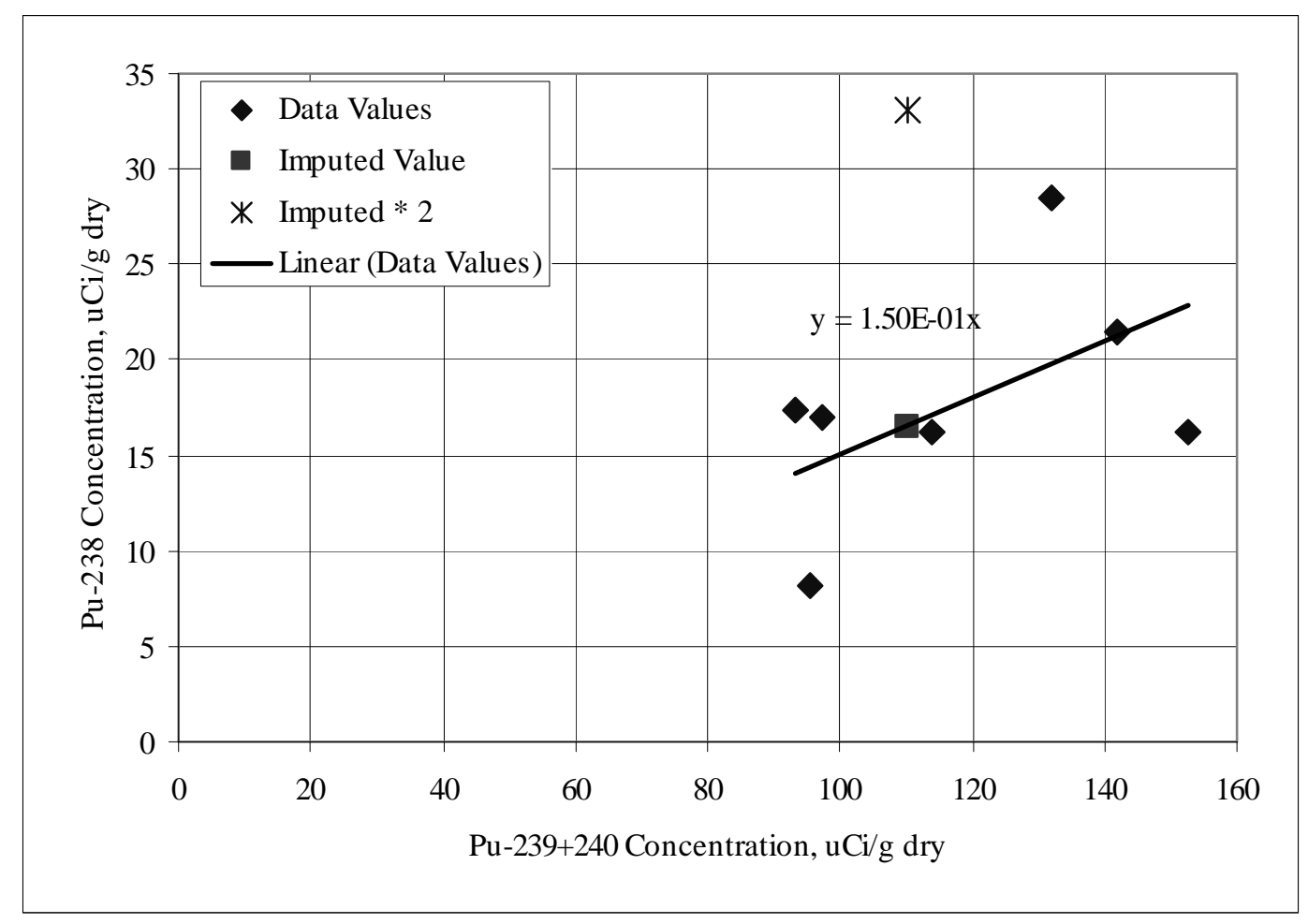

Figure 3-21. ${ }^{241} \mathrm{Am}$ Data and Imputations from ${ }^{239+240} \mathrm{Pu}$ Data for Canister Sludge.

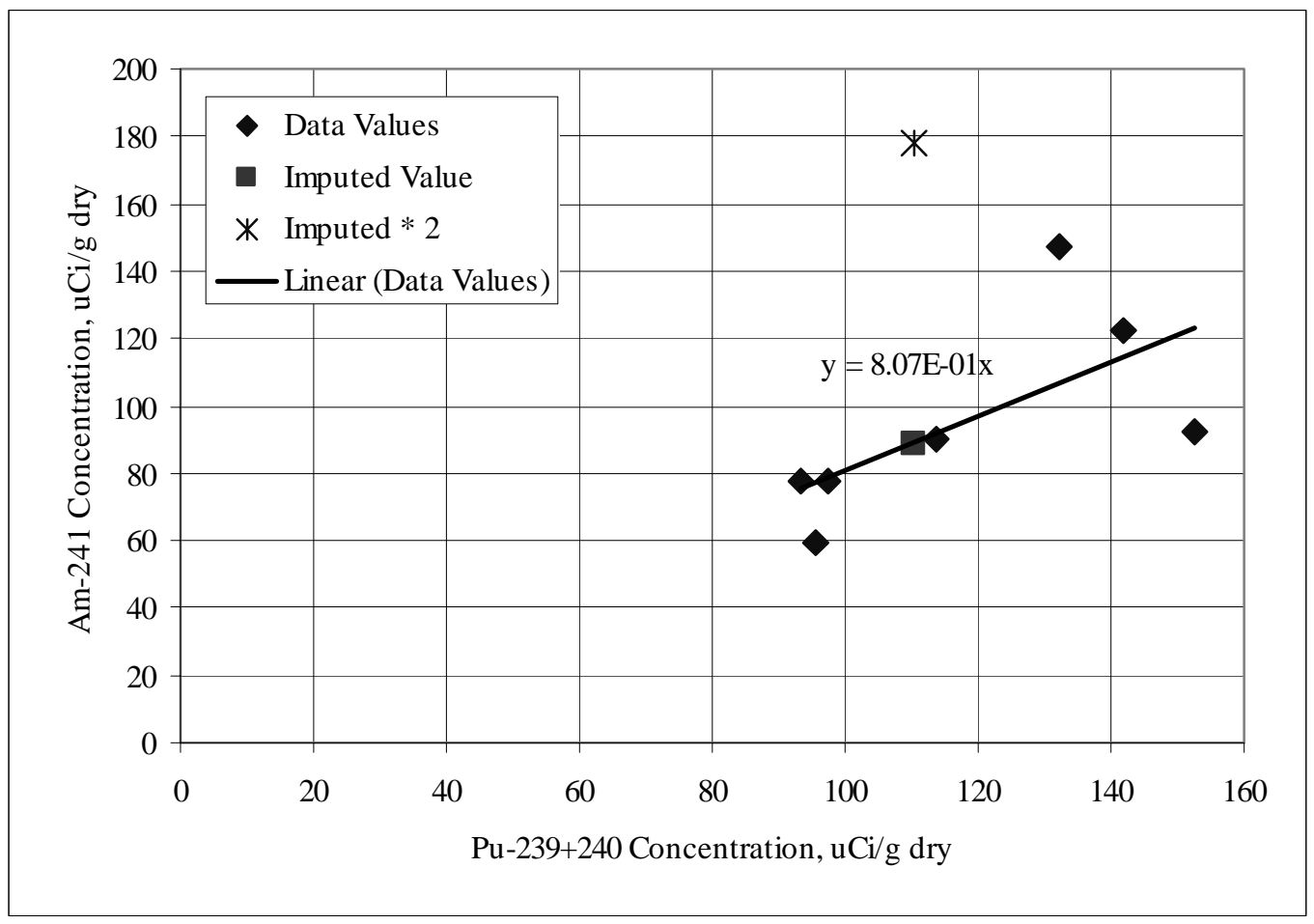


The Eu-152 and $\mathrm{Pu}-241$ were not measured in canister sludge. The imputations are based on the regressions obtained from Radnuc2A and shown in Table 3-3. The slopes from Table 3-3 (7.99E-03 for Eu-152, and 56.2 for $\mathrm{Pu}-241$ ) are multiplied by the measured concentration of the chosen nuclide (Eu-154 for $\mathrm{Eu}-152$, and $\mathrm{Pu}-238$ for $\mathrm{Pu}-241)$ to give the imputed values. The imputations are plotted in Figure 3-22 and Figure 3-23 along with the Radnuc2A values from which the slopes were calculated. As noted for Figure 3-9, the Radnuc2A could be better represented with a polynomial function rather than a straight line. The imputed values that have been doubled are larger than the Radnuc2A values, so the alternate case with doubled imputations is still bounding.

Figure 3-22. ${ }^{152} \mathrm{Eu}$ Imputations from ${ }^{154} \mathrm{Eu}$ Data for Canister Sludge.

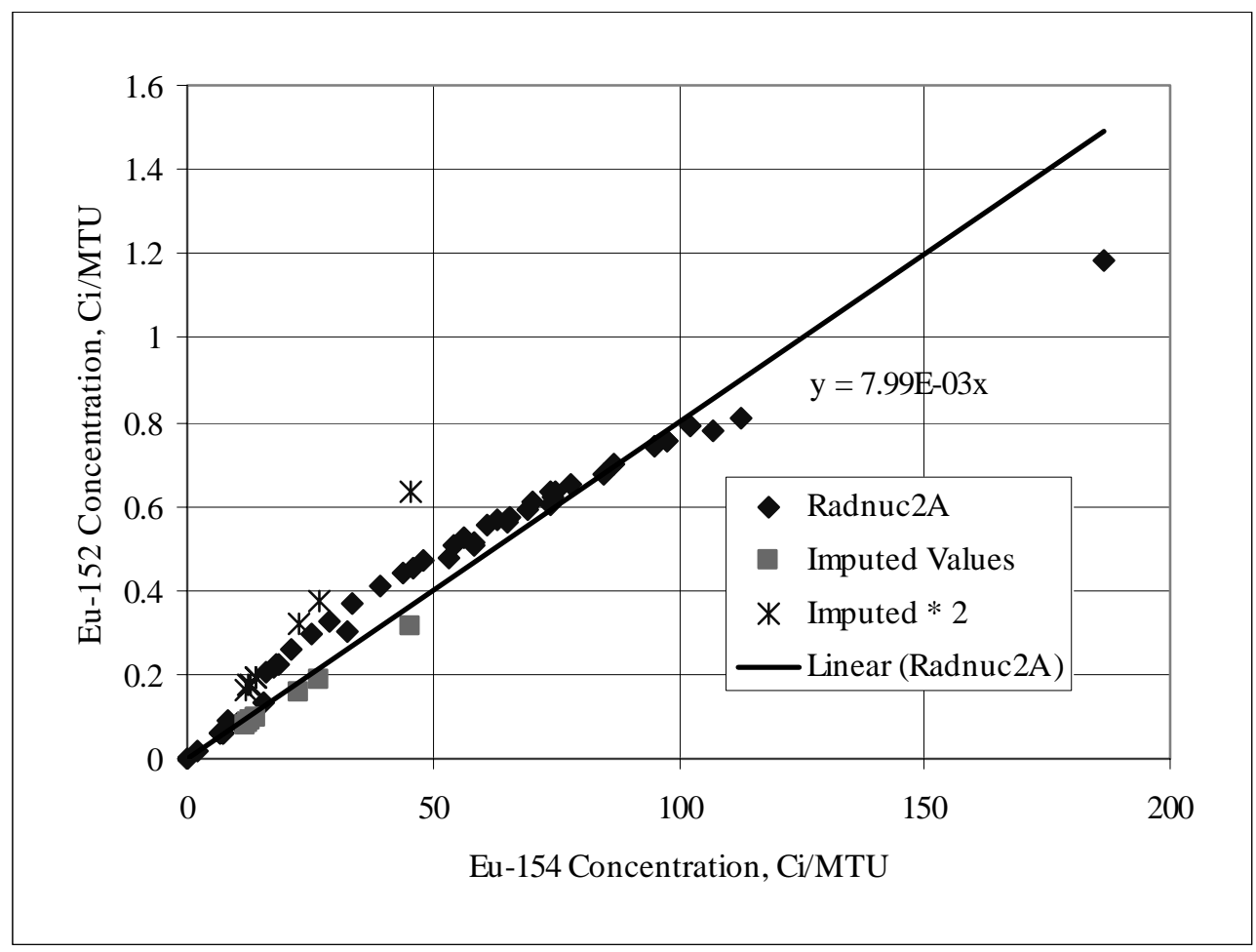


Figure 3-23. ${ }^{241} \mathrm{Pu}$ Imputations from ${ }^{238} \mathrm{Pu}$ Data and Imputations for Canister Sludge.

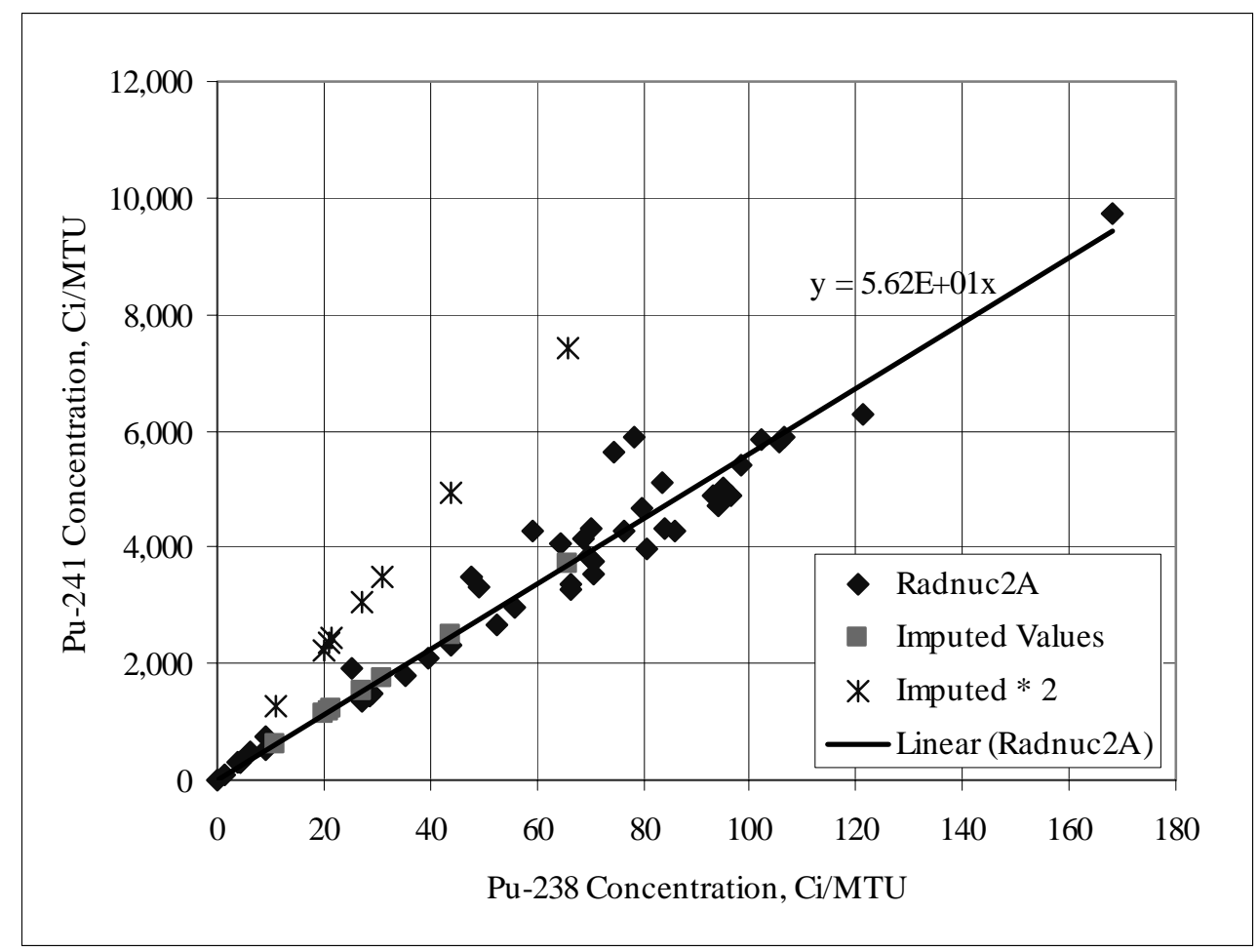

A complete listing of the canister sludge concentrations is given in Table 3-16. The values imputed for the stochastic analysis are marked with asterisks $(*)$. Also shown in the table are the mean values and standard deviations. These summary statistics include the influence of the imputed values. The mean values are used in later calculations to verify the stochastic calculation.

Table 3-16. Canister Sample Data with Imputed Values. (2 sheets)

\begin{tabular}{|c|c|c|c|c|c|}
\hline \multirow[b]{2}{*}{ Measured Quantity } & \multicolumn{5}{|c|}{ Sample Identity } \\
\hline & $96-05$ & $96-08$ & $96-13$ & $96-15$ & $96-04$ \\
\hline $\operatorname{Co-60}(\mu \mathrm{Ci} / \mathrm{mL})$ & $1.66 \mathrm{E}+00$ & $2.03 \mathrm{E}-01$ & $* 1.10 \mathrm{E}+00$ & $* 8.82 \mathrm{E}-01$ & $4.56 \mathrm{E}-01$ \\
\hline $\operatorname{Sr}-90(\mu \mathrm{Ci} / \mathrm{mL})$ & $4.07 \mathrm{E}+03$ & $2.75 \mathrm{E}+02$ & $3.29 \mathrm{E}+03$ & $2.28 \mathrm{E}+03$ & $2.21 \mathrm{E}+02$ \\
\hline Tc-99 $(\mu \mathrm{Ci} / \mathrm{mL})$ & $1.20 \mathrm{E}+01$ & $9.99 \mathrm{E}+00$ & $1.89 \mathrm{E}+01$ & $1.77 \mathrm{E}+00$ & $* 5.05 \mathrm{E}+00$ \\
\hline Cs-134 $(\mu \mathrm{Ci} / \mathrm{mL})$ & $4.48 \mathrm{E}-01$ & $* 1.54 \mathrm{E}-01$ & $* 5.35 \mathrm{E}-01$ & $* 5.11 \mathrm{E}-01$ & $* 1.53 \mathrm{E}-01$ \\
\hline Cs-137 $(\mu \mathrm{Ci} / \mathrm{mL})$ & $2.13 E+03$ & $3.45 \mathrm{E}+02$ & $1.20 \mathrm{E}+03$ & $1.15 \mathrm{E}+03$ & $3.42 \mathrm{E}+02$ \\
\hline Eu-152 $(\mu \mathrm{Ci} / \mathrm{mL})$ & $* 2.76 \mathrm{E}-01$ & $* 2.47 \mathrm{E}-02$ & $* 1.35 \mathrm{E}-01$ & $* 1.08 \mathrm{E}-01$ & $* 4.03 \mathrm{E}-02$ \\
\hline Eu-154 $(\mu \mathrm{Ci} / \mathrm{mL})$ & $3.46 \mathrm{E}+01$ & $3.10 \mathrm{E}+00$ & $1.69 \mathrm{E}+01$ & $1.35 \mathrm{E}+01$ & $5.04 \mathrm{E}+00$ \\
\hline Eu-155 $(\mu \mathrm{Ci} / \mathrm{mL})$ & $2.27 \mathrm{E}+01$ & $1.54 \mathrm{E}+00$ & $* 8.91 \mathrm{E}+00$ & $* 7.16 \mathrm{E}+00$ & $2.33 \mathrm{E}+00$ \\
\hline Np-237 $(\mu \mathrm{Ci} / \mathrm{mL})$ & $1.48 \mathrm{E}-02$ & $* 2.05 \mathrm{E}-03$ & 3.35E-02 & $1.80 \mathrm{E}-02$ & $3.04 \mathrm{E}-03$ \\
\hline Pu-238 $(\mu \mathrm{Ci} / \mathrm{mL})$ & $3.01 \mathrm{E}+01$ & $5.08 \mathrm{E}+00$ & $* 3.06 \mathrm{E}+01$ & $1.18 \mathrm{E}+01$ & $7.34 \mathrm{E}+00$ \\
\hline
\end{tabular}


SNF-10293, REV 0

Table 3-16. Canister Sample Data with Imputed Values. (2 sheets)

\begin{tabular}{|c|c|c|c|c|c|}
\hline Pu-239/240 $(\mu \mathrm{Ci} / \mathrm{mL})$ & $2.84 \mathrm{E}+02$ & $2.73 \mathrm{E}+01$ & $2.04 \mathrm{E}+02$ & $1.37 \mathrm{E}+02$ & $3.41 \mathrm{E}+01$ \\
\hline Pu-241 $(\mu \mathrm{Ci} / \mathrm{mL})$ & $* 1.69 \mathrm{E}+03$ & $* 2.85 \mathrm{E}+02$ & $* 1.72 \mathrm{E}+03$ & $* 6.64 \mathrm{E}+02$ & $* 4.12 \mathrm{E}+02$ \\
\hline Am-241 $(\mu \mathrm{Ci} / \mathrm{mL})$ & $1.72 \mathrm{E}+02$ & $2.26 \mathrm{E}+01$ & $* 1.64 \mathrm{E}+02$ & $8.57 \mathrm{E}+01$ & $3.79 \mathrm{E}+01$ \\
\hline Uranium $(\mathrm{kg} / \mathrm{L})$ & 1.51 & 0.12 & 1.45 & 1.07 & 0.111 \\
\hline Density (kg/L) & 2.34 & 1.19 & 2.46 & 1.85 & 1.20 \\
\hline \multirow[b]{2}{*}{ Measured Quantity } & \multicolumn{3}{|c|}{ Sample Identity } & \multirow[b]{2}{*}{ Mean } & \multirow{2}{*}{$\begin{array}{l}\text { Standard } \\
\text { Deviation }\end{array}$} \\
\hline & $96-06$ & $\mathrm{KC}-1$ & $\mathrm{KC}-2 / 3$ & & \\
\hline $\operatorname{Co-60}(\mu \mathrm{Ci} / \mathrm{mL})$ & $* 1.20 \mathrm{E}+00$ & $1.69 \mathrm{E}-01$ & 5.36E-01 & $7.76 \mathrm{E}-01$ & $5.25 \mathrm{E}-01$ \\
\hline $\operatorname{Sr}-90(\mu \mathrm{Ci} / \mathrm{mL})$ & $3.24 \mathrm{E}+03$ & $* 1.31 \mathrm{E}+03$ & $* 1.70 \mathrm{E}+03$ & $2.05 \mathrm{E}+03$ & $1.43 \mathrm{E}+03$ \\
\hline Tc-99 $(\mu \mathrm{Ci} / \mathrm{mL})$ & $* 1.47 \mathrm{E}+01$ & $* 4.67 \mathrm{E}+00$ & $* 1.54 \mathrm{E}+01$ & $1.03 \mathrm{E}+01$ & $6.02 \mathrm{E}+00$ \\
\hline Cs-134 $(\mu \mathrm{Ci} / \mathrm{mL})$ & $* 4.45 \mathrm{E}-01$ & $* 1.41 \mathrm{E}-01$ & $* 4.67 \mathrm{E}-01$ & $3.57 \mathrm{E}-01$ & $1.74 \mathrm{E}-01$ \\
\hline Cs-137 $(\mu \mathrm{Ci} / \mathrm{mL})$ & $9.98 \mathrm{E}+02$ & $3.17 \mathrm{E}+02$ & $1.05 \mathrm{E}+03$ & $9.40 \mathrm{E}+02$ & $6.14 \mathrm{E}+02$ \\
\hline $\mathrm{Eu}-152(\mu \mathrm{Ci} / \mathrm{mL})$ & $* 1.47 \mathrm{E}-01$ & $* 5.57 \mathrm{E}-02$ & $* 7.90 \mathrm{E}-02$ & $1.08 \mathrm{E}-01$ & $8.08 \mathrm{E}-02$ \\
\hline Eu-154 $(\mu \mathrm{Ci} / \mathrm{mL})$ & $1.85 \mathrm{E}+01$ & $6.96 \mathrm{E}+00$ & $9.89 \mathrm{E}+00$ & $1.36 \mathrm{E}+01$ & $1.01 \mathrm{E}+01$ \\
\hline Eu-155 $(\mu \mathrm{Ci} / \mathbf{m L})$ & $* 9.75 \mathrm{E}+00$ & $2.39 \mathrm{E}+00$ & $5.00 \mathrm{E}+00$ & $7.47 \mathrm{E}+00$ & $6.89 \mathrm{E}+00$ \\
\hline Np-237 $(\mu \mathrm{Ci} / \mathbf{m L})$ & $2.58 \mathrm{E}-02$ & $* 9.91 \mathrm{E}-03$ & $* 1.29 \mathrm{E}-02$ & $1.50 \mathrm{E}-02$ & $1.07 \mathrm{E}-02$ \\
\hline Pu-238 $(\mu \mathrm{Ci} / \mathrm{mL})$ & $2.91 \mathrm{E}+01$ & $1.73 \mathrm{E}+01$ & $1.97 \mathrm{E}+01$ & $1.89 \mathrm{E}+01$ & $1.03 \mathrm{E}+01$ \\
\hline $\mathrm{Pu}-239 / 240(\mu \mathrm{Ci} / \mathrm{mL})$ & $1.66 \mathrm{E}+02$ & $1.15 \mathrm{E}+02$ & $1.38 \mathrm{E}+02$ & $1.38 \mathrm{E}+02$ & $8.45 \mathrm{E}+01$ \\
\hline $\mathrm{Pu}-241(\mu \mathrm{Ci} / \mathrm{mL})$ & $* 1.63 \mathrm{E}+03$ & $* 9.74 \mathrm{E}+02$ & $* 1.10 \mathrm{E}+03$ & $1.06 \mathrm{E}+03$ & $5.78 \mathrm{E}+02$ \\
\hline Am-241 $(\mu \mathrm{Ci} / \mathrm{mL})$ & $1.33 \mathrm{E}+02$ & $9.86 \mathrm{E}+01$ & $1.10 \mathrm{E}+02$ & $1.03 \mathrm{E}+02$ & $5.41 \mathrm{E}+01$ \\
\hline Uranium $(\mathrm{kg} / \mathrm{L})$ & 1.35 & 0.556 & 0.722 & 0.860 & 0.571 \\
\hline Density (kg/L) & 2.34 & 1.49 & 2.12 & 1.87 & 0.523 \\
\hline
\end{tabular}

All values are in terms of the wet sludge concentration.

Imputed values are indicated with asterisks.

\subsubsection{Comparisons of Imputed Values with Detection Thresholds}

In seven cases, measurements from SNF-7765 Appendix D were quoted as "less-than" a detection threshold, as shown in Table 3-17. In six of these the imputed value was indeed less than the detection threshold, as would be expected. However, the imputed value for ${ }^{241} \mathrm{Am}$ in Sample 96-13 was 4.1 times greater than the threshold. In this case, it was decided to use the imputed value, because it would be conservative by overestimating the ${ }^{241} \mathrm{Am}$ concentration. 
Table 3-17. Nuclides in Canister Sludge Reported as Less-thans.

\begin{tabular}{|c|c|c|c|c|}
\hline Sample \# & Radionuclide & $\begin{array}{c}\text { Detection Threshold } \\
(\boldsymbol{\mu C i} / \mathbf{m L} \text { settled sludge })\end{array}$ & $\begin{array}{c}\text { Imputed value } \\
(\boldsymbol{\mu C i} / \mathbf{m L} \text { settled sludge })\end{array}$ & $\begin{array}{c}\text { Imputed/ } \\
\text { Threshold }\end{array}$ \\
\hline $96-08$ & ${ }^{237} \mathrm{~Np}$ & 0.0035 & 0.0021 & 0.59 \\
\hline $96-13$ & ${ }^{241} \mathrm{Am}$ & 40 & 164 & 4.10 \\
\hline $96-13$ & ${ }^{238} \mathrm{Pu}$ & 130 & 31 & 0.23 \\
\hline $96-13$ & ${ }^{60} \mathrm{Co}$ & 1.7 & 1.1 & 0.65 \\
\hline $96-13$ & ${ }^{155} \mathrm{Eu}$ & 12.2 & 8.9 & 0.73 \\
\hline $96-15$ & ${ }^{60} \mathrm{Co}$ & 2.39 & 0.88 & 0.37 \\
\hline $96-15$ & ${ }^{155} \mathrm{Eu}$ & 14.1 & 7.2 & 0.51 \\
\hline
\end{tabular}

\subsubsection{Canister Sludge Concentration Estimates}

Only eight canister sludge radionuclide measurement sets were available. While these could be applied to the 52 keys, another method for estimating canister sludge concentration is available. This relies on fuel inventories and a corrosion model described in HNF-8760.

The fuel inventories are calculated by the Radnuc2A software. Each key is the fuel discharged on a particular day. Because the positions of a group of fuel assemblies composing a key in the reactor during irradiation are known, it was possible to calculate the fission and activation product accumulation with a high degree of assurance. Thus Radnuc $2 \mathrm{~A}$ calculated the total amounts of various isotopes in each key. The files mentioned in HNF-8760 Section 7.4 are the source used in the stochastic analysis. These amounts are shown as activity per unit mass of uranium in canister sludge in Tables 3-18, 3-19, and 3-20. The estimated mass of uranium from HNF-8760 in the canister sludge is shown in the first column of numbers in Table 3-18. This uranium mass is compared with estimates from the sample data in Section 3.4.4.2. Additional information for comparison with Table 6 of HNF-8760 is provided in the last rows of these tables.

Table 3-18. Isotope Concentration in Canister Sludge from Radnuc2A: Co-60 to Cs-134. (3 sheets)

\begin{tabular}{|c|c|c|c|c|c|}
\hline Key Number & $\begin{array}{c}\text { Uranium } \\
(\mathbf{M T})\end{array}$ & $\begin{array}{c}\text { Co-60 } \\
(\mathbf{C i} / \mathbf{M T U})\end{array}$ & $\begin{array}{c}\text { Sr-90 } \\
(\mathbf{C i} / \mathbf{M T U})\end{array}$ & $\begin{array}{c}\text { Tc-99 } \\
(\mathbf{C i} / \mathbf{M T U})\end{array}$ & $\begin{array}{c}\text { Cs-134 } \\
(\mathbf{C i} / \mathbf{M T U})\end{array}$ \\
\hline$* 9621 *$ & na & $1.614 \mathrm{E}+00$ & $5.125 \mathrm{E}+03$ & $1.565 \mathrm{E}+00$ & $6.887 \mathrm{E}+00$ \\
\hline 10001 & $6.946 \mathrm{E}-04$ & $5.814 \mathrm{E}-01$ & $3.204 \mathrm{E}+03$ & $1.026 \mathrm{E}+00$ & $3.578 \mathrm{E}-01$ \\
\hline 10201 & $3.710 \mathrm{E}-01$ & $6.961 \mathrm{E}-01$ & $3.594 \mathrm{E}+03$ & $1.150 \mathrm{E}+00$ & $5.449 \mathrm{E}-01$ \\
\hline 10259 & $2.246 \mathrm{E}-01$ & $6.503 \mathrm{E}-01$ & $3.320 \mathrm{E}+03$ & $1.047 \mathrm{E}+00$ & $4.729 \mathrm{E}-01$ \\
\hline 10294 & $8.542 \mathrm{E}-01$ & $6.619 \mathrm{E}-01$ & $3.342 \mathrm{E}+03$ & $1.052 \mathrm{E}+00$ & $4.928 \mathrm{E}-01$ \\
\hline
\end{tabular}


Table 3-18. Isotope Concentration in Canister Sludge from Radnuc2A: Co-60 to Cs-134. (3 sheets)

\begin{tabular}{|c|c|c|c|c|c|}
\hline Key Number & $\begin{array}{l}\text { Uranium } \\
\text { (MT) }\end{array}$ & $\begin{array}{c}\text { Co-60 } \\
\text { (Ci/MTU) }\end{array}$ & $\begin{array}{c}\text { Sr-90 } \\
(\mathbf{C i} / \mathbf{M T U})\end{array}$ & $\begin{array}{c}\text { Tc-99 } \\
\text { (Ci/MTU) }\end{array}$ & $\begin{array}{c}\text { Cs-134 } \\
\text { (Ci/MTU) }\end{array}$ \\
\hline 10350 & $7.950 \mathrm{E}-02$ & $8.912 \mathrm{E}-01$ & $4.350 \mathrm{E}+03$ & $1.408 \mathrm{E}+00$ & $9.168 \mathrm{E}-01$ \\
\hline 10385 & $5.001 \mathrm{E}-01$ & $7.906 \mathrm{E}-01$ & $3.864 \mathrm{E}+03$ & $1.228 \mathrm{E}+00$ & $7.246 \mathrm{E}-01$ \\
\hline 10456 & $4.053 \mathrm{E}-01$ & $1.030 \mathrm{E}+00$ & $4.827 \mathrm{E}+03$ & $1.572 \mathrm{E}+00$ & $1.256 \mathrm{E}+00$ \\
\hline 10576 & $1.651 \mathrm{E}-02$ & 8.843E-01 & $4.070 \mathrm{E}+03$ & $1.285 \mathrm{E}+00$ & $9.438 \mathrm{E}-01$ \\
\hline 10602 & $5.659 \mathrm{E}-01$ & $1.118 \mathrm{E}+00$ & $5.006 \mathrm{E}+03$ & $1.621 \mathrm{E}+00$ & $1.520 \mathrm{E}+00$ \\
\hline 10679 & $5.172 \mathrm{E}-01$ & $1.220 \mathrm{E}+00$ & $5.311 \mathrm{E}+03$ & $1.724 \mathrm{E}+00$ & $1.852 \mathrm{E}+00$ \\
\hline 10742 & $3.280 \mathrm{E}-01$ & $1.139 \mathrm{E}+00$ & $4.907 \mathrm{E}+03$ & $1.568 \mathrm{E}+00$ & $1.625 \mathrm{E}+00$ \\
\hline 10764 & $2.022 \mathrm{E}-03$ & $7.653 \mathrm{E}-01$ & $3.367 \mathrm{E}+03$ & $1.026 \mathrm{E}+00$ & $7.220 \mathrm{E}-01$ \\
\hline 10770 & $3.027 \mathrm{E}-01$ & $1.302 \mathrm{E}+00$ & $5.506 \mathrm{E}+03$ & $1.784 \mathrm{E}+00$ & $2.139 \mathrm{E}+00$ \\
\hline 10832 & $7.157 \mathrm{E}-01$ & $1.087 \mathrm{E}+00$ & $4.595 \mathrm{E}+03$ & $1.446 \mathrm{E}+00$ & $1.504 \mathrm{E}+00$ \\
\hline 10982 & $2.942 \mathrm{E}-01$ & $1.461 \mathrm{E}+00$ & $5.782 \mathrm{E}+03$ & $1.856 \mathrm{E}+00$ & $2.814 \mathrm{E}+00$ \\
\hline 11036 & $1.239 \mathrm{E}-01$ & $1.415 \mathrm{E}+00$ & $5.535 \mathrm{E}+03$ & $1.759 \mathrm{E}+00$ & $2.656 \mathrm{E}+00$ \\
\hline 11109 & $2.965 \mathrm{E}-01$ & $1.547 \mathrm{E}+00$ & $5.891 \mathrm{E}+03$ & $1.878 \mathrm{E}+00$ & $3.230 \mathrm{E}+00$ \\
\hline 11183 & $4.345 \mathrm{E}-01$ & $1.372 \mathrm{E}+00$ & $5.177 \mathrm{E}+03$ & $1.613 \mathrm{E}+00$ & $2.570 \mathrm{E}+00$ \\
\hline 11372 & $4.509 \mathrm{E}-01$ & $1.667 \mathrm{E}+00$ & $5.887 \mathrm{E}+03$ & $1.840 \mathrm{E}+00$ & $3.954 \mathrm{E}+00$ \\
\hline 11449 & $3.260 \mathrm{E}-01$ & $1.730 \mathrm{E}+00$ & $5.963 \mathrm{E}+03$ & $1.858 \mathrm{E}+00$ & $4.334 \mathrm{E}+00$ \\
\hline 11540 & $8.717 \mathrm{E}-02$ & $2.001 \mathrm{E}+00$ & $6.653 E+03$ & $2.089 \mathrm{E}+00$ & $5.921 \mathrm{E}+00$ \\
\hline 11733 & $3.236 \mathrm{E}-03$ & $1.721 \mathrm{E}+00$ & $5.508 \mathrm{E}+03$ & $1.662 \mathrm{E}+00$ & $4.549 \mathrm{E}+00$ \\
\hline 11806 & $2.221 \mathrm{E}-01$ & $1.995 \mathrm{E}+00$ & $6.187 \mathrm{E}+03$ & $1.885 \mathrm{E}+00$ & $6.181 \mathrm{E}+00$ \\
\hline 11897 & $2.954 \mathrm{E}-01$ & $2.123 \mathrm{E}+00$ & $6.392 \mathrm{E}+03$ & $1.944 \mathrm{E}+00$ & $7.163 \mathrm{E}+00$ \\
\hline 11979 & $2.790 \mathrm{E}-01$ & $2.199 \mathrm{E}+00$ & $6.461 \mathrm{E}+03$ & $1.955 \mathrm{E}+00$ & $7.785 \mathrm{E}+00$ \\
\hline 12127 & $1.687 \mathrm{E}-01$ & $2.018 \mathrm{E}+00$ & $5.745 \mathrm{E}+03$ & $1.693 \mathrm{E}+00$ & $6.759 \mathrm{E}+00$ \\
\hline 12201 & $1.471 \mathrm{E}-01$ & $2.331 \mathrm{E}+00$ & $6.425 \mathrm{E}+03$ & $1.912 \mathrm{E}+00$ & $9.130 \mathrm{E}+00$ \\
\hline$* 12240 *$ & na & 7.683E-01 & $2.236 \mathrm{E}+03$ & $5.905 \mathrm{E}-01$ & 8.973E-01 \\
\hline 12285 & $4.471 \mathrm{E}-01$ & $1.989 \mathrm{E}+00$ & $5.436 \mathrm{E}+03$ & $1.572 \mathrm{E}+00$ & $6.747 \mathrm{E}+00$ \\
\hline 12480 & $2.608 \mathrm{E}-01$ & $2.151 \mathrm{E}+00$ & $5.550 \mathrm{E}+03$ & $1.586 \mathrm{E}+00$ & $8.198 \mathrm{E}+00$ \\
\hline 12565 & $1.364 \mathrm{E}-01$ & $2.406 \mathrm{E}+00$ & $6.008 \mathrm{E}+03$ & $1.724 \mathrm{E}+00$ & $1.045 \mathrm{E}+01$ \\
\hline 12639 & $1.698 \mathrm{E}-01$ & $2.181 \mathrm{E}+00$ & $5.385 \mathrm{E}+03$ & $1.515 \mathrm{E}+00$ & $8.702 \mathrm{E}+00$ \\
\hline 12852 & $9.615 \mathrm{E}-03$ & $3.699 \mathrm{E}+00$ & $8.252 \mathrm{E}+03$ & $2.419 \mathrm{E}+00$ & $2.629 \mathrm{E}+01$ \\
\hline 13015 & $5.850 \mathrm{E}-03$ & $2.182 \mathrm{E}+00$ & $4.869 \mathrm{E}+03$ & $1.317 \mathrm{E}+00$ & $9.482 \mathrm{E}+00$ \\
\hline 13649 & $3.116 \mathrm{E}-02$ & $2.685 \mathrm{E}+00$ & $4.979 \mathrm{E}+03$ & $1.289 \mathrm{E}+00$ & $1.624 \mathrm{E}+01$ \\
\hline 13685 & $3.411 \mathrm{E}-03$ & $3.031 \mathrm{E}+00$ & $5.526 \mathrm{E}+03$ & $1.443 \mathrm{E}+00$ & $2.066 \mathrm{E}+01$ \\
\hline$* 14065 *$ & na & $2.313 \mathrm{E}+00$ & $3.856 \mathrm{E}+03$ & 9.391E-01 & $1.268 \mathrm{E}+01$ \\
\hline 14355 & $8.570 \mathrm{E}-02$ & $4.368 \mathrm{E}+00$ & $6.460 \mathrm{E}+03$ & $1.641 \mathrm{E}+00$ & $4.912 \mathrm{E}+01$ \\
\hline
\end{tabular}


SNF-10293, REV 0

Table 3-18. Isotope Concentration in Canister Sludge from Radnuc2A: Co-60 to Cs-134. (3 sheets)

\begin{tabular}{|c|c|c|c|c|c|}
\hline Key Number & $\begin{array}{c}\text { Uranium } \\
(\mathbf{M T})\end{array}$ & $\begin{array}{c}\text { Co-60 } \\
(\mathbf{C i} / \mathbf{M T U})\end{array}$ & $\begin{array}{c}\text { Sr-90 } \\
(\mathbf{C i} / \mathbf{M T U})\end{array}$ & $\begin{array}{c}\text { Tc-99 } \\
(\mathbf{C i} / \mathbf{M T U})\end{array}$ & $\begin{array}{c}\text { Cs-134 } \\
(\mathbf{C i} / \mathbf{M T U})\end{array}$ \\
\hline 14356 & $7.212 \mathrm{E}-01$ & $4.272 \mathrm{E}+00$ & $6.330 \mathrm{E}+03$ & $1.602 \mathrm{E}+00$ & $4.699 \mathrm{E}+01$ \\
\hline$* 14722 *$ & na & $1.072 \mathrm{E}+00$ & $1.513 \mathrm{E}+03$ & $3.353 \mathrm{E}-01$ & $3.995 \mathrm{E}+00$ \\
\hline 15204 & $4.875 \mathrm{E}-02$ & $1.780 \mathrm{E}+00$ & $2.180 \mathrm{E}+03$ & $4.680 \mathrm{E}-01$ & $8.687 \mathrm{E}+00$ \\
\hline$* 15240 *$ & na & $1.199 \mathrm{E}+00$ & $1.453 \mathrm{E}+03$ & $3.111 \mathrm{E}-01$ & $5.969 \mathrm{E}+00$ \\
\hline 15316 & $1.173 \mathrm{E}-02$ & $1.848 \mathrm{E}+00$ & $2.190 \mathrm{E}+03$ & $4.667 \mathrm{E}-01$ & $9.604 \mathrm{E}+00$ \\
\hline 15399 & $3.748 \mathrm{E}-03$ & $6.547 \mathrm{E}-03$ & $7.569 \mathrm{E}+00$ & $1.605 \mathrm{E}-03$ & $3.565 \mathrm{E}-02$ \\
\hline$* 15444 *$ & na & $1.308 \mathrm{E}+00$ & $1.492 \mathrm{E}+03$ & $3.153 \mathrm{E}-01$ & $7.300 \mathrm{E}+00$ \\
\hline 15451 & $5.913 \mathrm{E}-03$ & $2.257 \mathrm{E}+00$ & $2.547 \mathrm{E}+03$ & $5.461 \mathrm{E}-01$ & $1.600 \mathrm{E}+01$ \\
\hline$* 15455 *$ & na & $1.322 \mathrm{E}+00$ & $1.503 \mathrm{E}+03$ & $3.174 \mathrm{E}-01$ & $7.424 \mathrm{E}+00$ \\
\hline$* 15457 *$ & na & $1.328 \mathrm{E}+00$ & $1.509 \mathrm{E}+03$ & $3.186 \mathrm{E}-01$ & $7.465 \mathrm{E}+00$ \\
\hline 15458 & $2.651 \mathrm{E}-02$ & $1.312 \mathrm{E}+00$ & $1.490 \mathrm{E}+03$ & $3.147 \mathrm{E}-01$ & $7.381 \mathrm{E}+00$ \\
\hline$* 15460 *$ & na & $1.274 \mathrm{E}+00$ & $1.447 \mathrm{E}+03$ & $3.054 \mathrm{E}-01$ & $7.174 \mathrm{E}+00$ \\
\hline$* 15461 *$ & na & $4.153 \mathrm{E}-01$ & $4.714 \mathrm{E}+02$ & $9.953 \mathrm{E}-02$ & $2.340 \mathrm{E}+00$ \\
\hline \hline \multicolumn{2}{|r|}{ Weighted Average } & $1.614 \mathrm{E}+00$ & $5.125 \mathrm{E}+03$ & $1.565 \mathrm{E}+00$ & $6.887 \mathrm{E}+00$ \\
\hline \hline \multicolumn{2}{|r|}{ Decayed 3 years } & $1.09 \mathrm{E}+00$ & $4.77 \mathrm{E}+03$ & $1.56 \mathrm{E}+00$ & $2.51 \mathrm{E}+00$ \\
\hline \hline \multicolumn{2}{|r|}{ Percent Difference } & $-0.2 \%$ & $0.1 \%$ & $0.3 \%$ & $0.1 \%$ \\
\hline
\end{tabular}

- Values for isotopic inventory in canister sludge are from ORIGEN2 calculations for N Reactor fuel using the Radnuc2A software. These have been documented in HNF-8760. Neutron exposures, fuel models, and corrosion assumptions are specific to each key. The effective date of the above activity concentrations is $5 / 31 / 1998$.

- The above inventories include the effect of the fuel segregation campaign.

- Keys marked with asterisks use the concentrations in fuel because of the absence of predictions for canister sludge. All canisters in these keys are identified as "Good" in Table 3-12.

- The "Weighted Average" uses the canister sludge uranium mass in each key as the weighting factor.

- The "Decayed 3 years" is the "Weighted Average" with 3 years of decay included for comparison with Table 6 of HNF-8760 Revision 1.

- The "Percent Difference" row shows the difference between the "Decayed 3 years" and Table 6 of HNF-8760 Revision 1. The percent differences are shown in the last row of numbers. Negative differences mean the HNF-8760 number is larger. 
SNF-10293, REV 0

Table 3-19. Isotope Concentration in Canister Sludge from Radnuc2A: Cs-137 to Np-237. (2 sheets)

\begin{tabular}{|c|c|c|c|c|c|}
\hline $\begin{array}{c}\text { Key } \\
\text { Number }\end{array}$ & $\begin{array}{c}\text { Cs-137 } \\
\text { (Ci/MTU) }\end{array}$ & $\begin{array}{c}\text { Eu-152 } \\
(\mathbf{C i} / \mathbf{M T U})\end{array}$ & $\begin{array}{c}\text { Eu-154 } \\
(\mathbf{C i} / \mathbf{M T U})\end{array}$ & $\begin{array}{c}\text { Eu-155 } \\
\text { (Ci/MTU) }\end{array}$ & $\begin{array}{c}\text { Np-237 } \\
\text { (Ci/MTU) }\end{array}$ \\
\hline$* 9621 *$ & $6.858 \mathrm{E}+03$ & $5.147 \mathrm{E}-01$ & $5.851 \mathrm{E}+01$ & $8.642 \mathrm{E}+00$ & $3.271 \mathrm{E}-02$ \\
\hline 10001 & $4.064 \mathrm{E}+03$ & $2.033 \mathrm{E}-01$ & $1.584 \mathrm{E}+01$ & $3.226 \mathrm{E}+00$ & $2.100 \mathrm{E}-02$ \\
\hline 10201 & $4.623 \mathrm{E}+03$ & $2.576 \mathrm{E}-01$ & $2.143 \mathrm{E}+01$ & $3.773 \mathrm{E}+00$ & $2.364 \mathrm{E}-02$ \\
\hline 10259 & $4.218 \mathrm{E}+03$ & $2.191 \mathrm{E}-01$ & $1.753 \mathrm{E}+01$ & $3.609 \mathrm{E}+00$ & $2.145 \mathrm{E}-02$ \\
\hline 10294 & $4.249 \mathrm{E}+03$ & $2.222 \mathrm{E}-01$ & $1.783 \mathrm{E}+01$ & $3.671 \mathrm{E}+00$ & $2.156 \mathrm{E}-02$ \\
\hline 10350 & $5.731 \mathrm{E}+03$ & $3.660 \mathrm{E}-01$ & $3.356 \mathrm{E}+01$ & $4.659 \mathrm{E}+00$ & 2.919E-02 \\
\hline 10385 & $4.996 \mathrm{E}+03$ & $2.984 \mathrm{E}-01$ & $2.516 \mathrm{E}+01$ & $4.215 \mathrm{E}+00$ & $2.523 \mathrm{E}-02$ \\
\hline 10456 & $6.455 \mathrm{E}+03$ & 4.397E-01 & $4.369 \mathrm{E}+01$ & $5.314 \mathrm{E}+00$ & $3.280 \mathrm{E}-02$ \\
\hline 10576 & $5.294 \mathrm{E}+03$ & $3.283 \mathrm{E}-01$ & $2.894 \mathrm{E}+01$ & $4.707 \mathrm{E}+00$ & $2.648 \mathrm{E}-02$ \\
\hline 10602 & $6.721 \mathrm{E}+03$ & 4.697E-01 & $4.800 \mathrm{E}+01$ & $5.762 \mathrm{E}+00$ & $3.388 \mathrm{E}-02$ \\
\hline 10679 & $7.191 \mathrm{E}+03$ & $5.230 \mathrm{E}-01$ & $5.620 \mathrm{E}+01$ & $6.243 \mathrm{E}+00$ & $3.619 \mathrm{E}-02$ \\
\hline 10742 & $6.557 \mathrm{E}+03$ & $4.558 \mathrm{E}-01$ & $4.621 \mathrm{E}+01$ & $5.914 \mathrm{E}+00$ & $3.272 \mathrm{E}-02$ \\
\hline 10764 & $4.266 \mathrm{E}+03$ & $2.261 \mathrm{E}-01$ & $1.874 \mathrm{E}+01$ & $4.320 \mathrm{E}+00$ & $2.100 \mathrm{E}-02$ \\
\hline 10770 & $7.487 \mathrm{E}+03$ & $5.564 \mathrm{E}-01$ & $6.123 \mathrm{E}+01$ & $6.651 \mathrm{E}+00$ & $3.751 \mathrm{E}-02$ \\
\hline 10832 & $6.070 \mathrm{E}+03$ & $4.076 \mathrm{E}-01$ & $3.949 \mathrm{E}+01$ & $5.725 \mathrm{E}+00$ & $3.002 \mathrm{E}-02$ \\
\hline 10982 & $7.901 \mathrm{E}+03$ & $6.094 \mathrm{E}-01$ & $7.006 \mathrm{E}+01$ & $7.452 \mathrm{E}+00$ & $3.914 \mathrm{E}-02$ \\
\hline 11036 & $7.506 \mathrm{E}+03$ & $5.650 \mathrm{E}-01$ & $6.291 \mathrm{E}+01$ & $7.280 \mathrm{E}+00$ & $3.696 \mathrm{E}-02$ \\
\hline 11109 & $8.060 \mathrm{E}+03$ & $6.311 \mathrm{E}-01$ & $7.371 \mathrm{E}+01$ & $7.901 \mathrm{E}+00$ & $3.961 \mathrm{E}-02$ \\
\hline 11183 & $6.937 \mathrm{E}+03$ & $5.057 \mathrm{E}-01$ & $5.402 \mathrm{E}+01$ & $7.171 \mathrm{E}+00$ & $3.371 \mathrm{E}-02$ \\
\hline 11372 & $8.027 \mathrm{E}+03$ & $6.346 \mathrm{E}-01$ & $7.475 \mathrm{E}+01$ & $8.594 \mathrm{E}+00$ & $3.876 \mathrm{E}-02$ \\
\hline 11449 & $8.147 \mathrm{E}+03$ & $6.517 \mathrm{E}-01$ & $7.786 \mathrm{E}+01$ & $8.917 \mathrm{E}+00$ & $3.918 \mathrm{E}-02$ \\
\hline 11540 & $9.228 \mathrm{E}+03$ & $7.885 \mathrm{E}-01$ & $1.022 \mathrm{E}+02$ & $1.017 \mathrm{E}+01$ & 4.434E-02 \\
\hline 11733 & $7.407 \mathrm{E}+03$ & $5.732 \mathrm{E}-01$ & $6.561 \mathrm{E}+01$ & $9.066 \mathrm{E}+00$ & $3.482 \mathrm{E}-02$ \\
\hline 11806 & $8.456 \mathrm{E}+03$ & $6.995 \mathrm{E}-01$ & $8.666 \mathrm{E}+01$ & $1.035 \mathrm{E}+01$ & $3.977 \mathrm{E}-02$ \\
\hline 11897 & $8.776 \mathrm{E}+03$ & 7.435E-01 & $9.497 \mathrm{E}+01$ & $1.099 \mathrm{E}+01$ & 4.111E-02 \\
\hline 11979 & $8.873 E+03$ & $7.575 \mathrm{E}-01$ & $9.749 \mathrm{E}+01$ & $1.140 \mathrm{E}+01$ & 4.134E-02 \\
\hline 12127 & $7.736 \mathrm{E}+03$ & $6.219 \mathrm{E}-01$ & $7.418 \mathrm{E}+01$ & $1.071 \mathrm{E}+01$ & $3.550 \mathrm{E}-02$ \\
\hline 12201 & $8.794 \mathrm{E}+03$ & $7.548 \mathrm{E}-01$ & $9.743 \mathrm{E}+01$ & $1.218 \mathrm{E}+01$ & $4.036 \mathrm{E}-02$ \\
\hline$* 12240 *$ & $2.682 \mathrm{E}+03$ & $9.018 \mathrm{E}-02$ & $8.190 \mathrm{E}+00$ & $5.451 \mathrm{E}+00$ & $1.193 \mathrm{E}-02$ \\
\hline 12285 & $7.244 \mathrm{E}+03$ & $5.671 \mathrm{E}-01$ & $6.529 \mathrm{E}+01$ & $1.069 \mathrm{E}+01$ & $3.279 \mathrm{E}-02$ \\
\hline 12480 & $7.399 \mathrm{E}+03$ & $5.901 \mathrm{E}-01$ & $6.926 \mathrm{E}+01$ & $1.161 \mathrm{E}+01$ & $3.310 \mathrm{E}-02$ \\
\hline 12565 & $8.099 \mathrm{E}+03$ & $6.789 \mathrm{E}-01$ & $8.459 \mathrm{E}+01$ & $1.285 \mathrm{E}+01$ & $3.618 \mathrm{E}-02$ \\
\hline 12639 & $7.137 \mathrm{E}+03$ & $5.635 \mathrm{E}-01$ & $6.524 \mathrm{E}+01$ & $1.188 \mathrm{E}+01$ & $3.154 \mathrm{E}-02$ \\
\hline 12852 & $1.165 \mathrm{E}+04$ & $1.184 \mathrm{E}+00$ & $1.866 \mathrm{E}+02$ & $1.898 \mathrm{E}+01$ & $5.183 \mathrm{E}-02$ \\
\hline 13015 & $6.337 \mathrm{E}+03$ & $4.778 \mathrm{E}-01$ & $5.342 \mathrm{E}+01$ & $1.228 \mathrm{E}+01$ & $2.723 \mathrm{E}-02$ \\
\hline
\end{tabular}


SNF-10293, REV 0

Table 3-19. Isotope Concentration in Canister Sludge from Radnuc2A: Cs-137 to Np-237. (2 sheets)

\begin{tabular}{|c|c|c|c|c|c|}
\hline $\begin{array}{c}\text { Key } \\
\text { Number }\end{array}$ & $\begin{array}{c}\text { Cs-137 } \\
(\mathbf{C i} / \mathbf{M T U})\end{array}$ & $\begin{array}{c}\text { Eu-152 } \\
(\mathbf{C i} / \mathbf{M T U})\end{array}$ & $\begin{array}{c}\text { Eu-154 } \\
(\mathbf{C i} / \mathbf{M T U})\end{array}$ & $\begin{array}{c}\text { Eu-155 } \\
(\mathbf{C i} / \mathbf{M T U})\end{array}$ & $\begin{array}{c}\text { Np-237 } \\
(\mathbf{C i} / \mathbf{M T U})\end{array}$ \\
\hline 13649 & $6.454 \mathrm{E}+03$ & $5.048 \mathrm{E}-01$ & $5.840 \mathrm{E}+01$ & $1.537 \mathrm{E}+01$ & $2.662 \mathrm{E}-02$ \\
\hline 13685 & $7.256 \mathrm{E}+03$ & $6.048 \mathrm{E}-01$ & $7.370 \mathrm{E}+01$ & $1.703 \mathrm{E}+01$ & $2.996 \mathrm{E}-02$ \\
\hline$* 14065 *$ & $4.807 \mathrm{E}+03$ & $3.042 \mathrm{E}-01$ & $3.231 \mathrm{E}+01$ & $1.442 \mathrm{E}+01$ & $1.920 \mathrm{E}-02$ \\
\hline 14355 & $8.624 \mathrm{E}+03$ & $8.071 \mathrm{E}-01$ & $1.126 \mathrm{E}+02$ & $2.447 \mathrm{E}+01$ & $3.432 \mathrm{E}-02$ \\
\hline 14356 & $8.423 \mathrm{E}+03$ & $7.792 \mathrm{E}-01$ & $1.071 \mathrm{E}+02$ & $2.402 \mathrm{E}+01$ & $3.348 \mathrm{E}-02$ \\
\hline$* 14722 *$ & $1.778 \mathrm{E}+03$ & $5.762 \mathrm{E}-02$ & $6.505 \mathrm{E}+00$ & $8.712 \mathrm{E}+00$ & $6.733 \mathrm{E}-03$ \\
\hline 15204 & $2.559 \mathrm{E}+03$ & $8.601 \mathrm{E}-02$ & $1.010 \mathrm{E}+01$ & $1.462 \mathrm{E}+01$ & $9.396 \mathrm{E}-03$ \\
\hline$* 15240 *$ & $1.705 \mathrm{E}+03$ & $5.747 \mathrm{E}-02$ & $6.765 \mathrm{E}+00$ & $9.854 \mathrm{E}+00$ & $6.247 \mathrm{E}-03$ \\
\hline 15316 & $2.570 \mathrm{E}+03$ & $8.714 \mathrm{E}-02$ & $1.032 \mathrm{E}+01$ & $1.522 \mathrm{E}+01$ & $9.371 \mathrm{E}-03$ \\
\hline 15399 & $8.879 \mathrm{E}+00$ & $3.031 \mathrm{E}-04$ & $3.615 \mathrm{E}-02$ & $5.403 \mathrm{E}-02$ & $3.220 \mathrm{E}-05$ \\
\hline$* 15444 *$ & $1.750 \mathrm{E}+03$ & $5.994 \mathrm{E}-02$ & $7.173 \mathrm{E}+00$ & $1.080 \mathrm{E}+01$ & $6.331 \mathrm{E}-03$ \\
\hline 15451 & $3.038 \mathrm{E}+03$ & $1.311 \mathrm{E}-01$ & $1.544 \mathrm{E}+01$ & $1.721 \mathrm{E}+01$ & $1.103 \mathrm{E}-02$ \\
\hline$* 15455 *$ & $1.764 \mathrm{E}+03$ & $6.043 \mathrm{E}-02$ & $7.239 \mathrm{E}+00$ & $1.092 \mathrm{E}+01$ & $6.374 \mathrm{E}-03$ \\
\hline$* 15457 *$ & $1.770 \mathrm{E}+03$ & $6.067 \mathrm{E}-02$ & $7.269 \mathrm{E}+00$ & $1.097 \mathrm{E}+01$ & $6.399 \mathrm{E}-03$ \\
\hline 15458 & $1.748 \mathrm{E}+03$ & $5.994 \mathrm{E}-02$ & $7.182 \mathrm{E}+00$ & $1.084 \mathrm{E}+01$ & $6.320 \mathrm{E}-03$ \\
\hline$* 15460 *$ & $1.697 \mathrm{E}+03$ & $5.818 \mathrm{E}-02$ & $6.974 \mathrm{E}+00$ & $1.052 \mathrm{E}+01$ & $6.130 \mathrm{E}-03$ \\
\hline$* 15461 *$ & $5.529 \mathrm{E}+02$ & $1.896 \mathrm{E}-02$ & $2.272 \mathrm{E}+00$ & $3.431 \mathrm{E}+00$ & $1.998 \mathrm{E}-03$ \\
\hline \hline $\begin{array}{c}\text { Weighted } \\
\text { Average }\end{array}$ & $6.858 \mathrm{E}+03$ & $5.147 \mathrm{E}-01$ & $5.851 \mathrm{E}+01$ & $8.642 \mathrm{E}+00$ & $3.271 \mathrm{E}-02$ \\
\hline $\begin{array}{c}\text { Decayed } \\
3 \text { years }\end{array}$ & $6.40 \mathrm{E}+03$ & $4.42 \mathrm{E}-01$ & $4.62 \mathrm{E}+01$ & $5.68 \mathrm{E}+00$ & $3.27 \mathrm{E}-02$ \\
\hline $\begin{array}{c}\text { Percent } \\
\text { Difference }\end{array}$ & $0.2 \%$ & $0.2 \%$ & $0.9 \%$ & $0.2 \%$ & $0.3 \%$ \\
\hline
\end{tabular}

- Values for isotopic inventory in canister sludge are from ORIGEN2 calculations for N Reactor fuel using the Radnuc2A software. These have been documented in HNF-8760. Neutron exposures, fuel models, and corrosion assumptions are specific to each key. The effective date of the above activity concentrations is $5 / 31 / 1998$.

- The above inventories include the effect of the fuel segregation campaign.

- Keys marked with asterisks use the concentrations in fuel because of the absence of predictions for canister sludge. All canisters in these keys are identified as "Good" in Table 3-12.

- The "Weighted Average" uses the canister sludge uranium mass in each key as the weighting factor.

- The "Decayed 3 years" is the "Weighted Average" with 3 years of decay included for comparison with Table 6 of HNF-8760 Revision 1.

- The "Percent Difference" row shows the difference between the "Decayed 3 years" and Table 6 of HNF-8760 Revision 1. The percent differences are shown in the last row of numbers. Negative differences mean the HNF-8760 number is larger. 
SNF-10293, REV 0

Table 3-20. Isotope Concentration in Canister Sludge from Radnuc2A: Pu-238 to Am-241. (2 sheets)

\begin{tabular}{|c|c|c|c|c|c|}
\hline Key Number & $\begin{array}{c}\text { Pu-238 } \\
(\mathrm{Ci} / \mathrm{MTU}) \\
\end{array}$ & $\begin{array}{c}\text { Pu-239 } \\
\text { (Ci/MTU) }\end{array}$ & $\begin{array}{c}\text { Pu-240 } \\
(\mathbf{C i} / \mathbf{M T U}) \\
\end{array}$ & $\begin{array}{c}\text { Pu-241 } \\
(\mathbf{C i} / \mathbf{M T U}) \\
\end{array}$ & $\begin{array}{c}\text { Am-241 } \\
\text { (Ci/MTU) }\end{array}$ \\
\hline$* 9621 *$ & $6.951 \mathrm{E}+01$ & $1.383 \mathrm{E}+02$ & $8.582 \mathrm{E}+01$ & $3.836 \mathrm{E}+03$ & $2.409 \mathrm{E}+02$ \\
\hline 10001 & $2.744 \mathrm{E}+01$ & $1.049 \mathrm{E}+02$ & $4.589 \mathrm{E}+01$ & $1.345 \mathrm{E}+03$ & $1.125 \mathrm{E}+02$ \\
\hline 10201 & $3.518 \mathrm{E}+01$ & $1.132 \mathrm{E}+02$ & $5.454 \mathrm{E}+01$ & $1.768 \mathrm{E}+03$ & $1.429 \mathrm{E}+02$ \\
\hline 10259 & $2.878 \mathrm{E}+01$ & $1.064 \mathrm{E}+02$ & $4.734 \mathrm{E}+01$ & $1.457 \mathrm{E}+03$ & $1.163 \mathrm{E}+02$ \\
\hline 10294 & $2.908 \mathrm{E}+01$ & $1.068 \mathrm{E}+02$ & $4.769 \mathrm{E}+01$ & $1.478 \mathrm{E}+03$ & $1.173 \mathrm{E}+02$ \\
\hline 10350 & $5.251 \mathrm{E}+01$ & $1.298 \mathrm{E}+02$ & $7.294 \mathrm{E}+01$ & $2.641 \mathrm{E}+03$ & $2.084 \mathrm{E}+02$ \\
\hline 10385 & $3.965 \mathrm{E}+01$ & $1.187 \mathrm{E}+02$ & $5.994 \mathrm{E}+01$ & $2.074 \mathrm{E}+03$ & $1.621 \mathrm{E}+02$ \\
\hline 10456 & $6.617 \mathrm{E}+01$ & $1.394 \mathrm{E}+02$ & $8.547 \mathrm{E}+01$ & $3.271 \mathrm{E}+03$ & $2.536 \mathrm{E}+02$ \\
\hline 10576 & $4.371 \mathrm{E}+01$ & $1.222 \mathrm{E}+02$ & $6.394 \mathrm{E}+01$ & $2.307 \mathrm{E}+03$ & $1.743 \mathrm{E}+02$ \\
\hline 10602 & $7.044 \mathrm{E}+01$ & $1.423 \mathrm{E}+02$ & $8.922 \mathrm{E}+01$ & $3.517 \mathrm{E}+03$ & $2.656 \mathrm{E}+02$ \\
\hline 10679 & $8.063 \mathrm{E}+01$ & $1.480 \mathrm{E}+02$ & $9.768 \mathrm{E}+01$ & $3.988 \mathrm{E}+03$ & $2.972 \mathrm{E}+02$ \\
\hline 10742 & $6.615 \mathrm{E}+01$ & $1.392 \mathrm{E}+02$ & $8.515 \mathrm{E}+01$ & $3.379 \mathrm{E}+03$ & $2.485 \mathrm{E}+02$ \\
\hline 10764 & $2.790 \mathrm{E}+01$ & $1.050 \mathrm{E}+02$ & $4.590 \mathrm{E}+01$ & $1.487 \mathrm{E}+03$ & $1.081 \mathrm{E}+02$ \\
\hline 10770 & $8.606 \mathrm{E}+01$ & $1.515 \mathrm{E}+02$ & $1.023 \mathrm{E}+02$ & $4.272 \mathrm{E}+03$ & $3.133 \mathrm{E}+02$ \\
\hline 10832 & $5.600 \mathrm{E}+01$ & $1.321 \mathrm{E}+02$ & $7.576 \mathrm{E}+01$ & $2.951 \mathrm{E}+03$ & $2.130 \mathrm{E}+02$ \\
\hline 10982 & $9.396 \mathrm{E}+01$ & $1.554 \mathrm{E}+02$ & $1.084 \mathrm{E}+02$ & $4.722 \mathrm{E}+03$ & $3.331 \mathrm{E}+02$ \\
\hline 11036 & $8.399 \mathrm{E}+01$ & $1.501 \mathrm{E}+02$ & $1.003 \mathrm{E}+02$ & $4.310 \mathrm{E}+03$ & $3.005 \mathrm{E}+02$ \\
\hline 11109 & $9.634 \mathrm{E}+01$ & $1.567 \mathrm{E}+02$ & $1.102 \mathrm{E}+02$ & $4.900 \mathrm{E}+03$ & $3.373 \mathrm{E}+02$ \\
\hline 11183 & $7.064 \mathrm{E}+01$ & $1.419 \mathrm{E}+02$ & $8.863 \mathrm{E}+01$ & $3.763 \mathrm{E}+03$ & $2.547 \mathrm{E}+02$ \\
\hline 11372 & $9.290 \mathrm{E}+01$ & $1.546 \mathrm{E}+02$ & $1.070 \mathrm{E}+02$ & $4.890 \mathrm{E}+03$ & $3.198 \mathrm{E}+02$ \\
\hline 11449 & $9.519 \mathrm{E}+01$ & $1.554 \mathrm{E}+02$ & $1.086 \mathrm{E}+02$ & $5.037 \mathrm{E}+03$ & $3.246 \mathrm{E}+02$ \\
\hline 11540 & $1.212 \mathrm{E}+02$ & $1.679 \mathrm{E}+02$ & $1.283 \mathrm{E}+02$ & $6.289 \mathrm{E}+03$ & $3.992 \mathrm{E}+02$ \\
\hline 11733 & $7.658 \mathrm{E}+01$ & $1.446 \mathrm{E}+02$ & $9.276 \mathrm{E}+01$ & $4.292 \mathrm{E}+03$ & $2.610 \mathrm{E}+02$ \\
\hline 11806 & $9.853 \mathrm{E}+01$ & $1.571 \mathrm{E}+02$ & $1.108 \mathrm{E}+02$ & $5.407 \mathrm{E}+03$ & $3.249 \mathrm{E}+02$ \\
\hline 11897 & $1.057 \mathrm{E}+02$ & $1.602 \mathrm{E}+02$ & $1.159 \mathrm{E}+02$ & $5.800 \mathrm{E}+03$ & $3.425 \mathrm{E}+02$ \\
\hline 11979 & $1.067 \mathrm{E}+02$ & $1.608 \mathrm{E}+02$ & $1.168 \mathrm{E}+02$ & $5.910 \mathrm{E}+03$ & $3.432 \mathrm{E}+02$ \\
\hline 12127 & $7.997 \mathrm{E}+01$ & $1.463 \mathrm{E}+02$ & $9.512 \mathrm{E}+01$ & $4.663 \mathrm{E}+03$ & $2.619 \mathrm{E}+02$ \\
\hline 12201 & $1.023 \mathrm{E}+02$ & $1.585 \mathrm{E}+02$ & $1.130 \mathrm{E}+02$ & $5.840 \mathrm{E}+03$ & $3.238 \mathrm{E}+02$ \\
\hline$* 12240 *$ & $9.128 \mathrm{E}+00$ & $6.974 \mathrm{E}+01$ & $1.950 \mathrm{E}+01$ & $5.152 \mathrm{E}+02$ & $2.784 \mathrm{E}+01$ \\
\hline 12285 & $6.871 \mathrm{E}+01$ & $1.394 \mathrm{E}+02$ & $8.545 \mathrm{E}+01$ & $4.158 \mathrm{E}+03$ & $2.256 \mathrm{E}+02$ \\
\hline 12480 & $7.009 \mathrm{E}+01$ & $1.402 \mathrm{E}+02$ & $8.648 \mathrm{E}+01$ & $4.327 \mathrm{E}+03$ & $2.254 \mathrm{E}+02$ \\
\hline 12565 & $8.349 \mathrm{E}+01$ & $1.482 \mathrm{E}+02$ & $9.754 \mathrm{E}+01$ & $5.092 \mathrm{E}+03$ & $2.610 \mathrm{E}+02$ \\
\hline 12639 & $6.423 \mathrm{E}+01$ & $1.361 \mathrm{E}+02$ & $8.110 \mathrm{E}+01$ & $4.078 \mathrm{E}+03$ & $2.052 \mathrm{E}+02$ \\
\hline 12852 & $1.681 \mathrm{E}+02$ & $1.849 \mathrm{E}+02$ & $1.584 \mathrm{E}+02$ & $9.717 \mathrm{E}+03$ & $4.720 \mathrm{E}+02$ \\
\hline 13015 & $4.940 \mathrm{E}+01$ & $1.238 \mathrm{E}+02$ & $6.654 \mathrm{E}+01$ & $3.334 \mathrm{E}+03$ & $1.541 \mathrm{E}+02$ \\
\hline
\end{tabular}


SNF-10293, REV 0

Table 3-20. Isotope Concentration in Canister Sludge from Radnuc2A: Pu-238 to Am-241. (2 sheets)

\begin{tabular}{|c|c|c|c|c|c|}
\hline Key Number & $\begin{array}{c}\text { Pu-238 } \\
(\mathbf{C i} / \mathbf{M T U})\end{array}$ & $\begin{array}{c}\text { Pu-239 } \\
(\mathbf{C i} / \mathbf{M T U})\end{array}$ & $\begin{array}{c}\text { Pu-240 } \\
(\mathbf{C i} / \mathbf{M T U})\end{array}$ & $\begin{array}{c}\text { Pu-241 } \\
(\mathbf{C i} / \mathbf{M T U})\end{array}$ & $\begin{array}{c}\text { Am-241 } \\
(\mathbf{C i} / \mathbf{M T U})\end{array}$ \\
\hline 13649 & $4.773 \mathrm{E}+01$ & $1.222 \mathrm{E}+02$ & $6.447 \mathrm{E}+01$ & $3.482 \mathrm{E}+03$ & $1.390 \mathrm{E}+02$ \\
\hline 13685 & $5.925 \mathrm{E}+01$ & $1.320 \mathrm{E}+02$ & $7.558 \mathrm{E}+01$ & $4.280 \mathrm{E}+03$ & $1.698 \mathrm{E}+02$ \\
\hline$* 14065 *$ & $2.515 \mathrm{E}+01$ & $9.884 \mathrm{E}+01$ & $4.031 \mathrm{E}+01$ & $1.902 \mathrm{E}+03$ & $6.821 \mathrm{E}+01$ \\
\hline 14355 & $7.817 \mathrm{E}+01$ & $1.434 \mathrm{E}+02$ & $9.081 \mathrm{E}+01$ & $5.886 \mathrm{E}+03$ & $1.985 \mathrm{E}+02$ \\
\hline 14356 & $7.455 \mathrm{E}+01$ & $1.413 \mathrm{E}+02$ & $8.785 \mathrm{E}+01$ & $5.645 \mathrm{E}+03$ & $1.902 \mathrm{E}+02$ \\
\hline$* 14722 *$ & $4.369 \mathrm{E}+00$ & $4.130 \mathrm{E}+01$ & $9.848 \mathrm{E}+00$ & $3.108 \mathrm{E}+02$ & $9.318 \mathrm{E}+00$ \\
\hline 15204 & $6.163 \mathrm{E}+00$ & $5.765 \mathrm{E}+01$ & $1.375 \mathrm{E}+01$ & $4.624 \mathrm{E}+02$ & $1.208 \mathrm{E}+01$ \\
\hline$* 15240 *$ & $4.099 \mathrm{E}+00$ & $3.832 \mathrm{E}+01$ & $9.138 \mathrm{E}+00$ & $3.088 \mathrm{E}+02$ & $7.985 \mathrm{E}+00$ \\
\hline 15316 & $6.159 \mathrm{E}+00$ & $5.749 \mathrm{E}+01$ & $1.371 \mathrm{E}+01$ & $4.679 \mathrm{E}+02$ & $1.183 \mathrm{E}+01$ \\
\hline 15399 & $2.122 \mathrm{E}-02$ & $1.977 \mathrm{E}-01$ & $4.712 \mathrm{E}-02$ & $1.626 \mathrm{E}+00$ & $4.010 \mathrm{E}-02$ \\
\hline$* 15444 *$ & $4.173 \mathrm{E}+00$ & $3.884 \mathrm{E}+01$ & $9.262 \mathrm{E}+00$ & $3.215 \mathrm{E}+02$ & $7.817 \mathrm{E}+00$ \\
\hline 15451 & $9.088 \mathrm{E}+00$ & $6.448 \mathrm{E}+01$ & $1.807 \mathrm{E}+01$ & $7.315 \mathrm{E}+02$ & $1.779 \mathrm{E}+01$ \\
\hline$* 15455 *$ & $4.202 \mathrm{E}+00$ & $3.910 \mathrm{E}+01$ & $9.325 \mathrm{E}+00$ & $3.241 \mathrm{E}+02$ & $7.855 \mathrm{E}+00$ \\
\hline$* 15457 *$ & $4.220 \mathrm{E}+00$ & $3.925 \mathrm{E}+01$ & $9.361 \mathrm{E}+00$ & $3.254 \mathrm{E}+02$ & $7.880 \mathrm{E}+00$ \\
\hline 15458 & $4.167 \mathrm{E}+00$ & $3.877 \mathrm{E}+01$ & $9.244 \mathrm{E}+00$ & $3.215 \mathrm{E}+02$ & $7.784 \mathrm{E}+00$ \\
\hline$* 15460 *$ & $4.043 \mathrm{E}+00$ & $3.762 \mathrm{E}+01$ & $8.972 \mathrm{E}+00$ & $3.120 \mathrm{E}+02$ & $7.547 \mathrm{E}+00$ \\
\hline$* 15461 *$ & $1.318 \mathrm{E}+00$ & $1.226 \mathrm{E}+01$ & $2.923 \mathrm{E}+00$ & $1.016 \mathrm{E}+02$ & $2.459 \mathrm{E}+00$ \\
\hline \hline $\begin{array}{c}\text { Weighted } \\
\text { Average }\end{array}$ & $6.951 \mathrm{E}+01$ & $1.383 \mathrm{E}+02$ & $8.582 \mathrm{E}+01$ & $3.836 \mathrm{E}+03$ & $2.409 \mathrm{E}+02$ \\
\hline $\begin{array}{c}\text { Decayed } \\
3 \text { years }\end{array}$ & $6.79 \mathrm{E}+01$ & $1.38 \mathrm{E}+02$ & $8.58 \mathrm{E}+01$ & $3.32 \mathrm{E}+03$ & $2.57 \mathrm{E}+02$ \\
\hline $\begin{array}{c}\text { Percent } \\
\text { Difference }\end{array}$ & $0.3 \%$ & $0.2 \%$ & $0.2 \%$ & $0.3 \%$ & $0.3 \%$ \\
\hline Var & $*$ & & & \\
\hline
\end{tabular}

- Values for isotopic inventory in canister sludge are from ORIGEN2 calculations for N Reactor fuel using the Radnuc2A software. These have been documented in HNF-8760. Neutron exposures, fuel models, and corrosion assumptions are specific to each key. The effective date of the above activity concentrations is $5 / 31 / 1998$.

- The above inventories include the effect of the fuel segregation campaign.

- Keys marked with asterisks use the concentrations in fuel because of the absence of predictions for canister sludge. All canisters in these keys are identified as "Good" in Table 3-12.

- The "Weighted Average" uses the canister sludge uranium mass in each key as the weighting factor.

- The "Decayed 3 years" is the "Weighted Average" with 3 years of decay included for comparison with Table 6 of HNF-8760 Revision 1.

- The "Percent Difference" row shows the difference between the "Decayed 3 years" and Table 6 of HNF-8760 Revision 1. The percent differences are shown in the last row of numbers. Negative differences mean the HNF-8760 number is larger. 
The canister sludge concentration estimates from HNF-8760 include the effect of the fuel segregation program and the depth of corrosion.

A "fuel segregation" program was carried on in the early 1980s in the K-East Basin. This program sorted fuel into one of four categories based on ${ }^{240} \mathrm{Pu}$ content expressed as weight percent of total Pu: <6 wt.\% was assigned to "Group A;" 6-9 wt.\% was assigned to "Group B;" 9-12 wt.\% was assigned to "Group C;" and > 12 wt.\% was assigned to "Group D." Group A fuel was preferentially removed from KE to Purex for dissolution, leaving the higher-exposure, higher- ${ }^{240} \mathrm{Pu}$ fuel behind.

The fuel segregation adjustment eliminates low exposure fuel assemblies, which have been removed from the $\mathrm{K}$ East Basin. This increases the average burnup of what was left behind and thus the activity concentrations of the isotopes in the fuel. The corrosion adjustment estimates the volume of fuel that has oxidized. It also considers that the surface layer of uranium, as well as the ends of the assemblies, experience higher burnup than other portions of the fuel. Thus small amounts of corrosion from good and fair condition fuel have slightly higher burnup than larger quantities from poor and bad condition fuel. The activity concentrations increase with increasing burnup.

The results from HNF-8760 are thus the mass of uranium that has oxidized, and the concentrations of the various isotopes in that uranium. This enables calculation of radionuclide concentrations in canister sludge using the measured uranium concentration in the sludge. The isotope concentrations in canister sludge are calculated by multiplying the uranium concentration by the activity per unit mass of uranium as generated by Radnuc2A for each key.

The stochastic analysis uses the measured concentration of uranium from the 8 canister sludge samples (multiplied by the canister sludge volume) to obtain the uranium mass rather than the uranium mass available from Radnuc2A. This approach is used because both the floor and canister sludge in K East Basin contains various non-fuel materials, such as corrosion products from fuel canisters, corrosion products from basin piping and structural materials, sand, and miscellaneous items. The measured uranium concentration takes this into account.

\subsubsection{Testing the Canister Sludge Concentration Estimates}

HNF-8760 does not list the isotope concentrations in canister sludge by key. These are given in the files mentioned in Section 7.4 of HNF-8760. To check these numbers, four comparisons are carried out. The first uses the estimates by key to calculate canister sludge concentrations shown in HNF-8760. The second compares the total uranium mass in canister sludge calculated from the estimates from HNF-8760 with the canister sludge data. The third comparison uses these total uranium masses to calculate canister sludge concentrations for the whole basin. The comparison is again between the estimates from HNF-8760 and the canister sludge data. The final comparison applies the HNF-8760 results to individual canister sludge samples. 
SNF-10293, REV 0

\subsubsection{Average Radionuclide Concentrations in Canister Sludge Uranium}

As a check on the canister sludge concentrations by key found in the files referred to in HNF-8760 Section 7.4, the values shown in Table 6 of HNF-8760 Revision 1 were calculated. These values are weighted sums of the isotope concentrations in each key. The weighting factors are the uranium mass by key shown in Table 3-18. The formula used to calculate these weighted averages is shown below. The sums are over the 52 keys.

$$
\text { Weighted Average Concentration }=\frac{\sum_{\mathrm{K}} \mathrm{MU}_{\mathrm{K}} \mathrm{C}_{\mathrm{K}}}{\sum_{\mathrm{K}} \mathrm{MU}_{\mathrm{K}}}
$$

where

$$
\begin{aligned}
M_{\mathrm{K}}= & \begin{array}{l}
\text { mass of uranium associated with canister sludge from the Kth key } \\
(\text { see Table 3-18) }
\end{array} \\
\mathrm{C}_{\mathrm{K}}= & \begin{array}{l}
\text { radionuclide concentration in canister sludge associated with the } \\
\text { Kth key }
\end{array}
\end{aligned}
$$

One additional consideration is that the effective date of the totals given in HNF-8760 is May 31, 2001, while the effective date of the concentrations shown in Tables 3-18, 3-19, and 3-20 is May 31, 1998. Three years of radioactive decay needs to be applied to the weighted totals in the tables to match the averages shown in HNF-8760. The decay factors for this amount of decay are shown in Table 3-21. The decay factors are calculated using the formula below. Note that more elegant formulas are given in many textbooks. The formula shown is mathematically equivalent to those formulas.

$$
\text { Decay Factor }=\left(\frac{1}{2}\right)^{\text {DecayTime } / \text { Haltlife }}
$$

In addition, the Pu-241 decays to Am-241, which complicates the calculation of Am-241 activity. The formula to calculate how much Am-241 is produced from Pu-241 decay is shown below. For 3 years of decay, the Am-241 ingrowth factor is 4.469E-03. This number is multiplied by the initial activity of Pu-241.

$$
{ }^{241} \text { Am Ingrowth Factor }=\frac{\left(\frac{1}{2}\right)^{\text {DecayTime } / \text { Haltife }(\mathrm{Pu})}-\left(\frac{1}{2}\right)^{\text {DecayTime } / \text { HaltLife (Am) }}}{1.0-\left(\frac{\text { Half Life }(\mathrm{Am})}{\text { Half Life }(\mathrm{Pu})}\right)}
$$


SNF-10293, REV 0

Table 3-21. Nuclide Half Lives and 3-year Decay Factors.

\begin{tabular}{|c|c|c|}
\hline Nuclide & Half Life (y) & 3-year Decay Factor \\
\hline${ }^{60} \mathrm{Co}$ & $5.271 \mathrm{E}+00$ & $6.740 \mathrm{E}-01$ \\
\hline${ }^{90} \mathrm{Sr}$ & $2.860 \mathrm{E}+01$ & $9.299 \mathrm{E}-01$ \\
\hline${ }^{99} \mathrm{Tc}$ & $2.130 \mathrm{E}+05$ & $1.000 \mathrm{E}+00$ \\
\hline${ }^{134} \mathrm{Cs}$ & $2.062 \mathrm{E}+00$ & $3.648 \mathrm{E}-01$ \\
\hline${ }^{137} \mathrm{Cs}$ & $3.017 \mathrm{E}+01$ & $9.334 \mathrm{E}-01$ \\
\hline${ }^{152} \mathrm{Eu}$ & $1.360 \mathrm{E}+01$ & $8.582 \mathrm{E}-01$ \\
\hline${ }^{154} \mathrm{Eu}$ & $8.800 \mathrm{E}+00$ & $7.895 \mathrm{E}-01$ \\
\hline${ }^{155} \mathrm{Eu}$ & $4.960 \mathrm{E}+00$ & $6.575 \mathrm{E}-01$ \\
\hline${ }^{237} \mathrm{~Np}$ & $2.140 \mathrm{E}+06$ & $1.000 \mathrm{E}+00$ \\
\hline${ }^{238} \mathrm{Pu}$ & $8.775 \mathrm{E}+01$ & $9.766 \mathrm{E}-01$ \\
\hline${ }^{239} \mathrm{Pu}$ & $2.413 \mathrm{E}+04$ & $9.999 \mathrm{E}-01$ \\
\hline${ }^{240} \mathrm{Pu}$ & $6.537 \mathrm{E}+03$ & $9.997 \mathrm{E}-01$ \\
\hline${ }^{241} \mathrm{Pu}$ & $1.440 \mathrm{E}+01$ & $8.655 \mathrm{E}-01$ \\
\hline${ }^{241} \mathrm{Am}$ & $4.322 \mathrm{E}+02$ & $9.952 \mathrm{E}-01$ \\
\hline
\end{tabular}

- The radioactive decay half-lives are from DOE/TIC-11026, Radioactive Decay Data Tables, D. C. Kocher, 1981.

- The "3-year Decay Factor" is multiplied by the initial activity of an isotope to calculate the activity that remains after 3 years of decay.

- Although the initial amount of ${ }^{241} \mathrm{Am}$ decreases by the factor shown, some ${ }^{241} \mathrm{Am}$ is produced by the decay of ${ }^{241} \mathrm{Pu}$ to ${ }^{241} \mathrm{Am}$. The amount produced is the initial ${ }^{241} \mathrm{Pu}$ activity times $4.469 \mathrm{E}-03$.

The weighted average radionuclide concentrations using the canister sludge estimates by key are listed in Tables 3-18, 3-19, and 3-20. In addition, the average concentrations after 3 years of decay are shown. Finally in these tables, the percent differences with the weighted averages listed in Table 6 of HNF-8760 are given. The differences between the decayed values and HNF-8760 are all less than 1 percent.

\subsubsection{Total Uranium Mass in Canister Sludge}

The uranium mass in canister sludge calculated using the methods of HNF-8760, and the uranium mass calculated from the canister sludge sample data are compared in Table 3-22. To calculate the "Total Uranium Mass" by key from the sample data, the mean canister sludge volume from Table $3-11$ is multiplied by the average uranium concentration $(0.860 \mathrm{~kg} / \mathrm{L})$ from Table 5-14. The total, shown in the last row of numbers, is 4.05 times smaller than the total shown for the HNF-8760 uranium masses. While this difference in total uranium mass in sludge is significant, it should have little effect on the stochastic calculation because only the isotope concentrations from HNF-8760 are used in the stochastic analysis. Comparisons with canisters sludge data in Appendix E show the Radnuc2A predictions are clearly conservative with respect to safety basis. 
SNF-10293, REV 0

Table 3-22. Canister Sludge Volume and Uranium Mass by Key. (2 sheets)

\begin{tabular}{|c|c|c|c|}
\hline Key Number & $\begin{array}{c}\text { Average Volume } \\
\text { (liters) }\end{array}$ & $\begin{array}{c}\text { Total Uranium Mass } \\
(\mathrm{kg})\end{array}$ & $\begin{array}{l}\text { HNF-8760 Uranium } \\
(\mathrm{kg})\end{array}$ \\
\hline 9621 & $3.003 \mathrm{E}+00$ & $2.584 \mathrm{E}+00$ & na \\
\hline 10001 & $1.289 \mathrm{E}+00$ & $1.109 \mathrm{E}+00$ & $6.946 \mathrm{E}-01$ \\
\hline 10201 & $3.860 \mathrm{E}+01$ & $3.322 \mathrm{E}+01$ & $3.710 \mathrm{E}+02$ \\
\hline 10259 & $5.934 \mathrm{E}+01$ & $5.106 \mathrm{E}+01$ & $2.246 \mathrm{E}+02$ \\
\hline 10294 & $1.514 \mathrm{E}+02$ & $1.303 \mathrm{E}+02$ & $8.542 \mathrm{E}+02$ \\
\hline 10350 & $7.019 \mathrm{E}+00$ & $6.040 \mathrm{E}+00$ & $7.950 \mathrm{E}+01$ \\
\hline 10385 & $1.223 \mathrm{E}+02$ & $1.052 \mathrm{E}+02$ & $5.001 \mathrm{E}+02$ \\
\hline 10456 & $8.776 \mathrm{E}+01$ & $7.552 \mathrm{E}+01$ & $4.053 \mathrm{E}+02$ \\
\hline 10576 & $7.023 \mathrm{E}+00$ & $6.043 \mathrm{E}+00$ & $1.651 \mathrm{E}+01$ \\
\hline 10602 & $1.099 \mathrm{E}+02$ & $9.455 \mathrm{E}+01$ & $5.659 \mathrm{E}+02$ \\
\hline 10679 & $1.087 \mathrm{E}+02$ & $9.358 \mathrm{E}+01$ & $5.172 \mathrm{E}+02$ \\
\hline 10742 & $7.903 \mathrm{E}+01$ & $6.801 \mathrm{E}+01$ & $3.280 \mathrm{E}+02$ \\
\hline 10764 & $3.596 \mathrm{E}+00$ & $3.094 \mathrm{E}+00$ & $2.022 \mathrm{E}+00$ \\
\hline 10770 & $6.801 \mathrm{E}+01$ & $5.852 \mathrm{E}+01$ & $3.027 \mathrm{E}+02$ \\
\hline 10832 & $1.903 \mathrm{E}+02$ & $1.637 \mathrm{E}+02$ & $7.157 \mathrm{E}+02$ \\
\hline 10982 & $8.227 \mathrm{E}+01$ & $7.080 \mathrm{E}+01$ & $2.942 \mathrm{E}+02$ \\
\hline 11036 & $3.999 \mathrm{E}+01$ & $3.441 \mathrm{E}+01$ & $1.239 \mathrm{E}+02$ \\
\hline 11109 & $8.658 \mathrm{E}+01$ & $7.450 \mathrm{E}+01$ & $2.965 \mathrm{E}+02$ \\
\hline 11183 & $1.390 \mathrm{E}+02$ & $1.196 \mathrm{E}+02$ & $4.345 \mathrm{E}+02$ \\
\hline 11372 & $1.179 \mathrm{E}+02$ & $1.015 \mathrm{E}+02$ & $4.509 \mathrm{E}+02$ \\
\hline 11449 & $9.540 \mathrm{E}+01$ & $8.209 \mathrm{E}+01$ & $3.260 \mathrm{E}+02$ \\
\hline 11540 & $3.135 \mathrm{E}+01$ & $2.698 \mathrm{E}+01$ & $8.717 \mathrm{E}+01$ \\
\hline 11733 & $1.289 \mathrm{E}+00$ & $1.109 \mathrm{E}+00$ & $3.236 \mathrm{E}+00$ \\
\hline 11806 & $7.366 \mathrm{E}+01$ & $6.338 \mathrm{E}+01$ & $2.221 \mathrm{E}+02$ \\
\hline 11897 & $1.020 \mathrm{E}+02$ & $8.778 \mathrm{E}+01$ & $2.954 \mathrm{E}+02$ \\
\hline 11979 & $9.674 \mathrm{E}+01$ & $8.324 \mathrm{E}+01$ & $2.790 \mathrm{E}+02$ \\
\hline 12127 & $6.445 \mathrm{E}+01$ & $5.546 \mathrm{E}+01$ & $1.687 \mathrm{E}+02$ \\
\hline 12201 & $5.228 \mathrm{E}+01$ & $4.499 \mathrm{E}+01$ & $1.471 \mathrm{E}+02$ \\
\hline 12240 & $8.649 \mathrm{E}-01$ & 7.442E-01 & na \\
\hline 12285 & $1.323 \mathrm{E}+02$ & $1.138 \mathrm{E}+02$ & $4.471 \mathrm{E}+02$ \\
\hline 12480 & $1.069 \mathrm{E}+02$ & $9.199 \mathrm{E}+01$ & $2.608 \mathrm{E}+02$ \\
\hline 12565 & $6.476 \mathrm{E}+01$ & $5.573 \mathrm{E}+01$ & $1.364 \mathrm{E}+02$ \\
\hline 12639 & $7.945 \mathrm{E}+01$ & $6.837 \mathrm{E}+01$ & $1.698 \mathrm{E}+02$ \\
\hline 12852 & $4.444 \mathrm{E}+00$ & $3.824 \mathrm{E}+00$ & $9.615 \mathrm{E}+00$ \\
\hline
\end{tabular}


Table 3-22. Canister Sludge Volume and Uranium Mass by Key. (2 sheets)

\begin{tabular}{|c|c|c|c|}
\hline Key Number & $\begin{array}{c}\text { Average Volume } \\
\text { (liters) }\end{array}$ & $\begin{array}{c}\text { Total Uranium Mass } \\
(\mathrm{kg})\end{array}$ & $\begin{array}{l}\text { HNF-8760 Uranium } \\
(\mathrm{kg})\end{array}$ \\
\hline 13015 & $2.290 \mathrm{E}+00$ & $1.971 \mathrm{E}+00$ & $5.850 \mathrm{E}+00$ \\
\hline 13649 & $1.174 \mathrm{E}+01$ & $1.010 \mathrm{E}+01$ & $3.116 \mathrm{E}+01$ \\
\hline 13685 & $3.019 \mathrm{E}+00$ & $2.598 \mathrm{E}+00$ & $3.411 \mathrm{E}+00$ \\
\hline 14065 & $2.883 \mathrm{E}-01$ & $2.481 \mathrm{E}-01$ & na \\
\hline 14355 & $2.649 \mathrm{E}+01$ & $2.280 \mathrm{E}+01$ & $8.570 \mathrm{E}+01$ \\
\hline 14356 & $1.146 \mathrm{E}+02$ & $9.859 \mathrm{E}+01$ & $7.212 \mathrm{E}+02$ \\
\hline 14722 & $2.883 \mathrm{E}-01$ & $2.481 \mathrm{E}-01$ & na \\
\hline 15204 & $1.016 \mathrm{E}+02$ & $8.746 \mathrm{E}+01$ & $4.875 \mathrm{E}+01$ \\
\hline 15240 & $5.766 \mathrm{E}-01$ & 4.962E-01 & na \\
\hline 15316 & $2.159 \mathrm{E}+01$ & $1.858 \mathrm{E}+01$ & $1.173 \mathrm{E}+01$ \\
\hline 15399 & $2.002 \mathrm{E}+00$ & $1.723 \mathrm{E}+00$ & $3.748 \mathrm{E}+00$ \\
\hline 15444 & $2.883 \mathrm{E}-01$ & $2.481 \mathrm{E}-01$ & na \\
\hline 15451 & $1.007 \mathrm{E}+01$ & $8.669 \mathrm{E}+00$ & $5.913 \mathrm{E}+00$ \\
\hline 15455 & $1.442 \mathrm{E}+00$ & $1.240 \mathrm{E}+00$ & na \\
\hline 15457 & $5.766 \mathrm{E}-01$ & $4.962 \mathrm{E}-01$ & na \\
\hline 15458 & $1.297 \mathrm{E}+02$ & $1.116 \mathrm{E}+02$ & $2.651 \mathrm{E}+01$ \\
\hline 15460 & $1.759 \mathrm{E}+01$ & $1.513 \mathrm{E}+01$ & na \\
\hline 15461 & $1.124 \mathrm{E}+01$ & $9.675 \mathrm{E}+00$ & na \\
\hline Total & $2.864 \mathrm{E}+03$ & $2.465 \mathrm{E}+03$ & $9.980 \mathrm{E}+03$ \\
\hline
\end{tabular}

- Canister sludge volumes are calculated from the mean sludge depths, the area factors and the number of canisters of each kind. The mean volumes are also shown in Table 3-11.

- The total uranium masses are calculated using the average uranium concentration in canister sludge, $0.860 \mathrm{~kg} / \mathrm{L}$, from Table 3-16.

- The uranium masses in canister sludge estimated using the methods in HNF-8760 are shown in the second last column. These are also listed in Table 3-18. The total uranium mass is a factor of 4.05 greater than the mass calculated using average canister sludge measurements.

Note that the total masses of uranium are all that can be compared in Table 3-22. The values shown for each key have different meanings. The uranium masses from HNF-8760 are obtained from theoretical considerations of how fuel corrodes and how to apply the qualitative fuel condition assignments. The uranium masses from the sample data use the average uranium canister concentration together with the canister sludge volume estimated from the sludge depth measurements. In actual canisters, the uranium concentration will differ from the average. The HNF-8760 approach attempts to describe this difference, while the measurement averages do not. Thus the values for each key should show considerable difference. 
The next section examines the effect of the uranium difference on the isotope concentrations.

\subsubsection{Uranium-Weighted Radionuclide Concentrations}

The average isotopic concentrations in canister sludge are shown in Table 3-23. They are the weighted sums of the isotope concentrations shown in Tables 3-18, 3-19, and 3-20. The weighting factors are the two columns of uranium mass given in Table 3-22. The same formula shown in Section 3.4.4.1 is also used here, except the uranium masses can be one of two estimates. "Method A" uses the uranium masses from the canister sludge sample data. "Method B" uses the uranium masses from HNF-8760. For each isotope, the two weighted averages differ by at most $6 \%$.

Table 3-23. K East Basin Canister Sludge Concentrations.

\begin{tabular}{|c|c|c|c|}
\hline Quantity, Units & Method A & Method B & $\begin{array}{c}\text { Percent } \\
\text { Differences }\end{array}$ \\
\hline Co-60, Ci/MTU & $1.643 \mathrm{E}+00$ & $1.614 \mathrm{E}+00$ & $-1.8 \%$ \\
\hline Sr-90, Ci/MTU & 4.927E+03 & $5.125 \mathrm{E}+03$ & $4.0 \%$ \\
\hline Tc-99, Ci/MTU & $1.486 \mathrm{E}+00$ & $1.565 \mathrm{E}+00$ & $5.3 \%$ \\
\hline Cs-134, Ci/MTU & $6.559 \mathrm{E}+00$ & $6.887 \mathrm{E}+00$ & $5.0 \%$ \\
\hline Cs-137, Ci/MTU & $6.584 \mathrm{E}+03$ & $6.858 \mathrm{E}+03$ & $4.2 \%$ \\
\hline Eu-152, Ci/MTU & 4.931E-01 & $5.147 \mathrm{E}-01$ & $4.4 \%$ \\
\hline Eu-154, Ci/MTU & $5.667 \mathrm{E}+01$ & $5.851 \mathrm{E}+01$ & $3.2 \%$ \\
\hline Eu-155, Ci/MTU & $9.167 \mathrm{E}+00$ & $8.642 \mathrm{E}+00$ & $-5.7 \%$ \\
\hline Np-237, Ci/MTU & $3.108 \mathrm{E}-02$ & $3.271 \mathrm{E}-02$ & $5.2 \%$ \\
\hline $\mathrm{Pu}-238, \mathrm{Ci} / \mathrm{MTU}$ & $6.642 \mathrm{E}+01$ & $6.951 \mathrm{E}+01$ & $4.7 \%$ \\
\hline $\mathrm{Pu}-239, \mathrm{Ci} / \mathrm{MTU}$ & $1.316 \mathrm{E}+02$ & $1.383 \mathrm{E}+02$ & $5.1 \%$ \\
\hline $\mathrm{Pu}-240, \mathrm{Ci} / \mathrm{MTU}$ & $8.139 E+01$ & $8.582 \mathrm{E}+01$ & $5.4 \%$ \\
\hline $\mathrm{Pu}-241, \mathrm{Ci} / \mathrm{MTU}$ & $3.678 \mathrm{E}+03$ & $3.836 \mathrm{E}+03$ & $4.3 \%$ \\
\hline Am-241, Ci/MTU & $2.277 \mathrm{E}+02$ & $2.409 \mathrm{E}+02$ & $5.8 \%$ \\
\hline
\end{tabular}

- The weighted isotopic concentrations (Ci/MTU) are computed from the isotopic concentrations shown in Tables 3-18, 3-19, and 3-20. The weighting factors are the uranium masses by key shown in Table 3-22. "Method A" uses the uranium masses computed from sludge depth and total uranium measurements. "Method B" uses the estimated uranium mass in sludge described in HNF-8760.

- The last column shows the percent differences between the two weighted sums. Negative values mean the HNF-8760 derived number is smaller than the number derived from sludge measurements. 


\section{SNF-10293, REV 0}

\subsubsection{Canister Sludge Sample Concentrations}

One more comparison will be carried out between the HNF-8760 results and the canister sludge sample measurements. The sludge concentrations estimated in HNF-8760 will be compared with the measured values. To compare HNF-8760 calculations to measurements, calculations and measurements are expressed in units of Ci/MTU (equivalent to $\mu \mathrm{Ci} / \mathrm{g} \mathrm{U}$ ). Measured radionuclide concentrations were converted to units of activity per metric ton of uranium in sludge by dividing by the uranium concentration. The radionuclide activity per unit mass of uranium has the advantage of being largely independent of sludge density, and largely independent of any dilution of fuel corrosion products in sludge by non-fuel material such as aluminum corrosion, iron oxide, sand, dirt, resin beads, insect parts, etc.

The discharge date of a fuel key is implicit in the key number, which is the number of days since September 23, 1944. Radnuc2A outputs in this document were decay-corrected to May 31, 1998. The sample results are valid for the time at which they were analyzed. Decay correction is only important for radionuclides with relatively short half-lives with respect to the time scale between discharge and date of interest, as shown in Table 3-21.

In 1996, nine fuel storage barrels (seven containing spent fuel elements) in the K East Basin were sampled to recover portions of the canister sludge to transport to the laboratories for analysis. These samples were taken with a single pull sludge sampler that handed the samples totally underwater. Consistent with the definition of "sludge" the samples taken were obtained using a vacuum probe with slots restricting the particles sizes recovered to 0.25 " or less. To obtain as representative a sample as possible, the vacuum probe was used to make individual axial traverses starting at the top of the sludge column in the canister going to the bottom of the canister, moving within the cusps between the fuel elements and/or canister barrel wall. A range of fuel canister barrels was selected for sampling including 1) those fabricated of stainless steel and aluminum and 2) those containing fuel with varying degrees of damage. Table 3-24 summarizes the canister barrels sampled, further detail can be found in Makenas 1997. Note that 96-01 contained unirradiated fuel (burnup 0), so that Radnuc2A predicts zero radionuclide concentrations in sludge.

In 1999 a second series of canister barrels were sampled in K East Basin for sludge, this time using the Consolidated Sludge Sampler HNF-4746 (Pitner 1999a). The Consolidated Sampler HNF-4016 (Baker 1999) can either take 1) "single pull" samples (where all sludge and water collected is placed in the sample container to send to the lab) or 2) "continuous draw" sludge samples that consolidate in one sample container sludge from a large number of basin locations. In the latter case the excess water is exhausted back to the basin pool through a special 5-micron stainless steel filter, while some fine particulate is lost through the filter the quantity is very small compared to the overall sample. The same sample vacuum probe was used here as in 1996. Table 3-25 summarizes the single pull sample taken, $\mathrm{KC}-1$, and the combined consolidated sample taken, KC-2/3. HNF-4746 and HNF-SD-SNF-TI-015 (Pearce 2001) Appendix D summarize data from these analyses.

A key parameter to the stochastic calculation model for the canister sludge source term is the condition of the fuel elements with respect to damage and degradation. To best validate and calibrate the source term model, the best estimate of classification of damage of the fuel elements 
in the barrels sampled is required. To provide this, a special assessment was performed of the condition of fuel in the barrels sampled for sludge. This was based on 1) the criteria for classifications as noted for the model, Table 5 of HNF-8760, and 2) the condition of the fuel elements---which was based on photographs taken of the top of the barrels and fuel elements from survey video recorded in 1994. The assessment was performed on the canister barrels sampled in 1996 and the single pull sample, designated KC-1, taken in 1999. The photographs of the top of the fuel and barrels are documented for the 1996 sludge samples in HNF-SP-1201 Appendix A, and for the 1999 samples in HNF-4016 Appendix A. Table 3-26 summarizes the results of these assessments. Additional detail about the categories used to assess fuel condition is provided in Appendix C.

The details of the calculations are given in Appendix E. It contains the canister sludge sample results in units of $\mathrm{Ci} / \mathrm{MTU}$, the output by key and fuel model for the samples, and the estimated canister sludge sample concentrations. In addition, the estimated and measured sample concentrations are plotted against one another for comparison. Ideally, they should fall on the line $\mathrm{Y}=\mathrm{X}$, which is also shown in the graphs.

Comparisons of Radnuc2A predictions to canister sludge measurements are summarized in the tables and figures in Appendix E. In general, the Radnuc2A predictions are larger than the measured concentrations, as evidenced on the graphs showing most of the points above the line $Y=X$. Two notable exceptions need further discussion. First, the Radnuc2A predicted values for the short-lived isotopes ${ }^{60} \mathrm{Co}$ and ${ }^{155} \mathrm{Eu}$ are somewhat lower than the measured values. From the tables given in Appendix E, none of the 1999 samples (KC-n) are below the line $\mathrm{Y}=\mathrm{X}$. Thus, if about 2 years of decay had been included on the 1996 samples the plotted values below the line would be closer to the line.

Second, the ${ }^{99} \mathrm{Tc}$ measured concentrations are about 20 times greater than the Radnuc $2 \mathrm{~A}$ estimates. The authors have not found a way to reconcile the large difference between the predictions and the measurements. Fortunately, ${ }^{99} \mathrm{Tc}$ is an insignificant contributor to both the heat generation rate and the inhalation dose.

It can be concluded that using predictions from Radnuc $2 \mathrm{~A}$ to represent canister sludge in the $\mathrm{K}$-East Basin should be conservative, that is, Radnuc2A predictions will exceed actual values in general, on the average, and with only Tc-99 as an exception. These results are reassuring that, for radionuclides that generate significant heat or dose, Radnuc2A predictions should be larger, that is, erring on the side of safety. 
SNF-10293, REV 0

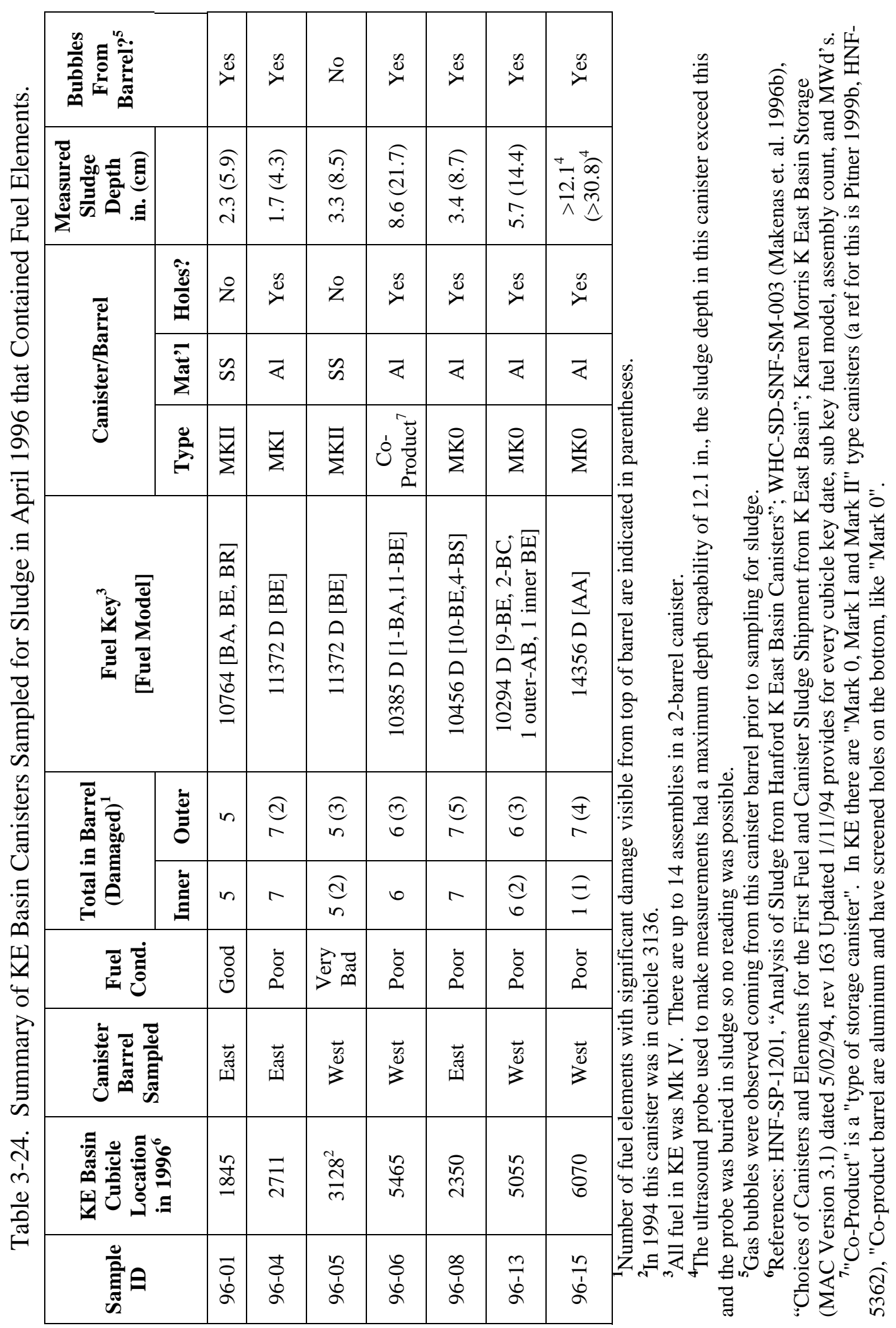




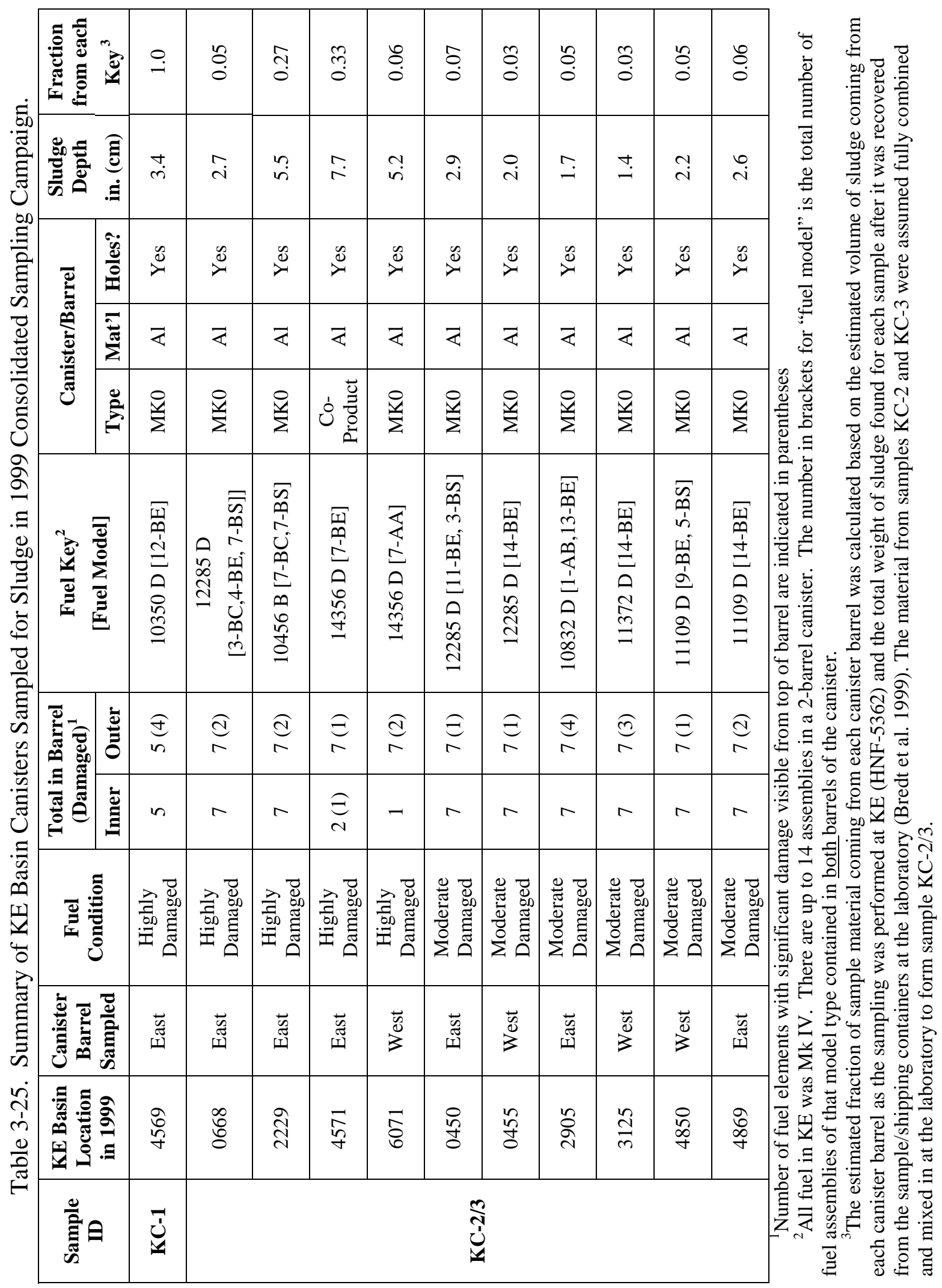


SNF-10293, REV 0

Table 3-26. Summary Table of Estimated Fuel Damage by Category in Barrels Sampled for Sludge KE Basin.

\begin{tabular}{|c|c|c|c|c|c|c|c|c|}
\hline Sample: & $96-01$ & $96-04$ & $96-05$ & $96-06$ & $96-08$ & $96-13$ & $96-15$ & KC-1 \\
\hline Barrel: & $1845 \mathrm{E}$ & $2711 \mathrm{E}$ & $3128 \mathrm{~W}$ & $5465 \mathrm{~W}$ & $2350 \mathrm{E}$ & $5055 \mathrm{~W}$ & $6070 \mathrm{~W}$ & $4569 \mathrm{E}$ \\
\hline \multicolumn{7}{|c|}{ Inner Elements } \\
\hline Intact & 5 & 7 & 4 & 6 & 7 & 4 & & 5 \\
\hline Breached & & & 1 & & & 1 & 1 & \\
\hline Defected & & & & & & 1 & & \\
\hline Bad & & & & & & & \\
\hline \multicolumn{7}{|l|}{} \\
\hline
\end{tabular}

Note 1. 96-05 in 1994, this canister was in cubicle 3136

Note 2. The category counts are based on a specific criteria inferred from model categories used for this analysis Additional information is provided in Appendix C.

\subsubsection{Stochastic Model for Canister Sludge Concentration}

The stochastic model for canister sludge radionuclide concentration uses the isotope concentrations derived in HNF-8760 together with the uranium concentration in canister sludge obtained from the sample measurements. Because the average radioactive inventory of each key is largely determined in HNF-8760, there is no need for probability distributions to describe the isotope concentrations. The range of possible isotopic concentrations in the canister sludge comes from the range of uranium concentrations in the canister sludge.

During the regression analyses for the canister sludge imputations, it was noted that the canister sludge density and the uranium concentration can be related to one another. With density as the independent variable and uranium concentration as the dependent variable, the regression slope is 1.03 and the $y$-intercept is $-1.07 \mathrm{~kg} / \mathrm{L}$ with $\mathrm{R}^{2}=0.887$. The slope and y-intercept are not statistically different from $1.0 \mathrm{~kg} / \mathrm{L}$ and $-1.0 \mathrm{~kg} / \mathrm{L}$. Thus, it was assumed that the uranium concentration could be calculated from the canister sludge density using the equation shown below.

$$
\text { Uranium Concentration }=\text { Sludge Density }-1.0 \mathrm{~kg} / \mathrm{L}
$$

The above formula predicts a mean uranium concentration of $0.873 \mathrm{~kg} / \mathrm{L}$ when the actual mean shown in Table 3-16 is $0.860 \mathrm{~kg} / \mathrm{L}$, a difference of $1.5 \%$. Note that the predicted value is larger. Within the stochastic model for canister sludge the estimated isotope concentrations, heat, dose, and total plutonium are proportional to the uranium concentration. Since the above equation 


\section{SNF-10293, REV 0}

estimates slightly higher uranium concentrations, the estimated quantities associated with the isotope concentrations, heat, dose, and total plutonium will also be slightly larger.

The measured wet density of the canister sludge and the uranium concentration are graphed in Figure 3-24. In this figure, the data values in Table 3-16 are shown as discrete points. The solid line is the equation shown above. The dashed lines show the upper and lower bounds for the uranium concentration that were selected for the stochastic model. The dashed lines were chosen to enclose the data values and keep the solid line as the average of the upper and lower bound.

Figure 3-24. Uranium Concentration Versus Canister Sludge Density.

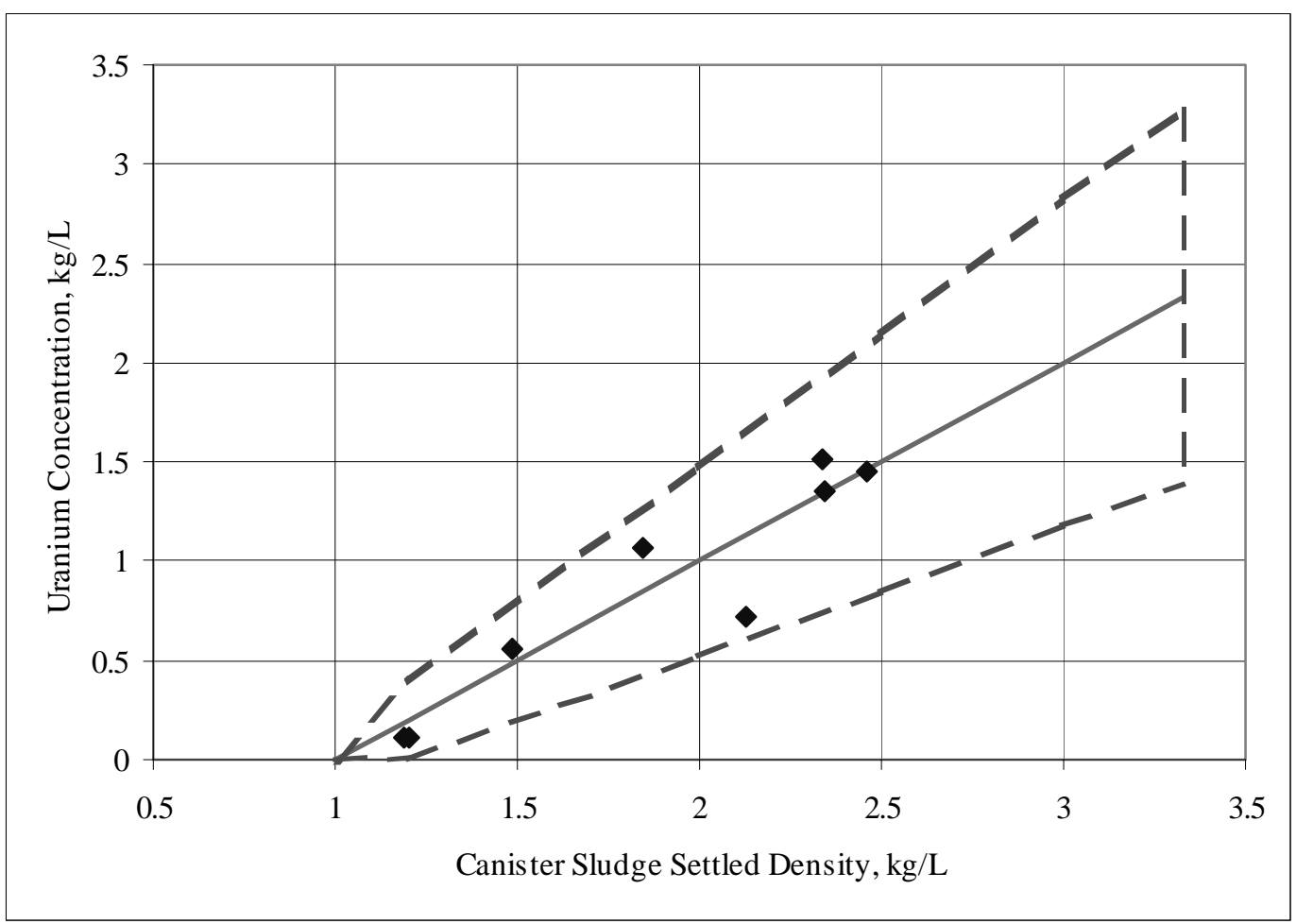

In the stochastic model the sludge density and uranium concentration are calculated using the following method. The first step is to calculate the sludge density by selecting at random one of the 8 values shown in Table 3-16. The chosen data value is adjusted by a factor generated from a uniform distribution with a mean value of 1.0. This factor allows for the variability of the data values without changing the mean. It does increase the standard deviation.

The magnitude of the variability factor applied to the canister sludge density data is calculated using the $99 \%$ confidence interval for the mean discussed in Section 3.2.2. The upper bounds for the measured and imputed quantities are shown in Table 3-27. The variability factor for canister sludge was selected to range from 0.4 to 1.6 based on this table. With this factor, the largest possible sludge density is $(2.458 \mathrm{~kg} / \mathrm{L}-1.0 \mathrm{~kg} / \mathrm{L})(1.6)+1.0 \mathrm{~kg} / \mathrm{L}=3.333 \mathrm{~kg} / \mathrm{L}$. This upper limit on sludge density is indicated in Figure 3-24. The variability factor is applied using the equation shown below to prevent the calculated sludge density from becoming less than $1.0 \mathrm{~kg} / \mathrm{L}$.

$$
\text { Stochastic Sludge Density }=(\text { Factor })(\text { Data Value }-1.0 \mathrm{~kg} / \mathrm{L})+1.0 \mathrm{~kg} / \mathrm{L}
$$


Table 3-27. Variability Factor for the Canister Sample Data.

\begin{tabular}{|c|c|}
\hline Measured Quantity & $\begin{array}{c}\text { Upper Bound for } \\
\text { Variability Factor }\end{array}$ \\
\hline Density & 1.55 \\
\hline Uranium & 1.60 \\
\hline Co-60 & 1.62 \\
\hline Sr-90 & 1.63 \\
\hline Tc-99 & 1.52 \\
\hline Cs-134 & 1.45 \\
\hline Cs-137 & 1.59 \\
\hline Eu-152 & 1.68 \\
\hline
\end{tabular}

\begin{tabular}{|c|c|}
\hline Measured Quantity & $\begin{array}{c}\text { Upper Bound for } \\
\text { Variability Factor }\end{array}$ \\
\hline $\mathrm{Eu}-154$ & 1.68 \\
\hline $\mathrm{Eu}-155$ & 1.84 \\
\hline $\mathrm{Np}-237$ & 1.65 \\
\hline $\mathrm{Pu}-238$ & 1.50 \\
\hline $\mathrm{Pu}-239 / 240$ & 1.56 \\
\hline $\mathrm{Pu}-241$ & 1.50 \\
\hline Am-241 & 1.48 \\
\hline Average & $\mathbf{1 . 5 9}$ \\
\hline
\end{tabular}

The upper bound on the variability factor is calculated from the $99 \%$ confidence level of the mean and the mean for each material.

The "Average" is the mean for the values shown in the table.

The second step is to calculate the uranium concentration. The uranium concentration is regarded as a uniform distribution with a mean value given by the assumed relation between uranium concentration and sludge density shown in the graph as a solid line. The upper and lower bounds are approximated from the sludge density using the equations below. These are shown in Figure 3-24 with dashed lines.

$$
\begin{array}{ll}
\text { Upper Bound }=1.3478 * \text { Density }-1.2174 \mathrm{~kg} / \mathrm{L} & \text { for Density } \geq 1.2 \mathrm{~kg} / \mathrm{L} \\
\text { Upper Bound }=2.0 * \text { Density }-2.0 \mathrm{~kg} / \mathrm{L} & \text { for Density }<1.2 \mathrm{~kg} / \mathrm{L} \\
\text { Lower Bound }=0.6522 * \text { Density }-0.7826 \mathrm{~kg} / \mathrm{L} & \text { for Density } \geq 1.2 \mathrm{~kg} / \mathrm{L} \\
\text { Lower Bound }=0.0 \mathrm{~kg} / \mathrm{L} & \text { for Density }<1.2 \mathrm{~kg} / \mathrm{L}
\end{array}
$$

This range of values was chosen to cover the observed spread in the uranium concentrations about the solid line in Figure 3-24. The mean value of the upper and lower bounds can be shown to be the solid line. The dashed lines appear to represent most of the variation in the uranium concentration. While there may be departures at small densities and uranium concentrations, these are associated with small isotope concentrations which should have little effect on the stochastic results. The area surrounded by dashed lines is the allowed range of values for sludge density and uranium concentration.

It could be argued that at lower sludge densities the uranium concentration shows greater variability than has been assumed in this simple model. However, the lower densities also lead to lower activity concentrations, heat generation rates and inhalation doses. Therefore the bounding stochastic results would hardly be affected. Thus, the simple model for uranium concentration is adequate for the stochastic analysis.

The third step is to calculate the activity concentration in canister sludge of each isotope in a key. The activity concentration of the isotopes of concern is the product of the randomly selected uranium concentration and the estimated canister sludge concentration from Radnuc $2 \mathrm{~A}$ for that 
key and isotope. The estimated canister sludge concentrations are shown in Tables 3-18, 3-19, and 3-20.

Four alternatives to the base case described above are discussed below. These represent the PDF for canister sludge concentration in other ways. The purpose of these alternate cases is to quantify the effect of different modeling assumptions on the stochastic results for sludge containers.

The first alternative to the base case uses canister sample data rather than the Radnuc $2 \mathrm{~A}$ estimates. The same method used on floor sludge concentration was applied to canister sludge. A random number from 1 to 8 is generated to select one of the 8 samples. Then a variability factor with a mean of 1.0 is calculated. The range of this variability factor is from 0.4 to 1.6. Then the various isotope concentrations are calculated by multiplying the variability factor times the sample concentrations. This method is also used to calculate the sludge density and uranium concentration. The result applies to the canister sludge in one key.

From the graphical comparisons in Appendix E, it is expected that this alternative will reduce the isotope concentrations for all nuclides except Tc-99. It is also expected that the heat generation rate and the inhalation dose will also decrease.

A second alternative to the base case is to use a uniform distribution for the canister sludge density rather than the density data. The uranium concentration is calculated using the same method that went into Figure 3-24. The isotope concentrations are calculated using Radnuc2A results. To preserve the mean and not allow sludge densities below $1.05 \mathrm{~kg} / \mathrm{L}$, the upper limit for this uniform distribution is $2.70 \mathrm{~kg} / \mathrm{L}$. The upper limit is calculated as the sum of the mean value $(1.873 \mathrm{~kg} / \mathrm{L})$ and the difference between the mean value and the lower bound $(1.873-1.05=0.823$ $\mathrm{kg} / \mathrm{L})$. Because this is significantly lower than the upper limit for the base case, the bounding stochastic results are expected to be lower also.

A third alternative is to decrease the range for the variability factor in the base case. This is expected to decrease the bounding results because of the decrease in the upper limit for the canister sludge density.

A fourth alternative is to use non-segregated canister sludge estimates (transmitted with the files mentioned in Section 7.4 of HNF-8760) rather than the values shown in Tables 3-18, 3-19, and 3-20. The non-segregated canister sludge estimates assume that the segregation campaign in $\mathrm{KE}$ Basin to remove the low irradiated fuel did not occur. Using material with less irradiation, and lower activity concentrations than the base case should lead to a reduction in all the stochastic results.

\subsection{HEAT AND DOSE}

Heat generation rates and inhalation dose factors are both calculated from the activity of the various radionuclides present in a volume of sludge. The conversion factors used in the calculation are shown in Table 3-28. To speed the calculation, the heat generation rate and inhalation dose factors are calculated for each floor sample and for each key and treated like another isotope in the calculations. For each key there is a given amount of heat (in watts) and dose (in rem) just as there is a given amount of each isotope (in curies). 
SNF-10293, REV 0

Table 3-28. Heat Generation Rate and Inhalation Dose Factors.

\begin{tabular}{|c|c|c|c|c|}
\hline \multirow{2}{*}{ Nuclide } & \multicolumn{2}{|c|}{ Heat Generation Rate Factor } & \multicolumn{2}{c|}{ Inhalation Dose Factor } \\
\cline { 2 - 5 } & $\mathbf{( W / C i )}$ & $\mathbf{( k e V / d e c a y )}$ & $\mathbf{( S v / B q )}$ & Lung Class \\
\hline Co-60 & $1.542 \mathrm{E}-02$ & $2,600.80$ & $5.91 \mathrm{E}-08$ & Y \\
\hline Sr-90 & $6.698 \mathrm{E}-03$ & $1,129.88$ & $6.70 \mathrm{E}-08$ & D \\
\hline Tc-99 & $5.015 \mathrm{E}-04$ & 84.60 & $2.25 \mathrm{E}-09$ & W \\
\hline Cs-134 & $1.020 \mathrm{E}-02$ & $1,720.24$ & $1.25 \mathrm{E}-08$ & D \\
\hline Cs-137 & $4.819 \mathrm{E}-03$ & 812.90 & $8.63 \mathrm{E}-09$ & D \\
\hline Eu-152 & $7.635 \mathrm{E}-03$ & $1,287.89$ & $5.97 \mathrm{E}-08$ & W \\
\hline Eu-154 & $9.082 \mathrm{E}-03$ & $1,532.00$ & $7.73 \mathrm{E}-08$ & W \\
\hline Eu-155 & $7.766 \mathrm{E}-04$ & 131.00 & $1.12 \mathrm{E}-08$ & W \\
\hline Np-237 & $3.103 \mathrm{E}-02$ & $5,234.10$ & $1.46 \mathrm{E}-04$ & W \\
\hline Pu-238 & $3.314 \mathrm{E}-02$ & $5,590.80$ & $1.06 \mathrm{E}-04$ & W \\
\hline Pu-239 & $3.108 \mathrm{E}-02$ & $5,242.78$ & $1.16 \mathrm{E}-04$ & W \\
\hline Pu-240 & $3.114 \mathrm{E}-02$ & $5,253.73$ & $1.16 \mathrm{E}-04$ & W \\
\hline Pu-241 & $3.182 \mathrm{E}-05$ & 5.37 & $2.23 \mathrm{E}-06$ & W \\
\hline Am-241 & $3.337 \mathrm{E}-02$ & $5,630.00$ & $1.20 \mathrm{E}-04$ & W \\
\hline
\end{tabular}

- Information about the short-half life progeny has been included with the parent nuclides. In particular, Sr-90 includes Y-90 (1.00), Cs-137 includes Ba-137m (0.9443), Np-237 includes Pa-233 (1.00), and Pu-241 includes U-237 (2.39E-05). Branching ratios are shown in parentheses.

- Energy per decay is taken from ENDF/B-VI (National Nuclear Data Center 2001). Neutrino energies are not included. Recoil energy of the progeny nuclide is also omitted.

- Inhalation Dose Factors are from Federal Guidance Report Number 11 (EPA-520/1-88-020, Eckerman et al. 1988). The worst-case solubility assumptions have been made. For Sr-90, the Y-90 has lung class Y.

The heating factors are calculated from the energy per decay values listed in Table 3-28. The energies of the radiation particles emitted when the nuclide decays are taken from ENDF/B-VI (National Nuclear Data Center 2001). The energy of the neutrinos as well as the recoil energy of the progeny nuclide has been omitted. The energy per decay is converted to watts per curie of activity using the formula shown below.

$$
\text { Heating Factor }=\left(1.602177 \times 10^{-16} \mathrm{~J} / \mathrm{keV}\right)\left(3.7 \times 10^{10} \mathrm{decay} / \mathrm{s} \text { per curie }\right)(\text { Decay Energy })
$$

The inhalation dose factors listed in Table 3-28 are from Federal Guidance Report Number 11 (Eckerman, Wolbarst, and Richardson1988). These are the same values used in HNF-SD-SNF-TI-059 Revision 2 (Rittmann 1999) for the Spent Nuclear Fuel Project safety basis documents. 


\subsection{URANIUM METAL}

Values for the mass of uranium metal in a sludge volume of interest are needed because uranium metal reacts with water to produce hydrogen gas and heat. The mass of metallic uranium in sludge is an important indicator of the hydrogen generation rate.

Values for the design basis and safety basis amounts of metallic uranium are given in SNF-7765 Appendix B for both floor sludge and canister sludge. The design basis represents an average while the safety basis is an upper bound that is several standard deviations above the mean. The distribution is likely peaked at lower values. However, because of the lack of detailed information, the metallic uranium content of sludge is assumed to be a uniform distribution that ranges from zero to the safety basis value. This will overestimate the uranium metal content of sludge containers in the stochastic analysis because the mean of this PDF is larger than the nominal value for uranium metal given in SNF-7765.

The uranium metal content is expressed as a mass fraction of the sludge. These fractions are shown in Table 3-29. The mass of floor sludge is multiplied by the uranium metal mass fraction for floor sludge to calculate the mass of uranium metal in a given volume of floor sludge. A similar calculation is performed for canister sludge. The range of possible uranium metal mass follows from the ranges used for sludge volume, sludge density, and metallic uranium fraction.

Table 3-29. Range of Uranium Metal Mass Fractions.

\begin{tabular}{|l|c|c|}
\hline \multicolumn{1}{|c|}{ Material } & Design Basis & Safety Basis \\
\hline Floor Sludge & $0.26 \%$ & $1.5 \%$ \\
\hline Canister Sludge & $2.1 \%$ & $5.0 \%$ \\
\hline
\end{tabular}

Uranium metal mass fractions are multiplied by the sludge mass to calculate the uranium metal mass present in a given volume of sludge.

Values are from SNF-7765, Section 4.3. Design Basis represents an average, while safety basis represents a maximum.

\subsection{PLUTONIUM WEIGHT PERCENT}

The plutonium weight percent is calculated to determine various quantities for accountability purposes. The plutonium weight percent is the mass of plutonium divided by the mass of sludge. It can be calculated by dividing the total plutonium concentration $(\mathrm{kg} / \mathrm{L})$ by the sludge density $(\mathrm{kg} / \mathrm{L})$. The total plutonium concentration is calculated using the specific activity of the plutonium isotopes, $\mathrm{Pu}-238, \mathrm{Pu}-239, \mathrm{Pu}-240$, and $\mathrm{Pu}-241$. Most of the mass is from $\mathrm{Pu}-239$. $\mathrm{Pu}-242$ has not been included because it is a minor contributor.

In canister sludge, the calculation of plutonium weight percent involves dividing the concentration of each plutonium isotope by the specific activity of the isotope and summing the results. The specific activities used in the stochastic analysis are listed in Table 3-30. The specific activities are calculated using half-life values in DOE/TIC-11026 (Kocher 1981). 
SNF-10293, REV 0

Table 3-30. Specific Activity of the Plutonium Isotopes.

\begin{tabular}{|c|c|}
\hline Isotope & $\begin{array}{c}\text { Specific Activity } \\
(\mathbf{C i} / \mathbf{g})\end{array}$ \\
\hline $\mathrm{Pu}-238$ & 17.11 \\
\hline $\mathrm{Pu}-239$ & 0.06197 \\
\hline $\mathrm{Pu}-240$ & 0.2278 \\
\hline $\mathrm{Pu}-241$ & 103.0 \\
\hline
\end{tabular}

In floor sludge sample results, the Pu-239 and Pu-240 were not separated in the chemical analysis. To calculate the plutonium weight percent in floor sludge, the stochastic analysis uses a range of specific activities for the $\mathrm{Pu}-239 / 240$ combination that were derived from the Radnuc2A results for canister sludge. The HNF-8760 calculations gave 4 data files listing the activity of each isotope of interest in each key. Two are for fuel and two are for canister sludge. The pairs are further separated into a fuel segregation and non-segregation cases. For each key in each data file, the Pu-239 and Pu-240 mass per MTU was calculated and summed to give the total Pu-239/240 mass per MTU. This was then divided into the combined Pu-239/240 activity per MTU to give the specific activity of the combination.

The next step is to construct cumulative distributions for the specific activity of the combination. The sludge mass or fuel mass was used as the weighting factor. These cumulative distributions are shown in Figure 3-25. The segregated sludge has the highest specific activity. It is the wavy line to the right of all the others. However, when calculating plutonium weight percent, the conservative approach tries to minimize the specific activity. Hence, the cumulative distributions for non-segregated sludge and both types of fuel were also calculated and plotted.

From the hodgepodge of lines in Figure 3-25 a bounding case was constructed. This is shown as the dashed line farthest to the left. Because it has a smaller specific activity than any of the four inventories, it is a bounding case. The distribution used in the stochastic analysis is also listed in Table 3-31. This representation for the Pu-239/240 specific activity will overestimate the plutonium weight percent, but is more realistic than simply using the specific activity of Pu-239 to represent the mixtures. The mean value for the specific activity probability distribution is $0.07805 \mathrm{Ci} / \mathrm{g}$. Note that the entire range of values for $\mathrm{Pu}-239 / 240$ specific activity $(0.07 \mathrm{Ci} / \mathrm{g}$ to $0.083 \mathrm{Ci} / \mathrm{g}$ ) is relatively small. As a result, this distribution will have little effect on the stochastic results. 
SNF-10293, REV 0

Table 3-31. Continuous Probability Distribution for Pu239/240 Specific Activity.

\begin{tabular}{|c|c|}
\hline $\begin{array}{c}\text { Specific Activity } \\
\text { (Ci/g) }\end{array}$ & $\begin{array}{c}\text { Cumulative } \\
\text { Probability }\end{array}$ \\
\hline 0.070 & 0.00 \\
\hline 0.072 & 0.25 \\
\hline 0.080 & 0.40 \\
\hline 0.083 & 1.00 \\
\hline
\end{tabular}

The distribution mean is $0.07805 \mathrm{Ci} / \mathrm{g}$.

Figure 3-25. Specific Activity of Pu-239 and Pu-240 Mixtures in Canister Sludge.

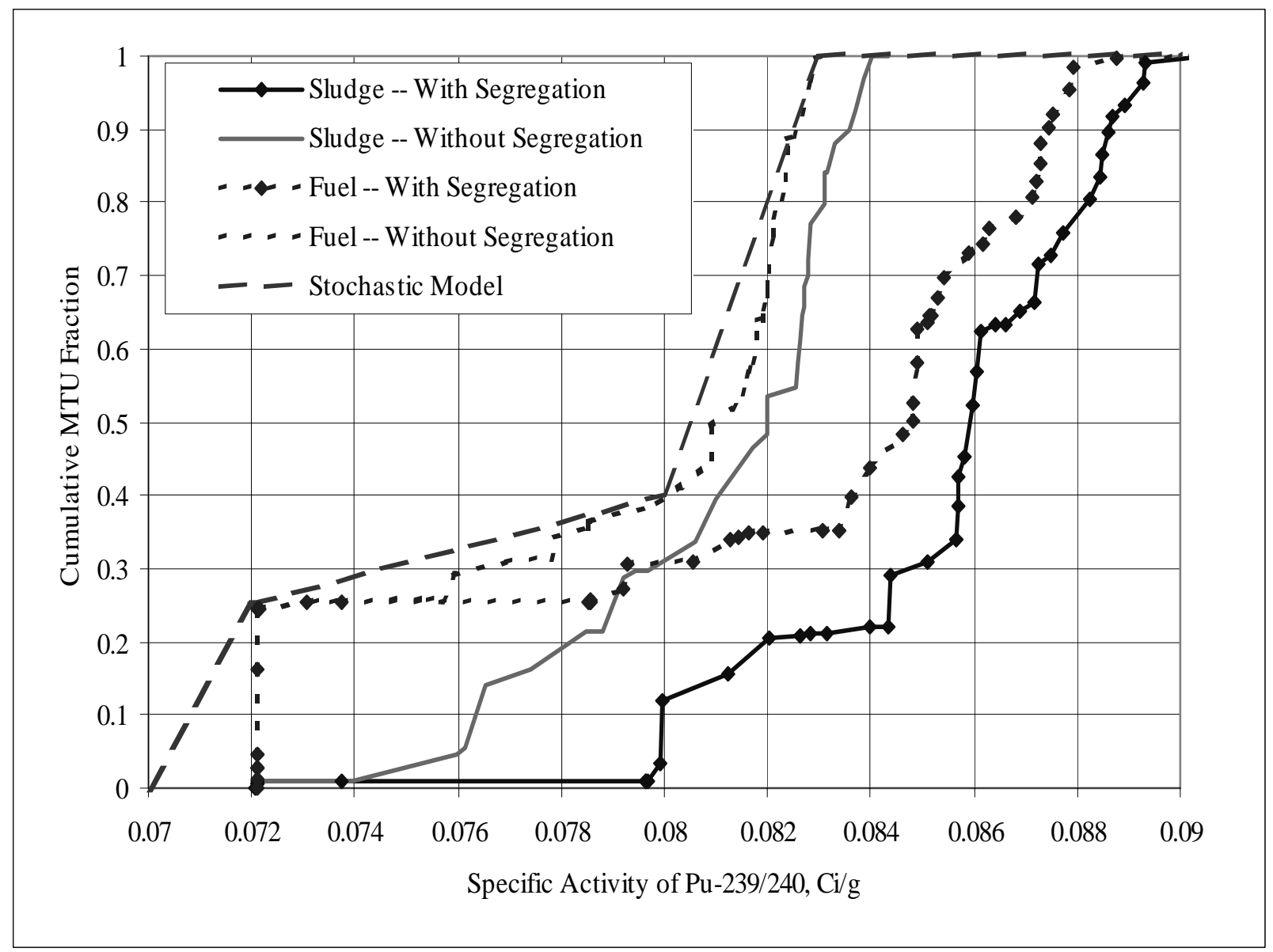




\subsection{CALCULATIONS}

The stochastic calculation uses Monte-Carlo techniques to randomly generate floor and canister sludge volumes and concentrations for each key in K East Basin. These keys are then retrieved in a random order and added to sludge containers. Each simulation gives values for the inventories in the containers that were filled. After many repetitions, a probability distribution is determined for each quantity of interest, e.g., isotope amounts, heat generation rate, and inhalation dose. Bounding values are selected from each spectrum. The typical bound $\left(95^{\text {th }}\right.$ percentile) is exceeded no more than $5 \%$ of the cases.

Most of the radioactivity is found in the canister sludge. These calculations assume that the sludge retrieval process is $100 \%$ efficient, i.e., that no sludge is left behind. However, the currently proposed retrieval process will remove most of the floor sludge but leave a portion of the canister sludge behind. Thus, the assumption of $100 \%$ removal of both floor and canister sludge leads to sludge container inventory estimates (both mean and bounding) that are high.

The PDFs for the stochastic calculations have been selected to preserve the mean values of the input data. The average values for these input parameters can be calculated by hand. A comparison of these hand calculated average input parameters to the stochastic calculations is provided as a check on the stochastic analysis approach.

\subsection{STOCHASTIC METHOD}

The stochastic method employed to model the distribution of various items of interest in filled sludge containers has two parts. The first part is generating the volumes and compositions of floor and canister sludge in each key using the PDFs discussed in Section 3.0. The floor and canister sludge amounts are combined to give the total amount of each item of interest in a key. The second part of the calculation is selecting the key retrieval order and filling the sludge containers.

The calculation of floor sludge amounts is shown in Figure 4-1. The floor sludge volume for this key is the mean value from Table 3-1 multiplied by a variability factor that ranges from 0.8 to 1.2, as shown in the upper portion of Figure 4-1. This volume is used to calculate the total amount of the other items from their concentration. The concentration calculation begins with the random selection of one of the 23 floor sample results. Then a variability factor is created that ranges from 0.2 to 1.8 . This factor is multiplied by all the materials in the sample to generate the concentrations of most of the materials. In particular, the floor sludge density, the uranium concentration, the concentration of the 13 isotopes, the heat generation rate per unit volume, and the inhalation dose per unit volume are all calculated this way. Two additional random numbers are needed to calculate the uranium metal concentration and the plutonium mass. The uranium metal concentration is calculated from the floor sludge density and a uniform distribution that ranges from 0 to $1.5 \%$. The total plutonium per unit volume is calculated from the $\mathrm{Pu}-238$, the $\mathrm{Pu}-239 / 240$, and the $\mathrm{Pu}-241$ concentrations. Pu-242 is not included because it is a minor contributor. The second random number is needed to calculate a value for the $\mathrm{Pu}-239 / 240$ specific activity. Each of these concentrations is multiplied by the floor sludge 
volume for this key to give the total amounts of the materials of interest shown in the tall box on the right side of Figure 4-1. Note that the input concentrations are all tallied for comparison with the input data.

Figure 4-1. Stochastic Calculation: Floor Sludge Amounts per Key.

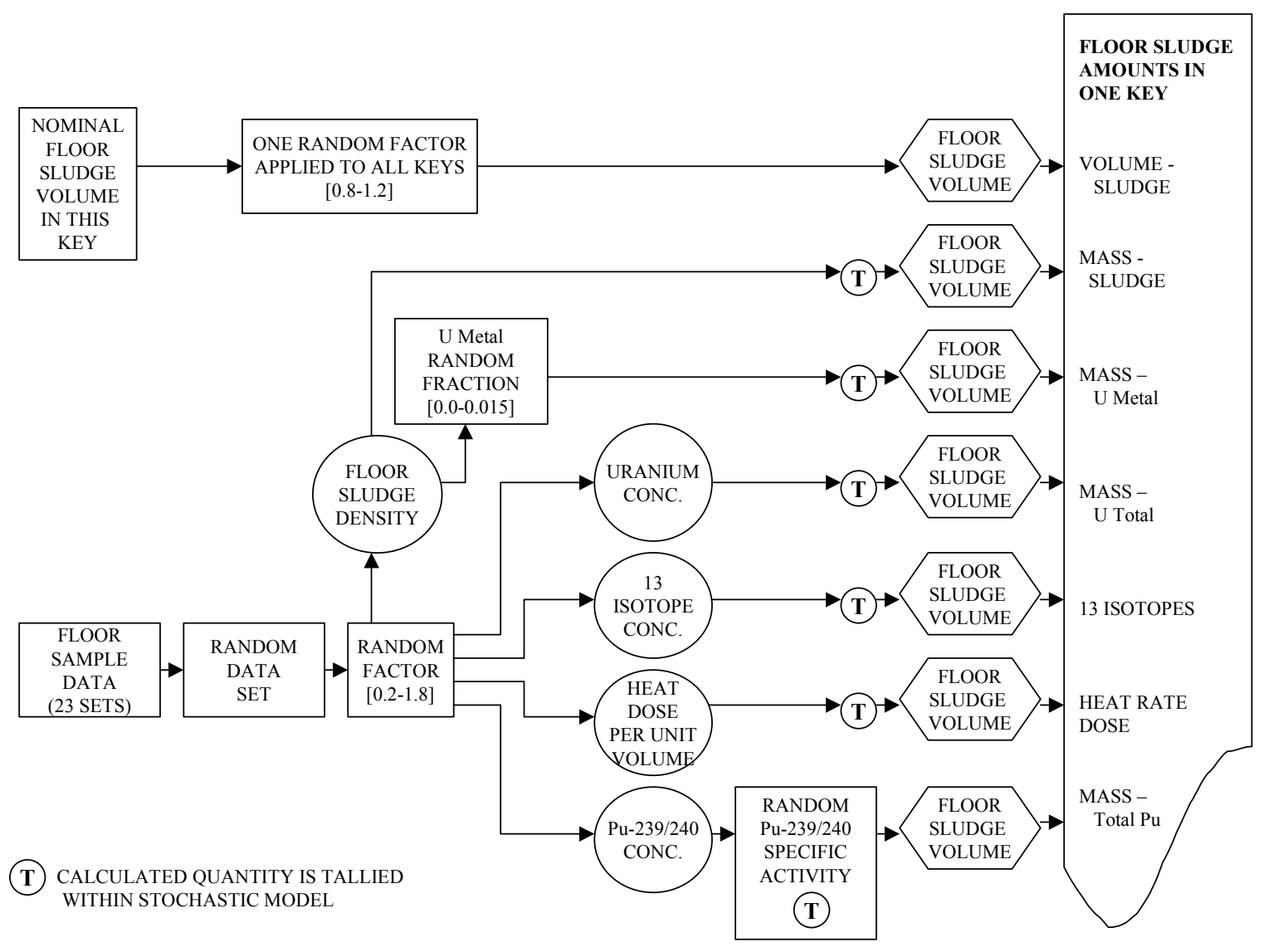


The calculation of canister sludge amounts is shown in Figures 4-2 and 4-3. The canister sludge volume is based on the canister sludge depth measurements. One of the depths for each fuel condition is selected at random. The depth is multiplied by a variability factor that ranges from 0.5 to 1.5. As shown in Figure 4-2, this is done for both types of fair condition fuel and the result is combined as described in Section 3.3.1. Note that the sludge depths are all tallied for comparison with the data. The depths are then multiplied by the appropriate depth-to-volume conversion factors based on the canister condition. These conversion factors are discussed in Section 3.3.1 and are taken from WHC-SD-SNF-TI-034. The result is the total volume of canister sludge in the key.

Figure 4-2. Stochastic Calculation: Canister Sludge Volume per Key.

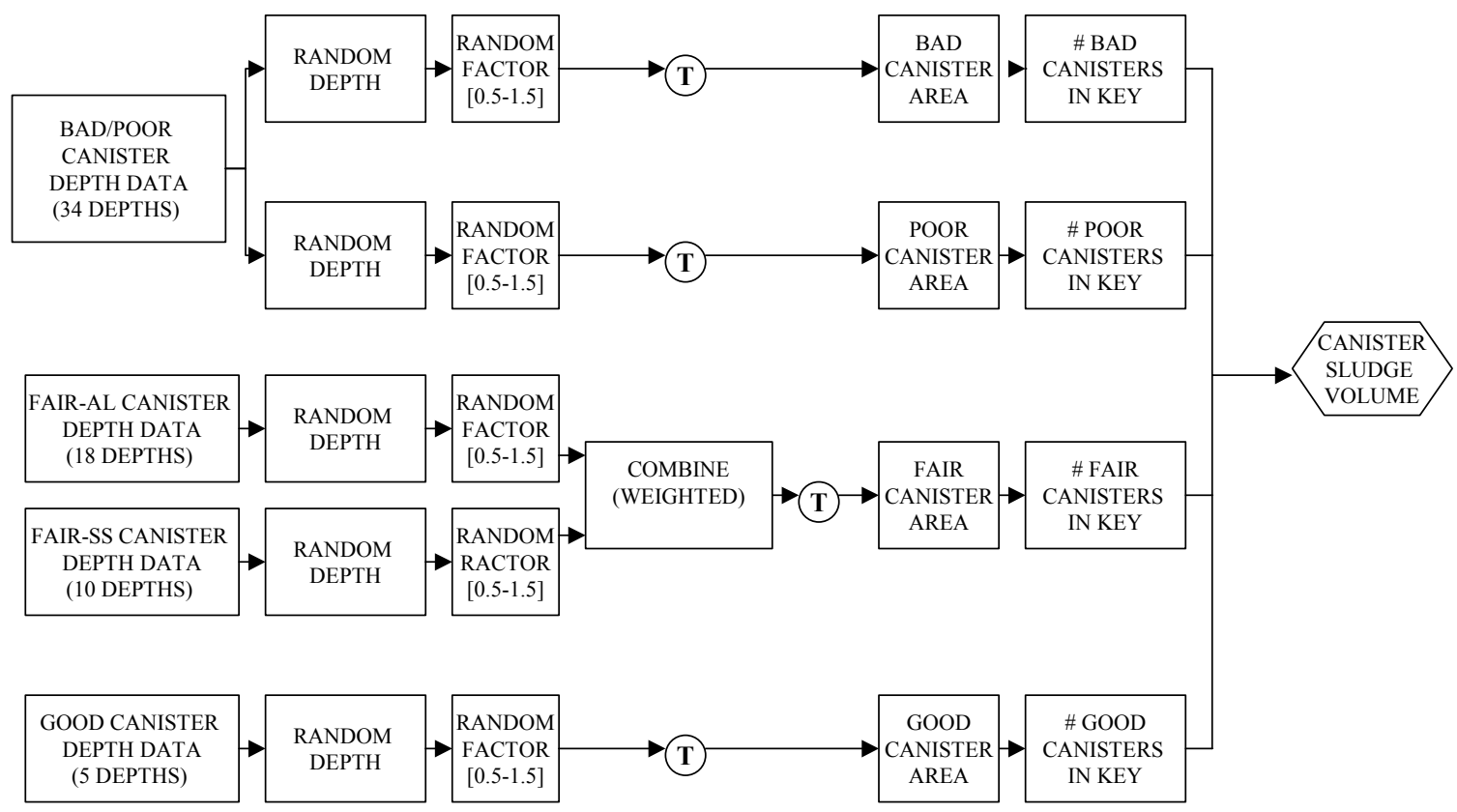

(T) CALCUlated QuANTity is TALLIED WITHIN STOCHASTIC MODEL 
This sludge volume is used to calculate the total amount of sludge mass, U Metal, uranium (total), Co-60, Sr-90, Tc-99, Cs-134, Cs-137, Er-152, Er-154, Np-237, Am-241, Pu-238, Pu-239, $\mathrm{Pu}-240, \mathrm{Pu}-241$, heat rate, dose, and total plutonium mass within a given key. The concentration calculation begins with the random selection of one of the 8 canister sample results for sludge density. Then a variability factor is created that ranges from 0.33 to 1.67 . This factor is multiplied by the selected density. The resulting sludge density is used in the calculation of the uranium metal and the uranium total concentrations. The uranium metal concentration is calculated from a uniform distribution that ranges from 0 to $5 \%$ of the sludge density. The total uranium concentration is calculated as a uniform distribution between the upper and lower bounds for uranium concentration, which depend on the sludge density. The randomly selected uranium concentration is then multiplied by the segregated sludge concentrations from HNF-8760 to generate the concentrations of most of the materials. In particular the concentration of the 13 isotopes, the plutonium mass per unit volume, the heat generation rate per unit volume, and the inhalation dose per unit volume are all calculated this way. Each of these concentrations is multiplied by the canister sludge volume for this key to give the total amounts of the materials of interest shown in the tall box on the right side of Figure 4-3. Note that selected input concentrations are tallied for comparison with the input data.

Figure 4-3. Stochastic Calculation: Canister Sludge Amounts per Key.

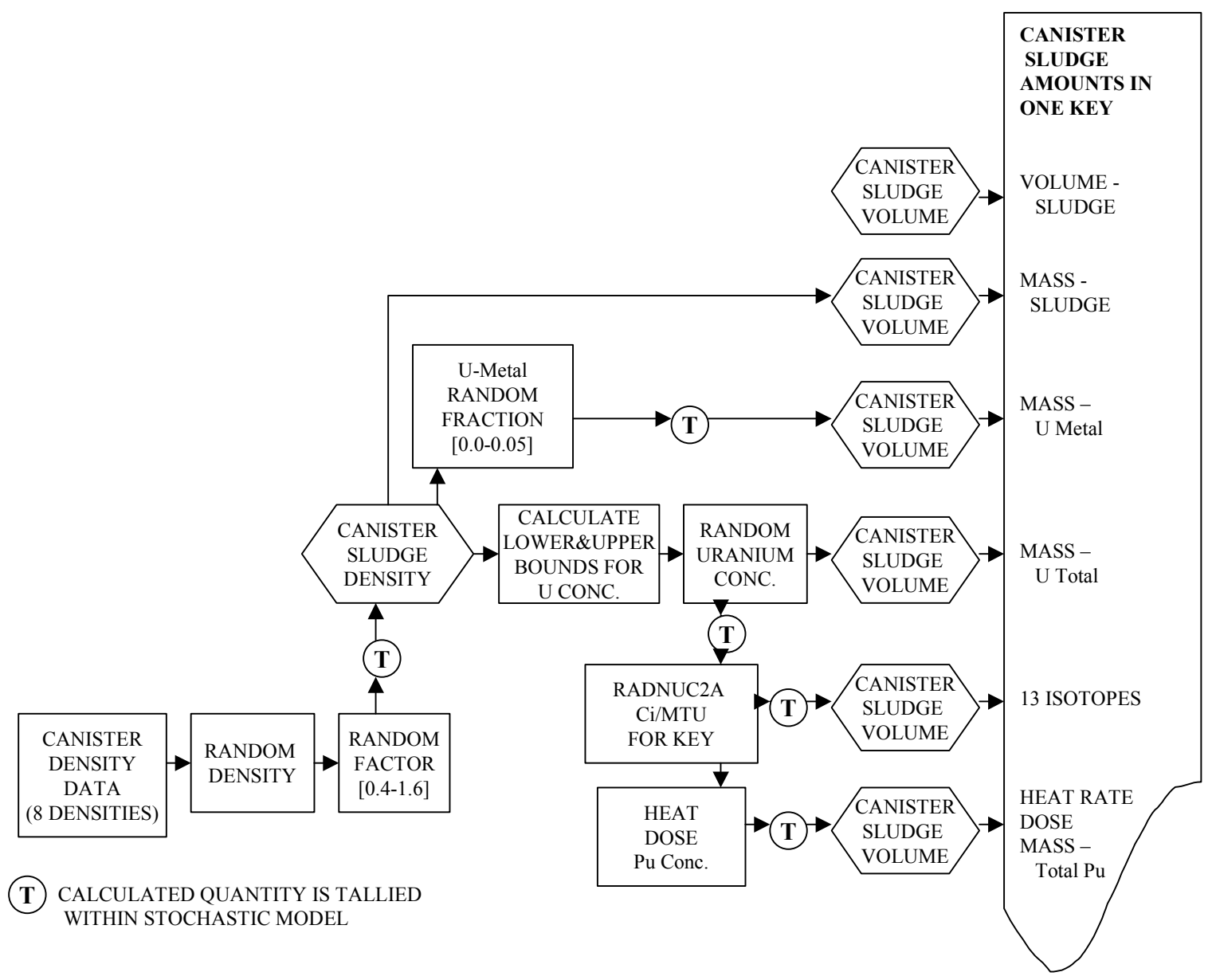


Having calculated the total amounts of floor and canister sludge for a particular key, the next step is to add these amounts together. It is assumed that all of the floor and canister sludge associated with a key will be placed in the sludge container. The calculation of the total amounts per key is shown in Figure 4-4.

The calculations shown in Figure 4-1 through Figure 4-4 are repeated until values are assigned to all 52 keys. Then the key retrieval calculations begin.

Figure 4-4. Stochastic Calculation: Total Sludge Amount per Key.

\begin{tabular}{|l|}
\hline FLOOR SLUDGE \\
AMOUNTS IN \\
ONE KEY \\
VOLUME - \\
SLUDGE \\
MASS - \\
SLUDGE \\
MASS - \\
U Metal \\
MASS - \\
U Total \\
13 ISOTOPES \\
HEAT RATE \\
DOSE \\
MASS - \\
Total Pu \\
\\
\end{tabular}
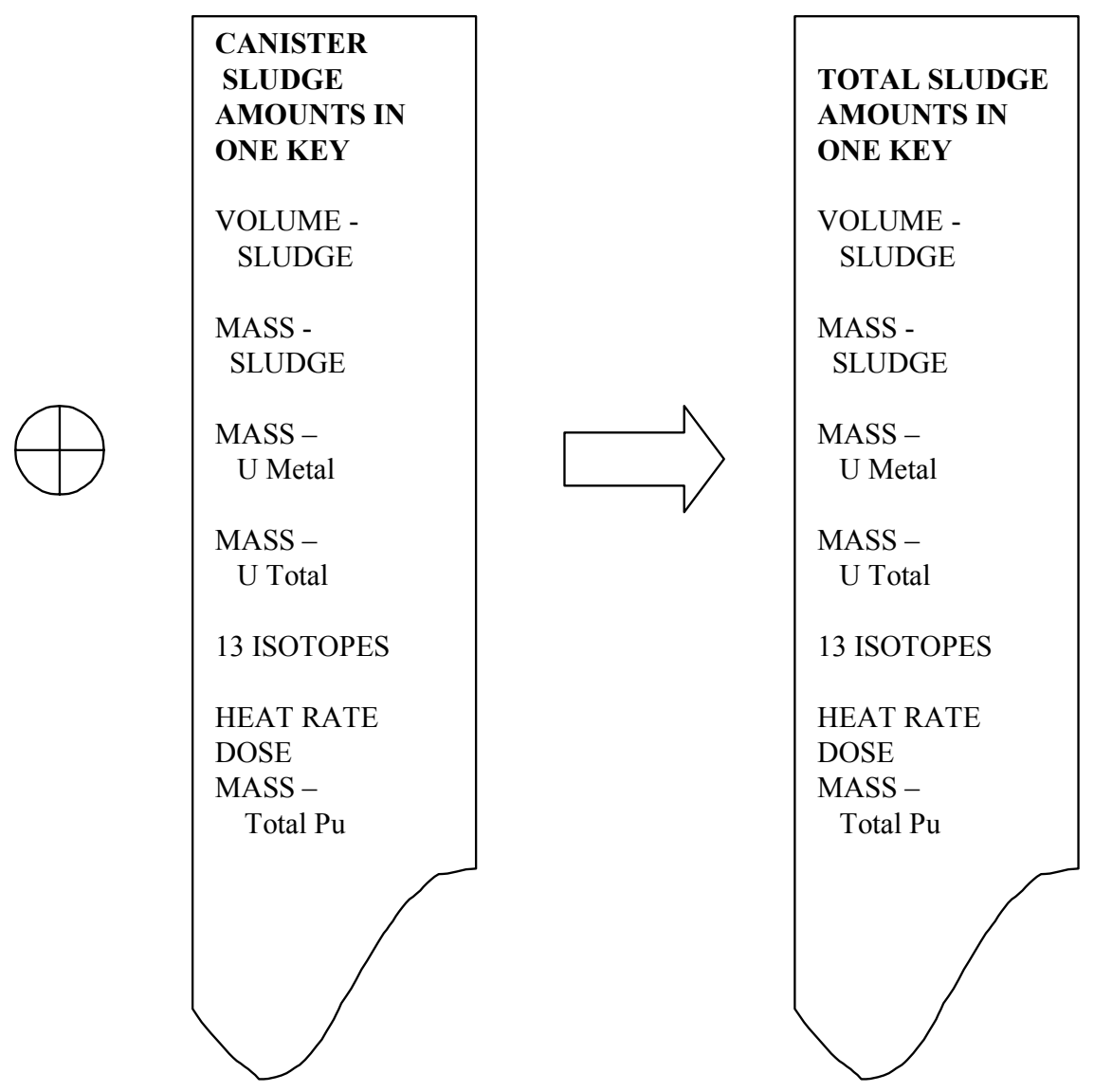
The process of selecting the retrieval sequence begins with the list of 52 keys in the order they were created. This is shown as the "DATA LIST" in Figure 4-5. A random number from 1 to 52 selects one of these keys to be the first retrieved from K East Basin. This key is at the top of the "PICK LIST" in Figure 4-5. This key is removed from the data list. The next iteration begins by generating a random number from 1 to 51 to select from the remaining keys in the data list. The selected key is transferred to the pick list. The iteration is repeated until there is one key left in the data list. This key is added to the bottom of the pick list without generating a random number. Thus, a total of 51 random numbers are used to rearrange the keys into a random retrieval sequence.

Figure 4-5. Stochastic Calculation: Generating a Retrieval Sequence.

DATA LIST


To fill the sludge containers with the amounts in each key, the first key in the pick list is added to the first container. If the container is not full, then the second key is added. This is repeated until the container is full. The extra from the last key added is placed in the next container. This process is illustrated in Figure 4-6. The key amounts are divided according to volume. If 30\% of the key volume goes into the next container, then $30 \%$ of the key amounts also go into the next container. If the last container is less than $90 \%$ full, it is ignored. The $90 \%$ criteria is an assumption that was tested and found to have little effect on the final results (see Section 5.8.7).

Figure 4-6. Stochastic Calculation: Filling Sludge Containers.

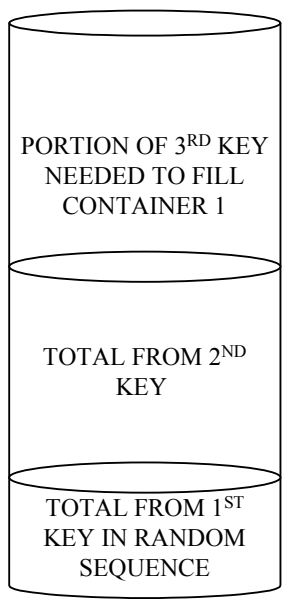

CONTAINER 1

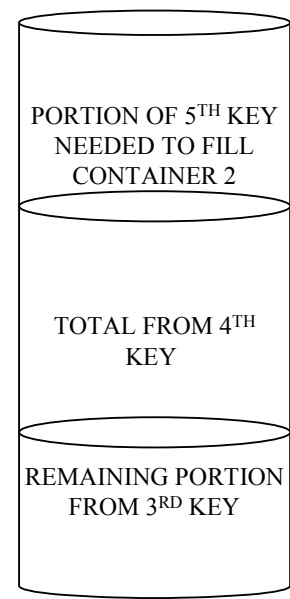

CONTAINER 2

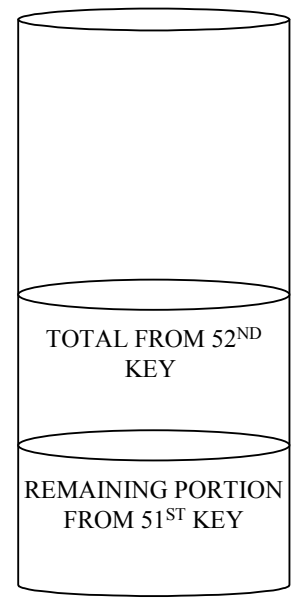

LAST CONTAINER 
The filled containers are tallied as illustrated in Figure 4-7. First the concentration of each item of interest is computed. The concentrations are computed as the amount (either activity or mass) divided by the volume of the container, or by the mass of sludge, or by the mass of uranium. Each concentration in each container is tallied using the same procedure. The magnitude of the concentration determines which bin it goes into. Once the bin is selected, the number of counts in that bin is increased by one.

Figure 4-7. Stochastic Calculation: Tallying Container Concentrations.

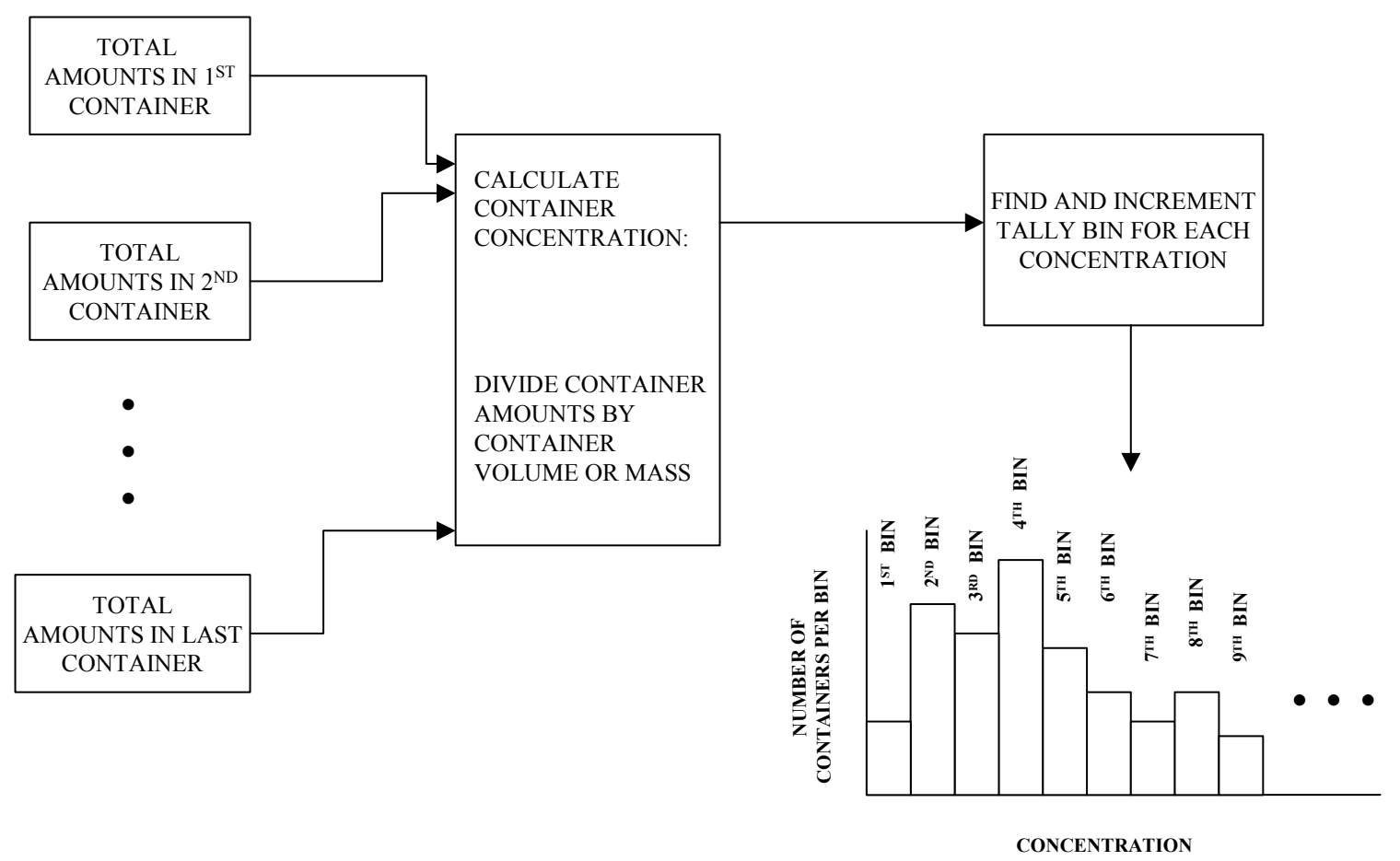

The basin is emptied (all keys retrieved) many times to obtain a good statistical representation in the tallies, i.e., many counts in each of the tally bins. Each time the basin is emptied, one or more containers of the specified volume are filled. The independent random events required to model the emptying of the basin are summarized in Table 4-1. The calculational steps discussed above are shown as three blocks in Table 4-1. The first two blocks list the random calculations for floor sludge and calculations for canister sludge. The random arrangement of the keys is the last block in Table 4-1. The block headings are in bold type. The total number of random events for each block is shown in parentheses.

In Table 4-1, the column labeled "Count" has the number of random events for each random variable during one retrieval sequence. Events with a count of 52 are generated independently for each key. There are 52 keys. The only event in the first part that affects all keys is the floor sludge volume adjustment factor.

The canister sludge depth calculation occurs for each of the four fuel conditions that apply for a given key (shown in Table 3-12). However, if there are no bad canisters in a given key, the depth of bad sludge will not be computed for that key. Hence, the number shown (171) is no 
simple multiple of 52. The random ordering of the keys has 51 random events to order the 52 keys for retrieval. Once 51 keys are selected, only one key remains to be taken.

Table 4-1. Overview of Random Events to Empty K East Basin.

\begin{tabular}{|c|c|c|}
\hline Random Variable & Method of Calculation & Count \\
\hline \multicolumn{3}{|c|}{ Floor Sludge Amounts (209) } \\
\hline $\begin{array}{l}\text { Volume Factor } \\
\{\text { Section } 3.1\}\end{array}$ & uniform from 0.8 to 1.2 & 1 \\
\hline $\begin{array}{l}\text { Sample Index } \\
\{\text { Section } 3.2\}\end{array}$ & discrete number from 1 to 23 & 52 \\
\hline $\begin{array}{c}\text { Sample Variability Factor } \\
\{\text { Section } 3.2\}\end{array}$ & uniform from 0.2 to 1.8 & 52 \\
\hline $\begin{array}{c}\text { Uranium Metal Fraction } \\
\{\text { Section } 3.6\} \\
\end{array}$ & uniform from 0 to 0.015 & 52 \\
\hline $\begin{array}{c}\mathrm{Pu}-239 / 240 \text { Specific Activity } \\
\{\text { Section } 3.7\}\end{array}$ & continuous from 0.070 to $0.083 \mathrm{Ci} / \mathrm{g}$ & 52 \\
\hline \multicolumn{3}{|c|}{ Canister Sludge Amounts (550) } \\
\hline $\begin{array}{l}\text { Sludge Depth Index } \\
\quad\{\text { Section } 3.3\}\end{array}$ & discrete (good, fair, poor, bad) & 171 \\
\hline $\begin{array}{c}\text { Sludge Depth Variability Factor } \\
\{\text { Section } 3.3\}\end{array}$ & uniform from 0.5 to 1.5 & 171 \\
\hline $\begin{array}{c}\text { Sludge Density Index } \\
\{\text { Section } 3.4\} \\
\end{array}$ & discrete number from 1 to 8 & 52 \\
\hline $\begin{array}{c}\text { Sludge Density Variability Factor } \\
\{\text { Section } 3.4\}\end{array}$ & uniform from 0.33 to 1.67 & 52 \\
\hline $\begin{array}{c}\text { Uranium Concentration Factor } \\
\qquad \text { Section } 3.4 .5\}\end{array}$ & uniform & 52 \\
\hline $\begin{array}{c}\text { Uranium Metal Fraction } \\
\{\text { Section } 3.6\}\end{array}$ & uniform from 0 to 0.050 & 52 \\
\hline \multicolumn{3}{|c|}{ Container Filling (51) } \\
\hline Key Retrieval Order & $\begin{array}{l}\text { discrete number from } 1 \text { to the number of keys } \\
\text { remaining in the basin }\end{array}$ & 51 \\
\hline \multicolumn{2}{|c|}{ Total Random Events to Empty K East Basin } & 810 \\
\hline
\end{tabular}


SNF-10293, REV 0

\subsection{PRESERVATION OF MEAN VALUES}

The calculation of mean results will be presented to illustrate the nature of the calculation and serve as a check on the stochastic results. The comparisons between calculated mean values and stochastic results are divided into three general areas, floor sludge input data, canister sludge input data, and container results. Where possible, the standard deviations are compared as well.

\subsubsection{Floor Sludge Input Data}

The floor sludge concentrations were tallied to verify that the stochastic distribution being used was representing the sample data and imputed values. The typical plot is shown in Figure 4-8. This figure shows the CDF for floor sludge density. The data values are shown as squares.

Figure 4-8. Stochastic Result for Floor Sludge Density.

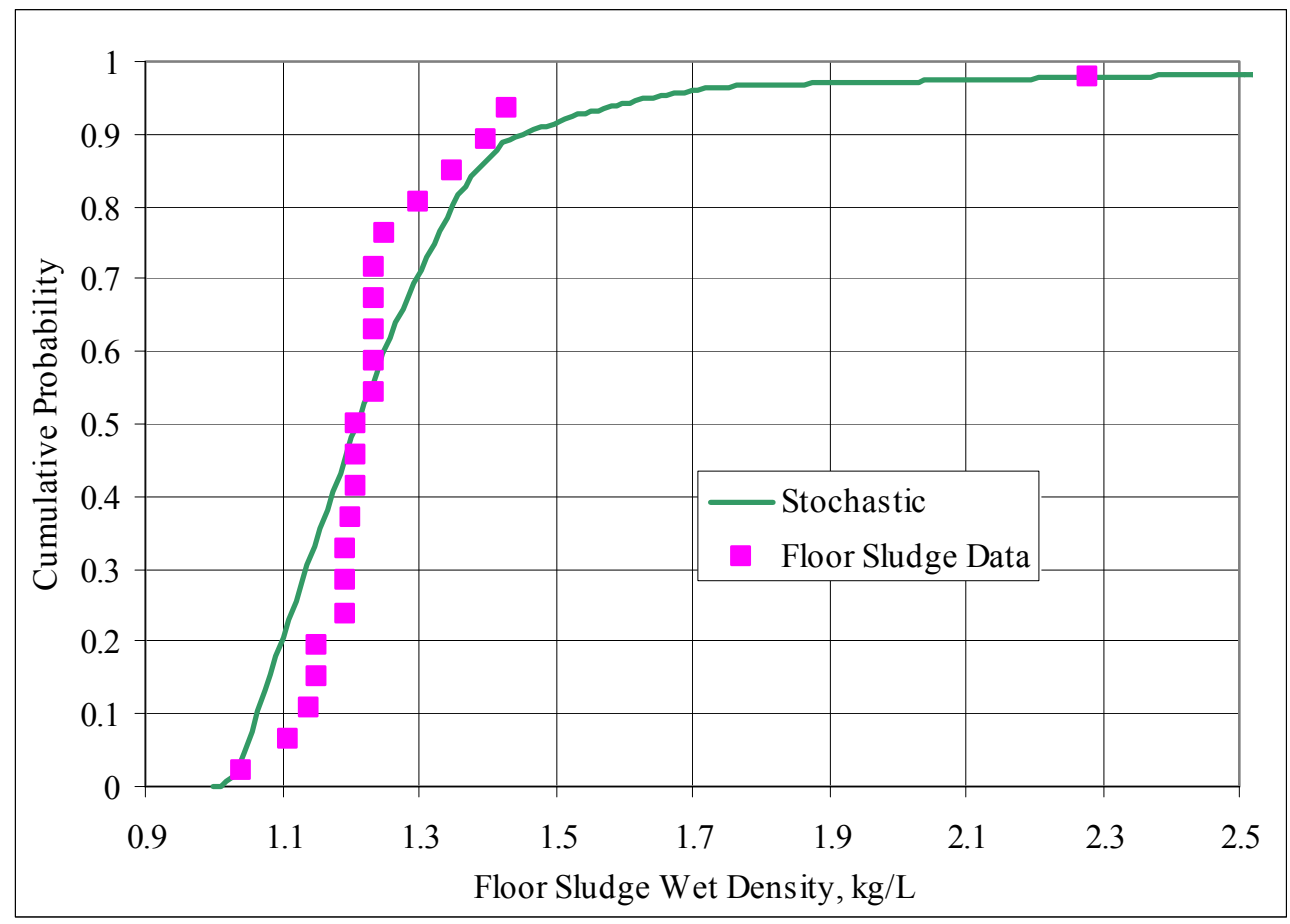

The mean values for floor sludge concentrations discussed in Sections 3.1 and 3.2 are listed in Table 4-2. The mean value for uranium metal is half the maximum shown in Table 3-29. Heat and dose values are computed as described in Section 3.5. The total plutonium is computed using the PDF for Pu-239/240 specific activity presented in Section 3.7. The percent differences between the sample mean and the stochastic mean is less than $0.5 \%$ in all cases. The standard deviations increase $10 \%$ to $20 \%$ in the stochastic analysis. The actual increase depends on how the data values are distributed between the maximum and minimum values. 
SNF-10293, REV 0

Table 4-2. Floor Sludge Comparisons -- Samples vs Stochastic.

\begin{tabular}{|c|c|c|c|c|c|}
\hline \multirow{2}{*}{ Component } & \multicolumn{2}{|c|}{ From Samples } & \multicolumn{2}{|c|}{ From Stochastic Analysis } & \multirow{2}{*}{$\begin{array}{c}\text { Percent } \\
\text { Difference: } \\
\text { Standard } \\
\text { Deviation }\end{array}$} \\
\hline & Mean & $\begin{array}{l}\text { Standard } \\
\text { Deviation }\end{array}$ & Mean & $\begin{array}{l}\text { Standard } \\
\text { Deviation }\end{array}$ & \\
\hline Co- $60, \mu \mathrm{Ci} / \mathrm{mL}$ & $8.14 \mathrm{E}-01$ & $7.00 \mathrm{E}-01$ & $8.14 \mathrm{E}-01$ & $8.42 \mathrm{E}-01$ & $20 \%$ \\
\hline $\mathrm{Sr}-90, \mu \mathrm{Ci} / \mathrm{mL}$ & $1.80 \mathrm{E}+02$ & $4.14 \mathrm{E}+02$ & $1.80 \mathrm{E}+02$ & $4.54 \mathrm{E}+02$ & $10 \%$ \\
\hline $\mathrm{Tc}-99, \mu \mathrm{Ci} / \mathrm{mL}$ & $6.99 \mathrm{E}-02$ & $9.10 \mathrm{E}-02$ & $6.99 \mathrm{E}-02$ & $1.03 \mathrm{E}-01$ & $13 \%$ \\
\hline $\mathrm{Cs}-134, \mu \mathrm{Ci} / \mathrm{mL}$ & $1.12 \mathrm{E}-01$ & $2.10 \mathrm{E}-01$ & $1.12 \mathrm{E}-01$ & $2.32 \mathrm{E}-01$ & $11 \%$ \\
\hline $\mathrm{Cs}-137, \mu \mathrm{Ci} / \mathrm{mL}$ & $2.55 \mathrm{E}+02$ & $4.68 \mathrm{E}+02$ & $2.55 \mathrm{E}+02$ & $5.19 \mathrm{E}+02$ & $11 \%$ \\
\hline $\mathrm{Eu}-152, \mu \mathrm{Ci} / \mathrm{mL}$ & $1.18 \mathrm{E}-02$ & $2.12 \mathrm{E}-02$ & $1.18 \mathrm{E}-02$ & $2.35 \mathrm{E}-02$ & $11 \%$ \\
\hline $\mathrm{Eu}-154, \mu \mathrm{Ci} / \mathrm{mL}$ & $1.47 \mathrm{E}+00$ & $2.65 \mathrm{E}+00$ & $1.47 \mathrm{E}+00$ & $2.94 \mathrm{E}+00$ & $11 \%$ \\
\hline $\mathrm{Eu}-155, \mu \mathrm{Ci} / \mathrm{mL}$ & $7.26 \mathrm{E}-01$ & $1.22 \mathrm{E}+00$ & $7.26 \mathrm{E}-01$ & $1.36 \mathrm{E}+00$ & $11 \%$ \\
\hline $\mathrm{Np}-237, \mu \mathrm{Ci} / \mathrm{mL}$ & $1.39 \mathrm{E}-03$ & $1.83 \mathrm{E}-03$ & $1.39 \mathrm{E}-03$ & $2.07 \mathrm{E}-03$ & $13 \%$ \\
\hline $\mathrm{Pu}-238, \mu \mathrm{Ci} / \mathrm{mL}$ & $1.87 \mathrm{E}+00$ & $2.81 \mathrm{E}+00$ & $1.87 \mathrm{E}+00$ & $3.15 \mathrm{E}+00$ & $12 \%$ \\
\hline $\mathrm{Pu}-239 / 240, \mu \mathrm{Ci} / \mathrm{mL}$ & $1.00 \mathrm{E}+01$ & $1.30 \mathrm{E}+01$ & $1.00 \mathrm{E}+01$ & $1.48 \mathrm{E}+01$ & $14 \%$ \\
\hline $\mathrm{Pu}-241, \mu \mathrm{Ci} / \mathrm{mL}$ & $1.05 \mathrm{E}+02$ & $1.58 \mathrm{E}+02$ & $1.05 \mathrm{E}+02$ & $1.77 \mathrm{E}+02$ & $12 \%$ \\
\hline $\mathrm{Am}-241, \mu \mathrm{Ci} / \mathrm{mL}$ & $1.02 \mathrm{E}+01$ & $1.65 \mathrm{E}+01$ & $1.02 \mathrm{E}+01$ & $1.84 \mathrm{E}+01$ & $11 \%$ \\
\hline Uranium, kg/L & $6.41 \mathrm{E}-02$ & $1.38 \mathrm{E}-01$ & $6.41 \mathrm{E}-02$ & $1.52 \mathrm{E}-01$ & $10 \%$ \\
\hline Density, kg/L & $1.27 \mathrm{E}+00$ & $2.37 \mathrm{E}-01$ & $1.27 \mathrm{E}+00$ & $2.84 \mathrm{E}-01$ & $20 \%$ \\
\hline Heat, $\mathrm{W} / \mathrm{m}^{3}$ & $3.18 \mathrm{E}+00$ & na & $3.18 \mathrm{E}+00$ & $6.63 \mathrm{E}+00$ & na \\
\hline Dose, $\mathrm{rem} / \mathrm{ml}$ & $1.05 \mathrm{E}+04$ & na & $1.05 \mathrm{E}+04$ & $1.70 \mathrm{E}+04$ & na \\
\hline $\mathrm{U}$ metal, kg/L & $9.52 \mathrm{E}-03$ & na & $9.52 \mathrm{E}-03$ & $6.02 \mathrm{E}-03$ & na \\
\hline Plutonium, kg/L & $1.29 \mathrm{E}-04$ & na & $1.30 \mathrm{E}-04$ & $1.92 \mathrm{E}-04$ & na \\
\hline $\begin{array}{c}\mathrm{Pu}-239 / 240 \text { Specific } \\
\text { Activity }\end{array}$ & $7.81 \mathrm{E}+01$ & na & $7.81 \mathrm{E}+01$ & $4.64 \mathrm{E}+00$ & na \\
\hline
\end{tabular}

- The isotope concentrations, uranium concentration, and sludge density were measured in the samples or imputed. The other items shown in the list were not measured.

- Mean isotope concentrations, density and uranium concentration in floor sludge are described in Sections 3.1 and 3.2. The heat generation rate and inhalation dose factor are computed using the conversion factors shown in Section 3.5. The uranium metal concentration is calculated using half the maximum shown in Table 3-29. The total plutonium concentration is computed using the specific activity PDF for Pu-239/240 described in Section 3.7 in addition to the specific activities for $\mathrm{Pu}-238$ and $\mathrm{Pu}-241$.

- Stochastic results are from tallies taken immediately after the input item is calculated (see Figure 4-1).

- Percent differences for the mean values are all less than $0.5 \%$. 


\section{SNF-10293, REV 0}

\subsubsection{Canister Sludge Input Data}

The canister sludge concentrations are tallied to verify that the PDFs being used represent the input data. Figure 4-3 shows where in the calculation of canister sludge amounts per key that the tallies occur. The canister sludge density, uranium concentration, and uranium metal concentration use the same PDF for each key. However, the isotope concentrations, heat, dose and total plutonium tallies are different for each key because the Radnuc2 A numbers depend on the key. Thus, these tallies are not simple distributions of data, but involve the superposition of 52 different distributions. Each of the 52 distributions is formed by the product of the total uranium PDF with the Radnuc2A concentration for that key.

The mean and standard deviation for canister sludge density and total uranium total concentration are shown in Table 3-16 and again in Table 4-3 as "Hand Calculated Sums" to distinguish them from the "Stochastic Results." The mean value for uranium metal concentration is the mean canister sludge density times the mean uranium metal fraction in canister sludge $(2.5 \%)$. The standard deviation for uranium metal concentration is not readily computed from the product of the two distributions. Thus in Table 4-3, the mean is shown, but not the standard deviation.

The mean for the canister sludge isotopic concentrations can be calculated by summing the isotope concentrations in Tables 3-18, 3-19, and 3-20, then multiplying by the average uranium concentration in canister sludge $(0.860 \mathrm{~kg} / \mathrm{L})$ and finally dividing by 52 . Because the isotope concentrations involve the uranium distribution and the relative amounts of the isotope in each key, the standard deviation could not be calculated by hand from the data. Thus in Table 4-3, the mean values are shown, but not the standard deviations.

Also shown in Table 4-3 are the stochastic results from the tallies. These match the expected mean values within $1.5 \%$. The difference arises from the method used to calculate uranium concentration in the base case stochastic analysis. As discussed in Section 3.4.5, the method leads to a slightly higher (1.5\%) mean value for the uranium concentration, which accounts for the difference noted in Table 4-3.

The standard deviations for uranium and density are from the sample data. The stochastic result for these standard deviations is $20 \%$ larger. The increase is due to the variability factor applied to each data point.

The lower portion of Table 4-3 compares values for canister sludge depth. Because the mean values for canister sludge depth are essentially the same, only the percent differences for the standard deviations are shown in Table 4-3. Because of the nature of the data for good fuel, the standard deviation reported in the stochastic analysis is less than the standard deviation calculated directly from the 5 data points. Figure 4-9 shows the cumulative tally for good condition fuel. The individual points show the cumulative data points used in Figure 3-13. 
Table 4-3. Canister Sludge Comparisons -- Hand Calculated Sums versus Stochastic.

\begin{tabular}{|c|c|c|c|c|c|}
\hline \multirow[b]{2}{*}{ Component } & \multicolumn{2}{|c|}{ Hand Calculated Sums } & \multicolumn{2}{|c|}{ Stochastic Results } & \multirow{2}{*}{$\begin{array}{c}\text { Percent } \\
\text { Difference: } \\
\text { Mean }\end{array}$} \\
\hline & Mean & $\begin{array}{l}\text { Standard } \\
\text { Deviation }\end{array}$ & Mean & $\begin{array}{l}\text { Standard } \\
\text { Deviation }\end{array}$ & \\
\hline $\mathrm{Co}-60, \mu \mathrm{Ci} / \mathrm{mL}$ & $1.41 \mathrm{E}+00$ & $\mathrm{Na}$ & $1.43 \mathrm{E}+00$ & $1.48 \mathrm{E}+00$ & $1.5 \%$ \\
\hline $\mathrm{Sr}-90, \mu \mathrm{Ci} / \mathrm{mL}$ & $3.72 \mathrm{E}+03$ & $\mathrm{Na}$ & $3.77 \mathrm{E}+03$ & $3.62 \mathrm{E}+03$ & $1.5 \%$ \\
\hline Tc- $99, \mu \mathrm{Ci} / \mathrm{mL}$ & $1.09 \mathrm{E}+00$ & $\mathrm{Na}$ & $1.11 \mathrm{E}+00$ & $1.10 \mathrm{E}+00$ & $1.6 \%$ \\
\hline $\mathrm{Cs}-134, \mu \mathrm{Ci} / \mathrm{mL}$ & $6.43 \mathrm{E}+00$ & $\mathrm{Na}$ & $6.53 \mathrm{E}+00$ & $1.19 \mathrm{E}+01$ & $1.5 \%$ \\
\hline $\mathrm{Cs}-137, \mu \mathrm{Ci} / \mathrm{mL}$ & $4.93 \mathrm{E}+03$ & na & $5.00 \mathrm{E}+03$ & $4.92 \mathrm{E}+03$ & $1.5 \%$ \\
\hline $\mathrm{Eu}-152, \mu \mathrm{Ci} / \mathrm{mL}$ & $3.63 \mathrm{E}-01$ & na & $3.68 \mathrm{E}-01$ & 4.14E-01 & $1.5 \%$ \\
\hline Eu-154, $\mu \mathrm{Ci} / \mathrm{mL}$ & $4.25 \mathrm{E}+01$ & na & $4.31 \mathrm{E}+01$ & $5.36 \mathrm{E}+01$ & $1.5 \%$ \\
\hline Eu- $155, \mu \mathrm{Ci} / \mathrm{mL}$ & $8.29 \mathrm{E}+00$ & na & $8.41 \mathrm{E}+00$ & $8.60 \mathrm{E}+00$ & $1.5 \%$ \\
\hline $\mathrm{Np}-237, \mu \mathrm{Ci} / \mathrm{mL}$ & $2.27 \mathrm{E}-02$ & na & $2.31 \mathrm{E}-02$ & $2.31 \mathrm{E}-02$ & $1.5 \%$ \\
\hline $\mathrm{Pu}-238, \mu \mathrm{Ci} / \mathrm{mL}$ & $4.72 \mathrm{E}+01$ & na & $4.79 \mathrm{E}+01$ & $5.68 \mathrm{E}+01$ & $1.5 \%$ \\
\hline $\mathrm{Pu}-239 / 240, \mu \mathrm{Ci} / \mathrm{mL}$ & $1.57 \mathrm{E}+02$ & na & $1.59 \mathrm{E}+02$ & $1.57 \mathrm{E}+02$ & $1.5 \%$ \\
\hline $\mathrm{Pu}-241, \mu \mathrm{Ci} / \mathrm{mL}$ & $2.68 \mathrm{E}+03$ & na & $2.72 \mathrm{E}+03$ & $3.20 \mathrm{E}+03$ & $1.5 \%$ \\
\hline $\mathrm{Am}-241, \mu \mathrm{Ci} / \mathrm{mL}$ & $1.58 \mathrm{E}+02$ & na & $1.60 \mathrm{E}+02$ & $1.88 \mathrm{E}+02$ & $1.5 \%$ \\
\hline Uranium, $\mathrm{kg} / \mathrm{L}$ & 0.860 & 0.571 & 0.874 & 0.684 & $1.5 \%$ \\
\hline Density, kg/L & 1.87 & 0.523 & 1.87 & 0.624 & $0.0 \%$ \\
\hline Heat, $\mathrm{W} / \mathrm{m}^{3}$ & $6.09 \mathrm{E}+01$ & na & $6.18 \mathrm{E}+01$ & $6.13 \mathrm{E}+01$ & $1.5 \%$ \\
\hline Dose, rem/ml & $1.79 \mathrm{E}+05$ & na & $1.82 \mathrm{E}+05$ & $1.98 \mathrm{E}+05$ & $1.5 \%$ \\
\hline $\mathrm{U}$ metal, $\mathrm{kg} / \mathrm{L}$ & $4.68 \mathrm{E}-02$ & na & $4.65 \mathrm{E}-02$ & $3.27 \mathrm{E}-02$ & $-0.7 \%$ \\
\hline Plutonium, kg/L & $1.87 \mathrm{E}-03$ & na & $1.90 \mathrm{E}-03$ & $1.82 \mathrm{E}-03$ & $1.5 \%$ \\
\hline \multicolumn{5}{|c|}{ Canister Sludge Depth, inches } & $\begin{array}{l}\text { Standard } \\
\text { Deviation }\end{array}$ \\
\hline Good & 0.465 & 1.04 & 0.465 & 0.959 & $-7.8 \%$ \\
\hline Fair & 1.61 & na & 1.61 & 0.893 & na \\
\hline Poor & 4.56 & 4.14 & 4.56 & 4.44 & $7.3 \%$ \\
\hline $\mathrm{Bad}$ & 4.56 & 4.14 & 4.56 & 4.44 & $7.4 \%$ \\
\hline
\end{tabular}

- Mean isotope concentrations in canister sludge are calculated from the sum of the Ci/MTU for each key (from Section 3.4) multiplied by the uranium concentration and divided by 52 (number of keys). This gives the average concentration per key, unweighted. The heat generation rate and inhalation dose factor are computed using the conversion factors shown in Section 3.5. The uranium metal concentration is calculated using half the maximum shown in Table 3-29. The total plutonium concentration is computed using the specific activities described in Section 3.7.

- Stochastic results are from tallies taken immediately after the input item is calculated (see Figure 4-3).

- Percent differences for the mean values are shown in the upper portion of the table. Percent differences for the standard deviations are only shown for canister sludge depths (the mean values are the same). Negative values signify that the stochastic result is smaller. 
Figure 4-9. Stochastic Result for Canister Sludge Depth (Good Fuel).

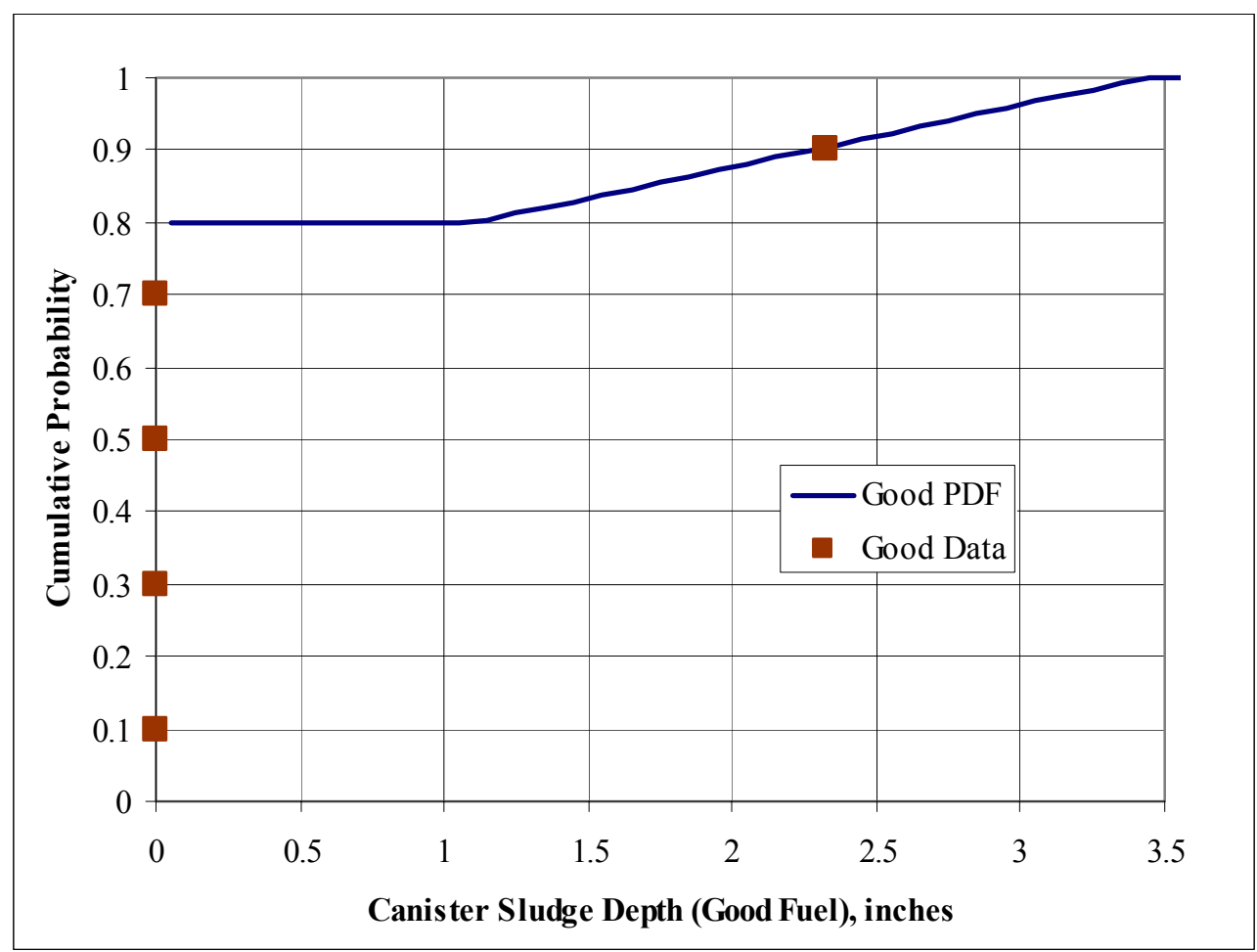

Comparing Figure 4-9 with Figure 3-13 shows the effect of using individual sludge depths along with a variability factor. For good fuel canister sludge, the greatest depth measured becomes a uniform distribution that starts at a depth of $0.5 \times 2.325$ in. and a probability of 0.80 . It ends at a depth of $1.5 \times 2.325 \mathrm{in}$. and a probability of 1.00 . The other $80 \%$ of the results have a depth of 0 .

The graph of the tally for fair fuel is shown in Figure 4-10. The stochastic result for aluminum and stainless steel canisters has been combined. The combination is weighted more strongly toward the aluminum; hence the curve is closer to the lower data. 
Figure 4-10. Stochastic Result for Canister Sludge Depth (Fair Fuel).

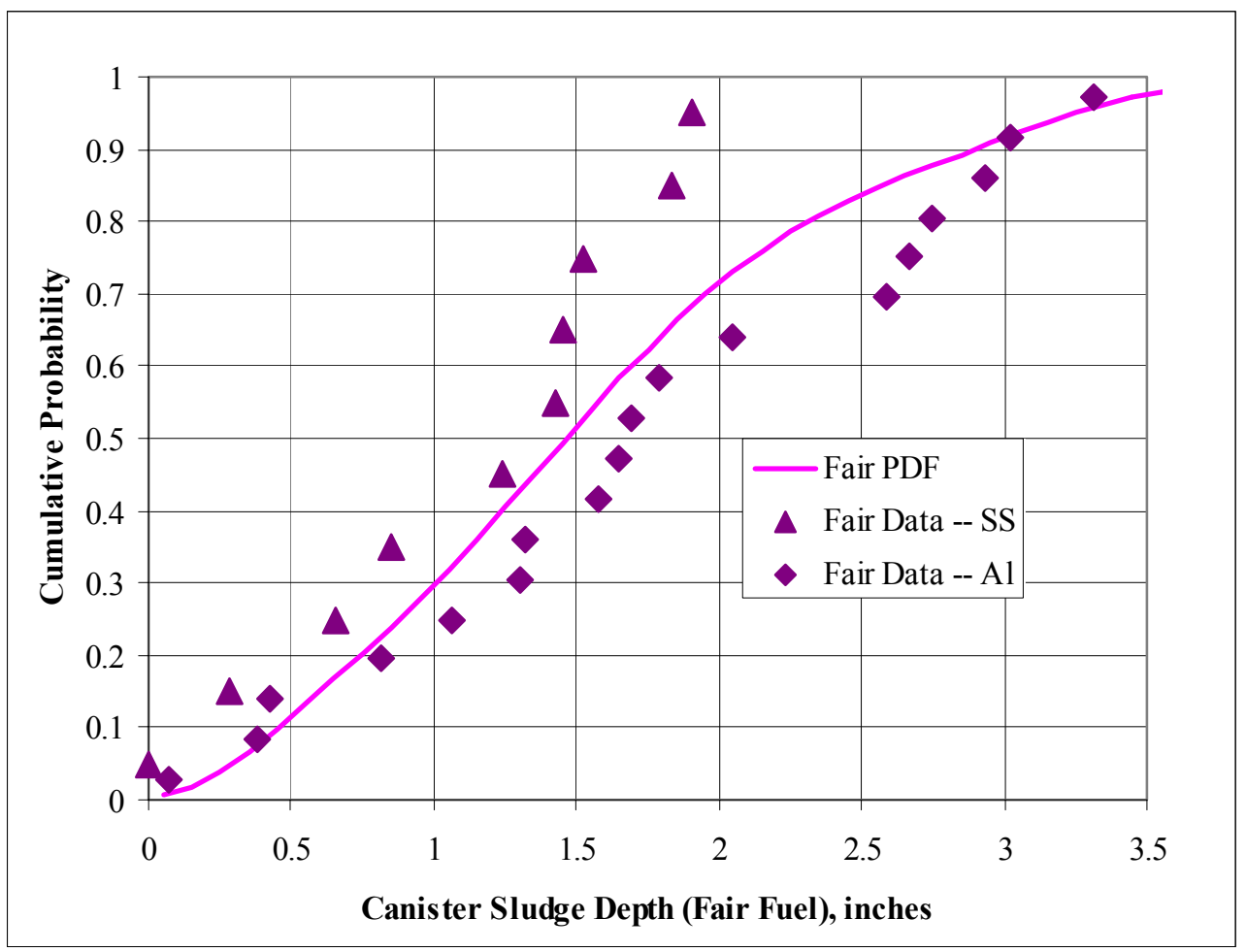

Figure 4-11 shows the stochastic results for bad and poor fuel along with the data values used for the input probability distribution. The graph for poor condition fuel is identical to Figure 4-11 because the bad and poor fuel are assumed equivalent.

The canister sludge density and uranium concentration were tallied two ways. The first is the individual tallies shown in Figure 4-12 and 4-13. The data values are shown as squares. The method for calculating uranium concentration produces a smooth curve through the data points. The second tally is a two dimensional array in which canister sludge density is the horizontal axis and uranium concentration is the vertical axis. This was done to verify that the uranium concentration is calculated from the sludge density as described in Section 3.4.5. The two dimension array has the same outline as Figure 3-24. In addition, the columns are uniform distributions between the lower and upper bounds. 
Figure 4-11. Stochastic Result for Canister Sludge Depth (Poor and Bad Fuel).

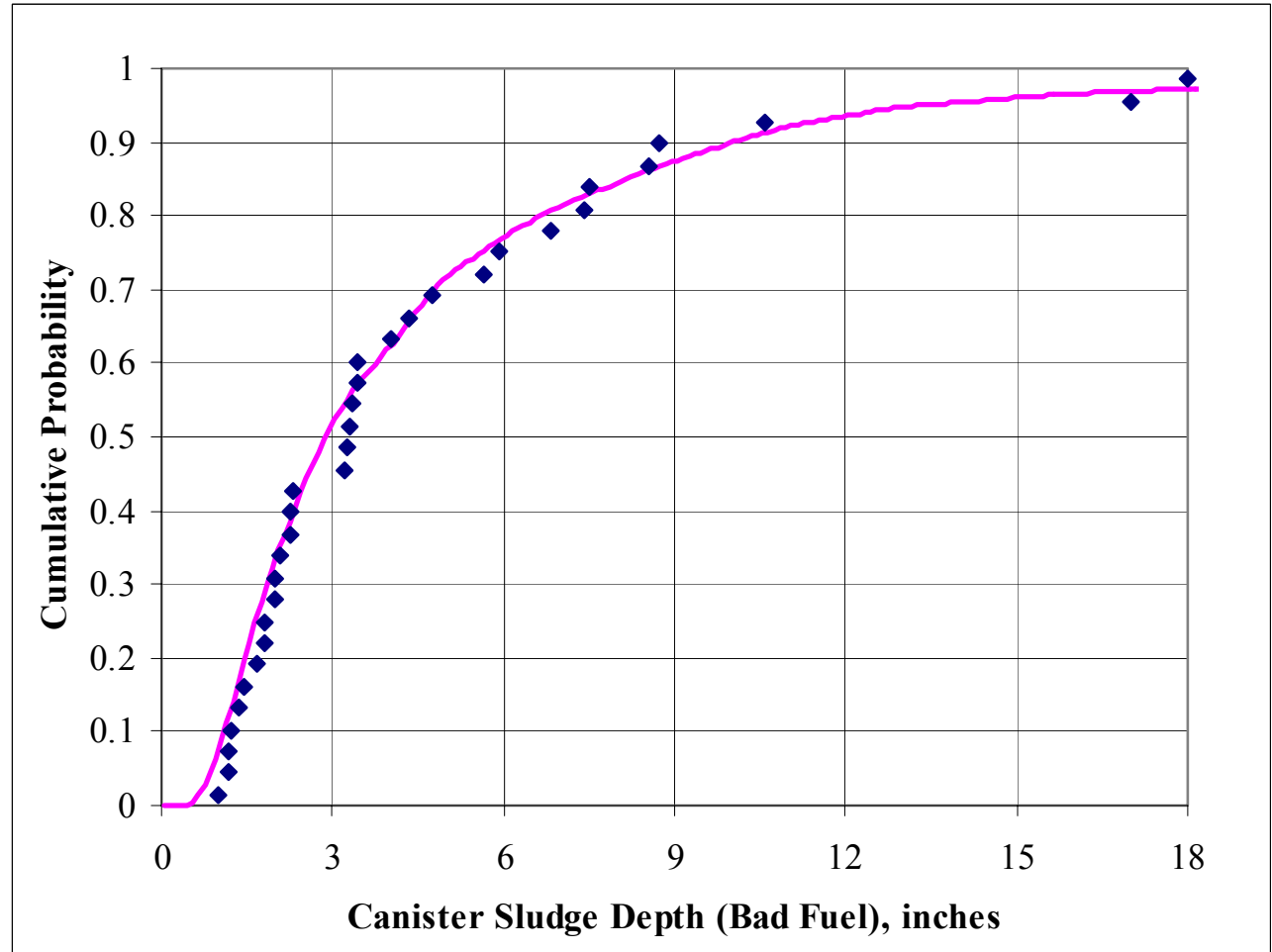

Figure 4-12. Stochastic Result for Canister Sludge Density.

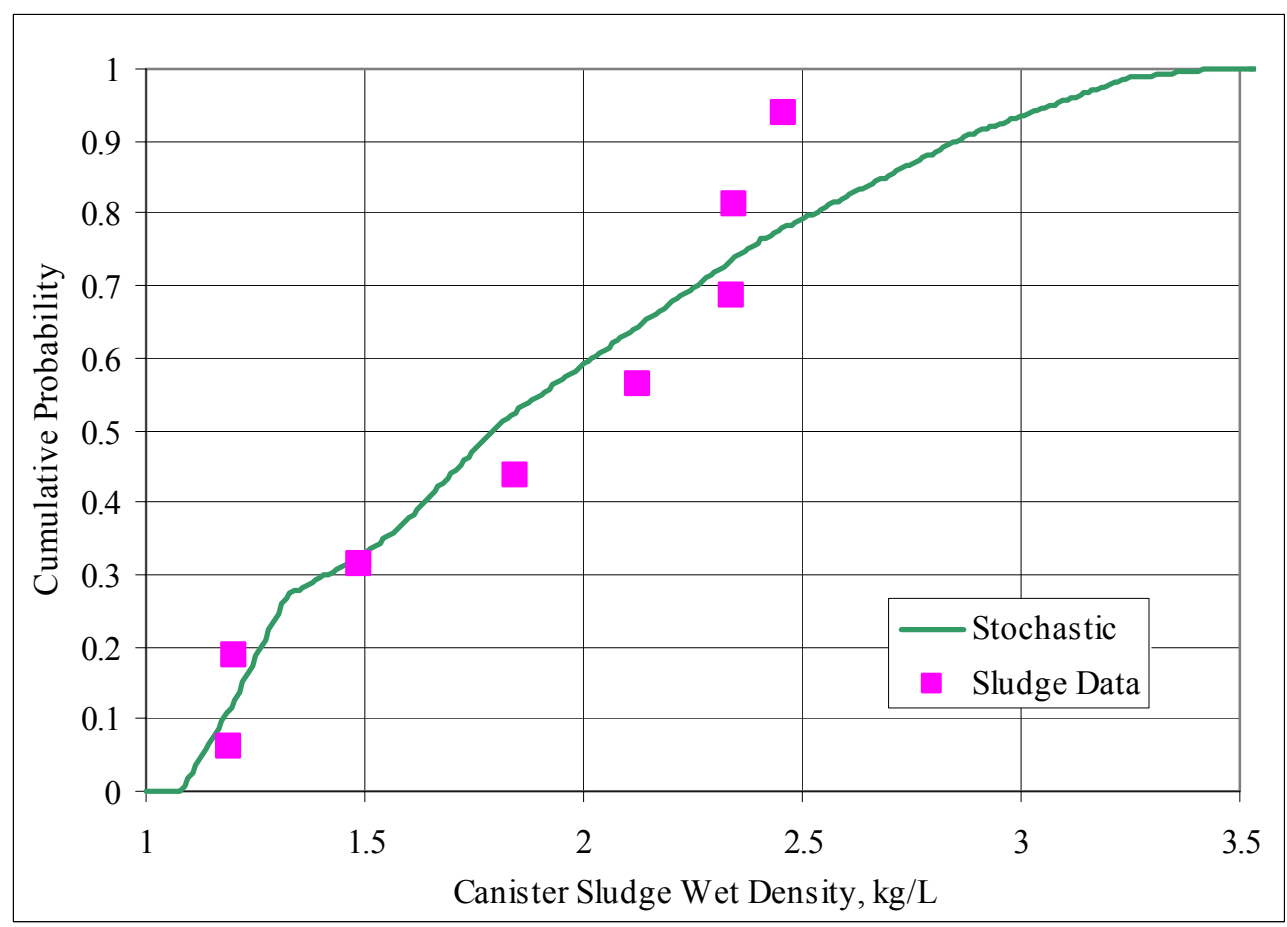


Figure 4-13. Stochastic Result for Canister Sludge Uranium Concentration.

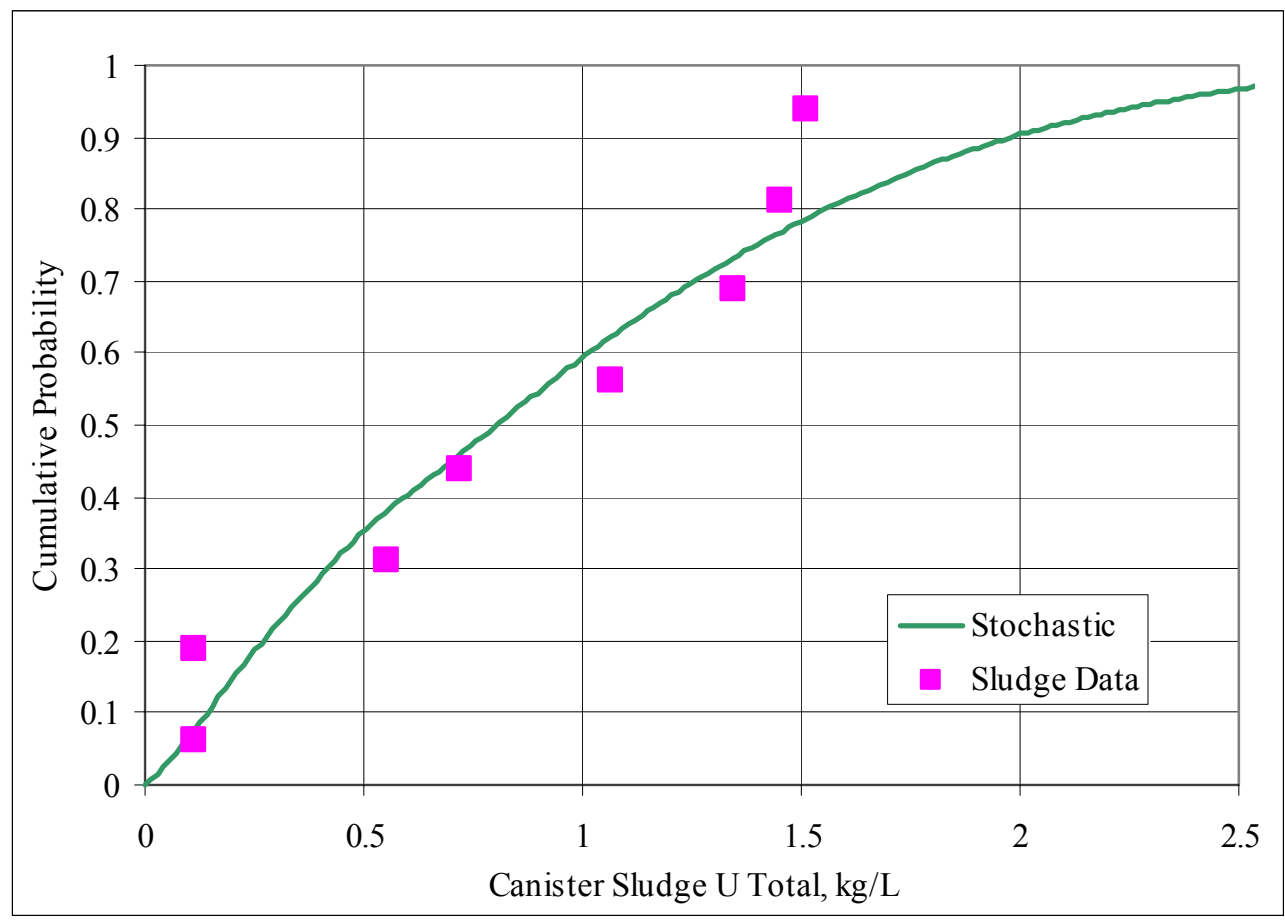

\subsubsection{Container Results}

The mean concentrations in the filled containers can be calculated using the mean floor sludge concentrations shown in Table 4-2 and the weighted mean canister sludge concentrations. The weighted mean canister sludge concentrations are calculated by multiplying the "Method A" concentrations shown in Table 3-23 by the average uranium concentration, $0.860 \mathrm{~kg} / \mathrm{L}$.

The relative amounts of canister and floor sludge are calculated from the average total volumes of each. The total floor sludge volume is $11.7 \mathrm{~m}^{3}$ (Table 3-1), while the total canister sludge volume is $2.86 \mathrm{~m}^{3}$ (Table $3-13$ ). Thus, the floor sludge is $80.3 \%$ of the total volume, and canister sludge is $19.7 \%$ of the total volume. The floor and canister sludge concentrations are combined using these fractions. In effect, all the sludge in cubicles containing fuel is mixed together to give the average concentration. This mixture is then put into containers. The resulting mean values for sludge containers are shown in Table 4-4.

The stochastic mean values are slightly higher than the calculated ones because of the slight difference in uranium concentration. When one distribution is divided by another distribution, as in the cases of a quantity per unit mass of another quantity, the stochastic result will not preserve the mean. Hence, there are small differences between the heat generation rate per MT sludge or per MTU. This is also true for inhalation dose per unit mass of soil inhaled, and plutonium weight percent. 
SNF-10293, REV 0

Table 4-4. Container Sludge Data Mean Values.

\begin{tabular}{|c|c|c|c|c|c|}
\hline \multirow{2}{*}{ Component } & \multicolumn{2}{|c|}{ Sludge Concentrations } & \multicolumn{2}{|c|}{ Container Mean Values } & \multirow{2}{*}{$\begin{array}{c}\text { Percent } \\
\text { Difference }\end{array}$} \\
\hline & Floor & Canister & Weighted & Stochastic & \\
\hline $\mathrm{Co}-60, \mu \mathrm{Ci} / \mathrm{mL}$ & $8.14 \mathrm{E}-01$ & $1.41 \mathrm{E}+00$ & 9.32E-01 & $9.35 \mathrm{E}-01$ & $0.3 \%$ \\
\hline $\mathrm{Sr}-90, \mu \mathrm{Ci} / \mathrm{mL}$ & $1.80 \mathrm{E}+02$ & $4.24 \mathrm{E}+03$ & $9.78 \mathrm{E}+02$ & $9.87 \mathrm{E}+02$ & $0.9 \%$ \\
\hline Tc-99, $\mu \mathrm{Ci} / \mathrm{mL}$ & $6.99 \mathrm{E}-02$ & $1.28 \mathrm{E}+00$ & $3.08 \mathrm{E}-01$ & $3.10 \mathrm{E}-01$ & $0.9 \%$ \\
\hline $\mathrm{Cs}-134, \mu \mathrm{Ci} / \mathrm{mL}$ & $1.12 \mathrm{E}-01$ & $5.64 \mathrm{E}+00$ & $1.20 \mathrm{E}+00$ & $1.21 \mathrm{E}+00$ & $1.1 \%$ \\
\hline $\mathrm{Cs}-137, \mu \mathrm{Ci} / \mathrm{mL}$ & $2.55 \mathrm{E}+02$ & $5.66 \mathrm{E}+03$ & $1.32 \mathrm{E}+03$ & $1.33 \mathrm{E}+03$ & $0.9 \%$ \\
\hline $\mathrm{Eu}-152, \mu \mathrm{Ci} / \mathrm{mL}$ & $1.18 \mathrm{E}-02$ & 4.24E-01 & $9.28 \mathrm{E}-02$ & 9.37E-02 & $1.0 \%$ \\
\hline $\mathrm{Eu}-154, \mu \mathrm{Ci} / \mathrm{mL}$ & $1.47 \mathrm{E}+00$ & $4.88 \mathrm{E}+01$ & $1.08 \mathrm{E}+01$ & $1.09 \mathrm{E}+01$ & $1.0 \%$ \\
\hline $\mathrm{Eu}-155, \mu \mathrm{Ci} / \mathrm{mL}$ & $7.26 \mathrm{E}-01$ & $7.89 \mathrm{E}+00$ & $2.13 \mathrm{E}+00$ & $2.15 \mathrm{E}+00$ & $0.9 \%$ \\
\hline $\mathrm{Np}-237, \mu \mathrm{Ci} / \mathrm{mL}$ & $1.39 \mathrm{E}-03$ & $2.67 \mathrm{E}-02$ & $6.37 \mathrm{E}-03$ & $6.43 \mathrm{E}-03$ & $0.9 \%$ \\
\hline $\mathrm{Pu}-238, \mu \mathrm{Ci} / \mathrm{mL}$ & $1.87 \mathrm{E}+00$ & $5.71 \mathrm{E}+01$ & $1.27 \mathrm{E}+01$ & $1.29 \mathrm{E}+01$ & $0.9 \%$ \\
\hline $\mathrm{Pu}-239 / 240, \mu \mathrm{Ci} / \mathrm{mL}$ & $1.00 \mathrm{E}+01$ & $1.83 \mathrm{E}+02$ & $4.41 \mathrm{E}+01$ & $4.44 \mathrm{E}+01$ & $0.9 \%$ \\
\hline $\mathrm{Pu}-241, \mu \mathrm{Ci} / \mathrm{mL}$ & $1.05 \mathrm{E}+02$ & $3.16 \mathrm{E}+03$ & $7.06 \mathrm{E}+02$ & $7.13 \mathrm{E}+02$ & $0.9 \%$ \\
\hline $\mathrm{Am}-241, \mu \mathrm{Ci} / \mathrm{mL}$ & $1.02 \mathrm{E}+01$ & $1.96 \mathrm{E}+02$ & $4.67 \mathrm{E}+01$ & $4.71 \mathrm{E}+01$ & $0.9 \%$ \\
\hline Uranium, kg/L & $6.41 \mathrm{E}-02$ & $8.60 \mathrm{E}-01$ & $2.21 \mathrm{E}-01$ & $2.23 \mathrm{E}-01$ & $0.9 \%$ \\
\hline Density, $\mathrm{kg} / \mathrm{L}$ & $1.27 \mathrm{E}+00$ & $1.87 \mathrm{E}+00$ & $1.39 \mathrm{E}+00$ & $1.39 \mathrm{E}+00$ & $0.0 \%$ \\
\hline Heat, $\mathrm{W} / \mathrm{m}^{3}$ & $3.18 \mathrm{E}+00$ & $7.05 \mathrm{E}+01$ & $1.64 \mathrm{E}+01$ & $1.66 \mathrm{E}+01$ & $0.9 \%$ \\
\hline Heat, W/MT & $2.50 \mathrm{E}+00$ & $3.76 \mathrm{E}+01$ & $1.18 \mathrm{E}+01$ & $1.16 \mathrm{E}+01$ & $-1.6 \%$ \\
\hline Heat, W/MTU & $4.96 \mathrm{E}+01$ & $8.19 \mathrm{E}+01$ & $7.44 \mathrm{E}+01$ & $7.54 \mathrm{E}+01$ & $1.4 \%$ \\
\hline Dose, $\mathrm{rem} / \mathrm{ml}$ & $1.05 \mathrm{E}+04$ & $2.15 \mathrm{E}+5$ & $5.08 \mathrm{E}+04$ & $5.12 \mathrm{E}+04$ & $0.9 \%$ \\
\hline Dose, rem/g & $8.25 \mathrm{E}+03$ & $1.15 \mathrm{E}+5$ & $3.66 \mathrm{E}+04$ & $3.61 \mathrm{E}+04$ & $-1.4 \%$ \\
\hline $\mathrm{U}$ metal, $\mathrm{kg} / \mathrm{L}$ & $9.52 \mathrm{E}-03$ & $4.68 \mathrm{E}-02$ & 1.69E-02 & $1.68 \mathrm{E}-02$ & $-0.6 \%$ \\
\hline $\mathrm{Pu}$ Mass Fraction & $1.02 \mathrm{E}-04$ & $2.16 \mathrm{E}-03$ & $3.82 \mathrm{E}-04$ & $3.77 \mathrm{E}-04$ & $-1.3 \%$ \\
\hline $\begin{array}{l}\text { Canister-to-Total } \\
\text { Volume Ratio }\end{array}$ & na & na & 0.197 & 0.196 & $-0.3 \%$ \\
\hline
\end{tabular}

- The Weighted Container Mean Values are computed by multiplying the Floor Sludge Concentration by 0.803 and multiplying the Canister Sludge Concentration by 0.197 and adding the two numbers.

Concentrations that are calculated by dividing by another density, such as "Dose, rem/g", must also be weighted by the density.

- The Stochastic Container Mean Values are results from the stochastic calculations.

- The "Percent Difference" column is calculated from the "Weighted" and "Stochastic" columns. A negative percent difference signifies that the stochastic result is smaller than the weighted sum of the floor and canister concentrations.

- The row labeled "Pu Mass Fraction" is the total plutonium mass in the container divided by the total mass of sludge in the container. This ratio is also known as a total Pu weight percent. A different name was chosen to avoid confusion since the numbers are fractions, not percents.

The sum of the volumes of sludge in individual keys has been tallied. The binned probabilities are shown in Figure 4-14. The horizontal scale is the sludge volume in cubic meters. The vertical scale is the probability per bin. Each bin is $0.050 \mathrm{~m}^{3}$ wide. The probability per bin is the number of results counted in one bin divided by the total number of cases. The values for floor 
sludge have been doubled to clearly separate the curve for floor sludge from the curve for the total.

The curve for canister sludge has a typical bell curve appearance. This is expected from the variety of random inputs (342 for each random composition). The curve for floor sludge follows a uniform distribution, as expected from the method used to adjust the floor sludge volume. The upper portion of the curve for floor sludge is not perfectly flat due to the small number of counts per bin (about 2,100). If the bins were wider, or the number of simulations larger, the curve would be flatter. The range of values for floor sludge volume matches the expected range, $80 \%$ to $120 \%$ of the mean. The minimum floor sludge volume is $9.37 \mathrm{~m}^{3}$ which is approximately 0.8 times the mean of $11.7 \mathrm{~m}^{3}$. The maximum is $14.05 \mathrm{~m}^{3}$ which is approximately 1.2 times the mean.

Figure 4-14. Stochastic Result for Sludge Total Volume in All Keys.

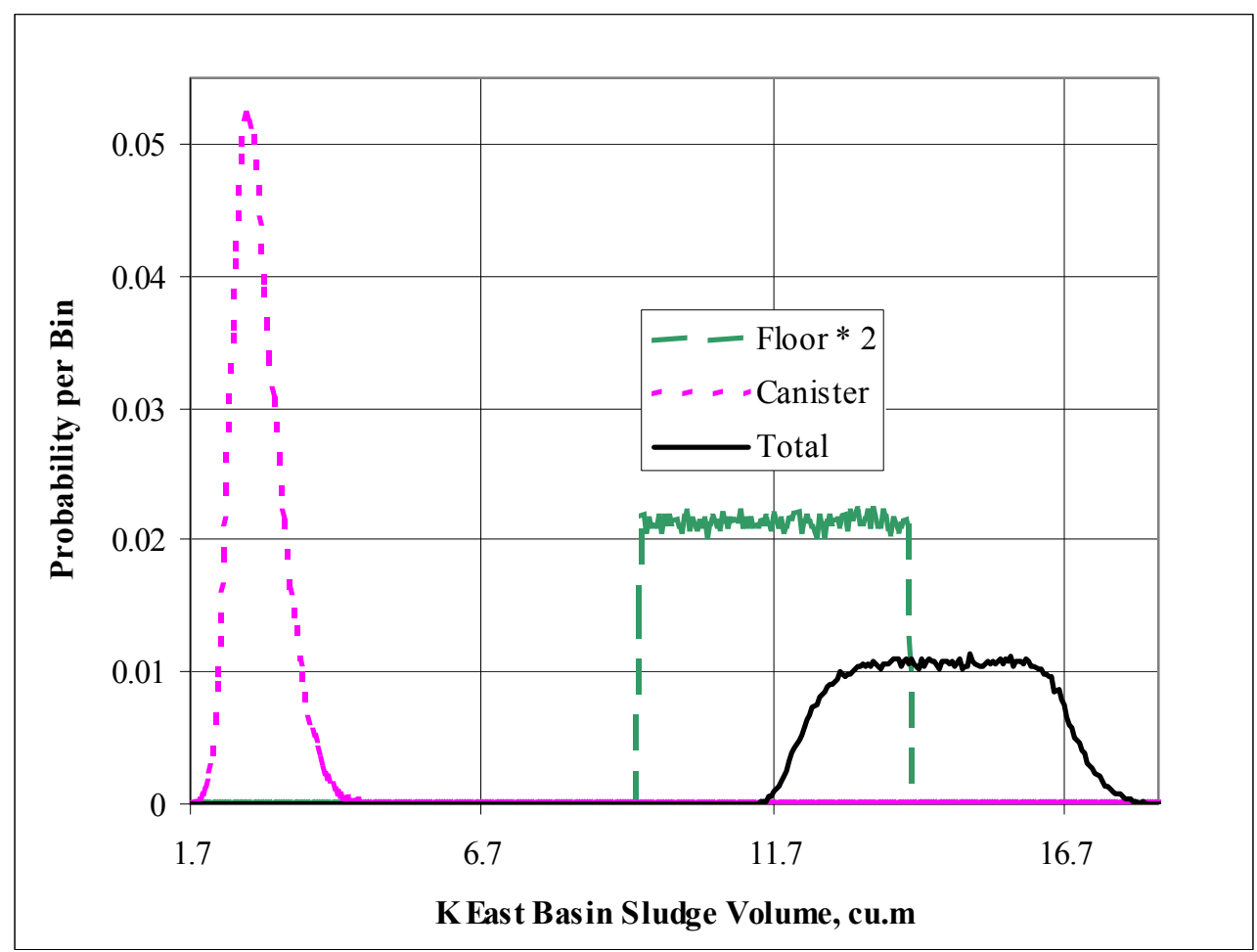

The numeric results from the stochastic curve are listed in Table 4-5. The total number of sludge compositions that were generated is 200,000 . The "Average Values" are the simple arithmetic means. Note that the mean and $50^{\text {th }}$ percentile values are close, indicating that the curves shown in Figure 4-14 are very symmetric. 
SNF-10293, REV 0

Table 4-5. Stochastic Results for the Total Sludge Volume $\left(\mathrm{m}^{3}\right)$.

\begin{tabular}{|c|c|c|c|}
\hline Type of Sludge & Floor & Canister & Total \\
\hline Average Values & $1.17 \mathrm{E}+01$ & $2.86 \mathrm{E}+00$ & $1.46 \mathrm{E}+01$ \\
\hline 95th Percentile & $1.38 \mathrm{E}+01$ & $3.63 \mathrm{E}+00$ & $1.68 \mathrm{E}+01$ \\
\hline 99th Percentile & $1.40 \mathrm{E}+01$ & $4.00 \mathrm{E}+00$ & $1.73 \mathrm{E}+01$ \\
\hline Standard Deviation & $1.35 \mathrm{E}+00$ & $4.14 \mathrm{E}-01$ & $1.41 \mathrm{E}+00$ \\
\hline Minimum Value & $9.37 \mathrm{E}+00$ & $1.65 \mathrm{E}+00$ & $1.12 \mathrm{E}+01$ \\
\hline Maximum Value & $1.405 \mathrm{E}+01$ & $4.89 \mathrm{E}+00$ & $1.85 \mathrm{E}+01$ \\
\hline Geometric Mean & $1.16 \mathrm{E}+01$ & $2.83 \mathrm{E}+00$ & $1.45 \mathrm{E}+01$ \\
\hline Geometric Std Dev & $1.12 \mathrm{E}+00$ & $1.15 \mathrm{E}+00$ & $1.10 \mathrm{E}+00$ \\
\hline
\end{tabular}

The standard deviation for a uniform distribution is calculated from the formula shown below. Values have been inserted to show the calculation for the floor sludge distribution. The theoretical result agrees very well with the observed stochastic result shown in Table 4-5.

$$
\text { Floor Sludge Standard Deviation }=\frac{\operatorname{Max}-\operatorname{Min}}{\sqrt{12}}=\frac{\left(14.05 \mathrm{~m}^{3}-9.37 \mathrm{~m}^{3}\right)}{\sqrt{12}}=1.35 \mathrm{~m}^{3}
$$

The geometric mean and standard deviation are also calculated for information. The primary use for the geometric mean and standard deviation is in the alternate case in which lognormal distributions are used to represent floor sludge concentrations. 
SNF-10293, REV 0

\subsection{STOCHASTIC RESULTS FOR THREE CONTAINER VOLUMES}

The results of the stochastic calculation are presented in this section. Tables with mean and bounding statistics are given along with a plot of the output PDF.

\subsection{CANISTER-TO-TOTAL VOLUME RATIO}

The ratio of the canister sludge volume to the total volume of sludge in a container is an indicator of potential heat generation rates within the container. A bounding value of $40 \%$ has been used. This quantity was tallied to examine the likelihood of extreme values. The stochastic result for probability distribution is shown in Figure 5-1. The vertical scale in this figure is the probability per bin. In this figure, each bin is 0.004 wide. The bin width was chosen so that the $99^{\text {th }}$ percentile value is near bin 120. The probability distributions shown in Figure 5-1 show the first 125 bins.

The curve for $0.5 \mathrm{~m}^{3}$ has the smallest peak height, and also has a peak at very low canister-tototal sludge ratios. This small ratio peak is because of the sludge in key 15458, the key with the most canisters. None of the canisters in key 15458 contain fuel in bad or poor condition, so the volume of canister sludge is low compared to the floor sludge associated with this key. The mean total volume is $1.628 \mathrm{~m}^{3}$, which is enough for three of the $0.5 \mathrm{~m}^{3}$ containers.

This peak at small canister-to-total sludge ratios illustrates a limitation of this stochastic analysis. As the container volume becomes small compared to the volume of sludge in the individual keys, certain ratios begin to dominate. The problem is that the stochastic modeling is carried out at the key volume level, so any container volume smaller than a significant fraction of the keys is likely to give unrealistic results. To accurately represent smaller container volumes, greater detail is needed in the stochastic model to better represent sludge at the individual canister level.

The curve for the $0.5 \mathrm{~m}^{3}$ container has a peak value around a ratio of 0.21 with a probability of 0.015 per bin. The curve for the $2.0 \mathrm{~m}^{3}$ container has a peak value around a ratio of 0.20 with a probability of 0.023 per bin. The curve for the $12 \mathrm{~m}^{3}$ container has a peak value around a ratio of 0.19 with a probability of nearly 0.05 per bin. The shape of the curves illustrates that larger container volumes make the extreme values less likely. With larger containers, the concentrations are usually closer to the mean. 
SNF-10293, REV 0

Figure 5-1. Stochastic Result for the Canister-to-Total Volume Ratio.

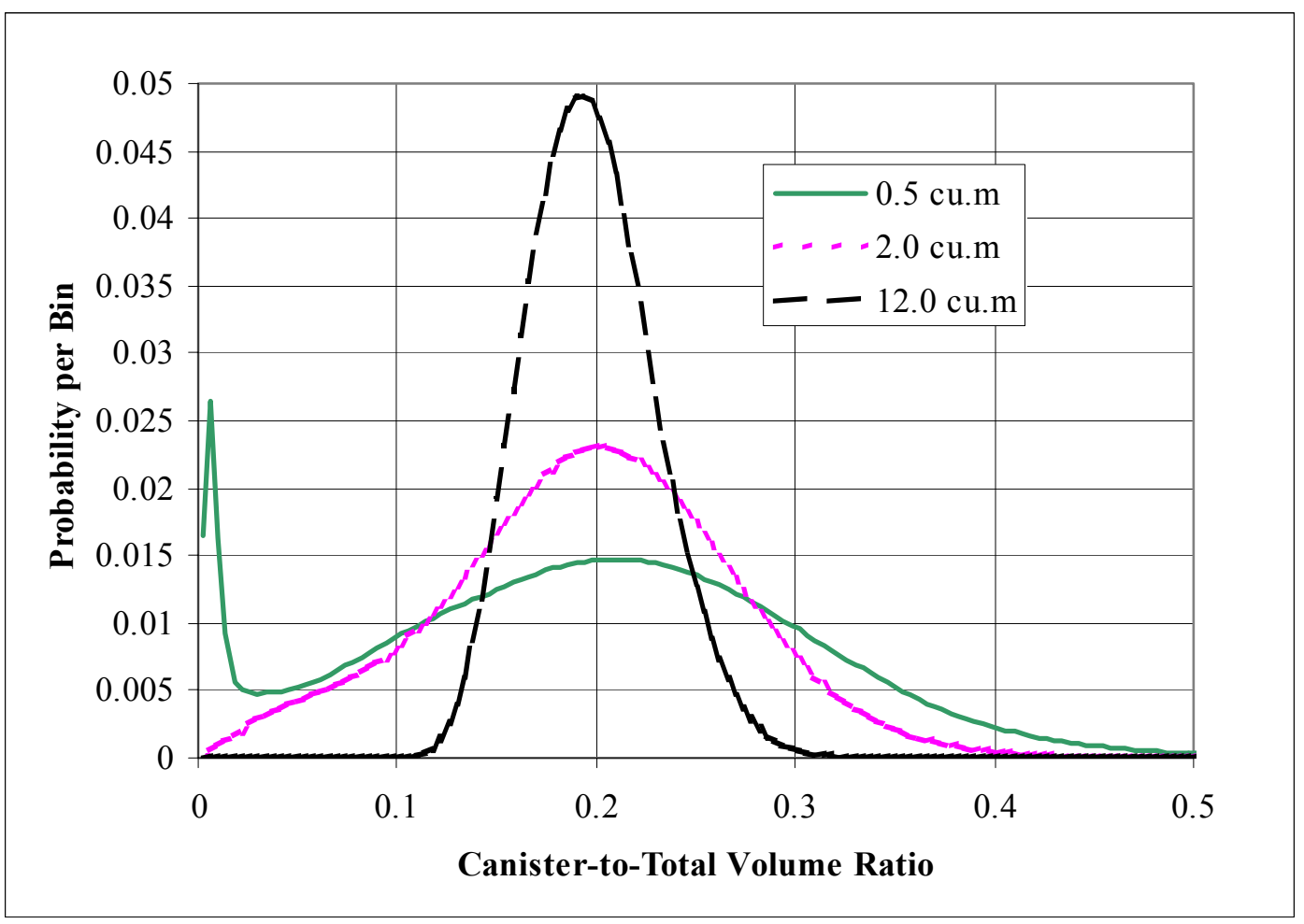

The numeric results from the stochastic analysis are summarized in Table 5-1. The "Total Count" is the total number of containers that were filled. For the $12 \mathrm{~m}^{3}$ volume, only one "container" is filled per key retrieval simulation. Hence, the total container count is 10 million. For the $2 \mathrm{~m}^{3}$ volume, there are 6 or 7 containers per simulation. For the $0.5 \mathrm{~m}^{3}$ volume, there are around 28 containers. These counts are the same for all the distributions discussed in Section 5.3.

Table 5-1. Stochastic Results for the Canister-to-Total Sludge Volume Ratio.

\begin{tabular}{|c|c|c|c|}
\hline Container Volume & $0.5 \mathrm{~m}^{3}$ & $2 \mathrm{~m}^{3}$ & $12 \mathrm{~m}^{3}$ \\
\hline Total Count & $287,461,350$ & $69,009,050$ & $10,000,000$ \\
\hline Average Values & 0.196 & 0.196 & 0.197 \\
\hline 95th Percentile & 0.371 & 0.313 & 0.253 \\
\hline 99th Percentile & 0.452 & 0.364 & 0.277 \\
\hline Standard Deviation & 0.107 & 0.072 & 0.032 \\
\hline Minimum Value & 0.000 & 0.000 & 0.086 \\
\hline Maximum Value & 0.780 & 0.568 & 0.362 \\
\hline Geometric Mean & 0.144 & 0.178 & 0.195 \\
\hline Geometric Std Dev. & 3.02 & 1.65 & 1.18 \\
\hline
\end{tabular}


The maximum and minimum values in Table 5-1 illustrate the effect of container size. The variability from container to container decreases with increasing volume.

\subsection{SLUDGE DENSITY}

Sludge density is calculated as the mass of sludge in a container divided by the volume of sludge in that container. The average sludge density in filled containers was tallied in the stochastic analysis. The distribution of densities is shown in Figure 5-2. As was noted in the curves for canister-to-total sludge volume ratios, the $0.5 \mathrm{~m}^{3}$ volume has the smallest peak and the greatest spread, while the $12 \mathrm{~m}^{3}$ volume has the tallest peak and the least spread. In addition, the peak value increases with increasing container volume.

The numeric results from the stochastic analysis are summarized in Table 5-2. Note that the distribution mean is the same for all three volumes. The increase in the peak with increasing volume is related to the skewing of the curve. The smallest volume is the most asymmetric as evidenced by having the largest $99^{\text {th }}$ percentile. The upper bounds $\left(95^{\text {th }}\right.$ and $99^{\text {th }}$ percentile) and the standard deviation decrease with increasing volume. The bin width in Figure 5-2 is $0.01 \mathrm{~kg} / \mathrm{L}$.

Figure 5-2. Stochastic Result for the Sludge Density.

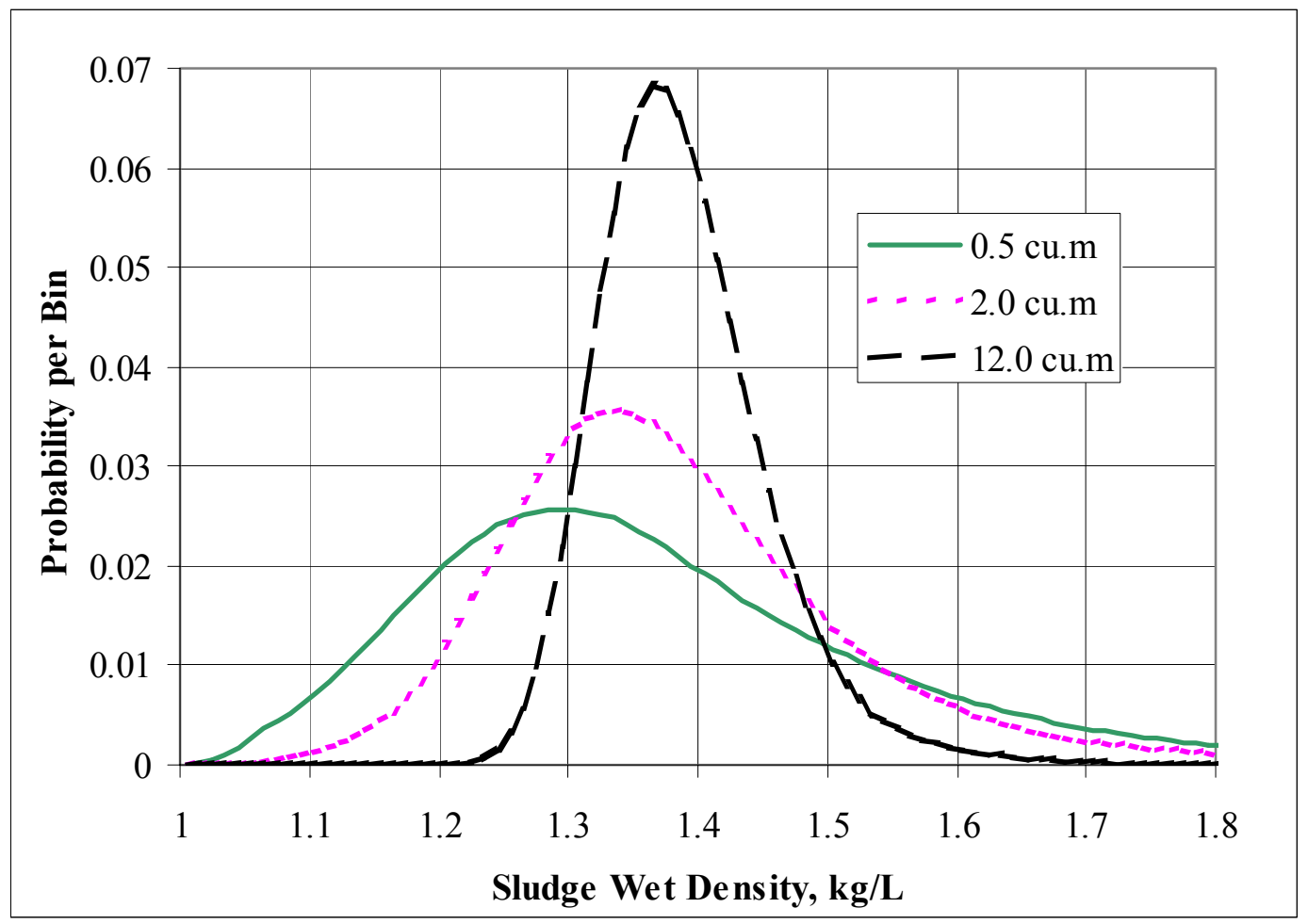


Table 5-2. Stochastic Results for the Sludge Density $(\mathrm{kg} / \mathrm{L})$.

\begin{tabular}{|c|c|c|c|}
\hline Container Volume & $0.5 \mathrm{~m}^{3}$ & $2 \mathrm{~m}^{3}$ & $12 \mathrm{~m}^{3}$ \\
\hline Total Count & $287,461,350$ & $69,009,050$ & $10,000,000$ \\
\hline Average Values & 1.39 & 1.39 & 1.39 \\
\hline 95th Percentile & 1.78 & 1.65 & 1.50 \\
\hline 99th Percentile & 2.28 & 1.89 & 1.59 \\
\hline Standard Deviation & 0.226 & 0.147 & 0.065 \\
\hline Minimum Value & 1.01 & 1.01 & 1.18 \\
\hline Maximum Value & 3.30 & 3.28 & 1.95 \\
\hline Geometric Mean & 1.37 & 1.38 & 1.39 \\
\hline Geometric Std Dev & 1.16 & 1.11 & 1.05 \\
\hline
\end{tabular}

\subsection{ISOTOPIC COMPOSITION}

Isotopic inventories in the various sludge container volumes were tallied as part of the stochastic analysis. The results are graphed and summarized in various tables in this section. The widths of the bins used in each tally are listed at the end of Appendix F. They were selected so that the $99^{\text {th }}$ percentile would be near bin number 120 .

Many of the graphs show a trend that the peak value increases with increasing container volume. Examination of the statistical summary table shows that the mean values are very nearly the same. The selection of the PDFs preserves the same mean values for all container volumes. The number of keys that are added to a container depends on the volume. With larger volumes, more keys are added. The decrease in the relative standard deviation for sums of PDFs mentioned in Section 2.0 makes the decreasing width of the peak understandable. With larger containers, more keys are summed and the relative standard deviation should decrease. The trend in the peak value is related to the skewing of the curve. The smallest volume has the longest tail towards large values. To keep the mean value the same, there must be more outcomes at lower values to offset the ones at higher values. Because there are fewer bins between the lowest possible value (usually zero) and the mean, the probability per bin must be greater. Hence, as the sludge volume decreases the relative standard deviation increases, i.e., the extreme values become more likely. The right half of the peak slumps toward high values while the left half of the peak pushes toward the lower limit. The net effect is that the peak shifts toward smaller values. 
Figure 5-3. Stochastic Result for the Co-60 Concentration.

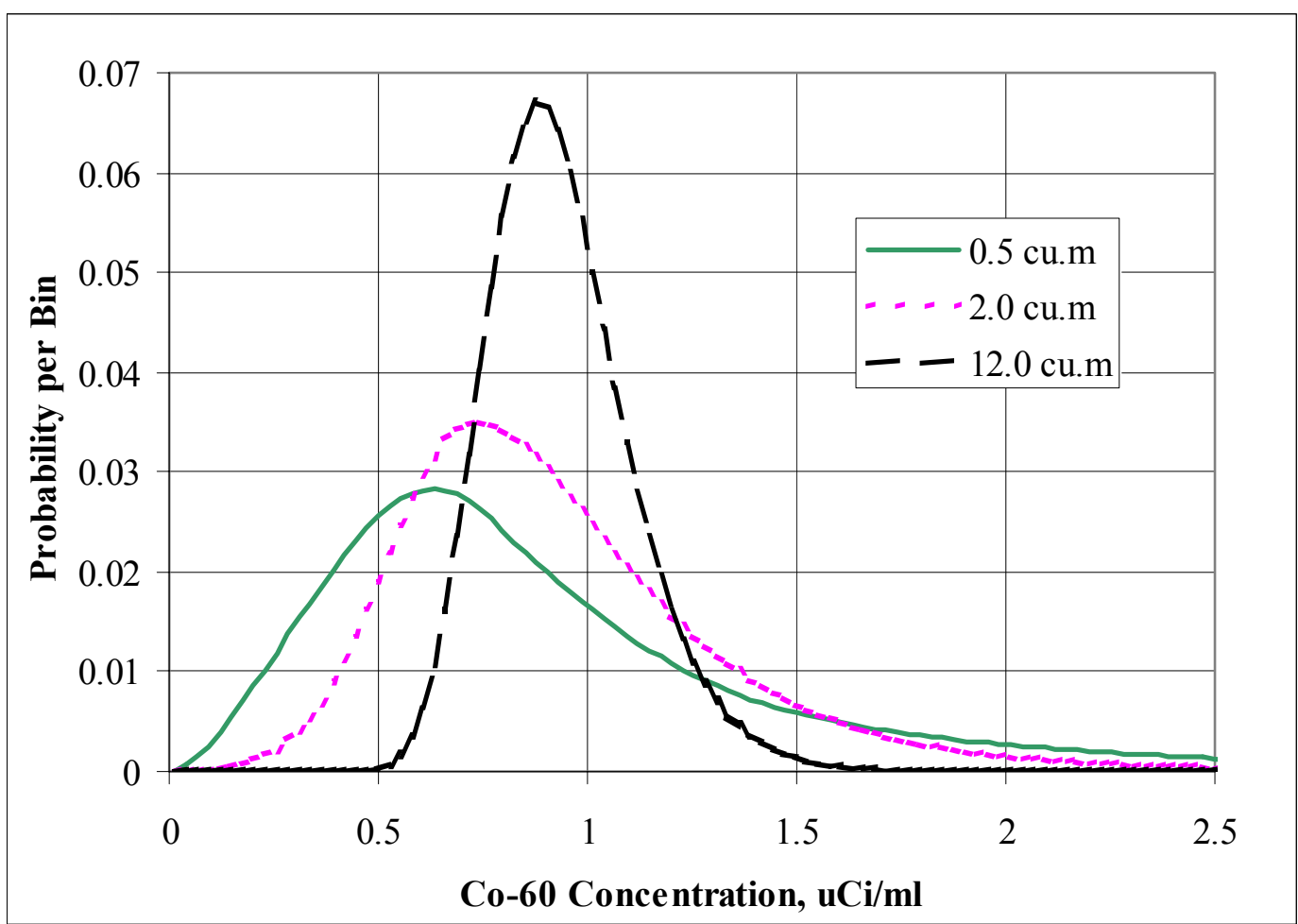

Table 5-3. Stochastic Results for the Co-60 Concentration $\left(\mathrm{Ci} / \mathrm{m}^{3}\right)$.

\begin{tabular}{|c|c|c|c|}
\hline Container Volume & $0.5 \mathrm{~m}^{3}$ & $2 \mathrm{~m}^{3}$ & $12 \mathrm{~m}^{3}$ \\
\hline Total Count & $287,461,350$ & $69,009,050$ & $10,000,000$ \\
\hline Average Values & $9.36 \mathrm{E}-01$ & $9.36 \mathrm{E}-01$ & $9.37 \mathrm{E}-01$ \\
\hline 95th Percentile & $2.19 \mathrm{E}+00$ & $1.67 \mathrm{E}+00$ & $1.25 \mathrm{E}+00$ \\
\hline 99th Percentile & $3.14 \mathrm{E}+00$ & $2.24 \mathrm{E}+00$ & $1.42 \mathrm{E}+00$ \\
\hline Standard Deviation & $6.11 \mathrm{E}-01$ & $3.95 \mathrm{E}-01$ & $1.71 \mathrm{E}-01$ \\
\hline Minimum Value & $1.73 \mathrm{E}-02$ & $2.33 \mathrm{E}-02$ & $3.75 \mathrm{E}-01$ \\
\hline Maximum Value & $1.13 \mathrm{E}+01$ & $5.54 \mathrm{E}+00$ & $2.12 \mathrm{E}+00$ \\
\hline Geometric Mean & $7.75 \mathrm{E}-01$ & $8.62 \mathrm{E}-01$ & $9.22 \mathrm{E}-01$ \\
\hline Geometric Std Dev & $1.93 \mathrm{E}+00$ & $1.52 \mathrm{E}+00$ & $1.20 \mathrm{E}+00$ \\
\hline
\end{tabular}


SNF-10293, REV 0

Figure 5-4. Stochastic Result for the Sr-90 Concentration.

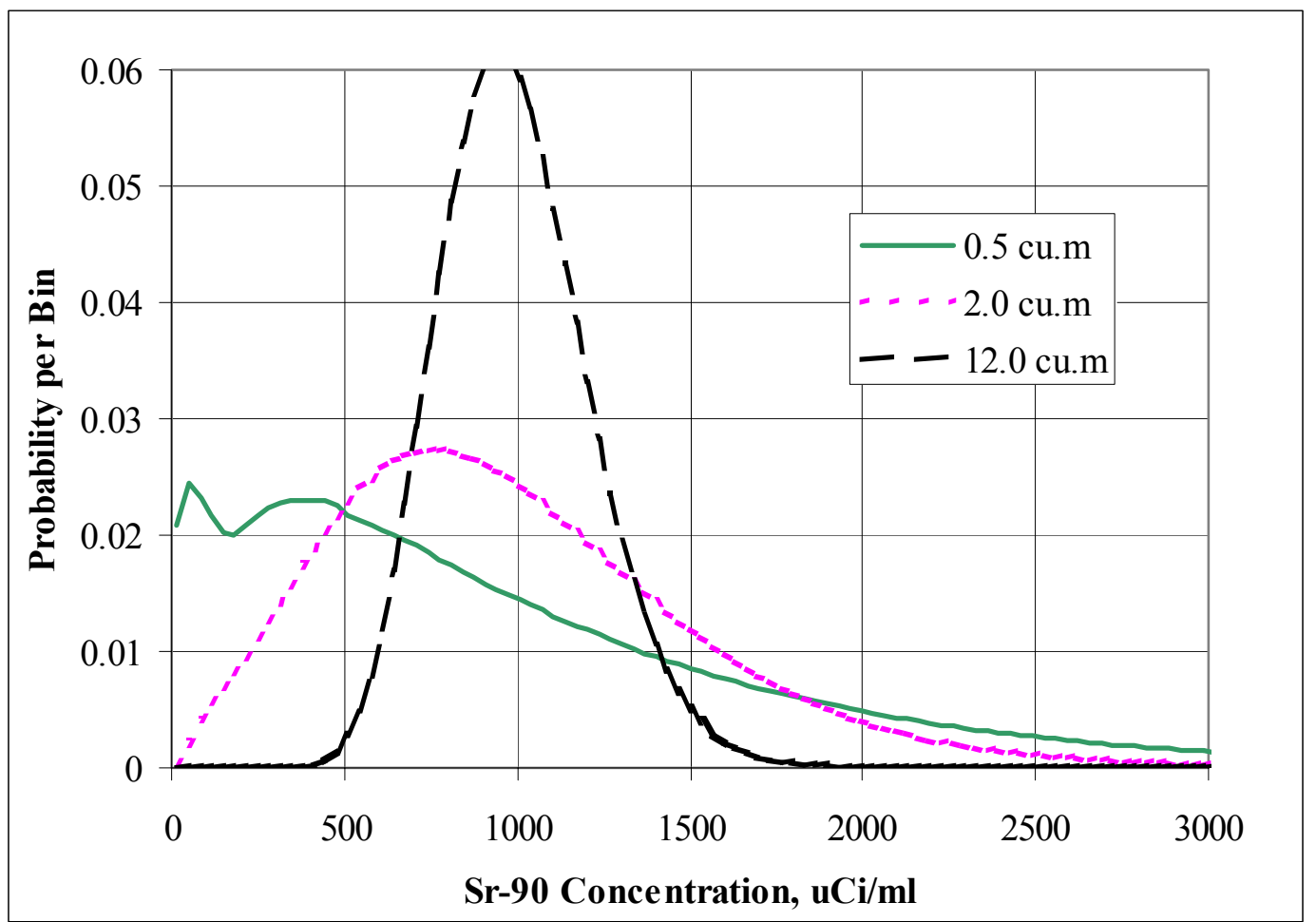

Table 5-4. Stochastic Results for the Sr-90 Concentration $\left(\mathrm{Ci} / \mathrm{m}^{3}\right)$.

\begin{tabular}{|c|c|c|c|}
\hline Container Volume & $0.5 \mathrm{~m}^{3}$ & $2 \mathrm{~m}^{3}$ & $12 \mathrm{~m}^{3}$ \\
\hline Total Count & $287,461,350$ & $69,009,050$ & $10,000,000$ \\
\hline Average Values & $9.90 \mathrm{E}+02$ & $9.87 \mathrm{E}+02$ & $9.93 \mathrm{E}+02$ \\
\hline 95th Percentile & $2.61 \mathrm{E}+03$ & $1.94 \mathrm{E}+03$ & $1.37 \mathrm{E}+03$ \\
\hline 99th Percentile & $3.73 \mathrm{E}+03$ & $2.49 \mathrm{E}+03$ & $1.55 \mathrm{E}+03$ \\
\hline Standard Deviation & $8.26 \mathrm{E}+02$ & $5.15 \mathrm{E}+02$ & $2.12 \mathrm{E}+02$ \\
\hline Minimum Value & $7.95 \mathrm{E}-01$ & $1.69 \mathrm{E}+00$ & $2.29 \mathrm{E}+02$ \\
\hline Maximum Value & $1.50 \mathrm{E}+04$ & $6.55 \mathrm{E}+03$ & $2.29 \mathrm{E}+03$ \\
\hline Geometric Mean & $6.45 \mathrm{E}+02$ & $8.43 \mathrm{E}+02$ & $9.70 \mathrm{E}+02$ \\
\hline Geometric Std Dev & $3.22 \mathrm{E}+00$ & $1.89 \mathrm{E}+00$ & $1.24 \mathrm{E}+00$ \\
\hline
\end{tabular}


Figure 5-5. Stochastic Result for the Tc-99 Concentration.

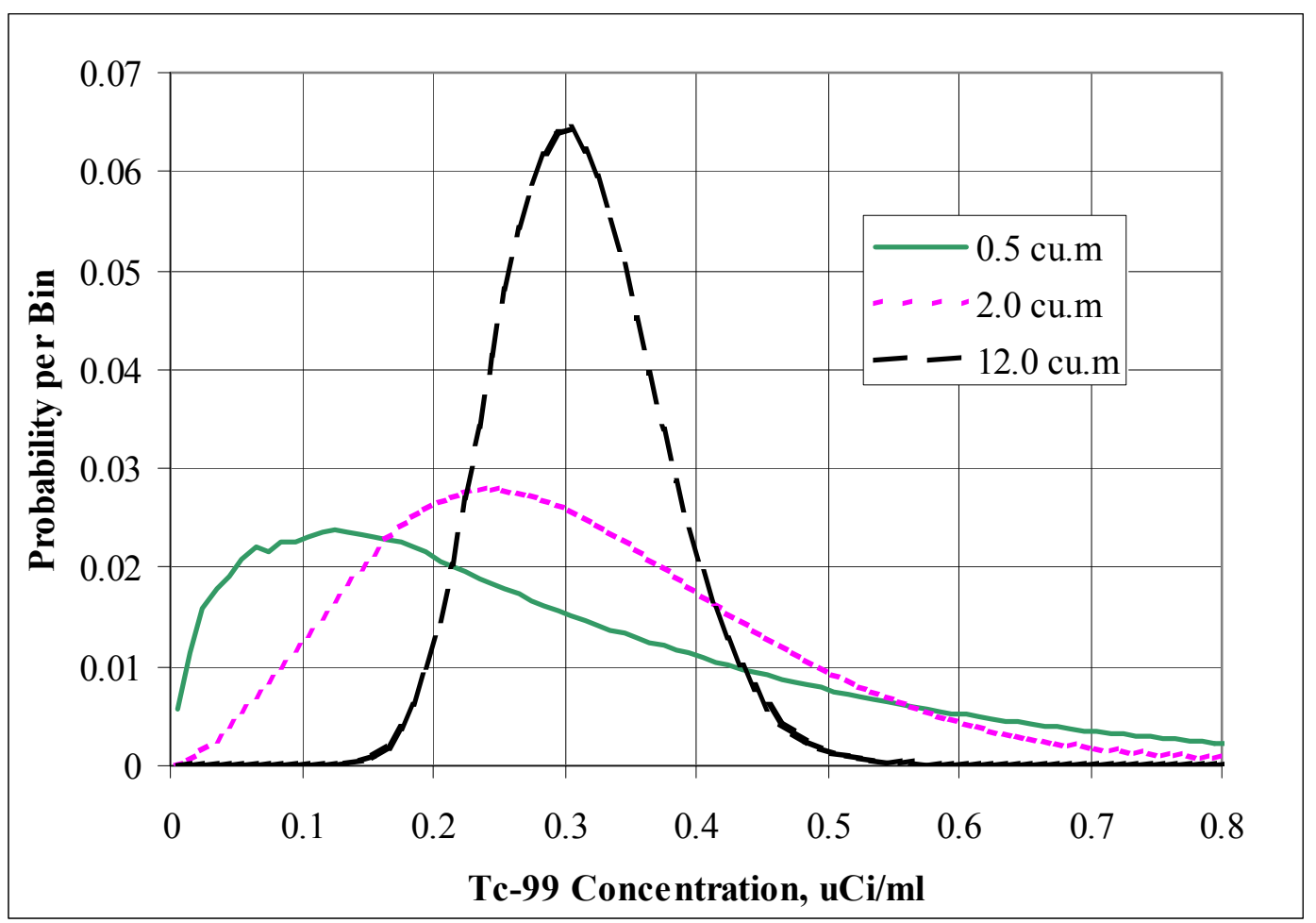

Table 5-5. Stochastic Results for the Tc-99 Concentration $\left(\mathrm{Ci} / \mathrm{m}^{3}\right)$.

\begin{tabular}{|c|c|c|c|}
\hline Container Volume & $0.5 \mathrm{~m}^{3}$ & $2 \mathrm{~m}^{3}$ & $12 \mathrm{~m}^{3}$ \\
\hline Total Count & $287,461,350$ & $69,009,050$ & $10,000,000$ \\
\hline Average Values & $3.11 \mathrm{E}-01$ & $3.10 \mathrm{E}-01$ & $3.12 \mathrm{E}-01$ \\
\hline 95th Percentile & $7.86 \mathrm{E}-01$ & $5.89 \mathrm{E}-01$ & $4.19 \mathrm{E}-01$ \\
\hline 99th Percentile & $1.13 \mathrm{E}+00$ & $7.48 \mathrm{E}-01$ & $4.71 \mathrm{E}-01$ \\
\hline Standard Deviation & $2.44 \mathrm{E}-01$ & $1.52 \mathrm{E}-01$ & $6.15 \mathrm{E}-02$ \\
\hline Minimum Value & $4.07 \mathrm{E}-04$ & $9.89 \mathrm{E}-04$ & $8.13 \mathrm{E}-02$ \\
\hline Maximum Value & $3.81 \mathrm{E}+00$ & $1.81 \mathrm{E}+00$ & $6.84 \mathrm{E}-01$ \\
\hline Geometric Mean & $2.21 \mathrm{E}-01$ & $2.72 \mathrm{E}-01$ & $3.06 \mathrm{E}-01$ \\
\hline Geometric Std Dev & $2.68 \mathrm{E}+00$ & $1.76 \mathrm{E}+00$ & $1.22 \mathrm{E}+00$ \\
\hline
\end{tabular}


Figure 5-6. Stochastic Result for the Cs-134 Concentration.

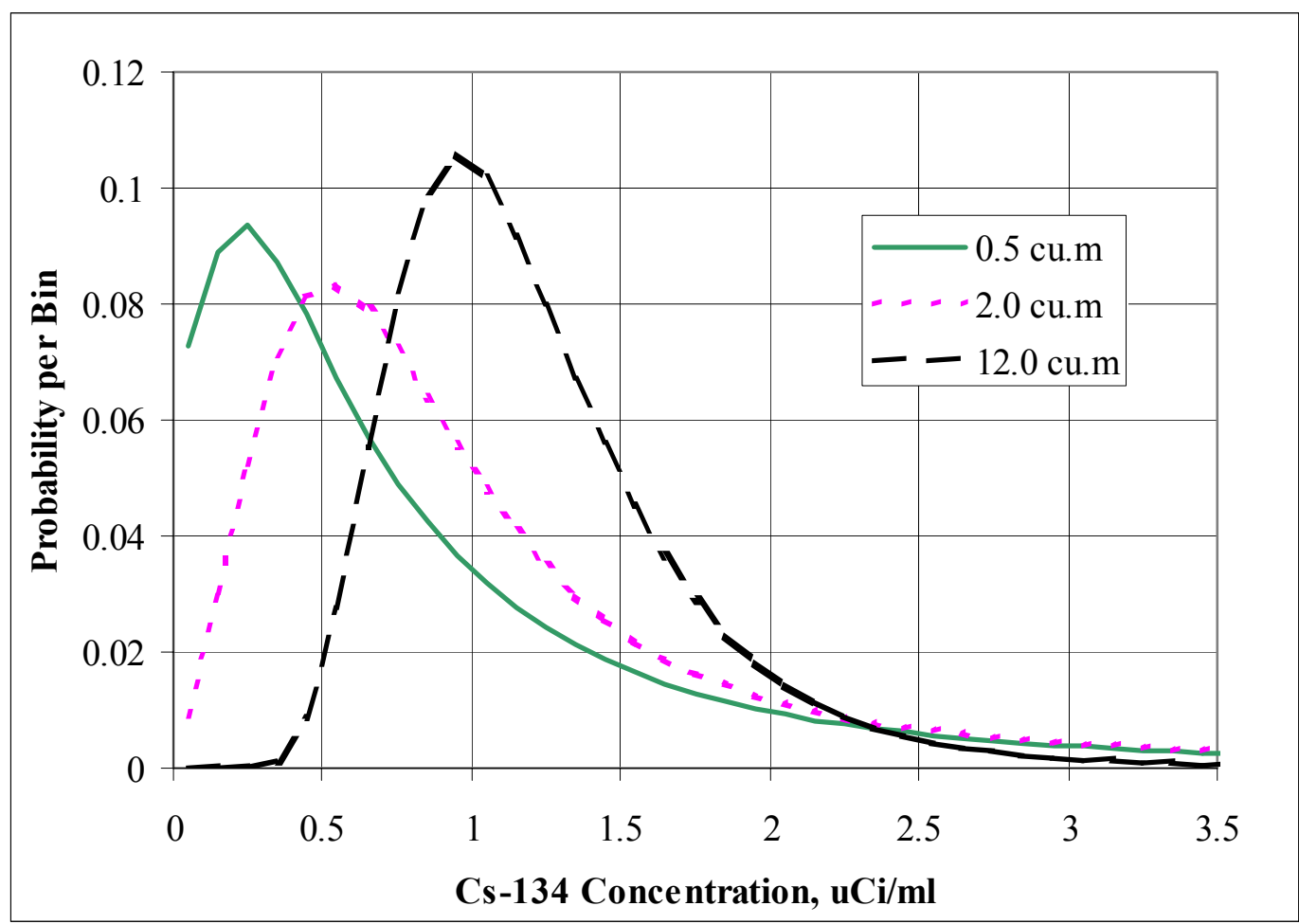

Table 5-6. Stochastic Results for the Cs- 134 Concentration $\left(\mathrm{Ci} / \mathrm{m}^{3}\right)$.

\begin{tabular}{|c|c|c|c|}
\hline Container Volume & $0.5 \mathrm{~m}^{3}$ & $2 \mathrm{~m}^{3}$ & $12 \mathrm{~m}^{3}$ \\
\hline Total Count & $287,461,164$ & $69,009,050$ & $10,000,000$ \\
\hline Average Values & $1.22 \mathrm{E}+00$ & $1.21 \mathrm{E}+00$ & $1.22 \mathrm{E}+00$ \\
\hline 95th Percentile & $3.88 \mathrm{E}+00$ & $3.50 \mathrm{E}+00$ & $2.13 \mathrm{E}+00$ \\
\hline 99th Percentile & $1.10 \mathrm{E}+01$ & $6.80 \mathrm{E}+00$ & $2.84 \mathrm{E}+00$ \\
\hline Standard Deviation & $2.33 \mathrm{E}+00$ & $1.32 \mathrm{E}+00$ & $4.85 \mathrm{E}-01$ \\
\hline Minimum Value & $1.37 \mathrm{E}-03$ & $2.94 \mathrm{E}-03$ & $2.06 \mathrm{E}-01$ \\
\hline Maximum Value & $1.12 \mathrm{E}+02$ & $3.86 \mathrm{E}+01$ & $7.31 \mathrm{E}+00$ \\
\hline Geometric Mean & $6.03 \mathrm{E}-01$ & $8.50 \mathrm{E}-01$ & $1.14 \mathrm{E}+00$ \\
\hline Geometric Std Dev & $3.93 \mathrm{E}+00$ & $2.47 \mathrm{E}+00$ & $1.46 \mathrm{E}+00$ \\
\hline
\end{tabular}


Figure 5-7. Stochastic Result for the Cs-137 Concentration.

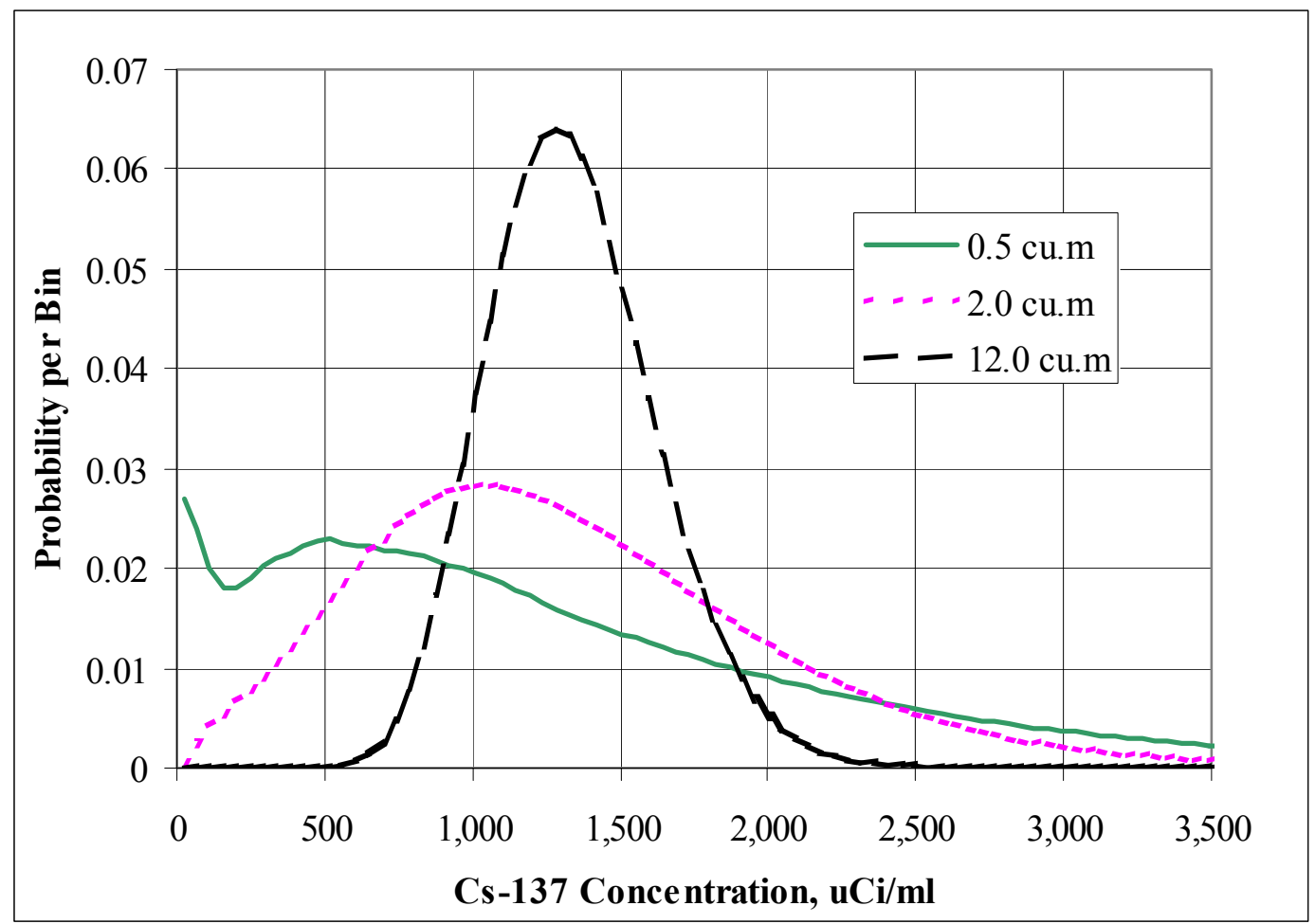

Table 5-7. Stochastic Results for the Cs- 137 Concentration $\left(\mathrm{Ci} / \mathrm{m}^{3}\right)$.

\begin{tabular}{|c|c|c|c|}
\hline Container Volume & $0.5 \mathrm{~m}^{3}$ & $2 \mathrm{~m}^{3}$ & $12 \mathrm{~m}^{3}$ \\
\hline Total Count & $287,461,350$ & $69,009,050$ & $10,000,000$ \\
\hline Average Values & $1.33 \mathrm{E}+03$ & $1.33 \mathrm{E}+03$ & $1.34 \mathrm{E}+03$ \\
\hline 95th Percentile & $3.45 \mathrm{E}+03$ & $2.58 \mathrm{E}+03$ & $1.82 \mathrm{E}+03$ \\
\hline 99th Percentile & $4.96 \mathrm{E}+03$ & $3.29 \mathrm{E}+03$ & $2.06 \mathrm{E}+03$ \\
\hline Standard Deviation & $1.09 \mathrm{E}+03$ & $6.78 \mathrm{E}+02$ & $2.78 \mathrm{E}+02$ \\
\hline Minimum Value & $2.55 \mathrm{E}+00$ & $3.77 \mathrm{E}+00$ & $3.43 \mathrm{E}+02$ \\
\hline Maximum Value & $2.00 \mathrm{E}+04$ & $8.74 \mathrm{E}+03$ & $3.05 \mathrm{E}+03$ \\
\hline Geometric Mean & $8.73 \mathrm{E}+02$ & $1.14 \mathrm{E}+03$ & $1.31 \mathrm{E}+03$ \\
\hline Geometric Std Dev & $3.25 \mathrm{E}+00$ & $1.87 \mathrm{E}+00$ & $1.24 \mathrm{E}+00$ \\
\hline
\end{tabular}


Figure 5-8. Stochastic Result for the Eu-152 Concentration.

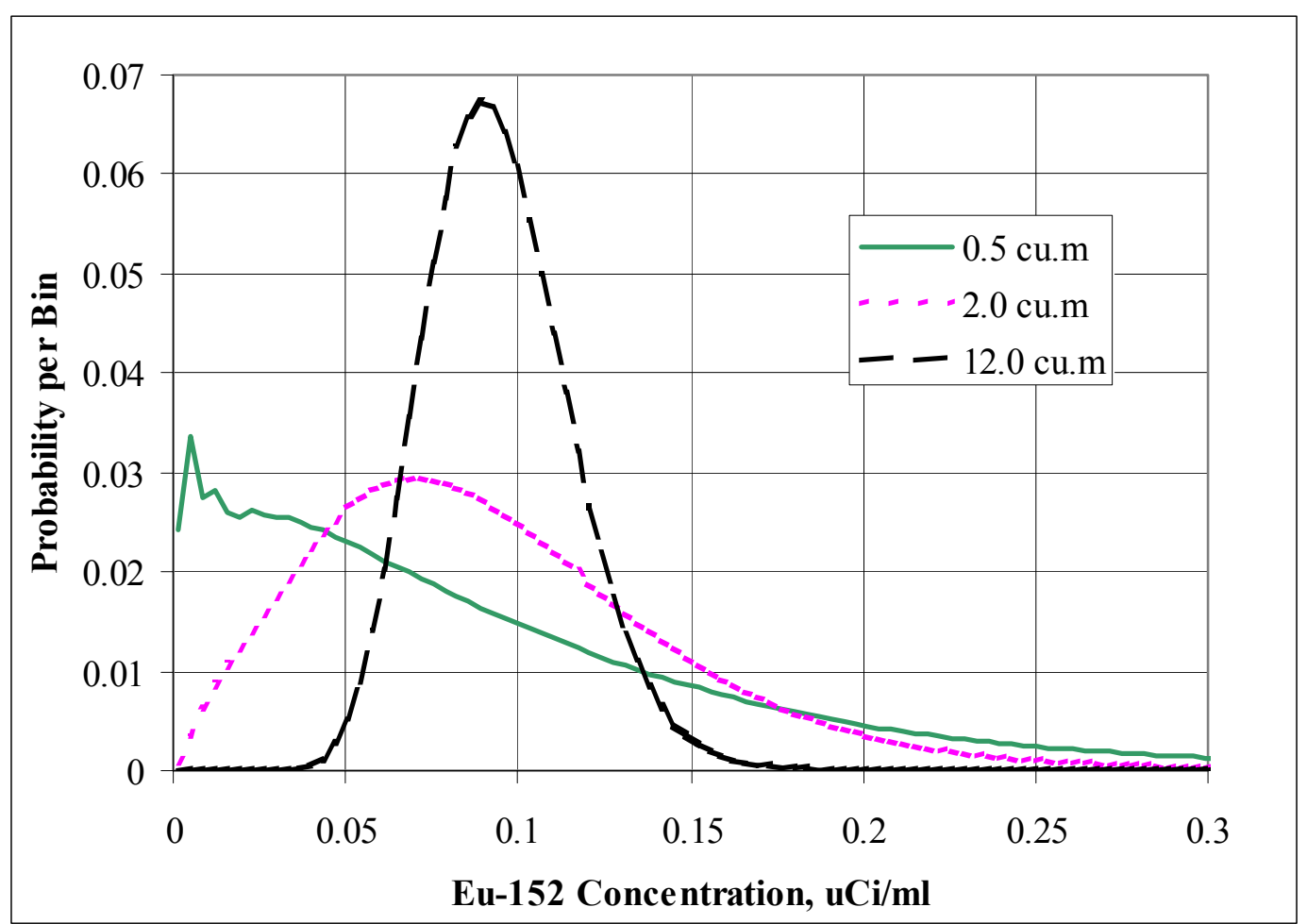

Table 5-8. Stochastic Results for the Eu-152 Concentration $\left(\mathrm{Ci} / \mathrm{m}^{3}\right)$.

\begin{tabular}{|c|c|c|c|}
\hline Container Volume & $0.5 \mathrm{~m}^{3}$ & $2 \mathrm{~m}^{3}$ & $12 \mathrm{~m}^{3}$ \\
\hline Total Count & $287,461,350$ & $69,009,050$ & $10,000,000$ \\
\hline Average Values & $9.40 \mathrm{E}-02$ & $9.37 \mathrm{E}-02$ & $9.43 \mathrm{E}-02$ \\
\hline 95th Percentile & $2.56 \mathrm{E}-01$ & $1.89 \mathrm{E}-01$ & $1.31 \mathrm{E}-01$ \\
\hline 99th Percentile & $3.84 \mathrm{E}-01$ & $2.48 \mathrm{E}-01$ & $1.49 \mathrm{E}-01$ \\
\hline Standard Deviation & $8.38 \mathrm{E}-02$ & $5.17 \mathrm{E}-02$ & $2.06 \mathrm{E}-02$ \\
\hline Minimum Value & $6.38 \mathrm{E}-05$ & $1.57 \mathrm{E}-04$ & $2.05 \mathrm{E}-02$ \\
\hline Maximum Value & $1.85 \mathrm{E}+00$ & $7.87 \mathrm{E}-01$ & $2.30 \mathrm{E}-01$ \\
\hline Geometric Mean & $5.89 \mathrm{E}-02$ & $7.86 \mathrm{E}-02$ & $9.21 \mathrm{E}-02$ \\
\hline Geometric Std Dev & $3.38 \mathrm{E}+00$ & $1.96 \mathrm{E}+00$ & $1.25 \mathrm{E}+00$ \\
\hline
\end{tabular}


Figure 5-9. Stochastic Result for the Eu-154 Concentration.

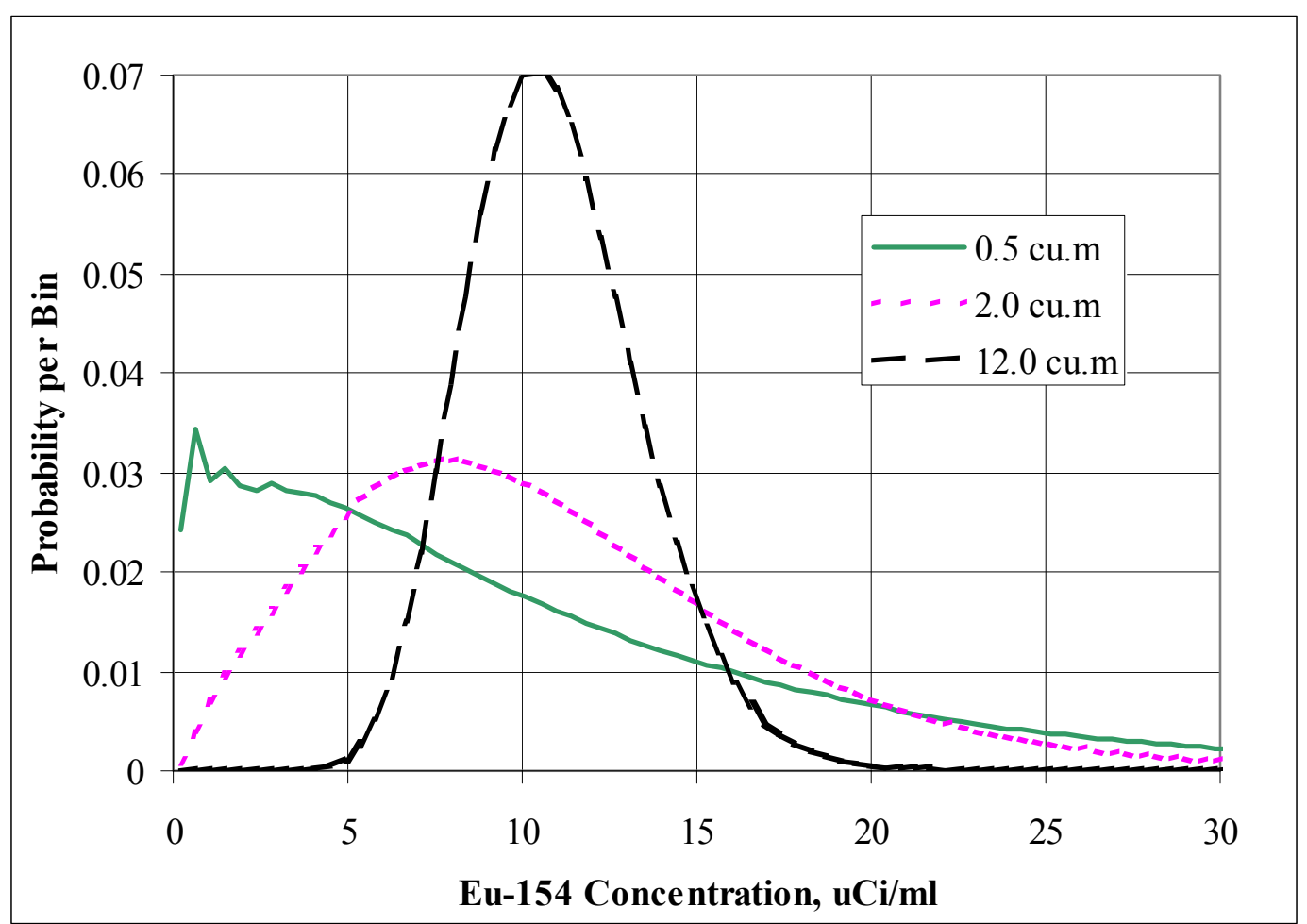

Table 5-9. Stochastic Results for the Eu-154 Concentration (Ci $\left./ \mathrm{m}^{3}\right)$.

\begin{tabular}{|c|c|c|c|}
\hline Container Volume & $0.5 \mathrm{~m}^{3}$ & $2 \mathrm{~m}^{3}$ & $12 \mathrm{~m}^{3}$ \\
\hline Total Count & $287,461,350$ & $69,009,050$ & $10,000,000$ \\
\hline Average Values & $1.09 \mathrm{E}+01$ & $1.09 \mathrm{E}+01$ & $1.09 \mathrm{E}+01$ \\
\hline 95th Percentile & $3.02 \mathrm{E}+01$ & $2.23 \mathrm{E}+01$ & $1.52 \mathrm{E}+01$ \\
\hline 99th Percentile & $4.68 \mathrm{E}+01$ & $2.98 \mathrm{E}+01$ & $1.74 \mathrm{E}+01$ \\
\hline Standard Deviation & $1.01 \mathrm{E}+01$ & $6.17 \mathrm{E}+00$ & $2.44 \mathrm{E}+00$ \\
\hline Minimum Value & $7.98 \mathrm{E}-03$ & $1.94 \mathrm{E}-02$ & $2.42 \mathrm{E}+00$ \\
\hline Maximum Value & $2.55 \mathrm{E}+02$ & $1.06 \mathrm{E}+02$ & $2.90 \mathrm{E}+01$ \\
\hline Geometric Mean & $6.80 \mathrm{E}+00$ & $9.07 \mathrm{E}+00$ & $1.07 \mathrm{E}+01$ \\
\hline Geometric Std Dev & $3.37 \mathrm{E}+00$ & $1.97 \mathrm{E}+00$ & $1.26 \mathrm{E}+00$ \\
\hline
\end{tabular}


Figure 5-10. Stochastic Result for the Eu-155 Concentration.

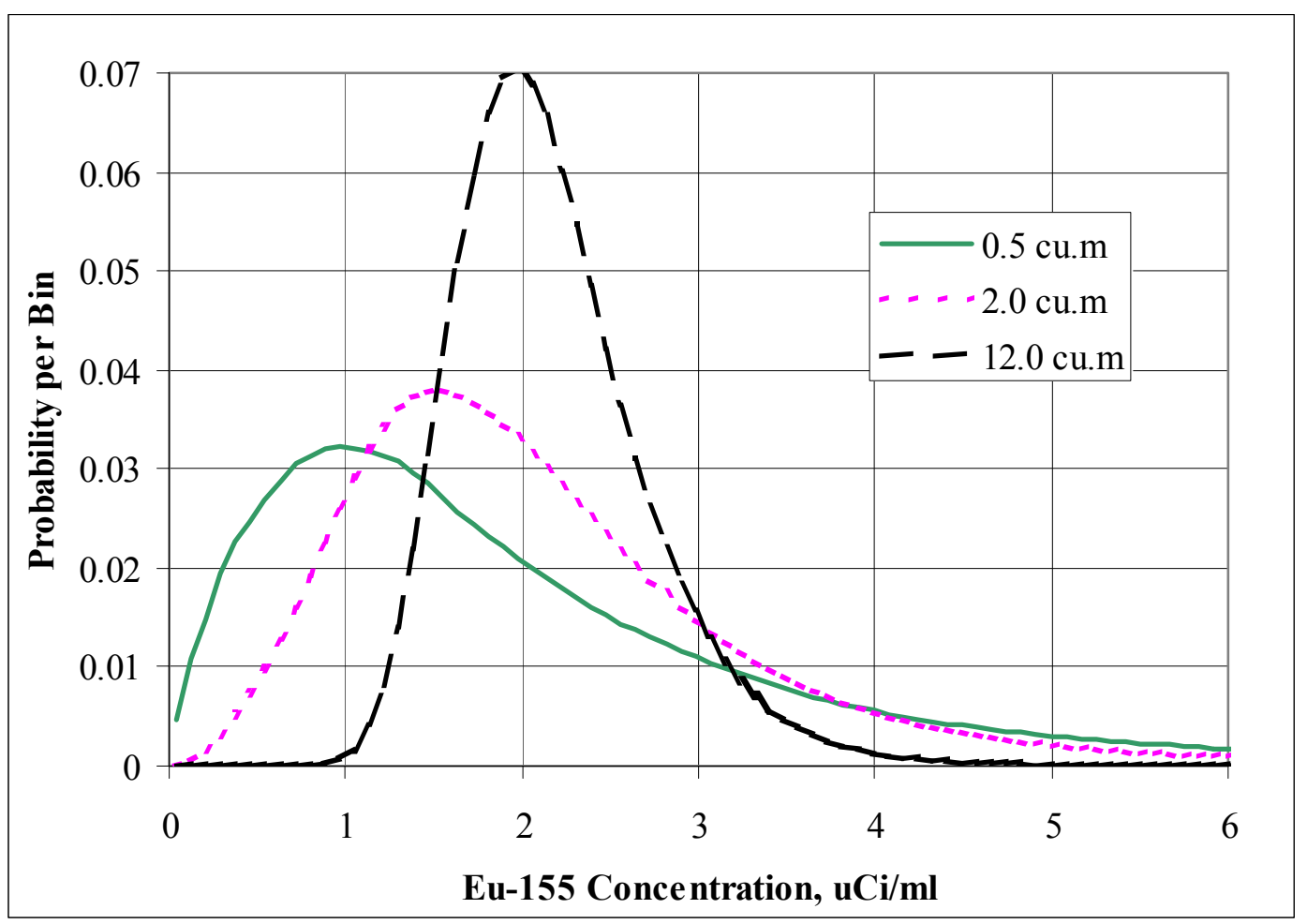

Table 5-10. Stochastic Results for the Eu-155 Concentration $\left(\mathrm{Ci} / \mathrm{m}^{3}\right)$.

\begin{tabular}{|c|c|c|c|}
\hline Container Volume & $0.5 \mathrm{~m}^{3}$ & $2 \mathrm{~m}^{3}$ & $12 \mathrm{~m}^{3}$ \\
\hline Total Count & $287,461,350$ & $69,009,050$ & $10,000,000$ \\
\hline Average Values & $2.16 \mathrm{E}+00$ & $2.15 \mathrm{E}+00$ & $2.16 \mathrm{E}+00$ \\
\hline 95th Percentile & $5.69 \mathrm{E}+00$ & $4.39 \mathrm{E}+00$ & $3.14 \mathrm{E}+00$ \\
\hline 99th Percentile & $9.26 \mathrm{E}+00$ & $6.48 \mathrm{E}+00$ & $3.73 \mathrm{E}+00$ \\
\hline Standard Deviation & $1.90 \mathrm{E}+00$ & $1.22 \mathrm{E}+00$ & $5.27 \mathrm{E}-01$ \\
\hline Minimum Value & $3.54 \mathrm{E}-03$ & $1.31 \mathrm{E}-02$ & $5.90 \mathrm{E}-01$ \\
\hline Maximum Value & $5.71 \mathrm{E}+01$ & $2.17 \mathrm{E}+01$ & $6.63 \mathrm{E}+00$ \\
\hline Geometric Mean & $1.55 \mathrm{E}+00$ & $1.86 \mathrm{E}+00$ & $2.10 \mathrm{E}+00$ \\
\hline Geometric Std Dev & $2.52 \mathrm{E}+00$ & $1.77 \mathrm{E}+00$ & $1.27 \mathrm{E}+00$ \\
\hline
\end{tabular}


SNF-10293, REV 0

Figure 5-11. Stochastic Result for the Np-237 Concentration.

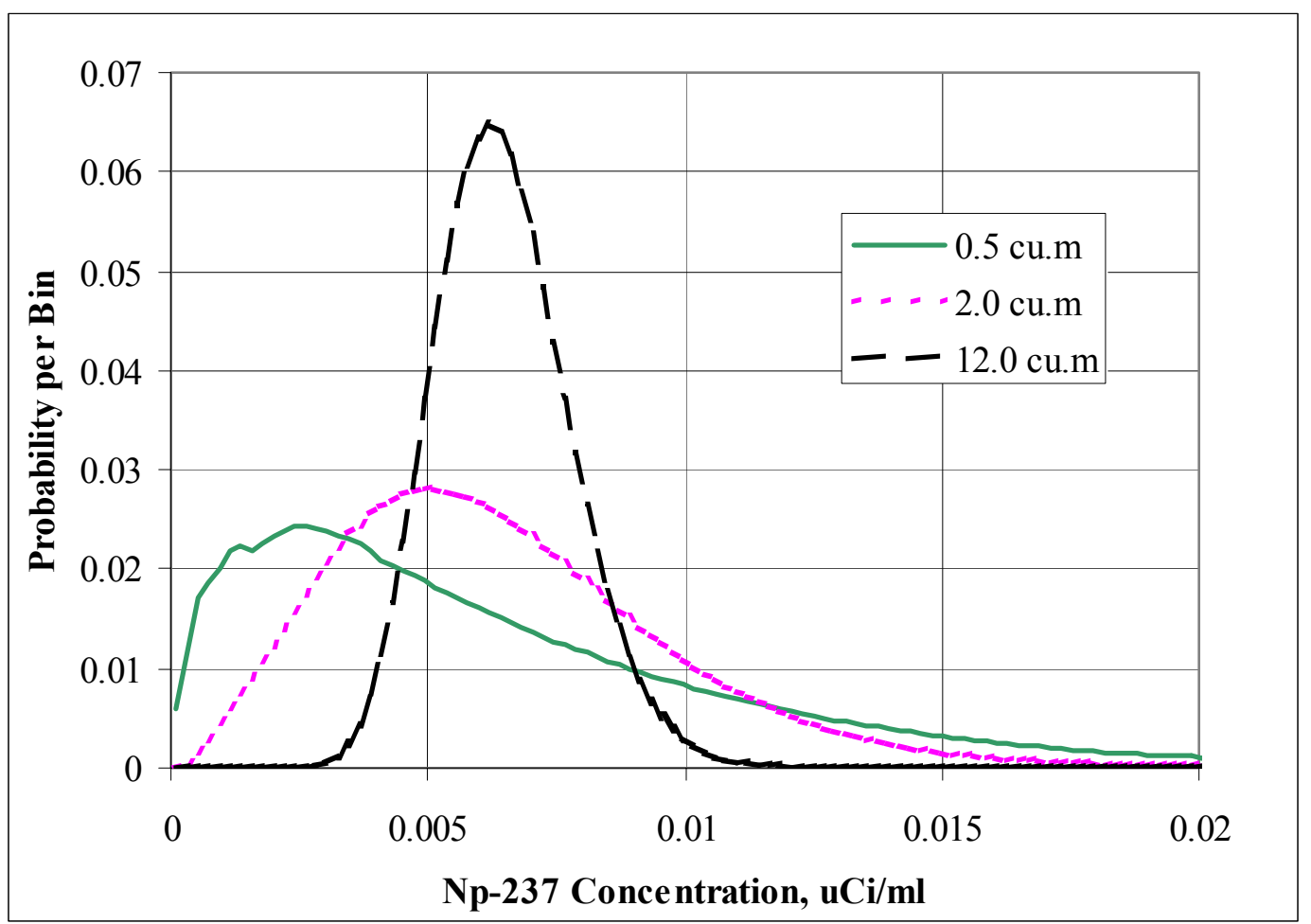

Table 5-11. Stochastic Results for the Np-237 Concentration $\left(\mathrm{Ci} / \mathrm{m}^{3}\right)$.

\begin{tabular}{|c|c|c|c|}
\hline Container Volume & $0.5 \mathrm{~m}^{3}$ & $2 \mathrm{~m}^{3}$ & $12 \mathrm{~m}^{3}$ \\
\hline Total Count & $287,461,350$ & $69,009,050$ & $10,000,000$ \\
\hline Average Values & $6.44 \mathrm{E}-03$ & $6.43 \mathrm{E}-03$ & $6.47 \mathrm{E}-03$ \\
\hline 95th Percentile & $1.64 \mathrm{E}-02$ & $1.23 \mathrm{E}-02$ & $8.71 \mathrm{E}-03$ \\
\hline 99th Percentile & $2.35 \mathrm{E}-02$ & $1.56 \mathrm{E}-02$ & $9.79 \mathrm{E}-03$ \\
\hline Standard Deviation & $5.10 \mathrm{E}-03$ & $3.17 \mathrm{E}-03$ & $1.28 \mathrm{E}-03$ \\
\hline Minimum Value & $8.27 \mathrm{E}-06$ & $1.99 \mathrm{E}-05$ & $1.69 \mathrm{E}-03$ \\
\hline Maximum Value & $7.97 \mathrm{E}-02$ & $3.77 \mathrm{E}-02$ & $1.42 \mathrm{E}-02$ \\
\hline Geometric Mean & $4.55 \mathrm{E}-03$ & $5.61 \mathrm{E}-03$ & $6.34 \mathrm{E}-03$ \\
\hline Geometric Std Dev & $2.71 \mathrm{E}+00$ & $1.78 \mathrm{E}+00$ & $1.22 \mathrm{E}+00$ \\
\hline
\end{tabular}


Figure 5-12. Stochastic Result for the Pu-238 Concentration.

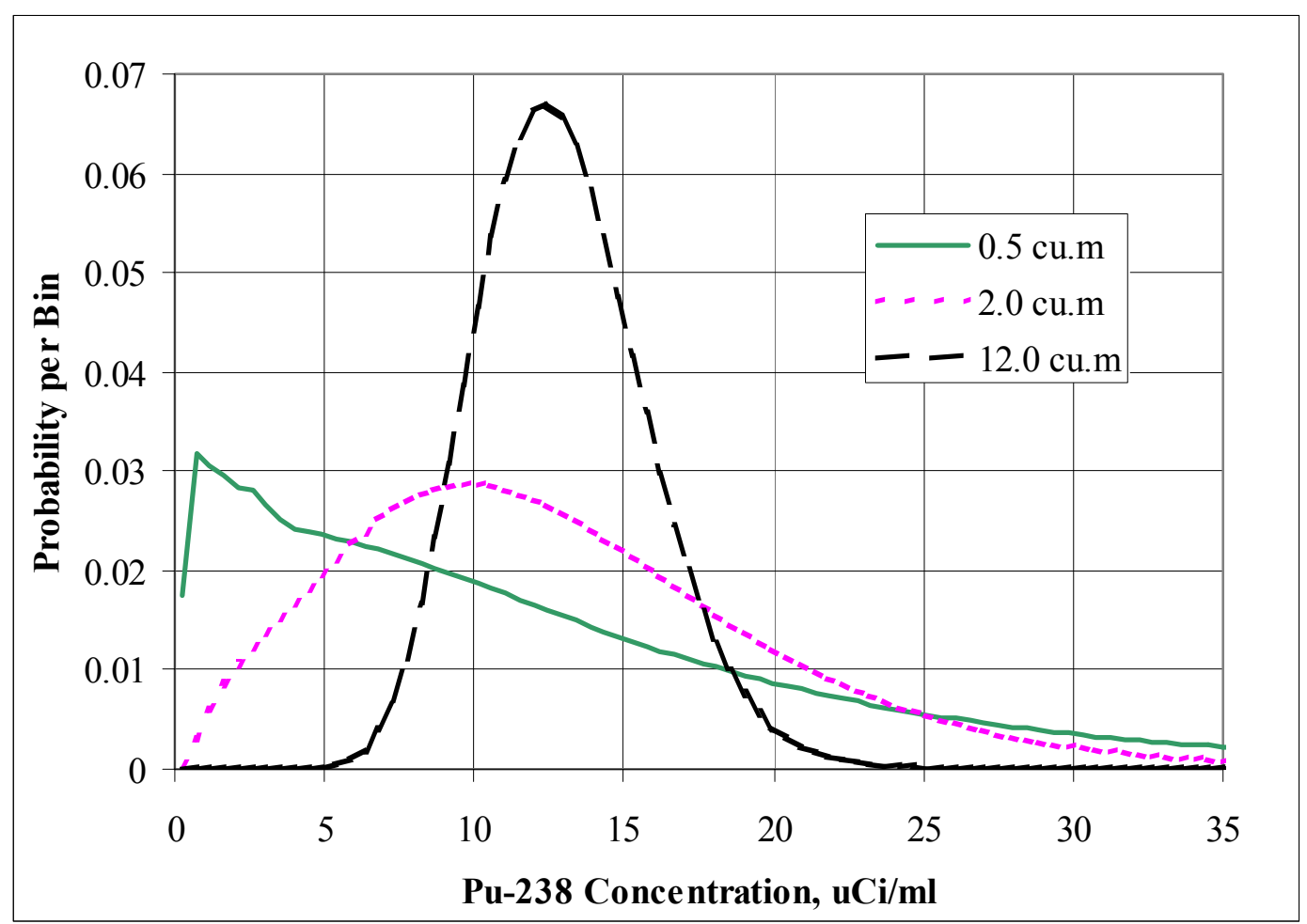

Table 5-12. Stochastic Results for the Pu-238 Concentration $\left(\mathrm{Ci} / \mathrm{m}^{3}\right)$.

\begin{tabular}{|c|c|c|c|}
\hline Container Volume & $0.5 \mathrm{~m}^{3}$ & $2 \mathrm{~m}^{3}$ & $12 \mathrm{~m}^{3}$ \\
\hline Total Count & $287,461,350$ & $69,009,050$ & $10,000,000$ \\
\hline Average Values & $1.29 \mathrm{E}+01$ & $1.29 \mathrm{E}+01$ & $1.29 \mathrm{E}+01$ \\
\hline 95th Percentile & $3.52 \mathrm{E}+01$ & $2.58 \mathrm{E}+01$ & $1.78 \mathrm{E}+01$ \\
\hline 99th Percentile & $5.22 \mathrm{E}+01$ & $3.35 \mathrm{E}+01$ & $2.02 \mathrm{E}+01$ \\
\hline Standard Deviation & $1.14 \mathrm{E}+01$ & $7.02 \mathrm{E}+00$ & $2.78 \mathrm{E}+00$ \\
\hline Minimum Value & $8.86 \mathrm{E}-03$ & $1.97 \mathrm{E}-02$ & $2.91 \mathrm{E}+00$ \\
\hline Maximum Value & $1.77 \mathrm{E}+02$ & $8.32 \mathrm{E}+01$ & $3.01 \mathrm{E}+01$ \\
\hline Geometric Mean & $8.16 \mathrm{E}+00$ & $1.08 \mathrm{E}+01$ & $1.26 \mathrm{E}+01$ \\
\hline Geometric Std Dev & $3.29 \mathrm{E}+00$ & $1.96 \mathrm{E}+00$ & $1.25 \mathrm{E}+00$ \\
\hline
\end{tabular}


Figure 5-13. Stochastic Result for the Pu-239/240 Concentration.

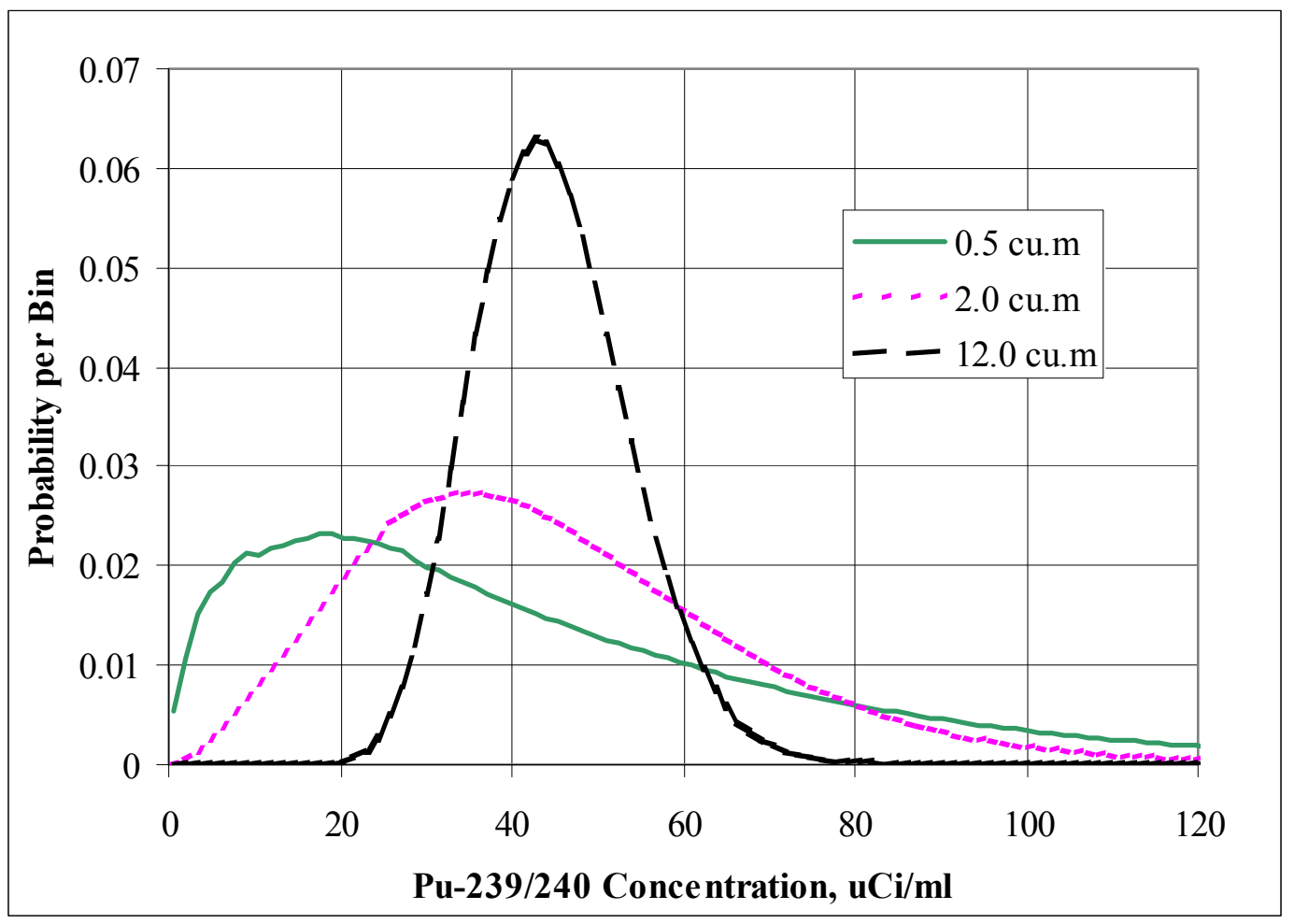

Table 5-13. Stochastic Results for the Pu-239/240 Concentration $\left(\mathrm{Ci} / \mathrm{m}^{3}\right)$.

\begin{tabular}{|c|c|c|c|}
\hline Container Volume & $0.5 \mathrm{~m}^{3}$ & $2 \mathrm{~m}^{3}$ & $12 \mathrm{~m}^{3}$ \\
\hline Total Count & $287,448,950$ & $68,997,500$ & $10,000,000$ \\
\hline Average Values & $4.46 \mathrm{E}+01$ & $4.44 \mathrm{E}+01$ & $4.47 \mathrm{E}+01$ \\
\hline 95th Percentile & $1.14 \mathrm{E}+02$ & $8.52 \mathrm{E}+01$ & $6.05 \mathrm{E}+01$ \\
\hline 99th Percentile & $1.65 \mathrm{E}+02$ & $1.09 \mathrm{E}+02$ & $6.82 \mathrm{E}+01$ \\
\hline Standard Deviation & $3.55 \mathrm{E}+01$ & $2.20 \mathrm{E}+01$ & $9.00 \mathrm{E}+00$ \\
\hline Minimum Value & $5.28 \mathrm{E}-02$ & $6.93 \mathrm{E}-02$ & $1.38 \mathrm{E}+01$ \\
\hline Maximum Value & $5.47 \mathrm{E}+02$ & $3.30 \mathrm{E}+02$ & $9.90 \mathrm{E}+01$ \\
\hline Geometric Mean & $3.15 \mathrm{E}+01$ & $3.88 \mathrm{E}+01$ & $4.38 \mathrm{E}+01$ \\
\hline Geometric Std Dev & $2.70 \mathrm{E}+00$ & $1.78 \mathrm{E}+00$ & $1.23 \mathrm{E}+00$ \\
\hline
\end{tabular}


Figure 5-14. Stochastic Result for the Pu-241 Concentration.

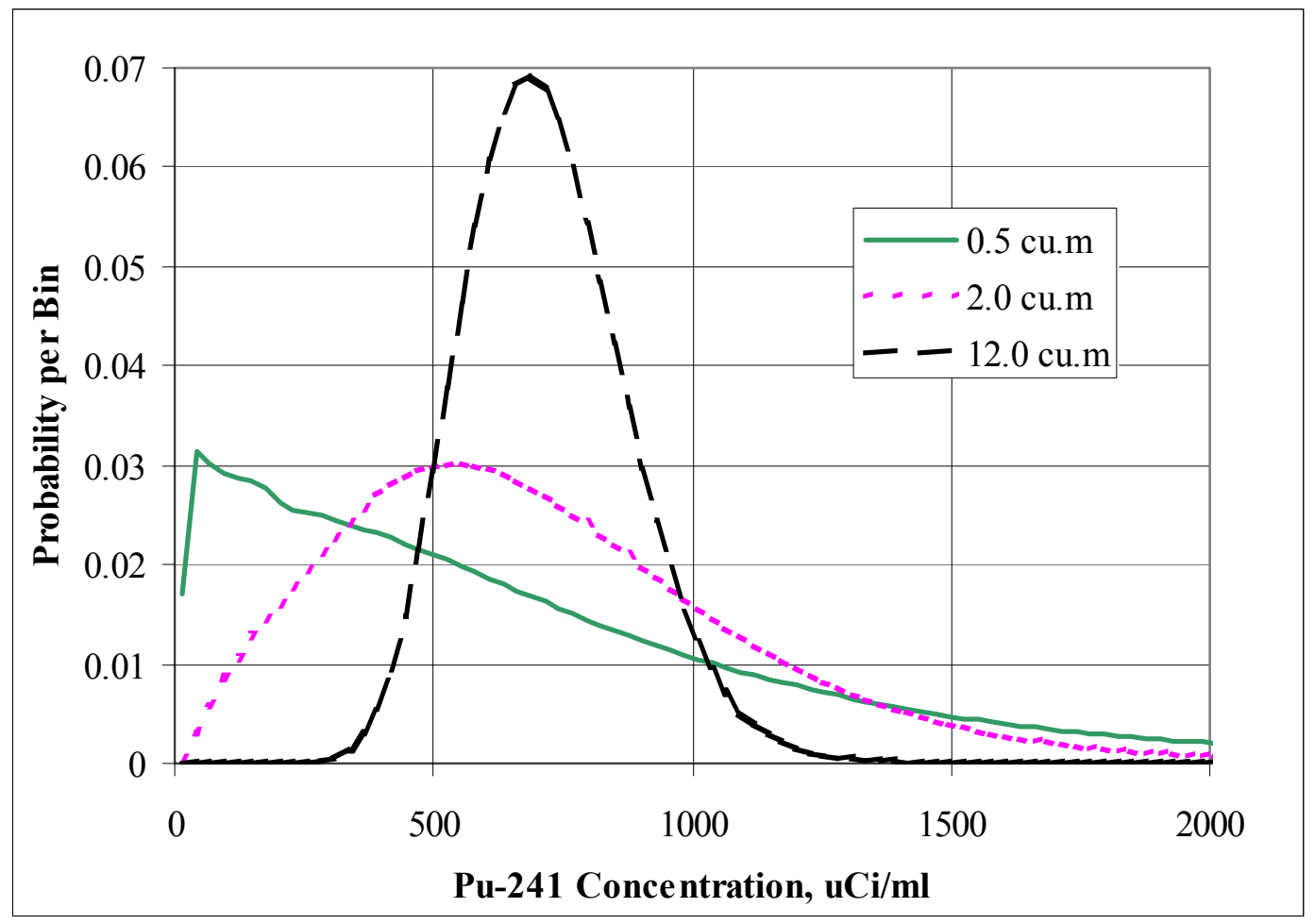

Table 5-14. Stochastic Results for the Pu-241 Concentration $\left(\mathrm{Ci} / \mathrm{m}^{3}\right)$.

\begin{tabular}{|c|c|c|c|}
\hline Container Volume & $0.5 \mathrm{~m}^{3}$ & $2 \mathrm{~m}^{3}$ & $12 \mathrm{~m}^{3}$ \\
\hline Total Count & $287,461,350$ & $69,009,050$ & $10,000,000$ \\
\hline Average Values & $7.15 \mathrm{E}+02$ & $7.13 \mathrm{E}+02$ & $7.18 \mathrm{E}+02$ \\
\hline 95th Percentile & $1.94 \mathrm{E}+03$ & $1.44 \mathrm{E}+03$ & $9.89 \mathrm{E}+02$ \\
\hline 99th Percentile & $2.91 \mathrm{E}+03$ & $1.88 \mathrm{E}+03$ & $1.12 \mathrm{E}+03$ \\
\hline Standard Deviation & $6.33 \mathrm{E}+02$ & $3.90 \mathrm{E}+02$ & $1.55 \mathrm{E}+02$ \\
\hline Minimum Value & $5.12 \mathrm{E}-01$ & $1.17 \mathrm{E}+00$ & $1.62 \mathrm{E}+02$ \\
\hline Maximum Value & $1.34 \mathrm{E}+04$ & $5.79 \mathrm{E}+03$ & $1.71 \mathrm{E}+03$ \\
\hline Geometric Mean & $4.58 \mathrm{E}+02$ & $6.00 \mathrm{E}+02$ & $7.01 \mathrm{E}+02$ \\
\hline Geometric Std Dev & $3.22 \mathrm{E}+00$ & $1.94 \mathrm{E}+00$ & $1.25 \mathrm{E}+00$ \\
\hline
\end{tabular}


Figure 5-15. Stochastic Result for the Am-241 Concentration.

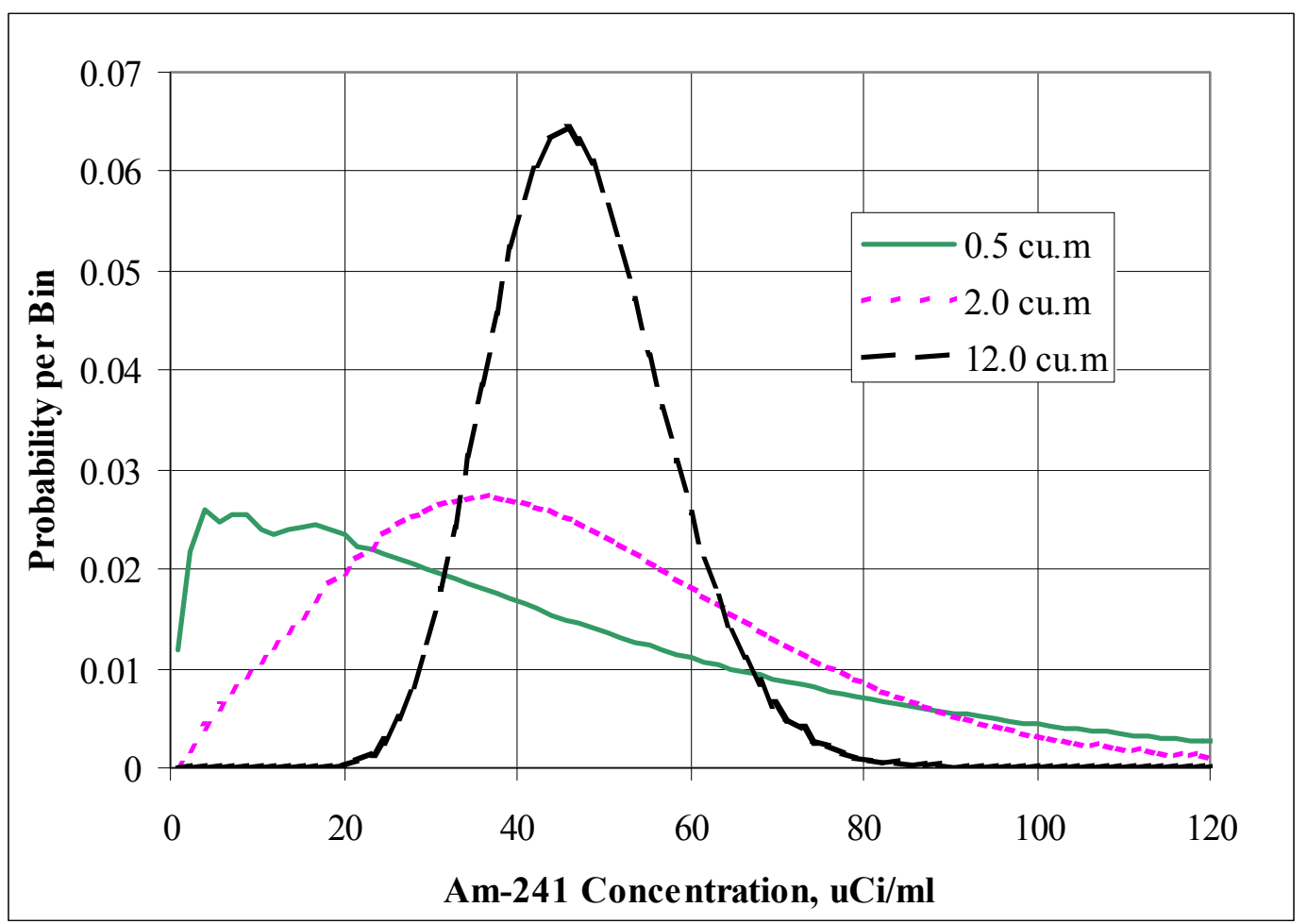

Table 5-15. Stochastic Results for the Am-241 Concentration $\left(\mathrm{Ci} / \mathrm{m}^{3}\right)$.

\begin{tabular}{|c|c|c|c|}
\hline Container Volume & $0.5 \mathrm{~m}^{3}$ & $2 \mathrm{~m}^{3}$ & $12 \mathrm{~m}^{3}$ \\
\hline Total Count & $287,461,350$ & $69,009,050$ & $10,000,000$ \\
\hline Average Values & $4.72 \mathrm{E}+01$ & $4.71 \mathrm{E}+01$ & $4.74 \mathrm{E}+01$ \\
\hline 95th Percentile & $1.26 \mathrm{E}+02$ & $9.29 \mathrm{E}+01$ & $6.47 \mathrm{E}+01$ \\
\hline 99th Percentile & $1.82 \mathrm{E}+02$ & $1.19 \mathrm{E}+02$ & $7.30 \mathrm{E}+01$ \\
\hline Standard Deviation & $4.01 \mathrm{E}+01$ & $2.48 \mathrm{E}+01$ & $9.87 \mathrm{E}+00$ \\
\hline Minimum Value & $4.61 \mathrm{E}-02$ & $7.38 \mathrm{E}-02$ & $1.13 \mathrm{E}+01$ \\
\hline Maximum Value & $5.78 \mathrm{E}+02$ & $2.82 \mathrm{E}+02$ & $1.06 \mathrm{E}+02$ \\
\hline Geometric Mean & $3.11 \mathrm{E}+01$ & $4.00 \mathrm{E}+01$ & $4.63 \mathrm{E}+01$ \\
\hline Geometric Std Dev & $3.08 \mathrm{E}+00$ & $1.90 \mathrm{E}+00$ & $1.24 \mathrm{E}+00$ \\
\hline
\end{tabular}




\subsection{HEAT GENERATION RATE}

The heat generation rate is tallied three ways during the simulation. The first is the total heat generated per unit volume of sludge. The second is the heat generated per unit mass of sludge. The third is the heat generated per unit mass of uranium (total). The second two tallies were necessary because they involve division of the first by another distribution, either total sludge mass or total uranium mass. The distribution of heat generation rates is shown in Figures 5-16, $5-17$, and 5-18. The corresponding numeric results from the stochastic analysis are summarized in Tables 5-16, 5-17, and 5-18. The bin widths are $0.56 \mathrm{~W} / \mathrm{m}^{3}, 0.32 \mathrm{~W} / \mathrm{MT}$, and $1.0 \mathrm{~W} / \mathrm{MTU}$.

Figure 5-16. Stochastic Result for Heat Generation per Unit Volume.

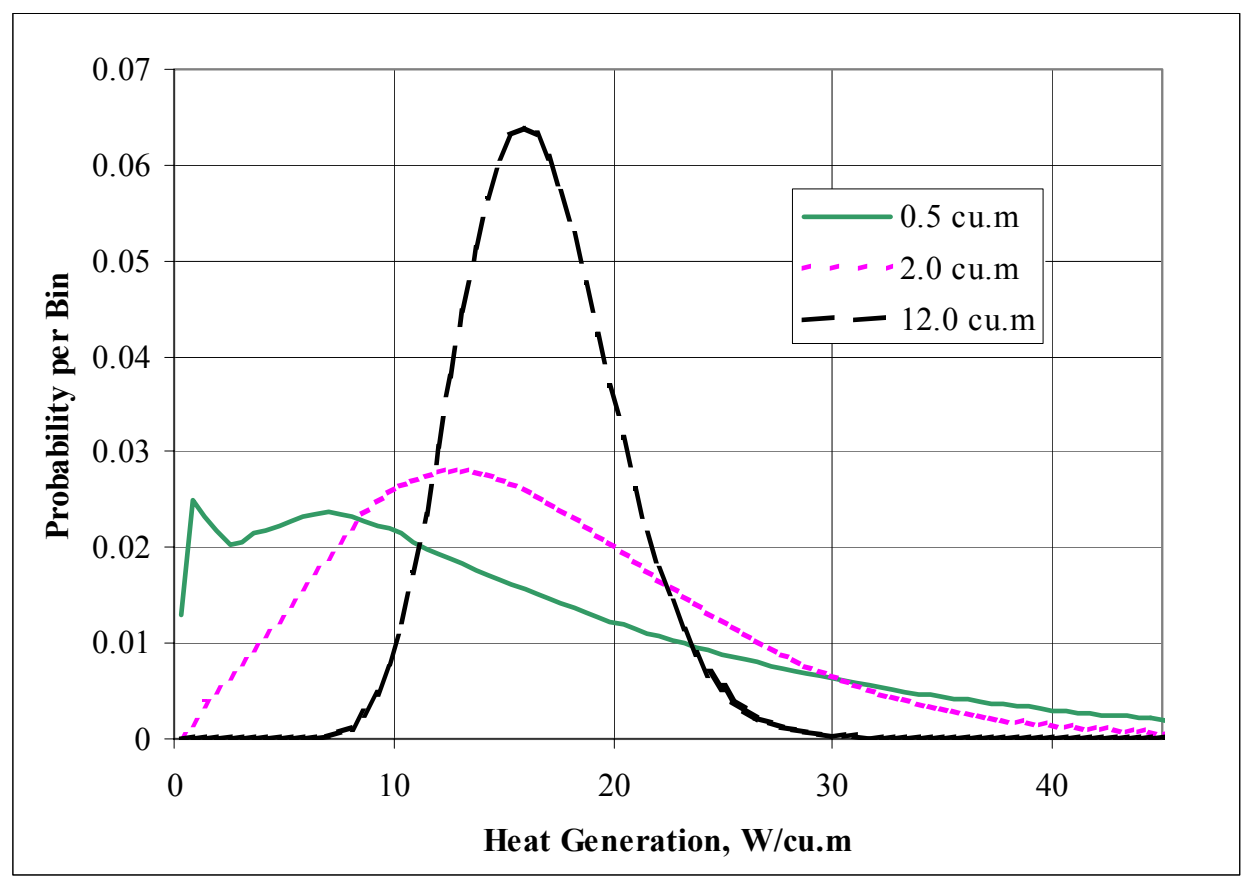

Table 5-16. Stochastic Results for Heat Generation Rate per Unit Volume $\left(\mathrm{W} / \mathrm{m}^{3}\right)$.

\begin{tabular}{|c|c|c|c|}
\hline Container Volume & $0.5 \mathrm{~m}^{3}$ & $2 \mathrm{~m}^{3}$ & $12 \mathrm{~m}^{3}$ \\
\hline Total Count & $287,461,350$ & $69,009,050$ & $10,000,000$ \\
\hline Average Values & $1.66 \mathrm{E}+01$ & $1.66 \mathrm{E}+01$ & $1.67 \mathrm{E}+01$ \\
\hline 95th Percentile & $4.32 \mathrm{E}+01$ & $3.22 \mathrm{E}+01$ & $2.27 \mathrm{E}+01$ \\
\hline 99th Percentile & $6.20 \mathrm{E}+01$ & $4.12 \mathrm{E}+01$ & $2.57 \mathrm{E}+01$ \\
\hline Standard Deviation & $1.36 \mathrm{E}+01$ & $8.48 \mathrm{E}+00$ & $3.46 \mathrm{E}+00$ \\
\hline Minimum Value & $5.67 \mathrm{E}-02$ & $1.07 \mathrm{E}-01$ & $4.06 \mathrm{E}+00$ \\
\hline Maximum Value & $2.39 \mathrm{E}+02$ & $1.06 \mathrm{E}+02$ & $3.76 \mathrm{E}+01$ \\
\hline Geometric Mean & $1.11 \mathrm{E}+01$ & $1.42 \mathrm{E}+01$ & $1.63 \mathrm{E}+01$ \\
\hline Geometric Std Dev & $3.03 \mathrm{E}+00$ & $1.85 \mathrm{E}+00$ & $1.24 \mathrm{E}+00$ \\
\hline
\end{tabular}


SNF-10293, REV 0

Figure 5-17. Stochastic Result for Heat Generation per Unit Mass of Sludge.

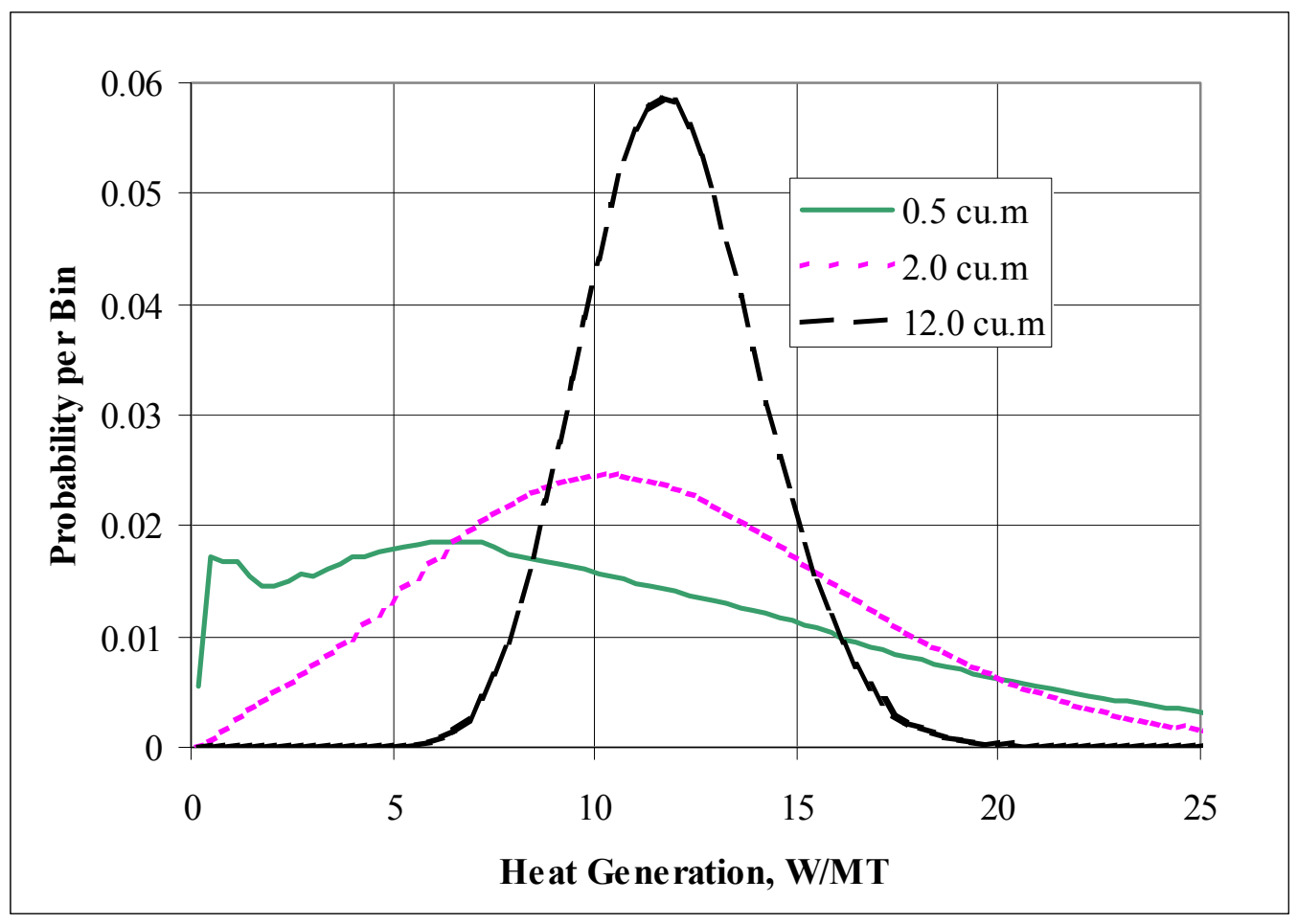

Table 5-17. Stochastic Results for Heat Generation per Unit Mass of Sludge (W/MT).

\begin{tabular}{|c|c|c|c|}
\hline Container Volume & $0.5 \mathrm{~m}^{3}$ & $2 \mathrm{~m}^{3}$ & $12 \mathrm{~m}^{3}$ \\
\hline Total Count & $287,461,350$ & $69,009,050$ & $10,000,000$ \\
\hline Average Values & $1.13 \mathrm{E}+01$ & $1.16 \mathrm{E}+01$ & $1.19 \mathrm{E}+01$ \\
\hline 95th Percentile & $2.65 \mathrm{E}+01$ & $2.09 \mathrm{E}+01$ & $1.56 \mathrm{E}+01$ \\
\hline 99th Percentile & $3.59 \mathrm{E}+01$ & $2.58 \mathrm{E}+01$ & $1.73 \mathrm{E}+01$ \\
\hline Standard Deviation & $7.98 \mathrm{E}+00$ & $5.22 \mathrm{E}+00$ & $2.14 \mathrm{E}+00$ \\
\hline Minimum Value & $5.60 \mathrm{E}-02$ & $9.64 \mathrm{E}-02$ & $3.30 \mathrm{E}+00$ \\
\hline Maximum Value & $8.45 \mathrm{E}+01$ & $5.51 \mathrm{E}+01$ & $2.39 \mathrm{E}+01$ \\
\hline Geometric Mean & $8.11 \mathrm{E}+00$ & $1.03 \mathrm{E}+01$ & $1.18 \mathrm{E}+01$ \\
\hline Geometric Std Dev & $2.72 \mathrm{E}+00$ & $1.73 \mathrm{E}+00$ & $1.20 \mathrm{E}+00$ \\
\hline
\end{tabular}


Figure 5-18. Stochastic Result for Heat Generation per Unit Mass of Uranium.

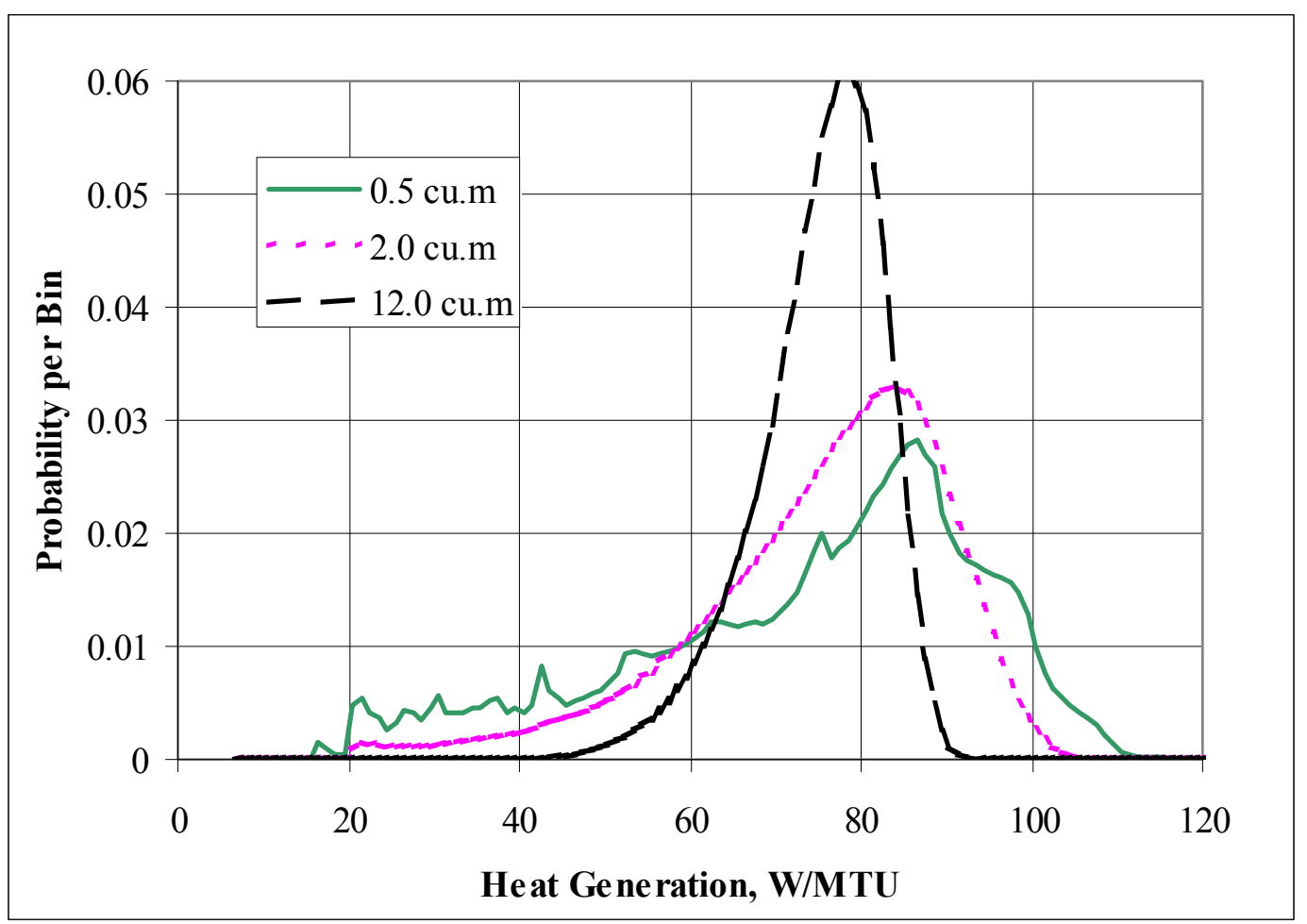

Table 5-18. Stochastic Results for Heat Generation per Unit Mass of Uranium (W/MTU).

\begin{tabular}{|c|c|c|c|}
\hline Container Volume & $0.5 \mathrm{~m}^{3}$ & $2 \mathrm{~m}^{3}$ & $12 \mathrm{~m}^{3}$ \\
\hline Total Count & $287,461,350$ & $69,009,050$ & $10,000,000$ \\
\hline Average Values & $7.41 \mathrm{E}+01$ & $7.54 \mathrm{E}+01$ & $7.51 \mathrm{E}+01$ \\
\hline 95th Percentile & $1.00 \mathrm{E}+02$ & $9.44 \mathrm{E}+01$ & $8.51 \mathrm{E}+01$ \\
\hline 99th Percentile & $1.07 \mathrm{E}+02$ & $9.91 \mathrm{E}+01$ & $8.79 \mathrm{E}+01$ \\
\hline Standard Deviation & $2.24 \mathrm{E}+01$ & $1.48 \mathrm{E}+01$ & $7.34 \mathrm{E}+00$ \\
\hline Minimum Value & $5.60 \mathrm{E}+00$ & $8.98 \mathrm{E}+00$ & $3.51 \mathrm{E}+01$ \\
\hline Maximum Value & $4.85 \mathrm{E}+02$ & $4.48 \mathrm{E}+02$ & $9.72 \mathrm{E}+01$ \\
\hline Geometric Mean & $7.01 \mathrm{E}+01$ & $7.36 \mathrm{E}+01$ & $7.47 \mathrm{E}+01$ \\
\hline Geometric Std Dev & $1.44 \mathrm{E}+00$ & $1.27 \mathrm{E}+00$ & $1.11 \mathrm{E}+00$ \\
\hline
\end{tabular}


SNF-10293, REV 0

\subsection{INHALATION DOSE FACTOR}

The inhalation dose factor is tallied two ways during the simulation. The first is the inhalation dose per unit volume of sludge. The second is the inhalation dose per unit mass of sludge. The second tally is necessary because the mass of sludge in a container is another distribution. The distribution of dose factors is shown in Figure 5-19 and Figure 5-20. The corresponding numeric results from the stochastic analysis are summarized in Table 5-19 and Table 5-20. The bin width in Figure 5-19 is 1,700 rem/ml while the bin width in Figure 5-20 is $1,000 \mathrm{rem} / \mathrm{g}$.

Figure 5-19. Stochastic Result for Inhalation Dose per Unit Volume.

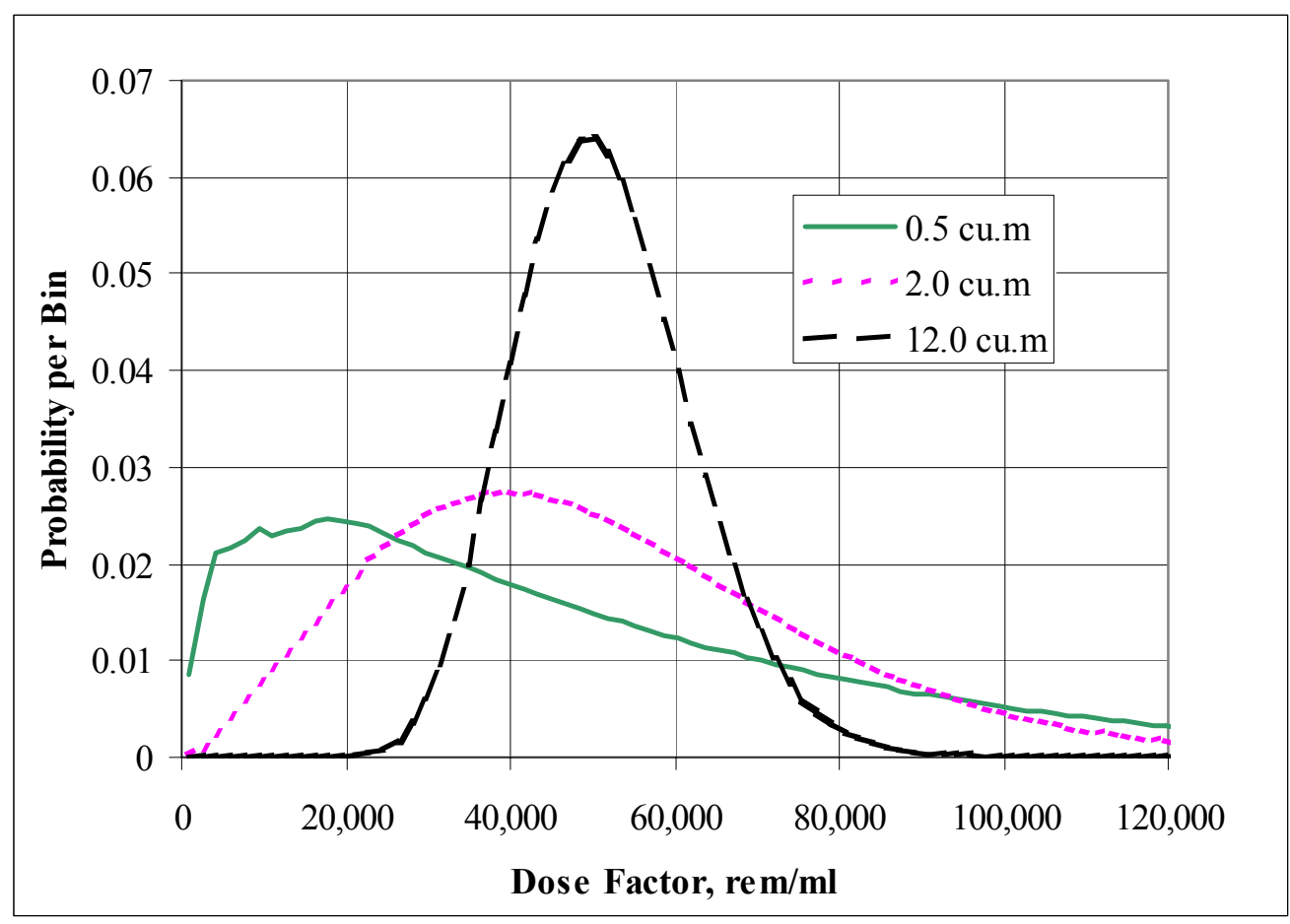

Table 5-19. Stochastic Results for Inhalation Dose per Unit Volume (rem/ml).

\begin{tabular}{|c|c|c|c|}
\hline Container Volume & $0.5 \mathrm{~m}^{3}$ & $2 \mathrm{~m}^{3}$ & $12 \mathrm{~m}^{3}$ \\
\hline Total Count & $287,461,350$ & $69,009,050$ & $10,000,000$ \\
\hline Average Values & $5.13 \mathrm{E}+04$ & $5.12 \mathrm{E}+04$ & $5.15 \mathrm{E}+04$ \\
\hline 95th Percentile & $1.34 \mathrm{E}+05$ & $9.95 \mathrm{E}+04$ & $6.98 \mathrm{E}+04$ \\
\hline 99th Percentile & $1.94 \mathrm{E}+05$ & $1.27 \mathrm{E}+05$ & $7.87 \mathrm{E}+04$ \\
\hline Standard Deviation & $4.23 \mathrm{E}+04$ & $2.62 \mathrm{E}+04$ & $1.05 \mathrm{E}+04$ \\
\hline Minimum Value & $5.85 \mathrm{E}+01$ & $1.31 \mathrm{E}+02$ & $1.26 \mathrm{E}+04$ \\
\hline Maximum Value & $6.20 \mathrm{E}+05$ & $2.99 \mathrm{E}+05$ & $1.16 \mathrm{E}+05$ \\
\hline Geometric Mean & $3.50 \mathrm{E}+04$ & $4.41 \mathrm{E}+04$ & $5.04 \mathrm{E}+04$ \\
\hline Geometric Std Dev & $2.89 \mathrm{E}+00$ & $1.84 \mathrm{E}+00$ & $1.23 \mathrm{E}+00$ \\
\hline
\end{tabular}


Figure 5-20. Stochastic Result for Inhalation Dose per Unit Mass of Sludge.

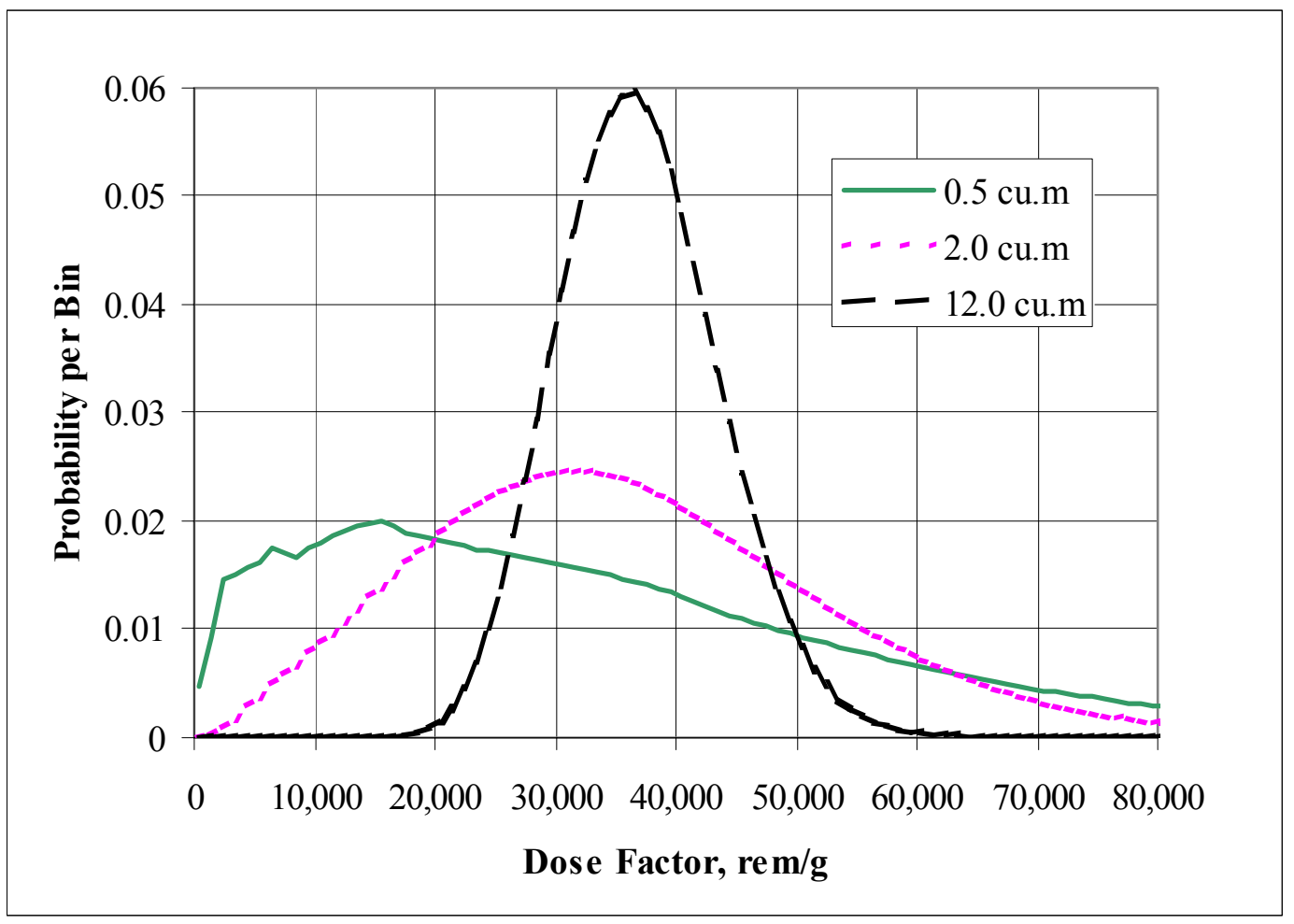

Table 5-20. Stochastic Results for Inhalation Dose per Unit Mass of Sludge $(\mathrm{rem} / \mathrm{g})$.

\begin{tabular}{|c|c|c|c|}
\hline Container Volume & $0.5 \mathrm{~m}^{3}$ & $2 \mathrm{~m}^{3}$ & $12 \mathrm{~m}^{3}$ \\
\hline Total Count & $287,461,350$ & $69,009,050$ & $10,000,000$ \\
\hline Average Values & $3.50 \mathrm{E}+04$ & $3.61 \mathrm{E}+04$ & $3.70 \mathrm{E}+04$ \\
\hline 95th Percentile & $8.36 \mathrm{E}+04$ & $6.55 \mathrm{E}+04$ & $4.83 \mathrm{E}+04$ \\
\hline 99th Percentile & $1.14 \mathrm{E}+05$ & $8.07 \mathrm{E}+04$ & $5.35 \mathrm{E}+04$ \\
\hline Standard Deviation & $2.52 \mathrm{E}+04$ & $1.64 \mathrm{E}+04$ & $6.58 \mathrm{E}+03$ \\
\hline Minimum Value & $5.60 \mathrm{E}+01$ & $1.17 \mathrm{E}+02$ & $1.02 \mathrm{E}+04$ \\
\hline Maximum Value & $2.49 \mathrm{E}+05$ & $1.57 \mathrm{E}+05$ & $7.53 \mathrm{E}+04$ \\
\hline Geometric Mean & $2.55 \mathrm{E}+04$ & $3.20 \mathrm{E}+04$ & $3.64 \mathrm{E}+04$ \\
\hline Geometric Std Dev & $2.61 \mathrm{E}+00$ & $1.73 \mathrm{E}+00$ & $1.20 \mathrm{E}+00$ \\
\hline
\end{tabular}

\subsection{URANIUM CONCENTRATION}

The total uranium concentration of the sludge in a container is tallied during the simulation. The distribution of densities is shown in Figure 5-21. The corresponding numeric results from the stochastic analysis are summarized in Table 5-21. The bin width in Figure $5-21$ is $0.0075 \mathrm{~kg} / \mathrm{L}$. 
Figure 5-21. Stochastic Result for Total Uranium Concentration.

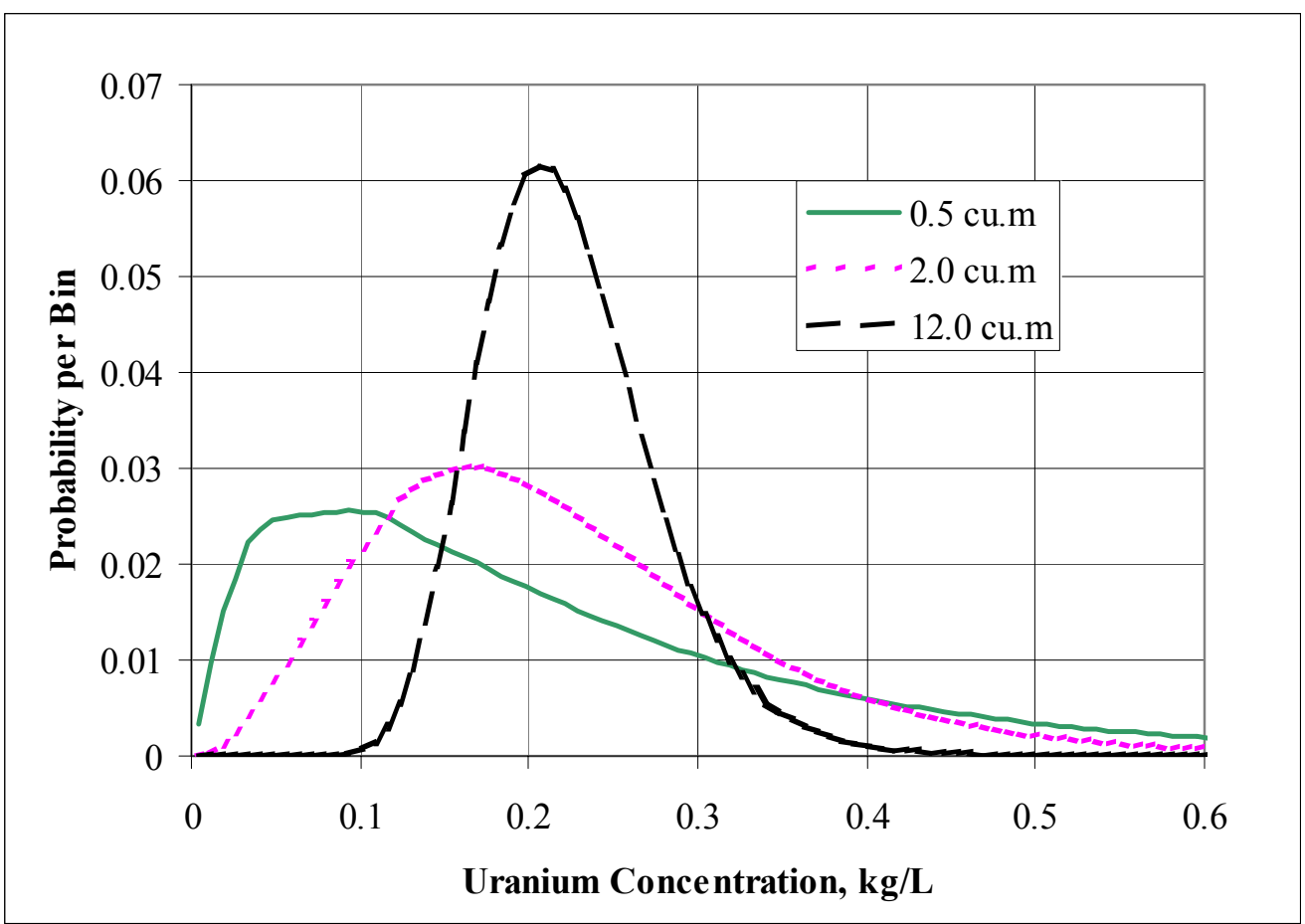

Table 5-21. Stochastic Results for Total Uranium Concentration $(\mathrm{kg} / \mathrm{L})$.

\begin{tabular}{|c|c|c|c|}
\hline Container Volume & $0.5 \mathrm{~m}^{3}$ & $2 \mathrm{~m}^{3}$ & $12 \mathrm{~m}^{3}$ \\
\hline Total Count & $287,461,350$ & $69,009,050$ & $10,000,000$ \\
\hline Average Values & $2.23 \mathrm{E}-01$ & $2.23 \mathrm{E}-01$ & $2.24 \mathrm{E}-01$ \\
\hline 95th Percentile & $5.81 \mathrm{E}-01$ & $4.39 \mathrm{E}-01$ & $3.17 \mathrm{E}-01$ \\
\hline 99th Percentile & $8.64 \mathrm{E}-01$ & $5.94 \mathrm{E}-01$ & $3.70 \mathrm{E}-01$ \\
\hline Standard Deviation & $1.81 \mathrm{E}-01$ & $1.17 \mathrm{E}-01$ & $5.13 \mathrm{E}-02$ \\
\hline Minimum Value & $3.78 \mathrm{E}-04$ & $1.37 \mathrm{E}-03$ & $6.28 \mathrm{E}-02$ \\
\hline Maximum Value & $2.38 \mathrm{E}+00$ & $1.75 \mathrm{E}+00$ & $6.50 \mathrm{E}-01$ \\
\hline Geometric Mean & $1.59 \mathrm{E}-01$ & $1.93 \mathrm{E}-01$ & $2.18 \mathrm{E}-01$ \\
\hline Geometric Std Dev & $2.61 \mathrm{E}+00$ & $1.77 \mathrm{E}+00$ & $1.26 \mathrm{E}+00$ \\
\hline
\end{tabular}

The uranium metal concentration is also tallied. This is the uranium metal mass divided by the sludge volume. The distribution of concentrations is shown in Figure 5-22. The corresponding numeric results from the stochastic analysis are listed in Table 5-22. The bin width in Figure $5-22$ is $0.00038 \mathrm{~kg} / \mathrm{L}$. 
Figure 5-22. Stochastic Result for Uranium Metal Concentration.

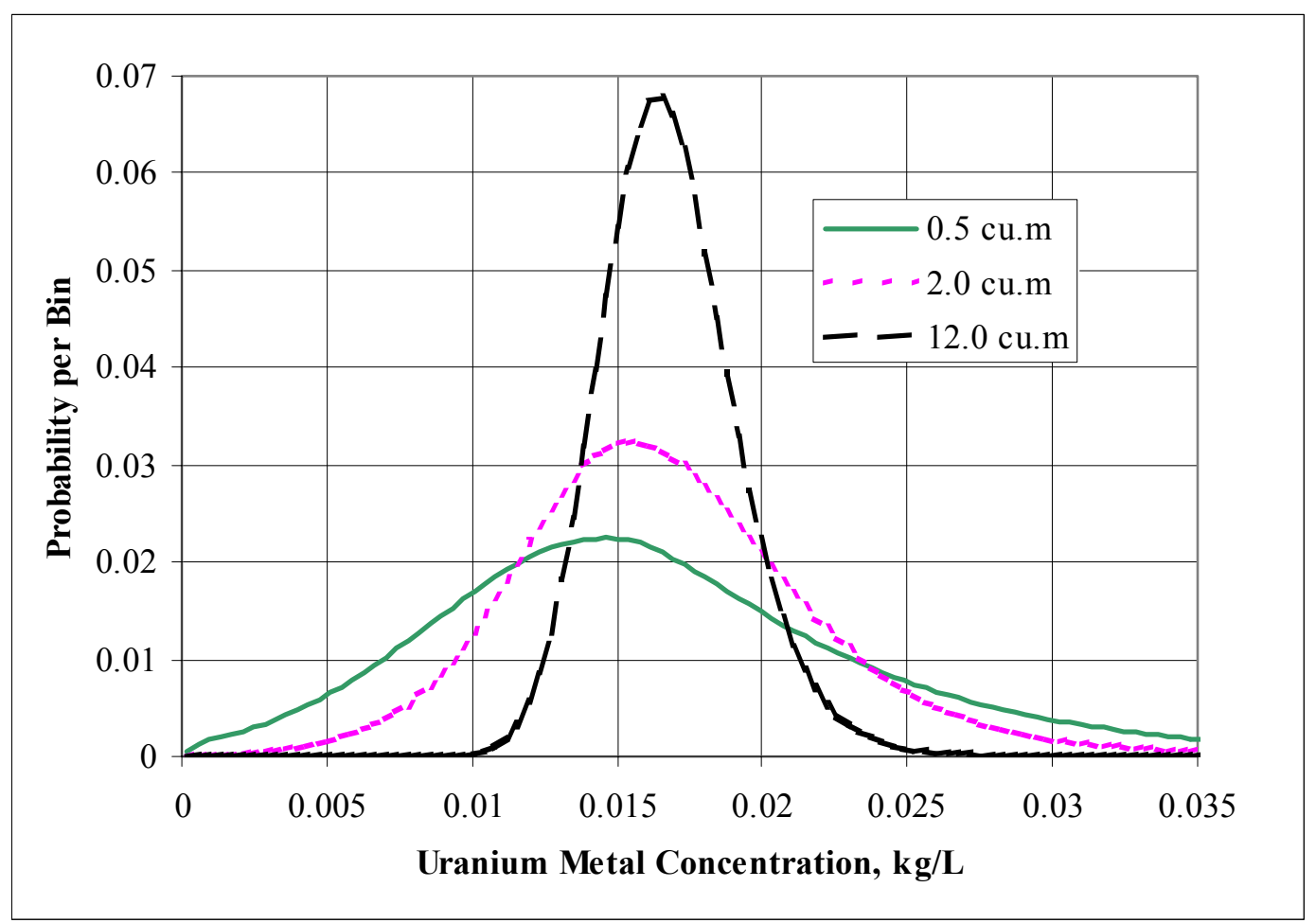

Table 5-22. Stochastic Results for Uranium Metal Concentration.

\begin{tabular}{|c|c|c|c|}
\hline Container Volume & $0.5 \mathrm{~m}^{3}$ & $2 \mathrm{~m}^{3}$ & $12 \mathrm{~m}^{3}$ \\
\hline Total Count & $287,461,350$ & $69,009,050$ & $10,000,000$ \\
\hline Average Values & $1.68 \mathrm{E}-02$ & $1.68 \mathrm{E}-02$ & $1.68 \mathrm{E}-02$ \\
\hline 95th Percentile & $3.20 \mathrm{E}-02$ & $2.60 \mathrm{E}-02$ & $2.09 \mathrm{E}-02$ \\
\hline 99th Percentile & $4.26 \mathrm{E}-02$ & $3.21 \mathrm{E}-02$ & $2.31 \mathrm{E}-02$ \\
\hline Standard Deviation & $8.22 \mathrm{E}-03$ & $5.30 \mathrm{E}-03$ & $2.33 \mathrm{E}-03$ \\
\hline Minimum Value & $1.17 \mathrm{E}-05$ & $1.39 \mathrm{E}-04$ & $7.29 \mathrm{E}-03$ \\
\hline Maximum Value & $1.21 \mathrm{E}-01$ & $7.58 \mathrm{E}-02$ & $3.41 \mathrm{E}-02$ \\
\hline Geometric Mean & $1.47 \mathrm{E}-02$ & $1.59 \mathrm{E}-02$ & $1.67 \mathrm{E}-02$ \\
\hline Geometric Std Dev & $1.79 \mathrm{E}+00$ & $1.40 \mathrm{E}+00$ & $1.15 \mathrm{E}+00$ \\
\hline
\end{tabular}


SNF-10293, REV 0

\subsection{PLUTONIUM WEIGHT PERCENT}

The plutonium weight percent in the sludge is tallied during the simulation. This is the total mass of plutonium in a container divided by the mass of sludge. The distribution is shown in Figure 5-23. The corresponding numeric results from the stochastic analysis are summarized in Table 5-23. Note that in the table, the numbers are shown as fractions rather than percents. The bin width in Figure 5-23 is $0.001 \%$.

Figure 5-23. Stochastic Result for Plutonium Weight Percent.

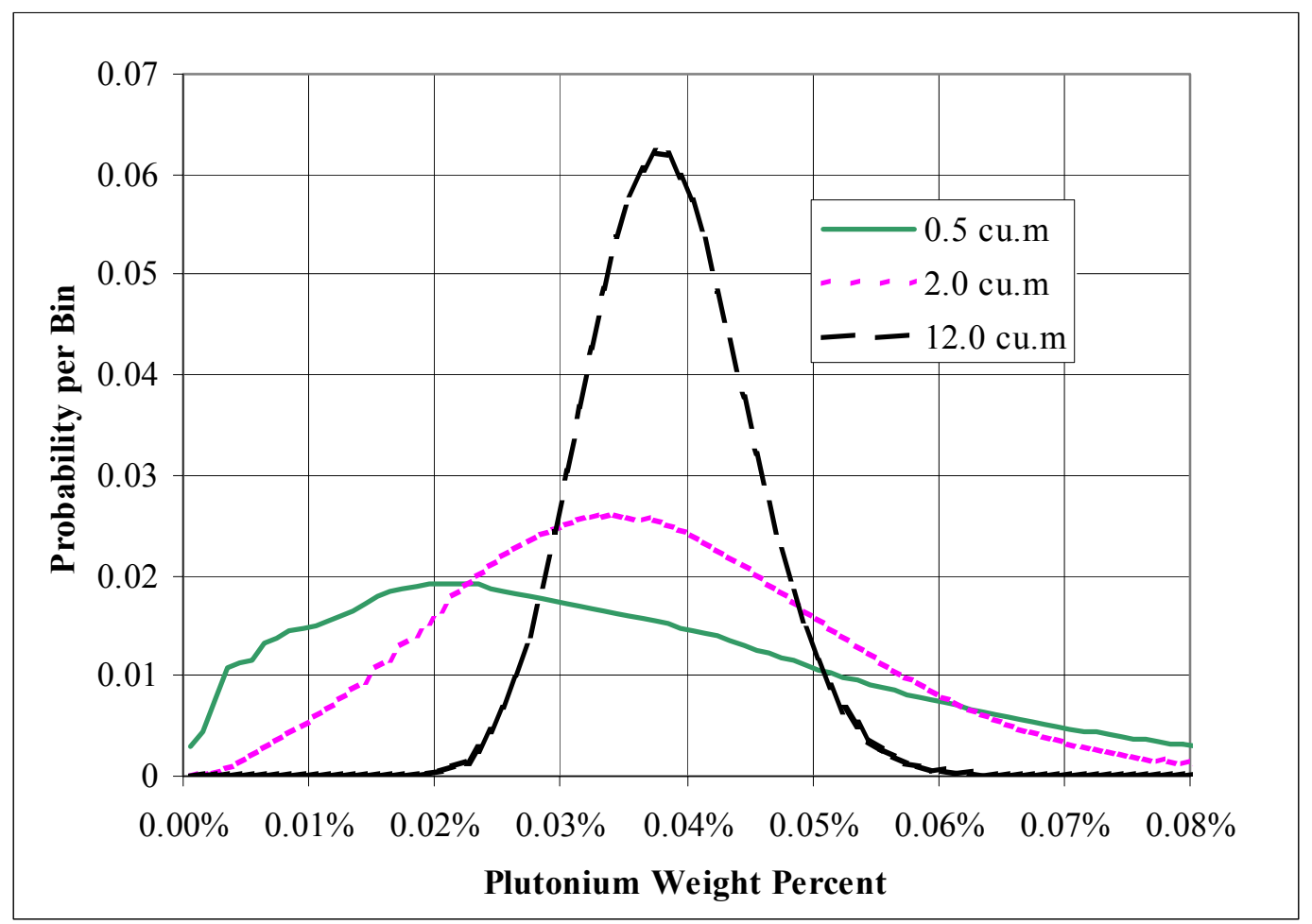

Table 5-23. Stochastic Results for Plutonium Weight Fraction.

\begin{tabular}{|c|c|c|c|}
\hline Container Volume & $0.5 \mathrm{~m}^{3}$ & $2 \mathrm{~m}^{3}$ & $12 \mathrm{~m}^{3}$ \\
\hline Total Count & $287,461,350$ & $69,009,050$ & $10,000,000$ \\
\hline Average Values & $3.66 \mathrm{E}-04$ & $3.77 \mathrm{E}-04$ & $3.86 \mathrm{E}-04$ \\
\hline 95th Percentile & $8.23 \mathrm{E}-04$ & $6.56 \mathrm{E}-04$ & $4.96 \mathrm{E}-04$ \\
\hline 99th Percentile & $1.10 \mathrm{E}-03$ & $7.99 \mathrm{E}-04$ & $5.47 \mathrm{E}-04$ \\
\hline Standard Deviation & $2.40 \mathrm{E}-04$ & $1.57 \mathrm{E}-04$ & $6.42 \mathrm{E}-05$ \\
\hline Minimum Value & $7.36 \mathrm{E}-07$ & $1.79 \mathrm{E}-06$ & $1.16 \mathrm{E}-04$ \\
\hline Maximum Value & $2.29 \mathrm{E}-03$ & $1.58 \mathrm{E}-03$ & $7.58 \mathrm{E}-04$ \\
\hline Geometric Mean & $2.82 \mathrm{E}-04$ & $3.41 \mathrm{E}-04$ & $3.81 \mathrm{E}-04$ \\
\hline Geometric Std Dev & $2.35 \mathrm{E}+00$ & $1.63 \mathrm{E}+00$ & $1.19 \mathrm{E}+00$ \\
\hline
\end{tabular}


SNF-10293, REV 0

\subsection{ALTERNATIVES TO THE BASE CASE}

Several additional cases were run to better understand the influence of some of the modeling choices. These cover both the selection of parameters and the shape of the PDF.

\subsubsection{Floor Sludge Volume}

Rather than represent the floor sludge volume as a uniform distribution that applies to all the keys at the same time, the floor sludge volume associated with each key (listed in Table 3-1) was allowed to vary independently. However, the variance was greater. The uniform distribution that applies to the entire basin (base case) has a range from $80 \%$ to $120 \%$ of the mean. As described in Section 3.1 the uniform distribution that was applied to the floor sludge volume for each key (this alternate case) ranges from $19 \%$ to $181 \%$ of the mean value. This range was set by an iterative process until the standard deviation on the total floor sludge volume was $10 \%$, or $1.17 \mathrm{~m}^{3}$.

Only two stochastic results changed significantly. The first is the standard deviation on the floor sludge volume associated with cubicles that have fuel. It decreased from $1.35 \mathrm{~m}^{3}$ to $1.17 \mathrm{~m}^{3}$. The second is the canister-to-total sludge volume ratio for the $0.5 \mathrm{~m}^{3}$ container, which increased from 0.371 to 0.410 . The results for mean and $95^{\text {th }}$ percentile changed no more than $5 \%$ for all container volumes. The graphs are essentially the same, except for the sludge volume tally. This is shown in Figure 5-24. Comparing this figure with Figure 4-11, the total volume of floor sludge in cubicles that have fuel has changed from a uniform distribution to one that resembles a normal distribution.

Figure 5-24. Stochastic Result for Total Sludge Volume with Independent Key Volumes.

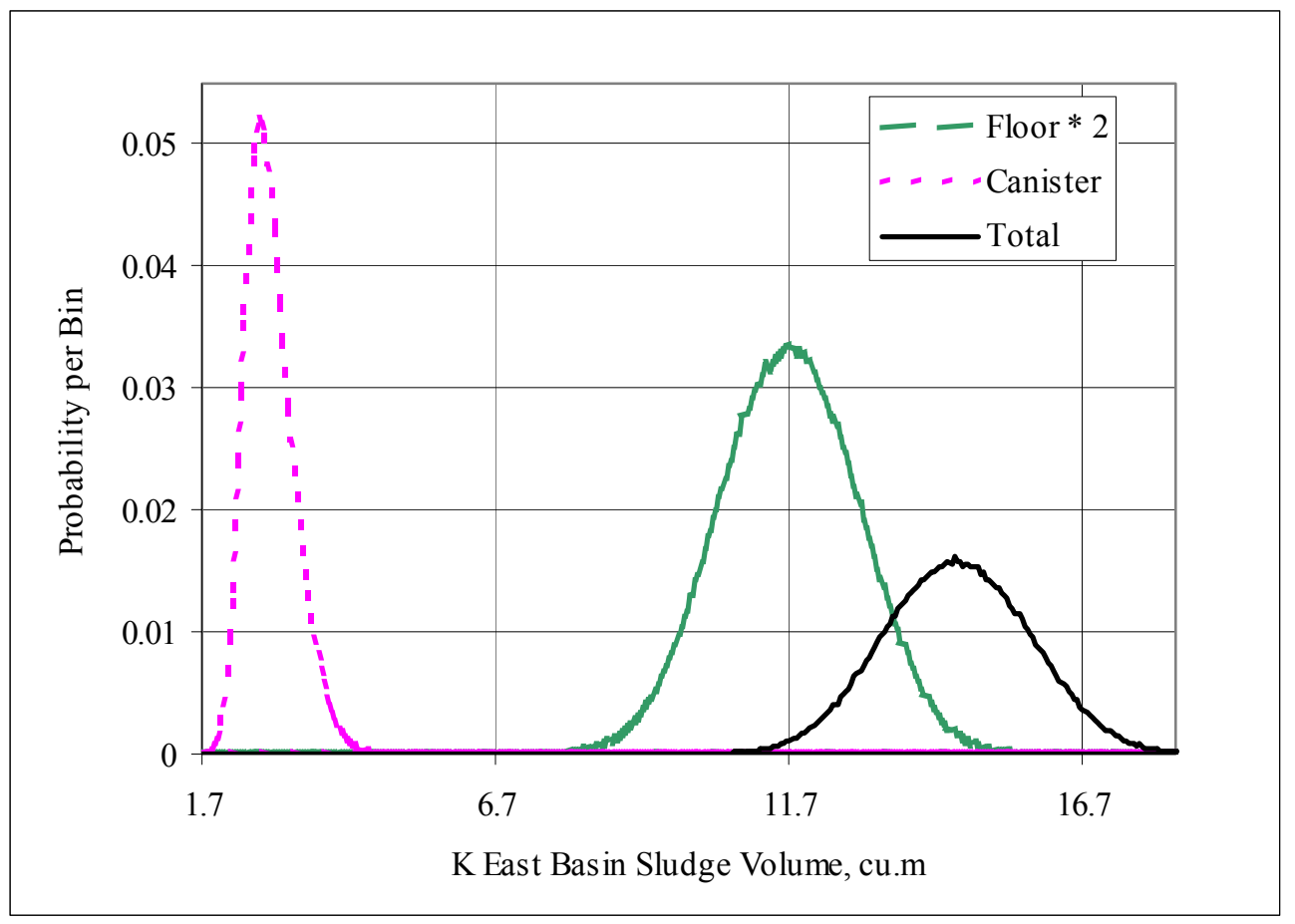


SNF-10293, REV 0

\subsubsection{Floor Sludge Isotopic Composition}

The four alternate cases described in Section 3.2.2 were run. The first reduces the variability factor to the range 0.9 to 1.1 . The second uses a piecewise continuous representation of the floor sludge compositions. The third uses a lognormal function to represent the floor sludge data. The fourth doubles the imputed values for floor sludge. Each of these alternate cases will be compared to the base case.

\subsubsection{Reduced Variability}

This case changes the variability factor for the floor sludge isotopic composition from the base case ( 0.2 to 1.8$)$ to 0.9 to 1.1 . An example CDF for the floor sludge density is shown in Figure 5-25. This can be compared with the base case CDF shown in Figure 4-8. The principal effect is to reduce the standard deviations of the floor sludge concentrations by as much as $18 \%$. However, floor sludge is a minor contributor to the container inventories.

Figure 5-25. Stochastic Result Using Reduced Variability for Floor Sludge Density.

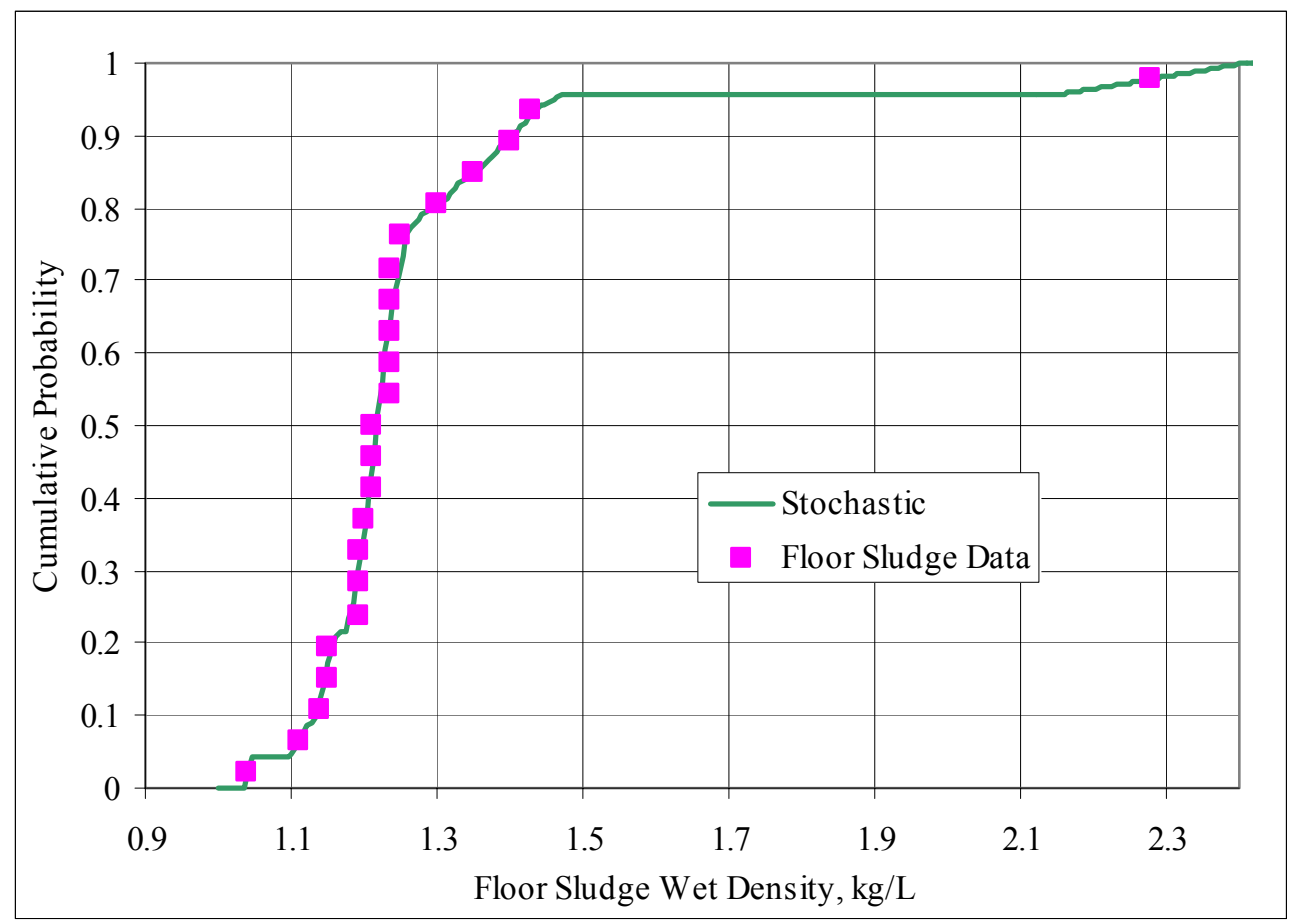

The container concentrations are largely the same as the base case. The largest decrease was for Co-60 because the average concentrations in floor sludge is within a factor of 2 of the average for canister sludge. The $95^{\text {th }}$ percentile Co- 60 concentration decreased by $9 \%, 7 \%$, and $4 \%$ in the $0.5 \mathrm{~m}^{3}, 2 \mathrm{~m}^{3}$, and $12 \mathrm{~m}^{3}$ containers, respectively. All other changes were less than $2 \%$.

\subsubsection{Piecewise Continuous Concentrations}

The floor sludge composition can be calculated from the 23 discrete values as a piecewise continuous cumulative distribution. A typical example of this CDF for floor sludge is shown in 
Figure 3-11. One random number is generated and used with the cumulative distributions for all 23 items of interest. This forces the distributions to be completely correlated. The mean values for all the floor sludge components remain the same, but the continuous distribution has a smaller standard deviation. Thus, it is expected that the stochastic results for the continuous distributions should have the same mean values but smaller $95^{\text {th }}$ and $99^{\text {th }}$ percentile values.

The results with the continuous distributions are largely the same as the reduced variability case. The floor sludge concentrations have the same mean values, and the standard deviations decreased as much as $20 \%$. The container mean values are the same. The largest decrease in the container concentrations is again for Co-60. The $95^{\text {th }}$ percentile Co-60 concentration decreased by $9 \%, 7 \%$, and $5 \%$ in the $0.5 \mathrm{~m}^{3}, 2 \mathrm{~m}^{3}$, and $12 \mathrm{~m}^{3}$ containers, respectively. All other changes were less than $3 \%$.

\subsubsection{Lognormal Cumulative Distributions}

As described in Section 3.2.2, the floor sludge concentrations were represented as cumulative lognormal functions using the geometric mean and geometric standard deviation of the measured and imputed values. Two stochastic CDFs are shown in Figures 5-26 and 5-27. Included in the graphs is a more exact lognormal CDF using the error function available in most spreadsheets. The points lie on the stochastically determined CDF. The good agreement between the stochastic tally and the theoretical function is partly due to the 52,000,000 values that are tallied. Figure 5-27 can be compared with Figure 4-8, which shows the floor sludge density for the base case. Note that the CDF for floor sludge density begins at $1.0 \mathrm{~kg} / \mathrm{L}$ rather than 0 .

Figure 5-26. Stochastic Result for Lognormal Floor Sludge Uranium Concentration.

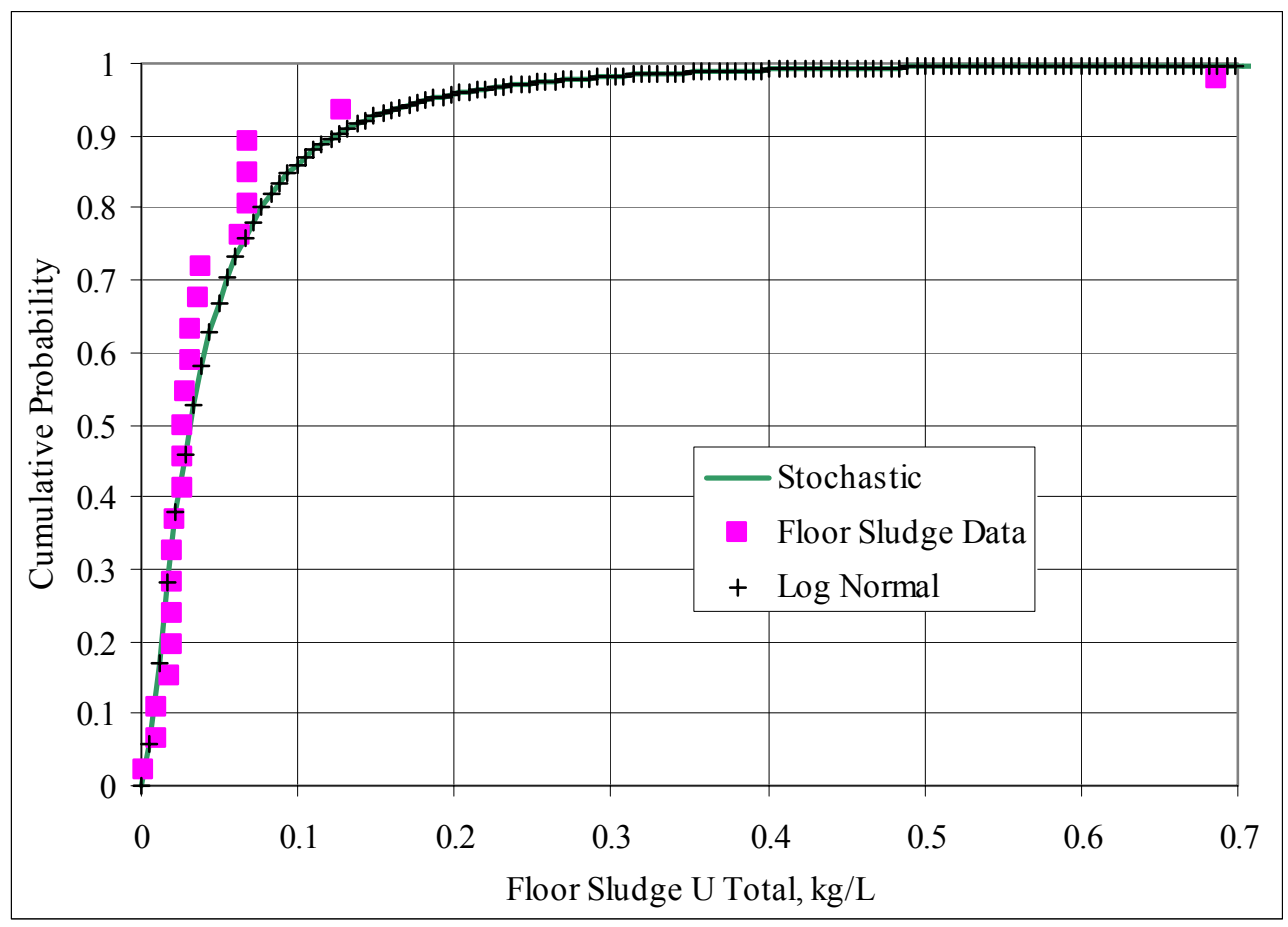


Figure 5-27. Stochastic Result for Lognormal Floor Sludge Density.

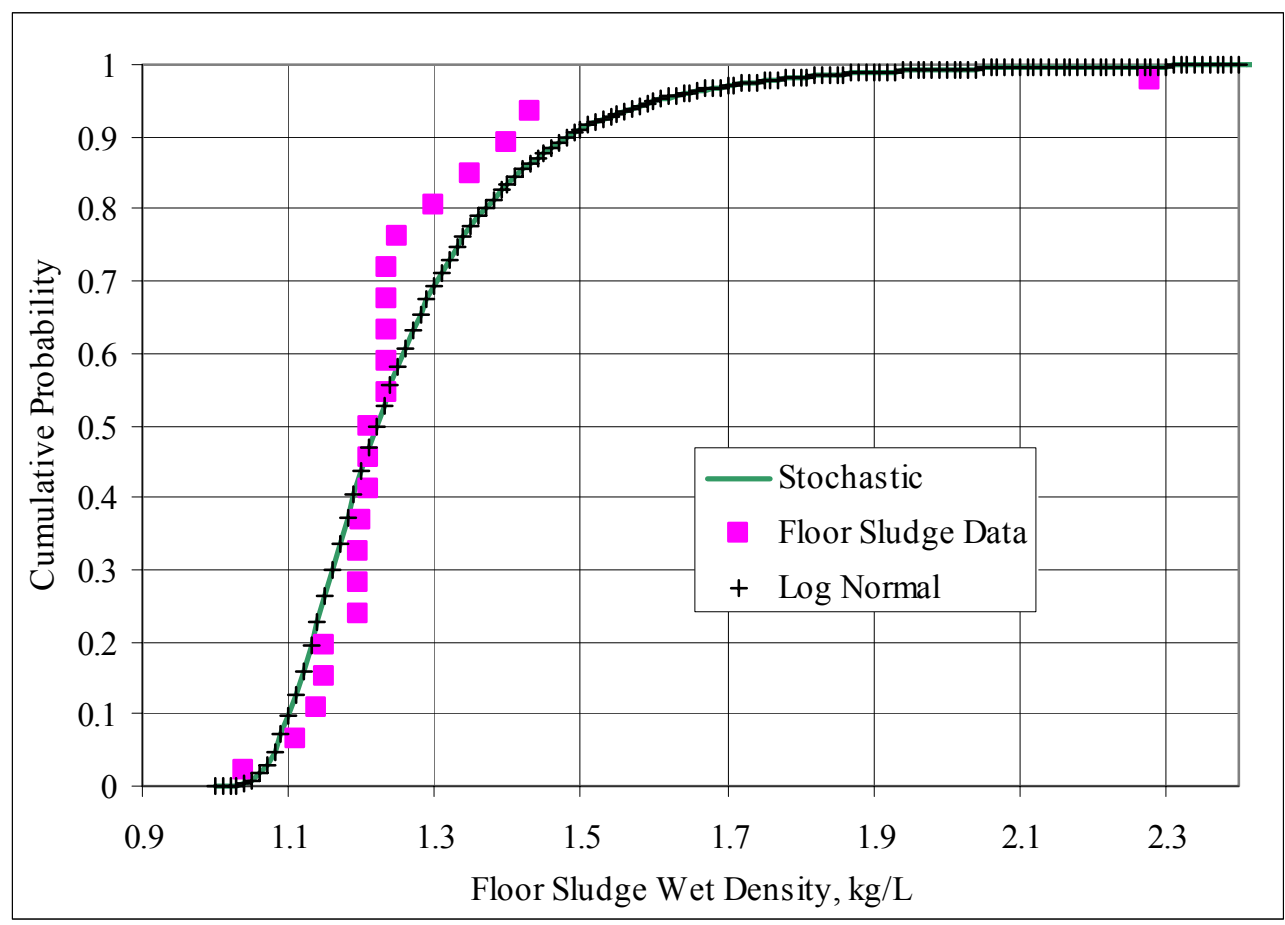

In this alternate case, the stochastic means for floor sludge concentrations differ from the data, with some larger and some smaller. The largest change was for the uranium concentration, which decreased $13 \%$. The standard deviations of the floor sludge concentrations all decreased. The largest decrease was for the uranium concentration, which decreased $48 \%$ (to about half the base case).

The container concentrations are largely unchanged, with most differences less than $3 \%$. Two concentrations that saw the greatest change were Co-60 and uranium. The $95^{\text {th }}$ percentile Co-60 concentration decreased by $11 \%, 6 \%$, and $2 \%$ in the $0.5 \mathrm{~m}^{3}, 2 \mathrm{~m}^{3}$, and $12 \mathrm{~m}^{3}$ containers, respectively. The $95^{\text {th }}$ percentile uranium concentration decreased by $9 \%, 8 \%$, and $6 \%$ in the $0.5 \mathrm{~m}^{3}, 2 \mathrm{~m}^{3}$, and $12 \mathrm{~m}^{3}$ containers, respectively.

\subsubsection{Double the Imputed Values}

This case uses the asterisks in the plots of data and imputed values for floor sludge from Section 3.2. The percent differences between the base case and doubled results for the container concentrations are shown in Table 5-24 for the $2 \mathrm{~m}^{3}$ container volumes. The other containers showed nearly the same differences. Items that changed less than $1 \%$ are not shown. The largest increases occur for the isotopes with many imputed values. 
SNF-10293, REV 0

Table 5-24. Percent Differences: Doubled Imputed Values Versus Base Case.

\begin{tabular}{|c|c|c|c|c|}
\hline & Mean & $\mathbf{9 5}^{\text {th }}$ Percent & 99 $^{\text {th }}$ Percent & Standard Deviation \\
\hline Sr-90 & $1.0 \%$ & LT & LT & LT \\
\hline Tc-99 & $18.1 \%$ & $14.6 \%$ & $15.3 \%$ & $10.7 \%$ \\
\hline Cs-134 & $6.6 \%$ & $2.5 \%$ & LT & LT \\
\hline Eu-152 & $10.0 \%$ & $7.4 \%$ & $6.2 \%$ & $4.8 \%$ \\
\hline Eu-155 & $4.9 \%$ & $2.4 \%$ & $1.3 \%$ & LT \\
\hline Np-237 & $13.5 \%$ & $12.1 \%$ & $13.0 \%$ & $10.0 \%$ \\
\hline Pu-238 & $4.5 \%$ & $4.2 \%$ & $3.5 \%$ & $3.9 \%$ \\
\hline Pu-241 & $11.8 \%$ & $7.9 \%$ & $6.2 \%$ & $4.6 \%$ \\
\hline Dose per $\mathrm{ml}$ & $1.8 \%$ & $1.4 \%$ & $1.1 \%$ & LT \\
\hline Dose per g & $1.7 \%$ & LT & LT & LT \\
\hline
\end{tabular}

- Percent differences that are positive indicate that the alternate case gave larger results than the base case. Items not shown had no difference greater than $1 \%$.

- $\quad \mathrm{LT}=$ difference is less than $1 \%$

- Above differences are for the $2 \mathrm{~m}^{3}$ volume. The other volumes showed nearly the same differences.

\subsubsection{Canister Sludge Depth}

Four alternate cases were run. The first reduces the variability factor to the range 0.9 to 1.1 . The second uses a piecewise continuous representation of the floor sludge compositions. The last two examine the influence of maximum and minimum sludge depths in the two canister barrels with off scale readings.

\subsubsection{Reduced Variability}

This case changes the variability factor for the canister sludge depth from the base case $(0.5$ to 1.5 ) to 0.9 to 1.1 . The principal effect is to reduce the standard deviations of the canister sludge depths by as much as $14 \%$. The container concentrations are largely the same as the base case. The largest decrease was for the canister-to-total sludge volume ratio. This ratio decreased 3.3\% and $2.5 \%$ in the $0.5 \mathrm{~m}^{3}$ and $2 \mathrm{~m}^{3}$ containers at the $95^{\text {th }}$ percentile. All other changes to the $95^{\text {th }}$ percentile results were less than $2 \%$.

\subsubsection{Piecewise Continuous Depth Distributions}

The canister sludge depths can be calculated from the discrete values for good, fair, and poor/bad fuel as continuous cumulative distributions. These distributions are shown in Figure 3-12 and Figure 3-13. The mean values are the same, but the distributions differ in their standard deviations Thus, it is expected that the stochastic results for the sludge containers should have the same mean values but smaller $95^{\text {th }}$ and $99^{\text {th }}$ percentile values. 
The results with the piecewise continuous distributions are largely the same as the base case. The canister sludge depths have the same mean values, and the standard deviations decreased as much as $15 \%$. The container mean values are the same. The container $95^{\text {th }}$ percentile values decreased at most 5\%. The stochastic results for the canister sludge depth distribution are shown in Figures 5-28, 5-29, and 5-30. The data points are also shown for reference, as they were in the base case depth distributions, Figure 4-8, 4-9, and 4-10. Compared with the base case, the piecewise continuous distributions for sludge depth follow the data points closer. The stochastic results for the base case shown in Figure 4-8 and Figure 4-10 are smoother, and extend to larger values.

Figure 5-28. Stochastic Result for Continuous Canister Sludge Depth (Good Fuel).

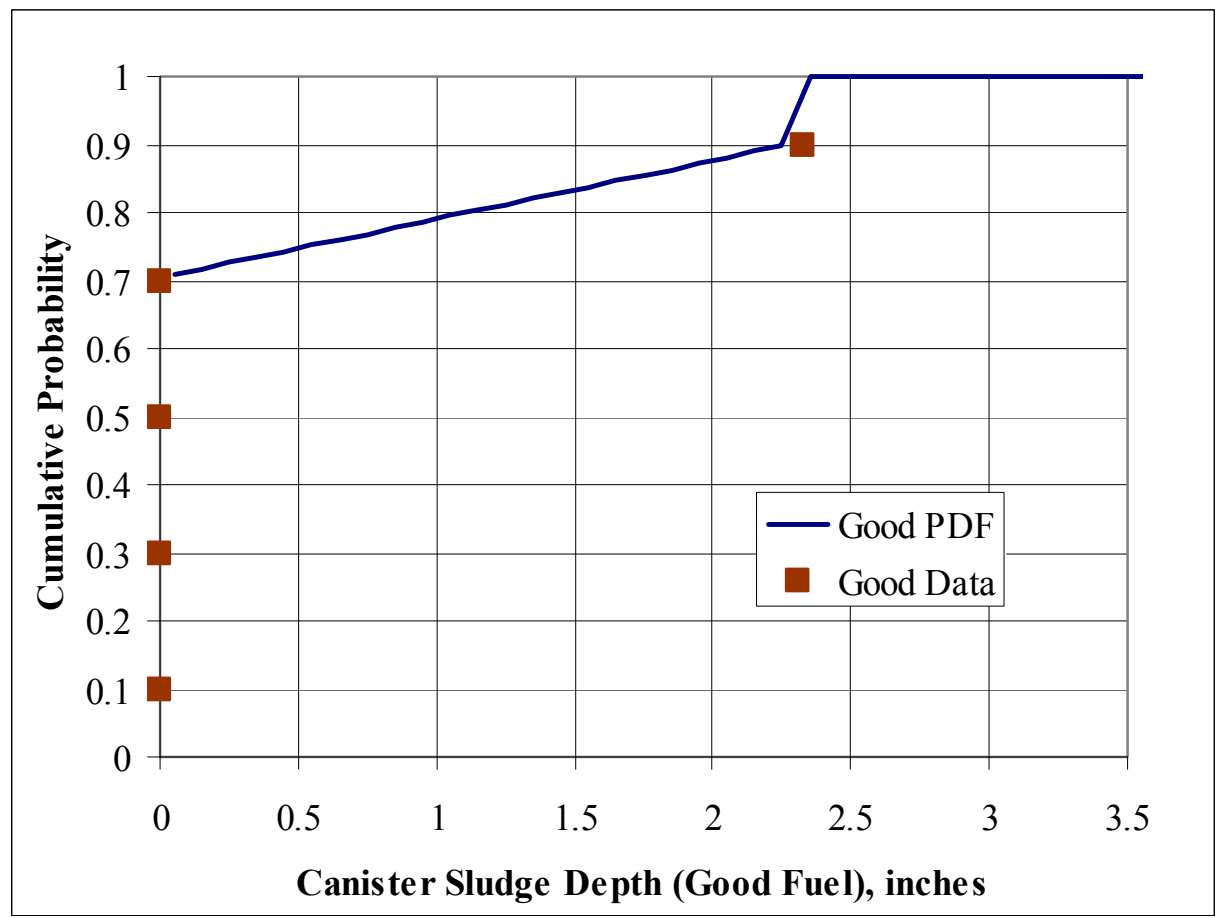


Figure 5-29. Stochastic Result for Continuous Canister Sludge Depth (Fair Fuel).

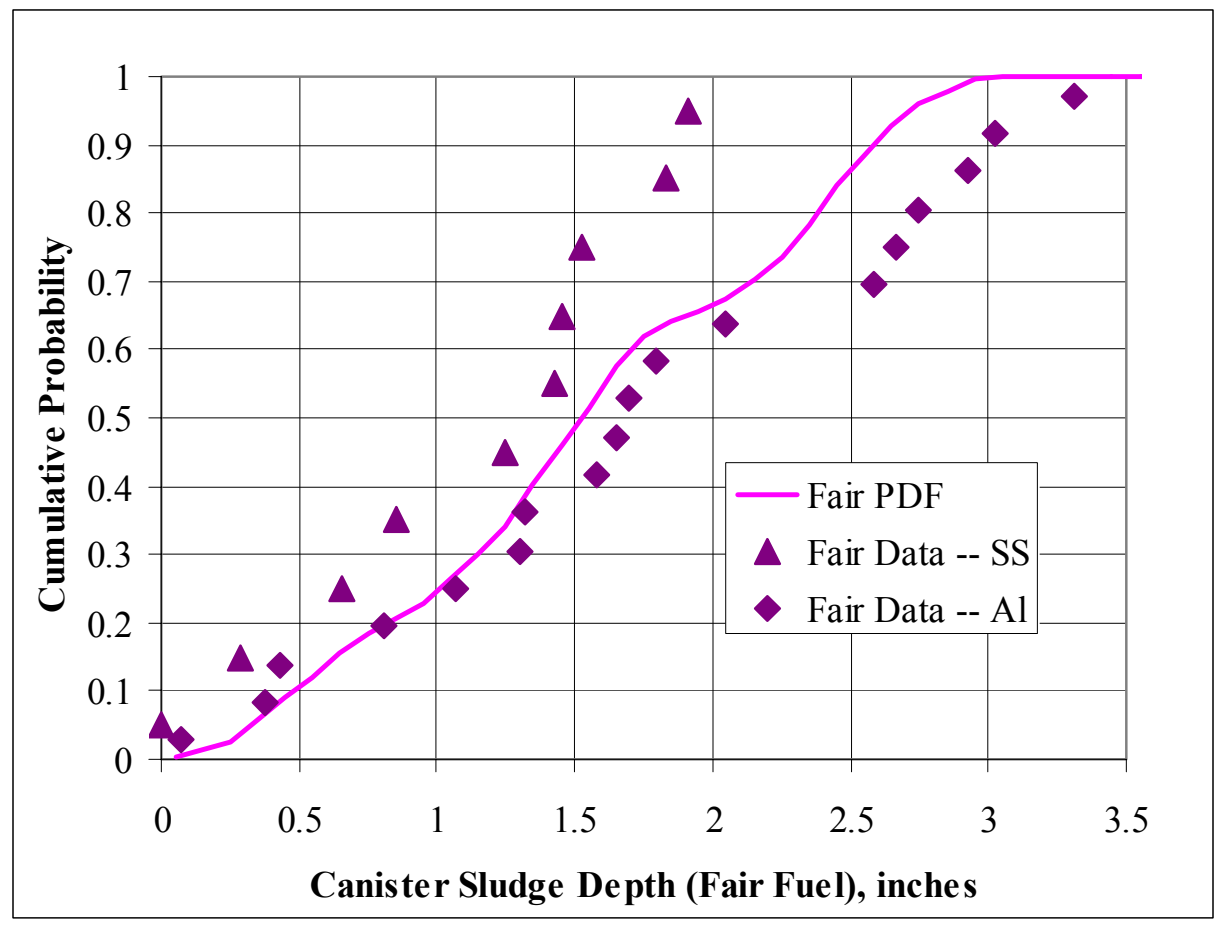

Figure 5-30. Stochastic Result for Continuous Canister Sludge Depth (Poor and Bad Fuel).

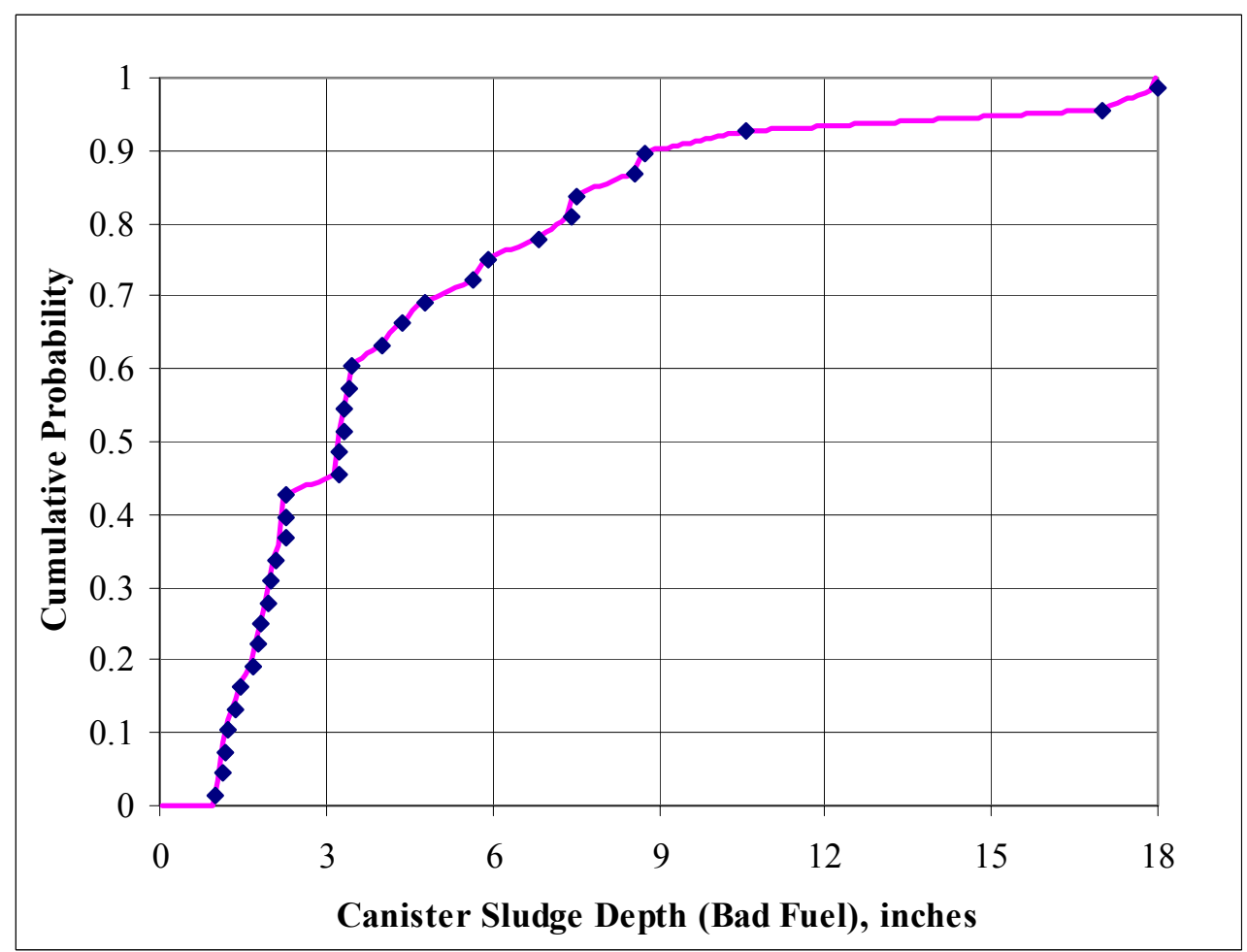


SNF-10293, REV 0

\subsubsection{Limiting Cases for Off Scale Depths}

This case changes the assumed values for the off scale depth readings in poor and bad fuel canisters. The minimum depths are 12 and 13 inches. The maximum depths are 19 and 20 inches. The container concentrations are largely the same as the base case. The mean values increased about $2 \%$ going from the smallest to the largest off scale depths. The $95^{\text {th }}$ percentile value increased about 3\%. The largest increase was for Cs-134 in the $12 \mathrm{~m}^{3}$ container. The $95^{\text {th }}$ percentile Cs-134 concentration increased 5\%. Because the base case is near the maximum, the increases in the $95^{\text {th }}$ percentile results for the maximum depth case are less than $2 \%$ for all items tracked.

\subsubsection{Canister Sludge Isotopic Composition}

Four alternate cases were run. The first uses the canister sample data rather than the isotopic concentrations from HNF-8760. This case includes an additional case in which imputed values for both floor and canister sludge are doubled. The second uses a uniform PDF to represent the canister sludge density rather than the sample data. The third reduces the variability factor to the range 0.9 to 1.1 . The fourth uses non-segregated canister sludge from Radnuc2A.

\subsubsection{Canister Sludge Data Rather than HNF-8760}

In this case the canister sludge data is treated like the floor sludge data. The variability factor is the same as used for sludge density (0.4 to 1.6). The uranium concentration is calculated from the data rather than the correlation with sludge density used for the base case. The stochastic result for the canister sludge uranium concentration is shown in Figure 5-31. This can be compared with the base case shown in Figure 4-13. In this alternate case the data is used with a variability factor. Thus, Figure 5-31 for uranium data resembles Figure 4-12, which shows sludge density. The other items tracked have similar graphs. Plutonium masses are calculated using the assumed PDF for $\mathrm{Pu}-239 / 240$ in addition to the specific activities for $\mathrm{Pu}-238$ and $\mathrm{Pu}-241$.

The stochastic results for containers show significant decreases for all nuclides except Tc-99. Ratios between the mean results using canister sludge data and the mean results for the base case are shown in Table 5-25. The canister-to-total ratios were not affected, nor were the sludge density. The Eu-155 concentration decreased by only 5\%. All other nuclide concentrations decreased much more. The Tc-99 increased by nearly a factor of 7 . Overall the heat generation rate is half the base case value, and the inhalation dose is $63 \%$ of the base case. Therefore, the use of HNF-8760 sludge concentration estimates (base case) leads to larger results than using the sample data. 
Figure 5-31. Stochastic Result Using Data for Canister Sludge Uranium Concentration.

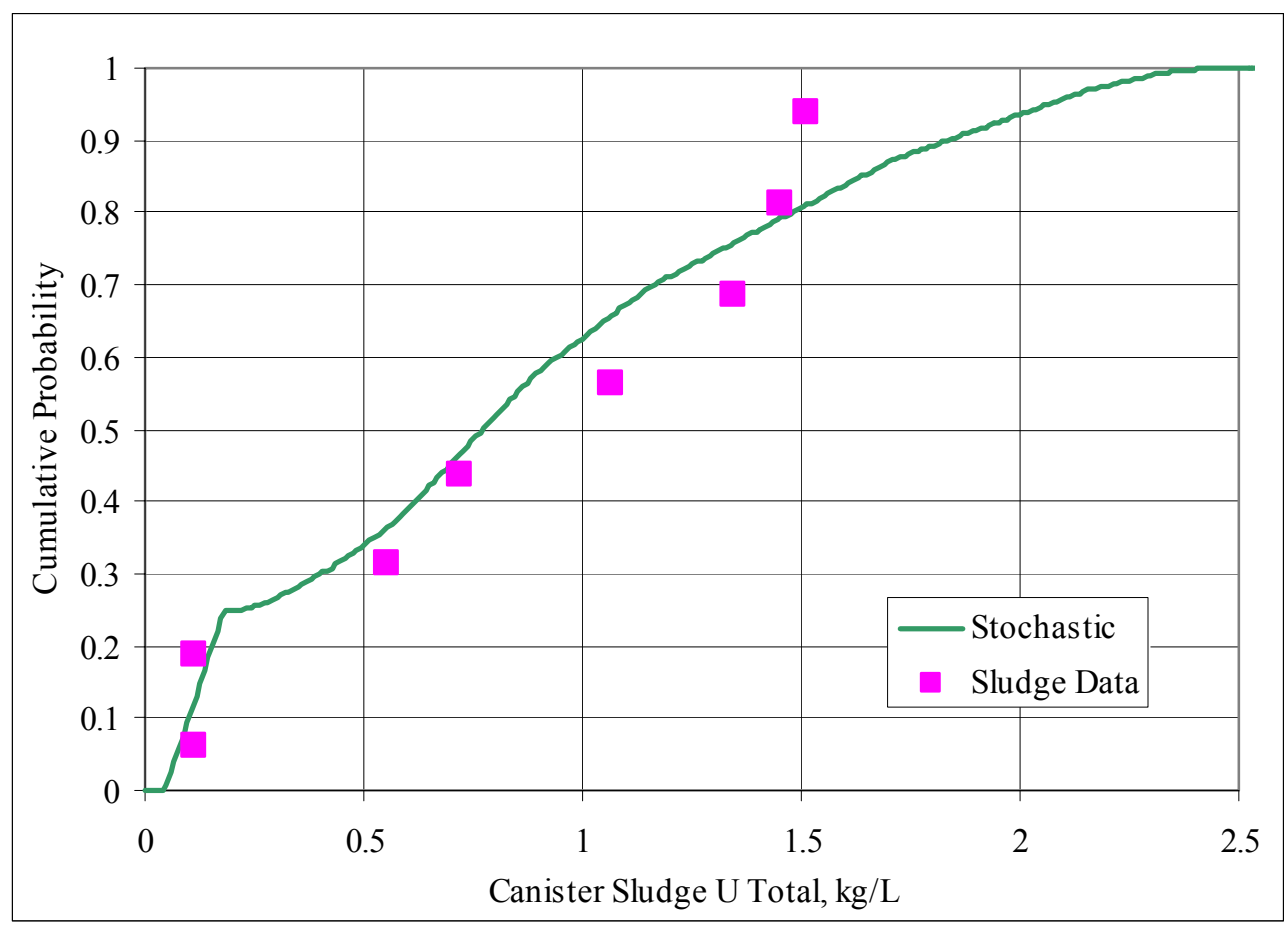

An additional case uses the canister sludge data along with imputed values that have been doubled. Both floor and canister sludge imputations are doubled. The Tc-99 ratio increases to 10 and the Eu-155 ratio increases to 1.3. All other items have lower concentrations than the base case. 
SNF-10293, REV 0

Table 5-25. Comparison Ratios: Canister Sludge Data Versus HNF-8760.

\begin{tabular}{|c|c|c|c|}
\hline Container Volume & $0.5 \mathrm{~m}^{3}$ & $2 \mathrm{~m}^{3}$ & $12 \mathrm{~m}^{3}$ \\
\hline Canister-to-Total Ratio & 1.00 & 1.00 & 1.00 \\
\hline Co-60 & 0.86 & 0.86 & 0.86 \\
\hline Sr-90 & 0.55 & 0.55 & 0.55 \\
\hline Tc-99 & 6.69 & 6.69 & 6.70 \\
\hline Cs-134 & 0.13 & 0.13 & 0.13 \\
\hline Cs-137 & 0.29 & 0.29 & 0.29 \\
\hline Eu-152 & 0.33 & 0.33 & 0.33 \\
\hline Eu-154 & 0.35 & 0.35 & 0.35 \\
\hline Eu-155 & 0.95 & 0.95 & 0.95 \\
\hline Np-237 & 0.63 & 0.63 & 0.63 \\
\hline Pu-238 & 0.40 & 0.40 & 0.40 \\
\hline $\mathrm{Pu}-239 / 240$ & 0.79 & 0.79 & 0.79 \\
\hline Pu-241 & 0.41 & 0.41 & 0.41 \\
\hline Am-241 & 0.60 & 0.60 & 0.60 \\
\hline Total Uranium & 0.99 & 0.99 & 0.99 \\
\hline Wet Density & 1.00 & 1.00 & 1.00 \\
\hline Heat Generation Rate & 0.47 & 0.47 & 0.47 \\
\hline Inhalation Dose & 0.63 & 0.63 & 0.63 \\
\hline Uranium Metal & 1.00 & 1.00 & 1.00 \\
\hline Pu Weight Percent & 0.85 & 0.85 & 0.85 \\
\hline Heat per MT & 0.46 & 0.47 & 0.47 \\
\hline Heat per MTU & 0.52 & 0.48 & 0.47 \\
\hline
\end{tabular}

The comparison ratios are calculated as the mean stochastic result using the canister sludge data divided by the base case mean results. 


\subsubsection{Uniform Canister Sludge Density}

The canister sludge wet density was also calculated using a uniform distribution that ranges from $1.05 \mathrm{~kg} / \mathrm{L}$ to $2.7 \mathrm{~kg} / \mathrm{L}$. This range of densities preserves the mean value of the canister sludge density distribution. The standard deviation decreases by $21 \%$. The stochastic result for the canister sludge density CDF is shown in Figure 5-32. This can be compared to the base case shown in Figure 4-12. The straight line indicates that the distribution is uniform.

Figure 5-32. Stochastic Result Using a Uniform Distribution for Canister Sludge Density.

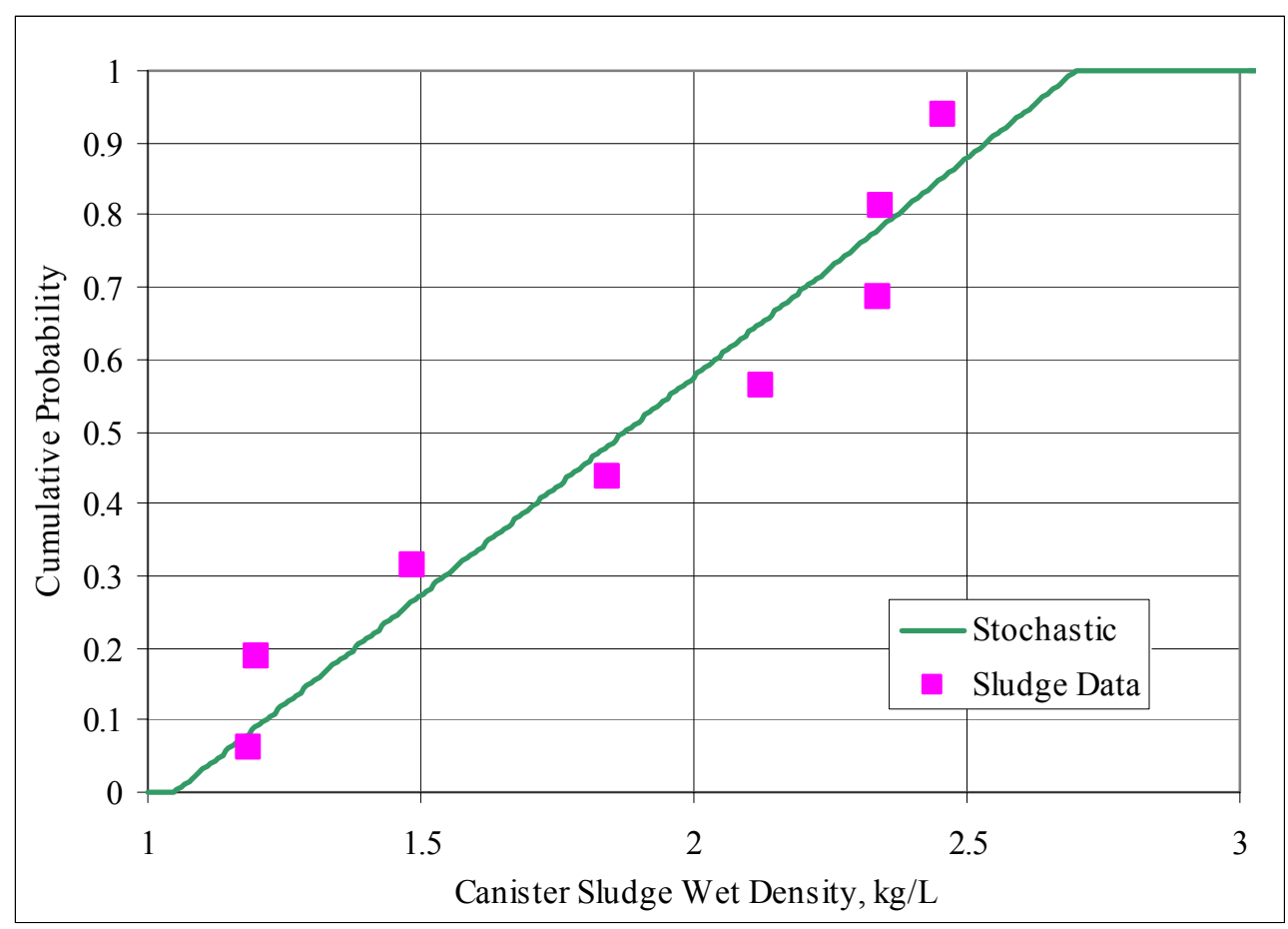

The container stochastic results have the same mean values as the base case. The $95^{\text {th }}$ percentile values decreased $7 \%$ for the $0.5 \mathrm{~m}^{3}$ container, $5 \%$ for the $2 \mathrm{~m}^{3}$ container, and $3 \%$ for the $12 \mathrm{~m}^{3}$ container. 


\subsubsection{Reduced Variability}

This case changes the variability factor for the canister sludge density from the base case $(0.3$ to 1.6) to 0.9 to 1.1 . The principal effect is to reduce the standard deviations of the canister sludge concentrations by as much as $21 \%$. An example for canister sludge density is shown in Figure 5-33. This can be compared to the base case shown in Figure 4-12.

Figure 5-33. Stochastic Result Using Reduced Variability for Canister Sludge Density.

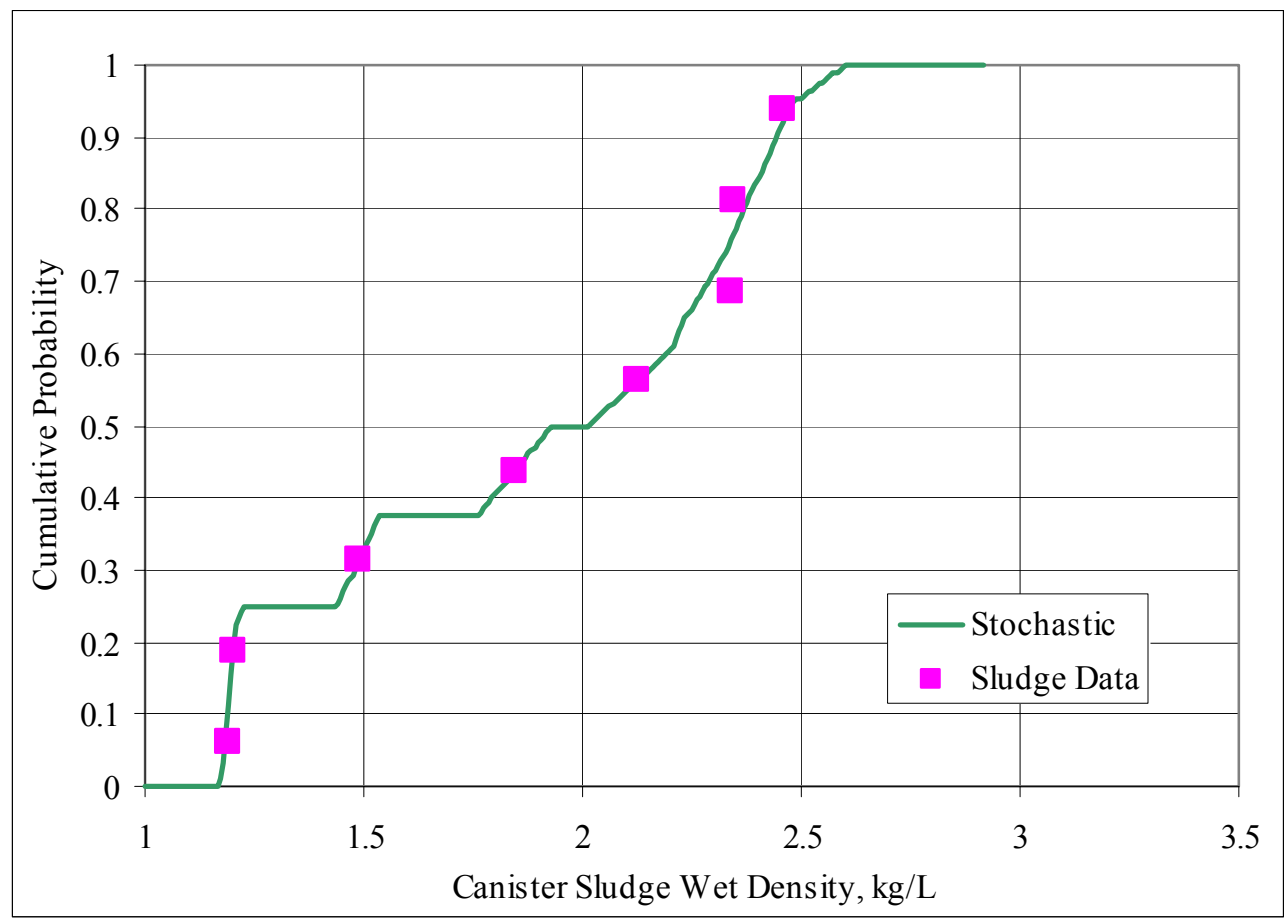

The container average concentrations are largely the same as the base case. The $95^{\text {th }}$ percentile values decrease by less than $8 \%$. The largest decrease was for total uranium concentration in the smallest container. The mean results are unchanged. However, the concentrations that are based on the amount per unit mass, namely, Pu mass fraction, heat generation per MT sludge, inhalation dose per gram all increased about $1.2 \%$.

\subsubsection{Non-Segregated Canister Sludge}

The effect of the fuel segregation assumption was examined for completeness. The nonsegregated fuel has a lower average concentration for all isotopes because the low burnup fuel is still present. Using the non-segregated fuel leads to lower radionuclide concentrations and other quantities of interest. The canister-to-total volume ratio, the uranium concentration (total and metal only), and the sludge wet density were not affected by the segregation assumption.

The stochastic results for non-segregated radionuclide concentrations decreased by as little as $7 \%$ (Co-60) to as much as 40\% (Cs-134, Eu-154 and Pu-238). The heat generation rates decreased by $22 \%$ while the inhalation dose factors decreased by $30 \%$. 


\section{SNF-10293, REV 0}

\subsubsection{Reduced Variability Factors}

The variability factors for floor sludge isotopic composition, canister sludge depth, and canister sludge density were reduced separately in previous cases. In this case, all three are reduced to the range 0.9 to 1.1 . The mean results are unchanged. However, the concentrations that are based on the amount per unit mass, namely, Pu weight percent, heat generation per MT sludge, inhalation dose per gram all increased about $1.4 \%$. The $95^{\text {th }}$ percentile values decreased $10 \%$ for the $0.5 \mathrm{~m}^{3}$ container, $8 \%$ for the $2 \mathrm{~m}^{3}$ container, and $5 \%$ for the $12 \mathrm{~m}^{3}$ container.

\subsubsection{One Retrieval Sequence}

Rather than allow any key retrieval sequence as in the base case, particular sequences were tested. The sequences tested are listed in Table 5-26. Also shown in the table are the results of comparisons with the base case. The percent increases are for the three container volumes $(0.5$, 2.0 , and $12 \mathrm{~m}^{3}$ ). They are the largest increase found for that sequence. In general, no tracked item increased by more than $19 \%$. The tracked item with the largest increase for each container is identified in parentheses.

Table 5-26. One Retrieval Sequence Comparisons with Base Case.

\begin{tabular}{|c|c|}
\hline Key Retrieval Order & $\begin{array}{l}\text { Increase in Stochastic Results from } \\
\text { Base Case: } 0.5-2-12 \mathrm{~m}^{3}\end{array}$ \\
\hline $\begin{array}{l}\text { Canister-to-Total Sludge Volume Ratio: Sort } \\
\text { keys by the ratio of canister sludge volume } \\
\text { divided by total sludge volume in the key. The } \\
\text { largest ratios are removed first. }\end{array}$ & $\begin{array}{l}\text { Average: } 1 \%-5 \%-16 \% \\
\text { (Pu-238- Pu-238 - Pu-238) } \\
\text { 95th Percent: } 5 \%-16 \%-12 \% \\
\text { (Ratio - Ratio - Pu-238) }\end{array}$ \\
\hline $\begin{array}{l}\text { Number of Bad, Poor, Fair, \& Good Canisters: } \\
\text { Sort keys by fuel condition. Keys with largest } \\
\text { number of bad canisters are removed first. }\end{array}$ & $\begin{array}{l}\text { Average: } 1 \%-5 \%-16 \% \\
\text { (Eu-154- Pu-238 - Pu-238) } \\
\text { 95th Percent: } 4 \%-11 \%-11 \% \\
\text { (Ratio - Ratio - Pu-238) }\end{array}$ \\
\hline $\begin{array}{l}\text { Canister Sludge Volume: Sort keys by the } \\
\text { canister sludge volume. Keys with the largest } \\
\text { volume are removed first. }\end{array}$ & $\begin{array}{l}\text { Average: } 1 \%-3 \%-3 \% \\
(P u-238-\mathrm{Pu}-238-\mathrm{Am}-241) \\
\text { 95th Percent: } 2 \%-6 \%-4 \% \\
(\mathrm{~Np}-237-\text { Ratio - Total U) }\end{array}$ \\
\hline $\begin{array}{l}\text { Average Canister Sludge Volume per Cubicle: } \\
\text { Sort keys by the ratio of canister sludge volume } \\
\text { divided by the total number of canisters in that } \\
\text { key. Keys with largest ratios are removed first. }\end{array}$ & $\begin{array}{l}\text { Average: } 1 \%-4 \%-6 \% \\
(\text { Pu-238 - Pu-238 - Eu-154) } \\
\text { 95th Percent: } 2 \%-3 \%-5 \% \\
\text { (Ratio - Eu-154- Pu-241) }\end{array}$ \\
\hline $\begin{array}{l}\text { Reverse Chronological Order (youngest first): } \\
\text { Sort keys by key number. Largest key number is } \\
\text { removed first. }\end{array}$ & $\begin{array}{l}\text { Average: } 1 \%-5 \%-15 \% \\
\text { (Cs-134-Cs-134-Cs-134) } \\
\text { 95th Percent: } 3 \%-19 \%-11 \% \\
(\mathrm{Pu}-238-\mathrm{Cs}-134-\mathrm{Cs}-134)\end{array}$ \\
\hline $\begin{array}{l}\text { Chronological Order (oldest first): Sort keys } \\
\text { by key number. Smallest key number is removed } \\
\text { first. }\end{array}$ & $\begin{array}{l}\text { Average: } 1 \%-5 \%-16 \% \\
\qquad(\mathrm{Pu}-238-\mathrm{Pu}-238-\mathrm{Pu}-238) \\
\text { 95th Percent: } 3 \%-17 \%-12 \% \\
\quad(\mathrm{Cs}-134-\mathrm{Cs}-134-\mathrm{Pu}-238)\end{array}$ \\
\hline \multicolumn{2}{|c|}{$\begin{array}{l}\text { The percent increases are shown for all three volumes with the } 0.5 \mathrm{~m}^{3} \text { volume first, the } 2 \mathrm{~m}^{3} \\
\text { volume second, and the } 12 \mathrm{~m}^{3} \text { volume third. Shown in parentheses is the item with the } \\
\text { largest increase for each volume. "Ratio" = canister-to-total sludge volume ratio }\end{array}$} \\
\hline
\end{tabular}


The largest increases are for the largest container. The reason for this effect is that the largest container discards the largest amount of sludge. The mean total volume of sludge is $14.6 \mathrm{~m}^{3}$. Thus the filling process for the $12 \mathrm{~m}^{3}$ container discards $2.6 \mathrm{~m}^{3}$ of sludge on the average. The $2 \mathrm{~m}^{3}$ container filling process discards $0.6 \mathrm{~m}^{3}$ and the $0.5 \mathrm{~m}^{3}$ container filling process discards $0.1 \mathrm{~m}^{3}$ each time the basin cubicles are emptied. Because there is one retrieval sequence, the same group of keys is affected each time the basin is emptied. The magnitude of the change is related to the volume discarded.

\subsubsection{Tally All Containers}

The base case tallies containers that are more than $90 \%$ full and discards the others. If all partially full containers are added to the tallies, then some bias is introduced, depending on how the concentrations of the partially filled containers are tallied. Two methods were used. One calculates the concentrations in partially filled containers using the container volume. This increases the number of low concentration containers, biasing the bounding results toward lower values. The other method calculates concentrations in partially filled containers using the sludge volume. This increases the number of high concentration containers, biasing the bounding results toward higher values.

The first method (container volume) decreased the mean values in the $0.5 \mathrm{~m}^{3}$ container by $2 \%$, the $2 \mathrm{~m}^{3}$ container by $6 \%$, and the $12 \mathrm{~m}^{3}$ container by $39 \%$. The large decrease for the large container is understandable because there is a partial container for nearly every full container. The $95^{\text {th }}$ percentile values decreased $1 \%, 4 \%$, and $21 \%$ in the three container volumes.

The second method (sludge volume) increased the mean values less than $2 \%$ for all three volumes. The $95^{\text {th }}$ percentile results increased by $0 \%, 3 \%$, and $26 \%$ in the three container volumes. Again the large increase for the $12 \mathrm{~m}^{3}$ container is because there is a partial container for nearly every full container.

An additional case was run using the $90 \%$ criteria where the partially filled containers that are counted (i.e., more than $90 \%$ full) base the concentration on the sludge volume rather than the container volume. There was no difference greater than $1 \%$ for the three volumes. 
SNF-10293, REV 0

This page intentionally left blank. 
SNF-10293, REV 0

\subsection{SOFTWARE VERIFICATION AND VALIDATION}

A stand-alone program was written to carry out the stochastic analysis. The program is written in the Pascal language as implemented by the Borland Pascal Compiler, Version 7.0. The software reads a data file, performs the requested number of simulated K East Basin retrievals and then writes the output file. The 10,000,000 retrieval simulations require about 6 hours of computer time on a $1 \mathrm{GHz}$ personal computer.

\subsection{USER GUIDE}

The program is named PDF-11. The " 11 " indicates it was the $11^{\text {th }}$ major version. The date of this version is July 26, 2002. To run the program, it is necessary to open a DOS window. At the prompt, type "PDF-11" to start it. With no command line input, the program shows the information in Figure 6-1.

Figure 6-1. Default Screen Output of PDF-11.

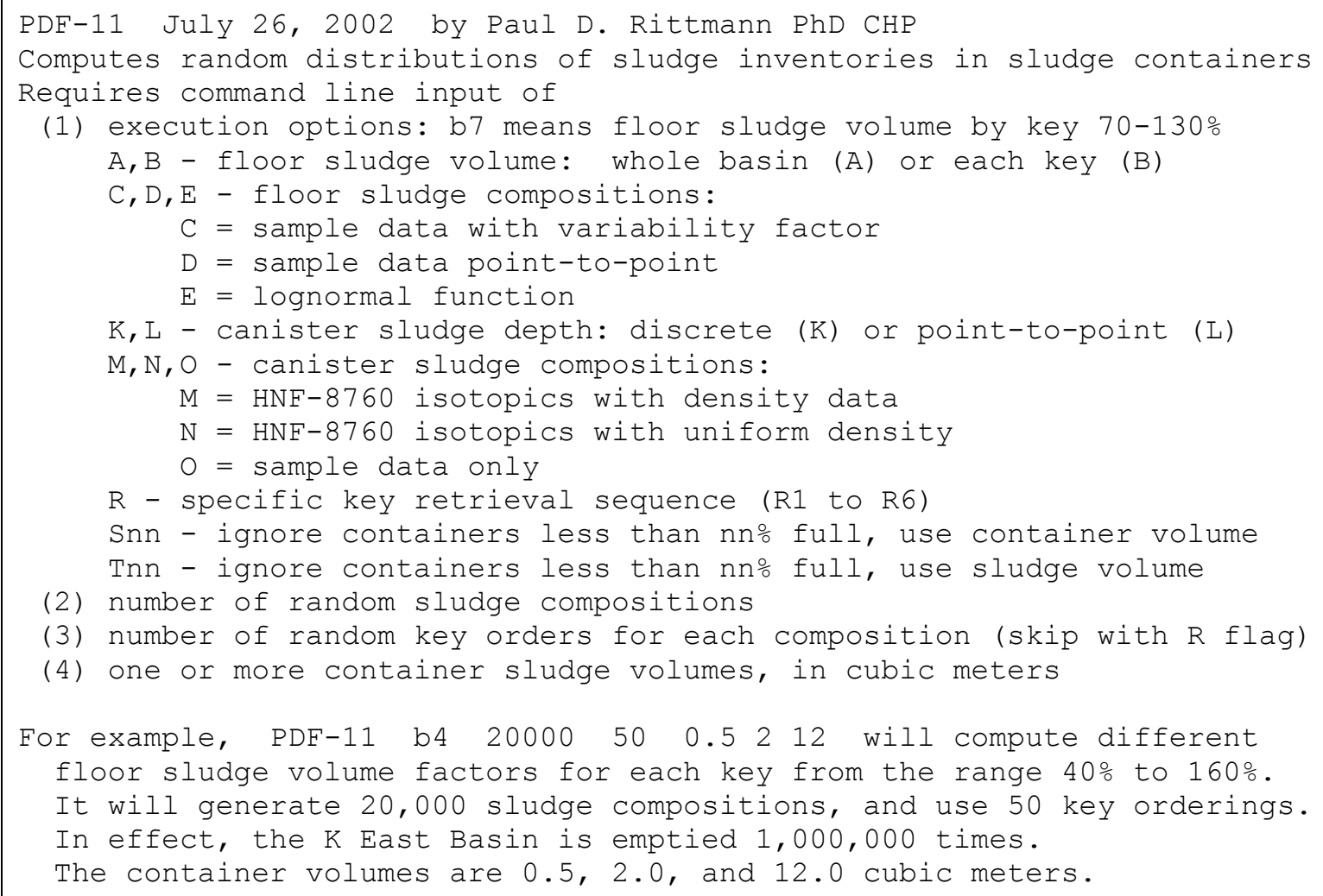

To actually run the simulations, there must be at least three items on the command line when the program starts. These are listed in Figure 6-1. The execution options are not required. If the first item on the command line is a number, the program assumes that all defaults will be used. The option characters may be followed by a number that replaces the default quantity with a new value. For example, using "A7" will change the floor sludge volume adjustment factor so it uses 
the range 0.7 to 1.3 rather than the default range of 0.8 to 1.2. More than one option may be invoked in a single run. For example, to change all the variability factors to the range 0.9 to 1.1 , the option string is "c9k9m9".

The first number on the command line is the number of sludge compositions to generate for $\mathrm{K}$ East Basin. This number is required for all execution options. It must be greater than 9 .

The second number is how many random key retrieval sequences to use with each composition. It must be greater than 2 . If the "R" option has been specified, then the program does not read this number from the command line.

The third number (or second number if the " $\mathrm{R}$ " option is used) is the volume of the sludge container. Up to four container volumes are allowed. The container volumes must be at least $0.1 \mathrm{~m}^{3}$. Note that valid command line input requires that sludge volumes with decimal points must have a number on both sides of the decimal point, or a read error will occur.

If the container volume is less than the sludge volume for a significant portion of the keys, the stochastic representation loses its credibility. A reasonable limit is a container volume that is greater than the total sludge volume per key for half of the 52 keys.

A cumulative plot of the total sludge volume per key is shown in Figure 6-2. The total sludge volumes include both floor and canister sludge using the averages discussed in Section 3.0. A reasonable limit is the median, or $50^{\text {th }}$ percentile value from Figure $6-2,0.2 \mathrm{~m}^{3}$. Half of the keys have a total sludge volume greater than this.

Figure 6-2. Cumulative Total Sludge Volume per Key.

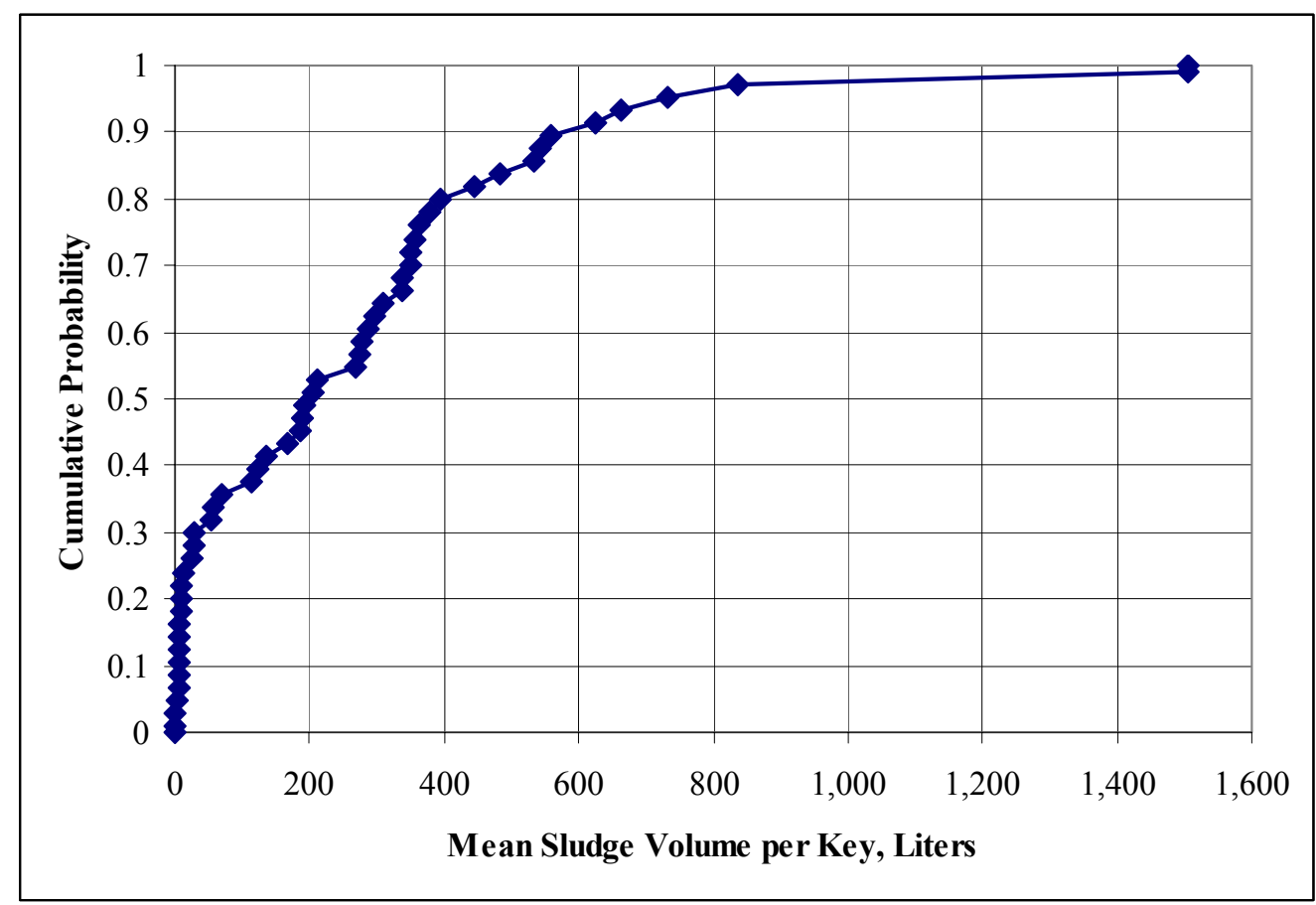


When the program runs, it reads the data files named PDF-11.DAT and PDF-11.HDR. These data files are listed in Appendix $F$ for reference. The program expects to find them in the current directory. If they are not in the directory, or are in use by another program, the run will end with an error message.

While the program is running, it displays how many compositions have been tested at this point. If the user wishes to end the run prematurely, pressing any button on the keyboard is sufficient. This will end the run as though a smaller number of compositions had been requested. The output tables are written to the file PDF-11.OUT in every case. If the output is worth saving, it should be copied to a file with another name to keep it from being replaced on the next PDF-11 run. The output file is a space-delimited text file that can be read into a spreadsheet to facilitate graphing the stochastic results, or converting the output concentrations into other units.

The output file is very long, typically exceeding 1.4 megabyte. Most of the length is because of the 66 tables of stochastic results, each of which has hundreds of lines. The output can be divided into three sections. The first section echoes certain data inputs and lists some cumulative probability distributions. These tables are of interest in checking that the input data file was read correctly. The second section shows the stochastically determined probability distributions in 43 tables. These include the $\mathrm{Pu}-239 / 240$ specific activity, canister sludge depth, total sludge volume in all keys, and the 38 concentrations in floor and canister sludge average over all keys. The third section shows the stochastic results for 23 quantities in each container volume.

The output begins with the program identifier (PDF-11 July 26, 2002). Then follows a summary of the stochastic model being used for this run. Included are the title lines from tables read from the data files (PDF-11.DAT and PDF-11.HDR). The first data file contains the sludge depth measurements shown in Appendix C. The average canister sludge depths are computed and displayed in the first portion of the output shown in Figure 6-3. This is done to verify that the program has correctly interpreted the depth measurements. The mean depths are then used to calculate the total sludge volume associated with each key as shown in Table 3-1. This table appears in the output file, as shown in Figure 6-4. 


\section{SNF-10293, REV 0}

Figure 6-3. First Block of Output from PDF-11 (Input Data).

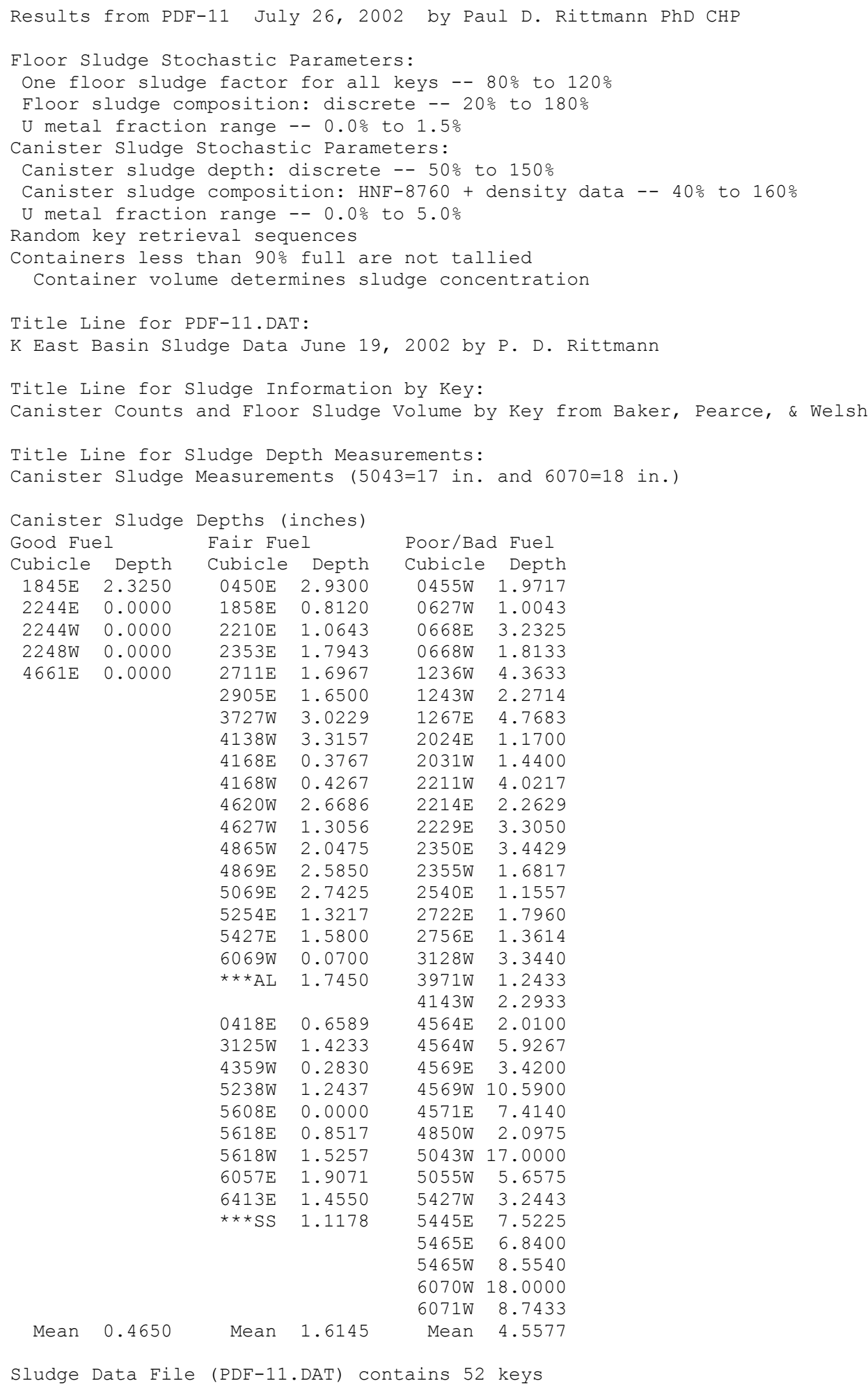




\section{SNF-10293, REV 0}

Figure 6-4. Second Block of Output from PDF-11 (Input Data).

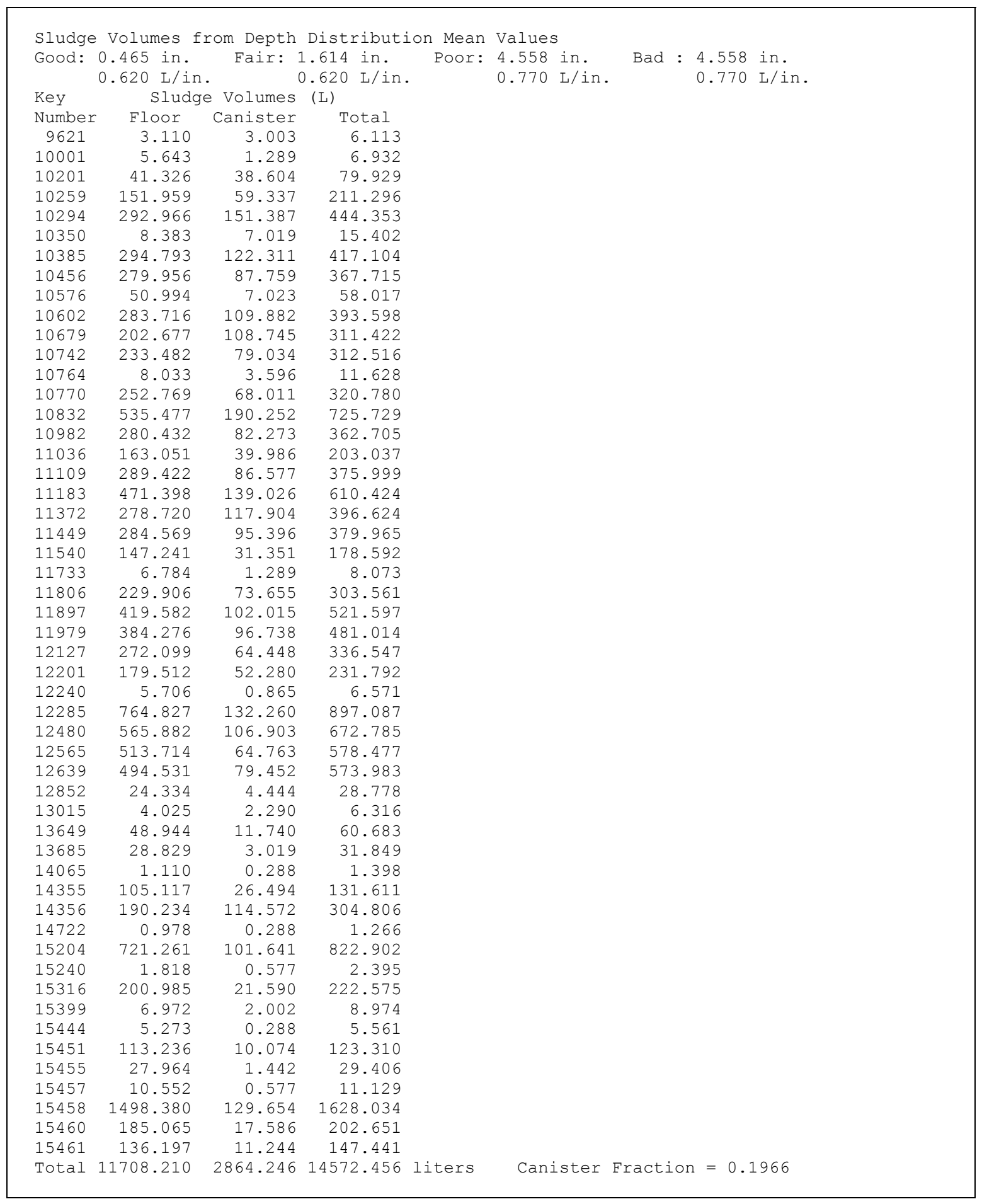


The sludge depth table shown in Figure 6-3 is sorted to construct the continuous cumulative distribution functions shown in Figure 3-12 and Figure 3-13. These functions are only used if a negative value is entered for the third container volume. (The default distribution is the data points modified by a variability factor.) This sorted table is shown in Figure 6-5.

Figure 6-5. Third Block of Output from PDF-11 (Input Data).

\begin{tabular}{|c|c|c|c|c|c|c|c|}
\hline \multicolumn{8}{|c|}{ Canister Sludge Cumulative Distributions (inches) } \\
\hline \multicolumn{2}{|c|}{ Good Fuel } & \multicolumn{2}{|c|}{ Fair Fuel } & \multicolumn{2}{|c|}{ Poor Fuel } & \multicolumn{2}{|l|}{ Bad Fuel } \\
\hline Depth & Prob & Depth & Prob & Depth & Prob & Depth & Prob \\
\hline 0.0000 & 0.1000 & 0.0700 & 0.0278 & 1.0043 & 0.0147 & 1.0043 & 0.0147 \\
\hline 0.0000 & 0.3000 & 0.3767 & 0.0833 & 1.1557 & 0.0441 & 1.1557 & 0.0441 \\
\hline 0.0000 & 0.5000 & 0.4267 & 0.1389 & 1.1700 & 0.0735 & 1.1700 & 0.0735 \\
\hline 0.0000 & 0.7000 & 0.8120 & 0.1944 & 1.2433 & 0.1029 & 1.2433 & 0.1029 \\
\hline 2.3250 & 0.9000 & 1.0643 & 0.2500 & 1.3614 & 0.1324 & 1.3614 & 0.1324 \\
\hline & & 1.3056 & 0.3056 & 1.4400 & 0.1618 & 1.4400 & 0.1618 \\
\hline & & 1.3217 & 0.3611 & 1.6817 & 0.1912 & 1.6817 & 0.1912 \\
\hline & & 1.5800 & 0.4167 & 1.7960 & 0.2206 & 1.7960 & 0.2206 \\
\hline & & 1.6500 & 0.4722 & 1.8133 & 0.2500 & 1.8133 & 0.2500 \\
\hline & & 1.6967 & 0.5278 & 1.9717 & 0.2794 & 1.9717 & 0.2794 \\
\hline & & 1.7943 & 0.5833 & 2.0100 & 0.3088 & 2.0100 & 0.3088 \\
\hline & & 2.0475 & 0.6389 & 2.0975 & 0.3382 & 2.0975 & 0.3382 \\
\hline & & 2.5850 & 0.6944 & 2.2629 & 0.3676 & 2.2629 & 0.3676 \\
\hline & & 2.6686 & 0.7500 & 2.2714 & 0.3971 & 2.2714 & 0.3971 \\
\hline & & 2.7425 & 0.8056 & 2.2933 & 0.4265 & 2.2933 & 0.4265 \\
\hline & & 2.9300 & 0.8611 & 3.2325 & 0.4559 & 3.2325 & 0.4559 \\
\hline & & 3.0229 & 0.9167 & 3.2443 & 0.4853 & 3.2443 & 0.4853 \\
\hline & & 3.3157 & 0.9722 & 3.3050 & 0.5147 & 3.3050 & 0.5147 \\
\hline & & 1.7450 & AL mean & 3.3440 & 0.5441 & 3.3440 & 0.5441 \\
\hline & & & & 3.4200 & 0.5735 & 3.4200 & 0.5735 \\
\hline & & 0.0000 & 0.0500 & 3.4429 & 0.6029 & 3.4429 & 0.6029 \\
\hline & & 0.2830 & 0.1500 & 4.0217 & 0.6324 & 4.0217 & 0.6324 \\
\hline & & 0.6589 & 0.2500 & 4.3633 & 0.6618 & 4.3633 & 0.6618 \\
\hline & & 0.8517 & 0.3500 & 4.7683 & 0.6912 & 4.7683 & 0.6912 \\
\hline & & 1.2437 & 0.4500 & 5.6575 & 0.7206 & 5.6575 & 0.7206 \\
\hline & & 1.4233 & 0.5500 & 5.9267 & 0.7500 & 5.9267 & 0.7500 \\
\hline & & 1.4550 & 0.6500 & 6.8400 & 0.7794 & 6.8400 & 0.7794 \\
\hline & & 1.5257 & 0.7500 & 7.4140 & 0.8088 & 7.4140 & 0.8088 \\
\hline & & 1.8300 & 0.8500 & 7.5225 & 0.8382 & 7.5225 & 0.8382 \\
\hline & & 1.1178 & SS mean & 8.5540 & 0.8676 & 8.5540 & 0.8676 \\
\hline & & & & 8.7433 & 0.8971 & 8.7433 & 0.8971 \\
\hline & & & & 10.5900 & 0.9265 & 10.5900 & 0.9265 \\
\hline & & & & 17.0000 & 0.9559 & 17.0000 & 0.9559 \\
\hline & & & & 18.0000 & 0.9853 & 18.0000 & 0.9853 \\
\hline Cum Ave & 0.4650 & Cum Ave & 1.6145 & Cum Ave & 4.5577 & Cum Ave & 4.5577 \\
\hline
\end{tabular}

The data file PDF-11.DAT also contains the isotopic concentrations for floor and canister sludge. The program reads the title lines and places them in the output file. The sorted activities are listed in the output file, as shown in Figure 6-6. This table has been split into three parts to make it readable. The first part shows the first 7 columns, while the second part shows the next 6 columns. The last part of Figure 6-6 shows the final 6 columns. Note that the program computes the last 4 columns. The first row of each part contains the mean values for the column below it. The second row has the standard deviations. The third and fourth rows have the geometric mean and geometric standard deviations. These four rows must match the summary values given in Tables 3-4, 3-5, and 3-6. 
Figure 6-6. Fourth Block of Output from PDF-11 (Input Data). (2 sheets)

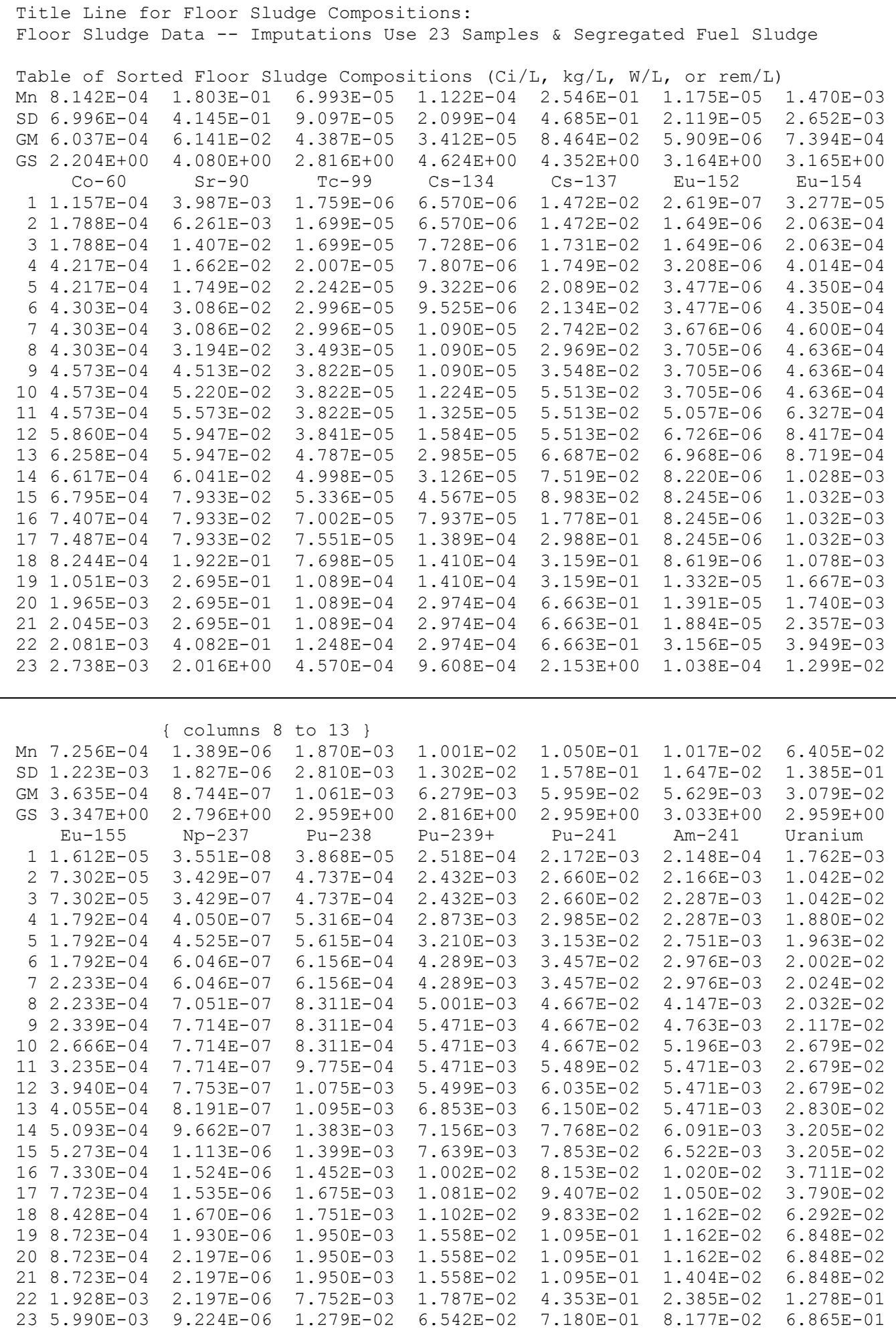


SNF-10293, REV 0

Figure 6-6. Fourth Block of Output from PDF-11 (Input Data). (2 sheets)

\begin{tabular}{|c|c|c|c|c|c|}
\hline \multicolumn{6}{|c|}{ columns 14 to 19} \\
\hline Mn & $1.269 \mathrm{E}+00$ & $3.179 \mathrm{E}-03$ & $1.046 \mathrm{E}+07$ & $3.172 \mathrm{E}-02$ & $1.294 \mathrm{E}-04$ \\
\hline $\mathrm{SD}$ & $2.368 \mathrm{E}-01$ & $6.000 \mathrm{E}-03$ & $1.516 \mathrm{E}+07$ & $5.920 \mathrm{E}-03$ & $1.684 \mathrm{E}-04$ \\
\hline GM & $1.220 \mathrm{E}+00$ & $1.501 \mathrm{E}-03$ & $6.219 \mathrm{E}+06$ & $3.134 \mathrm{E}-02$ & $8.112 \mathrm{E}-05$ \\
\hline GS & $1.842 \mathrm{E}+00$ & $3.022 \mathrm{E}+00$ & $2.897 \mathrm{E}+00$ & $1.159 \mathrm{E}+00$ & $2.817 \mathrm{E}+00$ \\
\hline & Density & Heat & Dose & U metal & Plutonium \\
\hline 1 & $1.040 \mathrm{E}+00$ & $3.268 E-04$ & $2.544 \mathrm{E}+05$ & $2.600 E-02$ & $3.249 \mathrm{E}-06$ \\
\hline 2 & $1.110 \mathrm{E}+00$ & $4.419 \mathrm{E}-04$ & $2.472 E+06$ & $2.775 \mathrm{E}-02$ & $3.145 \mathrm{E}-05$ \\
\hline 3 & $1.140 \mathrm{E}+00$ & $4.546 \mathrm{E}-04$ & $2.472 E+06$ & $2.850 \mathrm{E}-02$ & $3.145 \mathrm{E}-05$ \\
\hline 4 & $1.150 \mathrm{E}+00$ & $4.546 \mathrm{E}-04$ & $2.677 \mathrm{E}+06$ & $2.875 \mathrm{E}-02$ & $3.715 \mathrm{E}-05$ \\
\hline 5 & $1.150 \mathrm{E}+00$ & $5.834 \mathrm{E}-04$ & $3.057 \mathrm{E}+06$ & $2.875 \mathrm{E}-02$ & $4.145 \mathrm{E}-05$ \\
\hline 6 & $1.194 \mathrm{E}+00$ & $7.077 \mathrm{E}-04$ & $3.713 E+06$ & $2.985 \mathrm{E}-02$ & $5.532 \mathrm{E}-05$ \\
\hline 7 & $1.194 \mathrm{E}+00$ & $7.257 \mathrm{E}-04$ & $3.713 E+06$ & $2.985 \mathrm{E}-02$ & $5.532 \mathrm{E}-05$ \\
\hline 8 & $1.194 \mathrm{E}+00$ & $7.904 \mathrm{E}-04$ & $4.837 \mathrm{E}+06$ & $2.985 E-02$ & $6.466 \mathrm{E}-05$ \\
\hline 9 & $1.200 \mathrm{E}+00$ & $8.564 \mathrm{E}-04$ & $5.398 \mathrm{E}+06$ & $3.000 \mathrm{E}-02$ & $7.060 \mathrm{E}-05$ \\
\hline 10 & $1.210 \mathrm{E}+00$ & $9.892 \mathrm{E}-04$ & $5.509 \mathrm{E}+06$ & $3.025 \mathrm{E}-02$ & $7.060 \mathrm{E}-05$ \\
\hline 11 & $1.210 \mathrm{E}+00$ & $1.190 \mathrm{E}-03$ & $5.509 \mathrm{E}+06$ & $3.025 \mathrm{E}-02$ & $7.060 \mathrm{E}-05$ \\
\hline 12 & $1.210 \mathrm{E}+00$ & $1.190 \mathrm{E}-03$ & $5.509 \mathrm{E}+06$ & $3.025 E-02$ & $7.110 \mathrm{E}-05$ \\
\hline 13 & $1.235 \mathrm{E}+00$ & $1.190 \mathrm{E}-03$ & $6.193 E+06$ & $3.088 E-02$ & $8.846 \mathrm{E}-05$ \\
\hline 14 & $1.235 \mathrm{E}+00$ & $1.299 \mathrm{E}-03$ & $6.976 \mathrm{E}+06$ & $3.088 \mathrm{E}-02$ & $9.253 \mathrm{E}-05$ \\
\hline 15 & $1.235 \mathrm{E}+00$ & $1.506 \mathrm{E}-03$ & $7.362 \mathrm{E}+06$ & $3.088 \mathrm{E}-02$ & $9.871 \mathrm{E}-05$ \\
\hline 16 & $1.235 \mathrm{E}+00$ & $2.183 E-03$ & $1.068 \mathrm{E}+07$ & $3.088 \mathrm{E}-02$ & $1.293 \mathrm{E}-04$ \\
\hline 17 & $1.235 \mathrm{E}+00$ & $2.183 E-03$ & $1.084 \mathrm{E}+07$ & $3.088 \mathrm{E}-02$ & $1.396 \mathrm{E}-04$ \\
\hline 18 & $1.250 \mathrm{E}+00$ & $4.057 \mathrm{E}-03$ & $1.360 \mathrm{E}+07$ & $3.125 \mathrm{E}-02$ & $1.422 \mathrm{E}-04$ \\
\hline 19 & $1.300 \mathrm{E}+00$ & $4.797 E-03$ & $1.360 \mathrm{E}+07$ & $3.250 \mathrm{E}-02$ & $2.008 \mathrm{E}-04$ \\
\hline 20 & $1.350 \mathrm{E}+00$ & $5.976 \mathrm{E}-03$ & $1.360 \mathrm{E}+07$ & $3.375 \mathrm{E}-02$ & $2.008 \mathrm{E}-04$ \\
\hline 21 & $1.400 \mathrm{E}+00$ & $5.976 \mathrm{E}-03$ & $1.624 \mathrm{E}+07$ & $3.500 \mathrm{E}-02$ & $2.008 \mathrm{E}-04$ \\
\hline 22 & $1.430 \mathrm{E}+00$ & $5.976 \mathrm{E}-03$ & $2.059 \mathrm{E}+07$ & $3.575 E-02$ & $2.336 \mathrm{E}-04$ \\
\hline 23 & $2.280 \mathrm{E}+00$ & $2.925 E-02$ & $7.589 \mathrm{E}+07$ & $5.700 \mathrm{E}-02$ & $8.459 \mathrm{E}-04$ \\
\hline
\end{tabular}

The output for canister sludge data is shown in Figure 6-7. This table is divided into three parts as was done for the floor sludge sample data. The mean and standard deviations are for comparison with the values shown in Table 3-16.

The output for canister sludge isotopic compositions from HNF-8760 is shown in Figure 6-8 and lists mean values for canister sludge concentration that can be compared with tallies of canister sludge concentrations generated by the program. The first column is the HNF-8760 isotopic information by key weighted by the average canister sludge uranium per key. These are nearly the same as shown in the "Method A" column of Table 3-23. The difference is a factor of $0.860 \mathrm{~kg} / \mathrm{L}$, the concentration of uranium in canister sludge. The second column of numbers in the upper portion of Figure 6-8 is the sum of the HNF-8760 isotopic concentrations multiplied by the average uranium concentration. These are the mean values for the canister sludge input tallies for the base case. The final column shows the mean values for the canister sludge data. In the alternate case that uses the 8 sample results rather than the HNF-8760 isotopic concentrations, the canister sludge input tallies have the same mean values as the data. 


\section{SNF-10293, REV 0}

Figure 6-7. Fifth Block of Output from PDF-11 (Input Data).

\begin{tabular}{|c|c|c|c|c|c|c|c|}
\hline \multicolumn{8}{|c|}{ Canister Sludge Data -- Imputations Use 8 Samples \& Segregated Fuel Sludge } \\
\hline \multicolumn{3}{|c|}{ Table of Sorted Canister } & \multicolumn{5}{|c|}{ Sludge Compositions ( $\mathrm{Ci} / \mathrm{L}, \mathrm{kg} / \mathrm{L}, \mathrm{W} / \mathrm{L}$, or $\mathrm{rem} / \mathrm{L}$ ) } \\
\hline & $7.756 \mathrm{E}-04$ & $2.050 \mathrm{E}+00$ & $1.172 \mathrm{E}-02$ & $3.568 \mathrm{E}-04$ & $9.400 \mathrm{E}-01$ & $1.083 E-04$ & $1.355 \mathrm{E}-02$ \\
\hline & $5.254 \mathrm{E}-04$ & $1.427 \mathrm{E}+00$ & $6.664 \mathrm{E}-03$ & $1.745 E-04$ & $6.135 \mathrm{E}-01$ & $8.080 \mathrm{E}-05$ & $1.011 \mathrm{E}-02$ \\
\hline & $5.971 \mathrm{E}-04$ & $1.384 \mathrm{E}+00$ & $9.607 \mathrm{E}-03$ & $3.097 \mathrm{E}-04$ & 7. $623 \mathrm{E}-01$ & $8.454 \mathrm{E}-05$ & $1.058 \mathrm{E}-02$ \\
\hline & $2.310 \mathrm{E}+00$ & $3.091 E+00$ & $2.170 \mathrm{E}+00$ & $1.837 \mathrm{E}+00$ & $2.056 \mathrm{E}+00$ & $2.175 \mathrm{E}+00$ & $2.175 \mathrm{E}+00$ \\
\hline & $\mathrm{Co}-60$ & Sr-90 & $\mathrm{TC}-99$ & $\mathrm{Cs}-134$ & $\mathrm{Cs}-137$ & Eu-152 & Eu-154 \\
\hline 1 & $1.687 \mathrm{E}-04$ & $2.210 \mathrm{E}-01$ & $1.772 \mathrm{E}-03$ & $1.414 \mathrm{E}-04$ & $3.168 \mathrm{E}-01$ & $2.475 \mathrm{E}-05$ & $3.097 E-03$ \\
\hline 2 & $2.027 E-04$ & $2.746 \mathrm{E}-01$ & $7.868 \mathrm{E}-03$ & $1.529 \mathrm{E}-04$ & $3.425 E-01$ & $4.031 E-05$ & $5.043 E-03$ \\
\hline 3 & $4.558 \mathrm{E}-04$ & $1.314 \mathrm{E}+00$ & $9.596 \mathrm{E}-03$ & $1.538 \mathrm{E}-04$ & $3.446 \mathrm{E}-01$ & $5.565 \mathrm{E}-05$ & $6.963 E-03$ \\
\hline 4 & $5.364 \mathrm{E}-04$ & $1.705 \mathrm{E}+00$ & $9.991 E-03$ & $4.454 \mathrm{E}-04$ & $9.980 \mathrm{E}-01$ & $7.903 E-05$ & $9.888 E-03$ \\
\hline & $8.821 E-04$ & $2.283 E+00$ & $1.024 \mathrm{E}-02$ & $4.480 \mathrm{E}-04$ & $1.046 \mathrm{E}+00$ & $1.082 \mathrm{E}-04$ & 1. $354 \mathrm{E}-02$ \\
\hline 6 & 1.099E-03 & $3.238 \mathrm{E}+00$ & 1.201E-02 & $4.666 \mathrm{E}-04$ & $1.145 \mathrm{E}+00$ & $1.348 E-04$ & 1. $687 \mathrm{E}-02$ \\
\hline 7 & 1.202E-03 & $3.290 \mathrm{E}+00$ & $1.887 \mathrm{E}-02$ & $5.112 \mathrm{E}-04$ & 1.199E+00 & 1. $475 \mathrm{E}-04$ & $1.845 E-02$ \\
\hline \multicolumn{2}{|c|}{$81.658 \mathrm{E}-03$} & $4.075 \mathrm{E}+00$ & $2.339 E-02$ & $5.353 E-04$ & $2.128 \mathrm{E}+00$ & $2.763 \mathrm{E}-04$ & $3.458 E-02$ \\
\hline \multicolumn{8}{|c|}{ columns 8 to 13} \\
\hline $\mathrm{Mn}$ & $7.470 \mathrm{E}-03$ & $1.499 \mathrm{E}-05$ & $1.887 \mathrm{E}-02$ & 1. $382 \mathrm{E}-01$ & 1. $060 \mathrm{E}+00$ & $1.030 \mathrm{E}-01$ & $8.603 E-01$ \\
\hline & $6.889 E-03$ & $1.075 E-05$ & 1. $030 \mathrm{E}-02$ & $8.451 E-02$ & $5.784 \mathrm{E}-01$ & $5.412 \mathrm{E}-02$ & $5.710 E-01$ \\
\hline & $5.280 \mathrm{E}-03$ & $1.078 \mathrm{E}-05$ & $1.587 \mathrm{E}-02$ & $1.087 \mathrm{E}-01$ & $8.910 \mathrm{E}-01$ & $8.600 \mathrm{E}-02$ & $5.976 \mathrm{E}-01$ \\
\hline & $2.463 E+00$ & $2.679 \mathrm{E}+00$ & $1.974 \mathrm{E}+00$ & $2.302 E+00$ & $1.974 \mathrm{E}+00$ & $2.054 \mathrm{E}+00$ & $2.955 \mathrm{E}+00$ \\
\hline & Eu-155 & $N p-237$ & $\mathrm{Pu}-238$ & $\mathrm{Pu}-239+$ & $\mathrm{Pu}-241$ & Am-241 & Uranium \\
\hline 1 & $1.537 \mathrm{E}-03$ & $2.053 E-06$ & $5.082 E-03$ & $2.730 \mathrm{E}-02$ & $2.854 \mathrm{E}-01$ & $2.258 \mathrm{E}-02$ & $1.114 \mathrm{E}-01$ \\
\hline 2 & $2.331 E-03$ & $3.043 E-06$ & $7.345 E-03$ & $3.408 E-02$ & $4.124 \mathrm{E}-01$ & $3.792 \mathrm{E}-02$ & $1.153 E-01$ \\
\hline 3 & 2. 393E-03 & $9.907 \mathrm{E}-06$ & $1.182 \mathrm{E}-02$ & $1.148 \mathrm{E}-01$ & $6.635 E-01$ & $8.567 \mathrm{E}-02$ & $5.563 E-01$ \\
\hline 4 & $4.997 E-03$ & $1.286 \mathrm{E}-05$ & $1.734 \mathrm{E}-02$ & $1.374 \mathrm{E}-01$ & $9.735 \mathrm{E}-01$ & $9.861 \mathrm{E}-02$ & 7. 218E-01 \\
\hline 5 & $7.156 \mathrm{E}-03$ & $1.476 \mathrm{E}-05$ & $1.965 \mathrm{E}-02$ & $1.384 \mathrm{E}-01$ & $1.103 \mathrm{E}+00$ & 1.099E-01 & $1.067 \mathrm{E}+00$ \\
\hline 6 & $8.915 \mathrm{E}-03$ & $1.801 \mathrm{E}-05$ & $2.907 E-02$ & $1.663 \mathrm{E}-01$ & $1.632 \mathrm{E}+00$ & $1.331 E-01$ & $1.347 \mathrm{E}+00$ \\
\hline 7 & $9.750 \mathrm{E}-03$ & $2.578 E-05$ & $3.008 E-02$ & $2.039 E-01$ & 1. $689 \mathrm{E}+00$ & $1.645 \mathrm{E}-01$ & 1. $450 \mathrm{E}+00$ \\
\hline 8 & $2.268 \mathrm{E}-02$ & $3.349 \mathrm{E}-05$ & $3.060 E-02$ & $2.838 E-01$ & $1.718 \mathrm{E}+00$ & $1.719 E-01$ & $1.514 \mathrm{E}+00$ \\
\hline \multicolumn{8}{|c|}{ columns 14 to 19} \\
\hline $\mathrm{Mn}$ & $1.873 \mathrm{E}+00$ & $2.681 \mathrm{E}-02$ & $1.217 \mathrm{E}+08$ & $4.684 \mathrm{E}-02$ & $1.783 \mathrm{E}-03$ & & \\
\hline & $5.233 E-01$ & $1.696 \mathrm{E}-02$ & $6.838 \mathrm{E}+07$ & 1. $308 \mathrm{E}-02$ & $1.088 \mathrm{E}-03$ & & \\
\hline GM & 1. $680 \mathrm{E}+00$ & $2.059 \mathrm{E}-02$ & $9.939 \mathrm{E}+07$ & $4.510 \mathrm{E}-02$ & 1. $403 E-03$ & & \\
\hline & $2.342 \mathrm{E}+00$ & $2.398 \mathrm{E}+00$ & $2.141 \mathrm{E}+00$ & $1.352 \mathrm{E}+00$ & $2.299 \mathrm{E}+00$ & & \\
\hline & Density & Heat & Dose & $\mathrm{U}$ metal & Plutonium & & \\
\hline 1 & 1. $188 \mathrm{E}+00$ & $5.319 \mathrm{E}-03$ & $2.617 E+07$ & $2.970 \mathrm{E}-02$ & $3.528 E-04$ & & \\
\hline 2 & 1. 200E+00 & $5.772 \mathrm{E}-03$ & $3.781 \mathrm{E}+07$ & $3.000 \mathrm{E}-02$ & $4.411 E-04$ & & \\
\hline 3 & 1. $488 \mathrm{E}+00$ & $1.787 \mathrm{E}-02$ & $1.077 \mathrm{E}+08$ & $3.720 \mathrm{E}-02$ & $1.481 \mathrm{E}-03$ & & \\
\hline 4 & $1.845 \mathrm{E}+00$ & $2.524 \mathrm{E}-02$ & $1.082 \mathrm{E}+08$ & $4.612 \mathrm{E}-02$ & $1.768 \mathrm{E}-03$ & & \\
\hline 5 & $2.125 \mathrm{E}+00$ & $2.850 \mathrm{E}-02$ & $1.254 \mathrm{E}+08$ & $5.312 \mathrm{E}-02$ & $1.785 \mathrm{E}-03$ & & \\
\hline 6 & $2.340 \mathrm{E}+00$ & $3.733 E-02$ & $1.562 \mathrm{E}+08$ & $5.850 \mathrm{E}-02$ & $2.148 E-03$ & & \\
\hline 7 & $2.344 \mathrm{E}+00$ & $4.090 \mathrm{E}-02$ & $1.876 \mathrm{E}+08$ & $5.860 \mathrm{E}-02$ & $2.631 E-03$ & & \\
\hline 8 & $2.458 \mathrm{E}+00$ & $5.353 E-02$ & $2.249 E+08$ & $6.145 \mathrm{E}-02$ & $3.654 \mathrm{E}-03$ & & \\
\hline
\end{tabular}


Figure 6-8. Sixth Block of Output from PDF-11 (Input Data).

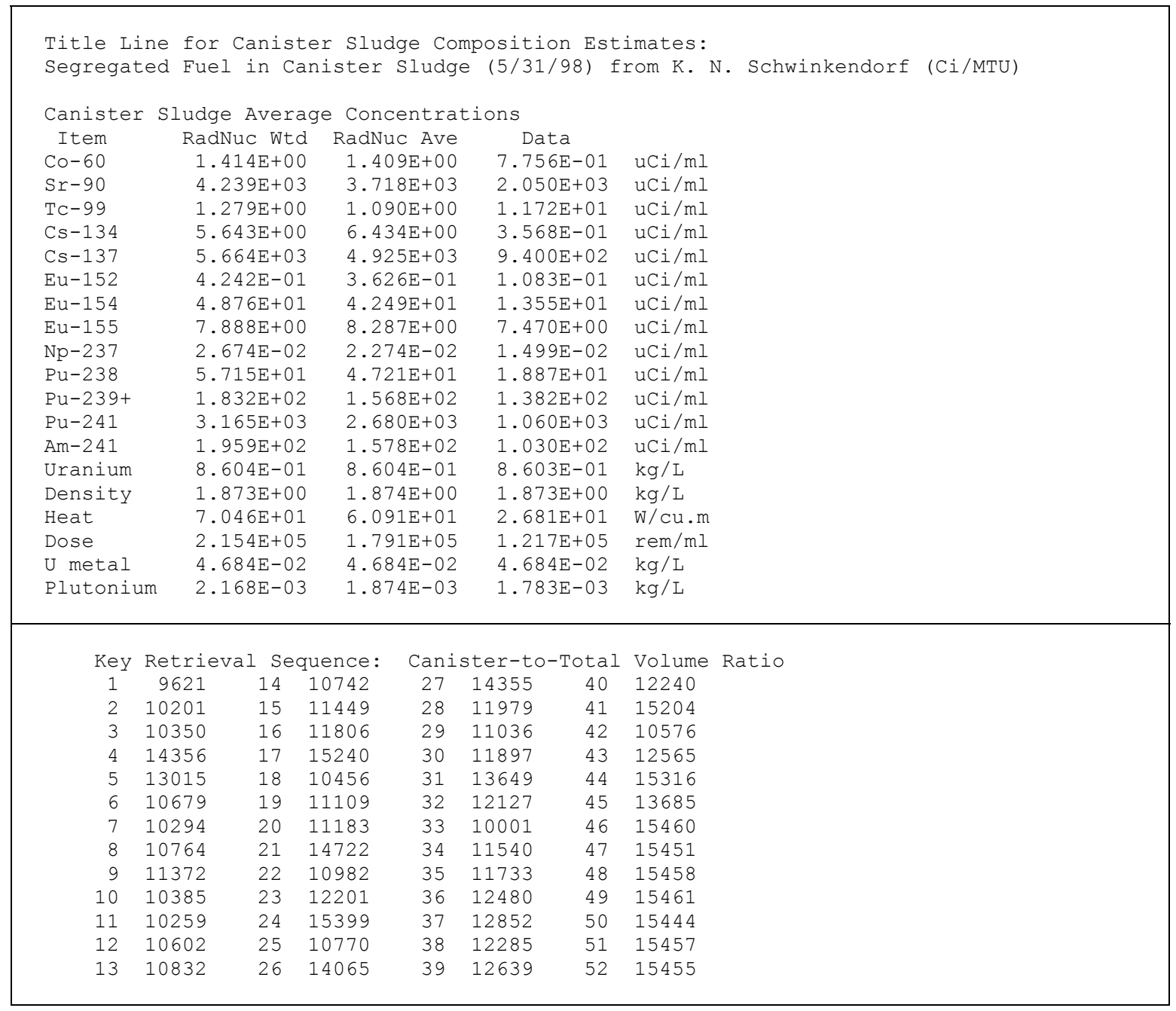

The lower portion of Figure 6-8 lists the key retrieval sequence using the "R1" option in which the keys are retrieved in the order of canister-to-total sludge volume ratio. The only time this table appears is when the " $\mathrm{R}$ " option is in use.

The echo of various input data items ends with the lists shown in Figure 6-8. Then follows a period of inactivity during which the sludge retrieval simulations are performed. The only output during the calculations occurs if a stochastically determined value is outside the range of the array in which it is tallied. An example message appears below.

\section{PDFout[4] needs more bins! 9.777E-02 1.000E-04 0.000E +00}

The first part of this message identifies the tally array that is too small. There are two main arrays: PDFinp and PFDout. The index tells which tracked item is involved. PDFout[4] refers to the Cs-134 concentration in filled sludge containers. The first number is the stochastic concentration, $0.09777 \mu \mathrm{Ci} / \mathrm{ml}$. The second is the bin width for this tally, $1.0 \times 10^{-4} \mu \mathrm{Ci} / \mathrm{ml}$. The 
third number is the lowest value tallied. The stochastic value should be tallied in bin 978 . Because there are only 900 bins available, this stochastic value cannot be tallied. The warning messages normally only appear for the Cs-134 tally due to large values with low probability. The skipped values are included in the reported mean, but not in the other summary statistics.

Once the stochastic calculations are completed, the final result tables are written to the output file. The first output is the total number of containers processed and skipped shown in Figure 6-9. The "Number of Sludge Compositions" may be less than the value entered on the command line when the program is started because of premature termination of the run. The "Number of Key Arrangements" is always the same number entered on the command line. The "Partial Containers Omitted" is the number of containers with less than $90 \%$ of the container volume. These are not included in the tallies. The "Partial Containers Counted" is the number of containers that are more than $90 \%$ but less than $100 \%$ full. The final row in Figure 6-9 shows the total number of containers filled and counted during the simulation.

Figure 6-9. Number of Containers Tallied During a PDF-11 Simulation.

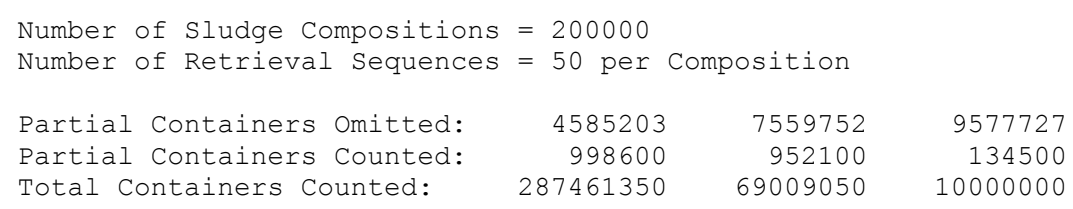

If the "R" option was not selected, then the next portion of the output shows the 2-dimensional distribution of key positions on the random retrieval list. After each key retrieval list is generated, the positions of the keys on the list are tallied. This serves as a check on the random number generator as well as the subroutine that rearranges the retrieval list. The 2-dimensional array has 2,704 positions $(2,704=52 * 52)$. The numbers in each position should be about the same, except for random fluctuations. These numbers are also tallied to verify that the resulting distribution of the 2,704 counts is roughly normal. As shown in Figure 6-10, the program first writes the 2-dimensional array. This large array is divided into three blocks corresponding to positions 1 to 17,18 to 34 , and 35 to 52 . The program then writes a tally of the counts in the 2,704 positions of the array. 
Figure 6-10. Two Dimension Tally of Key Positions on the Retrieval List.

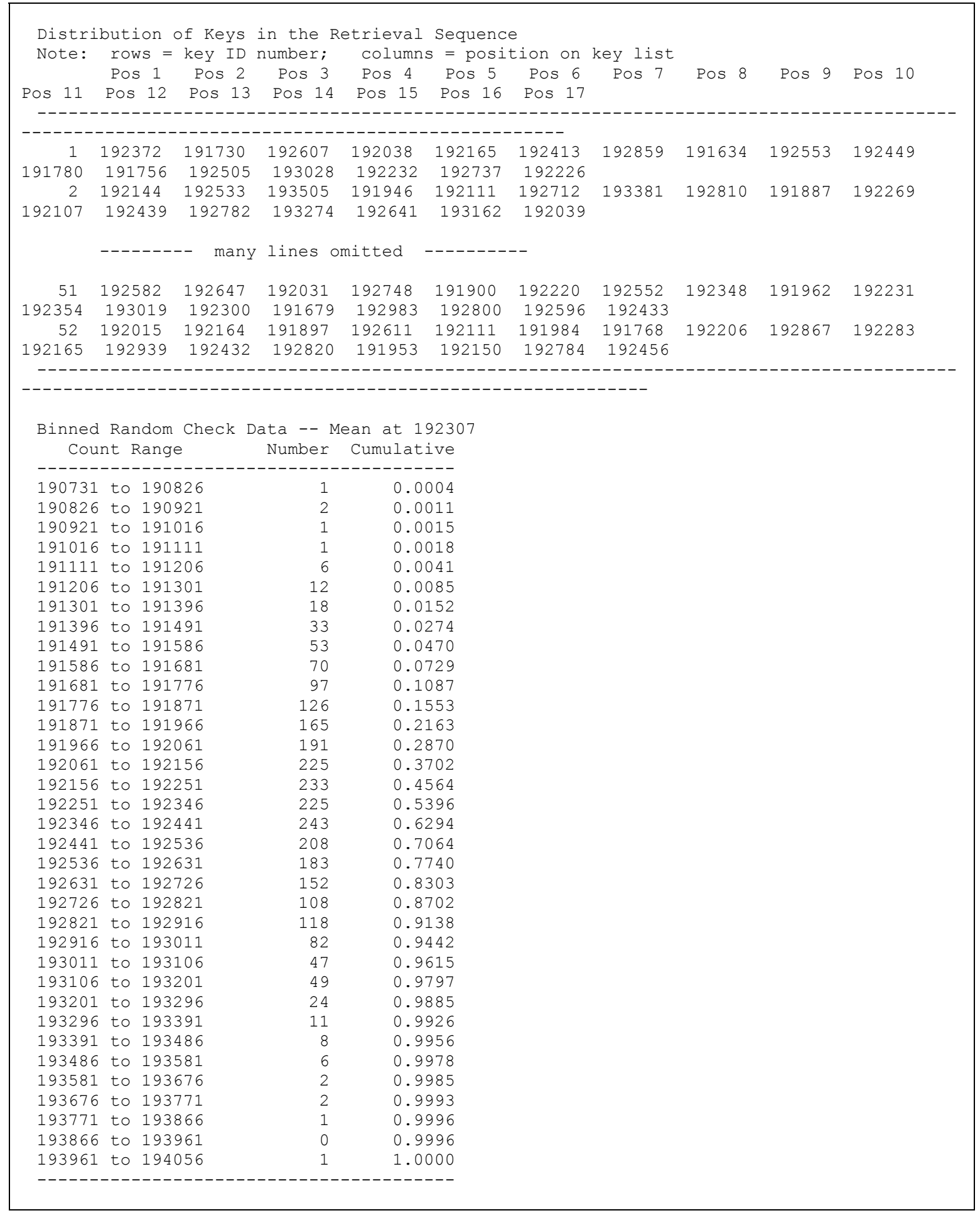

The next portion of the output lists the input parameter distributions. There are four general formats used. The first is a 2-dimensional array showing the canister sludge uranium 
concentration versus sludge density. The second is the table of $\mathrm{Pu}-239 / 240$ specific activity. The third is the sludge depth distribution for the four fuel conditions (good, fair, poor, and bad). The fourth is the total sludge volume distribution for the cubicles that have fuel.

The 2-dimensional array showing the canister sludge uranium concentration versus sludge density is shown in Figure 6-11. It serves to verify that the program has correctly implemented the procedure described in Section 3.4.5. The 2-dimensional array of counts should follow the general shape of Figure 3-24. In particular, the counts in any column should be roughly the same because each column is a uniform distribution. The array has 57 columns and 57 rows, hence the output splits it into 3 blocks. In addition, the vertical bin width (uranium concentration) is halved for the first 10 columns to permit closer examination of the distribution.

Figure 6-11. Two Dimension Tally of Canister Sludge Total Uranium Versus Density.

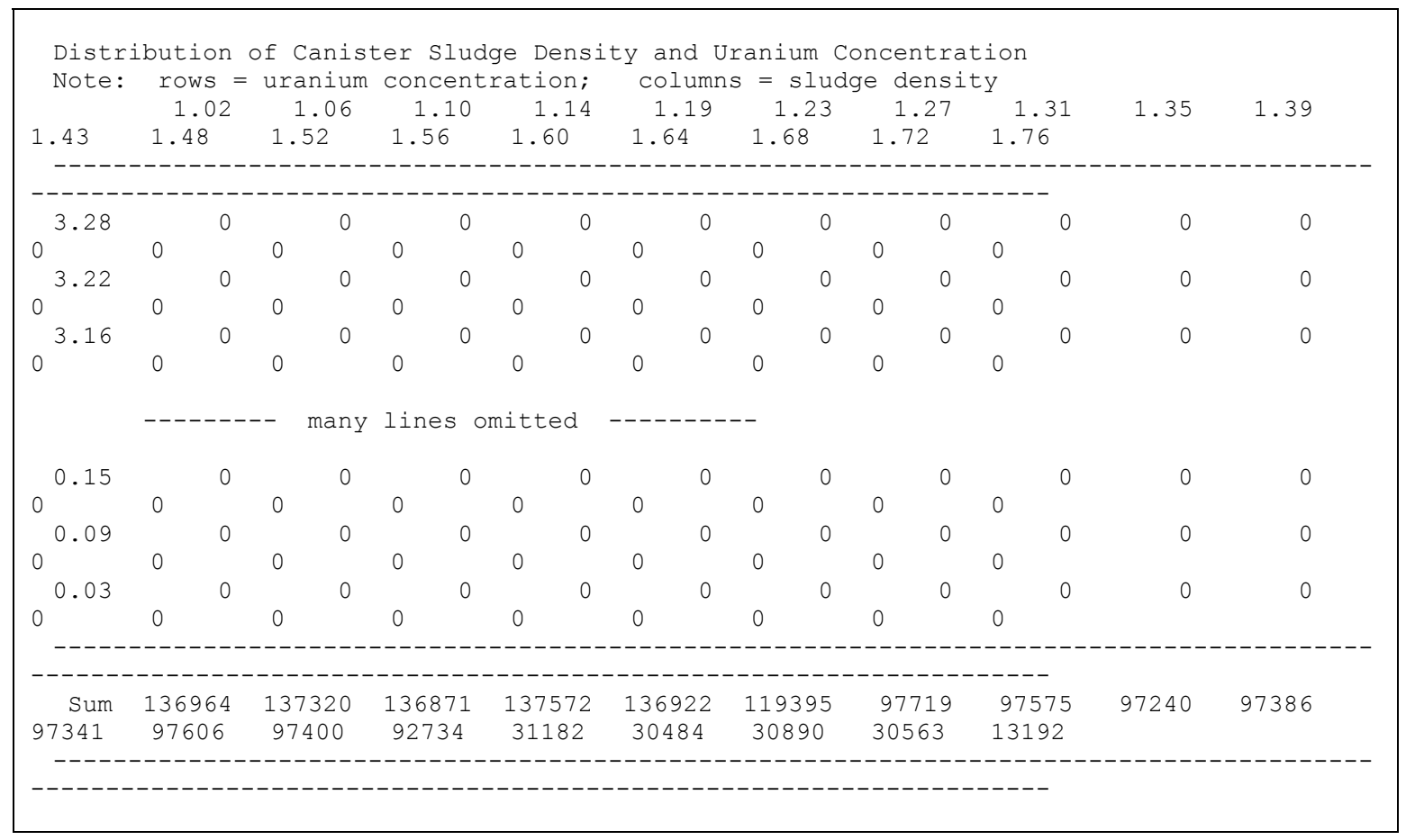

The table showing the tally for $\mathrm{Pu}-239 / 240$ specific activity is shown in Figure 6-12. This table shows the bin boundaries and the number of $\mathrm{Pu}-239 / 240$ specific activities that were found to lie in that bin. Two additional columns facilitate graphical presentation of results. The first extra column shows the bin midpoints. The last column shows the probability per bin. This is computed as the number of counts in a bin divided by the total number of counts.

The table ends with the summary statistics. The total count is the sum of all the bins. This number of bins between the lowest and the highest is shown in parentheses. The average is the arithmetic mean. The 95 and 99 percentile values are calculated from the cumulative distribution. The standard deviation is calculated from the histogram. The maximum and 
minimum values strongly depend on the number of simulated basin retrieval operations. It is possible for the maximum or minimum to not be listed in the output table. The tables are arrays with 300 positions. If a stochastic result is off the low or high end of the array, a message appears in the output file, but no bin receives a count. The off scale results are included in the calculation of the mean, but not in the calculation of the standard deviation. The final two statistics are the geometric mean and geometric standard deviation. These are calculated like the arithmetic mean and standard deviation except that the logarithms of the counts are used rather than the counts.

Figure 6-12. Tally for the Pu-239/240 Specific Activity.

\begin{tabular}{|c|c|c|c|}
\hline \multicolumn{4}{|c|}{ PDF Pu-239/240 Specific Activity } \\
\hline $\mathrm{Pu}-239+$ Spec.Act. Ci/g & Cnt-PDF & Midpoints & Prob-PDF \\
\hline $7.000 \mathrm{E}+01$ to $7.005 \mathrm{E}+01$ & 64855 & $7.003 \mathrm{E}+01$ & $6.236 \mathrm{E}-03$ \\
\hline $7.005 \mathrm{E}+01$ to $7.010 \mathrm{E}+01$ & 65363 & $7.007 \mathrm{E}+01$ & $6.285 \mathrm{E}-03$ \\
\hline $7.010 \mathrm{E}+01$ to $7.015 \mathrm{E}+01$ & 64923 & $7.013 \mathrm{E}+01$ & $6.243 E-03$ \\
\hline $7.015 \mathrm{E}+01$ to $7.020 \mathrm{E}+01$ & 64960 & $7.018 \mathrm{E}+01$ & $6.246 \mathrm{E}-03$ \\
\hline $7.020 \mathrm{E}+01$ to $7.025 \mathrm{E}+01$ & 64883 & $7.022 \mathrm{E}+01$ & $6.239 \mathrm{E}-03$ \\
\hline $7.025 \mathrm{E}+01$ to $7.030 \mathrm{E}+01$ & 64809 & $7.028 \mathrm{E}+01$ & $6.232 \mathrm{E}-03$ \\
\hline $7.030 \mathrm{E}+01$ to $7.035 \mathrm{E}+01$ & 64718 & $7.032 \mathrm{E}+01$ & $6.223 E-03$ \\
\hline $7.035 \mathrm{E}+01$ to $7.040 \mathrm{E}+01$ & 65005 & $7.038 \mathrm{E}+01$ & $6.250 \mathrm{E}-03$ \\
\hline $7.040 \mathrm{E}+01$ to $7.045 \mathrm{E}+01$ & 65296 & $7.043 \mathrm{E}+01$ & $6.278 \mathrm{E}-03$ \\
\hline $7.045 \mathrm{E}+01$ to $7.050 \mathrm{E}+01$ & 65331 & $7.047 \mathrm{E}+01$ & $6.282 \mathrm{E}-03$ \\
\hline $7.050 \mathrm{E}+01$ to $7.055 \mathrm{E}+01$ & 65149 & $7.053 E+01$ & $6.264 \mathrm{E}-03$ \\
\hline $7.055 \mathrm{E}+01$ to $7.060 \mathrm{E}+01$ & 64977 & $7.057 \mathrm{E}+01$ & $6.248 \mathrm{E}-03$ \\
\hline $7.060 \mathrm{E}+01$ to $7.065 \mathrm{E}+01$ & 64943 & $7.063 \mathrm{E}+01$ & $6.245 \mathrm{E}-03$ \\
\hline $7.065 \mathrm{E}+01$ to $7.070 \mathrm{E}+01$ & 64168 & $7.068 \mathrm{E}+01$ & $6.170 \mathrm{E}-03$ \\
\hline $7.070 \mathrm{E}+01$ to $7.075 \mathrm{E}+01$ & 64702 & $7.072 \mathrm{E}+01$ & $6.221 \mathrm{E}-03$ \\
\hline \multicolumn{4}{|c|}{$-------\quad$ many lines omitted ---------} \\
\hline $8.415 \mathrm{E}+01$ to $8.420 \mathrm{E}+01$ & 0 & $8.418 \mathrm{E}+01$ & $0.000 \mathrm{E}+00$ \\
\hline $8.420 \mathrm{E}+01$ to $8.425 \mathrm{E}+01$ & 0 & $8.422 E+01$ & $0.000 \mathrm{E}+00$ \\
\hline $8.425 \mathrm{E}+01$ to $8.430 \mathrm{E}+01$ & 0 & $8.428 E+01$ & $0.000 \mathrm{E}+00$ \\
\hline $8.430 \mathrm{E}+01$ to $8.435 \mathrm{E}+01$ & 0 & $8.432 E+01$ & $0.000 \mathrm{E}+00$ \\
\hline $8.435 \mathrm{E}+01$ to $8.440 \mathrm{E}+01$ & 0 & $8.438 \mathrm{E}+01$ & $0.000 \mathrm{E}+00$ \\
\hline $8.440 \mathrm{E}+01$ to $8.445 \mathrm{E}+01$ & 0 & $8.443 E+01$ & $0.000 \mathrm{E}+00$ \\
\hline $8.445 \mathrm{E}+01$ to $8.450 \mathrm{E}+01$ & 0 & $8.447 \mathrm{E}+01$ & $0.000 \mathrm{E}+00$ \\
\hline $8.450 \mathrm{E}+01$ to $8.455 \mathrm{E}+01$ & 0 & $8.453 \mathrm{E}+01$ & $0.000 \mathrm{E}+00$ \\
\hline $8.455 \mathrm{E}+01$ to $8.460 \mathrm{E}+01$ & 0 & $8.457 \mathrm{E}+01$ & $0.000 \mathrm{E}+00$ \\
\hline $8.460 \mathrm{E}+01$ to $8.465 \mathrm{E}+01$ & 0 & $8.463 \mathrm{E}+01$ & $0.000 \mathrm{E}+00$ \\
\hline $8.465 \mathrm{E}+01$ to $8.470 \mathrm{E}+01$ & 0 & $8.468 \mathrm{E}+01$ & $0.000 \mathrm{E}+00$ \\
\hline $8.470 \mathrm{E}+01$ to $8.475 \mathrm{E}+01$ & 0 & $8.472 \mathrm{E}+01$ & $0.000 \mathrm{E}+00$ \\
\hline $8.475 \mathrm{E}+01$ to $8.480 \mathrm{E}+01$ & 0 & $8.478 \mathrm{E}+01$ & $0.000 \mathrm{E}+00$ \\
\hline $8.480 \mathrm{E}+01$ to $8.485 \mathrm{E}+01$ & 0 & $8.482 \mathrm{E}+01$ & $0.000 \mathrm{E}+00$ \\
\hline $8.485 \mathrm{E}+01$ to $8.490 \mathrm{E}+01$ & 0 & $8.488 E+01$ & $0.000 \mathrm{E}+00$ \\
\hline $8.490 \mathrm{E}+01$ to $8.495 \mathrm{E}+01$ & 0 & $8.493 E+01$ & $0.000 \mathrm{E}+00$ \\
\hline $8.495 \mathrm{E}+01$ to $8.500 \mathrm{E}+01$ & 0 & $8.497 \mathrm{E}+01$ & $0.000 \mathrm{E}+00$ \\
\hline Total Count (260 bins) & 10400000 & & \\
\hline Average Value & $7.805 \mathrm{E}+01$ & & \\
\hline 95th Percentile & $8.275 E+01$ & & \\
\hline 99th Percentile & $8.295 \mathrm{E}+01$ & & \\
\hline Standard Deviation & $4.640 \mathrm{E}+00$ & & \\
\hline Minimum Value & $7.000 \mathrm{E}+01$ & & \\
\hline Maximum Value & $8.300 E+01$ & & \\
\hline Geometric Mean & $7.791 \mathrm{E}+01$ & & \\
\hline Geometric Std. Dev. & $1.063 \mathrm{E}+00$ & & \\
\hline
\end{tabular}


The table showing the sludge depth distribution for the four fuel conditions (good, fair, poor, and bad) is given in Figure 6-13. Note that poor fuel is assumed to have the same distribution as bad fuel in this analysis. The bin boundaries are shown along with the number of occurrences in each bin. The table ends with the summary statistics for each column. The mean is computed two ways. The first is calculated during the simulations. The stochastically determined values are summed. This is the exact mean and is used in the calculation of standard deviation. After the simulation, another mean is computed based on the midpoint of each bin. If these means differ by more than $1 \%$ then an extra row is printed showing the mean computed from the midpoints of the bins. For the tally of the canister sludge depth for good fuel, the two mean values differ appreciably. Hence, the extra line labeled "Binned Mean Value" in Figure 6-13.

Figure 6-13. Canister Sludge Depth Table Output from PDF-11.

\begin{tabular}{|c|c|c|c|c|}
\hline \multicolumn{5}{|l|}{ PDF Canister Sludge Depth } \\
\hline Sludge Depth Range, in & Good & Fair & Poor & Bad \\
\hline $0.000 \mathrm{E}+00$ to $1.000 \mathrm{E}-01$ & 7680545 & 58638 & 0 & 0 \\
\hline $1.000 \mathrm{E}-01$ to $2.000 \mathrm{E}-01$ & 0 & 89718 & 0 & 0 \\
\hline $2.000 \mathrm{E}-01$ to $3.000 \mathrm{E}-01$ & 0 & 174853 & 0 & 0 \\
\hline $3.000 \mathrm{E}-01$ to $4.000 \mathrm{E}-01$ & 0 & 216450 & 0 & 0 \\
\hline $4.000 \mathrm{E}-01$ to $5.000 \mathrm{E}-01$ & 0 & 276390 & 0 & 0 \\
\hline $5.000 \mathrm{E}-01$ to $6.000 \mathrm{E}-01$ & 0 & 277546 & 19809 & 11323 \\
\hline $6.000 \mathrm{E}-01$ to $7.000 \mathrm{E}-01$ & 0 & 282674 & 53572 & 30782 \\
\hline $7.000 \mathrm{E}-01$ to $8.000 \mathrm{E}-01$ & 0 & 284711 & 73483 & 42791 \\
\hline $8.000 \mathrm{E}-01$ to $9.000 \mathrm{E}-01$ & 0 & 294902 & 81732 & 47194 \\
\hline $9.000 \mathrm{E}-01$ to $1.000 \mathrm{E}+00$ & 0 & 312724 & 102711 & 59057 \\
\hline $1.000 \mathrm{E}+00$ to $1.100 \mathrm{E}+00$ & 0 & 331374 & 120562 & 69767 \\
\hline $1.100 \mathrm{E}+00$ to $1.200 \mathrm{E}+00$ & 30888 & 350488 & 137207 & 78942 \\
\hline $1.200 \mathrm{E}+00$ to $1.300 \mathrm{E}+00$ & 82547 & 361698 & 145313 & 83314 \\
\hline $1.300 \mathrm{E}+00$ to $1.400 \mathrm{E}+00$ & 83015 & 366201 & 144567 & 83730 \\
\hline $1.400 \mathrm{E}+00$ to $1.500 \mathrm{E}+00$ & 81966 & 372127 & 144156 & 83222 \\
\hline $1.500 \mathrm{E}+00$ to $1.600 \mathrm{E}+00$ & 82588 & 370984 & 130179 & 75677 \\
\hline \multicolumn{5}{|c|}{$-------\quad$ many lines omitted ---------} \\
\hline $2.860 \mathrm{E}+01$ to $2.870 \mathrm{E}+01$ & 0 & 0 & 0 & 0 \\
\hline $2.870 \mathrm{E}+01$ to $2.880 \mathrm{E}+01$ & 0 & 0 & 0 & 0 \\
\hline $2.880 \mathrm{E}+01$ to $2.890 \mathrm{E}+01$ & 0 & 0 & 0 & 0 \\
\hline $2.890 \mathrm{E}+01$ to $2.900 \mathrm{E}+01$ & 0 & 0 & 0 & 0 \\
\hline $2.900 \mathrm{E}+01$ to $2.910 \mathrm{E}+01$ & 0 & 0 & 0 & 0 \\
\hline $2.910 \mathrm{E}+01$ to $2.920 \mathrm{E}+01$ & 0 & 0 & 0 & 0 \\
\hline $2.920 \mathrm{E}+01$ to $2.930 \mathrm{E}+01$ & 0 & 0 & 0 & 0 \\
\hline $2.930 \mathrm{E}+01$ to $2.940 \mathrm{E}+01$ & 0 & 0 & 0 & 0 \\
\hline $2.940 \mathrm{E}+01$ to $2.950 \mathrm{E}+01$ & 0 & 0 & 0 & 0 \\
\hline $2.950 \mathrm{E}+01$ to $2.960 \mathrm{E}+01$ & 0 & 0 & 0 & 0 \\
\hline $2.960 \mathrm{E}+01$ to $2.970 \mathrm{E}+01$ & 0 & 0 & 0 & 0 \\
\hline $2.970 \mathrm{E}+01$ to $2.980 \mathrm{E}+01$ & 0 & 0 & 0 & 0 \\
\hline $2.980 \mathrm{E}+01$ to $2.990 \mathrm{E}+01$ & 0 & 0 & 0 & 0 \\
\hline $2.990 \mathrm{E}+01$ to $3.000 \mathrm{E}+01$ & 0 & 0 & 0 & 0 \\
\hline Total Count (270 bins) & 9600000 & 8200000 & 5200000 & 3000000 \\
\hline Average Value & $4.649 \mathrm{E}-01$ & $1.614 \mathrm{E}+00$ & $4.557 \mathrm{E}+00$ & $4.556 \mathrm{E}+00$ \\
\hline 95th Percentile & $2.906 \mathrm{E}+00$ & $3.304 \mathrm{E}+00$ & $1.340 \mathrm{E}+01$ & $1.340 \mathrm{E}+01$ \\
\hline 99th Percentile & $3.371 \mathrm{E}+00$ & $3.806 \mathrm{E}+00$ & $2.324 \mathrm{E}+01$ & $2.326 \mathrm{E}+01$ \\
\hline Standard Deviation & $9.590 \mathrm{E}-01$ & $8.931 \mathrm{E}-01$ & $4.439 \mathrm{E}+00$ & $4.443 \mathrm{E}+00$ \\
\hline Minimum Value & $0.000 \mathrm{E}+00$ & $2.772 \mathrm{E}-02$ & $5.022 \mathrm{E}-01$ & $5.022 \mathrm{E}-01$ \\
\hline Maximum Value & $3.487 \mathrm{E}+00$ & $4.523 E+00$ & $2.700 \mathrm{E}+01$ & $2.700 \mathrm{E}+01$ \\
\hline Geometric Mean & $1.068 \mathrm{E}-01$ & $1.320 \mathrm{E}+00$ & $3.190 \mathrm{E}+00$ & $3.189 \mathrm{E}+00$ \\
\hline Geometric Std. Dev. & $8.315 \mathrm{E}+00$ & $2.113 \mathrm{E}+00$ & $2.455 \mathrm{E}+00$ & $2.456 \mathrm{E}+00$ \\
\hline Binned Mean Value & $5.049 \mathrm{E}-01$ & $1.614 \mathrm{E}+00$ & $4.557 \mathrm{E}+00$ & $4.557 \mathrm{E}+00$ \\
\hline
\end{tabular}


The table showing the total sludge volume in all keys has 3 columns with counts for floor sludge, canister sludge, and the sum of the floor and canister. The total sludge volume table uses the same format as the canister sludge depth table, except that it has 3 columns rather than 4 . Thus, no example is presented.

The next 38 tables show the tallies of the input PDFs for floor and canister sludge concentration. They all follow the same format as shown in Figure 6-12 for Pu-239/240 specific activity. There are 19 tables for floor sludge and 19 tables for canister sludge. Each group of 19 tables includes the 13 isotopes (Co-60, Sr-90, Tc-99, Cs-134, Cs-137, Eu-152, Eu-154, Eu-155, Np-237, Pu-238, $\mathrm{Pu}-239 / 240, \mathrm{Pu}-241$, and $\mathrm{Am}-241$ ), uranium concentration, sludge density, heat generation rate, inhalation dose, uranium metal and total plutonium.

The tables of probability distributions for the floor sludge and canister sludge end with a summary table. The actual appearance of this information in the output file is shown in Figure 6-14. There are 46 lines of numbers in this table. The columns are the statistics presented in the previous 41 tables.

Figure 6-14. Summary Table for Various Input Distributions in the Output from PDF-11. (2 sheets)

\begin{tabular}{|c|c|c|c|c|c|c|}
\hline $\begin{array}{l}\text { Input PDF } \\
\text { Geom Mean }\end{array}$ & Average & 95 th & 99 th & Std Dev & Minimum & Maximum \\
\hline $\begin{array}{l}\mathrm{Pu}-239+\text { Spec. Act. Ci/g } \\
7.791 \mathrm{E}+01 \quad 1.063 \mathrm{E}+00\end{array}$ & $7.805 \mathrm{E}+01$ & $8.275 E+01$ & $8.295 \mathrm{E}+01$ & $4.640 \mathrm{E}+00$ & $7.000 \mathrm{E}+01$ & $8.300 \mathrm{E}+01$ \\
\hline $\begin{array}{l}\text { Sludge Depth Range, in } \\
1.067 \mathrm{E}-01 \quad 8.309 \mathrm{E}+00\end{array}$ & $4.644 \mathrm{E}-01$ & $2.905 \mathrm{E}+00$ & $3.371 \mathrm{E}+00$ & $9.584 \mathrm{E}-01$ & $0.000 \mathrm{E}+00$ & $3.487 \mathrm{E}+00$ \\
\hline $\begin{array}{l}\text { Sludge Depth Range, in } \\
\begin{array}{ll}1.320 \mathrm{E}+00 \quad 2.112 \mathrm{E}+00\end{array}\end{array}$ & $1.615 \mathrm{E}+00$ & $3.304 \mathrm{E}+00$ & $3.806 \mathrm{E}+00$ & $8.932 E-01$ & $2.772 \mathrm{E}-02$ & $4.533 \mathrm{E}+00$ \\
\hline $\begin{array}{l}\text { Sludge Depth Range, in } \\
\begin{array}{ll}3.190 \mathrm{E}+00 & 2.454 \mathrm{E}+00\end{array}\end{array}$ & $4.557 \mathrm{E}+00$ & $1.339 \mathrm{E}+01$ & $2.327 \mathrm{E}+01$ & $4.441 \mathrm{E}+00$ & $5.022 \mathrm{E}-01$ & $2.700 \mathrm{E}+01$ \\
\hline $\begin{array}{l}\text { Sludge Depth Range, in } \\
3.192 \mathrm{E}+00 \quad 2.455 \mathrm{E}+00\end{array}$ & $4.560 \mathrm{E}+00$ & $1.341 \mathrm{E}+01$ & $2.324 E+01$ & $4.441 \mathrm{E}+00$ & $5.022 \mathrm{E}-01$ & $2.700 \mathrm{E}+01$ \\
\hline $\begin{array}{l}\text { Floor Volume, cu.m } \\
\begin{array}{ll}1.163 \mathrm{E}+01 & 1.124 \mathrm{E}+00\end{array}\end{array}$ & $1.171 \mathrm{E}+01$ & $1.381 \mathrm{E}+01$ & $1.400 \mathrm{E}+01$ & $1.351 \mathrm{E}+00$ & $9.367 \mathrm{E}+00$ & $1.405 \mathrm{E}+01$ \\
\hline $\begin{array}{l}\text { Canister Vol, cu.m } \\
2.835 \mathrm{E}+00 \quad 1.152 \mathrm{E}+00\end{array}$ & $2.863 E+00$ & $3.620 \mathrm{E}+00$ & $3.987 \mathrm{E}+00$ & $4.115 \mathrm{E}-01$ & $1.592 \mathrm{E}+00$ & $5.211 \mathrm{E}+00$ \\
\hline $\begin{array}{l}\text { Total Volume, cu.m } \\
\begin{array}{ll}1.450 \mathrm{E}+01 & 1.103 \mathrm{E}+00\end{array}\end{array}$ & $1.457 \mathrm{E}+01$ & $1.678 \mathrm{E}+01$ & $1.732 \mathrm{E}+01$ & $1.413 \mathrm{E}+00$ & $1.134 \mathrm{E}+01$ & $1.868 \mathrm{E}+01$ \\
\hline $\begin{array}{l}\text { Co-60 uCi/ml Floor PDF } \\
\begin{array}{ll}5.259 \mathrm{E}-01 & 2.870 \mathrm{E}+00\end{array}\end{array}$ & $8.143 E-01$ & $2.908 \mathrm{E}+00$ & $3.929 \mathrm{E}+00$ & $8.428 \mathrm{E}-01$ & $2.314 \mathrm{E}-02$ & $4.928 \mathrm{E}+00$ \\
\hline $\begin{array}{l}\text { Sr-90 uCi/ml Floor PDF } \\
5.545 \mathrm{E}+01 \quad 6.327 \mathrm{E}+00\end{array}$ & $1.804 \mathrm{E}+02$ & $5.981 \mathrm{E}+02$ & $2.885 E+03$ & $4.542 \mathrm{E}+02$ & $7.974 \mathrm{E}-01$ & $3.629 \mathrm{E}+03$ \\
\hline $\begin{array}{l}\mathrm{TC}-99 \mathrm{uCi} / \mathrm{ml} \text { Floor PDF } \\
3.834 \mathrm{E}-02 \quad 3.648 \mathrm{E}+00\end{array}$ & $6.994 E-02$ & $1.896 \mathrm{E}-01$ & $6.539 \mathrm{E}-01$ & $1.032 \mathrm{E}-01$ & $3.518 \mathrm{E}-04$ & $8.226 \mathrm{E}-01$ \\
\hline $\begin{array}{l}\mathrm{Cs}-134 \mathrm{uCi} / \mathrm{ml} \text { Floor PDF } \\
2.927 \mathrm{E}-02 \quad 8.221 \mathrm{E}+00\end{array}$ & $1.123 \mathrm{E}-01$ & $4.817 \mathrm{E}-01$ & $1.375 \mathrm{E}+00$ & $2.321 \mathrm{E}-01$ & $1.314 \mathrm{E}-03$ & $1.729 \mathrm{E}+00$ \\
\hline $\begin{array}{l}\mathrm{Cs}-137 \mathrm{uCi} / \mathrm{ml} \text { Floor PDF } \\
7.289 \mathrm{E}+01 \quad 7.404 \mathrm{E}+00\end{array}$ & $2.548 E+02$ & $1.079 \mathrm{E}+03$ & $3.081 E+03$ & $5.184 \mathrm{E}+02$ & $2.944 \mathrm{E}+00$ & $3.875 \mathrm{E}+03$ \\
\hline $\begin{array}{l}\mathrm{Eu}-152 \mathrm{uCi} / \mathrm{ml} \text { Floor PDF } \\
5.242 \mathrm{E}-03 \quad 4.295 \mathrm{E}+00\end{array}$ & $1.175 \mathrm{E}-02$ & $4.261 \mathrm{E}-02$ & $1.485 E-01$ & $2.347 \mathrm{E}-02$ & $5.238 E-05$ & $1.868 \mathrm{E}-01$ \\
\hline $\begin{array}{l}\mathrm{Eu}-154 \mathrm{uCi} / \mathrm{ml} \text { Floor PDF } \\
6.559 \mathrm{E}-01 \quad 4.295 \mathrm{E}+00\end{array}$ & $1.470 \mathrm{E}+00$ & $5.332 \mathrm{E}+00$ & $1.859 \mathrm{E}+01$ & $2.937 \mathrm{E}+00$ & $6.554 \mathrm{E}-03$ & $2.338 \mathrm{E}+01$ \\
\hline $\begin{array}{l}\mathrm{Eu}-155 \mathrm{uCi} / \mathrm{ml} \text { Floor PDF } \\
3.211 \mathrm{E}-01 \quad 4.539 \mathrm{E}+00\end{array}$ & 7. $258 \mathrm{E}-01$ & $2.568 \mathrm{E}+00$ & $8.571 \mathrm{E}+00$ & $1.360 \mathrm{E}+00$ & $3.224 \mathrm{E}-03$ & $1.078 \mathrm{E}+01$ \\
\hline $\begin{array}{l}\mathrm{Np}-237 \mathrm{uCi} / \mathrm{ml} \text { Floor } \mathrm{PDF} \\
7.638 \mathrm{E}-04 \quad 3.629 \mathrm{E}+00\end{array}$ & $1.390 \mathrm{E}-03$ & $3.636 \mathrm{E}-03$ & $1.320 \mathrm{E}-02$ & $2.070 \mathrm{E}-03$ & $7.102 \mathrm{E}-06$ & $1.660 \mathrm{E}-02$ \\
\hline $\begin{array}{l}\mathrm{Pu}-238 \mathrm{uCi} / \mathrm{ml} \text { Floor } \mathrm{PDF} \\
9.369 \mathrm{E}-01 \quad 3.864 \mathrm{E}+00\end{array}$ & $1.870 \mathrm{E}+00$ & $8.486 \mathrm{E}+00$ & $1.830 \mathrm{E}+01$ & $3.147 \mathrm{E}+00$ & $7.736 \mathrm{E}-03$ & $2.302 \mathrm{E}+01$ \\
\hline
\end{tabular}


Figure 6-14. Summary Table for Various Input Distributions in the Output from PDF-11. (2 sheets)

\begin{tabular}{|c|c|c|c|c|c|c|c|}
\hline $\begin{array}{l}\mathrm{Pu}-239 / 240 \\
5.487 \mathrm{E}+00\end{array}$ & $\begin{array}{l}\text { Floor PDF } \\
3.650 \mathrm{E}+00\end{array}$ & $1.001 \mathrm{E}+01$ & $2.713 E+01$ & $9.361 \mathrm{E}+01$ & $1.477 \mathrm{E}+01$ & $5.036 \mathrm{E}-02$ & $1.178 \mathrm{E}+02$ \\
\hline $\begin{array}{l}\mathrm{Pu}-241 \mathrm{uCi} / \mathrm{m} \\
5.263 \mathrm{E}+01\end{array}$ & $\begin{array}{c}\mathrm{ml} \text { Floor PDF } \\
3.860 \mathrm{E}+00\end{array}$ & $1.050 \mathrm{E}+02$ & $4.765 \mathrm{E}+02$ & $1.027 \mathrm{E}+03$ & $1.767 \mathrm{E}+02$ & $4.344 \mathrm{E}-01$ & $1.292 \mathrm{E}+03$ \\
\hline $\begin{array}{l}A m-241 \mathrm{uCi} / \mathrm{m} \\
4.993 \mathrm{E}+00\end{array}$ & $\begin{array}{c}\text { ml Floor PDF } \\
3.945 \mathrm{E}+00\end{array}$ & $1.018 \mathrm{E}+01$ & $3.250 \mathrm{E}+01$ & $1.170 \mathrm{E}+02$ & $1.836 \mathrm{E}+01$ & $4.296 \mathrm{E}-02$ & $1.472 \mathrm{E}+02$ \\
\hline $\begin{array}{l}\text { Uranium } \mathrm{kg} / \mathrm{I} \\
2.721 \mathrm{E}-02\end{array}$ & $\begin{array}{l}\text { L Floor PDF } \\
4.248 \mathrm{E}+00\end{array}$ & $6.408 E-02$ & $1.899 \mathrm{E}-01$ & $9.823 \mathrm{E}-01$ & $1.521 \mathrm{E}-01$ & $3.524 \mathrm{E}-04$ & $1.236 \mathrm{E}+00$ \\
\hline $\begin{array}{l}\text { Density } \mathrm{kg} / \mathrm{I} \\
1.247 \mathrm{E}+00\end{array}$ & $\begin{array}{l}\text { L Floor PDF } \\
1.189 \mathrm{E}+00\end{array}$ & $1.269 \mathrm{E}+00$ & $1.635 \mathrm{E}+00$ & $2.832 \mathrm{E}+00$ & $2.838 \mathrm{E}-01$ & $1.008 \mathrm{E}+00$ & $3.304 \mathrm{E}+00$ \\
\hline $\begin{array}{l}\text { Heat W/cu.m } \\
1.293 \mathrm{E}+00\end{array}$ & $\begin{array}{l}\text { Floor PDF } \\
4.647 \mathrm{E}+00\end{array}$ & $3.180 \mathrm{E}+00$ & $9.996 \mathrm{E}+00$ & $4.186 \mathrm{E}+01$ & $6.631 E+00$ & $6.536 \mathrm{E}-02$ & $5.266 \mathrm{E}+01$ \\
\hline $\begin{array}{l}\text { Dose } \mathrm{rem} / \mathrm{ml} \\
5.468 \mathrm{E}+03\end{array}$ & $\begin{array}{l}\text { Floor PDF } \\
3.763 \mathrm{E}+00\end{array}$ & $1.047 \mathrm{E}+04$ & $2.885 E+04$ & $1.086 \mathrm{E}+05$ & $1.703 E+04$ & $5.088 E+01$ & $1.366 \mathrm{E}+05$ \\
\hline $\begin{array}{l}\text { U metal } \mathrm{kg} / \mathrm{I} \\
6.907 \mathrm{E}-03\end{array}$ & $\begin{array}{l}\text { L Floor PDF } \\
2.840 \mathrm{E}+00\end{array}$ & $9.517 \mathrm{E}-03$ & $1.860 \mathrm{E}-02$ & $2.567 \mathrm{E}-02$ & $6.020 E-03$ & $9.039 \mathrm{E}-10$ & $4.940 \mathrm{E}-02$ \\
\hline $\begin{array}{l}\mathrm{Pu} \mathrm{kg} / \mathrm{L} \\
7.131 \mathrm{E}-05\end{array}$ & $\begin{array}{l}\text { Floor PDF } \\
3.618 \mathrm{E}+00\end{array}$ & $1.299 \mathrm{E}-04$ & $3.549 \mathrm{E}-04$ & $1.210 \mathrm{E}-03$ & $1.923 \mathrm{E}-04$ & $6.120 \mathrm{E}-07$ & $1.695 \mathrm{E}-03$ \\
\hline $\begin{array}{l}C \circ-60 \\
8.029 \mathrm{E}-01\end{array}$ & $\begin{array}{c}\text { Canister PDF } \\
3.979 \mathrm{E}+00\end{array}$ & $1.431 E+00$ & $4.248 E+00$ & $6.695 \mathrm{E}+00$ & $1.445 E+00$ & $8.419 E-08$ & $1.425 \mathrm{E}+01$ \\
\hline $\begin{array}{l}\mathrm{Sr}-90 \\
2.089 \mathrm{E}+03\end{array}$ & $\begin{array}{c}\text { Canister PDF } \\
4.202 \mathrm{E}+00\end{array}$ & $3.774 \mathrm{E}+03$ & $1.102 \mathrm{E}+04$ & $1.484 \mathrm{E}+04$ & $3.527 \mathrm{E}+03$ & $9.733 \mathrm{E}-05$ & $2.682 \mathrm{E}+04$ \\
\hline $\begin{array}{l}\mathrm{TC}-99 \\
5.818 \mathrm{E}-01\end{array}$ & $\begin{array}{c}\text { Canister PDF } \\
4.524 \mathrm{E}+00\end{array}$ & $1.107 \mathrm{E}+00$ & $3.310 \mathrm{E}+00$ & $4.459 \mathrm{E}+00$ & $1.071 E+00$ & $2.064 E-08$ & $7.862 \mathrm{E}+00$ \\
\hline $\begin{array}{l}\mathrm{Cs}-134 \\
2.464 \mathrm{E}+00\end{array}$ & $\begin{array}{c}\text { Canister PDF } \\
5.767 \mathrm{E}+00\end{array}$ & $6.531 E+00$ & $2.357 E+01$ & $6.282 \mathrm{E}+01$ & $1.168 \mathrm{E}+01$ & $3.326 \mathrm{E}-07$ & $1.603 \mathrm{E}+02$ \\
\hline $\begin{array}{l}C s-137 \\
2.707 \mathrm{E}+03\end{array}$ & $\begin{array}{c}\text { Canister PDF } \\
4.315 \mathrm{E}+00\end{array}$ & $5.001 \mathrm{E}+03$ & $1.488 \mathrm{E}+04$ & $2.019 E+04$ & $4.793 E+03$ & $1.142 \mathrm{E}-04$ & $3.786 \mathrm{E}+04$ \\
\hline $\begin{array}{l}\mathrm{Eu}-152 \\
1.698 \mathrm{E}-01\end{array}$ & $\begin{array}{c}\text { Canister PDF } \\
5.231 \mathrm{E}+00\end{array}$ & $3.681 \mathrm{E}-01$ & $1.205 \mathrm{E}+00$ & $1.729 \mathrm{E}+00$ & $4.063 E-01$ & $3.898 E-09$ & $3.848 \mathrm{E}+00$ \\
\hline $\begin{array}{l}\mathrm{Eu}-154 \\
1.917 \mathrm{E}+01\end{array}$ & $\begin{array}{c}\text { Canister PDF } \\
5.262 \mathrm{E}+00\end{array}$ & $4.314 \mathrm{E}+01$ & $1.485 \mathrm{E}+02$ & $2.318 E+02$ & $5.250 \mathrm{E}+01$ & $4.649 \mathrm{E}-07$ & $6.065 E+02$ \\
\hline $\begin{array}{l}\mathrm{Eu}-155 \\
4.705 \mathrm{E}+00\end{array}$ & $\begin{array}{c}\text { Canister PDF } \\
4.029 \mathrm{E}+00\end{array}$ & $8.413 E+00$ & $2.501 \mathrm{E}+01$ & $3.852 \mathrm{E}+01$ & $8.386 \mathrm{E}+00$ & $6.948 E-07$ & $7.984 \mathrm{E}+01$ \\
\hline $\begin{array}{l}\mathrm{Np}-237 \\
1.206 \mathrm{E}-02\end{array}$ & $\begin{array}{c}\text { Canister PDF } \\
4.553 \mathrm{E}+00\end{array}$ & $2.309 \mathrm{E}-02$ & $6.946 \mathrm{E}-02$ & $9.397 \mathrm{E}-02$ & $2.254 \mathrm{E}-02$ & $4.141 \mathrm{E}-10$ & $1.685 \mathrm{E}-01$ \\
\hline $\begin{array}{l}\mathrm{Pu}-238 \\
1.999 \mathrm{E}+01\end{array}$ & $\begin{array}{c}\text { Canister PDF } \\
5.947 \mathrm{E}+00\end{array}$ & $4.793 E+01$ & $1.618 \mathrm{E}+02$ & $2.374 \mathrm{E}+02$ & $5.560 \mathrm{E}+01$ & $2.729 \mathrm{E}-07$ & $5.463 E+02$ \\
\hline $\begin{array}{l}\mathrm{Pu}-239+ \\
8.474 \mathrm{E}+01\end{array}$ & $\begin{array}{c}\text { Canister PDF } \\
4.441 \mathrm{E}+00\end{array}$ & $1.592 \mathrm{E}+02$ & $4.723 E+02$ & $6.347 \mathrm{E}+02$ & $1.525 \mathrm{E}+02$ & $3.148 E-06$ & $1.116 \mathrm{E}+03$ \\
\hline $\begin{array}{l}\mathrm{Pu}-241 \\
1.188 \mathrm{E}+03\end{array}$ & $\begin{array}{c}\text { Canister PDF } \\
5.540 \mathrm{E}+00\end{array}$ & $2.721 \mathrm{E}+03$ & $9.138 E+03$ & $1.336 \mathrm{E}+04$ & $3.133 E+03$ & $2.091 \mathrm{E}-05$ & $3.158 \mathrm{E}+04$ \\
\hline $\begin{array}{l}\mathrm{Am}-241 \\
6.059 \mathrm{E}+01\end{array}$ & $\begin{array}{c}\text { Canister PDF } \\
7.083 \mathrm{E}+00\end{array}$ & $1.602 \mathrm{E}+02$ & $5.408 E+02$ & $7.776 \mathrm{E}+02$ & $1.837 \mathrm{E}+02$ & $5.157 \mathrm{E}-07$ & $1.534 \mathrm{E}+03$ \\
\hline $\begin{array}{l}\text { Uranium } \\
5.768 \mathrm{E}-01\end{array}$ & $\begin{array}{c}\text { Canister PDF } \\
3.204 \mathrm{E}+00\end{array}$ & $8.735 E-01$ & $2.162 \mathrm{E}+00$ & $2.663 \mathrm{E}+00$ & $6.607 \mathrm{E}-01$ & $8.325 E-08$ & $3.274 \mathrm{E}+00$ \\
\hline $\begin{array}{l}\text { Density } \\
1.781 \mathrm{E}+00\end{array}$ & $\begin{array}{c}\text { Canister PDF } \\
1.380 \mathrm{E}+00\end{array}$ & $1.874 \mathrm{E}+00$ & $2.985 \mathrm{E}+00$ & $3.193 \mathrm{E}+00$ & $5.999 \mathrm{E}-01$ & $1.075 \mathrm{E}+00$ & $3.333 E+00$ \\
\hline $\begin{array}{l}\text { Heat/cu.m } \\
3.324 \mathrm{E}+01\end{array}$ & $\begin{array}{c}\text { Canister PDF } \\
4.342 \mathrm{E}+00\end{array}$ & $6.184 \mathrm{E}+01$ & $1.850 \mathrm{E}+02$ & $2.512 \mathrm{E}+02$ & $5.972 \mathrm{E}+01$ & $1.338 E-06$ & $4.738 E+02$ \\
\hline $\begin{array}{l}\text { Dose/ml } \\
8.503 \mathrm{E}+04\end{array}$ & $\begin{array}{c}\text { Canister PDF } \\
5.219 \mathrm{E}+00\end{array}$ & $1.818 \mathrm{E}+05$ & $5.808 E+05$ & $8.156 \mathrm{E}+05$ & $1.936 \mathrm{E}+05$ & $1.887 \mathrm{E}-03$ & $1.643 \mathrm{E}+06$ \\
\hline $\begin{array}{l}\text { U metal } \\
3.233 \mathrm{E}-02\end{array}$ & $\begin{array}{c}\text { Canister PDF } \\
3.028 \mathrm{E}+00\end{array}$ & $4.653 \mathrm{E}-02$ & $1.089 \mathrm{E}-01$ & $1.350 \mathrm{E}-01$ & $3.228 \mathrm{E}-02$ & $1.835 \mathrm{E}-09$ & $1.664 \mathrm{E}-01$ \\
\hline $\begin{array}{l}\mathrm{Pu} \mathrm{kg} / \mathrm{L} \\
1.041 \mathrm{E}-03\end{array}$ & $\begin{array}{c}\text { Canister PDF } \\
4.299 \mathrm{E}+00\end{array}$ & $1.903 E-03$ & $5.526 \mathrm{E}-03$ & $7.337 \mathrm{E}-03$ & $1.770 \mathrm{E}-03$ & $4.390 \mathrm{E}-11$ & $1.230 \mathrm{E}-02$ \\
\hline
\end{tabular}

The final block of tables in the output file show the results for the container volumes entered on the command line. The 23 tables are arranged as shown in Figure 6-15. Each column is labeled with the container volume. The line labeled "Total Count" shows the number of bins in the table. This number is at most 900 based on the array size used for the container sludge tallies. 
SNF-10293, REV 0

Figure 6-15. Example Container Distribution Table in the Output from PDF-11.

\begin{tabular}{|c|c|c|c|}
\hline \multicolumn{4}{|l|}{ Canister-to-Total Ratios } \\
\hline Canister/Total Ratios & $0.5 \mathrm{cu} . \mathrm{m}$ & $2.0 \mathrm{cu} \cdot \mathrm{m}$ & $12.0 \mathrm{cu} . \mathrm{m}$ \\
\hline $0.000 \mathrm{E}+00$ to $4.000 \mathrm{E}-03$ & 4785615 & 5181 & 0 \\
\hline $4.000 \mathrm{E}-03$ to $8.000 \mathrm{E}-03$ & 7571614 & 33683 & 0 \\
\hline $8.000 \mathrm{E}-03$ to $1.200 \mathrm{E}-02$ & 4666292 & 67597 & 0 \\
\hline 1.200E-02 to $1.600 \mathrm{E}-02$ & 2668982 & 92441 & 0 \\
\hline $1.600 \mathrm{E}-02$ to $2.000 \mathrm{E}-02$ & 1609865 & 116143 & 0 \\
\hline $2.000 \mathrm{E}-02$ to $2.400 \mathrm{E}-02$ & 1435659 & 141747 & 0 \\
\hline $2.400 \mathrm{E}-02$ to $2.800 \mathrm{E}-02$ & 1404592 & 167936 & 0 \\
\hline \multicolumn{2}{|c|}{-------- many lines omitted } & -------- & \\
\hline $1.580 \mathrm{E}+00$ to $1.584 \mathrm{E}+00$ & 0 & 0 & 0 \\
\hline $1.584 \mathrm{E}+00$ to $1.588 \mathrm{E}+00$ & 0 & 0 & 0 \\
\hline $1.588 \mathrm{E}+00$ to $1.592 \mathrm{E}+00$ & 0 & 0 & 0 \\
\hline $1.592 \mathrm{E}+00$ to $1.596 \mathrm{E}+00$ & 0 & 0 & 0 \\
\hline $1.596 \mathrm{E}+00$ to $1.600 \mathrm{E}+00$ & 0 & 0 & 0 \\
\hline Total Count (195 bins) & 287461350 & 69009050 & 10000000 \\
\hline Average Value & $1.963 E-01$ & $1.959 \mathrm{E}-01$ & $1.972 \mathrm{E}-01$ \\
\hline 95th Percentile & $3.714 \mathrm{E}-01$ & $3.129 \mathrm{E}-01$ & $2.527 \mathrm{E}-01$ \\
\hline 99th Percentile & $4.519 \mathrm{E}-01$ & $3.635 E-01$ & $2.773 \mathrm{E}-01$ \\
\hline Standard Deviation & $1.072 \mathrm{E}-01$ & $7.227 \mathrm{E}-02$ & $3.184 \mathrm{E}-02$ \\
\hline Minimum Value & $3.933 E-05$ & $1.277 \mathrm{E}-04$ & $8.562 \mathrm{E}-02$ \\
\hline Maximum Value & $7.795 \mathrm{E}-01$ & $5.684 \mathrm{E}-01$ & $3.617 \mathrm{E}-01$ \\
\hline Geometric Mean & 1. $438 \mathrm{E}-01$ & $1.784 \mathrm{E}-01$ & 1. $946 \mathrm{E}-01$ \\
\hline Geometric Std. Dev. & $3.021 E+00$ & $1.648 \mathrm{E}+00$ & $1.177 \mathrm{E}+00$ \\
\hline
\end{tabular}

The final result written to the output file is the summary statistics for each item tallied for the container inventory. There is one summary table for each volume. An example of these summary tables is shown in Figure 6-12. The 23 items are presented in the rows of the table. The columns are the statistics presented in the previous 23 tables. 
Figure 6-16. Summary of Container Inventories in the Output from PDF-11.

\begin{tabular}{|c|c|c|c|c|c|c|}
\hline $\begin{array}{cl}2.0 \text { cu.m } & \text { Volume } \\
\text { Geom Mean } & \text { Geom SD }\end{array}$ & Average & 95 th & 99 th & Std Dev & Minimum & Maximum \\
\hline Canister/Total Ratios & $1.959 \mathrm{E}-01$ & $3.129 \mathrm{E}-01$ & $3.635 \mathrm{E}-01$ & $7.227 \mathrm{E}-02$ & $1.277 \mathrm{E}-04$ & $5.684 \mathrm{E}-01$ \\
\hline $\begin{array}{ll}1.784 \mathrm{E}-01 & 1.648 \mathrm{E}+00\end{array}$ & & & & & & \\
\hline $\begin{array}{l}\mathrm{Co}-60 \mathrm{uCi} / \mathrm{ml} \\
8.615 \mathrm{E}-01 \quad 1.518 \mathrm{E}+00\end{array}$ & $9.356 \mathrm{E}-01$ & $1.673 \mathrm{E}+00$ & $2.239 \mathrm{E}+00$ & $3.946 \mathrm{E}-01$ & $2.328 \mathrm{E}-02$ & $5.539 \mathrm{E}+00$ \\
\hline $\mathrm{Sr}-90 \mathrm{uCi} / \mathrm{ml}$ & $9.871 \mathrm{E}+02$ & $1.938 \mathrm{E}+03$ & $2.492 \mathrm{E}+03$ & $5.153 \mathrm{E}+02$ & $1.694 \mathrm{E}+00$ & $6.548 \mathrm{E}+03$ \\
\hline $\mathrm{Tc}-99 \mathrm{uCi} / \mathrm{ml}$ & $3.102 \mathrm{E}-01$ & $5.889 \mathrm{E}-01$ & $7.482 \mathrm{E}-01$ & $1.515 \mathrm{E}-01$ & $9.891 \mathrm{E}-04$ & $1.808 \mathrm{E}+00$ \\
\hline $\begin{array}{l}2.715 \mathrm{E}-01 \quad 1.764 \mathrm{E}+00 \\
\mathrm{Cs}-134 \mathrm{uCi} / \mathrm{ml}\end{array}$ & $1.213 E+00$ & $3.499 \mathrm{E}+00$ & $6.800 \mathrm{E}+00$ & $1.315 \mathrm{E}+00$ & $2.937 E-03$ & $3.860 \mathrm{~F}+01$ \\
\hline $\begin{array}{ll}8.502 \mathrm{E}-01 & 2.469 \mathrm{E}+00\end{array}$ & 1. $\angle \perp \triangle D T U O$ & S. & . $.000 \mathrm{DT}$ & D. & & \\
\hline $\begin{array}{l}\mathrm{Cs}-137 \mathrm{uCi} / \mathrm{ml} \\
1.142 \mathrm{E}+03 \quad 1.868 \mathrm{E}+00\end{array}$ & $1.330 \mathrm{E}+03$ & $2.575 E+03$ & $3.293 E+03$ & $6.783 \mathrm{E}+02$ & $3.765 \mathrm{E}+00$ & $8.742 E+03$ \\
\hline Eu-152 uCi/mi & $9.369 \mathrm{E}-02$ & $1.893 \mathrm{E}-01$ & $2.481 E-01$ & $5.170 \mathrm{E}-02$ & $1.568 \mathrm{E}-04$ & $7.867 \mathrm{E}-01$ \\
\hline $\begin{array}{ll}7.858 \mathrm{E}-02 & 1.960 \mathrm{E}+00\end{array}$ & & & & & & \\
\hline $\begin{array}{l}\mathrm{Eu}-154 \mathrm{uCi} / \mathrm{ml} \\
9.065 \mathrm{E}+00 \quad 1.973 \mathrm{E}+00\end{array}$ & $1.086 \mathrm{E}+01$ & $2.232 \mathrm{E}+01$ & $2.977 \mathrm{E}+01$ & $6.169 \mathrm{E}+00$ & $1.939 \mathrm{E}-02$ & $1.060 \mathrm{E}+02$ \\
\hline Eu-155 uCi/ml & $2.154 \mathrm{E}+00$ & $4.394 \mathrm{E}+00$ & $6.484 \mathrm{E}+00$ & $1.217 \mathrm{E}+00$ & $1.306 \mathrm{E}-02$ & $2.165 \mathrm{E}+01$ \\
\hline $1.864 \mathrm{E}+00 \quad 1.767 \mathrm{E}+00$ & & & & & & \\
\hline $\mathrm{Np}-237 \mathrm{uCi} / \mathrm{ml}$ & $6.427 \mathrm{E}-03$ & 1. $225 \mathrm{E}-02$ & $1.559 \mathrm{E}-02$ & $3.166 \mathrm{E}-03$ & $1.994 \mathrm{E}-05$ & $3.767 \mathrm{E}-02$ \\
\hline $\mathrm{Pu}-238 \mathrm{uCi} / \mathrm{ml}$ & $1.285 \mathrm{E}+01$ & $2.584 \mathrm{E}+01$ & $3.348 \mathrm{E}+01$ & $7.015 \mathrm{E}+00$ & $1.974 \mathrm{E}-02$ & $8.323 E+01$ \\
\hline $1.079 \mathrm{E}+01 \quad 1.956 \mathrm{E}+00$ & & & & & & \\
\hline $\begin{array}{l}\mathrm{Pu}-239+\mathrm{uCi} / \mathrm{ml} \\
3.892 \mathrm{E}+01 \quad 1.761 \mathrm{E}+00\end{array}$ & $4.443 E+01$ & $8.424 \mathrm{E}+01$ & $1.070 \mathrm{E}+02$ & $2.165 E+01$ & $1.437 \mathrm{E}-01$ & $2.590 \mathrm{E}+02$ \\
\hline $\mathrm{Pu}-241 \mathrm{uCi} / \mathrm{ml}$ & $7.128 \mathrm{E}+02$ & $1.435 \mathrm{E}+03$ & $1.876 \mathrm{E}+03$ & $3.897 \mathrm{E}+02$ & $1.172 \mathrm{E}+00$ & $5.792 \mathrm{E}+03$ \\
\hline $6.003 \mathrm{E}+02 \quad 1.937 \mathrm{E}+00$ & & & & & & \\
\hline $\mathrm{Am}-241 \mathrm{uCi} / \mathrm{ml}$ & $4.708 \mathrm{E}+01$ & $9.288 \mathrm{E}+01$ & $1.189 \mathrm{E}+02$ & $2.482 \mathrm{E}+01$ & $7.376 \mathrm{E}-02$ & $2.819 \mathrm{E}+02$ \\
\hline $4.002 \mathrm{E}+01 \quad 1.902 \mathrm{E}+00$ & & & & & & \\
\hline Total Uranium (kg/L) & $2.226 \mathrm{E}-01$ & $4.386 \mathrm{E}-01$ & $5.940 \mathrm{E}-01$ & $1.169 \mathrm{E}-01$ & $1.369 \mathrm{E}-03$ & $1.745 \mathrm{E}+00$ \\
\hline $1.934 \mathrm{E}-01 \quad 1.774 \mathrm{E}+00$ & & & & & & \\
\hline $\begin{array}{l}\text { Sludge Density }(\mathrm{kg} / \mathrm{L}) \\
1.380 \mathrm{E}+00 \quad 1.105 \mathrm{E}+00\end{array}$ & $1.388 \mathrm{E}+00$ & $1.646 \mathrm{E}+00$ & $1.888 \mathrm{E}+00$ & $1.471 \mathrm{E}-01$ & $1.012 \mathrm{E}+00$ & $3.279 \mathrm{E}+00$ \\
\hline $\begin{array}{l}\text { Heating Rate }(\mathrm{W} / \mathrm{m} 3) \\
1.423 \mathrm{E}+01 \quad 1.849 \mathrm{E}+00\end{array}$ & $1.655 \mathrm{E}+01$ & $3.218 \mathrm{E}+01$ & $4.115 E+01$ & $8.475 \mathrm{E}+00$ & $1.065 \mathrm{E}-01$ & $1.059 \mathrm{E}+02$ \\
\hline $\begin{array}{ll}\text { Dose Factor } & (\mathrm{rem} / \mathrm{ml}) \\
4.409 \mathrm{E}+04 & 1.835 \mathrm{E}+00\end{array}$ & $5.117 E+04$ & $9.948 E+04$ & $1.271 \mathrm{E}+05$ & $2.618 \mathrm{E}+04$ & $1.308 \mathrm{E}+02$ & $2.991 \mathrm{E}+05$ \\
\hline $\begin{array}{l}\text { Uranium Metal }(\mathrm{kg} / \mathrm{L}) \\
1.591 \mathrm{E}-02 \quad 1.403 \mathrm{E}+00\end{array}$ & $1.676 \mathrm{E}-02$ & $2.601 \mathrm{E}-02$ & $3.206 \mathrm{E}-02$ & $5.300 \mathrm{E}-03$ & $1.388 \mathrm{E}-04$ & $7.576 \mathrm{E}-02$ \\
\hline $\begin{array}{l}\text { Pu Mass Fraction } \\
3.414 \mathrm{E}-04 \quad 1.628 \mathrm{E}+00\end{array}$ & $3.771 E-04$ & $6.560 \mathrm{E}-04$ & $7.989 E-04$ & $1.568 \mathrm{E}-04$ & $1.785 \mathrm{E}-06$ & $1.579 \mathrm{E}-03$ \\
\hline $\begin{array}{l}\text { Heating Rate (W/MT) } \\
1.032 \mathrm{E}+01 \quad 1.731 \mathrm{E}+00\end{array}$ & $1.164 \mathrm{E}+01$ & $2.094 \mathrm{E}+01$ & $2.577 \mathrm{E}+01$ & $5.223 \mathrm{E}+00$ & $9.636 \mathrm{E}-02$ & $5.512 \mathrm{E}+01$ \\
\hline $\begin{array}{l}\text { Heating Rate (W/MTU) } \\
7.360 \mathrm{E}+01 \quad 1.269 \mathrm{E}+00\end{array}$ & $7.541 \mathrm{E}+01$ & $9.442 \mathrm{E}+01$ & $9.910 \mathrm{E}+01$ & $1.477 \mathrm{E}+01$ & $8.984 \mathrm{E}+00$ & $4.480 \mathrm{E}+02$ \\
\hline $\begin{array}{ll}\text { Dose Factor } & (\mathrm{rem} / \mathrm{g}) \\
3.197 \mathrm{E}+04 & 1.728 \mathrm{E}+00\end{array}$ & $3.610 \mathrm{E}+04$ & $6.553 \mathrm{E}+04$ & $8.073 E+04$ & $1.640 \mathrm{E}+04$ & $1.170 \mathrm{E}+02$ & $1.572 \mathrm{E}+05$ \\
\hline
\end{tabular}

\subsection{METHOD OF CALCULATION}

This section summarizes the program flow and logic of PDF-11.PAS. The program is listed in Appendix $\mathrm{G}$ for reference. The program makes considerable use of arrays.

The program begins by checking the command line for the required input values. Having accepted the command line input, the program then reads the first data file, PDF-11.DAT. As shown in Appendix F, the data file begins with a title line followed by paragraphs describing the file. The title line is copied to the output file for reference. The program skips the other lines of information because they all begin with a blank space as the first character. 
The canister counts and floor sludge volume for each key are read into suitable arrays for later use. Then the canister sludge depth measurements are read, averaged and used to calculate the total floor and canister sludge volume for each key. The depths are sorted to construct the continuous cumulative distributions used in the stochastic calculation of canister sludge depth if the third volume is negative. The default is to use the actual depths modified by a variability factor obtained from a uniform distribution that ranges from 0.5 to 1.5 .

The floor sludge sample results and imputed values are read into arrays for use in the stochastic estimate of floor sludge amounts in each key. The program calculates four items that are derived from the information in the data file. The heat generation rate and inhalation dose factor are weighted sums of the activity concentrations. The weighting factors are listed in Table 3-28. The average uranium metal concentration is calculated from half the maximum value shown in Table 3-29. The plutonium weight percent is calculated using the specific activities of $\mathrm{Pu}-238$ and $\mathrm{Pu}-241$ along with the average specific activity for $\mathrm{Pu}-239 / \mathrm{Pu}-240$ mixtures shown in Table 3-31. The individual columns are then sorted and printed to the output file as shown in Figure 6-6.

The process for reading the floor sludge sample data is repeated to read the canister sludge sample data and imputed values. The sorted output is shown in Table 6-7.

The last table read from the data file is the canister sludge inventory estimates of activity per unit mass of uranium from Radnuc2A. These are used in the stochastic calculation of canister sludge amounts in each key.

The next step in preparing for the stochastic analysis is to initialize the arrays used to tally the results. The data file named PDF-11.HDR has the names of the tally arrays and values for the minimum and bin widths. The 46 tally arrays used for the input distributions have 300 bins. The 92 tally arrays for the container inventories of up to 4 container volumes have 900 bins. If a stochastic result is found to be outside the allowed range of bins, a message is added to the output file.

Having completed the initialization, the program then enters the stochastic calculation. The main loop is over the number of requested compositions. For each randomly determined composition the amount of the 19 items of interest in floor and canister sludge are calculated. The resulting average concentrations are then tallied.

There is another loop over the requested number of key retrieval sequences for that sludge composition. The amounts in each key are added to one container until it is full. Then the amounts in that container are tallied. Any excess is added to the next container. This is repeated until all the sludge associated with keys is retrieved.

The main calculation takes place in three procedures (subroutines). The first (Create_Sludge_Amounts) generates new floor and canister sludge compositions and tallies the averages. The second (Rearrange_Keys) generates random key retrieval sequences. The third (Fill_One) fills containers and increments the tally arrays according to the results for each container. 
The final sections of the program print the stochastic results to the output file as tables. The $95^{\text {th }}$, and $99^{\text {th }}$ percentile values are computed while the table is being written. The program adds the number of counts in each bin and compares this with the accumulated total. If there is a difference, the total count for that column will be printed as a negative number.

\subsection{SOFTWARE TESTING}

Testing was carried out using various methods. The first test method used is running the program in the "Debug" mode. In this mode, the values of the variables of interest can be examined while the program is executed line by line. This is particularly useful for examining the flow of execution, namely, what happens at the conditional statements. It was also utilized to verify that the random retrieval sequence used to fill containers would deal with the excess material properly.

The next method was to add tally arrays to check the randomness of the random key retrieval sequences. A 52 by 52 array was used to count the number of times each key was at a particular location in the retrieval order. If the order is truly random, then the numbers accumulated in the array should all be about the same. In addition, the variation between numbers should present a distribution that resembles a normal distribution. The results of one such tally are shown in Figure 6-17. The run used 10,000,000 key retrieval sequences. The expected number of counts in each position is $10,000,000 / 52=192,308$. The tally of the 2,704 bins ( 52 times 52 ) is shown in Figure 6-17. It is concluded that the random sequences are effectively random.

A similar check of the total floor sludge volume reinforces this confidence in the randomness of the random number generator. The floor sludge total should be a uniform distribution from $80 \%$ to $120 \%$ of the mean. The graph that shows the total amount of floor sludge, Figure 4-11, appears uniform. When the numbers in the bins were sorted and the cumulative distribution constructed, the resulting graph is shown in Figure 6-18.

A final check of the stochastic results occurs in the comparison between hand calculated mean values and the stochastically determined mean values. These comparisons are given in Section 4.2. The small differences that exist are readily explained by particular details of the calculations, such as using a calculated canister sludge uranium concentration rather than the data values. 
Figure 6-17. Statistical Variation in the Key Position Index.

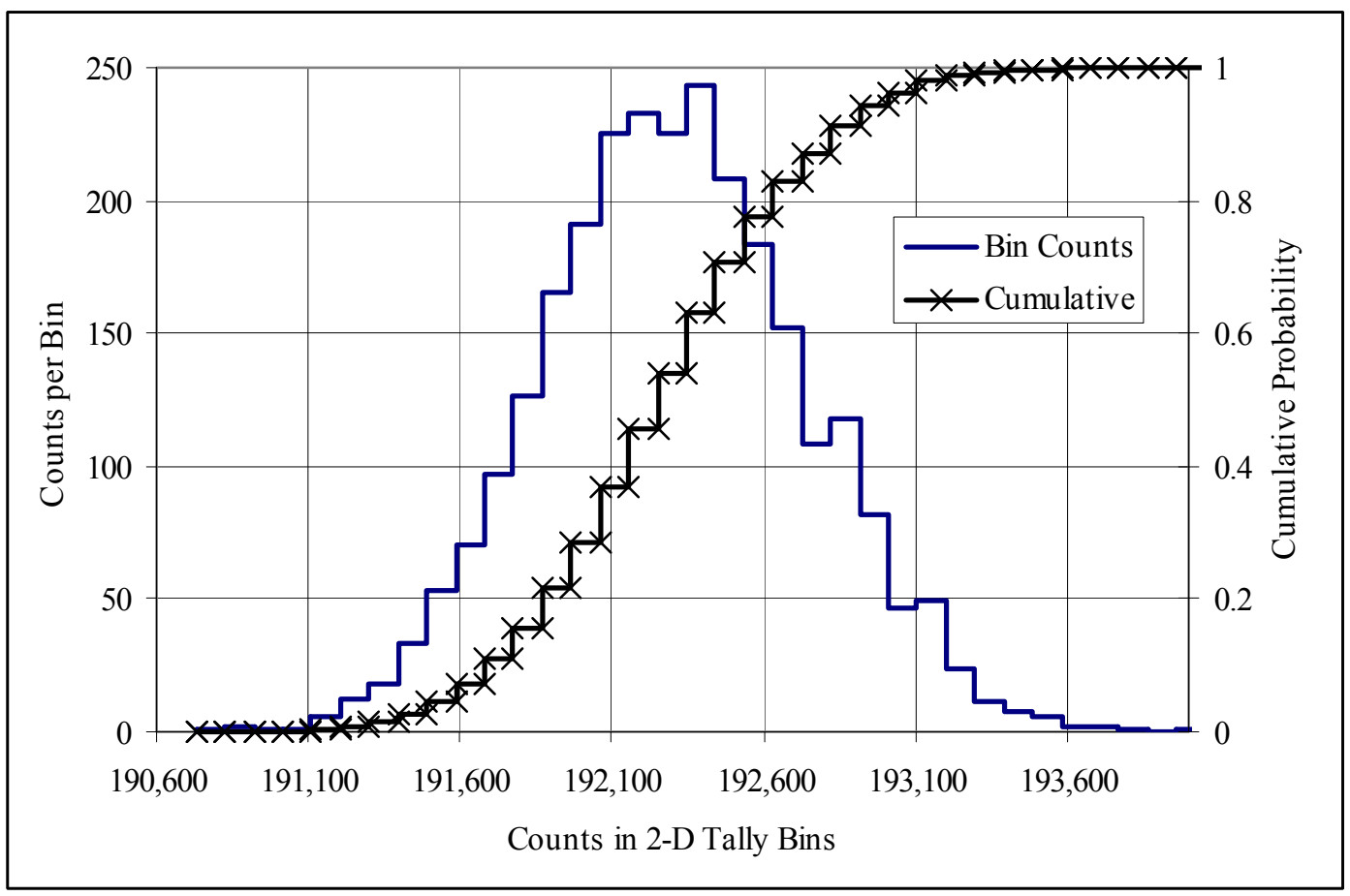

Figure 6-18. Statistical Variation in the Total Floor Sludge Counts.

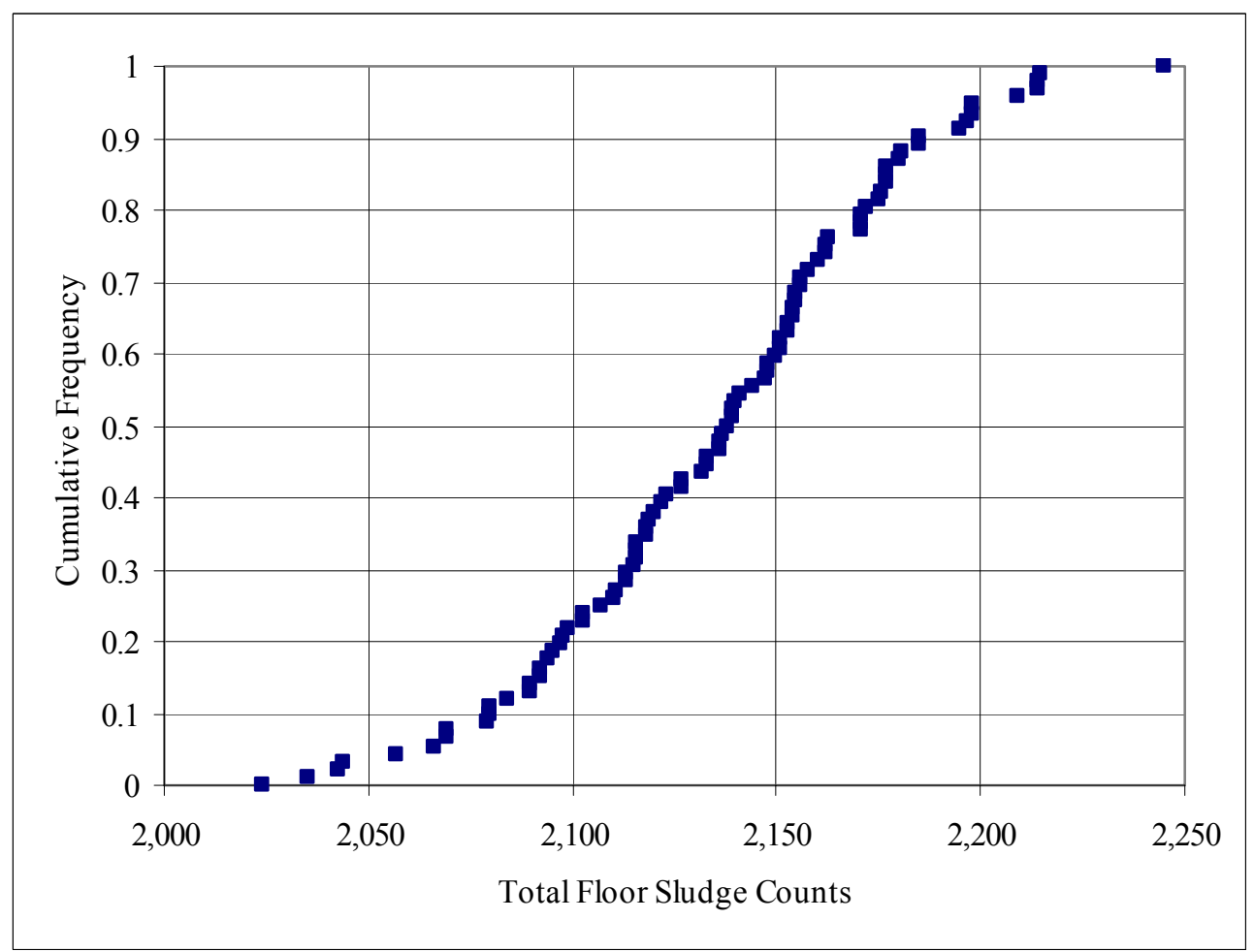




\subsection{CONCLUSIONS}

The goal of this analysis was to provide a safety basis characterization of the sludge in storage containers as it is retrieved from Hanford's 105-K East Basin. A stochastic modeling process was developed, documented, and implemented, and input data to the stochastic model were assembled and documented.

Input data consisted of measurements of sludge depths in canisters and in cubicles on the floor, as well as measurements sets of sludge density, uranium concentration, and 13 important radionuclides $\left({ }^{60} \mathrm{Co},{ }^{90} \mathrm{Sr},{ }^{99} \mathrm{Tc},{ }^{134} \mathrm{Cs},{ }^{137} \mathrm{Cs},{ }^{152} \mathrm{Eu},{ }^{154} \mathrm{Eu},{ }^{155} \mathrm{Eu},{ }^{237} \mathrm{~Np},{ }^{238} \mathrm{Pu},{ }^{239+240} \mathrm{Pu},{ }^{241} \mathrm{Pu}\right.$, $\left.{ }^{241} \mathrm{Am}\right)$.

For floor sludge, measurements of volumes and compositions were used directly. Radionuclide concentration results for particular radionuclides that were reported as "less than" values or not reported were replaced with imputed values. Imputed values were based on using linear regression to estimate the correlation between isotopes with measurements and those with missing or less than values.

For canister sludge, measurements of density and uranium concentration were used together with sludge concentration estimates developed in HNF-8760 (Schwinkendorf 2002). The HNF-8760 models include details of fuel composition and exposure for each key. Comparisons between the sludge concentrations estimated using HNF-8760 results and sample measurements show that the estimated concentrations are generally higher than the measurements. The only exception may be for Tc-99, for which the measurements were at much higher concentrations than the estimates.

The stochastic model developed, described, and implemented in this report statistically empties the K East Basin 10 million times into $0.5,2$, and $12 \mathrm{~m}^{3}$ containers filled on a key-by-key basis. For those containers, this report provides calculated probability distributions of canister-to-total volume ratios; sludge density; isotopic composition for 13 radionuclides and uranium; heat production; inhalation dose factors (rems per milliliter inhaled); and plutonium weight percents.

The utility of the probability distributions is to give plausible averages, upper bounds, and extreme values of parameters of interest without requiring the postulating and fitting of statistical functions to the limited data. The methods employed in this analysis accommodate the possibility of values outside the range of observed values, to the extent plausible, through the addition of uncertainty functions to stretch the data above and below observed values.

It is concluded that stochastic modeling of the compositions and volumes of mixtures of sludge yield useful results for a safety basis characterization. The benefit of the stochastic modeling approach is to clearly show all input data used, all assumptions that are made, and to produce results expressed in the form of statistical distributions. All of the variability of the input data are retained and contribute to the ranges in the output calculations. 
SNF-10293, REV 0

This page intentionally left blank. 


\subsection{ACKNOWLEDGMENTS}

The authors gratefully acknowledge the contributions of K. L. Pearce and T. L. Welsh in providing the detailed spreadsheet representations for the fuel condition, key locations, and floor sludge depth data used in this report. Kathleen Pearce provided invaluable input concerning the history of the sludge and the sampling. The authors wish to thank Ron Baker, Kevin N. Schwinkendorf, Warren D. Wittekind, and Steve Roblyer for helpful discussions on historical aspects of KE Basin fuel and sludge, for supporting the authors by assembling and checking new and critical input data concerning the spent fuel, and for the repeated runs of the Radnuc2A code. Many helpful plans and directions came from Brit E. Hey, Marty Plys, Ray Puigh, Joe Swenson, Jim Sloughter, and Ray Crawford. Terri L. Welsh, Denny Weier, and Scott Cooley provided statistical calculations, advice, feedback, and assistance. 
SNF-10293, REV 0

This page intentionally left blank. 


\section{SNF-10293, REV 0}

\subsection{REFERENCES}

Abramowitz, M., and I. A. Stegun, 1964, Handbook of Mathematical Functions, National Bureau of Standards Applied Mathematics Series 55, U. S. Department of Commerce, Washington, D.C.

Atwood, C. L., L. G. Blackwood, G. A. Harris, and C.A. Loehr, 1991, Recommended Methods for Statistical Analysis of Data Containing Less-Than-Detectable Measurements, EGGSARE--9247, Rev. 1, Idaho National Engineering Laboratory, Idaho Falls, Idaho.

Baker, R. B., 1995, Summary Status of K Basins Sludge Characterization, WHC-SD-SNF-TI-006, Rev. 0, Westinghouse Hanford Company, Richland, Washington.

Baker, R. B., 1999, Sampling and Analysis Plan for the Consolidated Sludge Samples from the Canisters and Floor of the 105-K East Basin, HNF-4016, Rev. 0, Duke Engineering and Services Hanford Inc., Richland, Washington.

Baker, R. B., 2001, Estimated Volumes of Sludge in the Hanford K East and K West Basins, HNF-8118, Rev. 0, Fluor Hanford Inc., Richland, Washington.

Barry, T. M. and W. J. Brattin, 1998, Distribution of Radon-222 In Community Groundwater Systems: Analysis of Type I Left-Censored Data with Single Censoring Point, Human and Ecological Risk Assessment 4(2):579-603.

Bredt, P. R., C. H. Delegard, A. J. Schmidt, and K. L. Silvers, 1999, Testing and Analysis of Consolidated Sludge Samples from the 105 K East Basin Floor and Canisters, PNNL-13341, Rev. 0, Pacific Northwest National Laboratories, Richland, Washington.

Eckerman, K. F., A. B. Wolbarst, and A. C. B. Richardson, 1988, Limiting Values of Radionuclide Intake and Air Concentration and Dose Conversion Factors for Inhalation, Submersion, and Ingestion, Federal Guidance Report No. 11, EPA-520/1-88-020, U.S. Environmental Protection Agency, Washington, D.C.

FH, 2001, Supporting Basis for Spent Nuclear Fuel Project Sludge Technical Databook, SNF-7765, Rev. 0, Fluor Hanford, Inc., Richland, Washington.

Gilbert, R.O., 1995, A Review of Statistical Methods for Data Sets with Multiple Censoring Points, PNL-10652, Rev. 0, Battelle, Pacific Northwest Laboratories, Richland, Washington.

Kocher, D. C., 1981, Radioactive Decay Data Tables, DOE/TIC-11026, Office of Scientific and Technical Information, U.S. Department of Energy, Washington, D.C. 
Makenas, B. J., T. L. Welsh, R. B. Baker, D. R. Hansen, and G. R. Golcar, 1996a, Analysis of Sludge from Hanford K East Basin Floor and Weasel Pit, WHC-SP-1182, Westinghouse Hanford Company, Richland, Washington.

Makenas, B. J., A. L. Pitner, R. B. Baker, 1996b, Choices of Canisters and Elements for the First Fuel and Canister Sludge Shipment from K East Basin, WHC-SD-SNF-SM-003, Rev. 0, Westinghouse Hanford Company, Richland, Washington.

Makenas, B. J., T. L. Welsh, R. B. Baker, E. W. Hoppe, A. J. Schmidt, J. Abrefah, J. M. Tingey, P. R. Bredt, and G. R. Golcar, 1997, Analysis of Sludge from Hanford K East Basin Canisters, HNF-SP-1201, U.S. Department of Energy, Richland, Washington.

Meling, 1994, Sludge Measurement Results for 105KE Basin, WHC-SD-WM-ANAL-037, Rev. 0, Westinghouse Hanford Company, Richland, Washington.

Mitchell, T. J., G. Ostrouchov, E. L. Frome, and G. D. Kerr, 1997, A Method for Estimating Occupational Radiation Dose to Individuals, Using Weekly Dosimetry Data, Radiation Research 147(2):195-207.

National Nuclear Data Center, 2001, Evaluated Nuclear Data File, Release VI. http://www.nndc.bnl.gov, ENDF/B-VI, Brookhaven National Laboratory, Upton, New York.

Packer, M. J., 1999, Single Use Letter Report for the Verification and Validation of RADNUC2A and ORIGEN S.2 Computer Codes, SNF-4503, Rev. 0, DE\&S Hanford, Richland, Washington.

Pan, W. and J. E. Connett, 2001, A multiple imputation approach to linear regression with clustered censored data, Lifetime.Data Anal. 7(2):111-123.

Pearce, K. L., 2001, Spent Nuclear Fuel Project Sludge Technical Databook, Volume 2, Sludge, HNF-SD-SNF-TI-015, Fluor Hanford Inc., Richland, Washington.

Pitner, A. L., 1995, K E Basin Underwater Visual Fuel Survey, WHC-SD-SNF-TI-012, Rev. 0, Westinghouse Hanford Company, Richland, Washington.

Pitner, A. L., 1996a, K East Fuel Cleanliness Evaluation, WHC-SD-SNF-TI-018, Rev. 0, Westinghouse Hanford Company, Richland, Washington.

Pitner, A. L., 1996b, K East Canister Sludge Survey, WHC-SD-SNF-TI-034, Rev. 0, Westinghouse Hanford Company, Richland, Washington. 
Pitner, A. L., 1999a, K East Basin Sludge Sampling 1999 Campaigns, HNF-4746, Rev.0, Numatec Hanford Corporation, Richland, Washington.

Pitner, A. L., 1999b, Revised Estimates of K East Basin Canister Type Distribution and Sludge Content, HNF-5362, Rev. 0, Numatec Hanford Corporation, Richland, Washington.

Rittmann, P. D., 1999, A Discussion on the Methodology for Calculating Radiological and Toxicological Consequences for the Spent Nuclear Fuel Project at the Hanford Site, HNF-SD-SNF-TI-059, Rev. 2, Fluor Daniel Northwest, Richland, Washington.

Schwarz, R. A., 1997, Modification to ORIGEN2 for Generating N Reactor Source Terms, PNNL-11555 UC-610 (3 Volumes), Pacific Northwest National Laboratory, Richland, Washington.

Schwinkendorf, K. N., W. D. Wittekind, and S. P. Roblyer, 2002, K East Basin Sludge CharacterizationUsing Exposure-Adjusted Radioisotopic Source Terms for N Reactor Fuel, HNF-8760, Rev. 1, Fluor Hanford, Richland, Washington.

Singh, A. K., A. Singh, and M. Englehardt, 1997, The Lognormal Distribution in Environmental Applications, EPA/600/R-97/006, U.S. Environmental Protection Agency, Washington, DC.

Strom, D. J., 1986, Estimating Individual and Collective Doses to Groups with 'Less than Detectable' Doses: A Method for Use in Epidemiologic Studies, Health Physics 51(4):437-445. 
SNF-10293, REV 0

This page intentionally left blank. 
SNF-10293, REV 0

APPENDIX A

K EAST BASIN FLOOR SLUDGE DEPTH 
SNF-10293, REV 0

This page intentionally left blank.

A-ii 
SNF-10293, REV 0

\section{APPENDIX A.}

\section{K EAST BASIN FLOOR SLUDGE DEPTHS (CM)}

Table A-1. East Bay (East Half) -- Cubicle 0101 is the South-East Corner of K East Basin. (3 sheets)

\begin{tabular}{|c|c|c|c|c|c|c|c|c|c|c|c|c|}
\hline & 1 & 2 & 3 & 4 & 5 & 6 & 7 & 8 & 9 & 10 & 11 & 12 \\
\hline 1 & 2.21 & 2.08 & 1.94 & 1.94 & 1.71 & 1.31 & $1.27 *$ & 1.28 & 1.30 & 1.10 & 0.96 & $0.95^{*}$ \\
\hline 2 & $2.22 *$ & 2.08 & $1.91 *$ & 1.95 & 2.02 & 1.53 & 1.32 & 1.35 & 1.39 & 1.20 & 1.00 & 0.96 \\
\hline 3 & 2.37 & 2.24 & 2.00 & 2.24 & 2.48 & 2.31 & 1.84 & 1.65 & 1.62 & 1.50 & 1.26 & 1.14 \\
\hline 4 & $2.54^{*}$ & $2.51^{*}$ & 2.38 & 2.51 & $2.54 *$ & 2.51 & 2.28 & 2.00 & 1.84 & 1.82 & 1.73 & 1.59 \\
\hline 5 & 2.58 & 2.63 & 2.65 & 2.56 & 2.54 & 2.53 & 2.38 & 2.08 & 1.93 & 1.91 & 1.90 & 1.88 \\
\hline 6 & 3.44 & $3.68^{*}$ & 3.22 & 2.59 & $2.54 *$ & 2.53 & 2.43 & 2.07 & 1.92 & $1.91^{*}$ & 1.91 & 1.93 \\
\hline 7 & 4.82 & 4.96 & 4.49 & 2.95 & 2.59 & 2.59 & 2.53 & 2.18 & 1.94 & 1.92 & 1.92 & 1.96 \\
\hline 8 & 5.05 & 5.08 & 5.00 & 4.00 & 3.05 & 2.84 & 2.68 & 2.30 & 2.06 & 1.97 & 1.98 & 2.07 \\
\hline 9 & 5.08 & 5.07 & 4.99 & 4.52 & 3.78 & 3.30 & 3.12 & 2.73 & 2.28 & 2.18 & 2.15 & 2.26 \\
\hline 10 & 5.56 & 5.41 & 5.33 & 5.07 & 4.55 & 4.22 & 3.70 & 3.22 & 2.83 & 2.48 & 2.38 & 2.45 \\
\hline 11 & 6.93 & 6.97 & 6.75 & 6.25 & 5.83 & 5.11 & 4.43 & 3.85 & 3.39 & 3.10 & 2.75 & 2.89 \\
\hline 12 & 7.54 & 7.59 & 7.51 & 7.24 & 6.67 & 5.94 & 5.21 & 4.57 & 3.91 & 3.60 & 3.37 & 3.12 \\
\hline 13 & 7.61 & $7.62 *$ & 7.60 & 7.46 & 7.07 & 6.46 & 5.77 & 5.13 & 4.61 & 4.06 & 3.64 & 3.68 \\
\hline 14 & 7.61 & $7.61 *$ & 7.60 & 7.48 & 7.15 & 6.71 & 6.12 & 5.52 & 4.75 & 4.63 & 4.23 & 4.05 \\
\hline 15 & 7.62 & $7.60^{*}$ & 7.57 & 7.44 & 7.21 & 6.65 & 6.33 & 5.68 & 5.28 & 4.89 & 4.80 & 4.12 \\
\hline 16 & 7.79 & 7.67 & 7.61 & 7.41 & 7.16 & 6.83 & 6.11 & 6.02 & 5.52 & 4.98 & 4.84 & 4.39 \\
\hline 17 & 8.37 & 8.04 & 7.89 & 7.50 & 7.11 & 6.50 & 6.23 & 5.28 & 5.42 & 5.01 & 4.53 & 4.47 \\
\hline 18 & 9.26 & $8.60^{*}$ & 8.30 & 7.63 & 7.13 & 7.34 & 5.63 & 5.51 & 4.52 & 4.65 & 4.52 & 4.05 \\
\hline 19 & 9.86 & 9.11* & 8.67 & 7.86 & 7.87 & 7.37 & 5.65 & 4.77 & 4.53 & 3.98 & 4.01 & 4.10 \\
\hline 20 & 10.10 & $9.52 *$ & 8.88 & 8.33 & 7.76 & 7.30 & 5.58 & 4.24 & 3.96 & 3.88 & 3.95 & 4.19 \\
\hline 21 & 10.20 & 9.83 & 8.81 & 7.92 & 7.37 & 6.31 & 5.94 & 4.22 & 3.92 & 3.79 & 3.81 & 4.07 \\
\hline 22 & $10.20 *$ & $9.85^{*}$ & 7.83 & 6.83 & 5.98 & 5.77 & 5.40 & 4.04 & 3.81 & 3.69 & 3.58 & 3.29 \\
\hline 23 & 9.98 & 8.41 & 5.64 & 5.61 & 5.48 & 5.48 & 5.52 & 4.46 & 3.85 & 3.52 & 3.57 & 3.34 \\
\hline 24 & $10.20 *$ & 7.64 & $5.08^{*}$ & 5.13 & 5.27 & 5.66 & 5.22 & 4.42 & 3.93 & 3.80 & 3.67 & 3.69 \\
\hline 25 & 9.61 & 7.06* & 5.37 & 5.37 & 5.19 & 5.05 & 4.88 & 4.41 & 4.20 & 3.83 & 3.79 & 3.70 \\
\hline 26 & 8.41 & 7.08 & $5.08^{*}$ & 5.17 & 5.31 & 5.02 & 4.48 & 4.27 & 3.97 & 3.96 & 3.93 & 4.15 \\
\hline 27 & 8.95 & 8.89 & 7.05 & 5.94 & 5.90 & 5.28 & 4.68 & 4.26 & 4.11 & 4.10 & 4.35 & 4.42 \\
\hline 28 & 11.50 & 9.48 & 8.52 & 7.56 & 5.95 & 4.32 & 3.81 & 3.62 & 3.59 & 4.09 & 4.36 & 4.44 \\
\hline 29 & $12.70^{*}$ & 10.40 & 8.70 & 7.82 & 5.25 & 3.85 & 3.01 & 3.07 & 3.32 & 3.88 & 4.33 & 4.45 \\
\hline 30 & 11.10 & 7.94 & 8.38 & 6.50 & 4.71 & 2.97 & 2.88 & 2.90 & 3.05 & 3.58 & 4.20 & 4.43 \\
\hline 31 & $12.10 *$ & 12.30 & 7.41 & 6.14 & 3.60 & 2.91 & $2.86^{*}$ & 2.87 & 3.01 & 3.43 & 4.03 & 4.41 \\
\hline 32 & 12.30 & $20.00 *$ & $3.18^{*}$ & 3.43 & 3.41 & 2.98 & 2.88 & 2.90 & 2.97 & 3.25 & 3.76 & 4.24 \\
\hline
\end{tabular}


Table A-1. East Bay (East Half) -- Cubicle 0101 is the South-East Corner of K East Basin. (3 sheets)

\begin{tabular}{|c|c|c|c|c|c|c|c|c|c|c|c|c|}
\hline & 1 & 2 & 3 & 4 & 5 & 6 & 7 & 8 & 9 & 10 & 11 & 12 \\
\hline 33 & $6.03 *$ & 11.70 & 6.96 & 5.72 & 3.42 & 3.04 & 2.91 & 2.88 & 2.96 & 3.21 & 3.62 & 4.13 \\
\hline 34 & .67 & 7.74 & 6.78 & 5.50 & 5.17 & 2.99 & 2.84 & 2.86 & 2.97 & 3.17 & 3.59 & 4.04 \\
\hline 35 & .76 & 3.40 & 2.09 & 1.94 & 2.37 & 4.02 & 2.81 & 2.72 & 3.01 & 3.16 & 3.38 & 3.63 \\
\hline 36 & 3.92 & 2.06 & $1.59^{*}$ & 1.71 & 1.94 & 2.34 & 3.93 & 2.58 & 2.75 & 3.12 & 3.26 & 3.42 \\
\hline 37 & 4.38 & 4.08 & 2.35 & 2.21 & 2.46 & 2.63 & 2.79 & 2.87 & 2.98 & 3.05 & 3.10 & 3.45 \\
\hline 38 & 4.63 & 4.76 & 4.47 & 3.53 & 3.19 & 2.99 & 2.95 & 2.99 & 3.00 & 2.97 & 2.99 & 3.04 \\
\hline 39 & 4.23 & 4.26 & 4.18 & 3.92 & 3.64 & 3.45 & 2.82 & 2.82 & 2.79 & 2.73 & 2.59 & 2.36 \\
\hline 40 & 3.85 & 3.81 & 3.85 & 3.87 & 3.79 & 3.65 & 3.52 & 3.93 & 2.77 & 2.64 & 2.33 & 1.90 \\
\hline 41 & 3.83 & 3.83 & 3.83 & 3.90 & 3.97 & 3.59 & 3.46 & 3.29 & 3.42 & 2.99 & 2.36 & 1.73 \\
\hline 42 & 3.83 & 3.81 & 3.82 & 3.89 & 3.96 & 4.00 & 3.53 & 3.40 & 3.16 & 2.84 & 2.18 & 1.50 \\
\hline 43 & 3.95 & 3.86 & 3.91 & 4.09 & 4.24 & 4.24 & 4.24 & 3.77 & 3.35 & 2.82 & 2.21 & 1.46 \\
\hline 44 & 4.74 & 4.58 & 4.51 & 4.56 & 4.67 & 4.70 & 4.25 & 4.06 & 3.49 & 2.98 & 2.27 & 1.51 \\
\hline 45 & 5.33 & 4.90 & 4.91 & 5.02 & 5.04 & 5.01 & 4.84 & 4.98 & 4.43 & 3.60 & 2.73 & 1.67 \\
\hline 46 & 6.18 & 4.76 & 4.92 & 5.22 & 5.54 & 5.20 & 5.07 & 4.87 & 4.59 & 3.93 & 2.59 & 2.04 \\
\hline 47 & $7.62 *$ & 6.22 & 5.53 & 5.55 & 5.53 & 5.70 & 5.60 & 5.48 & 4.97 & 3.96 & 3.38 & 2.74 \\
\hline 48 & 7.48 & 7.11 & 6.62 & 6.17 & 5.49 & 5.46 & 5.79 & 5.42 & 5.07 & 3.88 & 3.52 & 3.35 \\
\hline 49 & 7.60 & 7.52 & 7.60 & 6.60 & 6.47 & 5.84 & 5.31 & 5.65 & 4.29 & 3.96 & 3.82 & 3.57 \\
\hline 50 & $7.63^{*}$ & 7.61 & 7.59 & 7.53 & 6.71 & 6.35 & 5.63 & 5.20 & 4.32 & 4.12 & 3.82 & 3.65 \\
\hline 51 & 7.62 & 7.57 & 7.51 & 7.46 & 7.18 & 6.67 & 6.27 & 5.71 & 4.48 & 4.15 & 3.83 & 3.70 \\
\hline 52 & 7.42 & 7.33 & 7.35 & 7.27 & 7.23 & 7.08 & 6.37 & 5.15 & 4.33 & 3.88 & 3.76 & 3.70 \\
\hline 53 & 7.32 & 7.30 & 7.31 & 7.35 & 7.27 & 7.12 & 6.88 & 5.05 & 4.23 & 3.81 & 3.71 & 3.74 \\
\hline 54 & 7.31 & 7.31 & 7.30 & 7.37 & 7.41 & 6.93 & 6.17 & 5.09 & 4.16 & 3.77 & 3.69 & 3.76 \\
\hline 55 & 7.41 & 7.39 & 7.39 & 7.44 & 7.41 & 7.11 & 6.19 & 5.10 & 4.22 & 3.75 & 3.62 & 3.60 \\
\hline 56 & 7.90 & 7.84 & 7.72 & 7.74 & 7.64 & 7.79 & 6.33 & 5.15 & 4.39 & 3.82 & 3.57 & 3.38 \\
\hline 57 & 9.48 & 9.22 & 9.25 & 8.76 & 8.47 & 7.87 & 6.81 & 7.07 & 5.80 & 4.08 & 3.64 & 3.35 \\
\hline 58 & 12.70 & 12.40 & 2.00 & 11.60 & 10.00 & 9.07 & 8.34 & 8.19 & 5.45 & 4.48 & 3.79 & 3.50 \\
\hline 59 & 16.20 & 15.80 & 15.30 & 14.40 & 13.40 & 11.80 & 9.89 & 8.36 & 6.42 & 5.23 & 4.19 & 3.83 \\
\hline 60 & 18.30 & 18.10 & 17.60 & 16.60 & 15.60 & 14.80 & 12.00 & 9.29 & 7.55 & 5.59 & 4.84 & 4.38 \\
\hline 61 & 19.10 & 19.00 & 18.70 & 17.80 & 16.70 & 15.40 & 13.60 & 10.60 & 8.81 & 7.35 & 5.63 & 5.12 \\
\hline 62 & 19.30 & 19.30 & 19.00 & 18.20 & 16.90 & 16.30 & 13.50 & 12.80 & 9.84 & 8.41 & 7.32 & 5.92 \\
\hline 63 & $19.40^{*}$ & 19.30 & 19.00 & 18.10 & 16.80 & 16.80 & 14.20 & 12.50 & 11.20 & 9.12 & 8.09 & 6.32 \\
\hline 64 & 19.30 & 19.10 & 18.60 & 17.60 & 16.30 & 15.00 & 13.80 & 12.10 & 11.10 & 10.10 & 8.62 & 6.81 \\
\hline 65 & 18.40 & 17.90 & 17.40 & 16.40 & 15.00 & 14.00 & 12.30 & 11.40 & 10.70 & 10.00 & 7.30 & 6.80 \\
\hline 66 & 16.40 & 17.00 & 16.90 & 15.50 & 13.90 & 12.00 & 10.90 & 10.40 & 10.00 & 8.16 & 7.25 & 6.70 \\
\hline 67 & 15.30 & 17.90 & 17.60 & 15.20 & 11.90 & 9.92 & & 9.32 & 7.80 & 7.84 & 7.03 & 6.74 \\
\hline 68 & $14.30 *$ & 19.10 & 18.20 & 13.20 & 8.81 & 7.94 & 7.88 & 7.10 & 7.44 & 7.57 & 6.82 & 6.55 \\
\hline 69 & 15.10 & 17.50 & 15.50 & 8.28 & 5.93 & 5.74 & 6.26 & 6.65 & 7.11 & 7.23 & 7.13 & 6.57 \\
\hline
\end{tabular}


SNF-10293, REV 0

Table A-1. East Bay (East Half) -- Cubicle 0101 is the South-East Corner of K East Basin. (3 sheets)

\begin{tabular}{|c|c|c|c|c|c|c|c|c|c|c|c|c|}
\hline & 1 & 2 & 3 & 4 & 5 & 6 & 7 & 8 & 9 & 10 & 11 & 12 \\
\hline 70 & 11.50 & 12.30 & 10.70 & 8.25 & $5.40^{*}$ & 5.48 & 5.91 & 6.52 & 6.70 & 7.00 & 6.26 & 6.25 \\
\hline 71 & 5.02 & 6.88 & 10.30 & $10.80^{*}$ & 8.13 & 6.76 & 6.87 & 7.00 & 6.91 & 6.00 & 5.70 & 5.57 \\
\hline 72 & $4.45^{*}$ & 5.00 & 9.20 & 10.40 & 9.50 & 8.19 & 7.39 & 5.79 & 6.55 & 5.45 & 5.34 & 5.41 \\
\hline 73 & 4.60 & 5.20 & 7.69 & 9.11 & 9.03 & 8.24 & 7.61 & 6.78 & 6.00 & 5.43 & 5.28 & 5.48 \\
\hline 74 & 5.22 & 6.02 & 7.68 & 8.63 & 8.77 & 8.31 & 7.63 & 6.43 & 5.36 & 4.95 & 5.03 & 5.53 \\
\hline 75 & 5.16 & 5.80 & 8.44 & 9.11 & 9.06 & 8.63 & 7.70 & 6.06 & 4.95 & 4.58 & 4.56 & 5.43 \\
\hline 76 & $5.08^{*}$ & 5.43 & 8.86 & $9.21^{*}$ & 9.15 & 8.77 & 7.64 & 5.87 & 4.78 & 4.49 & $4.45^{*}$ & 4.75 \\
\hline
\end{tabular}

Asterisks mark locations that were measured in WHC-SD-WM-ANAL-037 (Meling 1994). 
SNF-10293, REV 0

Table A-2. East Bay, West Half (Floor Sludge Depths in cm).

(3 sheets)

\begin{tabular}{|c|c|c|c|c|c|c|c|c|c|c|}
\hline & 13 & 14 & 15 & 16 & 17 & 18 & 19 & 20 & 21 & 22 \\
\hline 1 & 0.96 & 1.16 & 1.90 & 2.98 & 3.25 & 3.20 & $3.18^{*}$ & 3.45 & 5.01 & $5.40^{*}$ \\
\hline 2 & 1.01 & 1.30 & 2.29 & 3.16 & 3.38 & 3.31 & 3.27 & 3.86 & 4.46 & $4.13^{*}$ \\
\hline 3 & 1.25 & 1.66 & 2.60 & 3.65 & 3.77 & 3.64 & 3.60 & 4.20 & 5.20 & 4.85 \\
\hline 4 & 1.70 & 2.15 & 2.92 & 3.56 & 3.97 & 3.63 & $3.49 *$ & 4.08 & 9.32 & 11.90 \\
\hline 5 & 2.00 & 2.49 & 3.22 & 3.79 & 4.35 & 4.37 & 4.36 & 5.36 & 11.80 & $13.00^{*}$ \\
\hline 6 & 2.11 & 2.72 & 3.50 & 3.93 & 4.68 & 4.99 & $5.08^{*}$ & 5.36 & 10.30 & 12.60 \\
\hline 7 & 2.20 & 2.71 & 3.37 & 3.79 & 4.13 & 4.71 & 4.92 & 5.12 & 7.36 & 10.00 \\
\hline 8 & 2.33 & 2.79 & 3.26 & 3.42 & 3.51 & 3.08 & 2.77 & 3.29 & 4.76 & 6.75 \\
\hline 9 & 2.63 & 2.91 & 3.14 & 3.39 & 3.07 & 2.64 & $2.54^{*}$ & 2.69 & 3.50 & 4.80 \\
\hline 10 & 2.75 & 2.90 & 3.20 & 3.18 & 3.10 & 3.04 & 3.02 & 3.09 & 3.37 & 4.02 \\
\hline 11 & 2.93 & 3.23 & 3.29 & 3.27 & 3.31 & 3.44 & $3.49 *$ & 3.46 & 3.47 & 3.80 \\
\hline 12 & 3.29 & 3.33 & 3.35 & 3.35 & 3.38 & 3.44 & 3.47 & 3.47 & 3.53 & 3.77 \\
\hline 13 & 3.47 & 3.44 & 3.44 & 3.41 & 3.37 & 3.40 & 3.42 & 3.50 & 3.57 & 3.83 \\
\hline 14 & 3.86 & 3.47 & 3.46 & 3.42 & 3.41 & 3.41 & 3.41 & 3.59 & 3.68 & 3.89 \\
\hline 15 & 3.71 & 3.63 & 3.79 & 3.68 & 3.56 & 3.52 & 3.51 & 3.77 & 3.89 & 4.08 \\
\hline 16 & 3.84 & 3.78 & 4.03 & 3.95 & 3.87 & 3.82 & 3.70 & 4.07 & & 4.42 \\
\hline 17 & 4.03 & 3.83 & 4.19 & 4.16 & 4.14 & 3.96 & 4.04 & 4.50 & 4.71 & 5.03 \\
\hline 18 & 4.06 & 4.00 & 4.24 & 4.24 & 4.00 & 4.07 & 4.49 & 4.91 & 5.48 & 5.80 \\
\hline 19 & 4.15 & 4.04 & 4.25 & 3.93 & 3.91 & 4.33 & 4.98 & 6.08 & 6.64 & 7.11 \\
\hline 20 & 4.32 & 4.11 & 3.93 & 3.92 & 4.07 & 4.56 & $5.6 /$ & 6.62 & 7.30 & 7.64 \\
\hline 21 & $4.45^{*}$ & 3.96 & 3.82 & 3.92 & 4.09 & 5.06 & 5.80 & 7.03 & 7.86 & 8.25 \\
\hline 22 & $2.86^{*}$ & $3.81 *$ & 3.73 & 3.65 & 4.01 & 4.77 & 6.15 & 7.59 & 8.37 & 8.68 \\
\hline 23 & 3.28 & 3.36 & 3.38 & 3.49 & 3.88 & 4.95 & 6.82 & 8.27 & 8.88 & 9.04 \\
\hline 24 & $3.81^{*}$ & $2.86^{*}$ & 3.00 & 3.29 & 3.84 & 5.36 & 7.41 & 8.71 & 9.13 & 9.19 \\
\hline 25 & 3.61 & 3.11 & 3.12 & 3.41 & 4.03 & 6.33 & 7.81 & 8.89 & 9.18 & $9.21^{*}$ \\
\hline 26 & 4.15 & 3.64 & 3.53 & 3.90 & 4.84 & 6.31 & 7.94 & 8.66 & 8.99 & 9.12 \\
\hline 27 & 4.44 & 4.34 & 4.28 & 4.58 & 5.26 & 6.45 & 7.60 & 8.26 & 8.36 & 8.32 \\
\hline 28 & $4.45^{*}$ & 4.44 & 4.52 & 5.00 & 5.76 & 6.32 & 7.30 & 8.01 & 8.25 & $8.26^{*}$ \\
\hline 29 & 4.45 & 4.46 & 4.65 & 5.41 & 5.95 & 6.23 & 6.74 & 7.68 & 8.14 & 8.23 \\
\hline 30 & 4.49 & 4.57 & 5.11 & 5.74 & 6.04 & 6.05 & 6.23 & 7.23 & 7.90 & 7.95 \\
\hline 31 & 4.61 & 4.83 & 5.47 & 5.95 & 6.03 & $6.03^{*}$ & 6.09 & 7.08 & 7.90 & 7.94* \\
\hline 32 & 4.64 & 4.99 & 5.65 & 5.96 & 6.04 & 6.05 & 6.20 & 7.13 & 7.80 & 7.90 \\
\hline 33 & 4.63 & 5.03 & 5.65 & 6.00 & 6.10 & 6.19 & 6.59 & 7.31 & 7.62 & 7.64 \\
\hline 34 & 4.54 & 4.94 & 5.66 & 5.97 & 6.19 & 6.46 & 7.00 & 7.46 & 7.61 & $7.62 *$ \\
\hline 35 & 4.34 & 5.02 & 5.46 & 5.83 & 6.17 & 6.57 & 7.12 & 7.47 & 7.59 & 7.61 \\
\hline 36 & 4.03 & 4.61 & 4.99 & 5.39 & 5.83 & 6.34 & 6.95 & 7.28 & 7.49 & 7.55 \\
\hline
\end{tabular}


Table A-2. East Bay, West Half (Floor Sludge Depths in cm). (3 sheets)

\begin{tabular}{|c|c|c|c|c|c|c|c|c|c|c|}
\hline & 13 & 14 & 15 & 16 & 17 & 18 & 19 & 20 & 21 & 22 \\
\hline 37 & 3.56 & 4.09 & 4.27 & 4.56 & 5.01 & 5.61 & 6.27 & 6.84 & 7.18 & 7.33 \\
\hline 38 & 2.92 & 2.78 & 3.09 & 3.19 & 3.52 & 3.76 & 4.96 & 5.80 & 6.42 & 6.77 \\
\hline 39 & 2.15 & 1.85 & 1.82 & 1.75 & 1.64 & 2.01 & 3.06 & 4.07 & 5.03 & 5.72 \\
\hline 40 & 1.48 & 1.16 & 0.96 & 0.75 & 0.74 & 0.89 & 1.47 & 2.31 & 3.36 & 4.33 \\
\hline 41 & 1.17 & 0.76 & 0.53 & 0.42 & 0.40 & 0.45 & 0.70 & 1.22 & 2.06 & 3.04 \\
\hline 42 & 0.94 & 0.58 & 0.40 & 0.34 & 0.33 & 0.34 & 0.45 & 0.75 & 1.33 & 2.14 \\
\hline 43 & 0.85 & 0.52 & 0.36 & 0.32 & $0.32 *$ & 0.33 & 0.39 & 0.59 & 1.02 & 1.66 \\
\hline 44 & 0.90 & 0.56 & 0.39 & 0.33 & 0.32 & 0.33 & 0.40 & 0.59 & 0.94 & 1.47 \\
\hline 45 & 1.12 & 0.72 & 0.48 & 0.39 & 0.36 & 0.39 & 0.46 & 0.67 & 1.00 & 1.45 \\
\hline 46 & 1.51 & 1.08 & 0.73 & 0.57 & 0.51 & 0.55 & 0.64 & 0.81 & 1.17 & 1.56 \\
\hline 47 & 2.20 & 1.74 & 1.27 & 1.04 & 0.91 & 0.92 & 0.99 & 1.15 & 1.45 & 1.77 \\
\hline 48 & 2.93 & 2.55 & 2.11 & 1.80 & & 1.52 & 1.02 & ד. & 1.82 & 2.04 \\
\hline 49 & 3.36 & 3.12 & 2.83 & 2.58 & 2.36 & 2.25 & 2.17 & $2.1 J$ & 2.25 & 2.39 \\
\hline 50 & 3.54 & 3.42 & 3.24 & 3.08 & 2.91 & 2.81 & 2.00 & 2.63 & 2.65 & 2.73 \\
\hline 51 & 3.62 & 3.51 & 3.41 & 3.29 & 3.17 & 3.10 & 0.01 & 2.96 & 2.95 & 2.99 \\
\hline 52 & 3.66 & 3.57 & 3.43 & 3.33 & 3.27 & 3.19 & 5.17 & 0.10 & 3.18 & 3.24 \\
\hline 53 & 3.76 & 3.59 & 3.34 & 3.25 & 3.23 & 3.23 & 0.21 & 3.31 & 3.38 & 3.44 \\
\hline 54 & $3.81^{*}$ & 3.34 & 3.08 & 3.12 & 3.17 & 3.23 & 3.32 & 3.41 & 3.63 & 3.67 \\
\hline 55 & 3.34 & $2.86^{*}$ & 2.91 & 3.03 & 3.13 & 3.22 & 3.36 & 3.50 & 3.68 & 3.87 \\
\hline 56 & 3.09 & 2.92 & 2.94 & 3.03 & 3.15 & 3.29 & 3.50 & 3.73 & 3.97 & 4.06 \\
\hline 57 & 3.16 & 3.05 & 3.05 & 3.12 & 3.24 & 3.41 & 3.69 & 3.84 & 4.11 & 4.38 \\
\hline 58 & 3.32 & 3.24 & 3.22 & 3.29 & 3.43 & 3.55 & 3.89 & 4.20 & 4.51 & 4.79 \\
\hline 59 & 3.62 & 3.62 & 3.51 & 3.61 & 3.65 & 4.03 & 4.38 & 4.72 & 5.03 & 5.31 \\
\hline 60 & 4.13 & 4.22 & 4.02 & 3.97 & 4.19 & 4.67 & 5.16 & 5.38 & 5.65 & 5.86 \\
\hline 61 & 4.86 & 5.07 & 4.48 & 4.70 & 4.97 & 5.52 & 5.91 & 6.15 & 6.25 & 6.62 \\
\hline 62 & 5.69 & 5.30 & 5.31 & 5.58 & 5.84 & 6.09 & 6.56 & 6.87 & 6.95 & 6.95 \\
\hline 63 & 6.02 & 6.01 & 6.49 & 6.36 & 6.56 & 6.71 & 7.10 & 7.15 & 7.19 & 7.22 \\
\hline 64 & 6.67 & 6.83 & 7.08 & 7.18 & 7.10 & 7.17 & 7.34 & 7.36 & 7.39 & 7.42 \\
\hline 65 & 6.75 & 6.74 & 7.22 & 7.32 & 7.40 & 7.37 & 7.43 & 7.44 & 7.48 & 7.40 \\
\hline 66 & 6.72 & 6.81 & 7.03 & 7.39 & 7.48 & 7.52 & 7.50 & 7.50 & 7.47 & 7.56 \\
\hline 67 & 6.63 & 6.78 & 7.02 & 7.29 & 7.55 & 7.59 & 7.55 & 7.44 & 7.62 & 7.76 \\
\hline 68 & 6.72 & 6.64 & 6.88 & 7.19 & 7.53 & $7.62^{*}$ & 7.53 & 7.42 & 7.80 & 7.91 \\
\hline 69 & 6.65 & 7.01 & 6.59 & 6.76 & 6.92 & 6.96 & 6.94 & 7.22 & 7.86 & $7.94 *$ \\
\hline 70 & 6.36 & 6.86 & 6.83 & 6.25 & 6.32 & $6.35^{*}$ & 6.35 & 6.74 & 7.56 & 7.80 \\
\hline 71 & 5.52 & 5.86 & 5.89 & 5.67 & 5.42 & 5.43 & 5.51 & 6.28 & 6.64 & 6.51 \\
\hline 72 & 5.47 & 5.56 & 5.41 & 5.09 & 4.61 & $4.45^{*}$ & 4.71 & 6.11 & 6.43 & $6.35^{*}$ \\
\hline 73 & 5.62 & 5.65 & 5.62 & 5.24 & 4.80 & 4.62 & 5.23 & 7.00 & 6.97 & 6.58 \\
\hline
\end{tabular}


SNF-10293, REV 0

Table A-2. East Bay, West Half (Floor Sludge Depths in cm). (3 sheets)

\begin{tabular}{|c|c|c|c|c|c|c|c|c|c|c|}
\hline & 13 & 14 & 15 & 16 & 17 & 18 & 19 & 20 & 21 & 22 \\
\hline 74 & 5.69 & 5.70 & 5.73 & 5.73 & 6.01 & 6.52 & 8.46 & 10.20 & 10.50 & 10.40 \\
\hline 75 & $5.72 *$ & 5.71 & 5.77 & 6.32 & 8.08 & 9.95 & 11.60 & 12.00 & 13.00 & 14.20 \\
\hline 76 & 5.62 & 5.69 & 5.97 & 7.18 & 9.30 & 11.30 & 12.00 & $12.10^{*}$ & 13.30 & $14.60 *$ \\
\hline
\end{tabular}

Asterisks mark locations that were measured in WHC-SD-WM-ANAL-037 (Meling 1994). 
Table A-3. Middle Bay, East Half. (3 sheets)

\begin{tabular}{|c|c|c|c|c|c|c|c|c|c|c|c|c|}
\hline & 23 & 24 & 25 & 26 & 27 & 28 & 29 & 30 & 31 & 32 & 33 & 34 \\
\hline 1 & 9.03 & 2.75 & $0.64 *$ & 0.82 & 0.98 & 0.41 & $32 *$ & 1.08 & $2.54^{*}$ & 2.46 & 2.76 & 4.21 \\
\hline 2 & $84^{*}$ & 7.65 & 1.42 & 1.33 & 1.37 & 0.83 & 0.51 & $0.32 *$ & 1.46 & 2.20 & 2.96 & 4.94 \\
\hline 3 & .18 & 7.65 & 4.18 & 2.65 & 2.20 & 2.15 & 2.17 & 0.96 & 1.60 & 2.82 & 3.72 & 5.53 \\
\hline 4 & 5.11 & 5.29 & 4.76 & 3.39 & 2.80 & 2.85 & $2.86^{*}$ & 2.77 & 3.64 & 4.31 & 4.37 & 5.05 \\
\hline 5 & $4.76^{*}$ & 4.81 & 4.75 & 4.01 & 3.41 & 3.20 & 3.08 & 3.60 & 4.38 & $4.45^{*}$ & 4.45 & 4.54 \\
\hline 6 & 4.78 & 4.75 & 4.54 & 4.04 & 3.85 & 4.21 & 5.14 & 5.73 & 5.14 & 4.51 & 4.53 & 4.49 \\
\hline 7 & 4.63 & 4.38 & 4.02 & 3.91 & 4.06 & 5.00 & 5.91 & $6.03 *$ & 5.91 & 5.14 & 4.82 & 4.72 \\
\hline 8 & 3.91 & 3.69 & 3.74 & 3.79 & 3.86 & 4.63 & 5.88 & 5.99 & 5.90 & 5.44 & 5.21 & 5.29 \\
\hline 9 & 2.99 & 3.01 & 3.63 & $3.81^{*}$ & 3.78 & 4.35 & 5.41 & 5.71 & 5.71 & 5.55 & 5.62 & 5.67 \\
\hline 10 & 2.20 & 1.82 & 1.89 & 3.24 & 3.45 & 3.77 & 4.47 & 5.08 & 5.38 & 5.49 & 5.78 & 6.04 \\
\hline 11 & 1.87 & 1.41 & $1.27 *$ & 1.55 & 2.62 & 3.38 & 4.56 & 5.03 & 5.52 & 5.59 & 5.50 & 5.45 \\
\hline 12 & 2.64 & 1.81 & 1.54 & 2.13 & 2.92 & 3.70 & 4.31 & 5.66 & 5.66 & 5.70 & 5.65 & 5.44 \\
\hline 13 & 4.12 & 3.27 & 3.46 & 3.92 & 3.85 & 3.80 & 4.36 & 5.36 & 5.85 & $5.72^{*}$ & 5.85 & 5.32 \\
\hline 14 & 6.02 & 3.68 & 3.39 & $4.13^{*}$ & 3.99 & 3.79 & 4.70 & 5.78 & 6.19 & $6.35^{*}$ & 6.19 & 5.85 \\
\hline 15 & 8.80 & 4.11 & $2.54^{*}$ & 3.35 & 3.59 & 3.20 & 3.74 & 5.54 & 6.22 & 6.32 & 6.25 & 5.75 \\
\hline 16 & $9.83^{*}$ & 7.76 & 3.12 & 2.83 & 2.41 & 2.52 & 3.22 & 4.48 & 5.75 & 6.10 & 5.95 & 5.45 \\
\hline 17 & 9.26 & 7.77 & 4.15 & 2.42 & $2.22 *$ & 2.28 & 2.05 & 3.67 & 4.91 & 5.56 & 5.46 & 5.04 \\
\hline 18 & 7.81 & 6.04 & 3.88 & 2.74 & 2.35 & 2.42 & 2.71 & 3.35 & 4.12 & 4.77 & 4.89 & 5.05 \\
\hline 19 & 5.79 & 4.38 & 3.58 & 3.45 & 3.26 & 3.03 & 3.11 & 3.35 & 3.85 & 4.27 & 4.78 & 4.75 \\
\hline 20 & 4.59 & 3.81 & 3.51 & $3.49 *$ & 3.48 & 3.41 & 3.40 & 3.66 & 3.78 & 4.11 & 4.29 & 4.56 \\
\hline 21 & 4.25 & 3.74 & 3.54 & 3.50 & 3.47 & 3.45 & 3.42 & 3.42 & 3.51 & 4.00 & 4.33 & 4.60 \\
\hline 22 & 3.93 & 3.88 & 3.72 & 3.68 & 3.67 & 3.63 & 3.57 & 3.46 & 3.40 & 3.51 & 3.96 & 4.04 \\
\hline 23 & 3.84 & 3.86 & 3.98 & 4.08 & 4.07 & 3.96 & 3.69 & 3.39 & 3.22 & 3.22 & 3.09 & 3.42 \\
\hline 24 & 3.97 & 3.78 & 3.86 & 4.23 & 4.36 & 4.21 & 3.58 & 3.14 & 2.84 & 2.81 & 2.34 & 2.70 \\
\hline 25 & 4.01 & 3.72 & 3.65 & 3.94 & $4.45^{*}$ & 4.21 & 3.01 & 2.47 & 2.30 & 1.90 & 2.18 & 2.61 \\
\hline 26 & 3.87 & 3.62 & 3.51 & $3.49^{*}$ & 3.82 & 2.99 & 1.79 & 1.77 & 1.94 & 2.17 & 2.41 & 2.79 \\
\hline 27 & 4.15 & 3.59 & 3.34 & 3.18 & 2.37 & 1.87 & $1.59^{*}$ & 1.63 & 1.79 & 2.03 & 2.35 & 2.70 \\
\hline 28 & 5.54 & 4.16 & 2.99 & 2.11 & $1.91^{*}$ & 1.90 & 1.67 & 1.65 & 1.67 & 1.79 & 2.00 & 2.35 \\
\hline 29 & 7.34 & 6.15 & 3.71 & 2.21 & 1.96 & 1.96 & 1.87 & 1.78 & 1.86 & 2.08 & 2.40 & 2.84 \\
\hline 30 & 8.19 & 7.87 & 5.89 & 3.37 & 2.38 & 2.21 & 2.15 & 2.05 & 2.11 & 2.26 & 2.66 & 3.04 \\
\hline 31 & $8.26^{*}$ & 8.19 & 7.31 & 5.03 & 3.24 & 2.70 & 2.53 & 2.40 & 2.42 & 2.52 & 2.70 & 2.79 \\
\hline 32 & 8.23 & 8.11 & 7.32 & 5.43 & 3.69 & 2.97 & 2.72 & 2.62 & 2.62 & 2.37 & 2.50 & 2.75 \\
\hline 33 & 8.02 & 7.68 & 6.63 & 4.68 & 3.29 & 2.70 & 2.59 & 2.58 & 2.60 & 2.66 & 2.57 & 2.83 \\
\hline 34 & 7.39 & 6.72 & 5.41 & 3.70 & 2.79 & 2.57 & 2.54 & 2.54 & 2.55 & 2.60 & 2.77 & 2.89 \\
\hline 35 & 6.41 & 5.53 & 4.23 & 3.12 & 2.62 & 2.54 & $2.54^{*}$ & 2.54 & 2.51 & 2.52 & 2.73 & 3.13 \\
\hline 36 & 5.45 & 4.56 & 3.54 & 2.80 & 2.51 & 2.50 & 2.52 & 2.48 & 2.37 & 2.37 & 2.68 & 3.16 \\
\hline 37 & 4.92 & 4.06 & 3.15 & 2.48 & 2.14 & 1.99 & 1.96 & 1.95 & 2.02 & 2.20 & 2.68 & 3.30 \\
\hline
\end{tabular}


Table A-3. Middle Bay, East Half. (3 sheets)

\begin{tabular}{|c|c|c|c|c|c|c|c|c|c|c|c|c|}
\hline & 23 & 24 & 25 & 26 & 27 & 28 & 29 & 30 & 31 & 32 & 33 & 34 \\
\hline 38 & 4.92 & 3.98 & 3.02 & 2.21 & 1.66 & 1.38 & 1.31 & 1.36 & 1.59 & 2.02 & 2.69 & 3.42 \\
\hline 39 & 5.43 & 4.46 & 3.30 & 2.20 & 1.52 & 1.29 & $127 *$ & 1.28 & 1.43 & 1.91 & 2.76 & 3.48 \\
\hline 40 & 6.30 & 5.46 & 4.15 & 2.65 & 1.68 & 1.34 & 1.29 & 1.32 & 1.50 & 2.04 & 2.86 & 3.52 \\
\hline 41 & 7.16 & 6.65 & 5.55 & 3.84 & 2.36 & 1.61 & 1.46 & 1.50 & 1.75 & 2.30 & 3.07 & 3.55 \\
\hline 42 & 7.68 & 7.48 & 6.89 & 5.58 & 3.99 & 2.70 & 2.17 & 1.99 & 2.24 & 2.73 & 3.28 & 3.69 \\
\hline 43 & 7.89 & 7.83 & 7.60 & 7.03 & 5.77 & 4.35 & 3.44 & 3.05 & 2.92 & 3.21 & 3.53 & 3.73 \\
\hline 44 & 7.94 & 7.92 & 7.86 & 7.57 & 6.85 & 5.78 & 4.78 & 4.11 & 3.84 & 3.71 & 3.86 & 3.88 \\
\hline 45 & 7.94* & 7.94 & 7.91 & 7.77 & 7.38 & 6.69 & 5.88 & 5.15 & 4.38 & 4.16 & 4.03 & 3.94 \\
\hline 46 & 7.94 & 7.93 & 7.93 & 7.85 & 7.61 & 7.15 & 6.52 & 5.93 & 5.19 & 4.69 & 4.34 & 4.14 \\
\hline 47 & 7.91 & 7.88 & 7.82 & 7.94 & 7.79 & 7.46 & 7.74 & 6.56 & 5.75 & 5.15 & 4.74 & 4.54 \\
\hline 48 & 7.76 & 7.71 & 7.65 & 7.55 & 8.06 & 8.94 & 8.15 & 7.08 & 6.28 & 5.65 & 5.37 & 4.92 \\
\hline 49 & 7.47 & 7.45 & 7.45 & 7.42 & 7.87 & 9.11 & 8.45 & 7.51 & 6.77 & 6.34 & 5.71 & 5.60 \\
\hline 50 & 7.33 & 7.33 & 7.34 & 7.55 & 7.59 & 7.54 & 8.64 & 7.77 & 7.23 & 6.3 & 6.22 & 6.04 \\
\hline 51 & 7.31 & 7.31 & 7.35 & 7.41 & 7.48 & 7.47 & 7.37 & 8.07 & 6.96 & 6.8 & 6.57 & 6.40 \\
\hline 52 & $7.31 *$ & 7.31 & 7.33 & 7.38 & 7.43 & 7.48 & 7.51 & 6.83 & 7.47 & 7.07 & 6.82 & 6.67 \\
\hline 53 & 7.30 & 7.29 & 7.34 & 7.40 & 7.45 & 7.57 & 8.06 & 8.26 & 7.77 & 7.13 & 6.9 & 6.86 \\
\hline 54 & 7.24 & 7.21 & 7.13 & 7.49 & 7.56 & 7.94 & 8.7 & 8.31 & 7.68 & 7.39 & 7.1 & 7.05 \\
\hline 55 & 7.02 & 6.97 & 6.84 & 6.70 & 7.40 & 8.93 & 8.57 & 8.13 & 7.75 & 7.36 & 7.27 & 7.20 \\
\hline 56 & 7.17 & 6.95 & 6.58 & 6.33 & 6.56 & 8.66 & 8.32 & 7.90 & 7.56 & 7.38 & 7.25 & 7.27 \\
\hline 57 & 7.97 & 7.34 & 6.40 & 6.05 & 5.99 & 6.19 & 8.18 & 7.80 & 7.51 & 7.36 & 7.31 & 7.29 \\
\hline 58 & $8.57^{*}$ & 6.90 & 5.67 & 5.43 & 5.50 & 5.82 & 6.25 & 7.75 & 7.49 & 7.37 & 7.31 & 7.30 \\
\hline 59 & $1.27 *$ & 2.95 & 4.27 & 4.75 & 5.05 & 5.52 & 6.04 & 6.58 & 6.95 & 7.19 & 7.27 & 7.29 \\
\hline 60 & 1.87 & 2.50 & 3.58 & 4.31 & 4.85 & 5.35 & 5.94 & 6.55 & 6.98 & 7.17 & 7.23 & 7.24 \\
\hline 61 & 2.73 & 3.00 & 3.54 & 4.24 & 4.80 & 5.31 & 5.96 & 6.55 & 7.00 & 7.20 & 7.17 & 7.25 \\
\hline 62 & 3.35 & 3.52 & 3.80 & 4.19 & 4.59 & 5.40 & 5.99 & 6.63 & 7.11 & 7.19 & 7.54 & 7.59 \\
\hline 63 & 3.90 & 4.06 & 4.12 & 4.39 & 4.70 & 4.94 & 6.04 & 5.97 & 6.88 & 7.88 & 8.29 & 8.30 \\
\hline 64 & 4.45 & 4.59 & 4.39 & 4.53 & 4.71 & 4.84 & 5.26 & 5.88 & 7.34 & 8.45 & 8.91 & 9.26 \\
\hline 65 & 4.96 & 4.15 & 4.23 & 4.32 & 4.43 & 4.70 & 5.02 & 6.48 & 7.52 & 8.28 & 9.47 & 9.91 \\
\hline 66 & 5.19 & 4.51 & 4.45 & 4.45 & 4.48 & & & 5.45 & 6.89 & & 9.65 & 10.10 \\
\hline 67 & 5.33 & 4.57 & 4.52 & 4.52 & 4.55 & & & 5.01 & 6.39 & & 9.63 & 10.10 \\
\hline 68 & 5.17 & 4.79 & 4.50 & 4.60 & 4.69 & & & 4.61 & & & & 9.74 \\
\hline 69 & 5.40 & & & 4.58 & $4.76^{*}$ & & & & & & & 6.71 \\
\hline 70 & & 5.38 & 4.26 & 3.15 & 3.03 & & & 3.48 & 4.23 & 4.95 & 4.58 & 3.96 \\
\hline 71 & $5.56^{*}$ & 5.49 & 4.14 & 1.65 & $1.27^{*}$ & 1.51 & 2.31 & 3.25 & 4.20 & 4.42 & 4.19 & 3.65 \\
\hline 72 & 5.52 & 5.30 & 3.97 & 1.85 & 1.40 & 1.56 & 2.23 & 3.19 & 4.13 & 4.53 & 4.59 & 4.56 \\
\hline 73 & 5.15 & 4.33 & 3.09 & 2.81 & 2.47 & 2.55 & 3.03 & 3.52 & 4.15 & 4.66 & 5.00 & 5.52 \\
\hline 74 & 5.76 & 4.01 & 1.88 & 2.98 & 4.05 & 4.11 & 4.10 & 4.18 & 4.28 & 4.54 & 4.84 & 5.10 \\
\hline 75 & $5.72^{*}$ & 4.24 & 1.27 & 4.47 & 5.93 & 5.77 & 5.12 & 4.62 & 4.31 & 4.35 & 4.19 & 3.80 \\
\hline
\end{tabular}


SNF-10293, REV 0

Table A-3. Middle Bay, East Half. (3 sheets)

\begin{tabular}{|c|c|c|c|c|c|c|c|c|c|c|c|c|}
\hline & 23 & 24 & 25 & 26 & 27 & 28 & 29 & 30 & 31 & 32 & 33 & 34 \\
\hline 76 & $9.21 *$ & 5.77 & 3.49 & $7.94 *$ & 7.22 & 6.13 & 5.36 & 4.72 & 4.32 & 4.05 & 3.78 & 3.31 \\
\hline
\end{tabular}

Asterisks mark locations that were measured in WHC-SD-WM-ANAL-037 (Meling 1994). 
SNF-10293, REV 0

Table A-4. Middle Bay, West Half. (3 sheets)

\begin{tabular}{|c|c|c|c|c|c|c|c|c|c|c|c|}
\hline & 35 & 36 & 37 & 38 & 39 & 40 & 41 & 42 & 43 & 44 & 45 \\
\hline 1 & 5.69 & 5.99 & 5.25 & 4.07 & 3.32 & 3.75 & $4.13 *$ & $0.95^{*}$ & $3.49^{*}$ & $2.22 *$ & $5.72 *$ \\
\hline 2 & 6.51 & 6.81 & 5.88 & 3.76 & 2.98 & 2.96 & 3.12 & 1.61 & 2.62 & 3.24 & 5.40 \\
\hline 3 & 6.80 & $6.99 *$ & 5.83 & 2.88 & 2.86 & $2.86^{*}$ & $2.86^{*}$ & $0.95^{*}$ & $2.22 *$ & 3.12 & $6.67^{*}$ \\
\hline 4 & 6.32 & 6.43 & 3.43 & $2.54^{*}$ & 2.58 & 2.74 & 2.85 & 2.16 & 1.71 & $0.64 *$ & 4.24 \\
\hline 5 & 4.85 & 3.97 & 2.27 & 1.84 & 2.04 & 2.31 & 2.89 & $3.18^{*}$ & 2.20 & $1.59 *$ & $6.35^{*}$ \\
\hline 6 & 4.03 & 1.90 & 1.20 & $0.95^{*}$ & 1.66 & $2.22 *$ & 2.45 & 3.08 & 3.34 & 3.52 & 4.32 \\
\hline 7 & 4.21 & 2.58 & 1.51 & 1.20 & 1.86 & 2.20 & 2.34 & 3.35 & 4.33 & $4.76^{*}$ & $3.18^{*}$ \\
\hline 8 & 5.19 & 4.80 & 3.39 & 2.49 & 2.26 & $2.22 *$ & 2.31 & 3.02 & 4.01 & 4.30 & 3.53 \\
\hline 9 & 6.16 & 6.25 & 5.66 & 3.96 & 2.90 & 2.53 & 2.79 & 2.67 & 2.52 & 2.99 & 3.28 \\
\hline 10 & 6.22 & $6.35^{*}$ & 5.97 & 4.67 & 5.11 & 5.58 & 4.66 & 1.75 & 0.87 & 1.23 & 1.91 \\
\hline 11 & 5.70 & 5.47 & 3.46 & 3.81 & 5.22 & $6.03 *$ & 5.03 & 1.26 & $0.64 *$ & 0.92 & 1.54 \\
\hline 12 & 4.30 & 2.96 & $2.54^{*}$ & 3.05 & 3.87 & $3.18^{*}$ & 3.70 & 1.93 & 0.97 & $0.95^{*}$ & 2.34 \\
\hline 13 & 4.19 & 3.16 & 3.34 & 4.93 & 4.41 & 3.99 & 3.77 & 2.91 & 1.75 & 2.36 & $3.81 *$ \\
\hline 14 & 4.87 & 4.49 & 5.38 & $5.72 *$ & 5.14 & $4.76^{*}$ & 4.62 & 3.70 & 2.73 & 2.74 & 3.42 \\
\hline 15 & 4.88 & 4.70 & 4.96 & 4.93 & 5.15 & 5.34 & 5.22 & 3.98 & 2.73 & $2.54^{*}$ & 3.18 \\
\hline 16 & 4.90 & 4.58 & 4.23 & $4.13^{*}$ & 5.09 & $6.03 *$ & 5.87 & 4.57 & 3.08 & 3.19 & $3.81 *$ \\
\hline 17 & 4.88 & 4.51 & 4.26 & 4.26 & 5.09 & 5.88 & 5.74 & 4.76 & 3.64 & 3.58 & 3.74 \\
\hline 18 & 4.76 & 4.56 & 4.50 & 4.12 & 5.09 & 5.48 & 5.35 & 4.75 & 4.04 & 3.77 & 3.71 \\
\hline 19 & 4.75 & 4.70 & 4.23 & 4.17 & 5.06 & 5.33 & 5.17 & 4.84 & 4.50 & 4.28 & 4.21 \\
\hline 20 & 4.71 & 4.34 & 4.35 & 4.16 & 5.24 & 5.46 & 5.29 & 5.35 & 5.39 & 5.51 & 5.57 \\
\hline 21 & 4.30 & 4.39 & 4.51 & 4.53 & 5.42 & 5.92 & 6.27 & 6.36 & 6.93 & 7.35 & 7.54 \\
\hline 22 & 4.21 & 4.49 & 4.88 & 5.20 & 5.96 & 6.35 & 7.08 & 7.80 & 8.15 & 8.54 & 8.64 \\
\hline 23 & 3.97 & 4.52 & 5.03 & 5.85 & 6.40 & 6.93 & 7.69 & 8.34 & 8.69 & 8.84 & 8.88 \\
\hline 24 & 3.75 & 4.40 & 5.35 & 6.03 & 6.56 & 7.33 & 8.03 & 8.55 & 8.80 & 8.88 & $8.89 *$ \\
\hline 25 & 3.27 & 4.30 & 5.29 & 6.11 & 6.75 & 7.52 & 8.15 & 8.58 & 8.79 & 8.87 & 8.88 \\
\hline 26 & 3.30 & 3.90 & 4.75 & 6.08 & 6.81 & 7.53 & 8.11 & 8.51 & 8.73 & 8.83 & 8.84 \\
\hline 27 & 3.23 & 3.92 & 4.64 & 5.73 & 6.65 & 7.47 & 8.02 & 8.40 & 8.62 & 8.73 & 8.76 \\
\hline 28 & 2.81 & 3.51 & 4.45 & 5.50 & 6.43 & 7.28 & 7.86 & 8.25 & 8.49 & 8.63 & 8.66 \\
\hline 29 & 3.41 & 3.71 & 4.55 & 5.82 & 6.33 & 7.13 & 7.81 & 8.22 & 8.49 & 8.65 & 8.70 \\
\hline 30 & 3.15 & 3.79 & 4.54 & 5.69 & 6.34 & 7.15 & 7.85 & 8.39 & 8.72 & 8.95 & 9.04 \\
\hline 31 & 3.24 & 3.82 & 4.54 & 5.43 & 6.37 & 7.35 & 8.03 & 8.71 & 9.09 & 9.31 & 9.40 \\
\hline 32 & 3.29 & 3.83 & 4.52 & 5.35 & 6.43 & 7.40 & 8.32 & 8.99 & 9.39 & 9.59 & 9.66 \\
\hline 33 & 3.21 & 3.83 & 4.40 & 5.16 & 6.23 & 7.35 & 8.44 & 9.21 & 9.60 & 9.75 & 9.79 \\
\hline 34 & 3.31 & 3.73 & 4.23 & 4.96 & 5.83 & 7.08 & 8.42 & 9.32 & 9.71 & 9.82 & 9.84 \\
\hline 35 & 3.41 & 3.74 & 4.01 & 4.52 & 5.23 & 6.53 & 8.14 & 9.27 & 9.73 & 9.83 & 9.84* \\
\hline 36 & 3.59 & 3.78 & 3.89 & 4.08 & 4.57 & 5.68 & 7.47 & 9.00 & 9.64 & 9.81 & 9.83 \\
\hline 37 & 3.69 & 3.80 & 3.83 & 3.89 & 4.13 & 4.92 & 6.62 & 8.35 & 9.32 & 9.66 & 9.75 \\
\hline
\end{tabular}


Table A-4. Middle Bay, West Half. (3 sheets)

\begin{tabular}{|c|c|c|c|c|c|c|c|c|c|c|c|}
\hline & 35 & 36 & 37 & 38 & 39 & 40 & 41 & 42 & 43 & 44 & 45 \\
\hline 38 & 3.74 & 3.81 & 3.81 & 3.82 & 3.93 & 4.48 & 5.74 & 7.30 & 8.55 & 9.19 & 9.40 \\
\hline 39 & 3.76 & 3.81 & $3.81 *$ & 3.82 & 3.88 & 4.28 & 5.11 & 6.38 & 7.59 & 8.29 & 8.62 \\
\hline 40 & 3.77 & 3.81 & 3.81 & 3.83 & 3.94 & 4.25 & 4.94 & 5.86 & 6.74 & 7.29 & 7.62 \\
\hline 41 & 3.77 & 3.82 & 3.83 & 3.88 & 4.00 & 4.33 & 4.93 & 5.70 & 6.40 & 6.76 & 6.93 \\
\hline 42 & 3.79 & 3.82 & 3.83 & 3.89 & 4.03 & 4.40 & 5.05 & 5.87 & 6.39 & 6.61 & 6.70 \\
\hline 43 & 3.83 & 3.81 & 3.81 & 3.84 & 3.98 & 4.43 & 5.23 & 6.08 & 6.51 & 6.64 & 6.67 \\
\hline 44 & 3.81 & 3.81 & $3.81^{*}$ & 3.82 & 3.89 & 4.56 & 5.50 & 6.28 & 6.60 & 6.66 & 6.67 \\
\hline 45 & 3.88 & 3.83 & 3.82 & 3.87 & 4.19 & 5.04 & 5.96 & 6.48 & 6.65 & 6.67 & $6.67 *$ \\
\hline 46 & 4.05 & 3.97 & 3.97 & 4.19 & 4.93 & 5.99 & 6.58 & 6.73 & 6.70 & 6.67 & 6.67 \\
\hline 47 & 4.37 & 4.41 & 4.60 & 5.24 & 6.27 & 6.94 & 7.12 & 7.09 & 6.90 & 6.74 & 6.69 \\
\hline 48 & 4.92 & 5.13 & 5.56 & 6.35 & 6.99 & 7.25 & 7.29 & 7.26 & 7.14 & 6.92 & 6.79 \\
\hline 49 & 5.46 & 5.79 & 6.22 & 6.81 & 7.17 & 7.29 & $7.30^{*}$ & 7.29 & 7.22 & 7.07 & 6.94 \\
\hline 50 & 6.03 & 6.26 & 6.55 & 6.92 & 7.16 & 7.27 & 7.29 & 7.27 & 7.20 & 7.10 & 7.04 \\
\hline 51 & 6.35 & 6.35 & 6.61 & 6.82 & 6.98 & 7.11 & 7.16 & 7.14 & 7.09 & 7.12 & 7.21 \\
\hline 52 & 6.57 & 6.44 & 6.42 & 6.57 & 6.56 & 6.57 & 6.61 & 6.69 & 6.93 & 7.26 & 7.50 \\
\hline 53 & 6.75 & 6.52 & 6.53 & 6.26 & 6.12 & 5.98 & 5.97 & 6.19 & 6.85 & 7.54 & 7.77 \\
\hline 54 & 6.96 & 6.82 & 6.51 & 6.13 & 5.88 & 5.77 & 5.74 & 5.86 & 6.83 & 7.80 & 7.92 \\
\hline 55 & 7.14 & 7.01 & 6.67 & 6.15 & 5.84 & 5.73 & $5.72 *$ & 5.77 & 6.83 & 7.90 & 7.94* \\
\hline 56 & 7.25 & 7.20 & 6.93 & 6.36 & 5.90 & 5.76 & 5.74 & 5.85 & 6.81 & 7.80 & 7.92 \\
\hline 57 & 7.30 & 7.28 & 7.14 & 6.63 & 6.07 & 5.86 & 5.84 & 6.05 & 6.76 & 7.52 & 7.76 \\
\hline 58 & $7.30 *$ & 7.29 & 7.20 & 6.78 & 6.19 & 5.89 & 5.87 & 6.10 & 6.56 & 7.10 & 7.38 \\
\hline 59 & 7.30 & 7.28 & 7.13 & 6.63 & 5.94 & 5.50 & 5.40 & 5.58 & 5.96 & 6.41 & 6.80 \\
\hline 60 & 7.25 & 7.18 & 6.86 & 6.15 & 5.21 & 4.61 & 4.42 & 4.58 & 4.97 & 5.55 & 6.00 \\
\hline 61 & 7.22 & 6.95 & 6.36 & 5.27 & 4.36 & 3.86 & 3.73 & 3.82 & 4.19 & 4.83 & 5.54 \\
\hline 62 & 7.37 & 6.90 & 5.98 & 4.76 & 3.87 & 3.57 & 3.51 & 3.57 & 3.87 & 4.60 & 5.51 \\
\hline 63 & 8.18 & 7.53 & 6.15 & 4.76 & 3.79 & 3.51 & $3.49 *$ & 3.52 & 3.86 & 4.90 & 6.09 \\
\hline 64 & 9.33 & 8.75 & 7.43 & 5.40 & 4.01 & 3.58 & 3.52 & 3.63 & 4.39 & 6.09 & 7.30 \\
\hline 65 & 9.96 & 9.71 & 8.81 & 6.62 & 4.73 & 3.98 & 3.88 & 4.41 & 6.06 & 7.82 & 8.40 \\
\hline 66 & 10.10 & 10.10 & 9.39 & 7.75 & 5.81 & 4.86 & 4.92 & 6.02 & 7.74 & 8.68 & 8.84 \\
\hline 67 & $10.20 *$ & 10.10 & 9.45 & 8.04 & 6.51 & 5.72 & 5.64 & 6.76 & 8.11 & 8.81 & $8.89 *$ \\
\hline 68 & 9.99 & 9.70 & 8.74 & 7.32 & 5.93 & 5.18 & 5.12 & 6.20 & 7.38 & 8.40 & 8.72 \\
\hline 69 & 6.75 & 6.68 & 6.48 & 5.87 & 4.99 & 4.22 & 4.12 & 4.97 & 5.48 & 5.64 & 5.66 \\
\hline 70 & 3.67 & 3.85 & 4.56 & 4.68 & 3.91 & 3.50 & 3.38 & 3.98 & 4.19 & 3.26 & 2.56 \\
\hline 71 & $3.49 *$ & 3.65 & 4.14 & 4.19 & 3.63 & 3.23 & 3.23 & 3.44 & 4.23 & 3.39 & $1.91 *$ \\
\hline 72 & 4.58 & 4.52 & 4.39 & 4.04 & 3.48 & 3.21 & $3.18^{*}$ & 3.28 & 4.80 & 6.75 & $8.26^{*}$ \\
\hline 73 & $5.72 *$ & 5.36 & 4.31 & 3.87 & 3.56 & 3.27 & 3.22 & 3.45 & 5.05 & 6.99 & 7.71 \\
\hline 74 & 4.96 & 3.27 & 3.12 & 3.39 & 3.54 & 3.47 & 3.48 & 3.88 & 5.00 & 6.31 & 6.82 \\
\hline 75 & 2.80 & $2.54 *$ & 2.63 & 3.02 & 3.43 & 3.65 & 3.77 & 4.17 & 4.94 & 5.69 & 6.13 \\
\hline
\end{tabular}


SNF-10293, REV 0

Table A-4. Middle Bay, West Half. (3 sheets)

\begin{tabular}{|c|c|c|c|c|c|c|c|c|c|c|c|}
\hline & 35 & 36 & 37 & 38 & 39 & 40 & 41 & 42 & 43 & 44 & 45 \\
\hline 76 & 2.76 & 2.60 & 2.67 & 2.95 & 3.35 & 3.67 & 4.18 & 4.34 & 4.86 & 5.36 & 5.63 \\
\hline
\end{tabular}
1994). 
SNF-10293, REV 0

Table A-5. West Bay, East Half. (3 sheets)

\begin{tabular}{|c|c|c|c|c|c|c|c|c|c|c|c|c|}
\hline & 46 & 47 & 48 & 49 & 50 & 51 & 52 & 53 & 54 & 55 & 56 & 57 \\
\hline 1 & $6.99 *$ & 5.07 & $3.18^{*}$ & 3.21 & 2.63 & $2.54^{*}$ & 2.53 & 2.28 & 2.19 & $2.22 *$ & 2.09 & 1.33 \\
\hline 2 & 5.73 & 5.05 & 3.39 & 3.28 & 2.75 & 2.57 & 2.50 & 2.19 & 2.03 & 2.06 & 1.65 & 1.28 \\
\hline 3 & 5.75 & 4.96 & 4.06 & 3.58 & 3.12 & 2.60 & 2.36 & 2.04 & 1.37 & 1.00 & 0.76 & 0.88 \\
\hline 4 & 5.13 & 4.96 & 4.62 & 3.72 & 3.70 & 3.09 & 2.39 & 1.76 & 1.09 & 0.82 & $0.64 *$ & 0.69 \\
\hline 5 & 5.08 & 5.04 & 4.90 & 4.24 & 3.66 & 3.57 & 2.61 & 1.72 & 1.06 & 0.69 & 0.66 & 0.73 \\
\hline 6 & $5.08^{*}$ & 5.06 & 4.85 & 4.31 & 3.80 & 3.36 & 2.40 & 1.50 & 1.03 & 0.88 & 0.87 & 0.93 \\
\hline 7 & 5.02 & 4.83 & 4.37 & 3.95 & 4.05 & 3.53 & 3.09 & 2.46 & 1.39 & 1.10 & 1.01 & 1.06 \\
\hline 8 & 3.94 & 3.44 & 3.50 & 3.72 & 3.48 & 3.32 & 3.19 & 2.85 & 2.26 & 1.45 & 1.27 & 1.27 \\
\hline 9 & 3.45 & $3.18^{*}$ & 3.34 & 3.41 & 3.20 & 3.20 & 3.21 & 3.06 & 2.67 & 2.17 & 1.53 & 1.49 \\
\hline 10 & 5.04 & 3.71 & 3.82 & 3.24 & $3.18^{*}$ & 3.18 & 3.15 & 3.00 & 2.07 & 2.37 & 1.98 & 1.60 \\
\hline 11 & 7.94 & 7.33 & 5.62 & 3.67 & 3.22 & 3.23 & 3.27 & 3.22 & 3.05 & 2.71 & 1.75 & 2.02 \\
\hline 12 & $8.09 *$ & 8.01 & 7.08 & 5.01 & 3.88 & 3.41 & 3.37 & 3.32 & 3.12 & 2.80 & 2.38 & 1.63 \\
\hline 13 & 8.07 & 7.98 & 7.34 & 6.06 & 4.87 & 3.83 & 3.68 & 3.51 & 3.02 & 2.84 & 2.52 & 1.89 \\
\hline 14 & 7.65 & 7.47 & 7.12 & 6.28 & 4.69 & 4.17 & 3.79 & 3.58 & 3.37 & 3.13 & 2.91 & 1.85 \\
\hline 15 & 6.30 & 6.33 & 6.39 & 5.82 & 4.82 & 4.41 & 3.61 & 3.30 & 3.30 & 3.27 & 3.15 & 2.53 \\
\hline 16 & 5.89 & 5.92 & 5.89 & 5.80 & 5.51 & 4.41 & 3.88 & 3.19 & 3.23 & 3.45 & 3.46 & 3.25 \\
\hline 17 & $5.88^{*}$ & 5.88 & 5.87 & 5.79 & 5.53 & 5.13 & 3.86 & 3.55 & 3.13 & 3.40 & $3.49 *$ & 3.33 \\
\hline 18 & 5.87 & 5.86 & 5.84 & 5.68 & 5.24 & 4.50 & 3.39 & 2.79 & 2.72 & 2.69 & 2.70 & 2.64 \\
\hline 19 & 5.85 & 5.82 & 5.77 & 5.57 & 5.08 & 4.36 & 3.40 & 2.58 & 2.30 & 2.00 & $1.91 *$ & 2.01 \\
\hline 20 & 5.73 & 5.76 & 5.54 & 5.26 & 5.01 & 4.45 & 3.09 & 2.54 & 2.25 & 1.93 & 1.92 & 1.94 \\
\hline 21 & 5.37 & 5.57 & 5.50 & 4.96 & 4.61 & 4.54 & 3.61 & 2.74 & 2.14 & 2.04 & 2.00 & 2.12 \\
\hline 22 & 4.58 & 5.20 & 5.26 & 5.28 & 4.65 & 4.37 & 3.72 & 3.03 & 2.49 & 2.23 & 2.43 & 2.56 \\
\hline 23 & 3.51 & 4.68 & 5.03 & 5.35 & 5.43 & 5.20 & 4.32 & 3.79 & 3.13 & 2.91 & 2.73 & 3.01 \\
\hline 24 & 3.62 & 4.04 & 4.83 & 5.52 & 5.87 & 5.80 & 5.52 & 4.51 & 4.28 & 3.59 & 3.20 & 2.94 \\
\hline 25 & 2.66 & 3.23 & 4.68 & 5.90 & 6.19 & 6.17 & 5.99 & 5.37 & 4.76 & 4.02 & 3.35 & 2.92 \\
\hline 26 & 1.84 & 2.40 & 4.82 & 6.20 & 6.33 & 6.32 & 6.12 & 5.70 & 4.99 & 4.12 & 3.41 & 2.90 \\
\hline 27 & $1.59 *$ & 2.50 & 5.20 & 6.31 & $6.35^{*}$ & 6.33 & 6.17 & 5.76 & 4.88 & 3.93 & 3.13 & 2.76 \\
\hline 28 & 6.78 & 6.77 & 6.54 & 6.37 & 6.35 & 6.34 & 6.40 & 6.20 & 5.56 & 4.31 & 3.55 & 3.00 \\
\hline 29 & $9.37^{*}$ & 9.13 & 8.11 & 6.97 & 6.48 & 6.36 & 6.24 & 6.19 & 5.47 & 4.49 & 3.44 & 3.38 \\
\hline 30 & 9.36 & 9.32 & 8.47 & 7.54 & 7.38 & 6.92 & 6.62 & 6.20 & 4.30 & 3.89 & 3.62 & 3.04 \\
\hline 31 & 9.30 & 9.18 & 8.89 & 7.65 & 7.04 & 7.10 & 6.63 & 5.46 & 5.09 & 4.01 & 3.55 & 3.26 \\
\hline 32 & 8.92 & 8.71 & 8.41 & 7.93 & 6.85 & 6.96 & 6.44 & 5.60 & 5.07 & 4.42 & 3.46 & 2.76 \\
\hline 33 & 7.58 & 7.42 & 7.21 & 6.92 & 6.58 & 6.27 & 5.43 & 4.66 & 4.10 & 3.27 & 2.91 & 2.69 \\
\hline 34 & 4.95 & 5.07 & 5.23 & 5.37 & 5.45 & 5.25 & 4.84 & 4.85 & 3.64 & 3.04 & 2.59 & 2.34 \\
\hline 35 & 2.57 & 2.81 & 3.26 & 3.79 & 4.66 & 4.80 & 4.07 & 3.73 & 3.35 & 2.92 & 2.57 & 2.62 \\
\hline 36 & 1.47 & 1.67 & 2.15 & 3.49 & 4.17 & 4.50 & 3.39 & 2.88 & 3.12 & 2.84 & 2.75 & 2.34 \\
\hline 37 & 1.05 & 1.23 & 2.34 & 3.35 & 4.09 & 3.08 & 3.19 & 2.85 & 2.52 & 2.87 & 2.52 & 2.33 \\
\hline
\end{tabular}


Table A-5. West Bay, East Half. (3 sheets)

\begin{tabular}{|c|c|c|c|c|c|c|c|c|c|c|c|c|}
\hline & 46 & 47 & 48 & 49 & 50 & 51 & 52 & 53 & 54 & 55 & 56 & 57 \\
\hline 38 & $0.95^{*}$ & 1.35 & 2.73 & 3.81 & 2.96 & 3.07 & 3.20 & 3.13 & 2.85 & 2.49 & 2.53 & 2.35 \\
\hline 39 & .35 & 3.73 & 4.41 & 3.12 & 3.23 & 3.17 & 3.21 & 3.23 & 2.83 & 2.71 & 2.57 & 2.28 \\
\hline 40 & $08 *$ & 6.15 & 6.37 & 3.68 & 3.49 & 3.25 & 3.21 & 3.10 & 3.03 & 3.62 & 2.77 & 2.57 \\
\hline 41 & $10.80 *$ & 9.36 & 7.79 & 6.62 & 6.32 & 5.36 & 4.83 & 4.51 & 4.21 & 3.29 & 2.99 & 2.95 \\
\hline 42 & 10.30 & 9.71 & 7.96 & 6.05 & 4.66 & 4.20 & 4.03 & 3.99 & 4.00 & 3.58 & 3.36 & 3.29 \\
\hline 43 & 9.34 & 8.63 & 6.57 & 4.17 & 3.38 & 3.22 & 3.38 & 3.61 & 3.50 & 3.47 & 3.37 & 3.34 \\
\hline 44 & 8.04 & 6.66 & 4.34 & 3.09 & 2.90 & 2.92 & 3.07 & 3.18 & 3.32 & 3.3 & 3.39 & 3.45 \\
\hline 45 & 6.99 & 5.44 & 3.62 & 2.92 & $2.86^{*}$ & 2.88 & & 3.03 & 3.21 & 3.30 & 3.36 & 3.47 \\
\hline 46 & 7.07 & 5.73 & 3.97 & 3.05 & 2.90 & 2.92 & 3.02 & 3.03 & 3.10 & 3.22 & 3.29 & 3.43 \\
\hline 47 & 7.83 & 7.16 & 5.50 & 3.93 & 3.35 & 3.25 & 3.37 & 3.19 & 3.11 & 3.0 & 3.14 & 3.39 \\
\hline 48 & 8.30 & 8.10 & 7.37 & 5.88 & 4.64 & 4.15 & 4.03 & 3.46 & 3.10 & 2.92 & 2.92 & 3.26 \\
\hline 49 & 8.41 & 8.37 & 8.17 & 7.19 & 5.85 & 4.87 & 3.98 & 3.63 & 3.22 & 2.77 & 3.10 & 3.11 \\
\hline 50 & $8.42 *$ & 8.40 & 8.31 & 7.49 & 6.02 & 4.89 & 3.52 & 3.35 & 2.64 & 2.85 & 2.97 & 3.16 \\
\hline 51 & 8.41 & 8.36 & 8.15 & 8.01 & 5.51 & 3.73 & 3.00 & 3.12 & 2.43 & 2.7 & 2.53 & 2.75 \\
\hline 52 & 8.33 & 8.13 & 7.55 & 6.72 & 4.94 & 3.10 & 2.36 & 2.10 & 2.81 & 2.5 & 2.55 & 2.62 \\
\hline 53 & 8.07 & 7.77 & 6.95 & 6.39 & 5.21 & 2.63 & 1.76 & 2.00 & 2.48 & 2.61 & $2.54^{*}$ & 2.67 \\
\hline 54 & 7.61 & 7.37 & 7.29 & 7.31 & 7.28 & 2.98 & $1.59^{*}$ & 1.66 & 2.67 & 3.3 & 2.96 & 3.51 \\
\hline 55 & 6.89 & 7.17 & 7.10 & 7.71 & 7.94* & 5.51 & 1.73 & 1.85 & 3.36 & 6.26 & 7.12 & 6.64 \\
\hline 56 & 6.68 & 6.56 & 6.76 & 7.26 & 7.24 & 2.76 & $1.27^{*}$ & 1.53 & 4.10 & 7.32 & $7.62 *$ & 7.48 \\
\hline 57 & 6.96 & 6.82 & 6.32 & 6.03 & 4.97 & 2.42 & 1.49 & 1.86 & 4.05 & 7.07 & 7.55 & 7.45 \\
\hline 58 & 7.08 & 6.92 & 6.43 & 5.60 & 4.21 & 2.75 & 2.17 & 2.11 & 4.17 & 5.96 & 6.84 & 6.98 \\
\hline 59 & 7.14 & 7.09 & 6.78 & 6.04 & 3.36 & 2.11 & 1.71 & 2.00 & 2.83 & 4.72 & 5.57 & 6.12 \\
\hline 60 & $7.15^{*}$ & 7.13 & 6.93 & 5.25 & 2.49 & 1.29 & 1.02 & 1.18 & 1.94 & 3.36 & 4.65 & 5.25 \\
\hline 61 & 7.14 & 7.10 & 6.80 & 5.02 & 2.25 & 1.05 & $0.95^{*}$ & 1.03 & 1.86 & 3.53 & 4.79 & 5.44 \\
\hline 62 & 7.07 & 6.93 & 6.48 & 5.29 & 3.24 & 1.50 & 1.08 & 1.34 & 2.77 & 4.92 & 5.67 & 5.82 \\
\hline 63 & 6.85 & 6.77 & 6.62 & 6.56 & 6.12 & 4.46 & 2.82 & 2.98 & 4.75 & 5.85 & 6.00 & 6.00 \\
\hline 64 & 6.38 & 6.52 & 7.00 & 7.44 & 7.54 & 7.21 & 5.98 & 5.06 & 5.65 & 6.01 & $6.03^{*}$ & 6.02 \\
\hline 65 & 5.54 & 6.07 & 7.06 & 7.57 & $7.62 *$ & 7.56 & & 6.04 & 6.04 & 6.01 & 6.03 & 6.03 \\
\hline 66 & 4.42 & 5.02 & 6.52 & 7.44 & 7.58 & 7.46 & & 6.23 & 6.10 & & 6.23 & 6.29 \\
\hline 67 & 3.68 & 4.06 & 5.44 & 6.79 & 7.15 & & & & & & 7.52 & 6.77 \\
\hline 68 & $3.49^{*}$ & 3.95 & 5.38 & 6.23 & 6.20 & & & & 6.79 & & & 7.73 \\
\hline 69 & & & 7.13 & 6.48 & 5.22 & & & & & & $7.62 *$ & 7.47 \\
\hline 70 & $11.10^{*}$ & 10.60 & 8.89 & 6.64 & 4.35 & & & 3.52 & 5.44 & 7.30 & 7.62 & 7.00 \\
\hline 71 & 10.90 & 10.50 & 9.02 & 6.29 & 3.95 & 3.24 & 3.10 & 3.26 & 4.38 & & 7.59 & 7.80 \\
\hline 72 & 10.20 & 9.64 & 8.09 & 5.81 & 4.07 & 3.36 & 3.24 & 3.44 & 4.55 & 6.35 & 7.47 & 7.79 \\
\hline 73 & 8.94 & 8.11 & 6.65 & 5.31 & 4.47 & 3.94 & 3.79 & 4.32 & 5.55 & 6.70 & 7.42 & 7.69 \\
\hline 74 & 7.43 & 6.43 & 5.36 & 4.76 & 4.57 & 4.55 & 4.87 & 5.84 & 6.93 & 7.40 & 7.16 & 7.76 \\
\hline 75 & 6.23 & 5.38 & 4.74 & 4.50 & 4.47 & 4.53 & 5.13 & 6.68 & 7.50 & 7.59 & 7.54 & 7.66 \\
\hline
\end{tabular}


SNF-10293, REV 0

Table A-5. West Bay, East Half. (3 sheets)

\begin{tabular}{|c|c|c|c|c|c|c|c|c|c|c|c|c|}
\hline & 46 & 47 & 48 & 49 & 50 & 51 & 52 & 53 & 54 & 55 & 56 & 57 \\
\hline 76 & 5.58 & 4.97 & 4.59 & 4.46 & $4.45 *$ & 4.48 & 5.09 & 6.94 & 7.58 & $7.62 *$ & 7.60 & 7.61 \\
\hline
\end{tabular}

Asterisks mark locations that were measured in WHC-SD-WM-ANAL-037 (Meling 1994). 
Table A-6. West Bay, West Half -- Cubicle 6776 is the North-West Corner of K East Basin. (3 sheets)

\begin{tabular}{|c|c|c|c|c|c|c|c|c|c|c|}
\hline & 58 & 59 & 60 & 61 & 62 & 63 & 64 & 65 & 66 & 67 \\
\hline 1 & $1.27^{*}$ & 1.28 & 1.46 & 1.58 & $1.59^{*}$ & 1.59 & 1.63 & 1.76 & 1.84 & 1.87 \\
\hline 2 & 1.27 & 1.29 & 1.43 & 1.57 & 1.59 & 1.60 & 1.69 & 1.84 & $1.89^{*}$ & 1.90 \\
\hline 3 & 1.13 & 1.34 & 1.48 & 1.54 & 1.59 & 1.66 & 1.81 & 1.89 & 1.91 & 1.91 \\
\hline 4 & 1.11 & 1.49 & 1.58 & 1.57 & 1.60 & 1.71 & 1.86 & 1.91 & 1.91 & $1.91 *$ \\
\hline 5 & 1.24 & 1.57 & $1.59^{*}$ & 1.59 & 1.60 & 1.72 & 1.86 & 1.90 & 1.91 & 1.91 \\
\hline 6 & 1.24 & 1.53 & 1.58 & 1.58 & 1.63 & 1.75 & 1.86 & 1.90 & 1.91 & 1.90 \\
\hline 7 & 1.28 & 1.47 & 1.54 & 1.57 & 1.64 & 1.75 & 1.86 & 1.91 & 1.92 & 1.89 \\
\hline 8 & 1.36 & 1.43 & 1.50 & 1.57 & 1.69 & 1.79 & 1.88 & 1.94 & 1.97 & 1.98 \\
\hline 9 & 1.47 & 1.49 & 1.47 & 1.66 & 1.67 & 1.78 & 1.91 & 2.04 & $2.07^{*}$ & 2.13 \\
\hline 10 & 1.48 & 1.42 & 1.38 & 1.50 & 1.66 & 1.78 & 2.00 & 2.12 & $2.29^{*}$ & 2.45 \\
\hline 11 & 1.46 & 1.35 & 1.30 & 1.39 & 1.54 & 1.80 & 1.99 & 2.32 & $2.64 *$ & 3.02 \\
\hline 12 & 1.62 & 1.28 & 1.25 & 1.29 & 1.43 & 1.69 & 2.10 & 2.63 & 3.28 & 4.87 \\
\hline 13 & 1.34 & 1.25 & 1.25 & 1.26 & 1.34 & 1.65 & 2.24 & 3.91 & 5.35 & 6.25 \\
\hline 14 & 1.33 & 1.21 & $1.27^{*}$ & 1.21 & 1.31 & 1.71 & 3.18 & 5.21 & 6.90 & 7.90 \\
\hline 15 & 1.47 & 1.09 & $0.95^{*}$ & 1.08 & 1.37 & 2.18 & 3.97 & 6.33 & 8.12 & 9.07 \\
\hline 16 & 1.95 & 1.43 & 1.32 & 1.42 & 1.74 & 2.50 & 4.75 & 8.77 & 10.20 & 10.70 \\
\hline 17 & 2.27 & 1.72 & $1.59^{*}$ & 1.72 & 2.15 & 3.81 & 8.01 & 10.70 & 11.50 & 11.60 \\
\hline 18 & 2.24 & 2.08 & $2.22 *$ & 2.10 & 2.41 & 5.19 & 9.76 & 11.80 & 12.00 & 12.00 \\
\hline 19 & 2.21 & 2.17 & 2.19 & 2.22 & 3.03 & 8.48 & 11.50 & 12.10 & 12.10 & 12.10 \\
\hline 20 & 2.33 & 2.51 & 2.57 & 3.16 & 5.48 & 9.52 & 12.10 & 12.40 & 12.40 & 12.40 \\
\hline 21 & 2.40 & 2.85 & 3.28 & 4.36 & 6.87 & 10.10 & 12.50 & 12.80 & 12.70 & 12.80 \\
\hline 22 & 2.91 & 3.41 & 4.16 & 5.79 & 8.74 & 12.00 & 13.60 & 14.10 & 14.20 & 14.20 \\
\hline 23 & 3.17 & 3.37 & 3.82 & 5.06 & 8.34 & 12.00 & 14.40 & 15.60 & 15.90 & 15.70 \\
\hline 24 & 2.84 & 2.73 & 2.92 & 3.65 & 6.03 & 10.50 & 14.10 & 15.50 & 15.80 & 15.60 \\
\hline 25 & 2.70 & 2.59 & 2.56 & 2.74 & 4.00 & 7.95 & 12.30 & 14.60 & 15.20 & 15.10 \\
\hline 26 & 2.65 & 2.55 & $2.54 *$ & 2.58 & 3.19 & 5.70 & 9.56 & 12.50 & 13.70 & 14.00 \\
\hline 27 & 2.60 & 2.58 & 2.56 & 2.64 & 3.15 & 4.71 & 7.18 & 9.84 & $11.00^{*}$ & 11.70 \\
\hline 28 & 2.90 & 2.73 & 2.70 & 2.86 & 3.29 & 4.12 & 5.34 & 6.67 & 7.88 & 8.87 \\
\hline 29 & 3.15 & 3.04 & 2.94 & 3.03 & 3.20 & 3.50 & 3.82 & 4.46 & 5.22 & 6.12 \\
\hline 30 & 3.18 & 2.92 & 2.95 & 2.95 & 2.87 & 2.88 & 2.77 & 3.09 & 3.52 & 4.19 \\
\hline 31 & 2.88 & 3.01 & 3.10 & 3.00 & 2.79 & 2.49 & 2.37 & 2.40 & 2.55 & 3.11 \\
\hline 32 & 2.79 & 2.96 & $3.18^{*}$ & 2.95 & 2.64 & 2.29 & 2.23 & 2.25 & 2.37 & 2.62 \\
\hline 33 & 2.61 & 2.44 & $2.22 *$ & 2.44 & 2.47 & 2.24 & $2.22 *$ & 2.23 & 2.28 & 2.35 \\
\hline 34 & 2.51 & 2.38 & 2.30 & 2.37 & 2.38 & 2.25 & 2.22 & 2.22 & 2.21 & 2.21 \\
\hline 35 & 2.24 & 2.20 & 2.37 & 2.37 & 2.29 & 2.19 & 2.16 & 2.13 & 2.09 & 2.02 \\
\hline 36 & 2.22 & 2.16 & 2.07 & 2.20 & 1.96 & 1.74 & 1.63 & 1.61 & 1.64 & 1.59 \\
\hline
\end{tabular}


Table A-6. West Bay, West Half -- Cubicle 6776 is the North-West Corner of K East Basin. (3 sheets)

\begin{tabular}{|c|c|c|c|c|c|c|c|c|c|c|}
\hline & 58 & 59 & 60 & 61 & 62 & 63 & 64 & 65 & 66 & 67 \\
\hline 37 & 2.18 & 2.04 & 2.05 & 1.71 & 1.29 & 0.97 & 0.86 & 0.91 & 1.07 & 1.19 \\
\hline 38 & .18 & 2.11 & 1.72 & 1.29 & 0.88 & 0.69 & 0.65 & 0.67 & 0.79 & 0.94 \\
\hline 39 & 2.27 & 1.99 & 1.57 & 1.12 & 0.77 & 0.65 & $0.64^{*}$ & 0.65 & 0.71 & 0.85 \\
\hline 40 & 2.46 & 2.21 & 1.78 & 1.25 & 0.84 & 0.68 & 0.65 & 0.66 & 0.74 & 0.84 \\
\hline 41 & 2.87 & 2.72 & 2.37 & 1.82 & 1.20 & 0.85 & 0.75 & 0.76 & 0.85 & 0.94 \\
\hline 42 & 3.21 & 3.18 & 3.07 & 2.73 & 2.10 & 1.46 & 1.14 & 1.09 & 1.15 & 1.15 \\
\hline 43 & 3.37 & 3.41 & 3.40 & 3.30 & 2.97 & 2.38 & 1.87 & 1.67 & 1.62 & 1.48 \\
\hline 44 & 3.44 & 3.48 & 3.48 & 3.46 & 3.33 & 2.95 & 2.46 & 2.24 & 2.10 & 1.80 \\
\hline 45 & 3.47 & 3.49 & $3.49^{*}$ & 3.48 & 3.39 & 3.09 & 2.65 & 2.48 & 2.35 & 2.16 \\
\hline 46 & 3.46 & 3.48 & 3.48 & 3.46 & 3.33 & 2.95 & & 2.34 & 2.29 & 2.19 \\
\hline 47 & 3.42 & 3.43 & 3.41 & 3.32 & 2.98 & 2.47 & & 2.03 & 2.14 & 2.18 \\
\hline 48 & 3.53 & 3.37 & 3.26 & 2.90 & 2.37 & 1.90 & & 1.77 & 1.91 & 2.12 \\
\hline 49 & 3.39 & 3.35 & 3.09 & 2.61 & 1.96 & 1.66 & & 1.64 & 1.77 & 2.06 \\
\hline 50 & 3.39 & 3.52 & 3.25 & 2.65 & 1.89 & 1.61 & 1.07 & 1.61 & 1.76 & 2.15 \\
\hline 51 & 3.12 & 4.04 & 4.08 & 3.27 & 2.21 & 1.71 & 1.62 & 1.68 & 1.97 & 2.56 \\
\hline 52 & 3.45 & 4.57 & 5.05 & 4.59 & 3.35 & 2.50 & 2.05 & 2.22 & 2.83 & 3.70 \\
\hline 53 & 3.80 & 5.44 & 5.90 & 5.79 & 5.07 & 4.07 & 3.77 & 4.21 & 5.10 & 5.87 \\
\hline 54 & 4.94 & 6.17 & 6.32 & 6.25 & 5.98 & 5.70 & 6.16 & 7.14 & 7.97 & 8.21 \\
\hline 55 & 6.12 & 6.32 & $6.35^{*}$ & 6.33 & 6.18 & 6.34 & 7.68 & 9.14 & 9.82 & 9.73 \\
\hline 56 & 6.68 & 6.30 & 6.34 & 6.27 & 6.00 & 6.13 & 8.01 & 10.10 & 10.70 & 10.50 \\
\hline 57 & 6.70 & 6.32 & 6.18 & 5.90 & 4.96 & 4.43 & 6.26 & 10.20 & 10.80 & 10.60 \\
\hline 58 & 6.64 & 6.12 & 5.69 & 4.85 & 3.44 & 2.51 & 2.43 & 6.29 & 10.20 & 9.80 \\
\hline 59 & 6.06 & 5.73 & 5.08 & 3.97 & 2.72 & 2.03 & $1.91 *$ & 2.43 & 5.47 & 5.54 \\
\hline 60 & 5.32 & 4.97 & 4.20 & 3.36 & 2.32 & 2.17 & 2.04 & 2.51 & 3.17 & 2.98 \\
\hline 61 & 5.25 & 4.93 & 4.49 & 3.50 & 3.14 & 2.66 & 2.59 & 2.90 & 2.90 & $2.86^{*}$ \\
\hline 62 & 5.76 & 5.47 & 4.79 & 4.78 & 3.58 & 3.31 & 3.15 & 3.07 & 2.94 & 2.88 \\
\hline 63 & 5.90 & 5.70 & 5.95 & 5.33 & 4.21 & 3.90 & 3.64 & 3.30 & 3.10 & 3.02 \\
\hline 64 & 5.97 & 5.78 & 6.63 & 6.26 & 5.85 & 4.32 & 3.87 & 3.78 & 3.46 & 3.55 \\
\hline 65 & 6.08 & 6.00 & 7.42 & 6.92 & 6.56 & 5.28 & 4.89 & 4.47 & 4.75 & 3.77 \\
\hline 66 & 6.08 & 5.98 & 7.91 & 6.88 & 6.48 & 5.73 & 5.44 & 5.92 & 4.57 & 4.23 \\
\hline 67 & 6.36 & 5.96 & 8.48 & 6.51 & 6.13 & 5.93 & 6.36 & 5.26 & 4.82 & 4.43 \\
\hline 68 & 6.10 & 5.60 & 9.14 & 6.48 & $6.03^{*}$ & 6.10 & 5.67 & 5.28 & 4.92 & 4.59 \\
\hline 69 & 7.43 & 5.16 & 15.40 & 6.79 & 6.03 & 6.22 & 6.38 & 6.38 & 6.17 & 4.22 \\
\hline 70 & 5.23 & $5.08^{*}$ & $19.10^{*}$ & $3.49^{*}$ & 4.85 & 6.21 & 6.37 & 6.23 & 4.33 & 3.96 \\
\hline 71 & 7.38 & 5.02 & 13.40 & 6.85 & 5.76 & 6.04 & & 5.58 & 4.05 & 3.59 \\
\hline 72 & 8.20 & 9.86 & 9.13 & 6.15 & 4.78 & 5.03 & 5.70 & 4.86 & 4.12 & 3.33 \\
\hline 73 & 8.62 & 8.72 & 6.67 & 4.76 & $4.45^{*}$ & 4.74 & 5.82 & 5.24 & 3.67 & 2.70 \\
\hline
\end{tabular}


SNF-10293, REV 0

Table A-6. West Bay, West Half -- Cubicle 6776 is the North-West Corner of K East Basin. (3 sheets)

\begin{tabular}{|c|c|c|c|c|c|c|c|c|c|c|}
\hline & 58 & 59 & 60 & 61 & 62 & 63 & 64 & 65 & 66 & 67 \\
\hline 74 & 8.05 & 7.72 & 6.27 & 5.18 & 4.88 & 6.07 & 6.82 & 5.97 & 2.96 & 2.26 \\
\hline 75 & 7.75 & 7.54 & 7.06 & 6.89 & 7.70 & 7.81 & $6.99 *$ & 6.44 & 2.42 & $2.22^{*}$ \\
\hline 76 & 7.68 & 6.80 & 7.53 & 7.96 & 8.64 & $8.89^{*}$ & 7.80 & 5.81 & 1.86 & $1.27^{*}$ \\
\hline
\end{tabular}

Asterisks mark locations that were measured in WHC-SD-WM-ANAL-037 (Meling 1994). 
SNF-10293, REV 0

APPENDIX B

EAST BASIN KEY LOCATIONS FROM 1994

B-i 
SNF-10293, REV 0

This page intentionally left blank.

B-ii 
SNF-10293, REV 0

APPENDIX B

EAST BASIN KEY LOCATIONS FROM 1994

Table B-1. East Bay (East Half) -- Cubicle 0101 is the South-East Corner of K East Basin. (3 sheets)

\begin{tabular}{|c|c|c|c|c|c|c|c|c|c|c|c|c|}
\hline & 1 & 2 & 3 & 4 & 5 & 6 & 7 & 8 & 9 & 10 & 11 & 12 \\
\hline 1 & & & & F9621 & F9621 & F9621 & & G5460 & & & & \\
\hline 2 & & & G2639 & & & & & G5460 & & & & \\
\hline 3 & & & G2639 & & & & & G5460 & & & & \\
\hline 4 & & & G2639 & & & & & G5460 & & & & \\
\hline 5 & G2565 & & G2639 & & F0770 & & F0770 & G5460 & & & & \\
\hline 6 & F2565 & & G2639 & G2480 & & & & G5460 & & & & \\
\hline 7 & G2565 & & G2639 & F2480 & & & & G5460 & & & & \\
\hline 8 & G2565 & & G2639 & F2480 & F0770 & & F0770 & G5460 & & & & \\
\hline 9 & G2565 & & G2639 & $\mathrm{G} 2480$ & & & F0770 & G5460 & & & & \\
\hline 10 & G2565 & & G2639 & & F0770 & & & G5460 & & & & \\
\hline 11 & G2565 & & G2639 & G2480 & F0770 & F2285 & G0770 & G5460 & & & & \\
\hline 12 & G2565 & & G2639 & G2480 & & $\mathrm{G} 2285$ & & G5460 & & & & \\
\hline 13 & F2565 & & F2639 & G2480 & & F2285 & F0770 & G5460 & & & & \\
\hline 14 & G2565 & & G2639 & G2480 & F0770 & & & G5460 & & & & \\
\hline 15 & & & P2639 & F2480 & & F2285 & & G5460 & & & & \\
\hline 16 & F2565 & & G2639 & $\mathrm{G} 2480$ & $\mathrm{P} 0770$ & F2285 & G0770 & G5460 & & & & \\
\hline 17 & G2565 & & G2639 & & F0770 & G2285 & & G5460 & P0742 & G2639 & G0679 & G2639 \\
\hline 18 & & & F2201 & F2480 & & G2285 & F0770 & G5460 & P0742 & G2639 & & \\
\hline 19 & G2565 & & F2201 & $\mathrm{G} 2480$ & & F2285 & & G5460 & F0742 & G2639 & & F2639 \\
\hline 20 & G2565 & & F2201 & F2480 & F0770 & G2285 & & G5460 & P0742 & & G0679 & G2639 \\
\hline 21 & G2565 & & G2639 & G2480 & F0770 & G2285 & G0770 & G5460 & & F2639 & & F2639 \\
\hline 22 & G2565 & & G2639 & $\mathrm{G} 2480$ & & & & G5460 & F0742 & F2639 & & \\
\hline 23 & G2565 & & & & & $\mathrm{G} 2285$ & & G5460 & P0742 & G2639 & F0679 & \\
\hline 24 & G2565 & & & $\mathrm{G} 2480$ & G0770 & G2285 & P0770 & G5460 & F0742 & G2639 & & F0679 \\
\hline 25 & $\mathrm{G} 2565$ & & & $\mathrm{G} 2480$ & & $\mathrm{G} 2285$ & & G5460 & P0742 & G2639 & F0679 & \\
\hline 26 & G2565 & & & $\mathrm{G} 2480$ & & $\mathrm{G} 2285$ & & G5460 & G0742 & F2639 & & G0679 \\
\hline 27 & G2565 & & & $\mathrm{G} 2480$ & F0770 & P2285 & & G5460 & & G1979 & F0679 & G0679 \\
\hline 28 & G2565 & & & $\mathrm{G} 2480$ & & $\mathrm{G} 2285$ & F0770 & G5460 & P0742 & G1979 & & \\
\hline 29 & F2565 & & & $\mathrm{G} 2480$ & & & & G5460 & G0742 & & F0679 & \\
\hline 30 & G2565 & & & & F0770 & $\mathrm{G} 2285$ & F0770 & G5460 & F0742 & G1979 & & F0679 \\
\hline 31 & G2565 & & & $\mathrm{G} 2480$ & & $\mathrm{G} 2285$ & & G5460 & P0742 & G1979 & F0679 & G0679 \\
\hline 32 & $\mathrm{G} 2565$ & & & $\mathrm{G} 2480$ & F0770 & G2285 & & G5460 & F0742 & & & G0679 \\
\hline
\end{tabular}

B-1 
Table B-1. East Bay (East Half) -- Cubicle 0101 is the South-East Corner of K East Basin. (3 sheets)

\begin{tabular}{|c|c|c|c|c|c|c|c|c|c|c|c|c|}
\hline & 1 & 2 & 3 & 4 & 5 & 6 & 7 & 8 & 9 & 10 & 11 & 12 \\
\hline 33 & & & & F2480 & & G2285 & F0770 & G5460 & F0742 & & F0679 & \\
\hline 34 & G2565 & & & G2480 & F0770 & & F0770 & F2201 & & P1979 & & P0679 \\
\hline 35 & G2565 & & & G2480 & & F2285 & & P4356 & F0742 & F1979 & F0679 & \\
\hline 36 & G2565 & & & G2480 & G0770 & F2285 & & F0385 & F0742 & F1979 & F0679 & B0679 \\
\hline 37 & G2565 & & F0832 & G2480 & & G2285 & & F0385 & F0742 & G1979 & & \\
\hline 38 & G2565 & & F0832 & F2480 & & F2285 & & G0385 & F0742 & & F4356 & \\
\hline 39 & G2565 & & G1540 & G2480 & P0770 & G2285 & & G0385 & & & & G0679 \\
\hline 40 & F2565 & & P0832 & F2480 & & G2285 & & P0385 & F0742 & G1979 & G0679 & F0679 \\
\hline 41 & & & F0832 & F2480 & P0770 & $\mathrm{G} 2285$ & F0742 & P0385 & F0742 & F1979 & F0679 & \\
\hline 42 & G2565 & & F0832 & & & F2285 & F0742 & F0385 & F0742 & & & \\
\hline 43 & F2565 & & F0832 & F2480 & F0770 & $\mathrm{G} 2285$ & & G0385 & F0742 & G1979 & F0679 & P0679 \\
\hline 44 & F2565 & & F0832 & F2480 & & & G0742 & G0385 & & F1979 & G0679 & F0679 \\
\hline 45 & F2565 & & F0832 & F2480 & G0770 & $\mathrm{G} 2285$ & F0742 & & G0742 & & & \\
\hline 46 & G2565 & & G0832 & F2480 & & G2285 & & F0385 & G0742 & & & \\
\hline 47 & & & G1540 & G2480 & F0770 & F2285 & F0742 & F0385 & F0742 & F1979 & F0679 & F0679 \\
\hline 48 & $\mathrm{G} 2565$ & & G0832 & F2480 & & G2285 & G0742 & G0385 & F0742 & & & G0679 \\
\hline 49 & F2565 & & F0832 & & & & G0742 & F0385 & F0742 & G1979 & & \\
\hline 50 & G2565 & & G0832 & F2285 & B0770 & F2285 & & G0385 & F0742 & G1979 & & F0679 \\
\hline 51 & F2565 & & P0832 & F2285 & G0770 & F2285 & G0742 & F0385 & & F1979 & & \\
\hline 52 & F2565 & & G0832 & G2285 & & G2285 & F0742 & F0385 & F0742 & & & P0679 \\
\hline 53 & G2565 & & G0832 & F2285 & G0770 & F2285 & & F0385 & P0742 & F1979 & & \\
\hline 54 & & & G0832 & & & F2285 & F0742 & G0385 & F0742 & & & \\
\hline 55 & G2565 & & G0832 & P2285 & & F2285 & F0742 & F0385 & & & & G0679 \\
\hline 56 & F2565 & & F0832 & F2285 & G0770 & & F0742 & F0385 & F0742 & F1979 & F4355 & F0679 \\
\hline 57 & F2565 & & G0832 & $\mathrm{G} 2285$ & & G2285 & F0742 & G0385 & F0742 & G1979 & G4355 & \\
\hline 58 & G2565 & & P0832 & F2285 & F0770 & F2285 & G0742 & G2480 & F0742 & & F4355 & \\
\hline 59 & G2565 & & G0832 & F2285 & & $\mathrm{G} 2285$ & & G2480 & G0742 & F1979 & & F0679 \\
\hline 60 & G2565 & & G1540 & G2285 & $\mathrm{P} 0770$ & F2285 & F0742 & F2480 & & G1979 & P4355 & F0679 \\
\hline 61 & G2565 & & G0832 & $\mathrm{G} 2285$ & F0770 & G2285 & G0742 & F2480 & & & & \\
\hline 62 & & & G0832 & & & G2285 & F0742 & & G0770 & & & F0679 \\
\hline 63 & G2565 & & F0832 & F2285 & F0770 & G2285 & G0742 & G2480 & & G2639 & & \\
\hline 64 & & & F0832 & F2285 & & & G0742 & $\mathrm{G} 2480$ & & G2639 & F4355 & \\
\hline 65 & G2565 & & F0770 & G2285 & F0770 & F2285 & F0742 & G2480 & F0770 & & & F0679 \\
\hline 66 & G2565 & & F1540 & $\mathrm{G} 2285$ & G0770 & G2285 & F0742 & $\mathrm{G} 2480$ & & G2639 & & \\
\hline 67 & G2565 & & F1540 & G2285 & & F2285 & & & G0770 & G2639 & F0742 & P0679 \\
\hline 68 & & & G0770 & $\mathrm{G} 2285$ & & B2285 & G0742 & $\mathrm{G} 2480$ & & G2639 & P0742 & \\
\hline 69 & G2565 & & G1540 & & G0770 & G2285 & & $\mathrm{G} 2480$ & & F2639 & B0742 & \\
\hline
\end{tabular}

B-2 
Table B-1. East Bay (East Half) -- Cubicle 0101 is the South-East Corner of K East Basin. (3 sheets)

\begin{tabular}{|c|c|c|c|c|c|c|c|c|c|c|c|c|}
\hline & 1 & 2 & 3 & 4 & 5 & 6 & 7 & 8 & 9 & 10 & 11 & 12 \\
\hline 70 & & & G1540 & G2285 & & P2285 & F0742 & F2480 & F0770 & & G0742 & \\
\hline 71 & & & G0770 & G2285 & & & G0742 & G2480 & & F2639 & & G0679 \\
\hline 72 & G2565 & & F0832 & G2285 & & F2285 & G0742 & G2480 & F0770 & F2639 & & \\
\hline 73 & & & G0770 & G2285 & G0770 & F2285 & & G2480 & & G2639 & & \\
\hline 74 & G2565 & & & & & & F2285 & & F2285 & & G2285 & F2285 \\
\hline 75 & & & & & & F2285 & F2285 & G2480 & G2285 & G2285 & G2285 & G2285 \\
\hline 76 & & & & & & & & F2285 & G2285 & G2285 & F2285 & \\
\hline
\end{tabular}

Keys are identified using the notation CXXXX, where $\mathrm{C}$ is the fuel condition $(\mathrm{G}, \mathrm{F}, \mathrm{P}$, or $\mathrm{B}$ for Good, Fair, Poor, or Bad), and XXXX is the last four digits of the key number.

Fuel condition identifiers are from WHC-SD-SNF-TI-012.

Key locations are from private communication with Karen Morris, K Basins Operations, 1995, "K East Basin Storage Map", MAC Version 3.1, Rev. 163, Updated 1/11/94, Report 5/02/94, 124 pages. 
SNF-10293, REV 0

Table B-2. East Bay, West Half (Floor Sludge Depths in cm).

(3 sheets)

\begin{tabular}{|c|c|c|c|c|c|c|c|c|c|c|}
\hline & 13 & 14 & 15 & 16 & 17 & 18 & 19 & 20 & 21 & 22 \\
\hline 1 & & & & & & & & & & \\
\hline 2 & & & & & & $\mathrm{G} 2480$ & F2480 & & G2480 & $\mathrm{G} 2480$ \\
\hline 3 & & & & & & $\mathrm{G} 2480$ & F2480 & $\mathrm{G} 2480$ & F2480 & $\mathrm{G} 2480$ \\
\hline 4 & & & & & F0770 & F0602 & & G1183 & F1183 & F0602 \\
\hline 5 & & & & & & F0602 & P0602 & G1183 & F1183 & F0602 \\
\hline 6 & & & & & & G0602 & & F1183 & F1183 & F0602 \\
\hline 7 & & & & & & G0602 & G0602 & F1183 & P1183 & G0576 \\
\hline 8 & & & & & F0770 & F0602 & & G1183 & F1183 & F0602 \\
\hline 9 & & & & & F0770 & F0602 & & F1183 & G1183 & F0602 \\
\hline 10 & & & & & & F0602 & G0602 & F1183 & F1183 & F0602 \\
\hline 11 & & & & & B0770 & F0602 & & F1183 & F1183 & B0456 \\
\hline 12 & & & & & & G2285 & & F1183 & F1183 & F0602 \\
\hline 13 & & & & & & G5461 & G0602 & G1183 & F1183 & F0602 \\
\hline 14 & & & & & $\mathrm{P} 0770$ & G5461 & G0602 & F1183 & F1183 & B0602 \\
\hline 15 & & & & & & G5461 & & G1183 & G1183 & B0602 \\
\hline 16 & & & & & F0770 & G5461 & F0602 & F1183 & F1183 & P0456 \\
\hline 17 & & F2201 & G2639 & & F0679 & G5461 & F0602 & F1183 & F1183 & F0602 \\
\hline 18 & F0679 & G2639 & G2639 & & B0679 & G5461 & F0602 & G1183 & F1183 & F0602 \\
\hline 19 & G0679 & G2639 & F2639 & F2201 & & G5461 & & F1183 & F1183 & G0602 \\
\hline 20 & F0679 & & G2639 & F2201 & & G5461 & F0602 & F1183 & F1183 & F0602 \\
\hline 21 & & F2201 & G2639 & & & G5461 & F0602 & G1183 & P1183 & F0602 \\
\hline 22 & & & G2639 & & & G5461 & G0602 & G1183 & F1183 & F0602 \\
\hline 23 & G0679 & G2201 & G2639 & G2201 & F0679 & G5461 & & G1183 & P1183 & F0602 \\
\hline 24 & & & G2639 & F2201 & F0679 & G5461 & G0602 & P1183 & F1183 & F0602 \\
\hline 25 & F0679 & F2201 & F2639 & & & G5461 & F0602 & F1183 & F1183 & F0602 \\
\hline 26 & F0679 & G2201 & G2639 & & G0679 & G5461 & F0602 & F1183 & F1183 & G0456 \\
\hline 27 & & & F2201 & & & G5461 & G0602 & F1183 & F1183 & G0602 \\
\hline 28 & & & G2201 & F2201 & & G5461 & F0602 & F1183 & F1183 & F0602 \\
\hline 29 & F0679 & G2201 & F2201 & G2201 & F0679 & G5461 & & G1183 & F1183 & B0456 \\
\hline 30 & & F2201 & G2201 & & F0679 & G5461 & G0602 & P1183 & F1183 & F0456 \\
\hline 31 & P0679 & & G2201 & & & G5461 & F0602 & F1183 & F1183 & G0576 \\
\hline 32 & & F2201 & G2639 & F2201 & & G5461 & F0602 & F1183 & F1183 & G0456 \\
\hline 33 & P0679 & F2201 & G2639 & & F0679 & G5461 & G0602 & F1183 & G1183 & G0576 \\
\hline 34 & G0679 & & G2639 & F2201 & B4356 & G5461 & P0602 & G1183 & F1183 & F0576 \\
\hline 35 & & G2201 & G2639 & & G0679 & G5461 & F0602 & G1183 & F1183 & F0456 \\
\hline 36 & B4356 & & G2201 & & & G5461 & & G1183 & G1183 & G0576 \\
\hline
\end{tabular}


Table B-2. East Bay, West Half (Floor Sludge Depths in cm). (3 sheets)

\begin{tabular}{|c|c|c|c|c|c|c|c|c|c|c|}
\hline & 13 & 14 & 15 & 16 & 17 & 18 & 19 & 20 & 21 & 22 \\
\hline 37 & F0679 & $\mathrm{G} 2201$ & & $\mathrm{G} 2201$ & & G5461 & G0602 & G1183 & F1183 & G0576 \\
\hline 38 & & F2201 & & G2201 & F0679 & G5461 & F0602 & F1183 & G1183 & F2285 \\
\hline 39 & B4356 & & & & G0679 & G5240 & G0602 & G1183 & G1183 & F0576 \\
\hline 40 & P0679 & & G2201 & & F0679 & G5240 & F0602 & G1183 & F1183 & G2285 \\
\hline 41 & & F2201 & & & B0679 & G5204 & & F1183 & F1183 & F0576 \\
\hline 42 & P4356 & & P1183 & F2201 & & G0764 & F0602 & F1183 & G1183 & G0576 \\
\hline 43 & & $\mathrm{G} 2201$ & F1183 & & & G0764 & P0602 & G1183 & F1183 & P1806 \\
\hline 44 & F0679 & G2201 & G1183 & & & G0764 & F0602 & F1183 & F1183 & G1183 \\
\hline 45 & F0679 & & F1183 & F2201 & F0679 & G0764 & F0602 & F1183 & G1183 & G1540 \\
\hline 46 & & G2201 & G1183 & G2201 & F0679 & G0764 & F0602 & F1183 & F1183 & G4722 \\
\hline 47 & F0679 & G2201 & F1183 & & & G0764 & G0602 & F1183 & G1183 & G4065 \\
\hline 48 & & & G1183 & G2201 & F0679 & G0764 & & G1183 & F1183 & G2240 \\
\hline 49 & P0679 & G2201 & G1183 & & & G0764 & F0602 & F1183 & G1183 & G2639 \\
\hline 50 & G4356 & & G1183 & G2201 & & G0764 & F0602 & F1183 & F1183 & F2639 \\
\hline 51 & & & G1183 & G2201 & & G5461 & B0602 & F1183 & F1183 & G2639 \\
\hline 52 & & G2201 & F1183 & & F0679 & G5461 & & G1183 & F1183 & G2639 \\
\hline 53 & G0679 & & G1183 & & & G5461 & F0602 & F1183 & G1183 & G2639 \\
\hline 54 & & G2201 & G1183 & & F0679 & G5461 & F0602 & F1183 & & F2639 \\
\hline 55 & G0679 & & F1183 & F2201 & & G5461 & F0602 & F1183 & & F2639 \\
\hline 56 & & G2201 & F1183 & F2201 & F0679 & G5461 & B0602 & F1183 & & F2639 \\
\hline 57 & & F2201 & G1183 & & & G5461 & & G1183 & & G2639 \\
\hline 58 & & & G1183 & & & F0742 & P0602 & G2480 & & F2639 \\
\hline 59 & G0679 & G2201 & F1183 & G2201 & F0679 & F2639 & P0602 & & & F2639 \\
\hline 60 & F0679 & & G1183 & F2201 & F0602 & G2639 & F0602 & F2480 & & F2639 \\
\hline 61 & F0679 & G2201 & G1183 & F2201 & F0602 & G2639 & P0602 & G2480 & & F2639 \\
\hline 62 & & & F1183 & & & G2639 & & G2480 & & G2639 \\
\hline 63 & F0679 & & F1183 & & & G2639 & P0602 & F2480 & F1372 & F2639 \\
\hline 64 & & & P1183 & & & G2639 & F0602 & F2480 & F1372 & G2639 \\
\hline 65 & & F2201 & G1183 & F2201 & & F2639 & P0602 & & & F2639 \\
\hline 66 & F0679 & & G1183 & & & F2639 & P0602 & F2480 & & G2639 \\
\hline 67 & P0679 & F2201 & & P2201 & P0602 & G2639 & & $\mathrm{G} 2480$ & F1372 & G2639 \\
\hline 68 & & & & & & G2639 & F0602 & F2480 & F1372 & F2639 \\
\hline 69 & P0679 & & & G2201 & & G2639 & G0602 & F2480 & & G5460 \\
\hline 70 & & F2201 & & & & G2639 & B0602 & & B1372 & G5460 \\
\hline 71 & & F2201 & & & & G2639 & & G2480 & & G5461 \\
\hline 72 & B0679 & F2201 & & & G0602 & F2639 & F0602 & F2480 & & G5461 \\
\hline 73 & P0679 & & & F2201 & & G2639 & F0602 & F2480 & F1372 & G5461 \\
\hline
\end{tabular}

B-5 
SNF-10293, REV 0

Table B-2. East Bay, West Half (Floor Sludge Depths in cm). (3 sheets)

\begin{tabular}{|c|c|c|c|c|c|c|c|c|c|c|}
\hline & 13 & 14 & 15 & 16 & 17 & 18 & 19 & 20 & 21 & 22 \\
\hline 74 & F2285 & G2285 & G2285 & G2285 & G2285 & F2639 & G2285 & F2285 & F2285 & G5461 \\
\hline 75 & F2285 & G2285 & & G2285 & G2285 & G2639 & G2285 & F2285 & G2285 & G5461 \\
\hline 76 & & & & & & G2639 & & & & G5461 \\
\hline
\end{tabular}


SNF-10293, REV 0

Table B-3. Middle Bay, East Half (3 sheets)

\begin{tabular}{|c|c|c|c|c|c|c|c|c|c|c|c|c|}
\hline & 23 & 24 & 25 & 26 & 27 & 28 & 29 & 30 & 31 & 32 & 33 & 34 \\
\hline 1 & G5458 & G5458 & & & & & & & & & & \\
\hline 2 & G5458 & G5458 & $\mathrm{G} 2480$ & G2480 & $\mathrm{G} 2480$ & F2480 & $\mathrm{G} 2480$ & & $\mathrm{G} 2480$ & & & \\
\hline 3 & G5458 & G5458 & G2480 & & F2480 & F2480 & F2480 & G2480 & $\mathrm{G} 2480$ & & & \\
\hline 4 & G5458 & G5458 & & & & F0456 & B4356 & & G1183 & & & \\
\hline 5 & G5458 & F5458 & & G1372 & & P0456 & F0832 & G2127 & G1183 & & & \\
\hline 6 & F5458 & G5458 & & P1372 & & G0456 & F0832 & F2127 & G1183 & & & \\
\hline 7 & G5458 & F5458 & F1372 & & & G0456 & P0832 & & G1183 & & & \\
\hline 8 & G5458 & G5458 & G1372 & F1372 & & F0456 & F0832 & F2127 & $\mathrm{P} 4356$ & & & \\
\hline 9 & G5458 & G5458 & & & F1449 & G0456 & & G2127 & G1183 & & & \\
\hline 10 & G5458 & G5458 & F1372 & & & F0456 & P0832 & G2127 & F1183 & & & \\
\hline 11 & G5458 & G5458 & & & F1372 & F0456 & & F2127 & F1183 & & & \\
\hline 12 & G5458 & G5458 & F1372 & F1979 & & F0456 & G0832 & F2127 & F1183 & & & \\
\hline 13 & G5458 & G5458 & F1372 & P1979 & P1372 & G0456 & F0832 & & & & & \\
\hline 14 & G5458 & G5458 & G1372 & & F1372 & G0456 & G0832 & G2127 & F1183 & & & \\
\hline 15 & G5458 & G5458 & & G1979 & F1372 & F0456 & G0832 & F2127 & F1183 & & & \\
\hline 16 & G5458 & G5458 & & & & F0456 & & F2127 & F1183 & & & \\
\hline 17 & G5458 & G5458 & & G1979 & & G0456 & G0832 & G2127 & F1183 & F0832 & F0832 & F1979 \\
\hline 18 & G5458 & G5458 & F1372 & F1979 & F1372 & F0456 & P0832 & F2127 & P1183 & F0832 & G0832 & F1979 \\
\hline 19 & G5458 & G5458 & F1372 & & & F0456 & F0832 & F2127 & F1183 & F0832 & F0832 & G2639 \\
\hline 20 & G5458 & G5458 & F1372 & & & F0456 & F0832 & F2127 & F1183 & F0832 & F0832 & F1979 \\
\hline 21 & G5458 & G5458 & F1372 & F1979 & F1372 & F0456 & & F2127 & F1183 & F0832 & G0832 & G2480 \\
\hline 22 & G5458 & G5458 & & G1979 & B4356 & F0456 & F0832 & G2127 & F1183 & F2127 & F0832 & G1979 \\
\hline 23 & G5458 & G5458 & F1372 & & F1372 & F0456 & P0832 & & F1183 & F0832 & F0832 & G1979 \\
\hline 24 & G5458 & G5458 & F1372 & F1979 & B4356 & B4356 & F0832 & $\mathrm{F} 2127$ & & F0832 & G0832 & G5458 \\
\hline 25 & G5458 & G5458 & & & & F0456 & G0832 & F2127 & F1372 & G0832 & P0832 & F5458 \\
\hline 26 & G5458 & G5458 & F1372 & & & F2285 & & F2127 & F1372 & G0832 & F0832 & F1979 \\
\hline 27 & G5458 & G5458 & F1372 & & F1372 & G5458 & F0832 & F2127 & & F0832 & F0832 & G1979 \\
\hline 28 & G5458 & G5458 & F1372 & & & G5458 & F0832 & G2127 & & F0832 & G0832 & F1979 \\
\hline 29 & G5458 & G5458 & & F2201 & F1372 & G5458 & F0832 & & F1372 & F2127 & F0832 & G5458 \\
\hline 30 & G5458 & G5458 & F1372 & P2201 & & G5458 & F0832 & F2127 & F1372 & G0832 & F0832 & G1979 \\
\hline 31 & G5458 & G5458 & & & F1372 & G5458 & G0832 & F2127 & F1372 & F0832 & F0832 & F1979 \\
\hline 32 & G5458 & G5458 & F1372 & G2201 & & G5458 & & F2127 & & F0832 & F0832 & G5458 \\
\hline 33 & G5458 & G5458 & & G2201 & F1372 & G5458 & P0832 & F2127 & F1372 & F0832 & F0832 & F1979 \\
\hline 34 & G5458 & G5458 & G1372 & & P1372 & G5458 & F0832 & F2127 & & G2127 & F0832 & F1979 \\
\hline 35 & G5458 & G5458 & & & & G5458 & & F2127 & F1372 & F0832 & F0832 & G5458 \\
\hline 36 & G5458 & G5458 & F1372 & F2201 & & G5458 & F0832 & F2127 & P1372 & F0832 & F0832 & F1979 \\
\hline 37 & G5458 & G5458 & & G2127 & F1372 & G5458 & F0832 & & & F0832 & G0832 & F1979 \\
\hline
\end{tabular}

B-7 
SNF-10293, REV 0

Table B-3. Middle Bay, East Half (3 sheets)

\begin{tabular}{|c|c|c|c|c|c|c|c|c|c|c|c|c|}
\hline & 23 & 24 & 25 & 26 & 27 & 28 & 29 & 30 & 31 & 32 & 33 & 34 \\
\hline 38 & G5458 & G5458 & F1372 & & F1372 & G5458 & F0832 & G2127 & & G2127 & G0832 & G5458 \\
\hline 39 & 5458 & G5458 & F1372 & G2127 & & G5458 & & G2127 & F1372 & F0832 & G0832 & G5458 \\
\hline 40 & G5458 & G5458 & 1372 & G2127 & & G5458 & F0832 & G2127 & F1372 & P0832 & F0832 & F1979 \\
\hline 41 & G5458 & G5458 & F1372 & G2127 & F1372 & G5458 & G0832 & G2127 & F1372 & F0832 & F0832 & F1979 \\
\hline 42 & G5458 & G5458 & & G2127 & & G5458 & F0832 & F2127 & F1372 & B0832 & F0832 & G5458 \\
\hline 43 & G5458 & F0456 & & G2127 & F1372 & G5458 & P0832 & G2127 & & $\mathrm{G} 2127$ & F0832 & F1979 \\
\hline 44 & P0456 & $\mathrm{F} 2480$ & P1372 & F2127 & F1372 & G5458 & F0832 & F2127 & F1372 & G0832 & F0832 & F1979 \\
\hline 45 & G2480 & G0456 & & $\mathrm{G} 2127$ & & G5458 & F0832 & G2127 & & F0832 & F0832 & G5458 \\
\hline 46 & F0456 & G2480 & F1372 & F2127 & F1372 & G5458 & P0832 & F4356 & & F0832 & F2480 & F1979 \\
\hline 47 & F0456 & F0456 & & & & G5458 & & & F1372 & F0832 & P2480 & F1979 \\
\hline 48 & F0456 & G0576 & & G2127 & & G5458 & F0832 & F2127 & F1372 & P0832 & F2480 & G5458 \\
\hline 49 & F0456 & P0456 & P1372 & G2127 & F1372 & G5458 & F0832 & F2127 & & F0832 & F2480 & G5458 \\
\hline 50 & B0456 & F0576 & F1372 & $\mathrm{G} 2127$ & & G5458 & F0832 & $\mathrm{G} 2127$ & G1372 & F0832 & F2480 & G1979 \\
\hline 51 & F0456 & F0456 & 1372 & G2127 & F1372 & G5458 & F0832 & $\mathrm{G} 2127$ & F1372 & F0832 & F2480 & G1979 \\
\hline 52 & F0456 & F0576 & & & F1372 & G5458 & P0832 & F2127 & F1372 & F0832 & F2480 & G1979 \\
\hline 53 & F0456 & F0456 & & F2127 & 1372 & F5458 & P0832 & F2127 & & F0832 & F2480 & G197 \\
\hline 54 & F0456 & F0456 & F1372 & & & G5458 & & & & F0832 & F2480 & \\
\hline 55 & P0456 & F0456 & F2480 & G2127 & & G5458 & F0832 & G2127 & F4355 & F0832 & $\mathrm{G} 2480$ & G1979 \\
\hline 56 & F0456 & F0456 & F2480 & F2127 & P1372 & G5458 & G0832 & & & F0832 & F2480 & G5458 \\
\hline 57 & G0456 & F0456 & F2480 & $\mathrm{G} 2127$ & F1372 & G5458 & P0832 & F2127 & & G0832 & $\mathrm{G} 2480$ & G545 \\
\hline 58 & F0456 & F0456 & $\mathrm{G} 2480$ & F2127 & & G5458 & P0832 & G2127 & & F0832 & F2480 & G1979 \\
\hline 59 & F0456 & F0456 & G2480 & F2127 & & G5458 & & $\mathrm{F} 2127$ & & G0832 & $\mathrm{G} 2480$ & G197 \\
\hline 60 & F0456 & F0456 & $\mathrm{G} 2480$ & G2127 & F1372 & G5458 & P0832 & F2127 & & F0832 & $\mathrm{F} 2480$ & P1979 \\
\hline 61 & F0456 & F0456 & & & & G5458 & G0832 & F2127 & & G0832 & G2480 & F2480 \\
\hline 62 & F0456 & F0456 & F2480 & G2127 & 1372 & G5458 & F0832 & & F4355 & F0832 & G2480 & G2480 \\
\hline 63 & F0456 & G0456 & G2480 & F2127 & F1372 & G5458 & F0832 & F2127 & G4355 & F0832 & & G1979 \\
\hline 64 & G0456 & G0456 & G2480 & F2127 & & G5458 & F0832 & F2127 & G4355 & F0832 & F2480 & G1979 \\
\hline 65 & G0456 & F0456 & F2480 & G2127 & & G5458 & & G2127 & G4355 & F0832 & G2480 & F0832 \\
\hline 66 & G0456 & F0456 & & F2127 & F1372 & G5458 & F0832 & F2127 & F4355 & F0832 & F2480 & F1979 \\
\hline 67 & G0456 & F0456 & G2480 & F2127 & F1372 & G5458 & F0832 & G2127 & G4355 & G0832 & G2480 & F0832 \\
\hline 68 & G0456 & G0456 & F2480 & $\mathrm{G} 2127$ & F1372 & G5458 & F0832 & & $\mathrm{P} 4355$ & F0832 & F2480 & G1979 \\
\hline 69 & F0456 & G0456 & F2480 & F2127 & & G5458 & F0832 & $\mathrm{G} 2127$ & G4355 & G0832 & F2480 & F1979 \\
\hline 70 & F0456 & F0456 & F2480 & & F1372 & G5458 & P0832 & F2127 & F4355 & F0832 & F2127 & F0832 \\
\hline 71 & G0456 & F0456 & G2480 & $\mathrm{G} 2127$ & & G5458 & & $\mathrm{G} 2127$ & F4355 & F0832 & F2480 & G1979 \\
\hline 72 & G0456 & G0456 & F2480 & $\mathrm{G} 2127$ & & G5458 & F0832 & F2127 & F4355 & G0832 & P2480 & G0832 \\
\hline 73 & F0456 & G0456 & G2480 & F2127 & F1372 & G5458 & P4356 & F2127 & F4355 & F0832 & F2480 & F1979 \\
\hline 74 & F0456 & F2285 & F2285 & F2285 & G2285 & G5458 & F2285 & $\mathrm{G} 2285$ & & F2285 & G2285 & F0832 \\
\hline 75 & F2285 & F2285 & & F2285 & F2285 & G5458 & G2285 & $\mathrm{G} 2285$ & & F2285 & G2285 & G228 \\
\hline
\end{tabular}

B-8 
SNF-10293, REV 0

Table B-3. Middle Bay, East Half (3 sheets)

\begin{tabular}{|l|l|l|l|l|l|l|l|l|l|l|l|l|}
\hline & 23 & 24 & 25 & 26 & 27 & 28 & 29 & 30 & 31 & 32 & 33 & 34 \\
\hline 76 & & & & & & G5458 & & & & & & \\
\hline
\end{tabular}

Keys are identified using the notation CXXXX, where $\mathrm{C}$ is the fuel condition $(\mathrm{G}, \mathrm{F}, \mathrm{P}$, or B for Good, Fair, Poor, or Bad), and XXXX is the last four digits of the key number.

Fuel condition identifiers are from WHC-SD-SNF-TI-012.

Key locations are from private communication with Karen Morris, K Basins Operations, 1995, "K East Basin Storage Map", MAC Version 3.1, Rev. 163, Updated 1/11/94, Report 5/02/94, 124 pages. 
SNF-10293, REV 0

Table B-4. Middle Bay, West Half. (3 sheets)

\begin{tabular}{|c|c|c|c|c|c|c|c|c|c|c|c|}
\hline & 35 & 36 & 37 & 38 & 39 & 40 & 41 & 42 & 43 & 44 & 45 \\
\hline 1 & & & & & & & & & & & \\
\hline 2 & & & & & & & & & & & \\
\hline 3 & & & & & & & & & & & \\
\hline 4 & & & & & & & & & & & \\
\hline 5 & & & & & & & & & & & \\
\hline 6 & & & & & & & & & & & \\
\hline 7 & & & & & & & & & & & \\
\hline 8 & & & & & & & & & & & \\
\hline 9 & & & & & & & & & & & \\
\hline 10 & & & & & G4356 & & & & & & \\
\hline 11 & & & & & & & & & & & \\
\hline 12 & & & & & & & & & & & \\
\hline 13 & & & & & & & & & & & \\
\hline 14 & & & & & & & & & & & \\
\hline 15 & & & & & & & & & & & \\
\hline 16 & & & & & & & & & & & \\
\hline 17 & F2639 & F0385 & G0982 & G0385 & $\mathrm{G} 2852$ & G5458 & G0982 & G5458 & G1897 & G5458 & G5458 \\
\hline 18 & G2639 & F0385 & F0982 & G0385 & F1979 & G5458 & G5458 & G5458 & G1449 & F1897 & G5458 \\
\hline 19 & F2639 & F0385 & F0982 & F0385 & F1979 & G5458 & F0982 & G5458 & F1449 & F1897 & G5458 \\
\hline 20 & G5458 & F0385 & & F0385 & F1979 & G5458 & F5458 & G5458 & G4355 & G5458 & G5458 \\
\hline 21 & G2639 & F0385 & F0982 & F0385 & F1979 & G5458 & F0982 & G5458 & P1449 & G1897 & G5458 \\
\hline 22 & F2639 & F0385 & G5458 & P0385 & F1979 & G5460 & G0982 & G5458 & F2852 & F1897 & G5458 \\
\hline 23 & G2639 & G0385 & G5458 & G0385 & F1979 & G5460 & F0982 & F1540 & F1449 & G1897 & G5458 \\
\hline 24 & G5458 & F0385 & G5458 & F0385 & F1979 & G5460 & G5458 & F1449 & G2852 & G5458 & G5458 \\
\hline 25 & F2639 & F0385 & G5458 & G5458 & F1979 & G5460 & F0982 & G5458 & F1449 & G1897 & G5458 \\
\hline 26 & P2639 & F0385 & F0982 & F0385 & F1979 & G5460 & F0982 & G5458 & F1449 & G5458 & G5458 \\
\hline 27 & G2639 & G0385 & F0982 & F0385 & F1979 & G5460 & G0982 & F1540 & F2565 & G1897 & G5458 \\
\hline 28 & G2639 & F0385 & G5458 & F0982 & G1979 & G5460 & G5458 & G5458 & F2285 & G1897 & G5458 \\
\hline 29 & F2639 & F0385 & F0982 & F0982 & F1979 & G5460 & F0982 & G5458 & G1449 & G1897 & G5458 \\
\hline 30 & F2639 & F0385 & G5458 & G5458 & F1979 & G5460 & G5458 & G5458 & G1449 & G1897 & G5458 \\
\hline 31 & F2639 & F0385 & F0982 & F0982 & F1979 & G5460 & P0982 & F1540 & G2285 & G1897 & G5458 \\
\hline 32 & G5458 & F0385 & F0982 & G5458 & F1979 & G5460 & F0982 & G5458 & F1449 & G1897 & G5458 \\
\hline 33 & F2639 & F0385 & F0982 & P0982 & F1979 & G5460 & G5458 & G5458 & G5458 & G1897 & G5458 \\
\hline 34 & F2480 & F0385 & G2852 & G5458 & F1979 & G5460 & F0982 & G5458 & G5458 & G1897 & G5458 \\
\hline 35 & F2480 & F0385 & F0982 & G5458 & F1979 & G5460 & G5458 & G1540 & G5458 & G1897 & G5458 \\
\hline 36 & F2639 & F0385 & G0982 & G0982 & G5458 & G5460 & F0982 & G1897 & G5458 & F1036 & G5458 \\
\hline 37 & $\mathrm{G} 2480$ & F0385 & $\mathrm{G} 2852$ & F0982 & P1979 & G5460 & G5458 & G5458 & G5458 & F1897 & G5458 \\
\hline
\end{tabular}

B-10 
Table B-4. Middle Bay, West Half. (3 sheets)

\begin{tabular}{|c|c|c|c|c|c|c|c|c|c|c|c|}
\hline & 35 & 36 & 37 & 38 & 39 & 40 & 41 & 42 & 43 & 44 & 45 \\
\hline 38 & G2480 & F0385 & F0982 & F0982 & F1979 & G5460 & F0982 & G1897 & G5458 & F1036 & F5458 \\
\hline 39 & G2480 & F0385 & F0982 & G5458 & F1979 & G5460 & G5458 & G5458 & G5458 & F1897 & G5458 \\
\hline 40 & G2480 & F0385 & F2852 & F0982 & G5458 & G5460 & G5458 & F1897 & G5458 & F1036 & G5458 \\
\hline 41 & G2480 & G0385 & F0982 & F0982 & G5458 & G5460 & F0982 & G5458 & G5458 & G1897 & G5458 \\
\hline 42 & G2480 & P0385 & F2480 & G5458 & G1979 & G5460 & F0982 & F1897 & G5460 & F1036 & G5458 \\
\hline 43 & G2639 & G0385 & F0982 & F0982 & F1979 & G5460 & B0982 & G5458 & G5460 & G1897 & G5458 \\
\hline 44 & F2480 & B0385 & F0982 & F0982 & G1979 & G5460 & G5458 & F1897 & G5458 & F1036 & G5458 \\
\hline 45 & F2480 & F0385 & F2480 & F0982 & F5458 & G5460 & G4356 & & G5458 & G1449 & G5458 \\
\hline 46 & G2639 & F0385 & G5458 & F0982 & G1979 & F1449 & F0982 & & G5458 & G1036 & G5458 \\
\hline 47 & F2480 & G0385 & F0982 & B4356 & F5458 & F1372 & G0982 & G1897 & G5458 & G1036 & G5458 \\
\hline 48 & G2480 & F0385 & G5458 & G5458 & G5458 & F1449 & F0982 & & G5458 & F1449 & G5458 \\
\hline 49 & G2480 & F0385 & F0982 & G5458 & F1979 & G1372 & F0982 & F1897 & G5458 & G1449 & G5458 \\
\hline 50 & G2480 & F0385 & F0982 & G5458 & G1979 & F1372 & F0982 & F1897 & G5458 & F1036 & G5458 \\
\hline 51 & F2639 & F0385 & F0982 & G5458 & G5458 & F1449 & G0982 & G5458 & G5458 & F1036 & G5458 \\
\hline 52 & G2480 & G5458 & G5458 & G5458 & G1979 & F1449 & F0982 & G1897 & G5458 & F1036 & G5458 \\
\hline 53 & F1979 & G5458 & F0982 & G5458 & G1979 & F1372 & F0982 & G1897 & G5458 & F1036 & G5458 \\
\hline 54 & G1979 & G5458 & P0982 & G5458 & G1979 & F1449 & F0982 & G1897 & G5458 & G1449 & G5458 \\
\hline 55 & F2639 & F4355 & F0982 & G5458 & G5458 & F1372 & F0982 & G5458 & G5458 & G1449 & G1540 \\
\hline 56 & F2639 & G5458 & G5458 & G5458 & G1979 & F1449 & G0982 & G5458 & G5458 & F4355 & F1449 \\
\hline 57 & F1979 & G5458 & F0982 & G5458 & G5458 & F1372 & F0982 & F1897 & G5458 & G4355 & F1449 \\
\hline 58 & G1979 & G4355 & & G5458 & F1979 & F1449 & F0982 & F1897 & G5458 & F4355 & F1540 \\
\hline 59 & G2639 & G5458 & F0982 & G5458 & F1979 & F1372 & F0982 & & F5458 & F1897 & F1540 \\
\hline 60 & G1979 & G5458 & G5458 & G5458 & G5458 & P1449 & G0982 & F1897 & G5458 & F4355 & F1540 \\
\hline 61 & G2639 & G5458 & F0982 & G5458 & G1979 & $\mathrm{P} 1372$ & F0982 & F1897 & G5458 & G1449 & G1540 \\
\hline 62 & G1979 & G5458 & F0982 & G5458 & G5458 & F1449 & F1806 & G1897 & G5458 & F4355 & G1449 \\
\hline 63 & G1979 & G5458 & G5458 & G5458 & G5458 & F1449 & F0982 & & G5458 & G1897 & G4356 \\
\hline 64 & G1979 & G5458 & G5458 & G5458 & F1979 & F1372 & F0982 & G5458 & G5458 & F1449 & B4356 \\
\hline 65 & G2639 & G5458 & G5458 & G5458 & F1979 & P1449 & G0001 & F1897 & G5458 & G1449 & F4356 \\
\hline 66 & G1979 & G5458 & G5458 & G5458 & F1979 & P1449 & F1806 & G1897 & G5458 & F1897 & F4356 \\
\hline 67 & F1979 & G5458 & G5458 & G5458 & G5458 & F1449 & F1806 & & G5458 & F0982 & F4356 \\
\hline 68 & G1979 & G5458 & G5458 & G5458 & F1979 & F1449 & F0001 & & G5458 & F1897 & F4356 \\
\hline 69 & G2639 & G5458 & G5458 & G5458 & G5458 & F1449 & P1806 & G1897 & G5458 & F1449 & B0350 \\
\hline 70 & G1979 & G5458 & G5458 & G5458 & F1979 & F1449 & F0764 & & G5458 & F1449 & G1733 \\
\hline 71 & F2639 & G5458 & G5458 & G5458 & P1979 & F1449 & & F1897 & G5458 & F0982 & P4356 \\
\hline 72 & G1979 & G5458 & G5458 & G5458 & G5458 & F1449 & & & G5458 & G0982 & F1733 \\
\hline 73 & F1979 & G5458 & G5458 & G5458 & F1979 & G1449 & & F1897 & G5458 & F0982 & B0350 \\
\hline 74 & G2285 & F2285 & G2285 & G2285 & F2285 & G2285 & & G2285 & G5458 & F1449 & F2285 \\
\hline 75 & F2285 & F2285 & G2285 & F2285 & G2285 & $\mathrm{G} 2285$ & F2285 & G2285 & G5458 & F2285 & F2285 \\
\hline
\end{tabular}

B-11 
SNF-10293, REV 0

Table B-4. Middle Bay, West Half. (3 sheets)

\begin{tabular}{|l|l|l|l|l|l|l|l|l|l|l|l|}
\hline & 35 & 36 & 37 & 38 & 39 & 40 & 41 & 42 & 43 & 44 & 45 \\
\hline 76 & & & & & & & & & G5458 & & \\
\hline
\end{tabular}


Table B-5. West Bay, East Half. (3 sheets)

\begin{tabular}{|c|c|c|c|c|c|c|c|c|c|c|c|c|}
\hline & 46 & 47 & 48 & 49 & 50 & 51 & 52 & 53 & 54 & 55 & 56 & 57 \\
\hline 1 & & & & & & & & & & & & \\
\hline 2 & 2480 & $\mathrm{G} 2480$ & & $\mathrm{G} 2480$ & $\mathrm{G} 2480$ & & F2480 & $\mathrm{G} 2480$ & & G2565 & F2565 & G2565 \\
\hline 3 & 2480 & $\mathrm{G} 2480$ & G2480 & $\mathrm{G} 2480$ & F2480 & F2480 & F2480 & F2480 & F2480 & F2565 & & F2565 \\
\hline 4 & G1183 & F1109 & F1897 & & & F1449 & & G0294 & & F0294 & & F0259 \\
\hline 5 & G2285 & & F1897 & F1109 & & G1449 & F1897 & G0294 & F1540 & G0294 & G1540 & F0259 \\
\hline 6 & F2285 & & & & F1540 & P1449 & F1897 & P0294 & & G0294 & G1540 & P4356 \\
\hline 7 & G1183 & F1109 & & F1109 & & G1449 & & G0294 & & F0294 & F1540 & F0259 \\
\hline 8 & G1183 & G1109 & F1897 & F1109 & & F1449 & F1897 & F0294 & F1540 & F0294 & F1540 & F0259 \\
\hline 9 & F1183 & & F1897 & F1109 & & F1449 & & F0294 & & F0294 & G3015 & G0259 \\
\hline 10 & F1183 & F1109 & P1897 & F1109 & & F1449 & F1897 & F0294 & & F0294 & F3015 & B4356 \\
\hline 11 & G1109 & F1109 & G1540 & F1109 & F1540 & F1449 & F1897 & B0294 & & B0294 & G1183 & F0259 \\
\hline 12 & F1183 & F1036 & & & & F1449 & F1897 & P0294 & F1540 & G0294 & F2852 & G4356 \\
\hline 13 & F1183 & & & G1109 & & F1449 & & B0294 & & F0294 & G2852 & F0259 \\
\hline 14 & F1183 & F1036 & & & & F1449 & G1897 & F0294 & F1540 & F0294 & F3015 & G0259 \\
\hline 15 & F1183 & & & G1109 & F1540 & F1449 & F1897 & F0294 & & F0294 & & G0259 \\
\hline 16 & F1109 & G1036 & G1897 & & & F1449 & & P4356 & & F0294 & G1449 & F0259 \\
\hline 17 & F1109 & & G1897 & F1109 & & G1449 & F1897 & F0294 & & B0294 & & F0259 \\
\hline 18 & F4356 & P1036 & G1897 & F1109 & F1540 & F1449 & P1897 & F0294 & & F0294 & F1449 & F4356 \\
\hline 19 & F1109 & F1036 & G5204 & & & F1449 & & G0294 & F1540 & P0294 & & G0259 \\
\hline 20 & F1109 & & G5204 & F1109 & & F1449 & F1897 & P0294 & & F0294 & F1449 & F4356 \\
\hline 21 & F4356 & F1036 & G5204 & F1109 & F0294 & F1449 & G1897 & F0294 & & F0294 & F1449 & P0259 \\
\hline 22 & G1109 & F1036 & G5204 & F1109 & F0294 & P1449 & & F0294 & F1540 & F0294 & & F0259 \\
\hline 23 & F1109 & F1036 & G5204 & P1109 & & G1449 & P1897 & F0294 & & F0294 & & F0259 \\
\hline 24 & F1109 & F1036 & G5204 & & F0294 & F1449 & & P0294 & G1540 & F0294 & F1449 & P4356 \\
\hline 25 & F4356 & F1036 & G5316 & F1109 & F0294 & G1449 & F1897 & F0294 & & F0294 & & F0259 \\
\hline 26 & G1109 & & G5316 & F1109 & P0294 & G1449 & F1897 & G0294 & & F0294 & F1449 & G0259 \\
\hline 27 & F1109 & F1036 & G5316 & P1109 & F0294 & F1449 & F1897 & F0294 & P0385 & B0294 & F1449 & G0259 \\
\hline 28 & F4356 & F1036 & G5316 & & F0294 & F1449 & F1897 & F0294 & F0385 & G0294 & & F0259 \\
\hline 29 & F1109 & F1036 & G5316 & F1109 & F0294 & F1449 & & F0294 & F0385 & F0294 & F1449 & B0259 \\
\hline 30 & P1109 & F1036 & G5316 & & & F2639 & & G0294 & G0385 & F0294 & & F0259 \\
\hline 31 & F1109 & F1036 & G5316 & & F0294 & F1449 & F1897 & F0294 & F0385 & G0294 & F1449 & F0259 \\
\hline 32 & G1109 & F1036 & G5316 & F1109 & & G5451 & G1897 & P0294 & F0385 & G0294 & F4356 & F0259 \\
\hline 33 & F1109 & & G5316 & G1109 & & G5451 & F1897 & F0294 & & F0294 & & F0259 \\
\hline 34 & F1109 & G1036 & G5316 & & & G5458 & & F0294 & G0385 & G0294 & & F0259 \\
\hline 35 & G1109 & F1036 & G5316 & F1109 & F0294 & G5458 & & B0294 & F0385 & G0294 & & F0259 \\
\hline 36 & G1109 & F1036 & G5316 & & F0294 & G5458 & F1897 & G0294 & F0385 & F0294 & & G0259 \\
\hline 37 & F1109 & G1036 & G5316 & F1109 & & G5458 & F1897 & F0294 & F0385 & G0294 & & F0259 \\
\hline
\end{tabular}

B-13 
Table B-5. West Bay, East Half. (3 sheets)

\begin{tabular}{|c|c|c|c|c|c|c|c|c|c|c|c|c|}
\hline & 46 & 47 & 48 & 49 & 50 & 51 & 52 & 53 & 54 & 55 & 56 & 57 \\
\hline 38 & & F1036 & G5316 & F1109 & F0294 & G5458 & F1897 & F0294 & G0385 & F0294 & G1897 & P0259 \\
\hline 39 & 109 & F1036 & G5316 & F1109 & & G5458 & & F0294 & G0385 & F0294 & & F0259 \\
\hline 40 & 1109 & & G5316 & F1109 & F0294 & G5458 & F1897 & P0294 & F0385 & F0294 & P1897 & F0259 \\
\hline 41 & 1109 & F1036 & G5316 & & G0294 & G5458 & F1897 & B0294 & F0385 & F0294 & & F0259 \\
\hline 42 & 1109 & F1036 & G5316 & P1109 & & G5458 & F4356 & F0294 & F0385 & F0294 & G1897 & G0259 \\
\hline 43 & 5451 & F1036 & G5316 & F1109 & B0294 & G5458 & & F0294 & F0385 & F0294 & F1897 & F0259 \\
\hline 44 & 5451 & F1036 & G5316 & F1109 & G0294 & G5458 & & G0294 & F0385 & G0294 & & F0259 \\
\hline 45 & 5451 & F1036 & G5316 & & & G5458 & F1449 & G5204 & P0385 & F0294 & & F0259 \\
\hline 46 & 5451 & 1036 & G5316 & G1109 & F0294 & G5458 & P1449 & G5204 & F0385 & F4356 & F1897 & F0259 \\
\hline 47 & 5451 & 036 & F5316 & & & G5458 & & G5204 & F0385 & G5204 & F1897 & G0259 \\
\hline 48 & 5451 & 036 & G5316 & F1109 & & G5458 & & G5204 & F0385 & G5204 & & F0259 \\
\hline 49 & 5444 & & G5316 & F1109 & & G5458 & & G5204 & F0385 & G5204 & G1897 & F0259 \\
\hline 50 & 5457 & 1897 & P1109 & F1109 & & G5458 & & G5204 & F0385 & G5204 & F1897 & G0259 \\
\hline 51 & 5457 & 897 & F1109 & F1109 & F0294 & G5458 & & G5204 & F0385 & G5204 & & F0259 \\
\hline 52 & 5451 & 897 & & & F0294 & G5458 & F1449 & G5204 & F0385 & G5204 & F1897 & F0259 \\
\hline 53 & G5451 & & & G1109 & F0294 & G5458 & & G5204 & P0385 & G5204 & & G0259 \\
\hline 54 & 5451 & & F1109 & & F0294 & G5458 & F1449 & G5204 & G0385 & G5204 & F1897 & F0259 \\
\hline 55 & G5451 & 1897 & 1109 & F1109 & B0294 & G5458 & F1449 & G5204 & F0385 & G5204 & & F0259 \\
\hline 56 & G5451 & 897 & & F1109 & F0294 & G5458 & & G5204 & F0385 & G5204 & G18 & G0259 \\
\hline 57 & G5451 & & F1109 & G5316 & F0294 & G5458 & & G5204 & F0385 & G5204 & & G0259 \\
\hline 58 & G5451 & 897 & & G5316 & F4356 & G5458 & & G5204 & F0385 & G5204 & G1897 & F0259 \\
\hline 59 & G5451 & & F1109 & G5316 & P0294 & G5458 & & G5204 & F0385 & G5204 & & G0259 \\
\hline 60 & G5451 & 897 & F1109 & G5316 & F0294 & G5458 & F1449 & G5204 & F0385 & G5204 & G1897 & F0259 \\
\hline 61 & G5458 & & & G5316 & F0294 & G5458 & & G5204 & F0385 & G5204 & G1897 & F0259 \\
\hline 62 & G5458 & 1897 & F1109 & G5316 & P0294 & G5458 & $\mathrm{G} 2240$ & G5204 & P0385 & G5204 & & G0259 \\
\hline 63 & G5458 & F1897 & & G5316 & F0294 & G5458 & F1449 & G5204 & P0385 & G5204 & & G025S \\
\hline 64 & G5458 & & & G5316 & F4356 & G5458 & $\mathrm{G} 2240$ & G5204 & G0385 & G5204 & F1897 & F0259 \\
\hline 65 & G5458 & 897 & 1109 & G5316 & G0294 & G5458 & & G5204 & B0385 & G5204 & & F0259 \\
\hline 66 & G5458 & F1897 & F1109 & G5316 & G0294 & G5458 & F1449 & G5204 & G0385 & G5204 & G1897 & F0259 \\
\hline 67 & G5458 & & & G5316 & F0294 & G5458 & & G5204 & F0385 & G5204 & G1897 & F0259 \\
\hline 68 & G5458 & G1897 & F1109 & F5316 & G0294 & G5458 & G1449 & G5204 & F0385 & G5204 & & F0259 \\
\hline 69 & G5458 & & F1109 & G5316 & F0294 & G5458 & & G5204 & F0385 & G5204 & G1897 & G0259 \\
\hline 70 & G5455 & 1897 & F1109 & G5316 & F0294 & G5458 & F1449 & G5204 & F0385 & G5204 & F1897 & F0259 \\
\hline 71 & G5455 & F1897 & F1109 & G5316 & F0294 & G5458 & & G5204 & F0385 & G5204 & & ת \\
\hline 72 & G5458 & & F1109 & F5399 & F0294 & G5458 & G1449 & G5204 & F0385 & G5204 & F1897 & F0259 \\
\hline 73 & G5455 & F1897 & F1109 & F5399 & F0294 & G5458 & F1449 & G5204 & & F5204 & & \\
\hline 74 & G5455 & G2285 & & G5451 & $\mathrm{G} 2285$ & G5458 & G2639 & G2639 & G2639 & G2639 & G2639 & \\
\hline 75 & G5455 & G2285 & G2285 & G2285 & G2285 & & G2639 & G2639 & & G2639 & G2639 & G263 \\
\hline
\end{tabular}


SNF-10293, REV 0

Table B-5. West Bay, East Half. (3 sheets)

\begin{tabular}{|l|l|l|l|l|l|l|l|l|l|l|l|l|}
\hline & 46 & 47 & 48 & 49 & 50 & 51 & 52 & 53 & 54 & 55 & 56 & 57 \\
\hline 76 & & & & & & & & & & & & \\
\hline
\end{tabular}

Keys are identified using the notation CXXXX, where $\mathrm{C}$ is the fuel condition (G, F, P, or B for Good, Fair, Poor, or Bad), and XXXX is the last four digits of the key number.

Fuel condition identifiers are from WHC-SD-SNF-TI-012.

Key locations are from private communication with Karen Morris, K Basins Operations, 1995, "K East Basin Storage Map", MAC Version 3.1, Rev. 163, Updated 1/11/94, Report 5/02/94, 124 pages. 
Table B-6. West Bay, West Half -- Cubicle 6776 is the North-West Corner of K East Basin. (3 sheets)

\begin{tabular}{|c|c|c|c|c|c|c|c|c|c|c|}
\hline & 58 & 59 & 60 & 61 & 62 & 63 & 64 & 65 & 66 & 67 \\
\hline 1 & & & & & & & & & & \\
\hline 2 & G5204 & F2565 & F2565 & & G5204 & F2565 & $\mathrm{G} 2565$ & G2565 & & \\
\hline 3 & G5204 & F2565 & G2565 & F2565 & G5204 & G5316 & F2565 & G2565 & & \\
\hline 4 & G5204 & G0259 & F1897 & G5451 & G5204 & G5316 & G1806 & G5204 & & \\
\hline 5 & G5204 & F0259 & & G5451 & G5204 & G5316 & & G5204 & & G2565 \\
\hline 6 & G5204 & & F1897 & G5451 & G5204 & F5316 & & G5204 & & G2565 \\
\hline 7 & G5204 & G0259 & F1897 & G5451 & G5204 & G5316 & F1806 & G5204 & & \\
\hline 8 & G5204 & G5458 & F1897 & G5451 & G5204 & G5316 & F1806 & G5204 & & G2565 \\
\hline 9 & G5204 & G5458 & F1897 & G5204 & G5204 & G5316 & & G5204 & & G2565 \\
\hline 10 & G5204 & G5458 & F1897 & G5204 & G5204 & G5316 & F1806 & G5204 & & G2565 \\
\hline 11 & G5204 & G5458 & & G5204 & G5204 & G5316 & & G5204 & & G2565 \\
\hline 12 & G5204 & G5458 & F1897 & G5316 & G5204 & G5316 & & G5204 & & G2565 \\
\hline 13 & G5204 & G5458 & F1806 & F5316 & G5204 & F5451 & F1806 & F5204 & & G2565 \\
\hline 14 & G5204 & G5458 & & G5316 & G5204 & G5316 & F1806 & G5204 & & G2565 \\
\hline 15 & G5204 & G5458 & & G5316 & G5204 & G5316 & & G5204 & & \\
\hline 16 & G5204 & G5458 & F1806 & G5316 & G5204 & G5451 & F1806 & G5204 & & G2565 \\
\hline 17 & G5204 & G5458 & & G5316 & G5204 & G5451 & F1806 & F5204 & & F2565 \\
\hline 18 & G5204 & G5458 & & G5316 & G5204 & G5451 & F1806 & G5204 & & \\
\hline 19 & G5204 & G2285 & & G5316 & G5204 & G5451 & & G5204 & & F2565 \\
\hline 20 & G5204 & F2285 & F1806 & G5204 & G5204 & G5451 & G1806 & F5204 & & G2565 \\
\hline 21 & G5204 & G2285 & F1806 & G5204 & G5204 & G5451 & & G5204 & & G2565 \\
\hline 22 & G5204 & F2285 & G1806 & G5204 & G5204 & G5316 & & G5204 & & G2565 \\
\hline 23 & G5204 & F2285 & & G5204 & G5204 & G5316 & G1806 & G5204 & & G2565 \\
\hline 24 & G5204 & & F1806 & G5204 & G5204 & G5316 & F1806 & G5204 & & F2565 \\
\hline 25 & G5204 & F2285 & G1806 & G5204 & G5204 & G5316 & F1806 & G5204 & & G2565 \\
\hline 26 & G5204 & F2285 & & G5204 & G5204 & G5316 & & G5204 & & F2565 \\
\hline 27 & G5204 & F2285 & F1806 & G5204 & G5204 & G5204 & & G5204 & & P2565 \\
\hline 28 & G5204 & G2285 & F1806 & G5204 & G5204 & G5204 & G1806 & G5204 & & G2565 \\
\hline 29 & G5204 & F2285 & & G5204 & G5204 & F5204 & G1806 & G5204 & & F2565 \\
\hline 30 & G5204 & & & F5204 & G5204 & G5204 & G1806 & G5204 & & \\
\hline 31 & G5204 & F2285 & G1806 & G5204 & G5204 & G5204 & & F5204 & & F2565 \\
\hline 32 & G5204 & G2285 & & G5204 & G5204 & G5204 & F1806 & G5204 & & F2565 \\
\hline 33 & G5204 & G2285 & & F5204 & G5204 & G5204 & & G5204 & & \\
\hline 34 & G5204 & F2285 & F1806 & G5204 & G5204 & G5204 & F1806 & G5204 & & G2565 \\
\hline 35 & G5204 & F2285 & F1806 & G5204 & G5204 & G5204 & F1806 & G5204 & & G2565 \\
\hline 36 & G5204 & & & G5204 & G5204 & G5204 & & G5204 & & \\
\hline
\end{tabular}


Table B-6. West Bay, West Half -- Cubicle 6776 is the North-West Corner of K East Basin. (3 sheets)

\begin{tabular}{|c|c|c|c|c|c|c|c|c|c|c|}
\hline & 58 & 59 & 60 & 61 & 62 & 63 & 64 & 65 & 66 & 67 \\
\hline 37 & G5204 & F2285 & F1806 & F5204 & G5204 & G5204 & G1806 & G5204 & & G2565 \\
\hline 38 & G5204 & G2285 & & G5204 & G2565 & G5204 & G1806 & G5204 & & G2565 \\
\hline 39 & G5204 & G2285 & F1806 & G5204 & G2565 & G5204 & & G5204 & & \\
\hline 40 & G5204 & G2285 & F1806 & G5204 & F1806 & G5204 & G1806 & G5204 & & \\
\hline 41 & G5204 & G2285 & F1806 & G5204 & G1540 & G5204 & & G5204 & & F2565 \\
\hline 42 & G5204 & G2285 & & G5204 & F1806 & G5204 & F1806 & G5204 & & \\
\hline 43 & G5204 & F2285 & & F5204 & F1540 & G5204 & & G5204 & & F2565 \\
\hline 44 & G5204 & F2285 & F1806 & G5204 & G1806 & G5204 & G1806 & G5204 & & G2565 \\
\hline 45 & G5204 & F2285 & & G5204 & F1806 & G5204 & G1806 & F5204 & & \\
\hline 46 & G5204 & & & G5204 & F1806 & G5204 & & G5204 & & \\
\hline 47 & G5204 & G2285 & F1806 & G5204 & F1540 & G5204 & & G5204 & & G2565 \\
\hline 48 & G5204 & G2285 & F1806 & G5204 & F4355 & G5204 & F1806 & F5204 & & \\
\hline 49 & G5204 & G2285 & G1806 & F5204 & G1540 & G5204 & & F5204 & & G2565 \\
\hline 50 & G5204 & G2285 & & G5204 & G4355 & G5204 & & G5204 & & G2565 \\
\hline 51 & G5204 & G2285 & F1806 & G5204 & F1540 & F5204 & F1806 & G5204 & & \\
\hline 52 & G5204 & & G1806 & G5204 & G4355 & G5204 & P1806 & G5204 & & \\
\hline 53 & G5204 & G2285 & & G5204 & F1540 & G5204 & & G5204 & & G2565 \\
\hline 54 & G5204 & & F1806 & G5204 & F1540 & G5204 & G1806 & G5204 & & G2565 \\
\hline 55 & G5204 & G2285 & F1806 & G5204 & G4355 & G5204 & G1806 & G5204 & & \\
\hline 56 & G5204 & G2285 & & G5204 & G3649 & G5204 & & G5204 & & \\
\hline 57 & G5204 & G2285 & F1806 & G5204 & F3649 & G5204 & F1806 & G5204 & & F2565 \\
\hline 58 & G5204 & F2285 & & G5204 & G3649 & F5204 & G1806 & G5204 & & \\
\hline 59 & G5204 & F2285 & & G5204 & F3649 & G5204 & & G5204 & & F2565 \\
\hline 60 & G5204 & & G1806 & G5204 & G3649 & G5204 & F1806 & G5204 & & F2565 \\
\hline 61 & G5204 & F2285 & G1806 & G5204 & F3649 & G5204 & F1806 & G5204 & & \\
\hline 62 & G5204 & F2285 & & P4356 & G3649 & G5204 & & G5204 & & G2565 \\
\hline 63 & G5204 & G2285 & F1806 & B0201 & F3649 & G5204 & G1806 & G5204 & & \\
\hline 64 & G5204 & G2285 & F1806 & B4356 & F3649 & G5204 & & G5204 & & \\
\hline 65 & G5204 & & F4356 & B0201 & F3649 & G5204 & G1806 & G5204 & & G2565 \\
\hline 66 & G5204 & F2285 & G4356 & B0201 & F3649 & G5204 & F1806 & G5204 & & \\
\hline 67 & G2565 & F2285 & G4356 & P0201 & G3649 & P0201 & & G5204 & & G2565 \\
\hline 68 & G1806 & F2285 & P4356 & G4356 & F3649 & B0201 & & G3685 & & F2565 \\
\hline 69 & G1806 & F2285 & F4356 & B4356 & F3649 & B0201 & & G3685 & & \\
\hline 70 & F1806 & F2285 & P4356 & F4356 & F3649 & B0201 & F1806 & F3685 & & G2565 \\
\hline 71 & F1806 & & P4356 & B4356 & G3649 & B0201 & & G3685 & & F2565 \\
\hline 72 & F1806 & G2285 & F1806 & F4356 & F1540 & B0201 & & G3685 & & \\
\hline 73 & G1806 & G2285 & & B4356 & F1540 & B0201 & G1806 & G3685 & & \\
\hline
\end{tabular}


SNF-10293, REV 0

Table B-6. West Bay, West Half -- Cubicle 6776 is the North-West Corner of K East Basin. (3 sheets)

\begin{tabular}{|c|c|c|c|c|c|c|c|c|c|c|}
\hline & 58 & 59 & 60 & 61 & 62 & 63 & 64 & 65 & 66 & 67 \\
\hline 74 & G2639 & G2639 & F1806 & F2639 & G2639 & F2639 & & G3685 & & \\
\hline 75 & G2639 & G2639 & G2639 & G2639 & F2639 & F2639 & & G3685 & & \\
\hline 76 & & & & & & & & & & \\
\hline
\end{tabular}


SNF-10293, REV 0

APPENDIX C

SUMMARY OF DAMAGE CONDITION CATEGORIES

C-i 
SNF-10293, REV 0

This page intentionally left blank.

C-ii 
SNF-10293, REV 0

\section{APPENDIX C SUMMARY OF DAMAGE CONDITION CATEGORIES}

Table C-1 summarizes the equivalence of fuel damage category terms used for individual fuel elements as well as fuel in a storage canister. The first two columns indicate the primary terms used, the third column relates the fuel condition terms used in the 1999 sludge sampling initiatives and referred to in Table 3-26 of the main text.

Categories of Fuel Element Damage

The fuel element damage categories are based on those derived in HNF-8760, Rev. 1 (Schwinkendorf and Wittekind 2002) that are linked to visual assessments made by Pitner (1998, HNF-2586). The authors of HNF-8760 interrelated Pitner's observation definitions into mathematical model parameters (see HNF-8760 Table 5). The basic qualitative fuel element damage categories used include:

Intact: No evident of breach or deposited sludge

Breached: Minor cladding rupture with no reacted fuel or deposited sludge visibly present

Defected: Definite evidence of cladding breach with reacted fuel egressing from element. Amount of exposed fuel may be significant, but there is no gross cladding splitting, element dilation, or fuel voiding.

Bad: Gross failure is evident with substantial element dilation, cladding splitting, fuel "mushrooming" or fuel voiding.

Categories of Damage for a Group of Fuel Assemblies in a Storage Canister

In Section 3.4.4.4 specific validation cases for canister sludge are presented. Here for the most accurate predictions of resulting sludge characteristics, the damage category for each fuel element in each canister barrel that was sampled for sludge at KE Basin is defined. There are only a limited number of canister barrels that have been sampled for sludge hence this detailed approach could be used. These fuel damage assessments per barrel (i.e., Table 3-26 of the main text) were then used in the models to predict the sludge characteristics for each barrel sampled. The resulting refined predictions for the parameters of interest (e.g., radioisotope concentrations) were then compared to the laboratory measurements for validation of the modeling methods being used in HNF-8067 and SNF-10293.

In addition to the assessment of fuel damage per element noted above, in 1995 Pitner also performed a survey of the general condition of fuel in each canister visible (1995, WHC-SD-TI012). Here fuel damage categories used ("Good, Fair, Poor and Bad") were primarily associated with the condition of the worst fuel elements in the group of fuel elements contained in the double-barreled canister (up to 14 fuel assemblies). Where the specific damage categories (Pitner, 1995) were: 
Good: All fuel elements in canister are basically intact. May have elements with minor cladding ruptures.

Fair: At least one fuel element in canister has a significant area of exposed fuel, such as a lose end cap, extruded fuel at the rupture point, or split cladding.

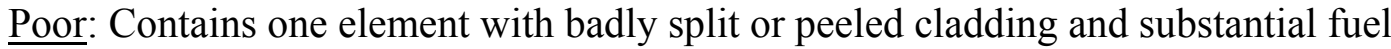
missing, or reacted fuel has mushroomed and dilated end of element.

Very Bad (or Bad): Multiple instances of "poor" elements in canister.

These survey terms and results are used with a simplifying assumption. The specific damage category terms used by Pitner for the canisters were retained, but in application to modeling the definitions were modified somewhat to simplify handling the data. Table C-1 provides an assumed equivalence between the fuel element damage categories used in HNF-8760 and overall damage categories for groups of fuel elements in a canister from earlier survey work (Pitner 1995 and 1998). Also, all fuel elements in a canister (up to 14 assemblies) are assumed to be at a single damage condition (i.e., a "good" canister having all "intact" fuel elements, a "fair" canister all "breached" elements, a "poor" canister all "defected" elements, and a "bad" canister all "bad" elements). These assumptions are judged to be 1) conservative for calculating the total amount of uranium fuel predicted to be converted from fuel to sludge and 2) reasonable approximations for calculating the other parameters being modeled (it is noted that location on a fuel element where the sludge comes from, as well as the overall volume of sludge coming from the element, has impact on the predicted parameters because of end/surface peaking factors present during irradiation, see HNF-8760).

Table C-1. Equivalence of Terms Used to Describe Degrees of Damaged Fuel

\begin{tabular}{|c|c|c|}
$\begin{array}{c}\text { Fuel Element } \\
\text { Damage } \\
\text { Categories }\end{array}$ & $\begin{array}{c}\text { Overall Damage } \\
\text { Categories for Group of } \\
\text { Fuel Elements in a } \\
\text { Canister }\end{array}$ & $\begin{array}{c}\text { General Fuel Condition } \\
\text { Terms from the 1999 Sludge } \\
\text { Sampling Campaign and } \\
\text { in Table 3-25 of Main Text }\end{array}$ \\
\hline Intact & Good & Good \\
\hline Breached & Fair & Moderately Damaged \\
\hline Defected & Poor* & Moderately Damaged \\
\hline Bad & Bad & Highly Damaged \\
\hline
\end{tabular}

* In some Spent Nuclear Fuel Project reports the "poor" category has been combined with the "Bad" category. An example is shown in Appendix D. 


\section{$\underline{\text { References }}$}

Pitner, AL, 1998, Summary of Fuel Damage Distributions in the K Basins, HNF-2586, Rev.0 Duke Engineering and Services Hanford, Richland Washington.

Pitner, AL, 1995, KE Basin Underwater Visual Fuel Survey, WHC-SD-SNF-TI-012, Rev. 0, Westinghouse Hanford Company, Richland, Washington.

Schwinkendorf, KN, and WD Wittekind, 2002, K East Basin Sludge Characterization Using Exposure-Adjusted Radioisotopic Source Terms for N Reactor Fuel, HNF-8760, Rev.1, Fluor Hanford, Richland, Washington. 
SNF-10293, REV 0

This page intentionally left blank. 
SNF-10293, REV 0

APPENDIX D

CANISTER SLUDGE DEPTH MEASUREMENTS

D-i 
SNF-10293, REV 0

This page intentionally left blank.

D-ii 
SNF-10293, REV 0

\section{APPENDIX D}

\section{CANISTER SLUDGE DEPTH MEASUREMENTS}

Canister Sludge Depth Measurements. (14 sheets)

\begin{tabular}{|c|c|c|c|c|c|c|}
\hline \multirow{2}{*}{$\begin{array}{c}\text { Canister } \\
\text { Metal }\end{array}$} & \multirow[b]{2}{*}{ Fuel Type } & \multirow{2}{*}{$\begin{array}{c}\text { Barrel } \\
\text { Location }\end{array}$} & \multirow{2}{*}{$\begin{array}{c}\text { Position in } \\
\text { Barrel }\end{array}$} & \multicolumn{3}{|c|}{ Canister Sludge Depth (inch) } \\
\hline & & & & Data & Mean $=0$ & Negative $=0$ \\
\hline $\mathrm{AL}$ & $\mathrm{Bad}$ & $0455 \mathrm{~W}$ & 1 & 1.19 & 1.19 & 1.19 \\
\hline $\mathrm{AL}$ & $\mathrm{Bad}$ & $0455 \mathrm{~W}$ & 2 & 1.16 & 1.16 & 1.16 \\
\hline $\mathrm{AL}$ & $\mathrm{Bad}$ & $0455 \mathrm{~W}$ & 3 & 1.81 & 1.81 & 1.81 \\
\hline $\mathrm{AL}$ & $\mathrm{Bad}$ & $0455 \mathrm{~W}$ & 4 & 3.03 & 3.03 & 3.03 \\
\hline $\mathrm{AL}$ & $\mathrm{Bad}$ & $0455 \mathrm{~W}$ & 5 & 2.66 & 2.66 & 2.66 \\
\hline $\mathrm{AL}$ & Bad & $0455 \mathrm{~W}$ & 6 & 1.98 & 1.98 & 1.98 \\
\hline \multicolumn{4}{|c|}{ 0455W Average } & 1.9717 & 1.9717 & 1.9717 \\
\hline $\mathrm{AL}$ & $\mathrm{Bad}$ & $0627 \mathrm{~W}$ & 1 & 0.63 & 0.63 & 0.63 \\
\hline $\mathrm{AL}$ & $\mathrm{Bad}$ & $0627 \mathrm{~W}$ & 2 & 0.82 & 0.82 & 0.82 \\
\hline $\mathrm{AL}$ & $\mathrm{Bad}$ & $0627 \mathrm{~W}$ & 3 & 1.14 & 1.14 & 1.14 \\
\hline $\mathrm{AL}$ & $\mathrm{Bad}$ & $0627 \mathrm{~W}$ & 5 & 2.23 & 2.23 & 2.23 \\
\hline $\mathrm{AL}$ & $\mathrm{Bad}$ & $0627 \mathrm{~W}$ & 6 & 0.74 & 0.74 & 0.74 \\
\hline $\mathrm{AL}$ & $\mathrm{Bad}$ & $0627 \mathrm{~W}$ & 11 & 1.04 & 1.04 & 1.04 \\
\hline \multirow[t]{2}{*}{$\mathrm{AL}$} & $\mathrm{Bad}$ & $0627 \mathrm{~W}$ & 12 & 0.43 & 0.43 & 0.43 \\
\hline & \multicolumn{3}{|c|}{ 0627W Average } & 1.0043 & 1.0043 & 1.0043 \\
\hline $\mathrm{AL}$ & $\mathrm{Bad}$ & $0668 \mathrm{E}$ & 1 & 2.74 & 2.74 & 2.74 \\
\hline $\mathrm{AL}$ & $\mathrm{Bad}$ & $0668 \mathrm{E}$ & 2 & 2.83 & 2.83 & 2.83 \\
\hline $\mathrm{AL}$ & $\mathrm{Bad}$ & $0668 \mathrm{E}$ & 3 & 2.13 & 2.13 & 2.13 \\
\hline \multirow[t]{2}{*}{$\mathrm{AL}$} & $\mathrm{Bad}$ & $0668 \mathrm{E}$ & 6 & 5.23 & 5.23 & 5.23 \\
\hline & \multicolumn{3}{|c|}{ 0668E Average } & 3.2325 & 3.2325 & 3.2325 \\
\hline $\mathrm{AL}$ & $\mathrm{Bad}$ & 0668W & 1 & 0.93 & 0.93 & 0.93 \\
\hline $\mathrm{AL}$ & $\mathrm{Bad}$ & $0668 \mathrm{~W}$ & 2 & 1.64 & 1.64 & 1.64 \\
\hline \multirow[t]{2}{*}{$\mathrm{AL}$} & $\mathrm{Bad}$ & $0668 \mathrm{~W}$ & 3 & 2.87 & 2.87 & 2.87 \\
\hline & \multicolumn{3}{|c|}{ 0668W Average } & 1.8133 & 1.8133 & 1.8133 \\
\hline $\mathrm{AL}$ & $\mathrm{Bad}$ & $1236 \mathrm{~W}$ & 1 & 4.03 & 4.03 & 4.03 \\
\hline $\mathrm{AL}$ & $\mathrm{Bad}$ & $1236 \mathrm{~W}$ & 2 & 4.63 & 4.63 & 4.63 \\
\hline $\mathrm{AL}$ & $\mathrm{Bad}$ & $1236 \mathrm{~W}$ & 7 & 4.43 & 4.43 & 4.43 \\
\hline
\end{tabular}


SNF-10293, REV 0

Canister Sludge Depth Measurements. (14 sheets)

\begin{tabular}{|c|c|c|c|c|c|c|}
\hline \multirow{2}{*}{$\begin{array}{c}\text { Canister } \\
\text { Metal }\end{array}$} & \multirow[b]{2}{*}{ Fuel Type } & \multirow{2}{*}{$\begin{array}{c}\text { Barrel } \\
\text { Location }\end{array}$} & \multirow{2}{*}{$\begin{array}{c}\text { Position in } \\
\text { Barrel }\end{array}$} & \multicolumn{3}{|c|}{ Canister Sludge Depth (inch) } \\
\hline & & & & Data & Mean $=0$ & Negative $=0$ \\
\hline & \multicolumn{3}{|c|}{ 1236W Average } & 4.3633 & 4.3633 & 4.3633 \\
\hline $\mathrm{AL}$ & $\mathrm{Bad}$ & $1243 \mathrm{~W}$ & 1 & 2.83 & 2.83 & 2.83 \\
\hline $\mathrm{AL}$ & $\mathrm{Bad}$ & $1243 \mathrm{~W}$ & 3 & 2.02 & 2.02 & 2.02 \\
\hline $\mathrm{AL}$ & $\mathrm{Bad}$ & $1243 \mathrm{~W}$ & 4 & 2.33 & 2.33 & 2.33 \\
\hline $\mathrm{AL}$ & $\mathrm{Bad}$ & $1243 \mathrm{~W}$ & 5 & 2.54 & 2.54 & 2.54 \\
\hline $\mathrm{AL}$ & $\mathrm{Bad}$ & $1243 \mathrm{~W}$ & 6 & 2.42 & 2.42 & 2.42 \\
\hline $\mathrm{AL}$ & $\mathrm{Bad}$ & $1243 \mathrm{~W}$ & 9 & 1.63 & 1.63 & 1.63 \\
\hline \multirow[t]{2}{*}{$\mathrm{AL}$} & $\mathrm{Bad}$ & $1243 \mathrm{~W}$ & 10 & 2.13 & 2.13 & 2.13 \\
\hline & & \multicolumn{2}{|c|}{ 1243W Average } & 2.2714 & 2.2714 & 2.2714 \\
\hline $\mathrm{AL}$ & $\mathrm{Bad}$ & $1267 \mathrm{E}$ & 1 & 2.79 & 2.79 & 2.79 \\
\hline $\mathrm{AL}$ & $\mathrm{Bad}$ & $1267 \mathrm{E}$ & 2 & 2.19 & 2.19 & 2.19 \\
\hline $\mathrm{AL}$ & Bad & $1267 \mathrm{E}$ & 3 & 2.79 & 2.79 & 2.79 \\
\hline $\mathrm{AL}$ & $\mathrm{Bad}$ & $1267 \mathrm{E}$ & 4 & 6.68 & 6.68 & 6.68 \\
\hline $\mathrm{AL}$ & $\mathrm{Bad}$ & $1267 \mathrm{E}$ & 5 & 6.78 & 6.78 & 6.78 \\
\hline \multirow[t]{2}{*}{$\mathrm{AL}$} & $\mathrm{Bad}$ & $1267 \mathrm{E}$ & 6 & 7.38 & 7.38 & 7.38 \\
\hline & & \multicolumn{2}{|c|}{ 1267E Average } & 4.7683 & 4.7683 & 4.7683 \\
\hline $\mathrm{AL}$ & $\mathrm{Bad}$ & $2024 \mathrm{E}$ & 1 & 0.42 & 0.42 & 0.42 \\
\hline $\mathrm{AL}$ & $\mathrm{Bad}$ & $2024 \mathrm{E}$ & 2 & 0.26 & 0.26 & 0.26 \\
\hline $\mathrm{AL}$ & $\mathrm{Bad}$ & $2024 \mathrm{E}$ & 3 & 0.13 & 0.13 & 0.13 \\
\hline $\mathrm{AL}$ & $\mathrm{Bad}$ & $2024 \mathrm{E}$ & 4 & 2.15 & 2.15 & 2.15 \\
\hline $\mathrm{AL}$ & $\mathrm{Bad}$ & $2024 \mathrm{E}$ & 5 & 2.34 & 2.34 & 2.34 \\
\hline \multirow[t]{2}{*}{$\mathrm{AL}$} & $\mathrm{Bad}$ & $2024 \mathrm{E}$ & 6 & 1.72 & 1.72 & 1.72 \\
\hline & & \multicolumn{2}{|c|}{ 2024E Average } & 1.1700 & 1.1700 & 1.1700 \\
\hline $\mathrm{AL}$ & $\mathrm{Bad}$ & $2031 \mathrm{~W}$ & 1 & 0.41 & 0.41 & 0.41 \\
\hline $\mathrm{AL}$ & $\mathrm{Bad}$ & $2031 \mathrm{~W}$ & 2 & 0.57 & 0.57 & 0.57 \\
\hline $\mathrm{AL}$ & $\mathrm{Bad}$ & $2031 \mathrm{~W}$ & 3 & 0.23 & 0.23 & 0.23 \\
\hline $\mathrm{AL}$ & $\mathrm{Bad}$ & $2031 \mathrm{~W}$ & 4 & 1.4 & 1.4 & 1.4 \\
\hline $\mathrm{AL}$ & $\mathrm{Bad}$ & $2031 \mathrm{~W}$ & 5 & 3.03 & 3.03 & 3.03 \\
\hline $\mathrm{AL}$ & $\mathrm{Bad}$ & $2031 \mathrm{~W}$ & 6 & 3 & 3 & 3 \\
\hline \multicolumn{4}{|c|}{ 2031W Average } & 1.4400 & 1.4400 & 1.4400 \\
\hline $\mathrm{AL}$ & $\mathrm{Bad}$ & $2211 \mathrm{~W}$ & 1 & 3.87 & 3.87 & 3.87 \\
\hline $\mathrm{AL}$ & $\mathrm{Bad}$ & $2211 \mathrm{~W}$ & 2 & 2.92 & 2.92 & 2.92 \\
\hline
\end{tabular}


SNF-10293, REV 0

Canister Sludge Depth Measurements. (14 sheets)

\begin{tabular}{|c|c|c|c|c|c|c|}
\hline \multirow{2}{*}{$\begin{array}{c}\text { Canister } \\
\text { Metal }\end{array}$} & \multirow[b]{2}{*}{ Fuel Type } & \multirow{2}{*}{$\begin{array}{c}\text { Barrel } \\
\text { Location }\end{array}$} & \multirow{2}{*}{$\begin{array}{c}\text { Position in } \\
\text { Barrel }\end{array}$} & \multicolumn{3}{|c|}{ Canister Sludge Depth (inch) } \\
\hline & & & & Data & Mean $=0$ & Negative $=0$ \\
\hline $\mathrm{AL}$ & $\mathrm{Bad}$ & $2211 \mathrm{~W}$ & 5 & 5.65 & 5.65 & 5.65 \\
\hline $\mathrm{AL}$ & $\mathrm{Bad}$ & $2211 \mathrm{~W}$ & 6 & 1.55 & 1.55 & 1.55 \\
\hline $\mathrm{AL}$ & $\mathrm{Bad}$ & $2211 \mathrm{~W}$ & 9 & 6.83 & 6.83 & 6.83 \\
\hline \multirow[t]{2}{*}{$\mathrm{AL}$} & $\mathrm{Bad}$ & $2211 \mathrm{~W}$ & 12 & 3.31 & 3.31 & 3.31 \\
\hline & \multicolumn{3}{|c|}{ 2211W Average } & 4.0217 & 4.0217 & 4.0217 \\
\hline $\mathrm{AL}$ & $\mathrm{Bad}$ & $2214 \mathrm{E}$ & 1 & 1.63 & 1.63 & 1.63 \\
\hline $\mathrm{AL}$ & $\mathrm{Bad}$ & $2214 \mathrm{E}$ & 2 & 1.84 & 1.84 & 1.84 \\
\hline $\mathrm{AL}$ & Bad & $2214 \mathrm{E}$ & 3 & 1.44 & 1.44 & 1.44 \\
\hline $\mathrm{AL}$ & Bad & $2214 \mathrm{E}$ & 4 & 4.57 & 4.57 & 4.57 \\
\hline $\mathrm{AL}$ & Bad & $2214 \mathrm{E}$ & 5 & 2.78 & 2.78 & 2.78 \\
\hline $\mathrm{AL}$ & $\mathrm{Bad}$ & $2214 \mathrm{E}$ & 6 & 1.6 & 1.6 & 1.6 \\
\hline \multirow[t]{2}{*}{$\mathrm{AL}$} & Bad & $2214 \mathrm{E}$ & 8 & 1.98 & 1.98 & 1.98 \\
\hline & \multicolumn{3}{|c|}{ 2214E Average } & 2.2629 & 2.2629 & 2.2629 \\
\hline $\mathrm{AL}$ & $\mathrm{Bad}$ & $2229 E$ & 1 & 2.32 & 2.32 & 2.32 \\
\hline $\mathrm{AL}$ & $\mathrm{Bad}$ & $2229 \mathrm{E}$ & 2 & 2.23 & 2.23 & 2.23 \\
\hline $\mathrm{AL}$ & $\mathrm{Bad}$ & $2229 E$ & 3 & 2.76 & 2.76 & 2.76 \\
\hline \multirow[t]{2}{*}{$\mathrm{AL}$} & $\mathrm{Bad}$ & $2229 E$ & 6 & 5.91 & 5.91 & 5.91 \\
\hline & \multicolumn{3}{|c|}{ 2229E Average } & 3.3050 & 3.3050 & 3.3050 \\
\hline $\mathrm{AL}$ & $\mathrm{Bad}$ & $2350 \mathrm{E}$ & 1 & 3.18 & 3.18 & 3.18 \\
\hline $\mathrm{AL}$ & $\mathrm{Bad}$ & $2350 \mathrm{E}$ & 2 & 5.92 & 5.92 & 5.92 \\
\hline $\mathrm{AL}$ & $\mathrm{Bad}$ & $2350 \mathrm{E}$ & 3 & 4.12 & 4.12 & 4.12 \\
\hline $\mathrm{AL}$ & $\mathrm{Bad}$ & $2350 \mathrm{E}$ & 4 & 2.8 & 2.8 & 2.8 \\
\hline $\mathrm{AL}$ & $\mathrm{Bad}$ & $2350 \mathrm{E}$ & 5 & 2.66 & 2.66 & 2.66 \\
\hline $\mathrm{AL}$ & $\mathrm{Bad}$ & $2350 \mathrm{E}$ & 6 & 3.13 & 3.13 & 3.13 \\
\hline \multirow[t]{2}{*}{$\mathrm{AL}$} & Bad & $2350 \mathrm{E}$ & 7 & 2.29 & 2.29 & 2.29 \\
\hline & \multicolumn{3}{|c|}{ 2350E Average } & 3.4429 & 3.4429 & 3.4429 \\
\hline $\mathrm{AL}$ & $\mathrm{Bad}$ & $2355 \mathrm{~W}$ & 1 & 1.26 & 1.26 & 1.26 \\
\hline $\mathrm{AL}$ & $\mathrm{Bad}$ & $2355 \mathrm{~W}$ & 2 & 1.74 & 1.74 & 1.74 \\
\hline $\mathrm{AL}$ & $\mathrm{Bad}$ & $2355 \mathrm{~W}$ & 3 & 2.74 & 2.74 & 2.74 \\
\hline $\mathrm{AL}$ & $\mathrm{Bad}$ & $2355 \mathrm{~W}$ & 4 & 1.87 & 1.87 & 1.87 \\
\hline $\mathrm{AL}$ & $\mathrm{Bad}$ & $2355 \mathrm{~W}$ & 5 & 1.26 & 1.26 & 1.26 \\
\hline $\mathrm{AL}$ & $\mathrm{Bad}$ & $2355 \mathrm{~W}$ & 6 & 1.22 & 1.22 & 1.22 \\
\hline
\end{tabular}


SNF-10293, REV 0

Canister Sludge Depth Measurements. (14 sheets)

\begin{tabular}{|c|c|c|c|c|c|c|}
\hline \multirow{2}{*}{$\begin{array}{c}\text { Canister } \\
\text { Metal }\end{array}$} & \multirow[b]{2}{*}{ Fuel Type } & \multirow{2}{*}{$\begin{array}{c}\text { Barrel } \\
\text { Location }\end{array}$} & \multirow{2}{*}{$\begin{array}{c}\text { Position in } \\
\text { Barrel }\end{array}$} & \multicolumn{3}{|c|}{ Canister Sludge Depth (inch) } \\
\hline & & & & Data & Mean $=0$ & Negative $=0$ \\
\hline & \multicolumn{3}{|c|}{ 2355W Average } & 1.6817 & 1.6817 & 1.6817 \\
\hline $\mathrm{AL}$ & Bad & $2540 \mathrm{E}$ & 1 & 0.72 & 0.72 & 0.72 \\
\hline $\mathrm{AL}$ & $\mathrm{Bad}$ & $2540 \mathrm{E}$ & 2 & 0.93 & 0.93 & 0.93 \\
\hline $\mathrm{AL}$ & $\mathrm{Bad}$ & $2540 \mathrm{E}$ & 3 & 0.82 & 0.82 & 0.82 \\
\hline $\mathrm{AL}$ & $\mathrm{Bad}$ & $2540 \mathrm{E}$ & 4 & 2.03 & 2.03 & 2.03 \\
\hline $\mathrm{AL}$ & $\mathrm{Bad}$ & $2540 \mathrm{E}$ & 5 & 1.4 & 1.4 & 1.4 \\
\hline $\mathrm{AL}$ & $\mathrm{Bad}$ & $2540 \mathrm{E}$ & 6 & 0.42 & 0.42 & 0.42 \\
\hline \multirow[t]{2}{*}{$\mathrm{AL}$} & $\mathrm{Bad}$ & $2540 \mathrm{E}$ & 9 & 1.77 & 1.77 & 1.77 \\
\hline & \multicolumn{3}{|c|}{ 2540E Average } & 1.1557 & 1.1557 & 1.1557 \\
\hline $\mathrm{AL}$ & Bad & $2722 \mathrm{E}$ & 1 & 1.21 & 1.21 & 1.21 \\
\hline $\mathrm{AL}$ & $\mathrm{Bad}$ & $2722 \mathrm{E}$ & 2 & 2.69 & 2.69 & 2.69 \\
\hline $\mathrm{AL}$ & Bad & $2722 \mathrm{E}$ & 4 & 2.06 & 2.06 & 2.06 \\
\hline $\mathrm{AL}$ & Bad & $2722 \mathrm{E}$ & 5 & 1.44 & 1.44 & 1.44 \\
\hline \multirow[t]{2}{*}{$\mathrm{AL}$} & $\mathrm{Bad}$ & $2722 \mathrm{E}$ & 6 & 1.58 & 1.58 & 1.58 \\
\hline & \multicolumn{3}{|c|}{ 2722E Average } & 1.7960 & 1.7960 & 1.7960 \\
\hline $\mathrm{AL}$ & $\mathrm{Bad}$ & $2756 \mathrm{E}$ & 1 & 0.9 & 0.9 & 0.9 \\
\hline $\mathrm{AL}$ & $\mathrm{Bad}$ & $2756 \mathrm{E}$ & 2 & 0.47 & 0.47 & 0.47 \\
\hline $\mathrm{AL}$ & $\mathrm{Bad}$ & $2756 \mathrm{E}$ & 3 & 0.79 & 0.79 & 0.79 \\
\hline $\mathrm{AL}$ & $\mathrm{Bad}$ & $2756 \mathrm{E}$ & 4 & 2.35 & 2.35 & 2.35 \\
\hline $\mathrm{AL}$ & $\mathrm{Bad}$ & $2756 \mathrm{E}$ & 5 & 2.13 & 2.13 & 2.13 \\
\hline $\mathrm{AL}$ & $\mathrm{Bad}$ & $2756 \mathrm{E}$ & 6 & 1.68 & 1.68 & 1.68 \\
\hline \multirow[t]{2}{*}{$\mathrm{AL}$} & $\mathrm{Bad}$ & $2756 \mathrm{E}$ & 8 & 1.21 & 1.21 & 1.21 \\
\hline & \multicolumn{3}{|c|}{ 2756E Average } & 1.3614 & 1.3614 & 1.3614 \\
\hline $\mathrm{AL}$ & $\mathrm{Bad}$ & $3128 \mathrm{~W}$ & 2 & 1.12 & 1.12 & 1.12 \\
\hline $\mathrm{AL}$ & $\mathrm{Bad}$ & $3128 \mathrm{~W}$ & 3 & 1.18 & 1.18 & 1.18 \\
\hline $\mathrm{AL}$ & $\mathrm{Bad}$ & $3128 \mathrm{~W}$ & 4 & 3.32 & 3.32 & 3.32 \\
\hline $\mathrm{AL}$ & $\mathrm{Bad}$ & $3128 W$ & 5 & 6.31 & 6.31 & 6.31 \\
\hline \multirow[t]{2}{*}{$\mathrm{AL}$} & $\mathrm{Bad}$ & $3128 \mathrm{~W}$ & 9 & 4.79 & 4.79 & 4.79 \\
\hline & \multicolumn{3}{|c|}{ 3128W Average } & 3.3440 & 3.3440 & 3.3440 \\
\hline $\mathrm{AL}$ & $\mathrm{Bad}$ & $3971 \mathrm{~W}$ & 1 & 1.08 & 1.08 & 1.08 \\
\hline $\mathrm{AL}$ & $\mathrm{Bad}$ & $3971 \mathrm{~W}$ & 2 & 1.2 & 1.2 & 1.2 \\
\hline $\mathrm{AL}$ & $\mathrm{Bad}$ & $3971 \mathrm{~W}$ & 3 & 1.31 & 1.31 & 1.31 \\
\hline
\end{tabular}


SNF-10293, REV 0

Canister Sludge Depth Measurements. (14 sheets)

\begin{tabular}{|c|c|c|c|c|c|c|}
\hline \multirow{2}{*}{$\begin{array}{c}\text { Canister } \\
\text { Metal }\end{array}$} & \multirow[b]{2}{*}{ Fuel Type } & \multirow{2}{*}{$\begin{array}{c}\text { Barrel } \\
\text { Location }\end{array}$} & \multirow{2}{*}{$\begin{array}{c}\text { Position in } \\
\text { Barrel }\end{array}$} & \multicolumn{3}{|c|}{ Canister Sludge Depth (inch) } \\
\hline & & & & Data & Mean $=0$ & Negative $=0$ \\
\hline $\mathrm{AL}$ & $\mathrm{Bad}$ & $3971 \mathrm{~W}$ & 4 & 0.81 & 0.81 & 0.81 \\
\hline $\mathrm{AL}$ & $\mathrm{Bad}$ & $3971 \mathrm{~W}$ & 5 & 1.18 & 1.18 & 1.18 \\
\hline \multirow[t]{2}{*}{$\mathrm{AL}$} & $\mathrm{Bad}$ & $3971 \mathrm{~W}$ & 6 & 1.88 & 1.88 & 1.88 \\
\hline & \multicolumn{3}{|c|}{ 3971W Average } & 1.2433 & 1.2433 & 1.2433 \\
\hline $\mathrm{AL}$ & $\mathrm{Bad}$ & $4143 \mathrm{~W}$ & 1 & 1.25 & 1.25 & 1.25 \\
\hline $\mathrm{AL}$ & $\mathrm{Bad}$ & $4143 \mathrm{~W}$ & 2 & 1.45 & 1.45 & 1.45 \\
\hline $\mathrm{AL}$ & $\mathrm{Bad}$ & $4143 \mathrm{~W}$ & 3 & 1.7 & 1.7 & 1.7 \\
\hline $\mathrm{AL}$ & $\mathrm{Bad}$ & $4143 \mathrm{~W}$ & 4 & 1.7 & 1.7 & 1.7 \\
\hline $\mathrm{AL}$ & $\mathrm{Bad}$ & $4143 \mathrm{~W}$ & 5 & 5.74 & 5.74 & 5.74 \\
\hline \multirow[t]{2}{*}{$\mathrm{AL}$} & $\mathrm{Bad}$ & $4143 \mathrm{~W}$ & 8 & 1.92 & 1.92 & 1.92 \\
\hline & \multicolumn{3}{|c|}{ 4143W Average } & 2.2933 & 2.2933 & 2.2933 \\
\hline $\mathrm{AL}$ & Bad & $4564 \mathrm{E}$ & 1 & 1.74 & 1.74 & 1.74 \\
\hline \multirow[t]{2}{*}{$\mathrm{AL}$} & Bad & $4564 \mathrm{E}$ & 2 & 2.28 & 2.28 & 2.28 \\
\hline & \multicolumn{3}{|c|}{ 4564E Average } & 2.0100 & 2.0100 & 2.0100 \\
\hline $\mathrm{AL}$ & $\mathrm{Bad}$ & $4564 W$ & 1 & 2.08 & 2.08 & 2.08 \\
\hline $\mathrm{AL}$ & $\mathrm{Bad}$ & $4564 \mathrm{~W}$ & 2 & 7.46 & 7.46 & 7.46 \\
\hline \multirow[t]{2}{*}{$\mathrm{AL}$} & $\mathrm{Bad}$ & $4564 \mathrm{~W}$ & 5 & 8.24 & 8.24 & 8.24 \\
\hline & \multicolumn{3}{|c|}{ 4564W Average } & 5.9267 & 5.9267 & 5.9267 \\
\hline \multirow[t]{2}{*}{$\mathrm{AL}$} & $\mathrm{Bad}$ & $4569 \mathrm{E}$ & 3 & 3.42 & 3.42 & 3.42 \\
\hline & \multicolumn{3}{|c|}{ 4569E Average } & 3.4200 & 3.4200 & 3.4200 \\
\hline \multirow[t]{2}{*}{$\mathrm{AL}$} & $\mathrm{Bad}$ & $4569 \mathrm{~W}$ & 3 & 10.59 & 10.59 & 10.59 \\
\hline & \multicolumn{3}{|c|}{ 4569W Average } & 10.5900 & 10.5900 & 10.5900 \\
\hline $\mathrm{AL}$ & $\mathrm{Bad}$ & $4571 \mathrm{E}$ & 2 & 9.39 & 9.39 & 9.39 \\
\hline $\mathrm{AL}$ & $\mathrm{Bad}$ & $4571 \mathrm{E}$ & 3 & 8.46 & 8.46 & 8.46 \\
\hline $\mathrm{AL}$ & Bad & $4571 \mathrm{E}$ & 4 & 5 & 5 & 5 \\
\hline $\mathrm{AL}$ & $\mathrm{Bad}$ & $4571 \mathrm{E}$ & 5 & 7.82 & 7.82 & 7.82 \\
\hline \multirow[t]{2}{*}{$\mathrm{AL}$} & $\mathrm{Bad}$ & $4571 \mathrm{E}$ & 6 & 6.4 & 6.4 & 6.4 \\
\hline & \multicolumn{3}{|c|}{ 4571E Average } & 7.4140 & 7.4140 & 7.4140 \\
\hline $\mathrm{AL}$ & $\mathrm{Bad}$ & $4850 \mathrm{~W}$ & 1 & 2.99 & 2.99 & 2.99 \\
\hline $\mathrm{AL}$ & $\mathrm{Bad}$ & $4850 \mathrm{~W}$ & 2 & 3.84 & 3.84 & 3.84 \\
\hline $\mathrm{AL}$ & $\mathrm{Bad}$ & $4850 \mathrm{~W}$ & 3 & 2.56 & 2.56 & 2.56 \\
\hline $\mathrm{AL}$ & $\mathrm{Bad}$ & $4850 \mathrm{~W}$ & 4 & 1.32 & 1.32 & 1.32 \\
\hline
\end{tabular}


SNF-10293, REV 0

Canister Sludge Depth Measurements. (14 sheets)

\begin{tabular}{|c|c|c|c|c|c|c|}
\hline \multirow{2}{*}{$\begin{array}{c}\text { Canister } \\
\text { Metal }\end{array}$} & \multirow[b]{2}{*}{ Fuel Type } & \multirow{2}{*}{$\begin{array}{c}\text { Barrel } \\
\text { Location }\end{array}$} & \multirow{2}{*}{$\begin{array}{c}\text { Position in } \\
\text { Barrel }\end{array}$} & \multicolumn{3}{|c|}{ Canister Sludge Depth (inch) } \\
\hline & & & & Data & Mean $=0$ & Negative $=0$ \\
\hline $\mathrm{AL}$ & $\mathrm{Bad}$ & $4850 \mathrm{~W}$ & 5 & 1.44 & 1.44 & 1.44 \\
\hline $\mathrm{AL}$ & $\mathrm{Bad}$ & 4850W & 6 & 1.4 & 1.4 & 1.4 \\
\hline $\mathrm{AL}$ & $\mathrm{Bad}$ & $4850 \mathrm{~W}$ & 11 & 1.74 & 1.74 & 1.74 \\
\hline \multirow[t]{2}{*}{$\mathrm{AL}$} & $\mathrm{Bad}$ & $4850 \mathrm{~W}$ & 12 & 1.49 & 1.49 & 1.49 \\
\hline & \multicolumn{3}{|c|}{ 4850W Average } & 2.0975 & 2.0975 & 2.0975 \\
\hline \multirow[t]{2}{*}{$\mathrm{AL}$} & $\mathrm{Bad}$ & $5043 \mathrm{~W}$ & 2 & 17.0 & 17.0 & 17.0 \\
\hline & \multicolumn{3}{|c|}{ 5043W Average } & 17.000 & 17.000 & 17.000 \\
\hline $\mathrm{AL}$ & $\mathrm{Bad}$ & $5055 \mathrm{~W}$ & 1 & 7.1 & 7.1 & 7.1 \\
\hline $\mathrm{AL}$ & Bad & $5055 \mathrm{~W}$ & 2 & 3.78 & 3.78 & 3.78 \\
\hline $\mathrm{AL}$ & $\mathrm{Bad}$ & $5055 \mathrm{~W}$ & 3 & 3.66 & 3.66 & 3.66 \\
\hline $\mathrm{AL}$ & $\mathrm{Bad}$ & $5055 \mathrm{~W}$ & 4 & 2.54 & 2.54 & 2.54 \\
\hline $\mathrm{AL}$ & Bad & $5055 \mathrm{~W}$ & 5 & 6.14 & 6.14 & 6.14 \\
\hline $\mathrm{AL}$ & $\mathrm{Bad}$ & $5055 \mathrm{~W}$ & 6 & 7.5 & 7.5 & 7.5 \\
\hline $\mathrm{AL}$ & $\mathrm{Bad}$ & $5055 \mathrm{~W}$ & 9 & 7.29 & 7.29 & 7.29 \\
\hline \multirow[t]{2}{*}{$\mathrm{AL}$} & $\mathrm{Bad}$ & $5055 \mathrm{~W}$ & 10 & 7.25 & 7.25 & 7.25 \\
\hline & \multicolumn{3}{|c|}{ 5055W Average } & 5.6575 & 5.6575 & 5.6575 \\
\hline $\mathrm{AL}$ & $\mathrm{Bad}$ & $5427 \mathrm{~W}$ & 1 & 6.7 & 6.7 & 6.7 \\
\hline $\mathrm{AL}$ & $\mathrm{Bad}$ & $5427 \mathrm{~W}$ & 2 & 5.78 & 5.78 & 5.78 \\
\hline $\mathrm{AL}$ & $\mathrm{Bad}$ & $5427 \mathrm{~W}$ & 3 & 6.97 & 6.97 & 6.97 \\
\hline $\mathrm{AL}$ & $\mathrm{Bad}$ & $5427 \mathrm{~W}$ & 4 & 0.91 & 0.91 & 0.91 \\
\hline $\mathrm{AL}$ & $\mathrm{Bad}$ & $5427 \mathrm{~W}$ & 6 & 0.93 & 0.93 & 0.93 \\
\hline $\mathrm{AL}$ & $\mathrm{Bad}$ & $5427 \mathrm{~W}$ & 10 & 0.68 & 0.68 & 0.68 \\
\hline \multirow[t]{2}{*}{$\mathrm{AL}$} & $\mathrm{Bad}$ & $5427 \mathrm{~W}$ & 12 & 0.74 & 0.74 & 0.74 \\
\hline & \multicolumn{3}{|c|}{ 5427W Average } & 3.2443 & 3.2443 & 3.2443 \\
\hline $\mathrm{AL}$ & Bad & $5445 \mathrm{E}$ & 1 & 3.75 & 3.75 & 3.75 \\
\hline $\mathrm{AL}$ & $\mathrm{Bad}$ & $5445 \mathrm{E}$ & 2 & 8.45 & 8.45 & 8.45 \\
\hline $\mathrm{AL}$ & $\mathrm{Bad}$ & $5445 \mathrm{E}$ & 3 & 11.74 & 11.74 & 11.74 \\
\hline \multirow[t]{2}{*}{$\mathrm{AL}$} & $\mathrm{Bad}$ & $5445 \mathrm{E}$ & 4 & 6.15 & 6.15 & 6.15 \\
\hline & \multicolumn{3}{|c|}{ 5445E Average } & 7.5225 & 7.5225 & 7.5225 \\
\hline \multirow[t]{2}{*}{$\mathrm{AL}$} & $\mathrm{Bad}$ & $5465 \mathrm{E}$ & 2 & 6.84 & 6.84 & 6.84 \\
\hline & \multicolumn{3}{|c|}{ 5465E Average } & 6.8400 & 6.8400 & 6.8400 \\
\hline $\mathrm{AL}$ & $\mathrm{Bad}$ & $5465 \mathrm{~W}$ & 1 & 10.03 & 10.03 & 10.03 \\
\hline
\end{tabular}


SNF-10293, REV 0

Canister Sludge Depth Measurements. (14 sheets)

\begin{tabular}{|c|c|c|c|c|c|c|}
\hline \multirow{2}{*}{$\begin{array}{l}\text { Canister } \\
\text { Metal }\end{array}$} & \multirow[b]{2}{*}{ Fuel Type } & \multirow{2}{*}{$\begin{array}{c}\text { Barrel } \\
\text { Location }\end{array}$} & \multirow{2}{*}{$\begin{array}{c}\text { Position in } \\
\text { Barrel }\end{array}$} & \multicolumn{3}{|c|}{ Canister Sludge Depth (inch) } \\
\hline & & & & Data & Mean $=0$ & Negative $=0$ \\
\hline $\mathrm{AL}$ & $\mathrm{Bad}$ & $5465 \mathrm{~W}$ & 3 & 8.79 & 8.79 & 8.79 \\
\hline $\mathrm{AL}$ & $\mathrm{Bad}$ & $5465 \mathrm{~W}$ & 5 & 9.27 & 9.27 & 9.27 \\
\hline $\mathrm{AL}$ & Bad & $5465 \mathrm{~W}$ & 6 & 9.59 & 9.59 & 9.59 \\
\hline \multirow[t]{2}{*}{$\mathrm{AL}$} & Bad & $5465 \mathrm{~W}$ & 7 & 5.09 & 5.09 & 5.09 \\
\hline & \multicolumn{3}{|c|}{ 5465W Average } & 8.5540 & 8.5540 & 8.5540 \\
\hline \multirow[t]{2}{*}{$\mathrm{AL}$} & $\mathrm{Bad}$ & 6070W & & 18 & 18 & 18 \\
\hline & \multicolumn{3}{|c|}{ 6070W Average } & 18.000 & 18.000 & 18.000 \\
\hline AL & $\mathrm{Bad}$ & $6071 \mathrm{~W}$ & 1 & 11.12 & 11.12 & 11.12 \\
\hline AL & Bad & $6071 \mathrm{~W}$ & 2 & 8.68 & 8.68 & 8.68 \\
\hline AL & Bad & $6071 \mathrm{~W}$ & 2 & 7.8 & 7.8 & 7.8 \\
\hline $\mathrm{AL}$ & $\mathrm{Bad}$ & $6071 \mathrm{~W}$ & 3 & 5.26 & 5.26 & 5.26 \\
\hline $\mathrm{AL}$ & $\mathrm{Bad}$ & $6071 \mathrm{~W}$ & 3 & 10.69 & 10.69 & 10.69 \\
\hline \multirow[t]{2}{*}{$\mathrm{AL}$} & $\mathrm{Bad}$ & $6071 \mathrm{~W}$ & 4 & 8.91 & 8.91 & 8.91 \\
\hline & \multicolumn{3}{|c|}{ 6071W Average } & 8.7433 & 8.7433 & 8.7433 \\
\hline $\mathrm{AL}$ & Fair & $0450 \mathrm{E}$ & 1 & 1.69 & 1.69 & 1.69 \\
\hline $\mathrm{AL}$ & Fair & $0450 \mathrm{E}$ & 2 & 1.11 & 1.11 & 1.11 \\
\hline $\mathrm{AL}$ & Fair & $0450 \mathrm{E}$ & 3 & 2.68 & 2.68 & 2.68 \\
\hline $\mathrm{AL}$ & Fair & $0450 \mathrm{E}$ & 4 & 4.5 & 4.5 & 4.5 \\
\hline $\mathrm{AL}$ & Fair & 0450E & 5 & 4.05 & 4.05 & 4.05 \\
\hline \multirow[t]{2}{*}{$\mathrm{AL}$} & Fair & 0450E & 6 & 3.55 & 3.55 & 3.55 \\
\hline & \multicolumn{3}{|c|}{ 0450E Average } & 2.9300 & 2.9300 & 2.9300 \\
\hline $\mathrm{AL}$ & Fair & $1858 \mathrm{E}$ & 1 & 0.63 & 0.63 & 0.63 \\
\hline $\mathrm{AL}$ & Fair & $1858 \mathrm{E}$ & 2 & 0.03 & 0.03 & 0.03 \\
\hline AL & Fair & $1858 \mathrm{E}$ & 4 & 1.14 & 1.14 & 1.14 \\
\hline $\mathrm{AL}$ & Fair & $1858 \mathrm{E}$ & 5 & 2.42 & 2.42 & 2.42 \\
\hline \multirow[t]{2}{*}{$\mathrm{AL}$} & Fair & $1858 \mathrm{E}$ & 9 & -0.16 & -0.16 & 0 \\
\hline & \multicolumn{3}{|c|}{ 1858E Average } & 0.8120 & 0.8120 & 0.8440 \\
\hline $\mathrm{AL}$ & Fair & $2210 \mathrm{E}$ & 1 & 0.55 & 0.55 & 0.55 \\
\hline $\mathrm{AL}$ & Fair & $2210 \mathrm{E}$ & 2 & 0.78 & 0.78 & 0.78 \\
\hline $\mathrm{AL}$ & Fair & $2210 \mathrm{E}$ & 3 & 0.89 & 0.89 & 0.89 \\
\hline $\mathrm{AL}$ & Fair & $2210 \mathrm{E}$ & 4 & 1.66 & 1.66 & 1.66 \\
\hline $\mathrm{AL}$ & Fair & $2210 \mathrm{E}$ & 5 & 1.87 & 1.87 & 1.87 \\
\hline
\end{tabular}


SNF-10293, REV 0

Canister Sludge Depth Measurements. (14 sheets)

\begin{tabular}{|c|c|c|c|c|c|c|}
\hline \multirow{2}{*}{$\begin{array}{c}\text { Canister } \\
\text { Metal }\end{array}$} & \multirow[b]{2}{*}{ Fuel Type } & \multirow{2}{*}{$\begin{array}{c}\text { Barrel } \\
\text { Location }\end{array}$} & \multirow{2}{*}{$\begin{array}{c}\text { Position in } \\
\text { Barrel }\end{array}$} & \multicolumn{3}{|c|}{ Canister Sludge Depth (inch) } \\
\hline & & & & Data & Mean $=0$ & Negative $=0$ \\
\hline $\mathrm{AL}$ & Fair & $2210 \mathrm{E}$ & 6 & 1.05 & 1.05 & 1.05 \\
\hline $\mathrm{AL}$ & Fair & $2210 \mathrm{E}$ & 8 & 0.65 & 0.65 & 0.65 \\
\hline \multicolumn{4}{|c|}{ 2210E Average } & 1.0643 & 1.0643 & 1.0643 \\
\hline $\mathrm{AL}$ & Fair & $2353 \mathrm{E}$ & 1 & 1.09 & 1.09 & 1.09 \\
\hline $\mathrm{AL}$ & Fair & $2353 \mathrm{E}$ & 2 & 1.02 & 1.02 & 1.02 \\
\hline $\mathrm{AL}$ & Fair & $2353 \mathrm{E}$ & 4 & 1.57 & 1.57 & 1.57 \\
\hline $\mathrm{AL}$ & Fair & $2353 \mathrm{E}$ & 5 & 1.35 & 1.35 & 1.35 \\
\hline $\mathrm{AL}$ & Fair & $2353 \mathrm{E}$ & 6 & 1.16 & 1.16 & 1.16 \\
\hline $\mathrm{AL}$ & Fair & $2353 \mathrm{E}$ & 10 & 3.12 & 3.12 & 3.12 \\
\hline $\mathrm{AL}$ & Fair & $2353 \mathrm{E}$ & 11 & 3.25 & 3.25 & 3.25 \\
\hline \multicolumn{4}{|c|}{ 2353E Average } & 1.7943 & 1.7943 & 1.7943 \\
\hline $\mathrm{AL}$ & Fair & $2711 \mathrm{E}$ & 1 & 0.31 & 0.31 & 0.31 \\
\hline $\mathrm{AL}$ & Fair & $2711 \mathrm{E}$ & 2 & 0.58 & 0.58 & 0.58 \\
\hline $\mathrm{AL}$ & Fair & $2711 \mathrm{E}$ & 3 & 0.54 & 0.54 & 0.54 \\
\hline $\mathrm{AL}$ & Fair & $2711 \mathrm{E}$ & 4 & 2.44 & 2.44 & 2.44 \\
\hline $\mathrm{AL}$ & Fair & $2711 \mathrm{E}$ & 5 & 3.9 & 3.9 & 3.9 \\
\hline $\mathrm{AL}$ & Fair & $2711 \mathrm{E}$ & 6 & 2.41 & 2.41 & 2.41 \\
\hline \multicolumn{4}{|c|}{ 2711E Average } & 1.6967 & 1.6967 & 1.6967 \\
\hline $\mathrm{AL}$ & Fair & $2905 \mathrm{E}$ & 2 & 1.83 & 1.83 & 1.83 \\
\hline $\mathrm{AL}$ & Fair & $2905 \mathrm{E}$ & 3 & 1.62 & 1.62 & 1.62 \\
\hline $\mathrm{AL}$ & Fair & $2905 \mathrm{E}$ & 4 & 1.9 & 1.9 & 1.9 \\
\hline $\mathrm{AL}$ & Fair & $2905 \mathrm{E}$ & 5 & 0.74 & 0.74 & 0.74 \\
\hline $\mathrm{AL}$ & Fair & $2905 \mathrm{E}$ & 6 & 2.16 & 2.16 & 2.16 \\
\hline \multicolumn{4}{|c|}{ 2905E Average } & 1.6500 & 1.6500 & 1.6500 \\
\hline $\mathrm{AL}$ & Fair & $3727 \mathrm{~W}$ & 1 & 4.06 & 4.06 & 4.06 \\
\hline $\mathrm{AL}$ & Fair & $3727 \mathrm{~W}$ & 2 & 7.2 & 7.2 & 7.2 \\
\hline $\mathrm{AL}$ & Fair & $3727 \mathrm{~W}$ & 3 & 2.63 & 2.63 & 2.63 \\
\hline $\mathrm{AL}$ & Fair & $3727 \mathrm{~W}$ & 4 & 2.17 & 2.17 & 2.17 \\
\hline $\mathrm{AL}$ & Fair & $3727 \mathrm{~W}$ & 5 & 1.64 & 1.64 & 1.64 \\
\hline $\mathrm{AL}$ & Fair & $3727 \mathrm{~W}$ & 6 & 1.69 & 1.69 & 1.69 \\
\hline $\mathrm{AL}$ & Fair & $3727 \mathrm{~W}$ & 11 & 1.77 & 1.77 & 1.77 \\
\hline \multicolumn{4}{|c|}{ 3727W Average } & 3.0229 & 3.0229 & 3.0229 \\
\hline
\end{tabular}

D-8 
SNF-10293, REV 0

Canister Sludge Depth Measurements. (14 sheets)

\begin{tabular}{|c|c|c|c|c|c|c|}
\hline \multirow{2}{*}{$\begin{array}{c}\text { Canister } \\
\text { Metal }\end{array}$} & \multirow[b]{2}{*}{ Fuel Type } & \multirow{2}{*}{$\begin{array}{c}\text { Barrel } \\
\text { Location }\end{array}$} & \multirow{2}{*}{$\begin{array}{c}\text { Position in } \\
\text { Barrel }\end{array}$} & \multicolumn{3}{|c|}{ Canister Sludge Depth (inch) } \\
\hline & & & & Data & Mean $=0$ & Negative $=0$ \\
\hline $\mathrm{AL}$ & Fair & $4138 \mathrm{~W}$ & 2 & 4.21 & 4.21 & 4.21 \\
\hline $\mathrm{AL}$ & Fair & 4138W & 3 & 2.69 & 2.69 & 2.69 \\
\hline $\mathrm{AL}$ & Fair & 4138W & 4 & 1.98 & 1.98 & 1.98 \\
\hline $\mathrm{AL}$ & Fair & $4138 \mathrm{~W}$ & 5 & 3.18 & 3.18 & 3.18 \\
\hline $\mathrm{AL}$ & Fair & 4138W & 6 & 3.61 & 3.61 & 3.61 \\
\hline AL & Fair & 4138W & 7 & 3.46 & 3.46 & 3.46 \\
\hline AL & Fair & $4138 W$ & 8 & 4.08 & 4.08 & 4.08 \\
\hline \multicolumn{4}{|c|}{ 4138W Average } & 3.3157 & 3.3157 & 3.3157 \\
\hline AL & Fair & $4168 \mathrm{E}$ & 3 & 0.48 & 0.48 & 0.48 \\
\hline $\mathrm{AL}$ & Fair & $4168 \mathrm{E}$ & 4 & 0.21 & 0.21 & 0.21 \\
\hline $\mathrm{AL}$ & Fair & $4168 \mathrm{E}$ & 6 & 0.44 & 0.44 & 0.44 \\
\hline \multicolumn{4}{|c|}{ 4168E Average } & 0.3767 & 0.3767 & 0.3767 \\
\hline $\mathrm{AL}$ & Fair & 4168W & 1 & 0.48 & 0.48 & 0.48 \\
\hline $\mathrm{AL}$ & Fair & $4168 \mathrm{~W}$ & 3 & 0.51 & 0.51 & 0.51 \\
\hline AL & Fair & $4168 W$ & 6 & 0.29 & 0.29 & 0.29 \\
\hline \multicolumn{4}{|c|}{ 4168W Average } & 0.4267 & 0.4267 & 0.4267 \\
\hline $\mathrm{AL}$ & Fair & $4620 W$ & 1 & 2.01 & 2.01 & 2.01 \\
\hline $\mathrm{AL}$ & Fair & $4620 \mathrm{~W}$ & 2 & 2.33 & 2.33 & 2.33 \\
\hline $\mathrm{AL}$ & Fair & $4620 \mathrm{~W}$ & 3 & 2.57 & 2.57 & 2.57 \\
\hline AL & Fair & $4620 \mathrm{~W}$ & 4 & 2.91 & 2.91 & 2.91 \\
\hline $\mathrm{AL}$ & Fair & $4620 \mathrm{~W}$ & 5 & 4.53 & 4.53 & 4.53 \\
\hline AL & Fair & $4620 \mathrm{~W}$ & 6 & 2.54 & 2.54 & 2.54 \\
\hline AL & Fair & $4620 \mathrm{~W}$ & 8 & 1.79 & 1.79 & 1.79 \\
\hline \multicolumn{4}{|c|}{ 4620W Average } & 2.6686 & 2.6686 & 2.6686 \\
\hline AL & Fair & $4627 \mathrm{~W}$ & 1 & 2.44 & 2.44 & 2.44 \\
\hline $\mathrm{AL}$ & Fair & $4627 \mathrm{~W}$ & 2 & 2.37 & 2.37 & 2.37 \\
\hline $\mathrm{AL}$ & Fair & $4627 \mathrm{~W}$ & 3 & 1.37 & 1.37 & 1.37 \\
\hline $\mathrm{AL}$ & Fair & $4627 \mathrm{~W}$ & 4 & 0.7 & 0.7 & 0.7 \\
\hline $\mathrm{AL}$ & Fair & $4627 \mathrm{~W}$ & 5 & 0.68 & 0.68 & 0.68 \\
\hline $\mathrm{AL}$ & Fair & $4627 \mathrm{~W}$ & 6 & 0.96 & 0.96 & 0.96 \\
\hline $\mathrm{AL}$ & Fair & $4627 \mathrm{~W}$ & 9 & 1.23 & 1.23 & 1.23 \\
\hline AL & Fair & $4627 \mathrm{~W}$ & 10 & 1.04 & 1.04 & 1.04 \\
\hline
\end{tabular}


SNF-10293, REV 0

Canister Sludge Depth Measurements. (14 sheets)

\begin{tabular}{|c|c|c|c|c|c|c|}
\hline \multirow{2}{*}{$\begin{array}{c}\text { Canister } \\
\text { Metal }\end{array}$} & \multirow[b]{2}{*}{ Fuel Type } & \multirow{2}{*}{$\begin{array}{c}\text { Barrel } \\
\text { Location }\end{array}$} & \multirow{2}{*}{$\begin{array}{c}\text { Position in } \\
\text { Barrel }\end{array}$} & \multicolumn{3}{|c|}{ Canister Sludge Depth (inch) } \\
\hline & & & & Data & Mean $=0$ & Negative $=0$ \\
\hline AL & Fair & $4627 \mathrm{~W}$ & 11 & 0.96 & 0.96 & 0.96 \\
\hline \multicolumn{4}{|c|}{ 4627W Average } & 1.3056 & 1.3056 & 1.3056 \\
\hline $\mathrm{AL}$ & Fair & $4865 \mathrm{~W}$ & 1 & 1.21 & 1.21 & 1.21 \\
\hline $\mathrm{AL}$ & Fair & $4865 \mathrm{~W}$ & 2 & 1.36 & 1.36 & 1.36 \\
\hline $\mathrm{AL}$ & Fair & $4865 \mathrm{~W}$ & 3 & 1.62 & 1.62 & 1.62 \\
\hline AL & Fair & $4865 \mathrm{~W}$ & 4 & 3.08 & 3.08 & 3.08 \\
\hline AL & Fair & $4865 \mathrm{~W}$ & 5 & 2.92 & 2.92 & 2.92 \\
\hline AL & Fair & $4865 \mathrm{~W}$ & 6 & 2.87 & 2.87 & 2.87 \\
\hline AL & Fair & $4865 \mathrm{~W}$ & 8 & 1.95 & 1.95 & 1.95 \\
\hline $\mathrm{AL}$ & Fair & $4865 \mathrm{~W}$ & 9 & 1.37 & 1.37 & 1.37 \\
\hline \multicolumn{4}{|c|}{ 4865W Average } & 2.0475 & 2.0475 & 2.0475 \\
\hline $\mathrm{AL}$ & Fair & 4869E & 1 & 2.26 & 2.26 & 2.26 \\
\hline $\mathrm{AL}$ & Fair & $4869 \mathrm{E}$ & 2 & 2.14 & 2.14 & 2.14 \\
\hline $\mathrm{AL}$ & Fair & $4869 \mathrm{E}$ & 3 & 1.56 & 1.56 & 1.56 \\
\hline $\mathrm{AL}$ & Fair & $4869 \mathrm{E}$ & 4 & 2.94 & 2.94 & 2.94 \\
\hline $\mathrm{AL}$ & Fair & $4869 \mathrm{E}$ & 5 & 3.63 & 3.63 & 3.63 \\
\hline $\mathrm{AL}$ & Fair & $4869 \mathrm{E}$ & 6 & 2.98 & 2.98 & 2.98 \\
\hline \multicolumn{4}{|c|}{ 4869E Average } & 2.5850 & 2.5850 & 2.5850 \\
\hline $\mathrm{AL}$ & Fair & $5069 \mathrm{E}$ & 1 & 4.16 & 4.16 & 4.16 \\
\hline $\mathrm{AL}$ & Fair & $5069 \mathrm{E}$ & 2 & 2.92 & 2.92 & 2.92 \\
\hline $\mathrm{AL}$ & Fair & $5069 \mathrm{E}$ & 3 & 4.06 & 4.06 & 4.06 \\
\hline $\mathrm{AL}$ & Fair & $5069 \mathrm{E}$ & 4 & 4.81 & 4.81 & 4.81 \\
\hline $\mathrm{AL}$ & Fair & $5069 \mathrm{E}$ & 5 & 1.12 & 1.12 & 1.12 \\
\hline AL & Fair & $5069 \mathrm{E}$ & 6 & 1.49 & 1.49 & 1.49 \\
\hline AL & Fair & $5069 \mathrm{E}$ & 9 & 0.89 & 0.89 & 0.89 \\
\hline $\mathrm{AL}$ & Fair & $5069 \mathrm{E}$ & 10 & 2.49 & 2.49 & 2.49 \\
\hline \multicolumn{4}{|c|}{ 5069E Average } & 2.7425 & 2.7425 & 2.7425 \\
\hline $\mathrm{AL}$ & Fair & $5254 \mathrm{E}$ & 2 & 0.82 & 0.82 & 0.82 \\
\hline $\mathrm{AL}$ & Fair & $5254 \mathrm{E}$ & 3 & 2.22 & 2.22 & 2.22 \\
\hline $\mathrm{AL}$ & Fair & $5254 \mathrm{E}$ & 4 & 0.98 & 0.98 & 0.98 \\
\hline $\mathrm{AL}$ & Fair & $5254 \mathrm{E}$ & 5 & 1.19 & 1.19 & 1.19 \\
\hline $\mathrm{AL}$ & Fair & $5254 \mathrm{E}$ & 6 & 1.5 & 1.5 & 1.5 \\
\hline
\end{tabular}


SNF-10293, REV 0

Canister Sludge Depth Measurements. (14 sheets)

\begin{tabular}{|c|c|c|c|c|c|c|}
\hline \multirow{2}{*}{$\begin{array}{c}\text { Canister } \\
\text { Metal }\end{array}$} & \multirow[b]{2}{*}{ Fuel Type } & \multirow{2}{*}{$\begin{array}{c}\text { Barrel } \\
\text { Location }\end{array}$} & \multirow{2}{*}{$\begin{array}{c}\text { Position in } \\
\text { Barrel }\end{array}$} & \multicolumn{3}{|c|}{ Canister Sludge Depth (inch) } \\
\hline & & & & Data & Mean $=0$ & Negative $=0$ \\
\hline \multirow[t]{2}{*}{$\mathrm{AL}$} & Fair & $5254 \mathrm{E}$ & 10 & 1.22 & 1.22 & 1.22 \\
\hline & \multicolumn{3}{|c|}{ 5254E Average } & 1.3217 & 1.3217 & 1.3217 \\
\hline $\mathrm{AL}$ & Fair & $5427 \mathrm{E}$ & 1 & 1.81 & 1.81 & 1.81 \\
\hline $\mathrm{AL}$ & Fair & $5427 \mathrm{E}$ & 2 & 1.87 & 1.87 & 1.87 \\
\hline $\mathrm{AL}$ & Fair & $5427 \mathrm{E}$ & 3 & 2.24 & 2.24 & 2.24 \\
\hline $\mathrm{AL}$ & Fair & $5427 \mathrm{E}$ & 4 & 1.32 & 1.32 & 1.32 \\
\hline $\mathrm{AL}$ & Fair & $5427 \mathrm{E}$ & 5 & 0.74 & 0.74 & 0.74 \\
\hline $\mathrm{AL}$ & Fair & $5427 \mathrm{E}$ & 6 & 1.41 & 1.41 & 1.41 \\
\hline $\mathrm{AL}$ & Fair & $5427 \mathrm{E}$ & 10 & 1.67 & 1.67 & 1.67 \\
\hline \multicolumn{4}{|c|}{ 5427E Average } & 1.5800 & 1.5800 & 1.5800 \\
\hline $\mathrm{AL}$ & Fair & $6069 \mathrm{~W}$ & 13 & 0.07 & 0.07 & 0.07 \\
\hline \multicolumn{4}{|c|}{ 6069W Average } & 0.0700 & 0.0700 & 0.0700 \\
\hline SS & Fair & $0418 \mathrm{E}$ & 1 & 0.74 & 0.74 & 0.74 \\
\hline $\mathrm{SS}$ & Fair & $0418 \mathrm{E}$ & 2 & 0.63 & 0.63 & 0.63 \\
\hline SS & Fair & $0418 \mathrm{E}$ & 3 & 1.14 & 1.14 & 1.14 \\
\hline SS & Fair & $0418 \mathrm{E}$ & 4 & 0.74 & 0.74 & 0.74 \\
\hline SS & Fair & $0418 \mathrm{E}$ & 5 & 0.43 & 0.43 & 0.43 \\
\hline $\mathrm{SS}$ & Fair & $0418 \mathrm{E}$ & 6 & 0.55 & 0.55 & 0.55 \\
\hline $\mathrm{SS}$ & Fair & $0418 \mathrm{E}$ & 10 & 1.14 & 1.14 & 1.14 \\
\hline $\mathrm{SS}$ & Fair & $0418 \mathrm{E}$ & 11 & 0.22 & 0.22 & 0.22 \\
\hline $\mathrm{SS}$ & Fair & $0418 \mathrm{E}$ & 12 & 0.34 & 0.34 & 0.34 \\
\hline \multicolumn{4}{|c|}{ 0418E Average } & 0.6589 & 0.6589 & 0.6589 \\
\hline SS & Fair & $3125 \mathrm{~W}$ & 1 & 1.09 & 1.09 & 1.09 \\
\hline SS & Fair & $3125 \mathrm{~W}$ & 2 & 1.48 & 1.48 & 1.48 \\
\hline SS & Fair & $3125 \mathrm{~W}$ & 3 & 1.46 & 1.46 & 1.46 \\
\hline $\mathrm{SS}$ & Fair & $3125 \mathrm{~W}$ & 4 & 1.68 & 1.68 & 1.68 \\
\hline $\mathrm{SS}$ & Fair & $3125 \mathrm{~W}$ & 5 & 1.44 & 1.44 & 1.44 \\
\hline SS & Fair & $3125 \mathrm{~W}$ & 6 & 1.39 & 1.39 & 1.39 \\
\hline \multicolumn{4}{|c|}{ 3125W Average } & 1.4233 & 1.4233 & 1.4233 \\
\hline $\mathrm{SS}$ & Fair & $4359 \mathrm{~W}$ & 1 & 0.25 & 0.25 & 0.25 \\
\hline $\mathrm{SS}$ & Fair & $4359 \mathrm{~W}$ & 3 & 0.4 & 0.4 & 0.4 \\
\hline SS & Fair & 4359W & 4 & -0.11 & -0.11 & 0 \\
\hline
\end{tabular}


SNF-10293, REV 0

Canister Sludge Depth Measurements. (14 sheets)

\begin{tabular}{|c|c|c|c|c|c|c|}
\hline \multirow{2}{*}{$\begin{array}{c}\text { Canister } \\
\text { Metal }\end{array}$} & \multirow[b]{2}{*}{ Fuel Type } & \multirow{2}{*}{$\begin{array}{c}\text { Barrel } \\
\text { Location }\end{array}$} & \multirow{2}{*}{$\begin{array}{c}\text { Position in } \\
\text { Barrel }\end{array}$} & \multicolumn{3}{|c|}{ Canister Sludge Depth (inch) } \\
\hline & & & & Data & Mean $=0$ & Negative $=0$ \\
\hline SS & Fair & $4359 \mathrm{~W}$ & 5 & 0.4 & 0.4 & 0.4 \\
\hline SS & Fair & 4359W & 6 & 0.19 & 0.19 & 0.19 \\
\hline SS & Fair & 4359W & 8 & 0.21 & 0.21 & 0.21 \\
\hline SS & Fair & 4359W & 9 & 0.38 & 0.38 & 0.38 \\
\hline SS & Fair & 4359W & 10 & 0.21 & 0.21 & 0.21 \\
\hline SS & Fair & 4359W & 11 & 0.43 & 0.43 & 0.43 \\
\hline SS & Fair & 4359W & 12 & 0.47 & 0.47 & 0.47 \\
\hline \multicolumn{4}{|c|}{ 4359W Average } & 0.2830 & 0.2830 & 0.2940 \\
\hline SS & Fair & $5238 \mathrm{~W}$ & 1 & 0.06 & 0.06 & 0.06 \\
\hline $\mathrm{SS}$ & Fair & $5238 \mathrm{~W}$ & 2 & 0.72 & 0.72 & 0.72 \\
\hline SS & Fair & $5238 \mathrm{~W}$ & 3 & 0.68 & 0.68 & 0.68 \\
\hline SS & Fair & $5238 \mathrm{~W}$ & 4 & 0.77 & 0.77 & 0.77 \\
\hline SS & Fair & $5238 \mathrm{~W}$ & 5 & 1.1 & 1.1 & 1.1 \\
\hline SS & Fair & $5238 \mathrm{~W}$ & 6 & 1.9 & 1.9 & 1.9 \\
\hline SS & Fair & $5238 \mathrm{~W}$ & 9 & 1.02 & 1.02 & 1.02 \\
\hline SS & Fair & $5238 \mathrm{~W}$ & 10 & 3.7 & 3.7 & 3.7 \\
\hline \multicolumn{4}{|c|}{ 5238W Average } & 1.2438 & 1.2438 & 1.2438 \\
\hline SS & Fair & $5608 \mathrm{E}$ & 1 & -0.32 & -0.2600 & 0 \\
\hline SS & Fair & $5608 \mathrm{E}$ & 2 & -0.06 & 0.0000 & 0 \\
\hline $\mathrm{SS}$ & Fair & $5608 \mathrm{E}$ & 3 & 0 & 0.0600 & 0 \\
\hline SS & Fair & $5608 \mathrm{E}$ & 7 & 0.07 & 0.1300 & 0.07 \\
\hline $\mathrm{SS}$ & Fair & $5608 \mathrm{E}$ & 8 & 0.01 & 0.0700 & 0.01 \\
\hline \multicolumn{4}{|c|}{ 5608E Average } & -0.0600 & 0.0000 & 0.0160 \\
\hline SS & Fair & $5618 \mathrm{E}$ & 2 & 1.12 & 1.12 & 1.12 \\
\hline SS & Fair & $5618 \mathrm{E}$ & 3 & 0.84 & 0.84 & 0.84 \\
\hline $\mathrm{SS}$ & Fair & $5618 \mathrm{E}$ & 4 & 0.84 & 0.84 & 0.84 \\
\hline $\mathrm{SS}$ & Fair & $5618 \mathrm{E}$ & 5 & 0.74 & 0.74 & 0.74 \\
\hline $\mathrm{SS}$ & Fair & $5618 \mathrm{E}$ & 6 & 0.7 & 0.7 & 0.7 \\
\hline $\mathrm{SS}$ & Fair & $5618 \mathrm{E}$ & 11 & 0.87 & 0.87 & 0.87 \\
\hline \multicolumn{4}{|c|}{ 5618E Average } & 0.8517 & 0.8517 & 0.8517 \\
\hline SS & Fair & $5618 W$ & 1 & 1.4 & 1.4 & 1.4 \\
\hline SS & Fair & $5618 W$ & 2 & 2.14 & 2.14 & 2.14 \\
\hline
\end{tabular}


SNF-10293, REV 0

Canister Sludge Depth Measurements. (14 sheets)

\begin{tabular}{|c|c|c|c|c|c|c|}
\hline \multirow{2}{*}{$\begin{array}{c}\text { Canister } \\
\text { Metal }\end{array}$} & \multirow[b]{2}{*}{ Fuel Type } & \multirow{2}{*}{$\begin{array}{c}\text { Barrel } \\
\text { Location }\end{array}$} & \multirow{2}{*}{$\begin{array}{c}\text { Position in } \\
\text { Barrel }\end{array}$} & \multicolumn{3}{|c|}{ Canister Sludge Depth (inch) } \\
\hline & & & & Data & Mean $=0$ & Negative $=0$ \\
\hline SS & Fair & $5618 \mathrm{~W}$ & 3 & 1.42 & 1.42 & 1.42 \\
\hline SS & Fair & $5618 \mathrm{~W}$ & 4 & 1.69 & 1.69 & 1.69 \\
\hline SS & Fair & $5618 \mathrm{~W}$ & 5 & 1.26 & 1.26 & 1.26 \\
\hline SS & Fair & $5618 \mathrm{~W}$ & 6 & 1.6 & 1.6 & 1.6 \\
\hline SS & Fair & $5618 \mathrm{~W}$ & 11 & 1.17 & 1.17 & 1.17 \\
\hline \multicolumn{4}{|c|}{ 5618W Average } & 1.5257 & 1.5257 & 1.5257 \\
\hline SS & Fair & $6057 \mathrm{E}$ & 1 & 0.68 & 0.68 & 0.68 \\
\hline SS & Fair & $6057 \mathrm{E}$ & 2 & 0.74 & 0.74 & 0.74 \\
\hline SS & Fair & $6057 \mathrm{E}$ & 3 & 0.68 & 0.68 & 0.68 \\
\hline SS & Fair & $6057 \mathrm{E}$ & 4 & 3.45 & 3.45 & 3.45 \\
\hline SS & Fair & $6057 \mathrm{E}$ & 5 & 2.83 & 2.83 & 2.83 \\
\hline SS & Fair & $6057 \mathrm{E}$ & 6 & 3.81 & 3.81 & 3.81 \\
\hline SS & Fair & $6057 \mathrm{E}$ & 8 & 1.16 & 1.16 & 1.16 \\
\hline \multicolumn{4}{|c|}{ 6057E Average } & 1.9071 & 1.9071 & 1.9071 \\
\hline SS & Fair & $6413 \mathrm{E}$ & 1 & 1.7 & 1.7 & 1.7 \\
\hline SS & Fair & $6413 \mathrm{E}$ & 2 & 1.53 & 1.53 & 1.53 \\
\hline SS & Fair & $6413 \mathrm{E}$ & 3 & 2.08 & 2.08 & 2.08 \\
\hline SS & Fair & $6413 \mathrm{E}$ & 4 & 1.38 & 1.38 & 1.38 \\
\hline SS & Fair & $6413 \mathrm{E}$ & 5 & 1.24 & 1.24 & 1.24 \\
\hline $\mathrm{SS}$ & Fair & $6413 \mathrm{E}$ & 6 & 1.1 & 1.1 & 1.1 \\
\hline $\mathrm{SS}$ & Fair & $6413 \mathrm{E}$ & 11 & 1.37 & 1.37 & 1.37 \\
\hline $\mathrm{SS}$ & Fair & $6413 \mathrm{E}$ & 12 & 1.24 & 1.24 & 1.24 \\
\hline \multicolumn{4}{|c|}{ 6413E Average } & 1.4550 & 1.4550 & 1.4550 \\
\hline SS & Fair & $6413 \mathrm{~W}$ & 3 & 2.04 & 2.04 & 2.04 \\
\hline SS & Fair & $6413 \mathrm{~W}$ & 4 & 2.04 & 2.04 & 2.04 \\
\hline $\mathrm{SS}$ & Fair & $6413 \mathrm{~W}$ & 5 & 1.61 & 1.61 & 1.61 \\
\hline $\mathrm{SS}$ & Fair & $6413 \mathrm{~W}$ & 6 & 2 & 2 & 2 \\
\hline SS & Fair & $6413 \mathrm{~W}$ & 11 & 1.46 & 1.46 & 1.46 \\
\hline \multicolumn{4}{|c|}{ 6413W Average } & 1.8300 & 1.8300 & 1.8300 \\
\hline $\mathrm{SS}$ & Good & $1845 \mathrm{E}$ & 1 & 2.45 & 2.45 & 2.45 \\
\hline SS & Good & $1845 \mathrm{E}$ & 2 & 2.75 & 2.75 & 2.75 \\
\hline SS & Good & $1845 \mathrm{E}$ & 3 & 1.95 & 1.95 & 1.95 \\
\hline
\end{tabular}


SNF-10293, REV 0

Canister Sludge Depth Measurements. (14 sheets)

\begin{tabular}{|c|c|c|c|c|c|c|}
\hline \multirow{2}{*}{$\begin{array}{c}\text { Canister } \\
\text { Metal }\end{array}$} & \multirow[b]{2}{*}{ Fuel Type } & \multirow{2}{*}{$\begin{array}{c}\text { Barrel } \\
\text { Location }\end{array}$} & \multirow{2}{*}{$\begin{array}{c}\text { Position in } \\
\text { Barrel }\end{array}$} & \multicolumn{3}{|c|}{ Canister Sludge Depth (inch) } \\
\hline & & & & Data & Mean $=0$ & Negative $=0$ \\
\hline \multirow[t]{2}{*}{ SS } & Good & $1845 \mathrm{E}$ & 4 & 2.15 & 2.15 & 2.15 \\
\hline & \multicolumn{3}{|c|}{ 1845E Average } & 2.3250 & 2.3250 & 2.3250 \\
\hline SS & Good & $2244 \mathrm{E}$ & 1 & -0.14 & -0.0720 & 0 \\
\hline SS & Good & $2244 \mathrm{E}$ & 2 & -0.06 & 0.0080 & 0 \\
\hline $\mathrm{SS}$ & Good & $2244 \mathrm{E}$ & 3 & 0.05 & 0.1180 & 0.05 \\
\hline $\mathrm{SS}$ & Good & $2244 \mathrm{E}$ & 4 & 0.26 & 0.3280 & 0.26 \\
\hline \multirow[t]{2}{*}{$\mathrm{SS}$} & Good & $2244 \mathrm{E}$ & 13 & -0.45 & -0.3820 & 0 \\
\hline & & \multicolumn{2}{|c|}{ 2244E Average } & -0.0680 & 0.0000 & 0.0620 \\
\hline SS & Good & $2244 W$ & 1 & -0.33 & -0.1700 & 0 \\
\hline $\mathrm{SS}$ & Good & $2244 \mathrm{~W}$ & 2 & -0.06 & 0.1000 & 0 \\
\hline $\mathrm{SS}$ & Good & $2244 \mathrm{~W}$ & 3 & -0.06 & 0.1000 & 0 \\
\hline $\mathrm{SS}$ & Good & $2244 \mathrm{~W}$ & 4 & 0.05 & 0.2100 & 0.05 \\
\hline $\mathrm{SS}$ & Good & $2244 \mathrm{~W}$ & 5 & -0.33 & -0.1700 & 0 \\
\hline $\mathrm{SS}$ & Good & $2244 \mathrm{~W}$ & 6 & -0.33 & -0.1700 & 0 \\
\hline SS & Good & $2244 \mathrm{~W}$ & 13 & -0.06 & 0.1000 & 0 \\
\hline \multicolumn{4}{|c|}{ 2244W Average } & -0.1600 & 0.0000 & 0.0071 \\
\hline $\mathrm{SS}$ & Good & $2248 \mathrm{~W}$ & 1 & -0.14 & -0.0583 & 0 \\
\hline $\mathrm{SS}$ & Good & $2248 \mathrm{~W}$ & 2 & -0.14 & -0.0583 & 0 \\
\hline $\mathrm{SS}$ & Good & $2248 \mathrm{~W}$ & 3 & -0.06 & 0.0217 & 0 \\
\hline SS & Good & $2248 W$ & 4 & 0.05 & 0.1317 & 0.05 \\
\hline SS & Good & $2248 W$ & 6 & -0.14 & -0.0583 & 0 \\
\hline $\mathrm{SS}$ & Good & $2248 \mathrm{~W}$ & 13 & -0.06 & 0.0217 & 0 \\
\hline \multicolumn{4}{|c|}{ 2248W Average } & -0.0817 & 0.0000 & 0.0083 \\
\hline $\mathrm{SS}$ & Good & $4661 \mathrm{E}$ & 1 & -0.12 & -0.0960 & 0 \\
\hline SS & Good & $4661 \mathrm{E}$ & 2 & 0.14 & 0.1640 & 0.14 \\
\hline $\mathrm{SS}$ & Good & $4661 \mathrm{E}$ & 3 & -0.12 & -0.0960 & 0 \\
\hline SS & Good & $4661 \mathrm{E}$ & 4 & -0.25 & -0.2260 & 0 \\
\hline $\mathrm{SS}$ & Good & $4661 \mathrm{E}$ & 8 & 0.23 & 0.2540 & 0.23 \\
\hline \multicolumn{4}{|c|}{ 4661E Average } & -0.0240 & 0.0000 & 0.0740 \\
\hline
\end{tabular}


SNF-10293, REV 0

APPENDIX E

COMPARISON OF THE CANISTER SLUDGE SAMPLES WITH RADNUC2A PREDICTIONS 
SNF-10293, REV 0

This page intentionally left blank.

E-ii 
SNF-10293, REV 0

\section{APPENDIX E \\ COMPARISON OF THE CANISTER SLUDGE SAMPLES WITH RADNUC2A PREDICTIONS}

The canister sludge sample measurements and imputed values are shown in Table E-1. The values in this table are the values shown in Table 3-16 divided by the uranium concentration reported for each sample. This gives concentrations that are largely independent of the presence of non-fuel materials in sludge.

The segregated fuel sludge compositions output from HNF-8760 are shown in Table E-2 and Table E-3. The predicted canister sample concentrations based on the isotope concentrations from HNF-8760 were calculated using the following approach. Tables 3-24 and 3-25 list the number of fuel assemblies of each fuel model representing the fuel in the KE Basin location for each sample. Since the identity of the fuel assemblies (i.e. fuel models) that have corroded in these locations is not known, it is assumed that each fuel assembly of a given fuel model has contributed equally to the canister sludge in these locations. Thus the activity concentrations in the sludge can be calculated using the key and fuel model identifiers in Tables 3-24 and 3-25 to select one or more rows from Tables E-2 and E-3 to represent this sample.

For samples 96-04, 96-05, 96-15, and $\mathrm{KC}-1$, the estimated canister sludge concentration is calculated in two steps. First, locate the key and fuel model in Tables E-2 and E-3. The identifier shown in Tables E-2 and E-3 is abbreviated by showing just the first and last letters. The abbreviations are used in Tables 3-24 and 3-25. The second step is to divide the activity by the uranium mass shown in Table E-2 for that key and fuel model. This gives the Ci/MTU shown in Table E-5.

For samples 96-06, 96-08, and 96-13, the estimated canister sludge concentration is calculated in three steps. The first step is to find the key and fuel model rows in Tables E-2 and E-3. The second step is to divide the activities by the uranium mass to convert to $\mathrm{Ci} / \mathrm{MTU}$. The third step is to combine the key models for that sample according to the number of canisters in the key. For example, sample 96-08 is from key 10456 which has $10 \mathrm{BE}$ canisters and $4 \mathrm{BS}$ canisters. The BE and BS rows are converted to $\mathrm{Ci} / \mathrm{MTU}$ and then added using the factor 10/14 on the BE concentrations and $4 / 14$ on the BS concentrations.

For sample $\mathrm{KC}-2 / 3$ the same three steps are followed, but the weighting factors are a little different. This sample was a composite of several canisters. Table E-4 shows the estimated fraction of the total sludge mass that went into the sample. These fractions must be further divided according to the relative number of canisters of the different fuel types. The resulting weighting factors are shown in the last column of Table E-4. These weighting factors are then multiplied by the $\mathrm{Ci} / \mathrm{MTU}$ of a given isotope. The sum is the estimated concentration for the isotope as shown in Table E-5. 
The canister sludge sample measurements and imputed values are compared with predictions using the HNF-8760 methods in Figure E-1 through Figure E-13. The Radnuc2A predictions generally overestimate the sludge concentrations. The notable exception is Tc-99, for which the predictions are an order of magnitude lower than the reported values. 
Table E-1. Canister Sample Data with Imputed Values (Ci/MTU).

\begin{tabular}{|c|c|c|c|c|c|}
\hline \multirow{2}{*}{ Measured Quantity } & \multicolumn{5}{|c|}{ Sample Identity } \\
\hline & $96-05$ & $96-08$ & $96-13$ & $96-15$ & $96-04$ \\
\hline Co-60 & $1.10 \mathrm{E}+00$ & $1.76 \mathrm{E}+00$ & $* 7.58 \mathrm{E}-01$ & $* 8.27 \mathrm{E}-01$ & $4.09 \mathrm{E}+00$ \\
\hline Sr-90 & $2.69 \mathrm{E}+03$ & $2.38 \mathrm{E}+03$ & $2.27 \mathrm{E}+03$ & $2.14 \mathrm{E}+03$ & $1.98 \mathrm{E}+03$ \\
\hline Тc-99 & $7.93 E+00$ & $8.67 \mathrm{E}+01$ & $1.30 \mathrm{E}+01$ & $1.66 \mathrm{E}+00$ & $7.06 \mathrm{E}+01$ \\
\hline Cs-134 & $2.96 \mathrm{E}-01$ & $* 1.33 \mathrm{E}+00$ & *3.69E-01 & $* 4.79 \mathrm{E}-01$ & $* 1.37 \mathrm{E}+00$ \\
\hline Cs-137 & $1.41 \mathrm{E}+03$ & $2.99 \mathrm{E}+03$ & $8.27 \mathrm{E}+02$ & $1.07 \mathrm{E}+03$ & $3.07 \mathrm{E}+03$ \\
\hline Eu-152 & $* 1.83 \mathrm{E}-01$ & $* 2.15 \mathrm{E}-01$ & $* 9.30 \mathrm{E}-02$ & $* 1.01 \mathrm{E}-01$ & $* 3.62 \mathrm{E}-01$ \\
\hline Eu-154 & $2.28 \mathrm{E}+01$ & $2.69 \mathrm{E}+01$ & $1.16 \mathrm{E}+01$ & $1.27 \mathrm{E}+01$ & $4.53 \mathrm{E}+01$ \\
\hline Eu-155 & $1.50 \mathrm{E}+01$ & $1.33 \mathrm{E}+01$ & $* 6.15 \mathrm{E}+00$ & $* 6.71 \mathrm{E}+00$ & $2.09 \mathrm{E}+01$ \\
\hline $\mathrm{Np}-237$ & $9.75 \mathrm{E}-03$ & $* 1.78 \mathrm{E}-02$ & $2.31 \mathrm{E}-02$ & $1.69 \mathrm{E}-02$ & $2.73 \mathrm{E}-02$ \\
\hline $\mathrm{Pu}-238$ & $1.99 \mathrm{E}+01$ & $4.41 \mathrm{E}+01$ & $* 2.11 \mathrm{E}+01$ & $1.11 \mathrm{E}+01$ & $6.59 \mathrm{E}+01$ \\
\hline $\mathrm{Pu}-239 / 240$ & $1.87 \mathrm{E}+02$ & $2.37 \mathrm{E}+02$ & $1.41 \mathrm{E}+02$ & $1.29 \mathrm{E}+02$ & $3.06 \mathrm{E}+02$ \\
\hline $\mathrm{Pu}-241$ & $* 1.12 \mathrm{E}+03$ & $* 2.48 \mathrm{E}+03$ & $* 1.19 \mathrm{E}+03$ & $* 6.22 \mathrm{E}+02$ & $* 3.70 \mathrm{E}+03$ \\
\hline Am-241 & $1.14 \mathrm{E}+02$ & $1.96 \mathrm{E}+02$ & $* 1.13 \mathrm{E}+02$ & $8.03 \mathrm{E}+01$ & $3.40 \mathrm{E}+02$ \\
\hline \multirow{2}{*}{ Measured Quantity } & \multicolumn{3}{|c|}{ Sample Identity } & \multirow{2}{*}{ Mean } & \multirow{2}{*}{$\begin{array}{l}\text { Standard } \\
\text { Deviation }\end{array}$} \\
\hline & $96-06$ & $\mathrm{KC}-1$ & $\mathrm{KC}-2 / 3$ & & \\
\hline Co-60 & $* 8.92 \mathrm{E}-01$ & $3.03 \mathrm{E}-01$ & 7.43E-01 & $1.31 \mathrm{E}+00$ & $1.20 \mathrm{E}+00$ \\
\hline Sr-90 & $2.40 \mathrm{E}+03$ & $* 2.36 \mathrm{E}+03$ & $* 2.36 \mathrm{E}+03$ & $2.32 \mathrm{E}+03$ & $2.07 \mathrm{E}+02$ \\
\hline Tc-99 & $* 7.12 \mathrm{E}+00$ & $* 1.84 \mathrm{E}+01$ & $* 3.24 \mathrm{E}+01$ & $2.97 \mathrm{E}+01$ & $3.19 \mathrm{E}+01$ \\
\hline Cs-134 & $* 3.31 \mathrm{E}-01$ & $* 2.54 \mathrm{E}-01$ & $* 6.46 \mathrm{E}-01$ & $6.35 \mathrm{E}-01$ & 4.60E-01 \\
\hline Cs-137 & $7.41 \mathrm{E}+02$ & $5.70 \mathrm{E}+02$ & $1.45 \mathrm{E}+03$ & $1.52 \mathrm{E}+03$ & $9.84 \mathrm{E}+02$ \\
\hline Eu-152 & $* 1.09 \mathrm{E}-01$ & $* 1.00 \mathrm{E}-01$ & $* 1.09 \mathrm{E}-01$ & $1.59 \mathrm{E}-01$ & $9.32 \mathrm{E}-02$ \\
\hline Eu-154 & $1.37 \mathrm{E}+01$ & $1.25 \mathrm{E}+01$ & $1.37 \mathrm{E}+01$ & $1.99 \mathrm{E}+01$ & $1.17 \mathrm{E}+01$ \\
\hline Eu-155 & $* 7.24 \mathrm{E}+00$ & $4.30 \mathrm{E}+00$ & $6.92 \mathrm{E}+00$ & $1.01 \mathrm{E}+01$ & $5.74 \mathrm{E}+00$ \\
\hline Np-237 & $1.91 \mathrm{E}-02$ & $* 1.78 \mathrm{E}-02$ & $* 1.78 \mathrm{E}-02$ & $1.87 \mathrm{E}-02$ & $5.06 \mathrm{E}-03$ \\
\hline $\mathrm{Pu}-238$ & $2.16 \mathrm{E}+01$ & $3.12 \mathrm{E}+01$ & $2.72 \mathrm{E}+01$ & $3.03 \mathrm{E}+01$ & $1.73 \mathrm{E}+01$ \\
\hline Pu-239/240 & $1.23 \mathrm{E}+02$ & $2.06 \mathrm{E}+02$ & $1.92 \mathrm{E}+02$ & $1.90 \mathrm{E}+02$ & $6.15 \mathrm{E}+01$ \\
\hline $\mathrm{Pu}-241$ & $* 1.21 \mathrm{E}+03$ & $* 1.75 \mathrm{E}+03$ & $* 1.53 \mathrm{E}+03$ & $1.70 \mathrm{E}+03$ & $9.74 \mathrm{E}+02$ \\
\hline Am-241 & $9.88 \mathrm{E}+01$ & $1.77 \mathrm{E}+02$ & $1.52 \mathrm{E}+02$ & $1.59 \mathrm{E}+02$ & $8.33 \mathrm{E}+01$ \\
\hline
\end{tabular}

All values are the canister sludge concentration divided by the appropriate uranium concentration. Imputed values are indicated with asterisks.

The mean and standard deviation are based on both measured and imputed values. 
Table E-2. Composition of Segregated Fuel Sludge by Key and Fuel Model (1 ${ }^{\text {st }}$ Part). (5 sheets)

\begin{tabular}{|c|c|c|c|c|c|c|c|c|c|}
\hline Key ID & $\begin{array}{c}\text { Fuel } \\
\text { Model }\end{array}$ & $\begin{array}{l}\text { xposure } \\
\text { IWd/MTU) }\end{array}$ & $\begin{array}{c}\text { Uranium } \\
\text { (MTU) }\end{array}$ & $\begin{array}{c}\text { Co-60 } \\
(\mathrm{Ci}) \\
\end{array}$ & $\begin{array}{c}\text { Sr-90 } \\
(\mathbf{C i}) \\
\end{array}$ & $\begin{array}{c}\text { Tc-99 } \\
\text { (Ci) }\end{array}$ & $\begin{array}{c}\text { Cs-134 } \\
\text { (Ci) }\end{array}$ & $\begin{array}{c}\text { Cs-137 } \\
\text { (Ci) }\end{array}$ & $\begin{array}{c}\text { Eu-152 } \\
\text { (Ci) }\end{array}$ \\
\hline & & & & & & & & & \\
\hline 10001 & & & & & & & & & \\
\hline 10001 & 309X09S & & 51F_-04 & 46E-04 & 8.04E-01 & $.57 \mathrm{E}-04$ & $8.98 \mathrm{E}-05$ & $1.02 \mathrm{E}+00$ & $5.10 \mathrm{E}-05$ \\
\hline 10001 & $9 \mathrm{E}$ & & & & & & & & $3.05 \mathrm{E}-05$ \\
\hline 10201 & $\mathrm{~A}$ & & & & -02 & & & & -02 \\
\hline 10201 & 309X09S & $.76 \mathrm{E}+03$ & & $32 \mathrm{E}-02$ & $2.73 \mathrm{E}+02$ & $8.85 \mathrm{E}-02$ & $4.73 \mathrm{E}-02$ & $3.56 \mathrm{E}+02$ & $2.18 \mathrm{E}-02$ \\
\hline 10201 & & $2.78 \mathrm{E}+03$ & & & $2.92 \mathrm{E}+02$ & & & $3.83 \mathrm{E}+02$ & $2.35 \mathrm{E}-02$ \\
\hline 10259 & 309X09A & & & & & & & & $2.96 \mathrm{E}-04$ \\
\hline 10259 & $309 \mathrm{X} 09 \mathrm{C}$ & & & & $7.29 \mathrm{E}+01$ & $2.28 \mathrm{E}-02$ & & $9.16 \mathrm{E}+01$ & $4.35 \mathrm{E}-03$ \\
\hline 10259 & B09X09E & & $00 \mathrm{~F}_{2} 01$ & 31E-01 & $69 \mathrm{E}+02$ & $2.11 \mathrm{E}-01$ & $9.63 \mathrm{E}-02$ & $51 \mathrm{E}+02$ & $.46 \mathrm{E}-02$ \\
\hline 10294 & 09A & $2.53 \mathrm{E}$ & & $65 \mathrm{~F}_{-} 03$ & $8.26 \mathrm{E}+00$ & $2.64 \mathrm{E}-03$ & $42 \mathrm{~F}_{2} 03$ & $07 \mathrm{E}+01$ & \\
\hline 10294 & $09 \mathrm{C}$ & & & & & & & $1.04 \mathrm{~L}+02$ & $5.06 \mathrm{E}-03$ \\
\hline 10294 & $09 \mathrm{~S}$ & & $9 \mathrm{E}-02$ & $6 \mathrm{E}-02$ & & $81 \mathrm{E}-02$ & $40 \mathrm{E}-02$ & $14 \mathrm{E}+02$ & $.29 \mathrm{E}-03$ \\
\hline 10294 & B09X09E & & $0 \cap 5 \mathrm{E} 01$ & 30F_01 & 03 & $8.42 \mathrm{E}-01$ & 94F-01 & $.40 \mathrm{E}+03$ & $.78 \mathrm{E}-01$ \\
\hline 10350 & & & & & & & & $4.52 \mathrm{E}+01$ & \\
\hline 10350 & $09 \mathrm{~S}$ & & & & $35 \mathrm{E}+01$ & $2.69 \mathrm{E}-02$ & $1.69 \mathrm{E}-02$ & $1.09 \mathrm{E}+02$ & $6.86 \mathrm{E}-03$ \\
\hline 10350 & 09X09E & $03 \mathrm{~F}+03$ & $97 F_{-} 02$ & $68 \mathrm{E}-02$ & $2.29 \mathrm{E}+02$ & $7.40 \mathrm{E}-02$ & $.79 \mathrm{E}-02$ & $3.01 \mathrm{E}+02$ & $1.92 \mathrm{E}-02$ \\
\hline 10385 & B09X09A & $3.33 \mathrm{E}+03$ & $644 \mathrm{~F}_{2} 03$ & $650 \mathrm{~F}_{-03}$ & $3.15 \mathrm{E}+01$ & $1.03 \mathrm{E}-02$ & 7.84E-03 & $4.22 \mathrm{E}+01$ & $2.89 \mathrm{E}-03$ \\
\hline 10385 & 09X09S & $59 \mathrm{E}+03$ & & $.06 \mathrm{E}-03$ & $2.47 \mathrm{~L} / 01$ & $7.86 \mathrm{E}-03$ & 4.70E-03 & $3.20 \mathrm{E}+01$ & $.93 \mathrm{E}-03$ \\
\hline 10385 & 09X09E & $2.54 \mathrm{E}+03$ & 7E-01 & 84E-01 & $1.88 \mathrm{E}+03$ & $5.96 \mathrm{E}-01$ & $3.50 \mathrm{E}-01$ & $.42 \mathrm{E}+03$ & $1.44 \mathrm{E}-01$ \\
\hline 10456 & B09X09A & $3.23 \mathrm{E}+03$ & $6.36 \mathrm{E}-03$ & $6.47 \mathrm{E}-03$ & $3.03 \mathrm{E}+01$ & $9.87 \mathrm{E}-03$ & $7.76 \mathrm{E}-03$ & $4.05 \mathrm{E}+01$ & $2.74 \mathrm{E}-03$ \\
\hline 10456 & & & & & & & & & \\
\hline 10456 & B09X09S & $3.19 \mathrm{E}+03$ & 88E-02 & 92E-02 & $2.31 \mathrm{E}+02$ & $7.50 \mathrm{E}-02$ & $84 \mathrm{E}-02$ & $08 \mathrm{E}+02$ & $.07 \mathrm{E}-02$ \\
\hline 10456 & B09X09E & $3.33 \mathrm{E}+03$ & $29 \mathrm{~F}_{-} 01$ & $3.46 \mathrm{E}-01$ & $1.62 \mathrm{E}+03$ & $5.28 \mathrm{E}-01$ & 4.29E-01 & $2.17 \mathrm{E}+03$ & $1.49 \mathrm{E}-01$ \\
\hline 10576 & B09X09S & $3.80 \mathrm{E}+03$ & $1.85 \mathrm{E}-04$ & & $1.04 \mathrm{E}+00$ & & & $1.43 \mathrm{E}+00$ & $1.06 \mathrm{E}-04$ \\
\hline 10576 & B09X09E & $2.66 \mathrm{E}+03$ & & $1.44 \mathrm{E}-02$ & $6.62 \mathrm{E}+01$ & $2.09 \mathrm{E}-02$ & $1.5 \angle \mathrm{E}-02$ & $0.00 \mathrm{E}+01$ & $5.32 \mathrm{E}-03$ \\
\hline 10602 & B09X09A & $3.31 \mathrm{E}+03$ & & $1.54 \mathrm{E}-02$ & $6.92 \mathrm{E}+01$ & $2.24 \mathrm{E}-02$ & $.06 \mathrm{E}-02$ & $.27 \mathrm{E}+01$ & $42 \mathrm{E}-03$ \\
\hline 10602 & B09X09C & $3.78 \mathrm{E}+03$ & $6.55 \mathrm{E}-04$ & 8.28E-04 & $3.67 \mathrm{E}+00$ & $1.21 \mathrm{E}-03$ & $1.28 \mathrm{E}-03$ & $5.01 \mathrm{E}+00$ & $3.73 \mathrm{E}-04$ \\
\hline 10602 & B09X09S & $3.79 \mathrm{E}+03$ & $2.00 \mathrm{~L}-02$ & $3.30 \mathrm{E}-02$ & $1.46 \mathrm{E}+02$ & $4.80 \mathrm{E}-02$ & $5.09 \mathrm{E}-02$ & $2.00 \mathrm{E}+02$ & $1.49 \mathrm{E}-02$ \\
\hline 10602 & B09X09E & $3.34 \mathrm{E}+03$ & $5.25 \mathrm{E}-01$ & $.83 \mathrm{E}-01$ & $2.61 \mathrm{E}+03$ & $8.46 \mathrm{E}-01$ & $7.88 \mathrm{E}-01$ & $3.51 \mathrm{E}+03$ & $2.44 \mathrm{E}-01$ \\
\hline 10679 & B09X09A & $3.89 \mathrm{E}+03$ & $1.06 \mathrm{E}-02$ & $1.42 \mathrm{E}-02$ & $6.13 \mathrm{E}+01$ & $2.01 \mathrm{E}-02$ & $2.36 \mathrm{E}-02$ & $8.41 \mathrm{E}+01$ & $6.41 \mathrm{E}-03$ \\
\hline 10679 & B09X09C & $2.51 \mathrm{E}+03$ & $2.49 \mathrm{E}-02$ & $2.15 \mathrm{E}-02$ & $9.64 \mathrm{E}+01$ & $3.00 \mathrm{E}-02$ & $2.28 \mathrm{E}-02$ & $1.24 \mathrm{E}+02$ & $7.51 \mathrm{E}-03$ \\
\hline 10679 & B09X09S & $2.84 \mathrm{E}+03$ & $2.57 \mathrm{E}-02$ & $2.50 \mathrm{E}-02$ & $1.11 \mathrm{E}+02$ & $3.50 \mathrm{E}-02$ & $2.98 \mathrm{E}-02$ & $1.45 \mathrm{E}+02$ & $9.35 \mathrm{E}-03$ \\
\hline
\end{tabular}


Table E-2. Composition of Segregated Fuel Sludge by Key and Fuel Model ( $1^{\text {st }}$ Part). (5 sheets)

\begin{tabular}{|c|c|c|c|c|c|c|c|c|c|}
\hline Key ID & $\begin{array}{c}\text { Fuel } \\
\text { Model }\end{array}$ & $\begin{array}{l}\text { Exposure } \\
\text { MWd/MTU) }\end{array}$ & $\begin{array}{c}\text { Uranium } \\
\text { (MTU) }\end{array}$ & $\begin{array}{c}\text { Co-60 } \\
\text { (Ci) }\end{array}$ & $\begin{array}{c}\text { Sr-90 } \\
\text { (Ci) }\end{array}$ & $\begin{array}{c}\text { Tc-99 } \\
\text { (Ci) }\end{array}$ & $\begin{array}{c}\text { Cs-134 } \\
\text { (Ci) }\end{array}$ & $\begin{array}{l}\text { Cs-137 } \\
\text { (Ci) }\end{array}$ & $\begin{array}{c}\text { Eu-152 } \\
\text { (Ci) }\end{array}$ \\
\hline 10679 & B09X09E & & & $5.70 \mathrm{E}-01$ & $.48 \mathrm{E}+03$ & 8.07E-01 & 8.82E-01 & $3.37 \mathrm{E}+03$ & $2.47 \mathrm{E}-01$ \\
\hline 10742 & $09 \mathrm{C}$ & & & & & & & +02 & \\
\hline 10742 & B09X09S & $93 \mathrm{E}+03$ & $1.10 \mathrm{E}-02$ & $.12 \mathrm{E}-02$ & $88 \mathrm{E}+01$ & $1.54 \mathrm{E}-02$ & $1.43 \mathrm{E}-02$ & $6.42 \mathrm{E}+01$ & $4.21 \mathrm{E}-03$ \\
\hline 10742 & B09X09E & $3.32 \mathrm{E}+03$ & $2.90 \mathrm{E}-01$ & $36 \mathrm{E}-01$ & $.45 \mathrm{E}+03$ & $4.63 \mathrm{E}-01$ & 4.87E-01 & $1.94 \mathrm{E}+03$ & $1.36 \mathrm{E}-01$ \\
\hline 10764 & B09X09A & & 4.64E-04 & $3.55 \mathrm{E}-04$ & $56 \mathrm{E}+00$ & & $3.35 \mathrm{E}-04$ & $1.98 \mathrm{E}+00$ & \\
\hline 10764 & B091 & & $7.42 \mathrm{I}$ & $5.68 \mathrm{E}-04$ & $0 \mathrm{E}+00$ & 04 & -04 & $17 \mathrm{E}+00$ & \\
\hline 10764 & B09X09E & $2.16 \mathrm{E}+03$ & $3.90 \mathrm{E}-04$ & $2.98 \mathrm{E}-04$ & $1.31 \mathrm{E}+00$ & $4.00 \mathrm{E}-04$ & $2.82 \mathrm{E}-04$ & $1.66 \mathrm{E}+00$ & $8.81 \mathrm{E}-05$ \\
\hline 10764 & $09 \mathrm{R}$ & & & $2.91 \mathrm{E}-04$ & $28 \mathrm{E}+00$ & $3.90 \mathrm{E}-04$ & $2.74 \mathrm{E}-04$ & $1.62 \mathrm{E}+00$ & \\
\hline 10764 & $\mathrm{~B} 07 \mathrm{X} 07 \mathrm{E}$ & & $4.68 \mathrm{E}-05$ & $3.58 \mathrm{E}-05$ & 58E-01 & $80 \mathrm{E}-05$ & $3.38 \mathrm{E}-05$ & $2.00 \mathrm{E}-01$ & \\
\hline 10770 & B09X09C & $02 \mathrm{E}+03$ & $1.07 \mathrm{E}-02$ & $1.14 \mathrm{E}-02$ & $92 \mathrm{E}+01$ & $1.55 \mathrm{E}-02$ & $1.53 \mathrm{E}-02$ & $0 \mathrm{E}+01$ & \\
\hline 10770 & B09X09S & $3.66 \mathrm{E}+03$ & $6.98 \mathrm{E}-02$ & $9.07 \mathrm{E}-02$ & $3.84 \mathrm{E}+02$ & $1.24 \mathrm{E}-01$ & $1.49 \mathrm{E}-01$ & $5.21 \mathrm{E}+02$ & \\
\hline 10770 & $69 \mathrm{E}$ & $\begin{array}{ll}0 \\
\end{array}$ & $2.22 \mathrm{E}-01$ & $2.92 \mathrm{E}-01$ & $23 \mathrm{E}+03$ & $4.00 \mathrm{E}-01$ & 4.84E-01 & $1.68 \mathrm{E}+03$ & $1.25 \mathrm{E}-01$ \\
\hline 10832 & B09X09A & $58 \mathrm{E}+03$ & $.05 \mathrm{E}-03$ & $.74 \mathrm{E}-03$ & $2.03 \mathrm{E}+01$ & $.27 \mathrm{E}-03$ & $.64 \mathrm{E}-03$ & $2.63 \mathrm{E}+01$ & $.63 \mathrm{E}-03$ \\
\hline 10832 & B09X09C & $2.66 \mathrm{E}+03$ & $2.76 \mathrm{E}-02$ & $2.67 \mathrm{E}-02$ & $1.14 \mathrm{E}+02$ & $3.53 \mathrm{E}-02$ & $3.25 \mathrm{E}-02$ & $1.48 \mathrm{E}+02$ & $9.31 \mathrm{E}-03$ \\
\hline & B09X09S & & & $5.09 \mathrm{E}-02$ & $13 \mathrm{E}+02$ & & & $2.85 \mathrm{E}+02$ & \\
\hline 10832 & 09X09E & & $6.41 \mathrm{E}-01$ & $6.96 \mathrm{E}-01$ & $4 \mathrm{E}+03$ & $25 \mathrm{E}-01$ & $61 \mathrm{E}-01$ & $3.89 \mathrm{E}+03$ & $2.61 \mathrm{E}-01$ \\
\hline 10982 & B09X09A & $30 \mathrm{~F}+03$ & $6.02 \mathrm{E}-03$ & $7.58 \mathrm{E}-03$ & $04 \mathrm{E}+01$ & $9.58 \mathrm{E}-03$ & $1.25 \mathrm{E}-02$ & $4.07 \mathrm{E}+01$ & $2.90 \mathrm{E}-03$ \\
\hline 10982 & B09X09C & & & $1.36 \mathrm{E}-02$ & $5.52 \mathrm{E}+01$ & & & $7.27 \mathrm{E}+01$ & \\
\hline 10982 & B09X09S & $0 / L T 05$ & $1.67 \mathrm{E}-02$ & $2.36 \mathrm{E}-02$ & $36 \mathrm{E}+01$ & $2.99 \mathrm{E}-02$ & $4.36 \mathrm{E}-02$ & $1.27 \mathrm{E}+02$ & $9.61 \mathrm{E}-03$ \\
\hline 10982 & 09X09E & & $9 \mathrm{E}-01$ & E-01 & $2 \mathrm{E}+03$ & $9 \mathrm{E}-01$ & $7.52 \mathrm{E}-01$ & $2.08 \mathrm{E}+03$ & \\
\hline 11036 & B09X09A & & $1.26 \mathrm{E}-04$ & $1.67 \mathrm{E}-04$ & 57E-01 & $2.07 \mathrm{E}-04$ & $2.93 \mathrm{E}-04$ & $8.83 \mathrm{E}-01$ & \\
\hline & & & & & & & & $3.17 \mathrm{E}+01$ & \\
\hline 11036 & B09X09S & $6 \mathrm{E}+03$ & 9 & 39E-02 & $+2 \mathrm{E}+01$ & 02 & 64E-02 & $7.37 \mathrm{E}+01$ & -03 \\
\hline 11036 & B09X09E & $3.63 \mathrm{E}+03$ & $1.10 \mathrm{E}-01$ & $1.55 \mathrm{E}-01$ & $6.08 \mathrm{E}+02$ & $1.93 \mathrm{E}-01$ & $2.92 \mathrm{E}-01$ & $8.24 \mathrm{E}+02$ & $6.21 \mathrm{E}-02$ \\
\hline & & & & & & & & & \\
\hline & B09X09C & $3.37 \mathrm{E}+03$ & & $1.59 \mathrm{E}-02$ & $6.12 \mathrm{E}+01$ & $1.92 \mathrm{E}-02$ & $2.87 \mathrm{E}-02$ & $8.21 \mathrm{E}+01$ & $5.97 \mathrm{E}-03$ \\
\hline 11109 & B09X & $3.97 \mathrm{E}+03$ & $2.13 \mathrm{E}-02$ & $3.41 \mathrm{E}-02$ & $1.30 \mathrm{E}+02$ & 4.15E-02 & 7.38E-02 & $1.78 \mathrm{E}+02$ & $1.42 \mathrm{E}-02$ \\
\hline 11109 & B09X09E & $3.85 \mathrm{E}+03$ & $2.61 \mathrm{E}-01$ & $4.03 \mathrm{E}-01$ & $1.54 \mathrm{E}+03$ & 4.90E-01 & 8.42E-01 & $2.10 \mathrm{E}+03$ & $1.65 \mathrm{E}-01$ \\
\hline & B09X09A & $2.29 \mathrm{E}+03$ & $4.82 \mathrm{E}-03$ & $4.56 \mathrm{E}-03$ & $1.77 \mathrm{E}+01$ & $5.27 \mathrm{E}-03$ & $5.80 \mathrm{E}-03$ & $2.25 \mathrm{E}+01$ & $1.30 \mathrm{E}-03$ \\
\hline & B09X09C & $3.24 \mathrm{E}+03$ & $2.64 \mathrm{E}-03$ & $3.50 \mathrm{E}-03$ & $1.33 \mathrm{E}+01$ & 4.11E-03 & $6.33 \mathrm{E}-03$ & $1.77 \mathrm{E}+01$ & $1.27 \mathrm{E}-03$ \\
\hline & B09X09S & $3.18 \mathrm{E}+03$ & $1.32 \mathrm{E}-02$ & $1.72 \mathrm{E}-02$ & $6.53 \mathrm{E}+01$ & 2.02E-02 & $3.05 \mathrm{E}-02$ & $8.68 \mathrm{E}+01$ & $6.15 \mathrm{E}-03$ \\
\hline 11183 & В09X09E & $3.36 \mathrm{E}+03$ & 4.14E-01 & $5.71 \mathrm{E}-01$ & $2.15 \mathrm{E}+03$ & $6.71 \mathrm{E}-01$ & $1.07 \mathrm{E}+00$ & $2.89 \mathrm{E}+03$ & $2.11 \mathrm{E}-01$ \\
\hline & B09X09A & $4.17 \mathrm{E}+03$ & 4.39E-03 & $8.17 \mathrm{E}-03$ & $2.86 \mathrm{E}+01$ & $9.06 \mathrm{E}-03$ & $2.17 \mathrm{E}-02$ & $3.96 \mathrm{E}+01$ & $3.31 \mathrm{E}-03$ \\
\hline 11372 & B09X09C & $3.02 \mathrm{E}+03$ & $8.65 \mathrm{E}-03$ & $1.15 \mathrm{E}-02$ & $4.13 \mathrm{E}+01$ & $1.25 \mathrm{E}-02$ & $2.15 \mathrm{E}-02$ & $5.45 \mathrm{E}+01$ & $3.82 \mathrm{E}-03$ \\
\hline
\end{tabular}


Table E-2. Composition of Segregated Fuel Sludge by Key and Fuel Model (1 ${ }^{\text {st }}$ Part). (5 sheets)

\begin{tabular}{|c|c|c|c|c|c|c|c|c|c|}
\hline Key ID & $\begin{array}{c}\text { Fuel } \\
\text { Model }\end{array}$ & $\begin{array}{l}\text { Exposure } \\
\text { MWd/MTU) }\end{array}$ & $\begin{array}{c}\text { Uranium } \\
\text { (MTU) }\end{array}$ & $\begin{array}{c}\text { Co-60 } \\
\text { (Ci) }\end{array}$ & $\begin{array}{c}\text { Sr-90 } \\
\text { (Ci) }\end{array}$ & $\begin{array}{c}\text { Tc-99 } \\
\text { (Ci) }\end{array}$ & $\begin{array}{c}\text { Cs-134 } \\
\text { (Ci) }\end{array}$ & $\begin{array}{l}\text { Cs-137 } \\
\text { (Ci) }\end{array}$ & $\begin{array}{c}\text { Eu-152 } \\
\text { (Ci) }\end{array}$ \\
\hline 11372 & B09X09S & $77 \mathrm{E}+03$ & $1.44 \mathrm{E}-02$ & $2.40 \mathrm{E}-02$ & $46 \mathrm{E}+01$ & 2.64E-02 & $5.67 \mathrm{E}-02$ & $1.15 \mathrm{E}+02$ & $9.11 \mathrm{E}-03$ \\
\hline 11372 & $9 \mathrm{E}$ & & & & & & & & \\
\hline 11449 & B09X09A & $99 \mathrm{E}+03$ & $.15 \mathrm{E}-03$ & $.75 \mathrm{E}-03$ & $97 \mathrm{E}+01$ & $6.19 \mathrm{E}-03$ & $.52 \mathrm{E}-02$ & $.72 \mathrm{E}+01$ & \\
\hline 11449 & B09X09C & $4.39 \mathrm{E}+02$ & $1.50 \mathrm{E}-03$ & $39 \mathrm{E}-04$ & $26 \mathrm{E}+00$ & $3.44 \mathrm{E}-04$ & $2.02 \mathrm{E}-04$ & $1.48 \mathrm{E}+00$ & $3.75 \mathrm{E}-05$ \\
\hline 11449 & $209 \mathrm{~S}$ & & $5.54 \mathrm{E}-02$ & $9.29 \mathrm{E}-02$ & $3.21 \mathrm{E}+02$ & & $2.25 \mathrm{E}-01$ & $4.37 \mathrm{E}+02$ & \\
\hline 11449 & B09X & & 2. & 4.65E-01 & $50 \mathrm{E}+03$ & 01 & & $19 \mathrm{E}+03$ & \\
\hline 11540 & B09X09A & $3.18 \mathrm{E}+03$ & $3.23 \mathrm{E}-03$ & 4.79E-03 & $1.63 \mathrm{E}+01$ & 4.94E-03 & $1.04 \mathrm{E}-02$ & $2.17 \mathrm{E}+01$ & $1.58 \mathrm{E}-03$ \\
\hline 11540 & 69C & & $4.29 \mathrm{E}-04$ & $6.46 \mathrm{E}-04$ & $20 \mathrm{E}+00$ & $6.67 \mathrm{E}-04$ & $1.42 \mathrm{E}-03$ & $2.93 \mathrm{E}+00$ & \\
\hline 11540 & $09 \mathrm{~S}$ & $49 \mathrm{E}+03$ & 9E-03 & $1.20 \mathrm{E}-02$ & $97 \mathrm{E}+01$ & 26E-02 & $3.81 \mathrm{E}-02$ & $.56 \mathrm{E}+01$ & -03 \\
\hline 11540 & B09X09E & $24 \mathrm{E}+03$ & 7.79E-02 & $1.57 \mathrm{E}-01$ & $22 \mathrm{E}+02$ & $1.64 \mathrm{E}-01$ & 4.66E-01 & $7.24 \mathrm{E}+02$ & $20 \mathrm{E}-02$ \\
\hline 11733 & B09X09S & $2.89 \mathrm{E}+03$ & $6.96 \mathrm{E}-04$ & $1.00 \mathrm{E}-03$ & $3.26 \mathrm{E}+00$ & $9.63 \mathrm{E}-04$ & $2.20 \mathrm{E}-03$ & $4.28 \mathrm{E}+00$ & $3.01 \mathrm{E}-04$ \\
\hline 11733 & $69 \mathrm{E}$ & $3.59 \mathrm{E}+03$ & $2.54 \mathrm{E}-03$ & 4.57E-03 & $46 \mathrm{E}+01$ & $4.42 \mathrm{E}-03$ & $1.25 \mathrm{E}-02$ & $1.97 \mathrm{E}+01$ & $1.55 \mathrm{E}-03$ \\
\hline 11806 & B09X09A & $86 \mathrm{E}+03$ & 29E-03 & $.82 \mathrm{E}-03$ & $54 \mathrm{E}+01$ & 51E-03 & $.09 \mathrm{E}-02$ & $.01 \mathrm{E}+01$ & $.42 \mathrm{E}-03$ \\
\hline 11806 & B09X09C & $3.41 \mathrm{E}+03$ & $2.45 \mathrm{E}-04$ & 4.29E-04 & $1.35 \mathrm{E}+00$ & $4.04 \mathrm{E}-04$ & $1.16 \mathrm{E}-03$ & $1.81 \mathrm{E}+00$ & \\
\hline & & & & $6.58 \mathrm{E}-03$ & $03 \mathrm{E}+01$ & & & $2.79 \mathrm{E}+01$ & \\
\hline 11806 & 09X09E & $3.87 \mathrm{E}+03$ & JE-01 & $31 \mathrm{E}-01$ & $4 \mathrm{E}+03$ & $4.08 \mathrm{E}-01$ & $1.34 \mathrm{E}+00$ & $1.83 \mathrm{E}+03$ & $1.51 \mathrm{E}-01$ \\
\hline 11897 & B09X09A & $40 \mathrm{~F}+03$ & $5.32 \mathrm{E}-05$ & $9.59 \mathrm{E}-05$ & $93 \mathrm{E}-01$ & $.73 \mathrm{E}-05$ & $2.72 \mathrm{E}-04$ & $3.93 \mathrm{E}-01$ & $3.05 \mathrm{E}-05$ \\
\hline 11897 & B09X09C & & & $6.20 \mathrm{E}-02$ & $1.90 \mathrm{E}+02$ & & & $2.54 \mathrm{E}+02$ & \\
\hline 11897 & B09X09S & $4 \mathrm{~L}+\mathrm{VJ}$ & & $4.01 \mathrm{E}-02$ & $21 \mathrm{E}+02$ & $3.67 \mathrm{E}-02$ & $1.34 \mathrm{E}-01$ & $1.66 \mathrm{E}+02$ & $1.40 \mathrm{E}-02$ \\
\hline 11897 & 09X09E & & 41E-01 & 25E-01 & $8 \mathrm{E}+03$ & E-01 & $.81 \mathrm{E}+00$ & $2.17 \mathrm{E}+03$ & \\
\hline 11979 & B09X09A & & 7.79E-04 & $1.45 \mathrm{E}-03$ & $31 \mathrm{E}+00$ & $1.28 \mathrm{E}-03$ & $4.30 \mathrm{E}-03$ & $5.78 \mathrm{E}+00$ & \\
\hline & & & & & & & & $.82 \mathrm{E}+02$ & $1.46 \mathrm{E}$ \\
\hline 11979 & B09X09S & $5 \mathrm{E}+03$ & 2 & 23E-02 & $54 \mathrm{E}+02$ & 02 & 78E-01 & $2.11 \mathrm{E}+02$ & 1.76 \\
\hline 11979 & B09X09E & $4.04 \mathrm{E}+03$ & $2.30 \mathrm{E}-01$ & $5.14 \mathrm{E}-01$ & $1.51 \mathrm{E}+03$ & $4.58 \mathrm{E}-01$ & $1.85 \mathrm{E}+00$ & $2.08 \mathrm{E}+03$ & $1.79 \mathrm{E}-01$ \\
\hline 12127 & & & & & & & & & \\
\hline 12127 & B09X09C & $2.48 \mathrm{E}+03$ & & $2.70 \mathrm{E}-03$ & $7.90 \mathrm{E}+00$ & $2.23 \mathrm{E}-03$ & $6.35 \mathrm{E}-03$ & $1.02 \mathrm{E}+01$ & $6.77 \mathrm{E}-04$ \\
\hline 12127 & B09X09S & $4.07 \mathrm{E}+03$ & $1.84 \mathrm{E}-02$ & $4.38 \mathrm{E}-02$ & $23 \mathrm{E}+02$ & $3.69 \mathrm{E}-02$ & $1.72 \mathrm{E}-01$ & $1.69 \mathrm{E}+02$ & $1.48 \mathrm{E}-02$ \\
\hline 12127 & B09X09E & $3.45 \mathrm{E}+03$ & $1.45 \mathrm{E}-01$ & $2.87 \mathrm{E}-01$ & $8.19 \mathrm{E}+02$ & $2.41 \mathrm{E}-01$ & $9.41 \mathrm{E}-01$ & $1.10 \mathrm{E}+03$ & $8.75 \mathrm{E}-02$ \\
\hline 12201 & B09X09A & $3.40 \mathrm{E}+03$ & $1.10 \mathrm{E}-03$ & $2.21 \mathrm{E}-03$ & $6.16 \mathrm{E}+00$ & $1.80 \mathrm{E}-03$ & $7.42 \mathrm{E}-03$ & $8.25 \mathrm{E}+00$ & $6.55 \mathrm{E}-04$ \\
\hline 12201 & B09X09C & $3.30 \mathrm{E}+03$ & $3.04 \mathrm{E}-03$ & $5.93 \mathrm{E}-03$ & $1.66 \mathrm{E}+01$ & 4.84E-03 & $1.94 \mathrm{E}-02$ & $2.22 \mathrm{E}+01$ & $1.73 \mathrm{E}-03$ \\
\hline 12201 & B09X09S & $3.96 \mathrm{E}+03$ & $3.75 \mathrm{E}-02$ & $8.88 \mathrm{E}-02$ & $2.44 \mathrm{E}+02$ & $7.28 \mathrm{E}-02$ & $3.53 \mathrm{E}-01$ & $3.35 \mathrm{E}+02$ & $2.90 \mathrm{E}-02$ \\
\hline 12201 & В09X09E & $3.90 \mathrm{E}+03$ & $1.06 \mathrm{E}-01$ & $2.46 \mathrm{E}-01$ & $6.78 \mathrm{E}+02$ & $2.02 \mathrm{E}-01$ & $9.63 \mathrm{E}-01$ & $9.28 \mathrm{E}+02$ & 7.97E-02 \\
\hline & B09X09A & $3.33 \mathrm{E}+03$ & $9.74 \mathrm{E}-05$ & $1.97 \mathrm{E}-04$ & 5.39E-01 & $1.56 \mathrm{E}-04$ & $6.81 \mathrm{E}-04$ & $7.20 \mathrm{E}-01$ & $5.68 \mathrm{E}-05$ \\
\hline 12285 & B09X09C & $2.73 E+03$ & $5.28 \mathrm{E}-03$ & $8.80 \mathrm{E}-03$ & $44 \mathrm{E}+01$ & $6.91 \mathrm{E}-03$ & 2.49E-02 & $3.18 \mathrm{E}+01$ & $2.26 \mathrm{E}-03$ \\
\hline
\end{tabular}


Table E-2. Composition of Segregated Fuel Sludge by Key and Fuel Model (1 ${ }^{\text {st }}$ Part). (5 sheets)

\begin{tabular}{|c|c|c|c|c|c|c|c|c|c|}
\hline Key ID & $\begin{array}{l}\text { Fuel } \\
\text { Model }\end{array}$ & $\begin{array}{l}\text { Exposure } \\
\text { MWd/MTU) }\end{array}$ & $\begin{array}{c}\text { Uranium } \\
\text { (MTU) }\end{array}$ & $\begin{array}{c}\mathrm{Co}-60 \\
\text { (Ci) }\end{array}$ & $\begin{array}{l}\text { Sr-90 } \\
\text { (Ci) }\end{array}$ & $\begin{array}{l}\text { Tc-99 } \\
\text { (Ci) }\end{array}$ & $\begin{array}{c}\text { Cs-134 } \\
\text { (Ci) }\end{array}$ & $\begin{array}{l}\text { Cs-137 } \\
\text { (Ci) }\end{array}$ & $\begin{array}{c}\text { Eu-152 } \\
\text { (Ci) }\end{array}$ \\
\hline 12285 & B09X09S & $3.77 \mathrm{E}+03$ & 4.22E-02 & $9.76 \mathrm{E}-02$ & $2.63 \mathrm{E}+02$ & 7.76E-02 & $3.85 \mathrm{E}-01$ & $3.59 \mathrm{E}+02$ & $3.03 \mathrm{E}-02$ \\
\hline 12285 & 09X09E & +03 & & E-01 & $14 \mathrm{E}+03$ & & $2.61 \mathrm{E}+00$ & +03 & -01 \\
\hline 12480 & B09X09A & $3.56 \mathrm{E}+03$ & $2.79 \mathrm{E}-03$ & $6.52 \mathrm{E}-03$ & $1.67 \mathrm{E}+01$ & 4.82E-03 & $2.70 \mathrm{E}-02$ & $2.25 \mathrm{E}+01$ & $1.88 \mathrm{E}-03$ \\
\hline 12480 & B09X09C & $2.91 \mathrm{E}+03$ & $1.19 \mathrm{E}-03$ & $2.27 \mathrm{E}-03$ & $5.91 \mathrm{E}+00$ & $1.66 \mathrm{E}-03$ & 7.62E-03 & $7.75 \mathrm{E}+00$ & $5.79 \mathrm{E}-04$ \\
\hline 12480 & B09X09S & $3.58 \mathrm{E}+03$ & & 4.22E-02 & $1.08 \mathrm{E}+02$ & $3.12 \mathrm{E}-02$ & $1.76 \mathrm{E}-01$ & $1.46 \mathrm{E}+02$ & $1.22 \mathrm{E}-02$ \\
\hline 12480 & B09X09E & & 01 & $10 \mathrm{E}-01$ & $1.32 \mathrm{E}+03$ & $76 \mathrm{E}-01$ & $1.93 \mathrm{E}+00$ & +03 & $39 \mathrm{E}-01$ \\
\hline 12565 & B09X09A & $3.30 \mathrm{E}+03$ & $1.02 \mathrm{E}-03$ & $2.27 \mathrm{E}-03$ & $5.70 \mathrm{E}+00$ & $1.62 \mathrm{E}-03$ & $9.07 \mathrm{E}-03$ & $7.60 \mathrm{E}+00$ & $6.11 \mathrm{E}-04$ \\
\hline 12565 & B09X09C & $3.15 \mathrm{E}+03$ & 4.89E-04 & $1.04 \mathrm{E}-03$ & $2.62 \mathrm{E}+00$ & 7.41E-04 & $3.96 \mathrm{E}-03$ & $3.47 \mathrm{E}+00$ & $2.72 \mathrm{E}-04$ \\
\hline 12565 & B09X09S & $3.11 \mathrm{E}+03$ & $1.28 \mathrm{E}-02$ & $2.68 \mathrm{E}-02$ & $6.78 \mathrm{E}+01$ & $1.91 \mathrm{E}-02$ & $1.01 \mathrm{E}-01$ & $8.96 \mathrm{E}+01$ & $6.98 \mathrm{E}-03$ \\
\hline 12565 & B09X09E & $3.61 \mathrm{E}+03$ & $1.22 \mathrm{E}-01$ & $2.98 \mathrm{E}-01$ & $7.43 \mathrm{E}+02$ & $2.14 \mathrm{E}-01$ & $1.31 \mathrm{E}+00$ & $1.00 \mathrm{E}+03$ & $.47 \mathrm{E}-02$ \\
\hline 12639 & B09X09S & $3.64 \mathrm{E}+03$ & $2.00 \mathrm{E}-02$ & $5.07 \mathrm{E}-02$ & $1.24 \mathrm{E}+02$ & $3.54 \mathrm{E}-02$ & $2.35 \mathrm{E}-01$ & $1.67 \mathrm{E}+02$ & $1.43 \mathrm{E}-02$ \\
\hline 12639 & B09X09E & $3.09 \mathrm{E}+03$ & $1.00 \mathrm{~L}-01$ & $3.19 \mathrm{E}-01$ & $7.90 \mathrm{E}+02$ & $2.22 \mathrm{E}-01$ & $1.24 \mathrm{E}+00$ & $1.04 \mathrm{E}+03$ & $8.14 \mathrm{E}-02$ \\
\hline 12639 & $\mathrm{~B} 07 \mathrm{X} 07 \mathrm{E}$ & $3.03 \mathrm{E}+03$ & $66 \mathrm{E}-05$ & $82 \mathrm{E}-04$ & $4.50 \mathrm{E}-01$ & $26 \mathrm{E}-04$ & $6.93 \mathrm{E}-04$ & $5.93 \mathrm{E}-01$ & $4.58 \mathrm{E}-05$ \\
\hline 12852 & B09X09E & $4.77 \mathrm{E}+03$ & & $3.56 \mathrm{E}-02$ & $7.93 \mathrm{E}+01$ & $2.33 \mathrm{E}-02$ & $2.53 \mathrm{E}-01$ & $1.12 \mathrm{E}+02$ & $1.14 \mathrm{E}-02$ \\
\hline 13015 & B09X09C & $2.12 \mathrm{E}+03$ & & $2.46 \mathrm{E}-03$ & $5.59 \mathrm{E}+00$ & $1.47 \mathrm{E}-03$ & $8.06 \mathrm{E}-03$ & $7.03 \mathrm{E}+00$ & 4.36E-04 \\
\hline 13015 & 09X09E & $2.96 \mathrm{E}+03$ & $40 \mathrm{E}-03$ & $1.03 \mathrm{E}-02$ & $2.29 \mathrm{E}+01$ & $6.24 \mathrm{E}-03$ & 4.74E-02 & $3.00 \mathrm{E}+01$ & $2.36 \mathrm{E}-03$ \\
\hline 13016 & B09X09S & $16 \mathrm{~F}+03$ & $1.48 \mathrm{E}-04$ & $2.54 \mathrm{E}-04$ & $5.75 \mathrm{E}-01$ & $1.51 \mathrm{E}-04$ & $8.46 \mathrm{E}-04$ & $7.26 \mathrm{E}-01$ & 4.57E- 05 \\
\hline 13371 & B09X09E & & & $1.49 \mathrm{E}-04$ & $3.13 \mathrm{E}-01$ & 7.57E-05 & $2.60 \mathrm{E}-04$ & 3.69E-01 & $1.08 \mathrm{E}-05$ \\
\hline 13649 & 09X09C & $2.14 \mathrm{E}+03$ & 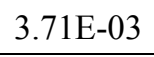 & $7.96 \mathrm{E}-03$ & $1.50 \mathrm{E}+01$ & $3.77 \mathrm{E}-03$ & $3.74 \mathrm{E}-02$ & $1.88 \mathrm{E}+01$ & $1.23 \mathrm{E}-03$ \\
\hline 13649 & 09X09E & $2.85 \mathrm{E}+03$ & $1 \mathrm{E}-02$ & $12 \mathrm{E}-02$ & $1.32 \mathrm{E}+02$ & $3.42 \mathrm{E}-02$ & $4.50 \mathrm{E}-01$ & $1.72 \mathrm{E}+02$ & $1.39 \mathrm{E}-02$ \\
\hline 13649 & B07X07E & $1.92 \mathrm{E}+03$ & $2.36 \mathrm{E}-03$ & 4.57E-03 & $8.62 \mathrm{E}+00$ & $2.15 \mathrm{E}-03$ & $1.92 \mathrm{E}-02$ & $1.07 \mathrm{E}+01$ & $6.40 \mathrm{E}-04$ \\
\hline 13685 & B09X09E & $3.01 \mathrm{E}+03$ & & $1.03 \mathrm{E}-02$ & $1.89 \mathrm{E}+01$ & 4.92E-03 & 7.05E-02 & $2.48 \mathrm{E}+01$ & $2.06 \mathrm{E}-03$ \\
\hline 14355 & 09X09C & $3.40 \mathrm{E}+03$ & $.23 \mathrm{E}-03$ & 37E-03 & $7.94 \mathrm{E}+00$ & $2.02 \mathrm{E}-03$ & $6.04 \mathrm{E}-02$ & $1.06 \mathrm{E}+01$ & $9.92 \mathrm{E}-04$ \\
\hline 14355 & B09X09S & $3.40 \mathrm{E}+03$ & 4.07E-03 & $1.78 \mathrm{E}-02$ & $2.63 \mathrm{E}+01$ & $6.67 \mathrm{E}-03$ & $2.00 \mathrm{E}-01$ & $3.51 \mathrm{E}+01$ & $3.28 \mathrm{E}-03$ \\
\hline & B09X09E & & & & & & & & $6.49 \mathrm{E}-02$ \\
\hline 14356 & B09X09A & $3.33 \mathrm{E}+03$ & $6.28 \mathrm{E}-01$ & $2.68 \mathrm{E}+00$ & $3.97 \mathrm{E}+03$ & $1.01 \mathrm{E}+00$ & $2.95 \mathrm{E}+01$ & $5.29 \mathrm{E}+03$ & $4.89 \mathrm{E}-01$ \\
\hline 14356 & B09X09S & $3.33 \mathrm{E}+03$ & $2.11 \mathrm{E}-02$ & $9.03 \mathrm{E}-02$ & $1.34 \mathrm{E}+02$ & 3.39E-02 & $9.93 \mathrm{E}-01$ & $1.78 \mathrm{E}+02$ & $1.65 \mathrm{E}-02$ \\
\hline 14356 & B09X09E & $3.33 \mathrm{E}+03$ & 7.22E-02 & 3.09E-01 & $4.57 \mathrm{E}+02$ & $1.16 \mathrm{E}-01$ & $3.40 \mathrm{E}+00$ & $6.09 \mathrm{E}+02$ & $5.63 \mathrm{E}-02$ \\
\hline 15204 & B09X09A & $9.10 \mathrm{E}+02$ & $7.16 \mathrm{E}-03$ & 1.19E-02 & $1.46 \mathrm{E}+01$ & $3.13 \mathrm{E}-03$ & 5.81E-02 & $1.71 \mathrm{E}+01$ & $5.75 \mathrm{E}-04$ \\
\hline 15204 & B09X09C & $6.89 \mathrm{E}+02$ & $1.58 \mathrm{E}-03$ & $2.08 \mathrm{E}-03$ & $2.54 \mathrm{E}+00$ & $5.46 \mathrm{E}-04$ & $1.01 \mathrm{E}-02$ & $2.98 \mathrm{E}+00$ & $1.00 \mathrm{E}-04$ \\
\hline 15204 & B09X09S & $1.02 \mathrm{E}+03$ & $1.01 \mathrm{E}-02$ & $1.83 \mathrm{E}-02$ & $2.24 \mathrm{E}+01$ & $4.82 \mathrm{E}-03$ & $8.94 \mathrm{E}-02$ & $2.63 \mathrm{E}+01$ & $8.86 \mathrm{E}-04$ \\
\hline 15204 & B09X09E & $1.02 \mathrm{E}+03$ & $2.98 \mathrm{E}-02$ & $5.43 \mathrm{E}-02$ & $6.64 \mathrm{E}+01$ & $1.43 \mathrm{E}-02$ & $2.65 \mathrm{E}-01$ & $7.80 \mathrm{E}+01$ & $2.62 \mathrm{E}-03$ \\
\hline 15204 & $\mathrm{~B} 07 \mathrm{X} 07 \mathrm{E}$ & $7.33 \mathrm{E}+02$ & $1.10 \mathrm{E}-04$ & $2.43 \mathrm{E}-04$ & $2.98 \mathrm{E}-01$ & $6.40 \mathrm{E}-05$ & 1.19E-03 & $3.50 \mathrm{E}-01$ & $1.18 \mathrm{E}-05$ \\
\hline 15316 & B09X09A & $9.84 \mathrm{E}+02$ & $6.03 \mathrm{E}-03$ & $1.11 \mathrm{E}-02$ & $1.32 \mathrm{E}+01$ & $2.81 \mathrm{E}-03$ & $5.78 \mathrm{E}-02$ & $1.55 \mathrm{E}+01$ & $5.25 \mathrm{E}-04$ \\
\hline
\end{tabular}




\section{SNF-10293, REV 0}

Table E-2. Composition of Segregated Fuel Sludge by Key and Fuel Model ( $1^{\text {st }}$ Part). ( 5 sheets)

\begin{tabular}{|c|c|c|c|c|c|c|c|c|c|}
\hline Key ID & $\begin{array}{c}\text { Fuel } \\
\text { Model }\end{array}$ & $\begin{array}{c}\text { Exposure } \\
\text { (MWd/MTU) }\end{array}$ & $\begin{array}{c}\text { Uranium } \\
\text { (MTU) }\end{array}$ & $\begin{array}{c}\text { Co-60 } \\
\text { (Ci) }\end{array}$ & $\begin{array}{c}\text { Sr-90 } \\
\text { (Ci) }\end{array}$ & $\begin{array}{c}\text { Tc-99 } \\
\text { (Ci) }\end{array}$ & $\begin{array}{c}\text { Cs-134 } \\
(\mathbf{C i})\end{array}$ & $\begin{array}{c}\text { Cs-137 } \\
\text { (Ci) }\end{array}$ & $\begin{array}{c}\text { Eu-152 } \\
\text { (Ci) }\end{array}$ \\
\hline 15316 & B09X09C & $7.76 \mathrm{E}+02$ & $1.12 \mathrm{E}-04$ & $1.69 \mathrm{E}-04$ & $2.01 \mathrm{E}-01$ & $4.27 \mathrm{E}-05$ & $8.80 \mathrm{E}-04$ & $2.35 \mathrm{E}-01$ & $7.98 \mathrm{E}-06$ \\
\hline 15316 & B09X09S & $9.50 \mathrm{E}+02$ & $2.60 \mathrm{E}-03$ & $4.67 \mathrm{E}-03$ & $5.53 \mathrm{E}+00$ & $1.18 \mathrm{E}-03$ & $2.43 \mathrm{E}-02$ & $6.49 \mathrm{E}+00$ & $2.20 \mathrm{E}-04$ \\
\hline 15316 & B09X09E & $1.03 \mathrm{E}+03$ & $2.99 \mathrm{E}-03$ & $5.71 \mathrm{E}-03$ & $6.76 \mathrm{E}+00$ & $1.44 \mathrm{E}-03$ & $2.97 \mathrm{E}-02$ & $7.94 \mathrm{E}+00$ & $2.69 \mathrm{E}-04$ \\
\hline 15399 & B09X09E & $1.69 \mathrm{E}+00$ & $3.75 \mathrm{E}-03$ & $2.45 \mathrm{E}-05$ & $2.84 \mathrm{E}-02$ & $6.01 \mathrm{E}-06$ & $1.34 \mathrm{E}-04$ & $3.33 \mathrm{E}-02$ & $1.14 \mathrm{E}-06$ \\
\hline 15451 & B09X09A & $1.30 \mathrm{E}+03$ & $4.77 \mathrm{E}-03$ & $1.19 \mathrm{E}-02$ & $1.34 \mathrm{E}+01$ & $2.88 \mathrm{E}-03$ & $8.61 \mathrm{E}-02$ & $1.60 \mathrm{E}+01$ & $7.06 \mathrm{E}-04$ \\
\hline 15451 & B09X09C & $1.33 \mathrm{E}+03$ & $6.52 \mathrm{E}-05$ & $1.67 \mathrm{E}-04$ & $1.88 \mathrm{E}-01$ & $4.05 \mathrm{E}-05$ & $1.25 \mathrm{E}-03$ & $2.25 \mathrm{E}-01$ & $1.03 \mathrm{E}-05$ \\
\hline 15451 & B09X09S & $4.57 \mathrm{E}+02$ & $3.08 \mathrm{E}-05$ & $3.06 \mathrm{E}-05$ & $3.49 \mathrm{E}-02$ & $7.36 \mathrm{E}-06$ & $1.72 \mathrm{E}-04$ & $4.09 \mathrm{E}-02$ & $1.40 \mathrm{E}-06$ \\
\hline 15451 & B07X07E & $5.61 \mathrm{E}+02$ & $1.05 \mathrm{E}-03$ & $1.26 \mathrm{E}-03$ & $1.43 \mathrm{E}+00$ & $3.02 \mathrm{E}-04$ & $7.04 \mathrm{E}-03$ & $1.68 \mathrm{E}+00$ & $5.75 \mathrm{E}-05$ \\
\hline 15458 & B09X09A & $7.34 \mathrm{E}+02$ & $4.26 \mathrm{E}-03$ & $6.49 \mathrm{E}-03$ & $7.37 \mathrm{E}+00$ & $1.56 \mathrm{E}-03$ & $3.65 \mathrm{E}-02$ & $8.64 \mathrm{E}+00$ & $2.96 \mathrm{E}-04$ \\
\hline 15458 & B09X09C & $4.44 \mathrm{E}+02$ & $5.21 \mathrm{E}-04$ & $5.04 \mathrm{E}-04$ & $5.72 \mathrm{E}-01$ & $1.21 \mathrm{E}-04$ & $2.83 \mathrm{E}-03$ & $6.71 \mathrm{E}-01$ & $2.30 \mathrm{E}-05$ \\
\hline 15458 & B09X09S & $6.11 \mathrm{E}+02$ & $8.08 \mathrm{E}-03$ & $1.05 \mathrm{E}-02$ & $1.19 \mathrm{E}+01$ & $2.51 \mathrm{E}-03$ & $5.88 \mathrm{E}-02$ & $1.39 \mathrm{E}+01$ & $4.78 \mathrm{E}-04$ \\
\hline 15458 & B09X09E & $5.99 \mathrm{E}+02$ & $1.35 \mathrm{E}-02$ & $1.72 \mathrm{E}-02$ & $1.95 \mathrm{E}+01$ & $4.12 \mathrm{E}-03$ & $9.67 \mathrm{E}-02$ & $2.29 \mathrm{E}+01$ & $7.85 \mathrm{E}-04$ \\
\hline 15458 & B09X09R & $4.13 \mathrm{E}+02$ & $7.47 \mathrm{E}-05$ & $6.77 \mathrm{E}-05$ & $7.69 \mathrm{E}-02$ & $1.62 \mathrm{E}-05$ & $3.81 \mathrm{E}-04$ & $9.02 \mathrm{E}-02$ & $3.09 \mathrm{E}-06$ \\
\hline 15458 & B07X07E & $4.20 \mathrm{E}+02$ & $7.49 \mathrm{E}-05$ & $6.89 \mathrm{E}-05$ & $7.83 \mathrm{E}-02$ & $1.65 \mathrm{E}-05$ & $3.88 \mathrm{E}-04$ & $9.18 \mathrm{E}-02$ & $3.15 \mathrm{E}-06$ \\
\hline
\end{tabular}

Values were generated using the methods described in HNF-8760. The effective date of these numbers is May 31, 1998.

The shortened form of the fuel model identifier uses only the first and last characters. 
Table E-3. Composition of Segregated Fuel Sludge by Key and Fuel Model (2 ${ }^{\text {nd }}$ Part). (5 sheets)

\begin{tabular}{|c|c|c|c|c|c|c|c|c|c|}
\hline Key ID & $\begin{array}{c}\text { Fuel } \\
\text { Model }\end{array}$ & $\begin{array}{c}\text { Eu-154 } \\
\text { (Ci) }\end{array}$ & $\begin{array}{c}\text { Eu-155 } \\
\text { (Ci) }\end{array}$ & $\begin{array}{c}\text { Np-237 } \\
\text { (Ci) }\end{array}$ & $\begin{array}{c}\text { Pu-238 } \\
\text { (Ci) }\end{array}$ & $\begin{array}{c}\text { Pu-239 } \\
\text { (Ci) }\end{array}$ & $\begin{array}{c}\text { Pu-240 } \\
\text { (Ci) }\end{array}$ & $\begin{array}{c}\text { Pu-241 } \\
\text { (Ci) }\end{array}$ & $\begin{array}{c}\text { Am-241 } \\
\text { (Ci) }\end{array}$ \\
\hline 10001 & B09X09A & $5.26 \mathrm{E}-04$ & $1.07 \mathrm{E}-04$ & $6.97 \mathrm{E}-07$ & $9.11 \mathrm{E}-04$ & $3.49 \mathrm{E}-03$ & $1.52 \mathrm{E}-03$ & 4.47E-02 & $3.74 \mathrm{E}-03$ \\
\hline 10001 & B09X09C & 4.13E-03 & $8.41 \mathrm{E}-04$ & $5.47 \mathrm{E}-06$ & $7.15 \mathrm{E}-03$ & $2.74 \mathrm{E}-02$ & $1.20 \mathrm{E}-02$ & $3.51 \mathrm{E}-01$ & $2.93 \mathrm{E}-02$ \\
\hline 10001 & B09X09S & $3.97 \mathrm{E}-03$ & $8.10 \mathrm{E}-04$ & $5.27 \mathrm{E}-06$ & $6.89 \mathrm{E}-03$ & $2.63 \mathrm{E}-02$ & $1.15 \mathrm{E}-02$ & $3.38 \mathrm{E}-01$ & $2.82 \mathrm{E}-02$ \\
\hline 10001 & B09X09E & $2.37 \mathrm{E}-03$ & 4.84E-04 & $3.15 \mathrm{E}-06$ & $4.11 \mathrm{E}-03$ & $1.57 \mathrm{E}-02$ & $6.88 \mathrm{E}-03$ & $2.02 \mathrm{E}-01$ & $1.69 \mathrm{E}-02$ \\
\hline 10201 & B09X09A & $4.00 \mathrm{E}+00$ & 8.21E-01 & 4.98E-03 & $6.65 \mathrm{E}+00$ & $2.48 \mathrm{E}+01$ & $1.10 \mathrm{E}+01$ & $3.34 \mathrm{E}+02$ & $2.70 \mathrm{E}+01$ \\
\hline 10201 & B09X09S & $1.90 \mathrm{E}+00$ & 2.79E-01 & $1.83 \mathrm{E}-03$ & $3.08 \mathrm{E}+00$ & $8.33 \mathrm{E}+00$ & $4.46 \mathrm{E}+00$ & $1.55 \mathrm{E}+02$ & $1.25 \mathrm{E}+01$ \\
\hline 10201 & B09X09E & $2.05 \mathrm{E}+00$ & $3.00 \mathrm{E}-01$ & $1.96 \mathrm{E}-03$ & $3.33 \mathrm{E}+00$ & $8.92 \mathrm{E}+00$ & $4.80 \mathrm{E}+00$ & $1.67 \mathrm{E}+02$ & $1.35 \mathrm{E}+01$ \\
\hline 10259 & B09X09A & $2.49 \mathrm{E}-02$ & $4.01 \mathrm{E}-03$ & $2.53 \mathrm{E}-05$ & 4.01E-02 & $1.18 \mathrm{E}-01$ & $6.03 \mathrm{E}-02$ & $2.06 \mathrm{E}+00$ & $1.65 \mathrm{E}-01$ \\
\hline 10259 & B09X09C & 3.39E-01 & $8.14 \mathrm{E}-02$ & 4.65E-04 & 5.63E-01 & $2.39 \mathrm{E}+00$ & $9.77 \mathrm{E}-01$ & $2.80 \mathrm{E}+01$ & $2.23 \mathrm{E}+00$ \\
\hline 10259 & B09X09E & $3.57 \mathrm{E}+00$ & $7.25 \mathrm{E}-01$ & $4.33 \mathrm{E}-03$ & $5.86 \mathrm{E}+00$ & $2.14 \mathrm{E}+01$ & $9.60 \mathrm{E}+00$ & $2.97 \mathrm{E}+02$ & $2.37 \mathrm{E}+01$ \\
\hline 10294 & B09X09A & $5.24 \mathrm{E}-02$ & 8.76E-03 & $5.42 \mathrm{E}-05$ & $8.41 \mathrm{E}-02$ & $2.56 \mathrm{E}-01$ & $1.28 \mathrm{E}-01$ & $4.37 \mathrm{E}+00$ & $3.47 \mathrm{E}-01$ \\
\hline 10294 & B09X09C & $3.98 \mathrm{E}-01$ & $9.30 \mathrm{E}-02$ & $5.29 \mathrm{E}-04$ & $6.54 \mathrm{E}-01$ & $2.70 \mathrm{E}+00$ & $1.12 \mathrm{E}+00$ & $3.28 \mathrm{E}+01$ & $2.60 \mathrm{E}+00$ \\
\hline 10294 & B09X09S & $5.13 \mathrm{E}-01$ & $9.57 \mathrm{E}-02$ & $5.76 \mathrm{E}-04$ & $8.30 \mathrm{E}-01$ & $2.79 \mathrm{E}+00$ & $1.32 \mathrm{E}+00$ & $4.27 \mathrm{E}+01$ & $3.39 \mathrm{E}+00$ \\
\hline 10294 & B09X09E & $1.43 \mathrm{E}+01$ & $2.94 \mathrm{E}+00$ & $1.73 \mathrm{E}-02$ & $2.33 \mathrm{E}+01$ & $8.55 \mathrm{E}+01$ & $3.82 \mathrm{E}+01$ & $1.18 \mathrm{E}+03$ & $9.39 \mathrm{E}+01$ \\
\hline 10350 & B09X09C & $3.03 \mathrm{E}-01$ & $3.59 \mathrm{E}-02$ & $2.31 \mathrm{E}-04$ & $4.68 \mathrm{E}-01$ & $9.80 \mathrm{E}-01$ & $6.04 \mathrm{E}-01$ & $2.28 \mathrm{E}+01$ & $1.81 \mathrm{E}+00$ \\
\hline 10350 & B09X09S & $6.13 \mathrm{E}-01$ & 8.96E-02 & $5.56 \mathrm{E}-04$ & $9.64 \mathrm{E}-01$ & $2.51 \mathrm{E}+00$ & $1.37 \mathrm{E}+00$ & $4.90 \mathrm{E}+01$ & $3.86 \mathrm{E}+00$ \\
\hline 10350 & B09X09E & $1.75 \mathrm{E}+00$ & $2.45 \mathrm{E}-01$ & $1.53 \mathrm{E}-03$ & $2.74 \mathrm{E}+00$ & $6.83 \mathrm{E}+00$ & $3.82 \mathrm{E}+00$ & $1.38 \mathrm{E}+02$ & $1.09 \mathrm{E}+01$ \\
\hline 10385 & B09X09A & $2.87 \mathrm{E}-01$ & $3.39 \mathrm{E}-02$ & $2.16 \mathrm{E}-04$ & $4.41 \mathrm{E}-01$ & $9.10 \mathrm{E}-01$ & $5.65 \mathrm{E}-01$ & $2.16 \mathrm{E}+01$ & $1.69 \mathrm{E}+00$ \\
\hline 10385 & B09X09S & $1.64 \mathrm{E}-01$ & 2.69E-02 & $1.62 \mathrm{E}-04$ & $2.57 \mathrm{E}-01$ & 7.57E-01 & $3.86 \mathrm{E}-01$ & $1.34 \mathrm{E}+01$ & $1.05 \mathrm{E}+00$ \\
\hline 10385 & B09X09E & $1.21 \mathrm{E}+01$ & $2.05 \mathrm{E}+00$ & $1.22 \mathrm{E}-02$ & $1.91 \mathrm{E}+01$ & $5.77 \mathrm{E}+01$ & $2.90 \mathrm{E}+01$ & $1.00 \mathrm{E}+03$ & $7.83 \mathrm{E}+01$ \\
\hline 10456 & B09X09A & $2.69 \mathrm{E}-01$ & $3.34 \mathrm{E}-02$ & $2.06 \mathrm{E}-04$ & $4.08 \mathrm{E}-01$ & $8.79 \mathrm{E}-01$ & $5.33 \mathrm{E}-01$ & $2.03 \mathrm{E}+01$ & $1.57 \mathrm{E}+00$ \\
\hline 10456 & B09X09C & $4.70 \mathrm{E}-01$ & $8.68 \mathrm{E}-02$ & $4.96 \mathrm{E}-04$ & 7.34E-01 & $2.38 \mathrm{E}+00$ & $1.15 \mathrm{E}+00$ & $3.86 \mathrm{E}+01$ & $2.97 \mathrm{E}+00$ \\
\hline 10456 & B09X09S & $2.02 \mathrm{E}+00$ & $2.55 \mathrm{E}-01$ & $1.56 \mathrm{E}-03$ & $3.07 \mathrm{E}+00$ & $6.71 \mathrm{E}+00$ & $4.03 \mathrm{E}+00$ & $1.53 \mathrm{E}+02$ & $1.18 \mathrm{E}+01$ \\
\hline 10456 & B09X09E & $1.50 \mathrm{E}+01$ & $1.78 \mathrm{E}+00$ & $1.10 \mathrm{E}-02$ & $2.26 \mathrm{E}+01$ & $4.65 \mathrm{E}+01$ & $2.89 \mathrm{E}+01$ & $1.11 \mathrm{E}+03$ & $8.64 \mathrm{E}+01$ \\
\hline 10576 & B09X09S & $1.18 \mathrm{E}-02$ & $1.18 \mathrm{E}-03$ & 7.24E-06 & $1.72 \mathrm{E}-02$ & $2.88 \mathrm{E}-02$ & $2.00 \mathrm{E}-02$ & 8.27E-01 & $6.29 \mathrm{E}-02$ \\
\hline 10576 & B09X09E & 4.66E-01 & 7.66E-02 & $4.30 \mathrm{E}-04$ & 7.05E-01 & $1.99 \mathrm{E}+00$ & $1.04 \mathrm{E}+00$ & $3.73 \mathrm{E}+01$ & $2.82 \mathrm{E}+00$ \\
\hline 10602 & B09X09A & $6.49 \mathrm{E}-01$ & 7.97E-02 & 4.67E-04 & $9.54 \mathrm{E}-01$ & $1.98 \mathrm{E}+00$ & $1.22 \mathrm{E}+00$ & $4.79 \mathrm{E}+01$ & $3.61 \mathrm{E}+00$ \\
\hline 10602 & B09X09C & 4.13E-02 & 4.19E-03 & $2.54 \mathrm{E}-05$ & $5.98 \mathrm{E}-02$ & $1.01 \mathrm{E}-01$ & 7.01E-02 & $2.89 \mathrm{E}+00$ & $2.19 \mathrm{E}-01$ \\
\hline 10602 & B09X09S & $1.65 \mathrm{E}+00$ & $1.67 \mathrm{E}-01$ & $1.01 \mathrm{E}-03$ & $2.39 \mathrm{E}+00$ & $4.03 \mathrm{E}+00$ & $2.79 \mathrm{E}+00$ & $1.16 \mathrm{E}+02$ & $8.74 \mathrm{E}+00$ \\
\hline 10602 & B09X09E & $2.48 \mathrm{E}+01$ & $3.01 \mathrm{E}+00$ & $1.77 \mathrm{E}-02$ & $3.65 \mathrm{E}+01$ & $7.44 \mathrm{E}+01$ & $4.64 \mathrm{E}+01$ & $1.82 \mathrm{E}+03$ & $1.38 \mathrm{E}+02$ \\
\hline 10679 & B09X09A & 7.29E-01 & 7.16E-02 & $4.25 \mathrm{E}-04$ & $1.04 \mathrm{E}+00$ & $1.67 \mathrm{E}+00$ & $1.19 \mathrm{E}+00$ & $5.01 \mathrm{E}+01$ & $3.75 \mathrm{E}+00$ \\
\hline 10679 & B09X09C & $6.41 \mathrm{E}-01$ & $1.16 \mathrm{E}-01$ & $6.16 \mathrm{E}-04$ & $9.55 \mathrm{E}-01$ & $2.92 \mathrm{E}+00$ & $1.45 \mathrm{E}+00$ & $5.18 \mathrm{E}+01$ & $3.83 \mathrm{E}+00$ \\
\hline 10679 & B09X09S & $8.61 \mathrm{E}-01$ & $1.32 \mathrm{E}-01$ & $7.24 \mathrm{E}-04$ & $1.27 \mathrm{E}+00$ & $3.27 \mathrm{E}+00$ & $1.79 \mathrm{E}+00$ & $6.67 \mathrm{E}+01$ & $4.95 \mathrm{E}+00$ \\
\hline 10679 & B09X09E & $2.68 \mathrm{E}+01$ & $2.91 \mathrm{E}+00$ & $1.70 \mathrm{E}-02$ & $3.84 \mathrm{E}+01$ & $6.87 \mathrm{E}+01$ & $4.61 \mathrm{E}+01$ & $1.89 \mathrm{E}+03$ & $1.41 \mathrm{E}+02$ \\
\hline
\end{tabular}


Table E-3. Composition of Segregated Fuel Sludge by Key and Fuel Model (2 ${ }^{\text {nd }}$ Part $)$. (5 sheets)

\begin{tabular}{|c|c|c|c|c|c|c|c|c|c|}
\hline Key ID & $\begin{array}{c}\text { Fuel } \\
\text { Model }\end{array}$ & $\begin{array}{c}\text { Eu-154 } \\
\text { (Ci) }\end{array}$ & $\begin{array}{c}\text { Eu-155 } \\
\text { (Ci) }\end{array}$ & $\begin{array}{l}\text { Np-237 } \\
\text { (Ci) }\end{array}$ & $\begin{array}{c}\text { Pu-238 } \\
\text { (Ci) }\end{array}$ & $\begin{array}{c}\text { Pu-239 } \\
\text { (Ci) }\end{array}$ & $\begin{array}{c}\text { Pu-240 } \\
\text { (Ci) }\end{array}$ & $\begin{array}{c}\text { Pu-241 } \\
\text { (Ci) }\end{array}$ & $\begin{array}{c}\text { Am-241 } \\
\text { (Ci) }\end{array}$ \\
\hline 10742 & B09X09C & 8.61E-01 & $1.40 \mathrm{E}-01$ & 7.43E-04 & $1.26 \mathrm{E}+00$ & $3.40 \mathrm{E}+00$ & $1.81 \mathrm{E}+00$ & $6.72 \mathrm{E}+01$ & $4.92 \mathrm{E}+00$ \\
\hline 10742 & B09X09S & $3.97 \mathrm{E}-01$ & 5.92E-02 & $3.19 \mathrm{E}-04$ & $5.75 \mathrm{E}-01$ & $1.42 \mathrm{E}+00$ & 7.95E-01 & $3.03 \mathrm{E}+01$ & $2.22 \mathrm{E}+00$ \\
\hline 10742 & B09X09E & $1.39 \mathrm{E}+01$ & $1.74 \mathrm{E}+00$ & $9.67 \mathrm{E}-03$ & $1.99 \mathrm{E}+01$ & $4.09 \mathrm{E}+01$ & $2.53 \mathrm{E}+01$ & $1.01 \mathrm{E}+03$ & $7.44 \mathrm{E}+01$ \\
\hline 10764 & B09X09A & 8.69E-03 & $2.00 \mathrm{E}-03$ & $9.74 \mathrm{E}-06$ & $1.29 \mathrm{E}-02$ & $4.87 \mathrm{E}-02$ & $2.13 \mathrm{E}-02$ & $6.90 \mathrm{E}-01$ & $5.01 \mathrm{E}-02$ \\
\hline 10764 & B09X09S & $1.39 \mathrm{E}-02$ & $3.21 \mathrm{E}-03$ & $1.56 \mathrm{E}-05$ & $2.07 \mathrm{E}-02$ & 7.79E-02 & $3.41 \mathrm{E}-02$ & $1.10 \mathrm{E}+00$ & 8.03E-02 \\
\hline 10764 & B09X09E & $7.31 \mathrm{E}-03$ & $1.68 \mathrm{E}-03$ & $8.19 \mathrm{E}-06$ & $1.09 \mathrm{E}-02$ & $4.09 \mathrm{E}-02$ & $1.79 \mathrm{E}-02$ & $5.80 \mathrm{E}-01$ & 4.22E-02 \\
\hline 10764 & B09X09R & 7.12E-03 & $1.64 \mathrm{E}-03$ & $7.98 \mathrm{E}-06$ & $1.06 \mathrm{E}-02$ & $3.99 \mathrm{E}-02$ & $1.74 \mathrm{E}-02$ & $5.65 \mathrm{E}-01$ & 4.11E-02 \\
\hline 10764 & $\mathrm{~B} 07 \mathrm{X} 07 \mathrm{E}$ & $8.77 \mathrm{E}-04$ & $2.02 \mathrm{E}-04$ & $9.83 \mathrm{E}-07$ & $1.31 \mathrm{E}-03$ & 4.91E-03 & $2.15 \mathrm{E}-03$ & $6.96 \mathrm{E}-02$ & $5.06 \mathrm{E}-03$ \\
\hline 10770 & B09X09C & $4.20 \mathrm{E}-01$ & $6.01 \mathrm{E}-02$ & $3.23 \mathrm{E}-04$ & $6.02 \mathrm{E}-01$ & $1.42 \mathrm{E}+00$ & $8.15 \mathrm{E}-01$ & $3.15 \mathrm{E}+01$ & $2.30 \mathrm{E}+00$ \\
\hline 10770 & B09X09S & $4.25 \mathrm{E}+00$ & 4.64E-01 & $2.61 \mathrm{E}-03$ & $5.97 \mathrm{E}+00$ & $1.06 \mathrm{E}+01$ & $7.12 \mathrm{E}+00$ & $2.97 \mathrm{E}+02$ & $2.18 \mathrm{E}+01$ \\
\hline 10770 & B09X09E & $1.39 \mathrm{E}+01$ & $1.49 \mathrm{E}+00$ & $8.42 \mathrm{E}-03$ & $1.95 \mathrm{E}+01$ & $3.39 \mathrm{E}+01$ & $2.31 \mathrm{E}+01$ & $9.65 \mathrm{E}+02$ & $7.08 \mathrm{E}+01$ \\
\hline 10832 & B09X09A & $1.44 \mathrm{E}-01$ & $2.55 \mathrm{E}-02$ & $1.29 \mathrm{E}-04$ & $2.07 \mathrm{E}-01$ & $6.04 \mathrm{E}-01$ & $3.08 \mathrm{E}-01$ & $1.13 \mathrm{E}+01$ & $8.15 \mathrm{E}-01$ \\
\hline 10832 & B09X09C & 8.33E-01 & $1.43 \mathrm{E}-01$ & 7.27E-04 & $1.20 \mathrm{E}+00$ & $3.37 \mathrm{E}+00$ & $1.75 \mathrm{E}+00$ & $6.51 \mathrm{E}+01$ & $4.69 \mathrm{E}+00$ \\
\hline 10832 & B09X09S & $2.08 \mathrm{E}+00$ & $2.64 \mathrm{E}-01$ & $1.42 \mathrm{E}-03$ & $2.91 \mathrm{E}+00$ & $5.99 \mathrm{E}+00$ & $3.71 \mathrm{E}+00$ & $1.50 \mathrm{E}+02$ & $1.08 \mathrm{E}+01$ \\
\hline 10832 & B09X09E & $2.52 \mathrm{E}+01$ & $3.67 \mathrm{E}+00$ & $1.92 \mathrm{E}-02$ & $3.58 \mathrm{E}+01$ & $8.46 \mathrm{E}+01$ & $4.85 \mathrm{E}+01$ & $1.89 \mathrm{E}+03$ & $1.36 \mathrm{E}+02$ \\
\hline 10982 & B09X09A & $3.02 \mathrm{E}-01$ & 3.95E-02 & $2.00 \mathrm{E}-04$ & 4.11E-01 & $8.47 \mathrm{E}-01$ & $5.23 \mathrm{E}-01$ & $2.15 \mathrm{E}+01$ & $1.51 \mathrm{E}+00$ \\
\hline 10982 & $\mathrm{~B} 09 \mathrm{X} 09 \mathrm{C}$ & $4.75 \mathrm{E}-01$ & 7.22E-02 & $3.56 \mathrm{E}-04$ & $6.56 \mathrm{E}-01$ & $1.58 \mathrm{E}+00$ & $8.93 \mathrm{E}-01$ & $3.53 \mathrm{E}+01$ & $2.47 \mathrm{E}+00$ \\
\hline 10982 & B09X09S & $1.08 \mathrm{E}+00$ & $1.21 \mathrm{E}-01$ & $6.29 \mathrm{E}-04$ & $1.45 \mathrm{E}+00$ & $2.54 \mathrm{E}+00$ & $1.72 \mathrm{E}+00$ & $7.37 \mathrm{E}+01$ & $5.19 \mathrm{E}+00$ \\
\hline 10982 & B09X09E & $1.88 \mathrm{E}+01$ & $1.96 \mathrm{E}+00$ & $1.03 \mathrm{E}-02$ & $2.51 \mathrm{E}+01$ & $4.08 \mathrm{E}+01$ & $2.88 \mathrm{E}+01$ & $1.26 \mathrm{E}+03$ & $8.88 \mathrm{E}+01$ \\
\hline 11036 & B09X09A & $6.83 \mathrm{E}-03$ & $8.68 \mathrm{E}-04$ & $4.33 \mathrm{E}-06$ & $9.19 \mathrm{E}-03$ & $1.81 \mathrm{E}-02$ & $1.15 \mathrm{E}-02$ & $4.80 \mathrm{E}-01$ & $3.34 \mathrm{E}-02$ \\
\hline 11036 & B09X09C & $2.52 \mathrm{E}-01$ & $3.10 \mathrm{E}-02$ & $1.56 \mathrm{E}-04$ & $3.37 \mathrm{E}-01$ & $6.45 \mathrm{E}-01$ & 4.15E-01 & $1.75 \mathrm{E}+01$ & $1.22 \mathrm{E}+00$ \\
\hline 11036 & B09X09S & $6.27 \mathrm{E}-01$ & 7.13E-02 & $3.63 \mathrm{E}-04$ & $8.35 \mathrm{E}-01$ & $1.47 \mathrm{E}+00$ & 9.89E-01 & $4.27 \mathrm{E}+01$ & $2.98 \mathrm{E}+00$ \\
\hline 11036 & B09X09E & $6.91 \mathrm{E}+00$ & 7.99E-01 & 4.06E-03 & $9.23 \mathrm{E}+00$ & $1.65 \mathrm{E}+01$ & $1.10 \mathrm{E}+01$ & $4.74 \mathrm{E}+02$ & $3.30 \mathrm{E}+01$ \\
\hline 11109 & B09X09A & $3.04 \mathrm{E}-01$ & 2.64E-02 & $1.37 \mathrm{E}-04$ & $3.91 \mathrm{E}-01$ & $5.08 \mathrm{E}-01$ & $4.05 \mathrm{E}-01$ & $1.91 \mathrm{E}+01$ & $1.32 \mathrm{E}+00$ \\
\hline 11109 & B09X09C & 6.37E-01 & 8.27E-02 & $4.01 \mathrm{E}-04$ & $8.44 \mathrm{E}-01$ & $1.68 \mathrm{E}+00$ & $1.06 \mathrm{E}+00$ & $4.46 \mathrm{E}+01$ & $3.06 \mathrm{E}+00$ \\
\hline 11109 & B09X09S & $1.70 \mathrm{E}+00$ & $1.73 \mathrm{E}-01$ & $8.77 \mathrm{E}-04$ & $2.21 \mathrm{E}+00$ & $3.42 \mathrm{E}+00$ & $2.47 \mathrm{E}+00$ & $1.11 \mathrm{E}+02$ & $7.67 \mathrm{E}+00$ \\
\hline 11109 & B09X09E & $1.92 \mathrm{E}+01$ & $2.06 \mathrm{E}+00$ & $1.03 \mathrm{E}-02$ & $2.51 \mathrm{E}+01$ & $4.09 \mathrm{E}+01$ & $2.87 \mathrm{E}+01$ & $1.28 \mathrm{E}+03$ & $8.80 \mathrm{E}+01$ \\
\hline 11183 & B09X09A & $1.13 \mathrm{E}-01$ & $2.55 \mathrm{E}-02$ & $1.08 \mathrm{E}-04$ & $1.54 \mathrm{E}-01$ & $5.29 \mathrm{E}-01$ & $2.43 \mathrm{E}-01$ & $8.69 \mathrm{E}+00$ & $5.84 \mathrm{E}-01$ \\
\hline 11183 & B09X09C & $1.32 \mathrm{E}-01$ & $1.84 \mathrm{E}-02$ & 8.57E-05 & $1.73 \mathrm{E}-01$ & $3.66 \mathrm{E}-01$ & $2.22 \mathrm{E}-01$ & $9.32 \mathrm{E}+00$ & $6.30 \mathrm{E}-01$ \\
\hline 11183 & B09X09S & $6.34 \mathrm{E}-01$ & $9.06 \mathrm{E}-02$ & $4.21 \mathrm{E}-04$ & 8.34E-01 & $1.81 \mathrm{E}+00$ & $1.08 \mathrm{E}+00$ & $4.51 \mathrm{E}+01$ & $3.05 \mathrm{E}+00$ \\
\hline 11183 & B09X09E & $2.26 \mathrm{E}+01$ & $2.98 \mathrm{E}+00$ & $1.40 \mathrm{E}-02$ & $2.95 \mathrm{E}+01$ & $5.89 \mathrm{E}+01$ & $3.70 \mathrm{E}+01$ & $1.57 \mathrm{E}+03$ & $1.06 \mathrm{E}+02$ \\
\hline 11372 & B09X09A & 4.19E-01 & $4.15 \mathrm{E}-02$ & $1.92 \mathrm{E}-04$ & $5.15 \mathrm{E}-01$ & 7.31E-01 & 5.53E-01 & $2.63 \mathrm{E}+01$ & $1.73 \mathrm{E}+00$ \\
\hline 11372 & B09X09C & $3.87 \mathrm{E}-01$ & $6.11 \mathrm{E}-02$ & $2.61 \mathrm{E}-04$ & 4.92E-01 & $1.15 \mathrm{E}+00$ & $6.58 \mathrm{E}-01$ & $2.75 \mathrm{E}+01$ & $1.79 \mathrm{E}+00$ \\
\hline 11372 & B09X09S & $1.07 \mathrm{E}+00$ & $1.24 \mathrm{E}-01$ & $5.57 \mathrm{E}-04$ & $1.33 \mathrm{E}+00$ & $2.22 \mathrm{E}+00$ & $1.54 \mathrm{E}+00$ & $7.02 \mathrm{E}+01$ & $4.59 \mathrm{E}+00$ \\
\hline
\end{tabular}


Table E-3. Composition of Segregated Fuel Sludge by Key and Fuel Model (2 ${ }^{\text {nd }}$ Part $)$. (5 sheets)

\begin{tabular}{|c|c|c|c|c|c|c|c|c|c|}
\hline Key ID & $\begin{array}{c}\text { Fuel } \\
\text { Model }\end{array}$ & $\begin{array}{c}\text { Eu-154 } \\
\text { (Ci) }\end{array}$ & $\begin{array}{c}\text { Eu-155 } \\
\text { (Ci) }\end{array}$ & $\begin{array}{l}\text { Np-237 } \\
\text { (Ci) }\end{array}$ & $\begin{array}{c}\text { Pu-238 } \\
\text { (Ci) }\end{array}$ & $\begin{array}{c}\text { Pu-239 } \\
\text { (Ci) }\end{array}$ & $\begin{array}{c}\text { Pu-240 } \\
\text { (Ci) }\end{array}$ & $\begin{array}{c}\text { Pu-241 } \\
\text { (Ci) }\end{array}$ & $\begin{array}{c}\text { Am-241 } \\
\text { (Ci) }\end{array}$ \\
\hline 11372 & B09X09E & $3.18 \mathrm{E}+01$ & $3.65 \mathrm{E}+00$ & $1.65 \mathrm{E}-02$ & $3.96 \mathrm{E}+01$ & $6.56 \mathrm{E}+01$ & $4.55 \mathrm{E}+01$ & $2.08 \mathrm{E}+03$ & $1.36 \mathrm{E}+02$ \\
\hline 11449 & B09X09A & $2.75 \mathrm{E}-01$ & $2.94 \mathrm{E}-02$ & $1.31 \mathrm{E}-04$ & $3.34 \mathrm{E}-01$ & $5.09 \mathrm{E}-01$ & $3.70 \mathrm{E}-01$ & $1.75 \mathrm{E}+01$ & $1.13 \mathrm{E}+00$ \\
\hline 11449 & B09X09C & $3.24 \mathrm{E}-03$ & $2.56 \mathrm{E}-03$ & $6.91 \mathrm{E}-06$ & $4.18 \mathrm{E}-03$ & 4.24E-02 & $1.01 \mathrm{E}-02$ & $2.07 \mathrm{E}-01$ & $1.31 \mathrm{E}-02$ \\
\hline 11449 & B09X09S & $4.01 \mathrm{E}+00$ & $4.81 \mathrm{E}-01$ & $2.10 \mathrm{E}-03$ & $4.92 \mathrm{E}+00$ & $8.44 \mathrm{E}+00$ & $5.74 \mathrm{E}+00$ & $2.63 \mathrm{E}+02$ & $1.69 \mathrm{E}+01$ \\
\hline 11449 & B09X09E & $2.11 \mathrm{E}+01$ & $2.39 \mathrm{E}+00$ & $1.05 \mathrm{E}-02$ & $2.58 \mathrm{E}+01$ & $4.17 \mathrm{E}+01$ & $2.93 \mathrm{E}+01$ & $1.36 \mathrm{E}+03$ & $8.78 \mathrm{E}+01$ \\
\hline 11540 & $\mathrm{~B} 09 \mathrm{X} 09 \mathrm{~A}$ & $1.68 \mathrm{E}-01$ & $2.54 \mathrm{E}-02$ & $1.03 \mathrm{E}-04$ & $2.06 \mathrm{E}-01$ & $4.43 \mathrm{E}-01$ & $2.65 \mathrm{E}-01$ & $1.16 \mathrm{E}+01$ & 7.29E-01 \\
\hline 11540 & B09X09C & $2.31 \mathrm{E}-02$ & $3.42 \mathrm{E}-03$ & $1.39 \mathrm{E}-05$ & $2.83 \mathrm{E}-02$ & 5.94E-02 & $3.60 \mathrm{E}-02$ & $1.58 \mathrm{E}+00$ & $9.98 \mathrm{E}-02$ \\
\hline 11540 & B09X09S & $6.67 \mathrm{E}-01$ & $6.05 \mathrm{E}-02$ & $2.68 \mathrm{E}-04$ & 7.86E-01 & $9.85 \mathrm{E}-01$ & 7.97E-01 & $4.01 \mathrm{E}+01$ & $2.55 \mathrm{E}+00$ \\
\hline 11540 & B09X09E & $8.05 \mathrm{E}+00$ & 7.97E-01 & $3.48 \mathrm{E}-03$ & $9.55 \mathrm{E}+00$ & $1.32 \mathrm{E}+01$ & $1.01 \mathrm{E}+01$ & $4.95 \mathrm{E}+02$ & $3.14 \mathrm{E}+01$ \\
\hline 11733 & B09X09S & $3.05 \mathrm{E}-02$ & $5.43 \mathrm{E}-03$ & $1.99 \mathrm{E}-05$ & $3.63 \mathrm{E}-02$ & 8.93E-02 & 4.95E-02 & $2.14 \mathrm{E}+00$ & $1.29 \mathrm{E}-01$ \\
\hline 11733 & B09X09E & $1.82 \mathrm{E}-01$ & $2.39 \mathrm{E}-02$ & $9.27 \mathrm{E}-05$ & $2.12 \mathrm{E}-01$ & $3.78 \mathrm{E}-01$ & $2.51 \mathrm{E}-01$ & $1.18 \mathrm{E}+01$ & $7.15 \mathrm{E}-01$ \\
\hline 11806 & B09X09A & $1.43 \mathrm{E}-01$ & $2.62 \mathrm{E}-02$ & 9.33E-05 & $1.68 \mathrm{E}-01$ & 4.20E-01 & $2.31 \mathrm{E}-01$ & $1.00 \mathrm{E}+01$ & $5.98 \mathrm{E}-01$ \\
\hline 11806 & B09X09C & $1.58 \mathrm{E}-02$ & $2.27 \mathrm{E}-03$ & $8.44 \mathrm{E}-06$ & $1.83 \mathrm{E}-02$ & $3.52 \mathrm{E}-02$ & $2.24 \mathrm{E}-02$ & $1.04 \mathrm{E}+00$ & $6.22 \mathrm{E}-02$ \\
\hline 11806 & B09X09S & $3.04 \mathrm{E}-01$ & $3.39 \mathrm{E}-02$ & $1.32 \mathrm{E}-04$ & $3.44 \mathrm{E}-01$ & $5.09 \mathrm{E}-01$ & $3.74 \mathrm{E}-01$ & $1.86 \mathrm{E}+01$ & $1.12 \mathrm{E}+00$ \\
\hline 11806 & B09X09E & $1.88 \mathrm{E}+01$ & $2.24 \mathrm{E}+00$ & $8.60 \mathrm{E}-03$ & $2.14 \mathrm{E}+01$ & $3.39 \mathrm{E}+01$ & $2.40 \mathrm{E}+01$ & $1.17 \mathrm{E}+03$ & $7.04 \mathrm{E}+01$ \\
\hline 11897 & B09X09A & $3.48 \mathrm{E}-03$ & $5.08 \mathrm{E}-04$ & $1.83 \mathrm{E}-06$ & $3.94 \mathrm{E}-03$ & 7.63E-03 & $4.83 \mathrm{E}-03$ & $2.27 \mathrm{E}-01$ & $1.33 \mathrm{E}-02$ \\
\hline 11897 & $\mathrm{~B} 09 \mathrm{X} 09 \mathrm{C}$ & $2.14 \mathrm{E}+00$ & $3.31 \mathrm{E}-01$ & $1.18 \mathrm{E}-03$ & $2.44 \mathrm{E}+00$ & $5.00 \mathrm{E}+00$ & $3.06 \mathrm{E}+00$ & $1.41 \mathrm{E}+02$ & $8.31 \mathrm{E}+00$ \\
\hline 11897 & B09X09S & $1.77 \mathrm{E}+00$ & $2.08 \mathrm{E}-01$ & $7.75 \mathrm{E}-04$ & $1.97 \mathrm{E}+00$ & $3.03 \mathrm{E}+00$ & $2.18 \mathrm{E}+00$ & $1.09 \mathrm{E}+02$ & $6.41 \mathrm{E}+00$ \\
\hline 11897 & B09X09E & $2.41 \mathrm{E}+01$ & $2.71 \mathrm{E}+00$ & $1.02 \mathrm{E}-02$ & $2.68 \mathrm{E}+01$ & $3.93 \mathrm{E}+01$ & $2.90 \mathrm{E}+01$ & $1.46 \mathrm{E}+03$ & $8.64 \mathrm{E}+01$ \\
\hline 11979 & B09X09A & 5.19E-02 & $7.68 \mathrm{E}-03$ & $2.67 \mathrm{E}-05$ & $5.78 \mathrm{E}-02$ & $1.12 \mathrm{E}-01$ & 7.07E-02 & $3.35 \mathrm{E}+00$ & $1.94 \mathrm{E}-01$ \\
\hline 11979 & B09X09C & $1.73 \mathrm{E}+00$ & $2.40 \mathrm{E}-01$ & $8.45 \mathrm{E}-04$ & $1.92 \mathrm{E}+00$ & $3.47 \mathrm{E}+00$ & $2.27 \mathrm{E}+00$ & $1.10 \mathrm{E}+02$ & $6.35 \mathrm{E}+00$ \\
\hline 11979 & B09X09S & $2.21 \mathrm{E}+00$ & $2.72 \mathrm{E}-01$ & 9.79E-04 & $2.43 \mathrm{E}+00$ & $3.87 \mathrm{E}+00$ & $2.73 \mathrm{E}+00$ & $1.36 \mathrm{E}+02$ & $7.89 \mathrm{E}+00$ \\
\hline 11979 & B09X09E & $2.32 \mathrm{E}+01$ & $2.66 \mathrm{E}+00$ & $9.68 \mathrm{E}-03$ & $2.54 \mathrm{E}+01$ & $3.74 \mathrm{E}+01$ & $2.75 \mathrm{E}+01$ & $1.40 \mathrm{E}+03$ & $8.13 \mathrm{E}+01$ \\
\hline 12127 & B09X09A & $2.21 \mathrm{E}-01$ & $3.69 \mathrm{E}-02$ & $1.19 \mathrm{E}-04$ & $2.41 \mathrm{E}-01$ & $5.15 \mathrm{E}-01$ & $3.07 \mathrm{E}-01$ & $1.44 \mathrm{E}+01$ & $8.09 \mathrm{E}-01$ \\
\hline 12127 & B09X09C & $6.48 \mathrm{E}-02$ & $1.51 \mathrm{E}-02$ & 4.59E-05 & $7.25 \mathrm{E}-02$ & $2.18 \mathrm{E}-01$ & $1.08 \mathrm{E}-01$ & $4.60 \mathrm{E}+00$ & $2.56 \mathrm{E}-01$ \\
\hline 12127 & B09X09S & $1.95 \mathrm{E}+00$ & $2.27 \mathrm{E}-01$ & $7.82 \mathrm{E}-04$ & $2.07 \mathrm{E}+00$ & $3.01 \mathrm{E}+00$ & $2.23 \mathrm{E}+00$ & $1.16 \mathrm{E}+02$ & $6.54 \mathrm{E}+00$ \\
\hline 12127 & B09X09E & $1.03 \mathrm{E}+01$ & $1.53 \mathrm{E}+00$ & $5.04 \mathrm{E}-03$ & $1.11 \mathrm{E}+01$ & $2.10 \mathrm{E}+01$ & $1.34 \mathrm{E}+01$ & $6.52 \mathrm{E}+02$ & $3.66 \mathrm{E}+01$ \\
\hline 12201 & B09X09A & 7.67E-02 & $1.18 \mathrm{E}-02$ & $3.76 \mathrm{E}-05$ & 8.18E-02 & $1.57 \mathrm{E}-01$ & $9.95 \mathrm{E}-02$ & $4.86 \mathrm{E}+00$ & $2.69 \mathrm{E}-01$ \\
\hline 12201 & B09X09C & $1.99 \mathrm{E}-01$ & $3.18 \mathrm{E}-02$ & $1.01 \mathrm{E}-04$ & $2.13 \mathrm{E}-01$ & $4.27 \mathrm{E}-01$ & 2.64E-01 & $1.28 \mathrm{E}+01$ & 7.04E-01 \\
\hline 12201 & B09X09S & $3.78 \mathrm{E}+00$ & 4.63E-01 & $1.54 \mathrm{E}-03$ & $3.96 \mathrm{E}+00$ & $6.01 \mathrm{E}+00$ & $4.34 \mathrm{E}+00$ & $2.25 \mathrm{E}+02$ & $1.25 \mathrm{E}+01$ \\
\hline 12201 & B09X09E & $1.03 \mathrm{E}+01$ & $1.29 \mathrm{E}+00$ & $4.26 \mathrm{E}-03$ & $1.08 \mathrm{E}+01$ & $1.67 \mathrm{E}+01$ & $1.19 \mathrm{E}+01$ & $6.16 \mathrm{E}+02$ & $3.42 \mathrm{E}+01$ \\
\hline 12285 & B09X09A & $6.61 \mathrm{E}-03$ & $1.06 \mathrm{E}-03$ & $3.26 \mathrm{E}-06$ & $6.94 \mathrm{E}-03$ & $1.38 \mathrm{E}-02$ & $8.55 \mathrm{E}-03$ & 4.19E-01 & $2.27 \mathrm{E}-02$ \\
\hline 12285 & B09X09C & $2.32 \mathrm{E}-01$ & $4.86 \mathrm{E}-02$ & $1.43 \mathrm{E}-04$ & $2.48 \mathrm{E}-01$ & $6.54 \mathrm{E}-01$ & $3.47 \mathrm{E}-01$ & $1.58 \mathrm{E}+01$ & $8.52 \mathrm{E}-01$ \\
\hline 12285 & B09X09S & $3.84 \mathrm{E}+00$ & $5.14 \mathrm{E}-01$ & $1.63 \mathrm{E}-03$ & $3.98 \mathrm{E}+00$ & $6.52 \mathrm{E}+00$ & $4.51 \mathrm{E}+00$ & $2.32 \mathrm{E}+02$ & $1.26 \mathrm{E}+01$ \\
\hline
\end{tabular}


Table E-3. Composition of Segregated Fuel Sludge by Key and Fuel Model (2 ${ }^{\text {nd }}$ Part $)$. (5 sheets)

\begin{tabular}{|c|c|c|c|c|c|c|c|c|c|}
\hline Key ID & $\begin{array}{c}\text { Fuel } \\
\text { Model }\end{array}$ & $\begin{array}{c}\text { Eu-154 } \\
\text { (Ci) }\end{array}$ & $\begin{array}{c}\text { Eu-155 } \\
\text { (Ci) }\end{array}$ & $\begin{array}{l}\text { Np-237 } \\
\text { (Ci) }\end{array}$ & $\begin{array}{c}\text { Pu-238 } \\
\text { (Ci) }\end{array}$ & $\begin{array}{c}\text { Pu-239 } \\
\text { (Ci) }\end{array}$ & $\begin{array}{c}\text { Pu-240 } \\
\text { (Ci) }\end{array}$ & $\begin{array}{c}\text { Pu-241 } \\
\text { (Ci) }\end{array}$ & $\begin{array}{c}\text { Am-241 } \\
\text { (Ci) }\end{array}$ \\
\hline 12285 & B09X09E & $2.51 \mathrm{E}+01$ & $4.22 \mathrm{E}+00$ & $1.29 \mathrm{E}-02$ & $2.65 \mathrm{E}+01$ & $5.52 \mathrm{E}+01$ & $3.33 \mathrm{E}+01$ & $1.61 \mathrm{E}+03$ & $8.74 \mathrm{E}+01$ \\
\hline 12480 & B09X09A & $2.32 \mathrm{E}-01$ & $3.48 \mathrm{E}-02$ & $1.01 \mathrm{E}-04$ & $2.33 \mathrm{E}-01$ & 4.14E-01 & $2.73 \mathrm{E}-01$ & $1.41 \mathrm{E}+01$ & 7.34E-01 \\
\hline 12480 & B09X09C & $6.27 \mathrm{E}-02$ & $1.25 \mathrm{E}-02$ & $3.45 \mathrm{E}-05$ & $6.43 \mathrm{E}-02$ & $1.54 \mathrm{E}-01$ & 8.59E-02 & $4.10 \mathrm{E}+00$ & $2.13 \mathrm{E}-01$ \\
\hline 12480 & B09X09S & $1.51 \mathrm{E}+00$ & $2.25 \mathrm{E}-01$ & $6.55 \mathrm{E}-04$ & $1.52 \mathrm{E}+00$ & $2.68 \mathrm{E}+00$ & $1.77 \mathrm{E}+00$ & $9.15 \mathrm{E}+01$ & $4.78 \mathrm{E}+00$ \\
\hline 12480 & B09X09E & $1.63 \mathrm{E}+01$ & $2.76 \mathrm{E}+00$ & $7.84 \mathrm{E}-03$ & $1.65 \mathrm{E}+01$ & $3.33 \mathrm{E}+01$ & $2.04 \mathrm{E}+01$ & $1.02 \mathrm{E}+03$ & $5.31 \mathrm{E}+01$ \\
\hline 12565 & B09X09A & 7.23E-02 & $1.22 \mathrm{E}-02$ & $3.38 \mathrm{E}-05$ & 7.19E-02 & $1.43 \mathrm{E}-01$ & 8.84E-02 & $4.48 \mathrm{E}+00$ & $2.29 \mathrm{E}-01$ \\
\hline 12565 & B09X09C & $3.12 \mathrm{E}-02$ & $5.65 \mathrm{E}-03$ & $1.54 \mathrm{E}-05$ & $3.12 \mathrm{E}-02$ & $6.66 \mathrm{E}-02$ & $3.96 \mathrm{E}-02$ & $1.97 \mathrm{E}+00$ & $1.01 \mathrm{E}-01$ \\
\hline 12565 & B09X09S & 7.94E-01 & $1.46 \mathrm{E}-01$ & $3.98 \mathrm{E}-04$ & 7.95E-01 & $1.73 \mathrm{E}+00$ & $1.02 \mathrm{E}+00$ & $5.03 \mathrm{E}+01$ & $2.57 \mathrm{E}+00$ \\
\hline 12565 & B09X09E & $1.06 \mathrm{E}+01$ & $1.59 \mathrm{E}+00$ & 4.49E-03 & $1.05 \mathrm{E}+01$ & $1.83 \mathrm{E}+01$ & $1.22 \mathrm{E}+01$ & $6.38 \mathrm{E}+02$ & $3.27 \mathrm{E}+01$ \\
\hline 12639 & B09X09S & $1.82 \mathrm{E}+00$ & $2.71 \mathrm{E}-01$ & $7.45 \mathrm{E}-04$ & $1.76 \mathrm{E}+00$ & $3.02 \mathrm{E}+00$ & $2.03 \mathrm{E}+00$ & $1.08 \mathrm{E}+02$ & $5.44 \mathrm{E}+00$ \\
\hline 12639 & B09X09E & $9.26 \mathrm{E}+00$ & $1.75 \mathrm{E}+00$ & $4.61 \mathrm{E}-03$ & $9.14 \mathrm{E}+00$ & $2.01 \mathrm{E}+01$ & $1.17 \mathrm{E}+01$ & $5.85 \mathrm{E}+02$ & $2.94 \mathrm{E}+01$ \\
\hline 12639 & B07X07E & $5.15 \mathrm{E}-03$ & 9.95E-04 & $2.61 \mathrm{E}-06$ & $5.09 \mathrm{E}-03$ & $1.15 \mathrm{E}-02$ & $6.61 \mathrm{E}-03$ & $3.27 \mathrm{E}-01$ & $1.64 \mathrm{E}-02$ \\
\hline 12852 & B09X09E & $1.79 \mathrm{E}+00$ & $1.83 \mathrm{E}-01$ & $4.98 \mathrm{E}-04$ & $1.62 \mathrm{E}+00$ & $1.78 \mathrm{E}+00$ & $1.52 \mathrm{E}+00$ & $9.34 \mathrm{E}+01$ & $4.54 \mathrm{E}+00$ \\
\hline 13015 & B09X09C & $4.32 \mathrm{E}-02$ & $1.47 \mathrm{E}-02$ & $3.00 \mathrm{E}-05$ & 4.12E-02 & $1.51 \mathrm{E}-01$ & $6.51 \mathrm{E}-02$ & $2.81 \mathrm{E}+00$ & $1.29 \mathrm{E}-01$ \\
\hline 13015 & B09X09E & $2.69 \mathrm{E}-01$ & $5.71 \mathrm{E}-02$ & $1.29 \mathrm{E}-04$ & $2.48 \mathrm{E}-01$ & $5.73 \mathrm{E}-01$ & $3.24 \mathrm{E}-01$ & $1.67 \mathrm{E}+01$ & 7.73E-01 \\
\hline 13016 & B09X09S & 4.54E-03 & $1.51 \mathrm{E}-03$ & $3.10 \mathrm{E}-06$ & 4.32E-03 & $1.55 \mathrm{E}-02$ & $6.78 \mathrm{E}-03$ & $2.95 \mathrm{E}-01$ & $1.36 \mathrm{E}-02$ \\
\hline 13371 & B09X09E & $1.09 \mathrm{E}-03$ & $1.17 \mathrm{E}-03$ & $1.52 \mathrm{E}-06$ & $9.58 \mathrm{E}-04$ & $9.32 \mathrm{E}-03$ & $2.22 \mathrm{E}-03$ & $5.87 \mathrm{E}-02$ & $2.47 \mathrm{E}-03$ \\
\hline 13649 & B09X09C & $1.29 \mathrm{E}-01$ & $4.81 \mathrm{E}-02$ & 7.72E-05 & $1.08 \mathrm{E}-01$ & $3.87 \mathrm{E}-01$ & $1.68 \mathrm{E}-01$ & $7.91 \mathrm{E}+00$ & $3.14 \mathrm{E}-01$ \\
\hline 13649 & B09X09E & $1.63 \mathrm{E}+00$ & $4.03 \mathrm{E}-01$ & $7.08 \mathrm{E}-04$ & $1.32 \mathrm{E}+00$ & $3.19 \mathrm{E}+00$ & $1.75 \mathrm{E}+00$ & $9.66 \mathrm{E}+01$ & $3.86 \mathrm{E}+00$ \\
\hline 13649 & B07X07E & $6.53 \mathrm{E}-02$ & $2.85 \mathrm{E}-02$ & $4.39 \mathrm{E}-05$ & $5.54 \mathrm{E}-02$ & $2.29 \mathrm{E}-01$ & $9.09 \mathrm{E}-02$ & $3.98 \mathrm{E}+00$ & $1.58 \mathrm{E}-01$ \\
\hline 13685 & B09X09E & $2.51 \mathrm{E}-01$ & $5.81 \mathrm{E}-02$ & $1.02 \mathrm{E}-04$ & $2.02 \mathrm{E}-01$ & $4.50 \mathrm{E}-01$ & $2.58 \mathrm{E}-01$ & $1.46 \mathrm{E}+01$ & $5.79 \mathrm{E}-01$ \\
\hline 14355 & B09X09C & $1.38 \mathrm{E}-01$ & $3.01 \mathrm{E}-02$ & 4.22E-05 & $9.60 \mathrm{E}-02$ & $1.76 \mathrm{E}-01$ & $1.12 \mathrm{E}-01$ & $7.23 \mathrm{E}+00$ & $2.44 \mathrm{E}-01$ \\
\hline 14355 & B09X09S & 4.58E-01 & $9.95 \mathrm{E}-02$ & $1.40 \mathrm{E}-04$ & $3.18 \mathrm{E}-01$ & $5.84 \mathrm{E}-01$ & 3.69E-01 & $2.39 \mathrm{E}+01$ & $8.08 \mathrm{E}-01$ \\
\hline 14355 & B09X09E & $9.05 \mathrm{E}+00$ & $1.97 \mathrm{E}+00$ & $2.76 \mathrm{E}-03$ & $6.29 \mathrm{E}+00$ & $1.15 \mathrm{E}+01$ & $7.30 \mathrm{E}+00$ & $4.73 \mathrm{E}+02$ & $1.60 \mathrm{E}+01$ \\
\hline 14356 & B09X09A & $6.72 \mathrm{E}+01$ & $1.51 \mathrm{E}+01$ & $2.10 \mathrm{E}-02$ & $4.68 \mathrm{E}+01$ & $8.87 \mathrm{E}+01$ & $5.52 \mathrm{E}+01$ & $3.54 \mathrm{E}+03$ & $1.19 \mathrm{E}+02$ \\
\hline 14356 & B09X09S & $2.26 \mathrm{E}+00$ & $5.08 \mathrm{E}-01$ & 7.08E-04 & $1.58 \mathrm{E}+00$ & $2.99 \mathrm{E}+00$ & $1.86 \mathrm{E}+00$ & $1.19 \mathrm{E}+02$ & $4.02 \mathrm{E}+00$ \\
\hline 14356 & B09X09E & $7.74 \mathrm{E}+00$ & $1.74 \mathrm{E}+00$ & $2.42 \mathrm{E}-03$ & $5.39 \mathrm{E}+00$ & $1.02 \mathrm{E}+01$ & $6.35 \mathrm{E}+00$ & $4.08 \mathrm{E}+02$ & $1.37 \mathrm{E}+01$ \\
\hline 15204 & B09X09A & $6.75 \mathrm{E}-02$ & $9.77 \mathrm{E}-02$ & $6.28 \mathrm{E}-05$ & 4.12E-02 & $3.85 \mathrm{E}-01$ & 9.19E-02 & $3.09 \mathrm{E}+00$ & $8.08 \mathrm{E}-02$ \\
\hline 15204 & B09X09C & $1.18 \mathrm{E}-02$ & $1.71 \mathrm{E}-02$ & $1.10 \mathrm{E}-05$ & 7.19E-03 & $6.72 \mathrm{E}-02$ & $1.60 \mathrm{E}-02$ & 5.39E-01 & $1.41 \mathrm{E}-02$ \\
\hline 15204 & B09X09S & $1.04 \mathrm{E}-01$ & $1.51 \mathrm{E}-01$ & $9.67 \mathrm{E}-05$ & $6.34 \mathrm{E}-02$ & $5.93 \mathrm{E}-01$ & $1.42 \mathrm{E}-01$ & $4.76 \mathrm{E}+00$ & $1.24 \mathrm{E}-01$ \\
\hline 15204 & B09X09E & $3.08 \mathrm{E}-01$ & $4.46 \mathrm{E}-01$ & $2.86 \mathrm{E}-04$ & $1.88 \mathrm{E}-01$ & $1.76 \mathrm{E}+00$ & 4.19E-01 & $1.41 \mathrm{E}+01$ & $3.68 \mathrm{E}-01$ \\
\hline 15204 & B07X07E & $1.38 \mathrm{E}-03$ & $2.00 \mathrm{E}-03$ & $1.28 \mathrm{E}-06$ & $8.42 \mathrm{E}-04$ & $7.88 \mathrm{E}-03$ & $1.88 \mathrm{E}-03$ & $6.32 \mathrm{E}-02$ & $1.65 \mathrm{E}-03$ \\
\hline 15316 & B09X09A & $6.22 \mathrm{E}-02$ & $9.17 \mathrm{E}-02$ & 5.64E-05 & $3.71 \mathrm{E}-02$ & $3.46 \mathrm{E}-01$ & $8.26 \mathrm{E}-02$ & $2.82 \mathrm{E}+00$ & $7.12 \mathrm{E}-02$ \\
\hline 15316 & B09X09C & $9.45 \mathrm{E}-04$ & $1.39 \mathrm{E}-03$ & $8.58 \mathrm{E}-07$ & $5.64 \mathrm{E}-04$ & $5.27 \mathrm{E}-03$ & $1.26 \mathrm{E}-03$ & 4.29E-02 & $1.08 \mathrm{E}-03$ \\
\hline
\end{tabular}


Table E-3. Composition of Segregated Fuel Sludge by Key and Fuel Model ( ${ }^{\text {nd }}$ Part). (5 sheets)

\begin{tabular}{|c|c|c|c|c|c|c|c|c|c|}
\hline Key ID & $\begin{array}{c}\text { Fuel } \\
\text { Model }\end{array}$ & $\begin{array}{c}\text { Eu-154 } \\
(\mathbf{C i})\end{array}$ & $\begin{array}{c}\text { Eu-155 } \\
(\mathbf{C i})\end{array}$ & $\begin{array}{c}\text { Np-237 } \\
(\mathbf{C i})\end{array}$ & $\begin{array}{c}\text { Pu-238 } \\
(\mathbf{C i})\end{array}$ & $\begin{array}{c}\text { Pu-239 } \\
(\mathbf{C i})\end{array}$ & $\begin{array}{c}\text { Pu-240 } \\
(\mathbf{C i})\end{array}$ & $\begin{array}{c}\text { Pu-241 } \\
(\mathbf{C i})\end{array}$ & $\begin{array}{c}\text { Am-241 } \\
(\mathbf{C i})\end{array}$ \\
\hline 15316 & B09X09S & $2.61 \mathrm{E}-02$ & $3.85 \mathrm{E}-02$ & $2.37 \mathrm{E}-05$ & $1.56 \mathrm{E}-02$ & $1.45 \mathrm{E}-01$ & $3.46 \mathrm{E}-02$ & $1.18 \mathrm{E}+00$ & $2.99 \mathrm{E}-02$ \\
\hline 15316 & B09X09E & $3.19 \mathrm{E}-02$ & $4.70 \mathrm{E}-02$ & $2.89 \mathrm{E}-05$ & $1.90 \mathrm{E}-02$ & $1.78 \mathrm{E}-01$ & $4.23 \mathrm{E}-02$ & $1.45 \mathrm{E}+00$ & $3.65 \mathrm{E}-02$ \\
\hline 15399 & B09X09E & $1.36 \mathrm{E}-04$ & $2.03 \mathrm{E}-04$ & $1.21 \mathrm{E}-07$ & $7.95 \mathrm{E}-05$ & $7.41 \mathrm{E}-04$ & $1.77 \mathrm{E}-04$ & $6.10 \mathrm{E}-03$ & $1.50 \mathrm{E}-04$ \\
\hline 15451 & B09X09A & $8.31 \mathrm{E}-02$ & $8.99 \mathrm{E}-02$ & $5.82 \mathrm{E}-05$ & $4.89 \mathrm{E}-02$ & $3.38 \mathrm{E}-01$ & $9.64 \mathrm{E}-02$ & $3.95 \mathrm{E}+00$ & $9.61 \mathrm{E}-02$ \\
\hline 15451 & B09X09C & $1.20 \mathrm{E}-03$ & $1.25 \mathrm{E}-03$ & $8.19 \mathrm{E}-07$ & $7.10 \mathrm{E}-04$ & $4.73 \mathrm{E}-03$ & $1.38 \mathrm{E}-03$ & $5.77 \mathrm{E}-02$ & $1.40 \mathrm{E}-03$ \\
\hline 15451 & B09X09S & $1.68 \mathrm{E}-04$ & $2.53 \mathrm{E}-04$ & $1.48 \mathrm{E}-07$ & $9.75 \mathrm{E}-05$ & $9.07 \mathrm{E}-04$ & $2.16 \mathrm{E}-04$ & $7.51 \mathrm{E}-03$ & $1.82 \mathrm{E}-04$ \\
\hline 15451 & B07X07E & $6.88 \mathrm{E}-03$ & $1.04 \mathrm{E}-02$ & $6.07 \mathrm{E}-06$ & $4.00 \mathrm{E}-03$ & $3.72 \mathrm{E}-02$ & $8.87 \mathrm{E}-03$ & $3.08 \mathrm{E}-01$ & $7.48 \mathrm{E}-03$ \\
\hline 15458 & B09X09A & $3.55 \mathrm{E}-02$ & $5.36 \mathrm{E}-02$ & $3.12 \mathrm{E}-05$ & $2.06 \mathrm{E}-02$ & $1.92 \mathrm{E}-01$ & $4.57 \mathrm{E}-02$ & $1.59 \mathrm{E}+00$ & $3.85 \mathrm{E}-02$ \\
\hline 15458 & B09X09C & $2.76 \mathrm{E}-03$ & $4.16 \mathrm{E}-03$ & $2.43 \mathrm{E}-06$ & $1.60 \mathrm{E}-03$ & $1.49 \mathrm{E}-02$ & $3.55 \mathrm{E}-03$ & $1.23 \mathrm{E}-01$ & $2.99 \mathrm{E}-03$ \\
\hline 15458 & B09X09S & $5.73 \mathrm{E}-02$ & $8.64 \mathrm{E}-02$ & $5.04 \mathrm{E}-05$ & $3.32 \mathrm{E}-02$ & $3.09 \mathrm{E}-01$ & $7.37 \mathrm{E}-02$ & $2.56 \mathrm{E}+00$ & $6.21 \mathrm{E}-02$ \\
\hline 15458 & B09X09E & $9.41 \mathrm{E}-02$ & $1.42 \mathrm{E}-01$ & $8.28 \mathrm{E}-05$ & $5.46 \mathrm{E}-02$ & $5.08 \mathrm{E}-01$ & $1.21 \mathrm{E}-01$ & $4.21 \mathrm{E}+00$ & $1.02 \mathrm{E}-01$ \\
\hline 15458 & B09X09R & $3.71 \mathrm{E}-04$ & $5.59 \mathrm{E}-04$ & $3.26 \mathrm{E}-07$ & $2.15 \mathrm{E}-04$ & $2.00 \mathrm{E}-03$ & $4.77 \mathrm{E}-04$ & $1.66 \mathrm{E}-02$ & $4.02 \mathrm{E}-04$ \\
\hline 15458 & B07X07E & $3.77 \mathrm{E}-04$ & $5.69 \mathrm{E}-04$ & $3.32 \mathrm{E}-07$ & $2.19 \mathrm{E}-04$ & $2.04 \mathrm{E}-03$ & $4.85 \mathrm{E}-04$ & $1.69 \mathrm{E}-02$ & $4.09 \mathrm{E}-04$ \\
\hline
\end{tabular}

Values were generated using the methods described in HNF-8760. The effective date of these numbers is May 31, 1998.

The shortened form of the fuel model identifier uses only the first and last characters. 
SNF-10293, REV 0

Table E-4. Weighting Factors for Sample KC-2/3.

\begin{tabular}{|c|c|c|c|c|}
\hline Key & Fuel Model & Canister Count & Sludge Fraction & Weighting Factor \\
\hline 10456 & BC & 7 & 0.27 & 0.135 \\
\hline 10456 & BS & 7 & & 0.135 \\
\hline 10832 & BA & 1 & 0.05 & 0.0035714 \\
\hline 10832 & BE & 13 & & 0.0464286 \\
\hline 11109 & BS & 5 & 0.05 & 0.0178571 \\
\hline 11109 & BE & 9 & & 0.0321429 \\
\hline 11109 & BE & 14 & 0.06 & 0.06 \\
\hline 11372 & BE & 14 & 0.03 & 0.03 \\
\hline 12285 & BC & 3 & 0.05 & 0.0107143 \\
\hline 12285 & BE & 4 & & 0.0142857 \\
\hline 12285 & BS & 7 & & 0.025 \\
\hline 12285 & BE & 11 & 0.07 & 0.055 \\
\hline 12285 & BS & 3 & & 0.015 \\
\hline 12285 & BE & 14 & 0.03 & 0.03 \\
\hline 14356 & BA & 7 & 0.06 & 0.06 \\
\hline 14356 & BE & 7 & 0.33 & 0.33 \\
\hline \multicolumn{2}{|r|}{} & Total in Column: & 1.00 & 1.00 \\
\hline
\end{tabular}

The sludge fractions are based on the estimated weight of sludge from the canisters that were sampled.

The weighting factors are the adjusted sludge fraction, according to the numbers of canisters of the fuel types for that key. 
SNF-10293, REV 0

\begin{tabular}{|c|c|c|c|c|c|}
\hline \multicolumn{6}{|c|}{ Table E-5. Radnuc2A Predicted Sample Concentrations (Ci/MTU). } \\
\hline \multirow[b]{2}{*}{ Measured Quantity } & \multicolumn{5}{|c|}{ Sample Identity } \\
\hline & $96-05$ & $96-08$ & 96-13 & $96-15$ & $96-04$ \\
\hline Co-60 & $1.67 \mathrm{E}+00$ & $1.04 \mathrm{E}+00$ & $6.61 \mathrm{E}-01$ & $4.27 \mathrm{E}+00$ & $1.67 \mathrm{E}+00$ \\
\hline Sr-90 & $5.90 \mathrm{E}+03$ & $4.86 \mathrm{E}+03$ & $3.34 \mathrm{E}+03$ & $6.33 \mathrm{E}+03$ & $5.90 \mathrm{E}+03$ \\
\hline Tc-99 & $1.85 \mathrm{E}+00$ & $1.58 \mathrm{E}+00$ & $1.05 \mathrm{E}+00$ & $1.60 \mathrm{E}+00$ & $1.85 \mathrm{E}+00$ \\
\hline Cs-134 & $3.97 \mathrm{E}+00$ & $1.27 \mathrm{E}+00$ & $4.93 \mathrm{E}-01$ & $4.70 \mathrm{E}+01$ & $3.97 \mathrm{E}+00$ \\
\hline Cs-137 & $8.05 \mathrm{E}+03$ & $6.51 \mathrm{E}+03$ & $4.25 \mathrm{E}+03$ & $8.42 \mathrm{E}+03$ & $8.05 \mathrm{E}+03$ \\
\hline Eu-152 & $6.37 \mathrm{E}-01$ & 4.45E-01 & $2.22 \mathrm{E}-01$ & 7.79E-01 & $6.37 \mathrm{E}-01$ \\
\hline Eu-154 & $7.52 \mathrm{E}+01$ & $4.42 \mathrm{E}+01$ & $1.79 \mathrm{E}+01$ & $1.07 \mathrm{E}+02$ & $7.52 \mathrm{E}+01$ \\
\hline Eu-155 & $8.62 \mathrm{E}+00$ & $5.35 \mathrm{E}+00$ & $3.67 \mathrm{E}+00$ & $2.40 \mathrm{E}+01$ & $8.62 \mathrm{E}+00$ \\
\hline $\mathrm{Np}-237$ & $3.89 \mathrm{E}-02$ & $3.31 \mathrm{E}-02$ & $2.15 \mathrm{E}-02$ & $3.35 \mathrm{E}-02$ & $3.89 \mathrm{E}-02$ \\
\hline $\mathrm{Pu}-238$ & $9.34 \mathrm{E}+01$ & $6.70 \mathrm{E}+01$ & $2.91 \mathrm{E}+01$ & $7.45 \mathrm{E}+01$ & $9.34 \mathrm{E}+01$ \\
\hline $\mathrm{Pu}-239 / 240$ & $2.62 \mathrm{E}+02$ & $2.27 \mathrm{E}+02$ & $1.54 \mathrm{E}+02$ & $2.29 \mathrm{E}+02$ & $2.62 \mathrm{E}+02$ \\
\hline $\mathrm{Pu}-241$ & $4.91 \mathrm{E}+03$ & $3.31 \mathrm{E}+03$ & $1.48 \mathrm{E}+03$ & $5.65 \mathrm{E}+03$ & $4.91 \mathrm{E}+03$ \\
\hline Am-241 & $3.21 \mathrm{E}+02$ & $2.57 \mathrm{E}+02$ & $1.17 \mathrm{E}+02$ & $1.90 \mathrm{E}+02$ & $3.21 \mathrm{E}+02$ \\
\hline \multirow[b]{2}{*}{ Measured Quantity } & \multicolumn{3}{|c|}{ Sample Identity } & \multirow[b]{2}{*}{ Mean } & \multirow{2}{*}{$\begin{array}{l}\text { Standard } \\
\text { Deviation }\end{array}$} \\
\hline & $96-06$ & KC-1 & $\mathrm{KC}-2 / 3$ & & \\
\hline Co-60 & $8.07 \mathrm{E}-01$ & $8.88 \mathrm{E}-01$ & $2.49 \mathrm{E}+00$ & $1.69 \mathrm{E}+00$ & $1.21 \mathrm{E}+00$ \\
\hline Sr-90 & $3.94 \mathrm{E}+03$ & $4.34 \mathrm{E}+03$ & $5.49 \mathrm{E}+03$ & $5.01 \mathrm{E}+03$ & $1.07 \mathrm{E}+03$ \\
\hline Tc-99 & $1.25 \mathrm{E}+00$ & $1.40 \mathrm{E}+00$ & $1.56 \mathrm{E}+00$ & $1.52 \mathrm{E}+00$ & $2.75 \mathrm{E}-01$ \\
\hline Cs-134 & $7.59 \mathrm{E}-01$ & $9.09 \mathrm{E}-01$ & $2.02 \mathrm{E}+01$ & $9.82 \mathrm{E}+00$ & $1.64 \mathrm{E}+01$ \\
\hline Cs-137 & $5.11 \mathrm{E}+03$ & $5.71 \mathrm{E}+03$ & $7.32 \mathrm{E}+03$ & $6.68 \mathrm{E}+03$ & $1.54 \mathrm{E}+03$ \\
\hline Eu-152 & $3.09 \mathrm{E}-01$ & $3.64 \mathrm{E}-01$ & $5.95 \mathrm{E}-01$ & 4.99E-01 & $1.93 \mathrm{E}-01$ \\
\hline Eu-154 & $2.65 \mathrm{E}+01$ & $3.32 \mathrm{E}+01$ & $7.31 \mathrm{E}+01$ & $5.66 \mathrm{E}+01$ & $3.07 \mathrm{E}+01$ \\
\hline Eu-155 & $4.29 \mathrm{E}+00$ & $4.65 \mathrm{E}+00$ & $1.37 \mathrm{E}+01$ & $9.11 \mathrm{E}+00$ & $6.87 \mathrm{E}+00$ \\
\hline Np-237 & $2.58 \mathrm{E}-02$ & $2.91 \mathrm{E}-02$ & $3.27 \mathrm{E}-02$ & $3.17 \mathrm{E}-02$ & $6.02 \mathrm{E}-03$ \\
\hline $\mathrm{Pu}-238$ & $4.17 \mathrm{E}+01$ & $5.20 \mathrm{E}+01$ & $6.94 \mathrm{E}+01$ & $6.51 \mathrm{E}+01$ & $2.31 \mathrm{E}+01$ \\
\hline $\mathrm{Pu}-239 / 240$ & $1.82 \mathrm{E}+02$ & $2.02 \mathrm{E}+02$ & $2.24 \mathrm{E}+02$ & $2.18 \mathrm{E}+02$ & $3.73 \mathrm{E}+01$ \\
\hline $\mathrm{Pu}-241$ & $2.16 \mathrm{E}+03$ & $2.62 \mathrm{E}+03$ & $4.36 \mathrm{E}+03$ & $3.68 \mathrm{E}+03$ & $1.50 \mathrm{E}+03$ \\
\hline Am-241 & $1.69 \mathrm{E}+02$ & $2.07 \mathrm{E}+02$ & $2.19 \mathrm{E}+02$ & $2.25 \mathrm{E}+02$ & $7.16 \mathrm{E}+01$ \\
\hline
\end{tabular}

Values shown are based on the HNF-8760 isotope concentrations shown in Table E-2 and Table E-3. 
Figure E-1. Comparison of Co-60 Data and Imputed Values with Predictions.

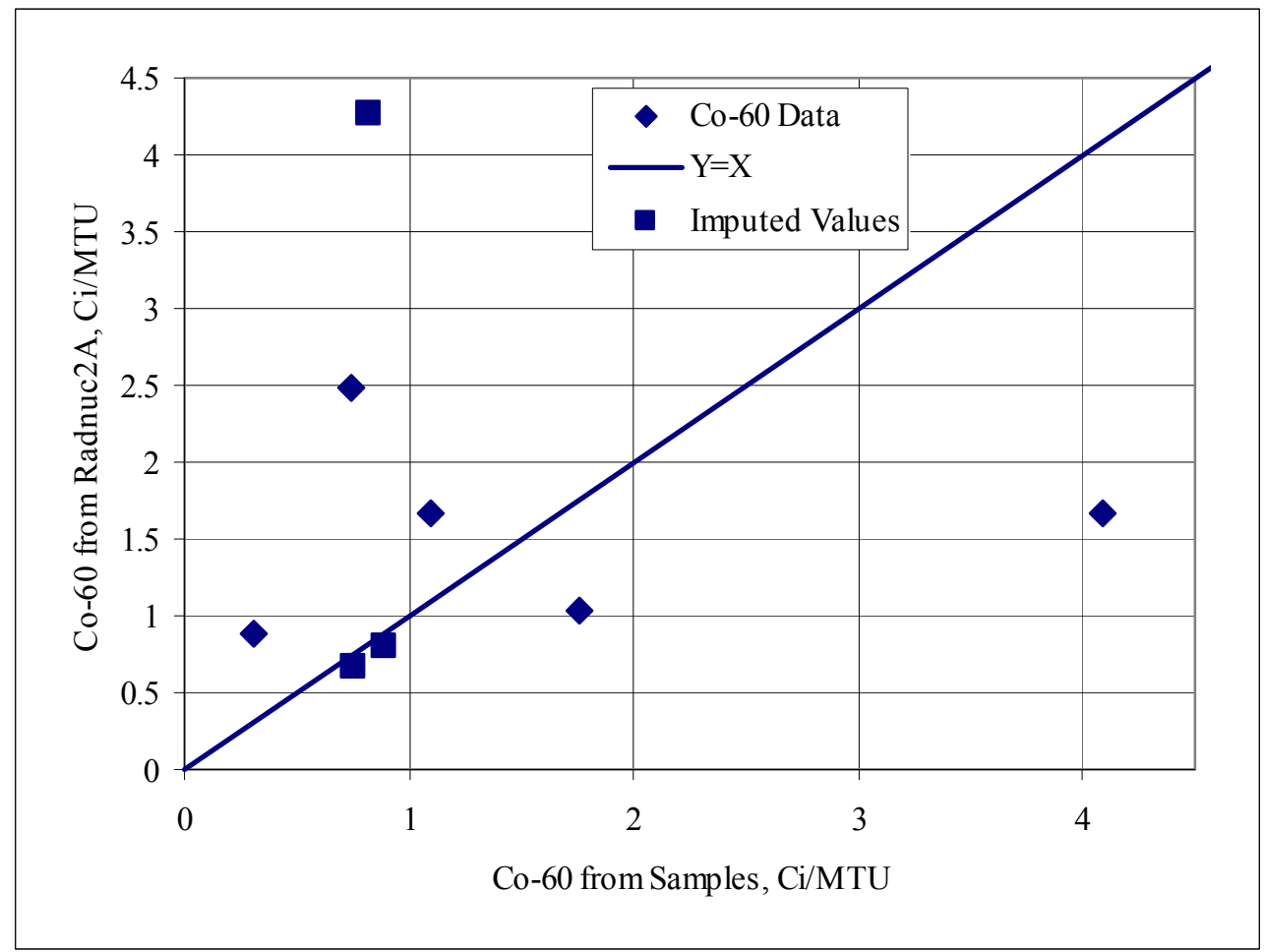

Figure E-2. Comparison of Sr-90 Data and Imputed Values with Predictions.

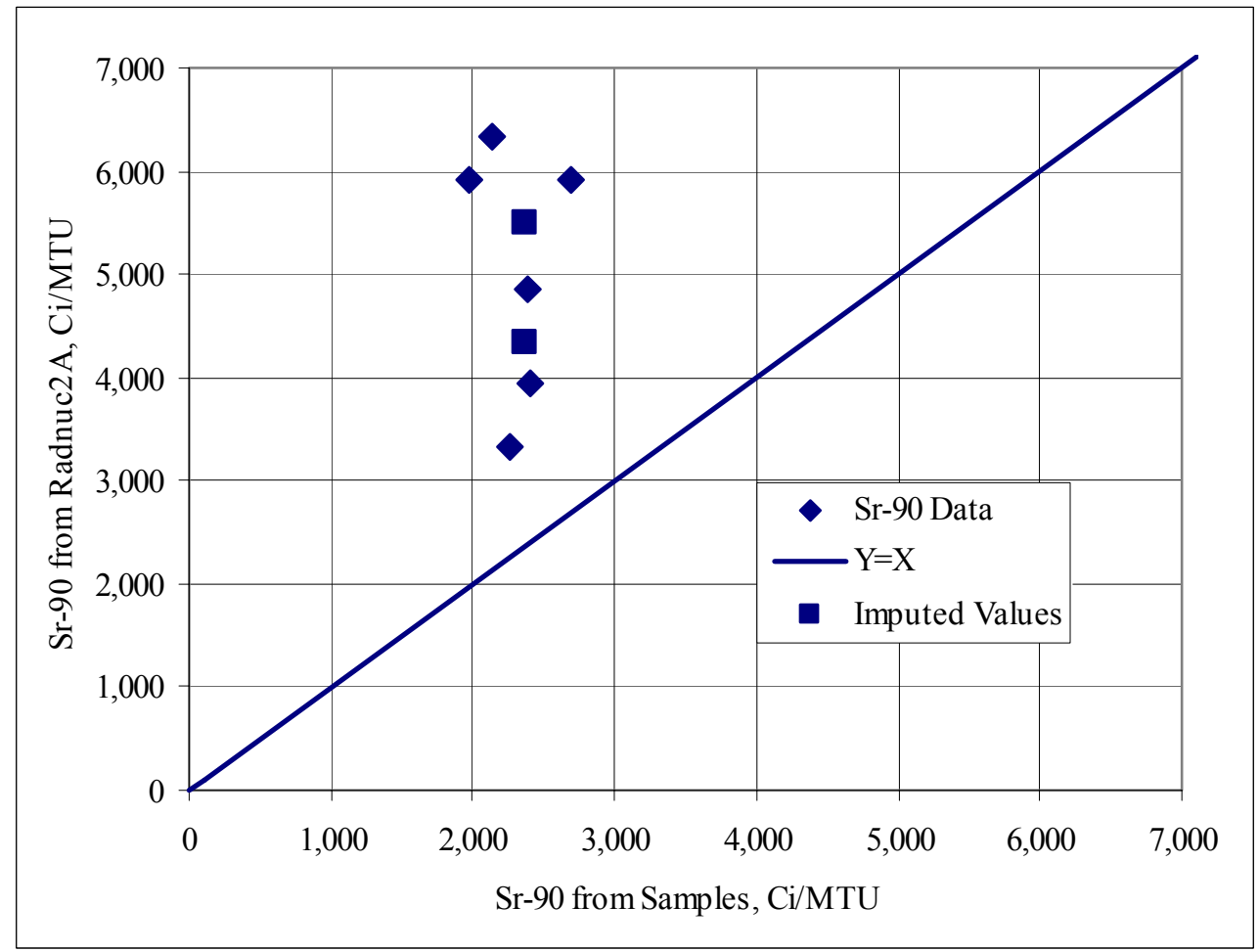


Figure E-3. Comparison of Tc-99 Data and Imputed Values with Predictions.

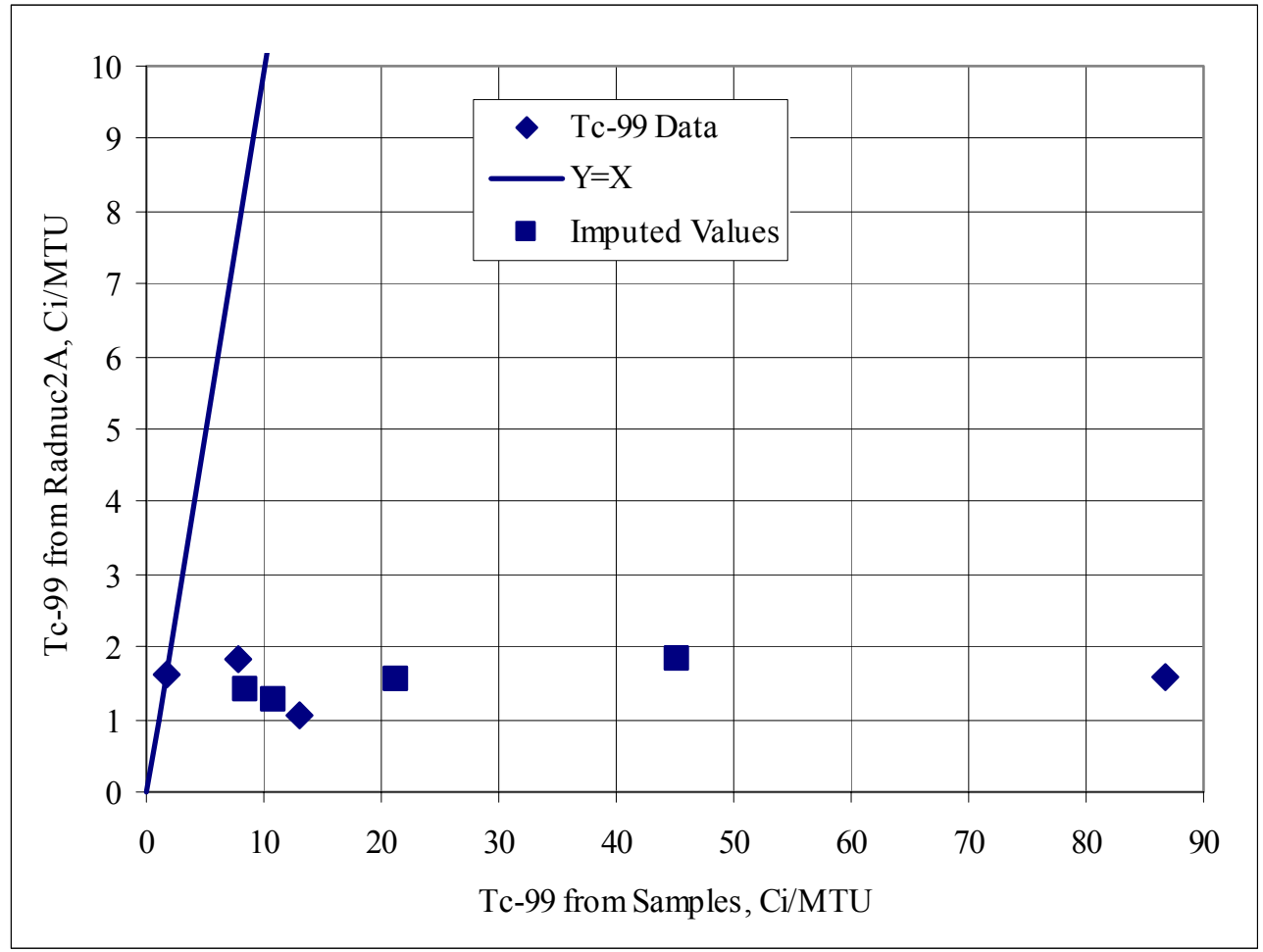

Figure E-4. Comparison of Cs-134 Data and Imputed Values with Predictions.

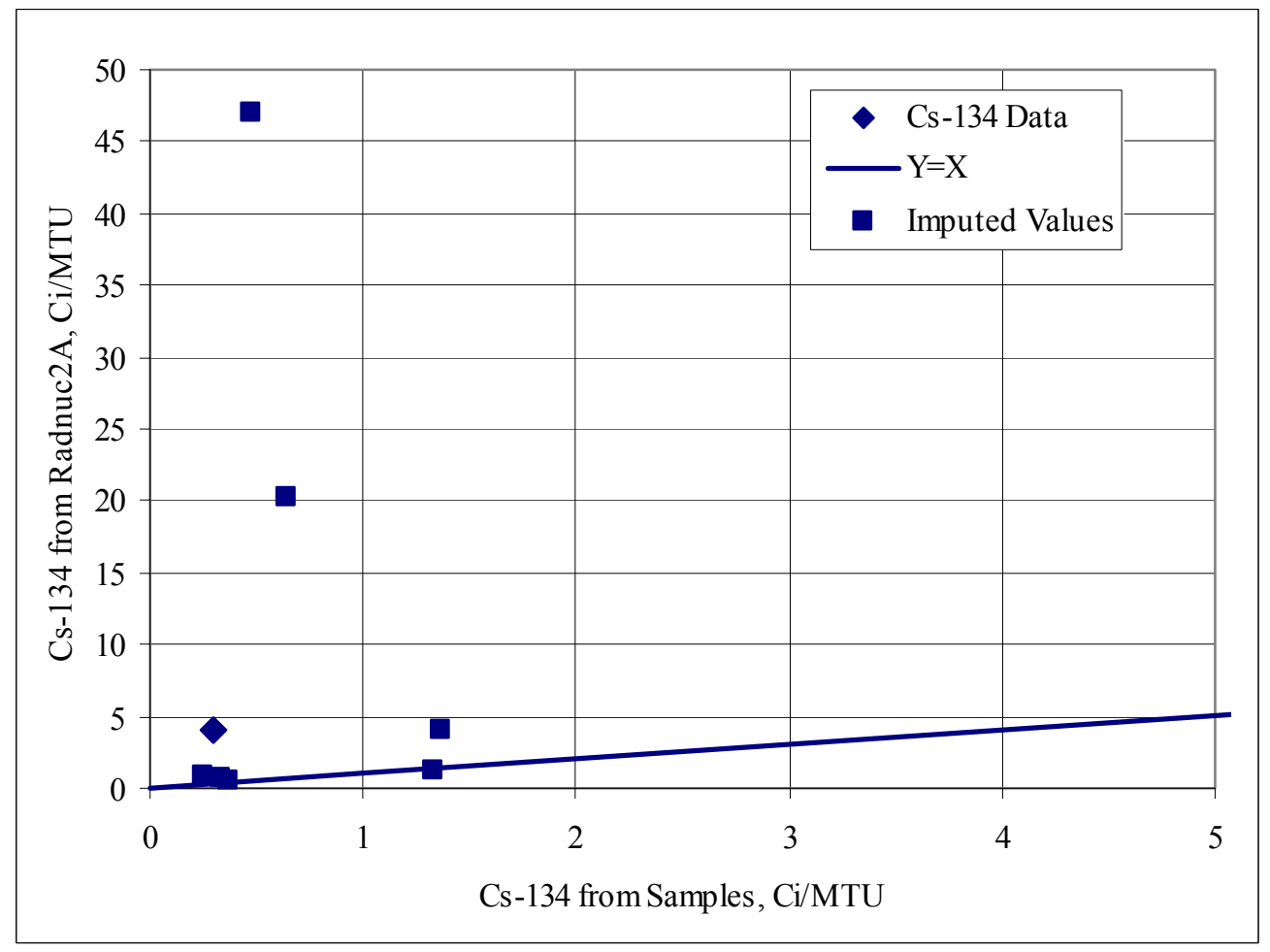


Figure E-5. Comparison of Cs-137 Data with Predictions.

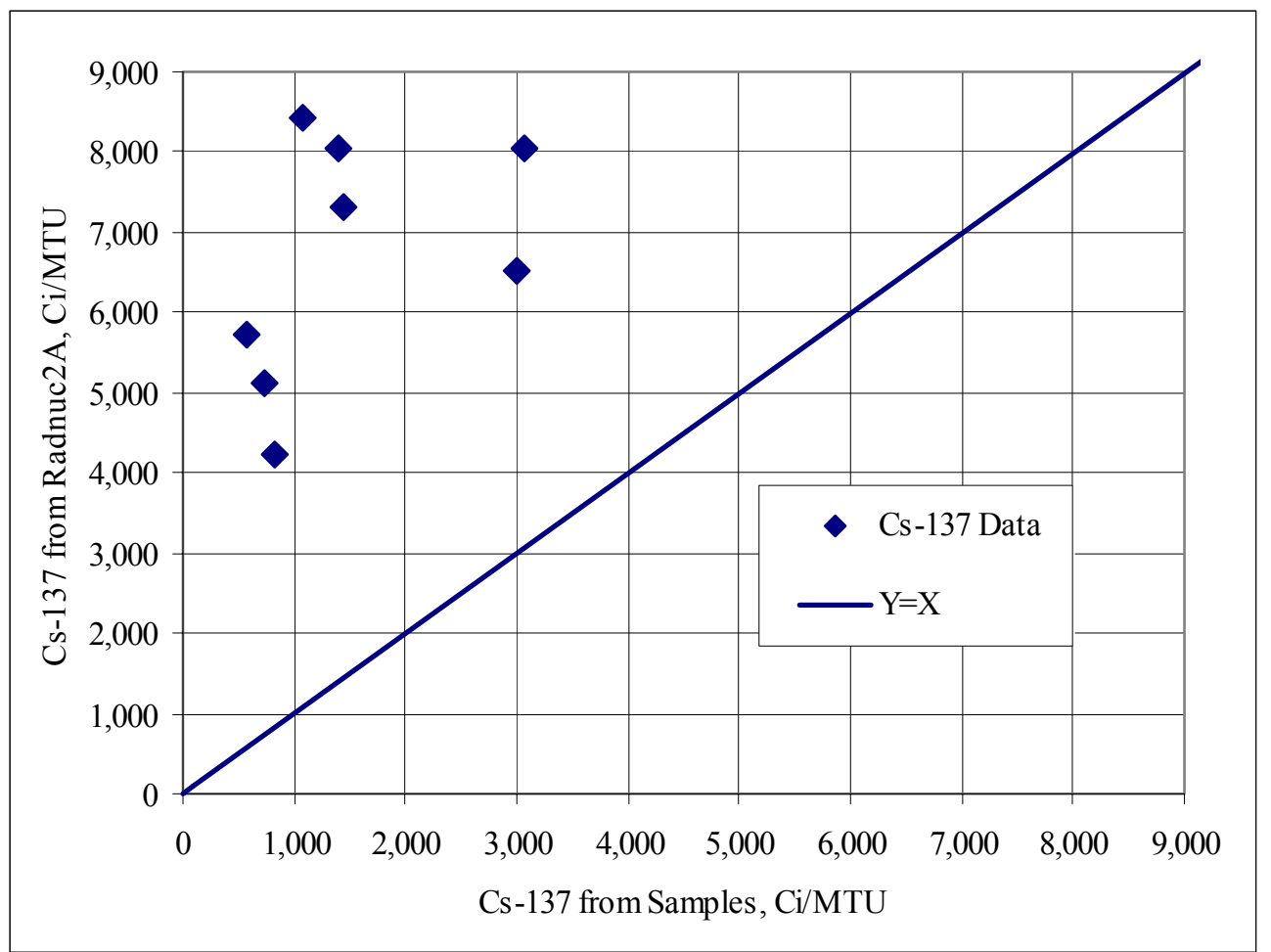

Figure E-6. Comparison of Eu-152 Data and Imputed Values with Predictions.

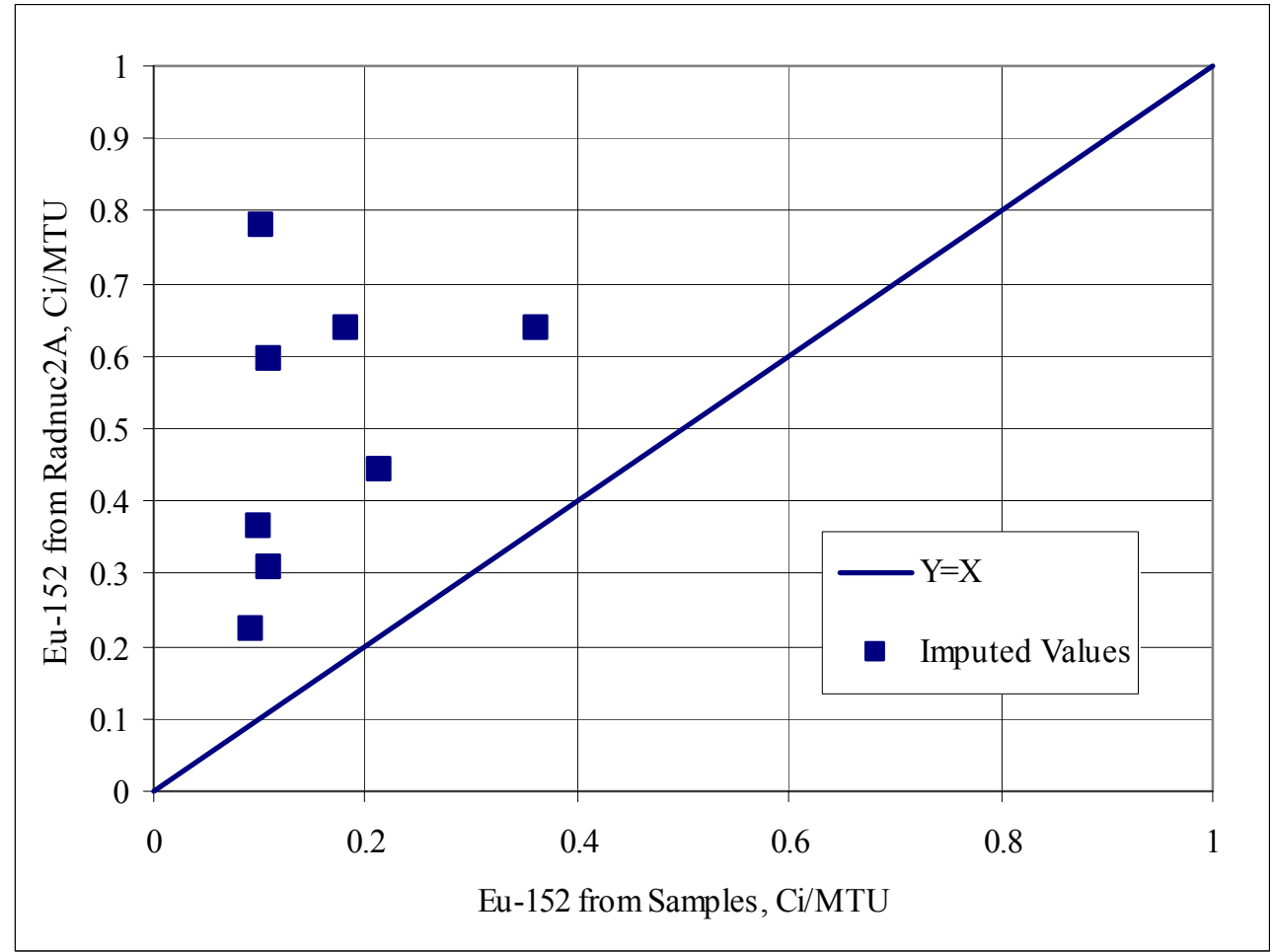


Figure E-7. Comparison of Eu-154 Data with Predictions.

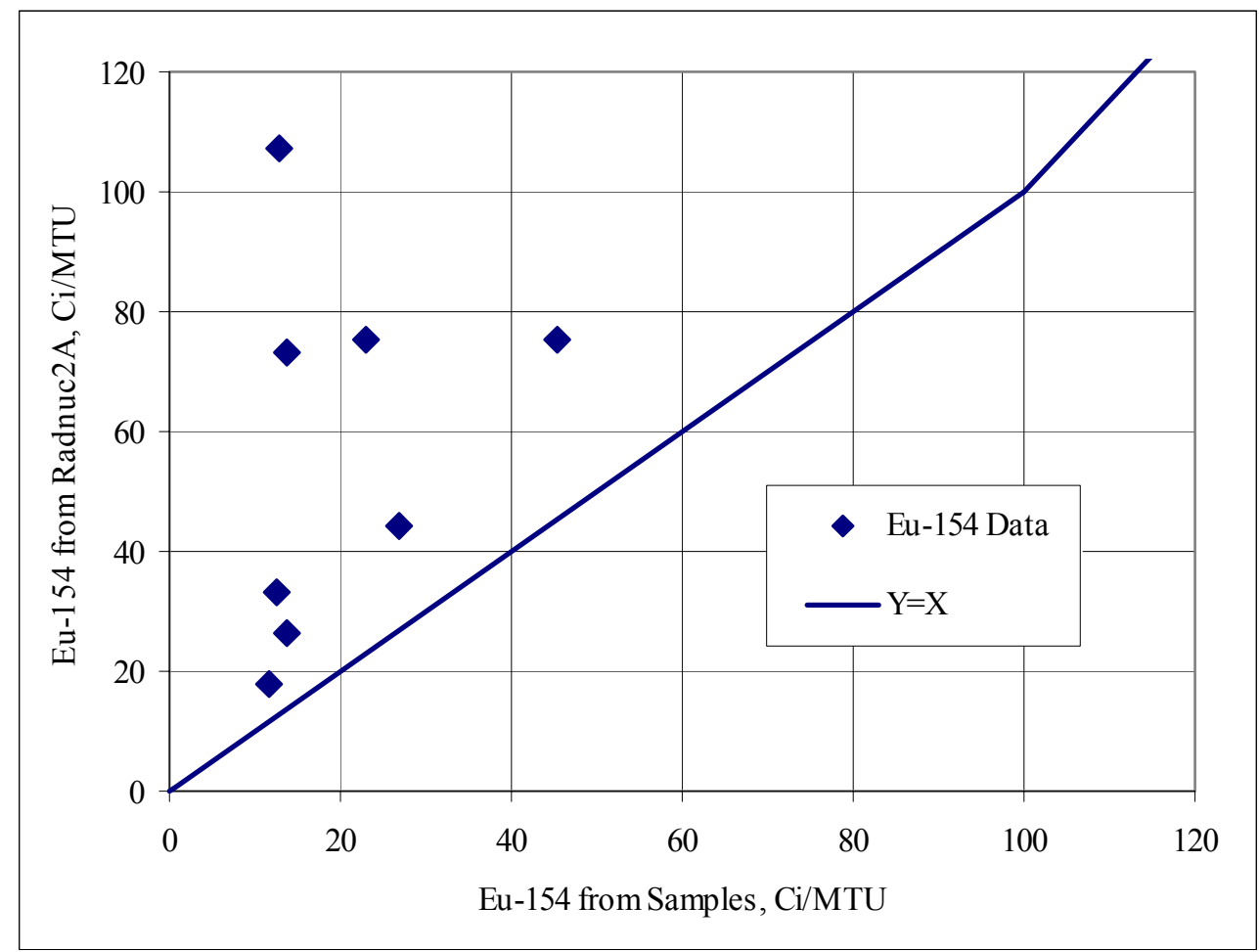

Figure E-8. Comparison of Eu-155 Data and Imputed Values with Predictions.

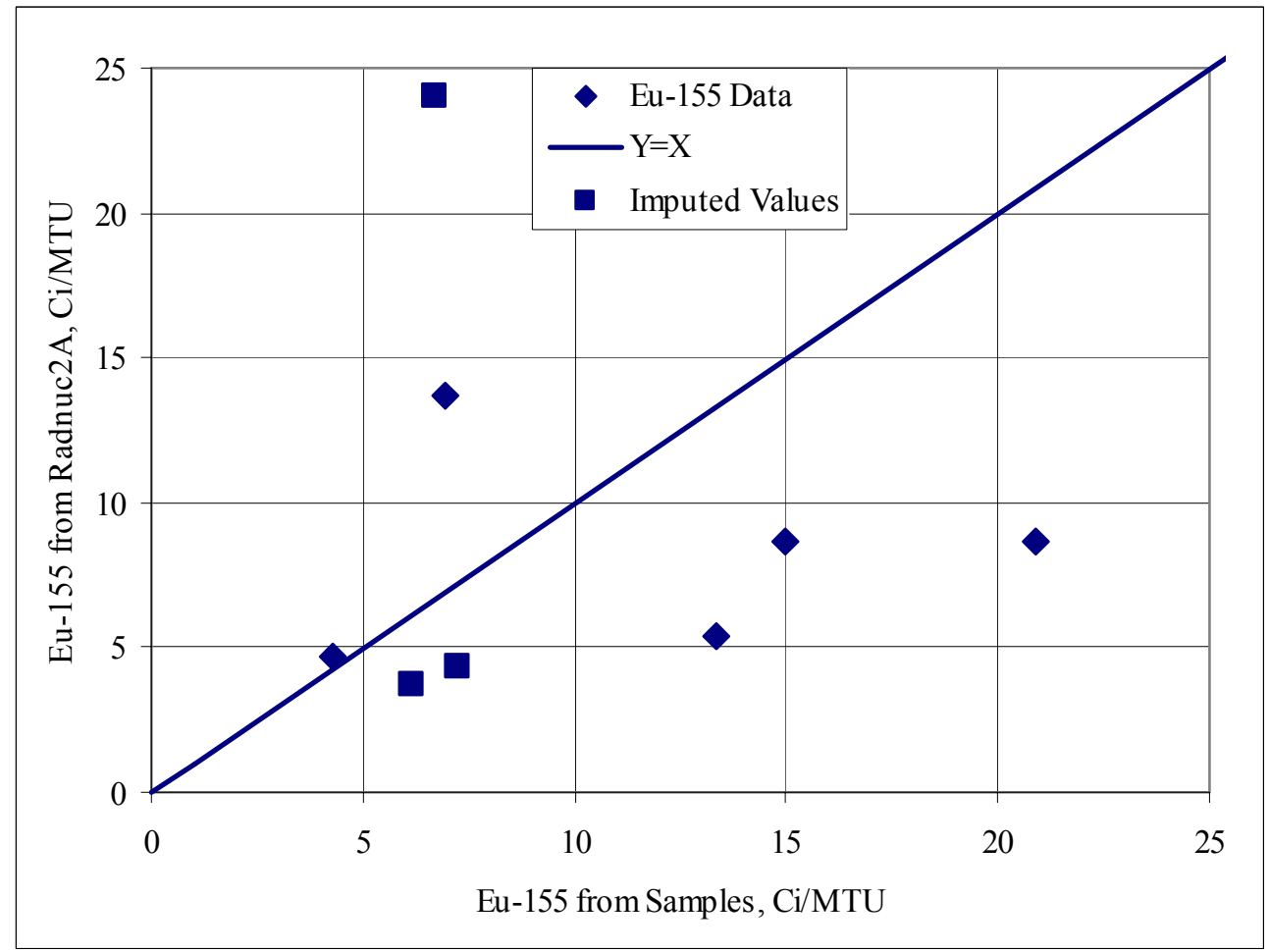


Figure E-9. Comparison of Np-237 Data and Imputed Values with Predictions.

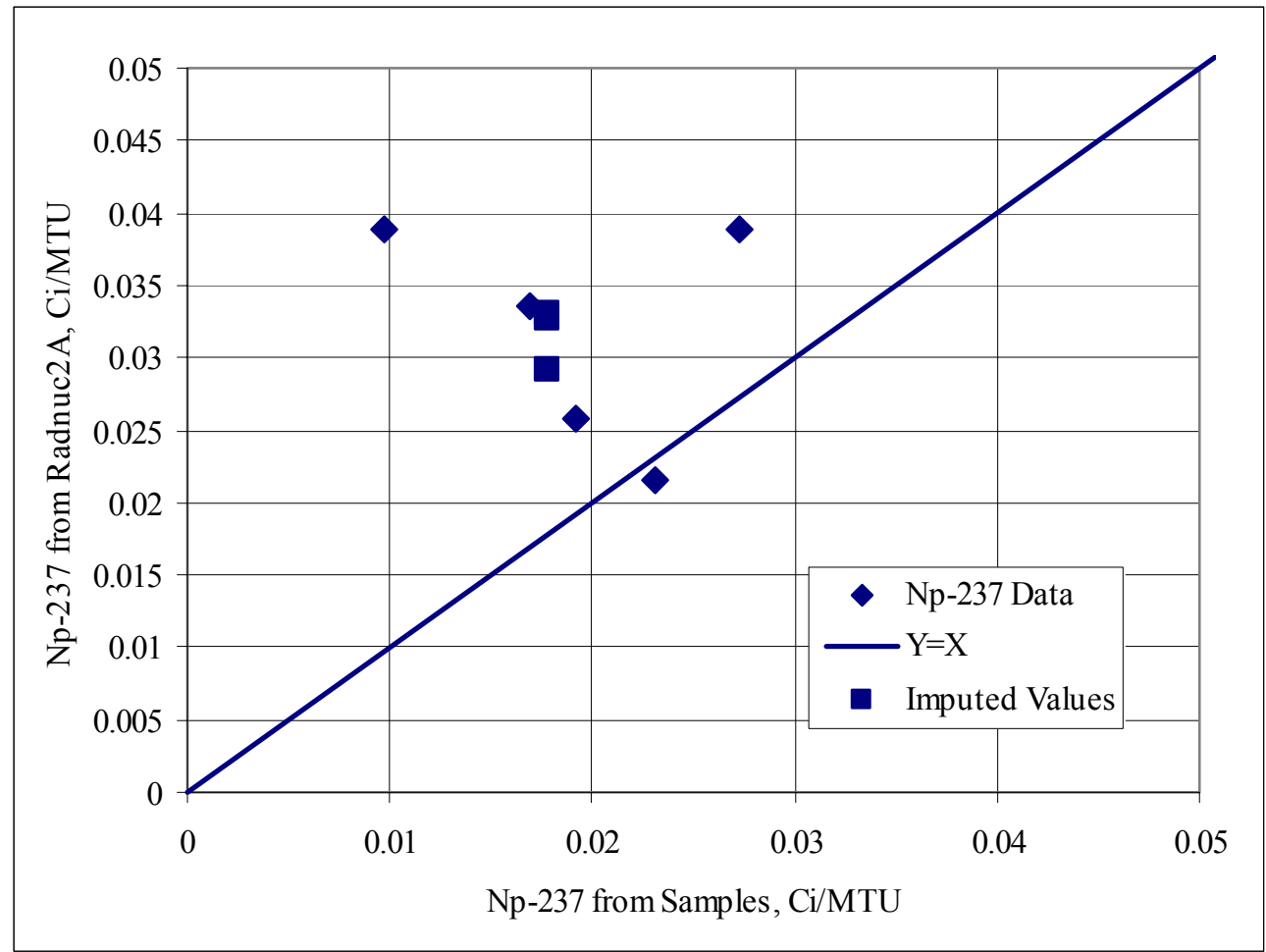

Figure E-10. Comparison of Pu-238 Data and Imputed Values with Predictions.

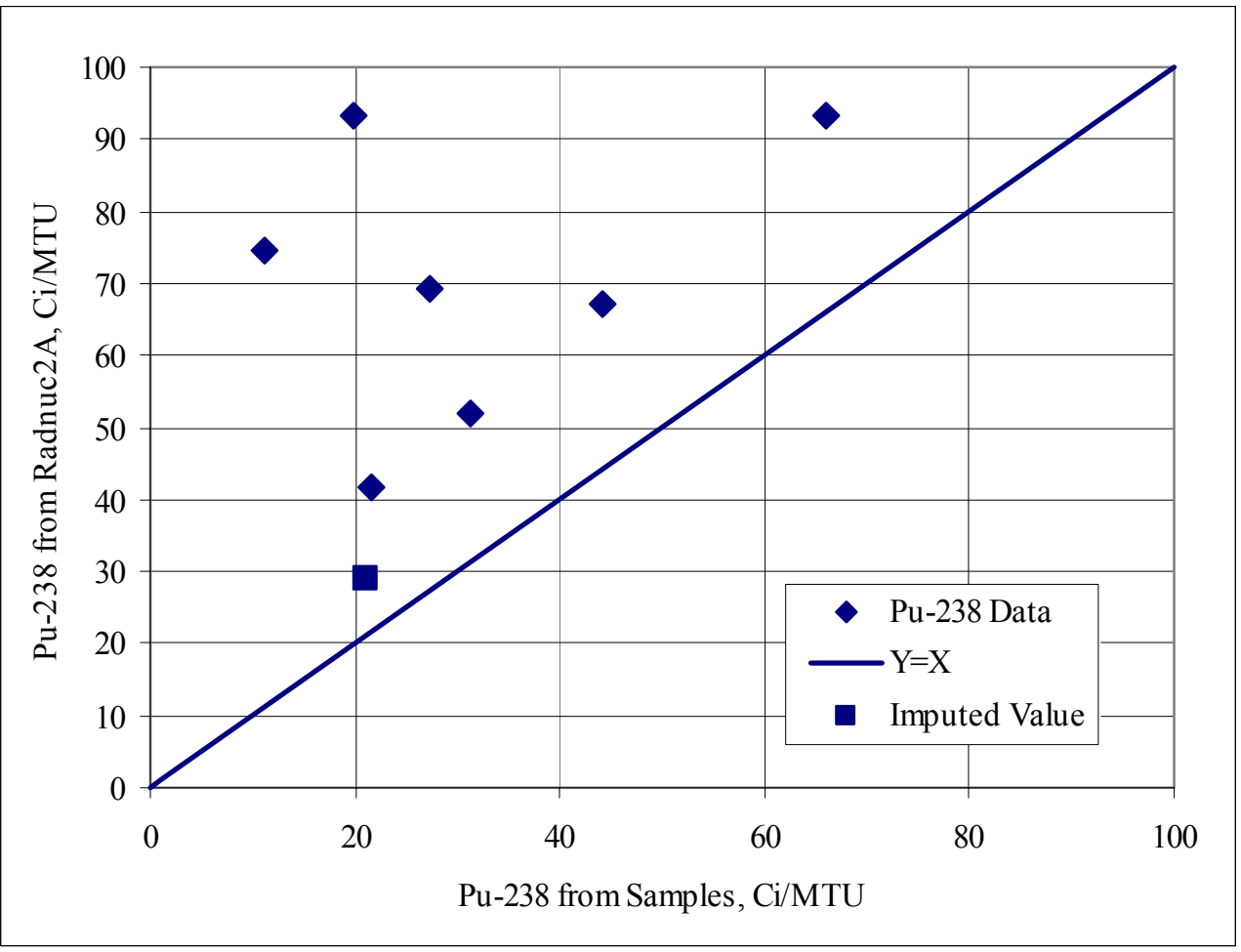


Figure E-11. Comparison of Pu-239/240 Data with Predictions.

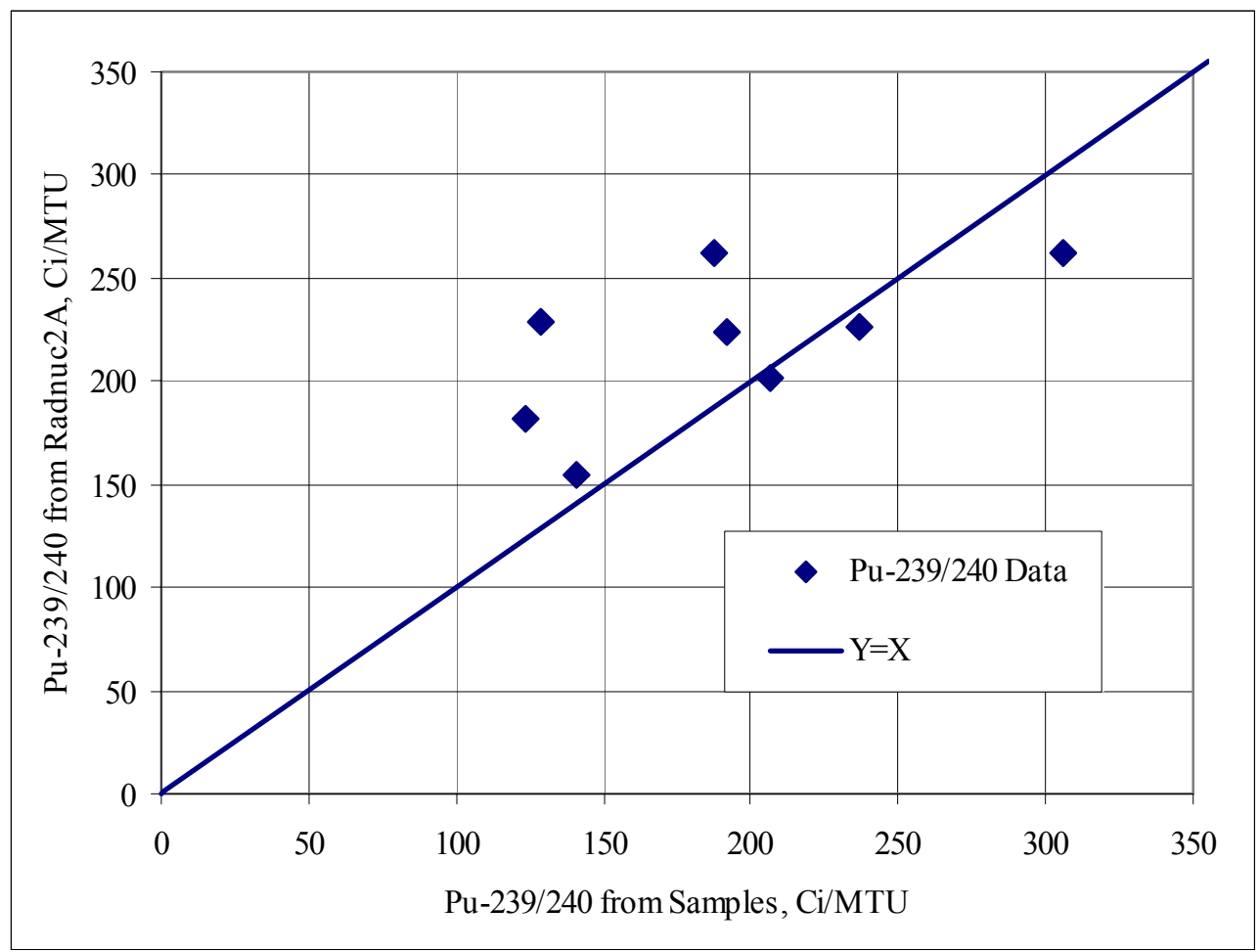

Figure E-12. Comparison of Pu-241 Imputed Values with Predictions.

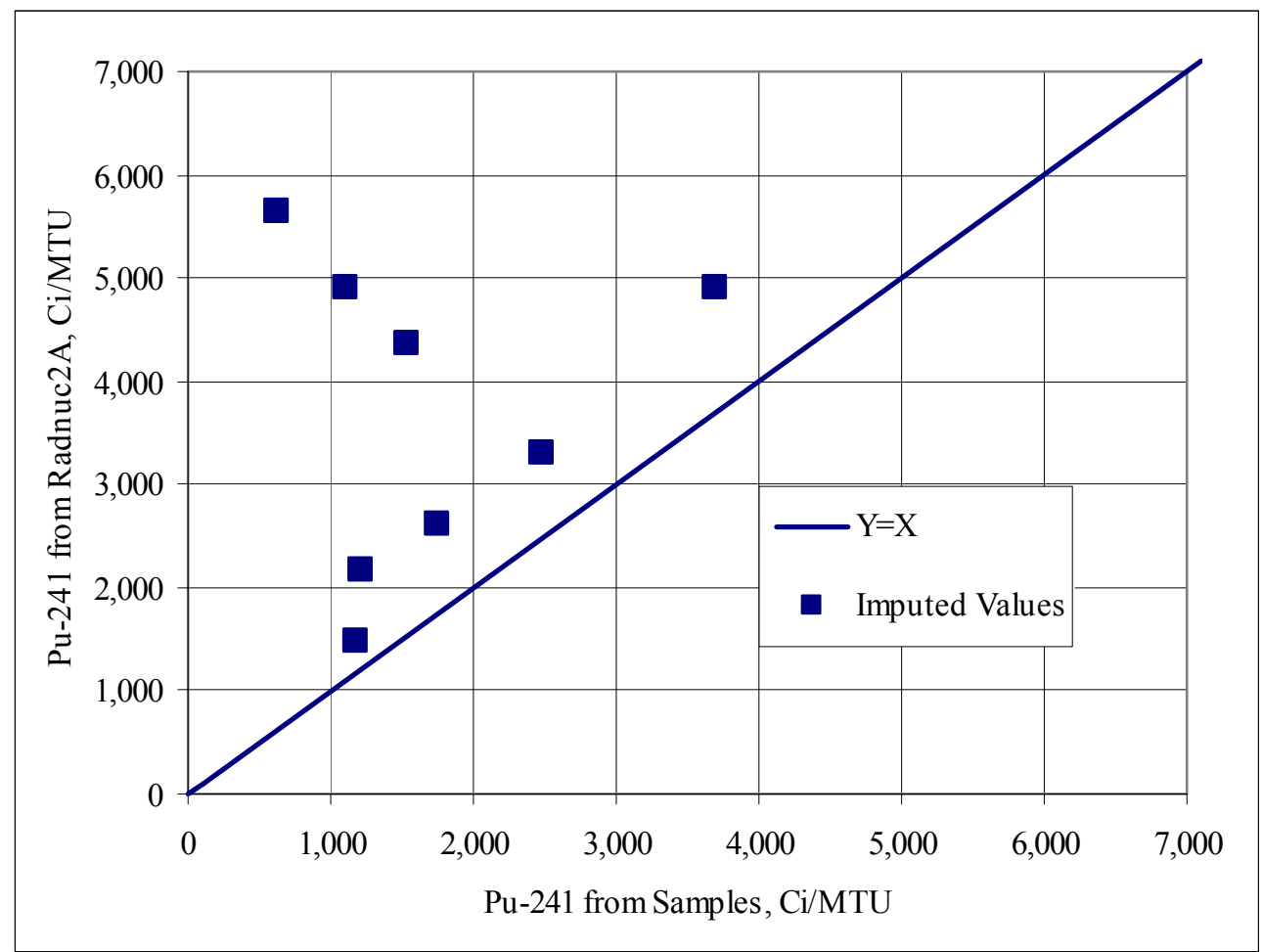


Figure E-13. Comparison of Am-241 Data and Imputed Values with Predictions.

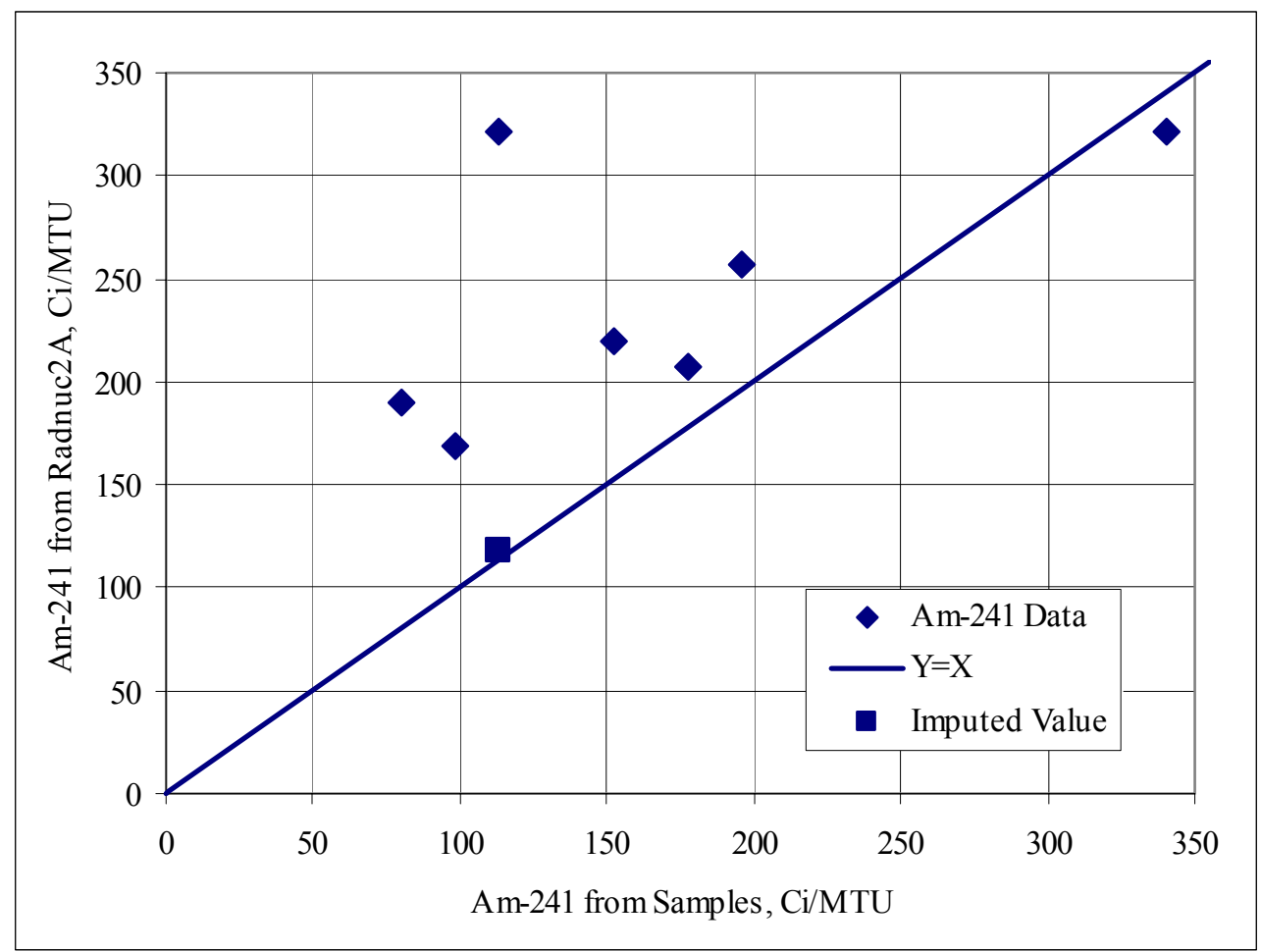


SNF-10293, REV 0

APPENDIX F

LISTING OF THE DATA FILES, PDF-11.DAT AND PDF-11.HDR 
SNF-10293, REV 0

This page intentionally left blank. 
APPENDIX F

\section{LISTING OF THE DATA FILES, PDF-11.DAT AND PDF-11.HDR}

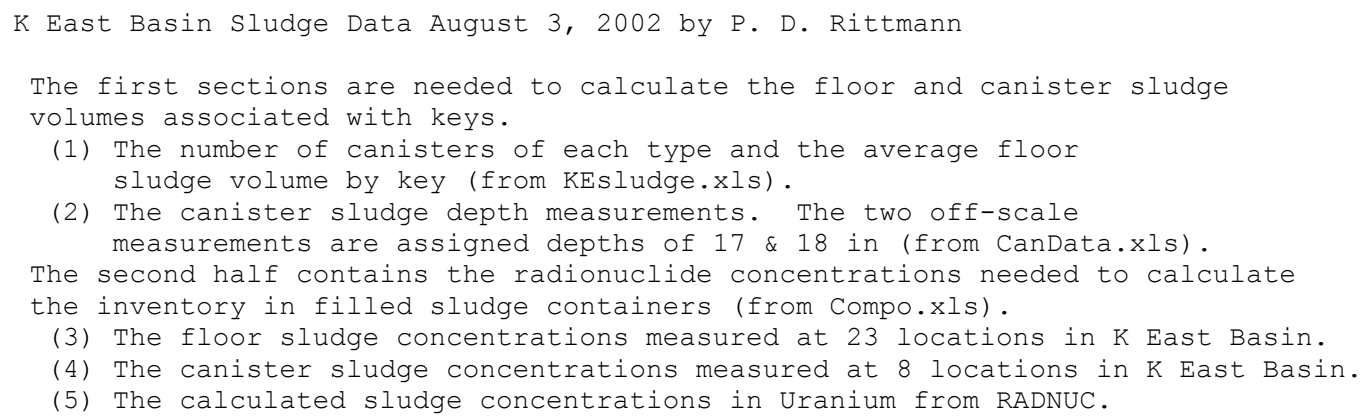

(1) The number of canisters of each type and the average floor sludge volume by key (from KEsludge.xls).

(2) The canister sludge depth measurements. The two off-scale measurements are assigned depths of 17 \& 18 in (from CanData.xls).

The second half contains the radionuclide concentrations needed to calculate the inventory in filled sludge containers (from Compo.xls).

(3) The floor sludge concentrations measured at 23 locations in $\mathrm{K}$ East Basin.

(4) The canister sludge concentrations measured at 8 locations in $\mathrm{K}$ East Basin.

(5) The calculated sludge concentrations in Uranium from RADNUC.

\begin{tabular}{|c|c|c|c|c|c|c|c|c|c|c|}
\hline KE Basin & Number & of $\mathrm{Ca}$ & isters & $\mathrm{of} \mathrm{Eac}$ & Type & Volume & Floor $\mathrm{S}$ & dge (lite & & \\
\hline Key \# & Good & Fair & Poor & Bad & Total & Good & Fair & Poor & $\mathrm{Bad}$ & Total \\
\hline 9621 & 0 & 3 & 0 & 0 & 3 & 0 & 3.10992 & 0 & 0 & 3.10992 \\
\hline 10001 & 1 & 1 & 0 & 0 & 2 & 2.43276 & 3.21024 & 0 & 0 & 5.64300 \\
\hline 10201 & 0 & 0 & 2 & 9 & 11 & 0 & 0 & 7.79988 & 33.5257 & 41.3256 \\
\hline 10259 & 20 & 43 & 2 & 1 & 66 & 49.8402 & 97.1969 & 2.80269 & 2.11926 & 151.959 \\
\hline 10294 & 23 & 78 & 10 & 9 & 120 & 54.1822 & 193.747 & 22.2585 & 22.7789 & 292.966 \\
\hline 10350 & 0 & 0 & 0 & 2 & 2 & 0 & 0 & 0 & 8.38299 & 8.38299 \\
\hline 10385 & 23 & 77 & 9 & 2 & 111 & 62.1796 & 204.91 & 21.5274 & 6.17595 & 294.793 \\
\hline 10456 & 23 & 53 & 5 & 3 & 84 & 75.6726 & 173.315 & 18.8288 & 12.1387 & 279.956 \\
\hline 10576 & 7 & 5 & 0 & 0 & 12 & 31.5444 & 19.4495 & 0 & 0 & 50.9939 \\
\hline 10602 & 18 & 52 & 10 & 5 & 85 & 60.0378 & 175.815 & 34.8023 & 13.0604 & 283.716 \\
\hline 10679 & 21 & 50 & 11 & 4 & 85 & 50.486 & 113.186 & 30.6728 & 8.3322 & 202.677 \\
\hline 10742 & 17 & 39 & 9 & 1 & 66 & 76.9266 & 127.256 & 24.8292 & 4.47051 & 233.482 \\
\hline 10764 & 9 & 1 & 0 & 0 & 10 & 5.91324 & 2.11926 & 0 & 0 & 8.0325 \\
\hline 10770 & 17 & 35 & 6 & 2 & 61 & 83.7108 & 138.322 & 24.453 & 6.28254 & 252.769 \\
\hline 10832 & 38 & 109 & 19 & 1 & 167 & 140.805 & 329.244 & 63.7157 & 1.71171 & 535.477 \\
\hline 10982 & 11 & 65 & 3 & 1 & 80 & 40.291 & 224.51 & 12.3519 & 3.27921 & 280.432 \\
\hline 11036 & 5 & 35 & 1 & 0 & 41 & 16.07 & 143.307 & 3.67422 & 0 & 163.051 \\
\hline 11109 & 12 & 62 & 6 & 0 & 80 & 36.993 & 227.061 & 25.3684 & 0 & 289.422 \\
\hline 11183 & 55 & 95 & 8 & 0 & 158 & 146.554 & 292.467 & 32.3777 & 0 & 471.398 \\
\hline 11372 & 6 & 81 & 9 & 1 & 97 & 19.4809 & 227.582 & 26.9171 & 4.74012 & 278.72 \\
\hline 11449 & 20 & 65 & 7 & 0 & 92 & 71.552 & 193.498 & 19.5185 & 0 & 284.569 \\
\hline 11540 & 15 & 27 & 0 & 0 & 42 & 57.8746 & 89.3663 & 0 & 0 & 147.241 \\
\hline 11733 & 1 & 1 & 0 & 0 & 2 & 1.60512 & 5.17902 & 0 & 0 & 6.78414 \\
\hline 11806 & 28 & 55 & 3 & 0 & 86 & 81.7025 & 143.294 & 4.90941 & 0 & 229.906 \\
\hline 11897 & 43 & 72 & 5 & 0 & 120 & 177.773 & 228.347 & 13.4617 & 0 & 419.582 \\
\hline 11979 & 49 & 65 & 5 & 0 & 119 & 171.052 & 199.373 & 13.8504 & 0 & 384.276 \\
\hline 12127 & 43 & 52 & 0 & 0 & 95 & 122.898 & 149.201 & 0 & 0 & 272.099 \\
\hline 12201 & 32 & 36 & 2 & 0 & 70 & 70.8673 & 101.961 & 6.68382 & 0 & 179.512 \\
\hline 12240 & 3 & 0 & 0 & 0 & 3 & 5.7057 & 0 & 0 & 0 & 5.7057 \\
\hline 12285 & 108 & 87 & 3 & 1 & 199 & 419.795 & 328.642 & 11.4114 & 4.97838 & 764.827 \\
\hline 12480 & 93 & 73 & 2 & 0 & 168 & 316.624 & 243.408 & 5.84991 & 0 & 565.882 \\
\hline 12565 & 84 & 37 & 1 & 0 & 122 & 377.551 & 128.827 & 7.3359 & 0 & 513.714 \\
\hline 12639 & 95 & 45 & 2 & 0 & 142 & 340.865 & 146.85 & 6.81549 & 0 & 494.531 \\
\hline 12852 & 5 & 3 & 0 & 0 & 8 & 15.3427 & 8.99118 & 0 & 0 & 24.3339 \\
\hline 13015 & 1 & 2 & 0 & 0 & 3 & 0.95931 & 3.06603 & 0 & 0 & 4.02534 \\
\hline 13649 & 6 & 10 & 0 & 0 & 16 & 17.0732 & 31.8704 & 0 & 0 & 48.9436 \\
\hline 13685 & 7 & 1 & 0 & 0 & 8 & 24.9233 & 3.90621 & 0 & 0 & 28.8295 \\
\hline 14065 & 1 & 0 & 0 & 0 & 1 & 1.10979 & 0 & 0 & 0 & 1.10979 \\
\hline 14355 & 12 & 16 & 2 & 0 & 30 & 42.8178 & 55.7215 & 6.57723 & 0 & 105.117 \\
\hline 14356 & 8 & 21 & 12 & 14 & 55 & 28.3404 & 77.7167 & 42.626 & 41.5513 & 190.234 \\
\hline 14722 & 1 & 0 & 0 & 0 & 1 & 0.97812 & 0 & 0 & 0 & 0.97812 \\
\hline 15204 & 297 & 16 & 0 & 0 & 313 & 681.929 & 39.3317 & 0 & 0 & 721.261 \\
\hline 15240 & 2 & 0 & 0 & 0 & 2 & 1.8183 & 0 & 0 & 0 & 1.8183 \\
\hline 15316 & 61 & 4 & 0 & 0 & 65 & 191.743 & 9.24198 & 0 & 0 & 200.985 \\
\hline 15399 & 0 & 2 & 0 & 0 & 2 & 0 & 6.97224 & 0 & 0 & 6.97224 \\
\hline
\end{tabular}


SNF-10293, REV 0

$\begin{array}{rrrrrrrrrrr}15444 & 1 & 0 & 0 & 0 & 1 & 5.27307 & 0 & 0 & 0 & 5.27307 \\ 15451 & 28 & 2 & 0 & 0 & 30 & 106.345 & 6.89073 & 0 & 0 & 0 \\ 15455 & 5 & 0 & 0 & 0 & 5 & 27.9642 & 0 & 0 & 0 \\ 15457 & 2 & 0 & 0 & 0 & 2 & 10.5524 & 0 & 0 \\ 15458 & 415 & 10 & 0 & 0 & 425 & 1463.73 & 34.648 & 0 & 10.5524 \\ 15460 & 61 & 0 & 0 & 0 & 61 & 185.065 & 0 & 0 & 0 \\ 15461 & 39 & 0 & 0 & 0 & 39 & 136.197 & 0 & 0 & 0\end{array}$

Canister Sludge Measurements (5043=17 in. and 6070=18 in.)

Fuel Canister Thickness Fuel

Condition Location Position (inches) Material

Bad

Bad $0455 \mathrm{~W}$

Bad $\quad 0455 \mathrm{~W}$

Bad $\quad 0455 \mathrm{~W}$

Bad $\quad 0455 \mathrm{~W}$

Bad $0455 \mathrm{~W}$

Bad $\quad 0627 \mathrm{~W}$

Bad $\quad 0627 \mathrm{~W}$

Bad $0627 \mathrm{~W}$

Bad $\quad 0627 \mathrm{~W}$

Bad $0627 \mathrm{~W}$

Bad $0627 \mathrm{~W}$

Bad $0627 \mathrm{~W}$

Bad $0668 \mathrm{E}$

Bad $0668 \mathrm{E}$

Bad $0668 \mathrm{E}$

Bad $0668 \mathrm{E}$

Bad $0668 \mathrm{~W}$

Bad $\quad 0668 \mathrm{~W}$

Bad $\quad 0668 \mathrm{~W}$

Bad 1236W

Bad 1236W

Bad 1236W

Bad 1243W

Bad 1243W

Bad 1243W

Bad 1243W

Bad 1243W

Bad 1243W

Bad 1243W

Bad $1267 \mathrm{E}$

Bad 1267E

Bad $1267 \mathrm{E}$

Bad $1267 \mathrm{E}$

Bad $1267 \mathrm{E}$

Bad $1267 \mathrm{E}$

Bad 2024E

Bad 2024E

Bad 2024E

Bad 2024E

Bad 2024E

Bad 2024E

Bad 2031W

Bad 2031W

Bad 2031W

Bad 2031W

Bad 2031W

Bad 2031W

Bad 2211W

Bad 2211W

Bad 2211W

Bad 2211W

Bad 2211W

Bad 2211W

Bad 2214E

Bad 2214E

Bad 2214E

Bad 2214E

Bad 2214E

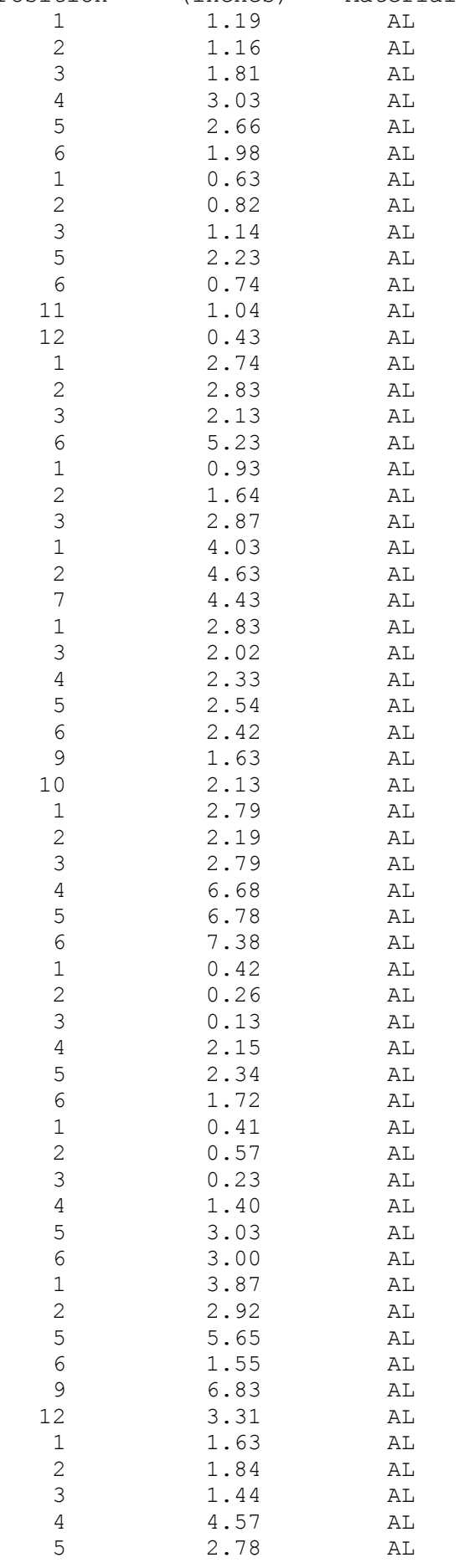


SNF-10293, REV 0

\begin{tabular}{|c|c|c|c|c|}
\hline $\mathrm{Bad}$ & $2214 \mathrm{E}$ & 6 & 1.60 & $\mathrm{AL}$ \\
\hline Bad & $2214 \mathrm{E}$ & 8 & 1.98 & $\mathrm{AL}$ \\
\hline Bad & $2229 E$ & 1 & 2.32 & $\mathrm{AL}$ \\
\hline Bad & $2229 E$ & 2 & 2.23 & $\mathrm{AL}$ \\
\hline Bad & $2229 E$ & 3 & 2.76 & $\mathrm{AL}$ \\
\hline Bad & $2229 E$ & 6 & 5.91 & $\mathrm{AL}$ \\
\hline Bad & $2350 \mathrm{E}$ & 1 & 3.18 & $\mathrm{AL}$ \\
\hline Bad & $2350 \mathrm{E}$ & 2 & 5.92 & $\mathrm{AL}$ \\
\hline $\mathrm{Bad}$ & $2350 \mathrm{E}$ & 3 & 4.12 & AL \\
\hline Bad & $2350 \mathrm{E}$ & 4 & 2.80 & $\mathrm{AL}$ \\
\hline Bad & $2350 \mathrm{E}$ & 5 & 2.66 & $\mathrm{AL}$ \\
\hline Bad & $2350 \mathrm{E}$ & 6 & 3.13 & $\mathrm{AL}$ \\
\hline Bad & $2350 \mathrm{E}$ & 7 & 2.29 & $\mathrm{AL}$ \\
\hline Bad & $2355 \mathrm{~W}$ & 1 & 1.26 & $\mathrm{AL}$ \\
\hline Bad & $2355 \mathrm{~W}$ & 2 & 1.74 & $\mathrm{AL}$ \\
\hline Bad & $2355 \mathrm{~W}$ & 3 & 2.74 & $\mathrm{AL}$ \\
\hline Bad & $2355 \mathrm{~W}$ & 4 & 1.87 & $\mathrm{AL}$ \\
\hline Bad & $2355 \mathrm{~W}$ & 5 & 1.26 & $\mathrm{AL}$ \\
\hline Bad & $2355 \mathrm{~W}$ & 6 & 1.22 & $\mathrm{AL}$ \\
\hline Bad & $2540 \mathrm{E}$ & 1 & 0.72 & $\mathrm{AL}$ \\
\hline Bad & $2540 \mathrm{E}$ & 2 & 0.93 & $\mathrm{AL}$ \\
\hline Bad & $2540 \mathrm{E}$ & 3 & 0.82 & $\mathrm{AL}$ \\
\hline Bad & $2540 \mathrm{E}$ & 4 & 2.03 & $\mathrm{AL}$ \\
\hline Bad & $2540 \mathrm{E}$ & 5 & 1.40 & $\mathrm{AL}$ \\
\hline Bad & $2540 \mathrm{E}$ & 6 & 0.42 & $\mathrm{AL}$ \\
\hline Bad & $2540 \mathrm{E}$ & 9 & 1.77 & $\mathrm{AL}$ \\
\hline $\mathrm{Bad}$ & $2722 E$ & 1 & 1.21 & $\mathrm{AL}$ \\
\hline Bad & $2722 \mathrm{E}$ & 2 & 2.69 & $\mathrm{AL}$ \\
\hline Bad & $2722 \mathrm{E}$ & 4 & 2.06 & $\mathrm{AL}$ \\
\hline Bad & $2722 \mathrm{E}$ & 5 & 1.44 & $\mathrm{AL}$ \\
\hline Bad & $2722 E$ & 6 & 1.58 & $\mathrm{AL}$ \\
\hline Bad & $2756 \mathrm{E}$ & 1 & 0.90 & $\mathrm{AL}$ \\
\hline Bad & $2756 \mathrm{E}$ & 2 & 0.47 & $\mathrm{AL}$ \\
\hline Bad & $2756 \mathrm{E}$ & 3 & 0.79 & $\mathrm{AL}$ \\
\hline Bad & $2756 \mathrm{E}$ & 4 & 2.35 & $\mathrm{AL}$ \\
\hline Bad & $2756 \mathrm{E}$ & 5 & 2.13 & $\mathrm{AL}$ \\
\hline $\mathrm{Bad}$ & $2756 \mathrm{E}$ & 6 & 1.68 & $\mathrm{AL}$ \\
\hline $\mathrm{Bad}$ & $2756 \mathrm{E}$ & 8 & 1.21 & $\mathrm{AL}$ \\
\hline Bad & $3128 \mathrm{~W}$ & 2 & 1.12 & $\mathrm{AL}$ \\
\hline $\mathrm{Bad}$ & $3128 \mathrm{~W}$ & 3 & 1.18 & $\mathrm{AL}$ \\
\hline Bad & $3128 \mathrm{~W}$ & 4 & 3.32 & $\mathrm{AL}$ \\
\hline $\mathrm{Bad}$ & $3128 \mathrm{~W}$ & 5 & 6.31 & $\mathrm{AL}$ \\
\hline $\mathrm{Bad}$ & $3128 \mathrm{~W}$ & 9 & 4.79 & $\mathrm{AL}$ \\
\hline $\mathrm{Bad}$ & $3971 \mathrm{~W}$ & 1 & 1.08 & $\mathrm{AL}$ \\
\hline Bad & $3971 \mathrm{~W}$ & 2 & 1.20 & $\mathrm{AL}$ \\
\hline Bad & $3971 \mathrm{~W}$ & 3 & 1.31 & $\mathrm{AL}$ \\
\hline Bad & $3971 \mathrm{~W}$ & 4 & 0.81 & $\mathrm{AL}$ \\
\hline Bad & $3971 \mathrm{~W}$ & 5 & 1.18 & $\mathrm{AL}$ \\
\hline Bad & $3971 \mathrm{~W}$ & 6 & 1.88 & $\mathrm{AL}$ \\
\hline Bad & $4143 \mathrm{~W}$ & 1 & 1.25 & $\mathrm{AL}$ \\
\hline Bad & $4143 W$ & 2 & 1.45 & $\mathrm{AL}$ \\
\hline $\mathrm{Bad}$ & $4143 W$ & 3 & 1.70 & $\mathrm{AL}$ \\
\hline Bad & $4143 W$ & 4 & 1.70 & $\mathrm{AL}$ \\
\hline Bad & $4143 \mathrm{~W}$ & 5 & 5.74 & $\mathrm{AL}$ \\
\hline Bad & $4143 W$ & 8 & 1.92 & $\mathrm{AL}$ \\
\hline Bad & $4564 \mathrm{E}$ & 1 & 1.74 & $\mathrm{AL}$ \\
\hline Bad & $4564 \mathrm{E}$ & 2 & 2.28 & $\mathrm{AL}$ \\
\hline Bad & $4564 \mathrm{~W}$ & 1 & 2.08 & $\mathrm{AL}$ \\
\hline Bad & $4564 \mathrm{~W}$ & 2 & 7.46 & $\mathrm{AL}$ \\
\hline Bad & $4564 \mathrm{~W}$ & 5 & 8.24 & $\mathrm{AL}$ \\
\hline Bad & $4569 \mathrm{E}$ & 3 & 3.42 & $\mathrm{AL}$ \\
\hline Bad & $4569 \mathrm{~W}$ & 3 & 10.59 & $\mathrm{AL}$ \\
\hline Bad & $4571 E$ & 2 & 9.39 & $\mathrm{AL}$ \\
\hline Bad & $4571 E$ & 3 & 8.46 & $\mathrm{AL}$ \\
\hline Bad & $4571 E$ & 4 & 5.00 & $\mathrm{AL}$ \\
\hline Bad & $4571 E$ & 5 & 7.82 & $\mathrm{AL}$ \\
\hline $\mathrm{Bad}$ & $4571 \mathrm{E}$ & 6 & 6.40 & $\mathrm{AL}$ \\
\hline $\mathrm{Bad}$ & $4850 \mathrm{~W}$ & 1 & 2.99 & $\mathrm{AL}$ \\
\hline $\mathrm{Bad}$ & $4850 \mathrm{~W}$ & 2 & 3.84 & $\mathrm{AL}$ \\
\hline Bad & $4850 \mathrm{~W}$ & 3 & 2.56 & $\mathrm{AL}$ \\
\hline
\end{tabular}

F-3 
SNF-10293, REV 0

\begin{tabular}{|c|c|c|c|c|}
\hline $\mathrm{Bad}$ & $4850 \mathrm{~W}$ & 4 & 1.32 & $\mathrm{AL}$ \\
\hline Bad & $4850 \mathrm{~W}$ & 5 & 1.44 & $\mathrm{AL}$ \\
\hline Bad & $4850 \mathrm{~W}$ & 6 & 1.40 & $\mathrm{AL}$ \\
\hline Bad & $4850 \mathrm{~W}$ & 11 & 1.74 & $\mathrm{AL}$ \\
\hline Bad & $4850 \mathrm{~W}$ & 12 & 1.49 & $\mathrm{AL}$ \\
\hline Bad & $5043 W$ & 2 & 17.00 & $\mathrm{AL}$ \\
\hline Bad & $5055 \mathrm{~W}$ & 1 & 7.10 & $\mathrm{AL}$ \\
\hline Bad & $5055 \mathrm{~W}$ & 2 & 3.78 & $\mathrm{AL}$ \\
\hline $\mathrm{Bad}$ & $5055 \mathrm{~W}$ & 3 & 3.66 & AL \\
\hline Bad & $5055 \mathrm{~W}$ & 4 & 2.54 & $\mathrm{AL}$ \\
\hline Bad & $5055 \mathrm{~W}$ & 5 & 6.14 & $\mathrm{AL}$ \\
\hline Bad & $5055 \mathrm{~W}$ & 6 & 7.50 & $\mathrm{AL}$ \\
\hline $\mathrm{Bad}$ & $5055 \mathrm{~W}$ & 9 & 7.29 & $\mathrm{AL}$ \\
\hline Bad & $5055 \mathrm{~W}$ & 10 & 7.25 & $\mathrm{AL}$ \\
\hline Bad & $5427 \mathrm{~W}$ & 1 & 6.70 & $\mathrm{AL}$ \\
\hline Bad & $5427 \mathrm{~W}$ & 2 & 5.78 & $\mathrm{AL}$ \\
\hline Bad & $5427 \mathrm{~W}$ & 3 & 6.97 & $\mathrm{AL}$ \\
\hline Bad & $5427 \mathrm{~W}$ & 4 & 0.91 & $\mathrm{AL}$ \\
\hline Bad & $5427 \mathrm{~W}$ & 6 & 0.93 & $\mathrm{AL}$ \\
\hline Bad & $5427 \mathrm{~W}$ & 10 & 0.68 & $\mathrm{AL}$ \\
\hline Bad & $5427 \mathrm{~W}$ & 12 & 0.74 & $\mathrm{AL}$ \\
\hline Bad & $5445 \mathrm{E}$ & 1 & 3.75 & $\mathrm{AL}$ \\
\hline Bad & $5445 \mathrm{E}$ & 2 & 8.45 & $\mathrm{AL}$ \\
\hline Bad & $5445 \mathrm{E}$ & 3 & 11.74 & $\mathrm{AL}$ \\
\hline Bad & $5445 E$ & 4 & 6.15 & $\mathrm{AL}$ \\
\hline Bad & $5465 \mathrm{E}$ & 2 & 6.84 & $\mathrm{AL}$ \\
\hline $\mathrm{Bad}$ & $5465 \mathrm{~W}$ & 1 & 10.03 & $\mathrm{AL}$ \\
\hline Bad & $5465 \mathrm{~W}$ & 3 & 8.79 & $\mathrm{AL}$ \\
\hline Bad & $5465 \mathrm{~W}$ & 5 & 9.27 & $\mathrm{AL}$ \\
\hline Bad & $5465 W$ & 6 & 9.59 & $\mathrm{AL}$ \\
\hline Bad & $5465 \mathrm{~W}$ & 7 & 5.09 & $\mathrm{AL}$ \\
\hline Bad & $6070 \mathrm{~W}$ & 1 & 18.00 & $\mathrm{AL}$ \\
\hline Bad & $6071 \mathrm{~W}$ & 1 & 11.12 & $\mathrm{AL}$ \\
\hline Bad & $6071 \mathrm{~W}$ & 2 & 8.68 & $\mathrm{AL}$ \\
\hline Bad & $6071 \mathrm{~W}$ & 2 & 7.80 & $\mathrm{AL}$ \\
\hline Bad & $6071 \mathrm{~W}$ & 3 & 5.26 & $\mathrm{AL}$ \\
\hline Bad & $6071 \mathrm{~W}$ & 3 & 10.69 & $\mathrm{AL}$ \\
\hline Bad & $6071 \mathrm{~W}$ & 4 & 8.91 & $\mathrm{AL}$ \\
\hline Fair & $0450 \mathrm{E}$ & 1 & 1.69 & $\mathrm{AL}$ \\
\hline Fair & $0450 \mathrm{E}$ & 2 & 1.11 & $\mathrm{AL}$ \\
\hline Fair & $0450 \mathrm{E}$ & 3 & 2.68 & $\mathrm{AL}$ \\
\hline Fair & $0450 \mathrm{E}$ & 4 & 4.50 & $\mathrm{AL}$ \\
\hline Fair & $0450 \mathrm{E}$ & 5 & 4.05 & $\mathrm{AL}$ \\
\hline Fair & $0450 \mathrm{E}$ & 6 & 3.55 & $\mathrm{AL}$ \\
\hline Fair & $1858 \mathrm{E}$ & 1 & 0.63 & $\mathrm{AL}$ \\
\hline Fair & $1858 \mathrm{E}$ & 2 & 0.03 & $\mathrm{AL}$ \\
\hline Fair & $1858 \mathrm{E}$ & 4 & 1.14 & $\mathrm{AL}$ \\
\hline Fair & $1858 \mathrm{E}$ & 5 & 2.42 & $\mathrm{AL}$ \\
\hline Fair & $1858 \mathrm{E}$ & 9 & -0.16 & $\mathrm{AL}$ \\
\hline Fair & $2210 E$ & 1 & 0.55 & $\mathrm{AL}$ \\
\hline Fair & $2210 \mathrm{E}$ & 2 & 0.78 & $\mathrm{AL}$ \\
\hline Fair & $2210 \mathrm{E}$ & 3 & 0.89 & $\mathrm{AL}$ \\
\hline Fair & $2210 \mathrm{E}$ & 4 & 1.66 & $\mathrm{AL}$ \\
\hline Fair & $2210 \mathrm{E}$ & 5 & 1.87 & $\mathrm{AL}$ \\
\hline Fair & $2210 \mathrm{E}$ & 6 & 1.05 & $\mathrm{AL}$ \\
\hline Fair & $2210 \mathrm{E}$ & 8 & 0.65 & $\mathrm{AL}$ \\
\hline Fair & $2353 E$ & 1 & 1.09 & $\mathrm{AL}$ \\
\hline Fair & $2353 E$ & 2 & 1.02 & $\mathrm{AL}$ \\
\hline Fair & $2353 E$ & 4 & 1.57 & $\mathrm{AL}$ \\
\hline Fair & $2353 E$ & 5 & 1.35 & $\mathrm{AL}$ \\
\hline Fair & $2353 E$ & 6 & 1.16 & $\mathrm{AL}$ \\
\hline Fair & $2353 E$ & 10 & 3.12 & $\mathrm{AL}$ \\
\hline Fair & $2353 E$ & 11 & 3.25 & $\mathrm{AL}$ \\
\hline Fair & $2711 E$ & 1 & 0.31 & $\mathrm{AL}$ \\
\hline Fair & $2711 E$ & 2 & 0.58 & $\mathrm{AL}$ \\
\hline Fair & $2711 E$ & 3 & 0.54 & $\mathrm{AL}$ \\
\hline Fair & $2711 \mathrm{E}$ & 4 & 2.44 & $\mathrm{AL}$ \\
\hline Fair & $2711 E$ & 5 & 3.90 & $\mathrm{AL}$ \\
\hline Fair & $2711 \mathrm{E}$ & 6 & 2.41 & $\mathrm{AL}$ \\
\hline Fair & $2905 E$ & 2 & 1.83 & $\mathrm{AL}$ \\
\hline
\end{tabular}


SNF-10293, REV 0

\begin{tabular}{|c|c|c|c|c|}
\hline Fair & $2905 E$ & 3 & 1.62 & $\mathrm{AL}$ \\
\hline Fair & $2905 E$ & 4 & 1.90 & $\mathrm{AL}$ \\
\hline Fair & $2905 E$ & 5 & 0.74 & $\mathrm{AL}$ \\
\hline Fair & $2905 E$ & 6 & 2.16 & $\mathrm{AL}$ \\
\hline Fair & $3727 \mathrm{~W}$ & 1 & 4.06 & $\mathrm{AL}$ \\
\hline Fair & $3727 \mathrm{~W}$ & 2 & 7.20 & $\mathrm{AL}$ \\
\hline Fair & $3727 \mathrm{~W}$ & 3 & 2.63 & $\mathrm{AL}$ \\
\hline Fair & $3727 \mathrm{~W}$ & 4 & 2.17 & $\mathrm{AL}$ \\
\hline Fair & $3727 \mathrm{~W}$ & 5 & 1.64 & $\mathrm{AL}$ \\
\hline Fair & $3727 \mathrm{~W}$ & 6 & 1.69 & $\mathrm{AL}$ \\
\hline Fair & $3727 \mathrm{~W}$ & 11 & 1.77 & $\mathrm{AL}$ \\
\hline Fair & $4138 \mathrm{~W}$ & 2 & 4.21 & $\mathrm{AL}$ \\
\hline Fair & $4138 \mathrm{~W}$ & 3 & 2.69 & AL \\
\hline Fair & $4138 \mathrm{~W}$ & 4 & 1.98 & $\mathrm{AL}$ \\
\hline Fair & $4138 \mathrm{~W}$ & 5 & 3.18 & AL \\
\hline Fair & $4138 \mathrm{~W}$ & 6 & 3.61 & $\mathrm{AL}$ \\
\hline Fair & $4138 \mathrm{~W}$ & 7 & 3.46 & $\mathrm{AL}$ \\
\hline Fair & $4138 \mathrm{~W}$ & 8 & 4.08 & $\mathrm{AL}$ \\
\hline Fair & $4168 \mathrm{E}$ & 3 & 0.48 & $\mathrm{AL}$ \\
\hline Fair & $4168 \mathrm{E}$ & 4 & 0.21 & $\mathrm{AL}$ \\
\hline Fair & $4168 \mathrm{E}$ & 6 & 0.44 & $\mathrm{AL}$ \\
\hline Fair & $4168 \mathrm{~W}$ & 1 & 0.48 & $\mathrm{AL}$ \\
\hline Fair & $4168 \mathrm{~W}$ & 3 & 0.51 & $\mathrm{AL}$ \\
\hline Fair & $4168 \mathrm{~W}$ & 6 & 0.29 & $\mathrm{AL}$ \\
\hline Fair & $4620 \mathrm{~W}$ & 1 & 2.01 & $\mathrm{AL}$ \\
\hline Fair & $4620 \mathrm{~W}$ & 2 & 2.33 & $\mathrm{AL}$ \\
\hline Fair & $4620 \mathrm{~W}$ & 3 & 2.57 & $\mathrm{AL}$ \\
\hline Fair & $4620 \mathrm{~W}$ & 4 & 2.91 & $\mathrm{AL}$ \\
\hline Fair & $4620 \mathrm{~W}$ & 5 & 4.53 & $\mathrm{AL}$ \\
\hline Fair & $4620 \mathrm{~W}$ & 6 & 2.54 & $\mathrm{AL}$ \\
\hline Fair & $4620 \mathrm{~W}$ & 8 & 1.79 & $\mathrm{AL}$ \\
\hline Fair & $4627 \mathrm{~W}$ & 1 & 2.44 & $\mathrm{AL}$ \\
\hline Fair & $4627 \mathrm{~W}$ & 2 & 2.37 & $\mathrm{AL}$ \\
\hline Fair & $4627 \mathrm{~W}$ & 3 & 1.37 & $\mathrm{AL}$ \\
\hline Fair & $4627 \mathrm{~W}$ & 4 & 0.70 & $\mathrm{AL}$ \\
\hline Fair & $4627 \mathrm{~W}$ & 5 & 0.68 & $\mathrm{AL}$ \\
\hline Fair & $4627 \mathrm{~W}$ & 6 & 0.96 & $\mathrm{AL}$ \\
\hline Fair & $4627 \mathrm{~W}$ & 9 & 1.23 & $\mathrm{AL}$ \\
\hline Fair & $4627 \mathrm{~W}$ & 10 & 1.04 & $\mathrm{AL}$ \\
\hline Fair & $4627 \mathrm{~W}$ & 11 & 0.96 & $\mathrm{AL}$ \\
\hline Fair & $4865 \mathrm{~W}$ & 1 & 1.21 & $\mathrm{AL}$ \\
\hline Fair & $4865 \mathrm{~W}$ & 2 & 1.36 & $\mathrm{AL}$ \\
\hline Fair & $4865 \mathrm{~W}$ & 3 & 1.62 & $\mathrm{AL}$ \\
\hline Fair & $4865 \mathrm{~W}$ & 4 & 3.08 & $\mathrm{AL}$ \\
\hline Fair & $4865 \mathrm{~W}$ & 5 & 2.92 & $\mathrm{AL}$ \\
\hline Fair & $4865 \mathrm{~W}$ & 6 & 2.87 & $\mathrm{AL}$ \\
\hline Fair & $4865 \mathrm{~W}$ & 8 & 1.95 & $\mathrm{AL}$ \\
\hline Fair & $4865 \mathrm{~W}$ & 9 & 1.37 & $\mathrm{AL}$ \\
\hline Fair & $4869 \mathrm{E}$ & 1 & 2.26 & $\mathrm{AL}$ \\
\hline Fair & $4869 \mathrm{E}$ & 2 & 2.14 & $\mathrm{AL}$ \\
\hline Fair & $4869 \mathrm{E}$ & 3 & 1.56 & $\mathrm{AL}$ \\
\hline Fair & $4869 \mathrm{E}$ & 4 & 2.94 & $\mathrm{AL}$ \\
\hline Fair & $4869 \mathrm{E}$ & 5 & 3.63 & $\mathrm{AL}$ \\
\hline Fair & $4869 \mathrm{E}$ & 6 & 2.98 & $\mathrm{AL}$ \\
\hline Fair & $5069 \mathrm{E}$ & 1 & 4.16 & $\mathrm{AL}$ \\
\hline Fair & $5069 \mathrm{E}$ & 2 & 2.92 & $\mathrm{AL}$ \\
\hline Fair & $5069 \mathrm{E}$ & 3 & 4.06 & $\mathrm{AL}$ \\
\hline Fair & $5069 \mathrm{E}$ & 4 & 4.81 & $\mathrm{AL}$ \\
\hline Fair & $5069 \mathrm{E}$ & 5 & 1.12 & $\mathrm{AL}$ \\
\hline Fair & $5069 \mathrm{E}$ & 6 & 1.49 & $\mathrm{AL}$ \\
\hline Fair & $5069 \mathrm{E}$ & 9 & 0.89 & $\mathrm{AL}$ \\
\hline Fair & $5069 \mathrm{E}$ & 10 & 2.49 & $\mathrm{AL}$ \\
\hline Fair & $5254 \mathrm{E}$ & 2 & 0.82 & $\mathrm{AL}$ \\
\hline Fair & $5254 \mathrm{E}$ & 3 & 2.22 & $\mathrm{AL}$ \\
\hline Fair & $5254 \mathrm{E}$ & 4 & 0.98 & $\mathrm{AL}$ \\
\hline Fair & $5254 \mathrm{E}$ & 5 & 1.19 & $\mathrm{AL}$ \\
\hline Fair & $5254 \mathrm{E}$ & 6 & 1.50 & $\mathrm{AL}$ \\
\hline Fair & $5254 \mathrm{E}$ & 10 & 1.22 & $\mathrm{AL}$ \\
\hline Fair & $5427 \mathrm{E}$ & 1 & 1.81 & $\mathrm{AL}$ \\
\hline Fair & $5427 \mathrm{E}$ & 2 & 1.87 & $\mathrm{AL}$ \\
\hline
\end{tabular}


SNF-10293, REV 0

\begin{tabular}{|c|c|c|c|c|}
\hline Fair & $5427 \mathrm{E}$ & 3 & 2.24 & $\mathrm{AL}$ \\
\hline Fair & $5427 E$ & 4 & 1.32 & $\mathrm{AL}$ \\
\hline Fair & $5427 \mathrm{E}$ & 5 & 0.74 & $\mathrm{AL}$ \\
\hline Fair & $5427 \mathrm{E}$ & 6 & 1.41 & $\mathrm{AL}$ \\
\hline Fair & $5427 E$ & 10 & 1.67 & $\mathrm{AL}$ \\
\hline Fair & $6069 \mathrm{~W}$ & 13 & 0.07 & $\mathrm{AL}$ \\
\hline Fair & $0418 E$ & 1 & 0.74 & SS \\
\hline Fair & $0418 \mathrm{E}$ & 2 & 0.63 & SS \\
\hline Fair & $0418 \mathrm{E}$ & 3 & 1.14 & SS \\
\hline Fair & $0418 \mathrm{E}$ & 4 & 0.74 & SS \\
\hline Fair & $0418 \mathrm{E}$ & 5 & 0.43 & SS \\
\hline Fair & $0418 \mathrm{E}$ & 6 & 0.55 & SS \\
\hline Fair & $0418 \mathrm{E}$ & 10 & 1.14 & SS \\
\hline Fair & $0418 \mathrm{E}$ & 11 & 0.22 & SS \\
\hline Fair & $0418 \mathrm{E}$ & 12 & 0.34 & SS \\
\hline Fair & $3125 \mathrm{~W}$ & 1 & 1.09 & SS \\
\hline Fair & $3125 \mathrm{~W}$ & 2 & 1.48 & SS \\
\hline Fair & $3125 \mathrm{~W}$ & 3 & 1.46 & SS \\
\hline Fair & $3125 \mathrm{~W}$ & 4 & 1.68 & SS \\
\hline Fair & $3125 \mathrm{~W}$ & 5 & 1.44 & SS \\
\hline Fair & $3125 \mathrm{~W}$ & 6 & 1.39 & SS \\
\hline Fair & $4359 \mathrm{~W}$ & 1 & 0.25 & SS \\
\hline Fair & $4359 \mathrm{~W}$ & 3 & 0.40 & SS \\
\hline Fair & $4359 \mathrm{~W}$ & 4 & -0.11 & SS \\
\hline Fair & $4359 \mathrm{~W}$ & 5 & 0.40 & SS \\
\hline Fair & $4359 \mathrm{~W}$ & 6 & 0.19 & SS \\
\hline Fair & $4359 \mathrm{~W}$ & 8 & 0.21 & SS \\
\hline Fair & $4359 \mathrm{~W}$ & 9 & 0.38 & SS \\
\hline Fair & $4359 \mathrm{~W}$ & 10 & 0.21 & SS \\
\hline Fair & $4359 \mathrm{~W}$ & 11 & 0.43 & SS \\
\hline Fair & $4359 \mathrm{~W}$ & 12 & 0.47 & SS \\
\hline Fair & $5238 \mathrm{~W}$ & 1 & 0.06 & SS \\
\hline Fair & $5238 W$ & 2 & 0.72 & SS \\
\hline Fair & $5238 W$ & 3 & 0.68 & SS \\
\hline Fair & $5238 W$ & 4 & 0.77 & SS \\
\hline Fair & $5238 W$ & 5 & 1.10 & SS \\
\hline Fair & $5238 \mathrm{~W}$ & 6 & 1.90 & SS \\
\hline Fair & $5238 \mathrm{~W}$ & 9 & 1.02 & SS \\
\hline Fair & $5238 \mathrm{~W}$ & 10 & 3.70 & SS \\
\hline Fair & $5608 \mathrm{E}$ & 1 & -0.32 & SS \\
\hline Fair & $5608 \mathrm{E}$ & 2 & -0.06 & SS \\
\hline Fair & $5608 \mathrm{E}$ & 3 & 0 & SS \\
\hline Fair & $5608 \mathrm{E}$ & 7 & 0.07 & SS \\
\hline Fair & $5608 \mathrm{E}$ & 8 & 0.01 & SS \\
\hline Fair & $5618 \mathrm{E}$ & 2 & 1.12 & SS \\
\hline Fair & $5618 \mathrm{E}$ & 3 & 0.84 & SS \\
\hline Fair & $5618 \mathrm{E}$ & 4 & 0.84 & SS \\
\hline Fair & $5618 \mathrm{E}$ & 5 & 0.74 & SS \\
\hline Fair & $5618 \mathrm{E}$ & 6 & 0.70 & SS \\
\hline Fair & $5618 \mathrm{E}$ & 11 & 0.87 & SS \\
\hline Fair & $5618 \mathrm{~W}$ & 1 & 1.40 & SS \\
\hline Fair & $5618 \mathrm{~W}$ & 2 & 2.14 & SS \\
\hline Fair & $5618 \mathrm{~W}$ & 3 & 1.42 & SS \\
\hline Fair & $5618 \mathrm{~W}$ & 4 & 1.69 & SS \\
\hline Fair & $5618 \mathrm{~W}$ & 5 & 1.26 & SS \\
\hline Fair & $5618 \mathrm{~W}$ & 6 & 1.60 & SS \\
\hline Fair & $5618 \mathrm{~W}$ & 11 & 1.17 & SS \\
\hline Fair & $6057 \mathrm{E}$ & 1 & 0.68 & SS \\
\hline Fair & $6057 \mathrm{E}$ & 2 & 0.74 & SS \\
\hline Fair & $6057 \mathrm{E}$ & 3 & 0.68 & SS \\
\hline Fair & $6057 \mathrm{E}$ & 4 & 3.45 & SS \\
\hline Fair & $6057 \mathrm{E}$ & 5 & 2.83 & SS \\
\hline Fair & $6057 \mathrm{E}$ & 6 & 3.81 & SS \\
\hline Fair & $6057 \mathrm{E}$ & 8 & 1.16 & SS \\
\hline Fair & $6413 E$ & 1 & 1.70 & SS \\
\hline Fair & $6413 E$ & 2 & 1.53 & SS \\
\hline Fair & $6413 \mathrm{E}$ & 3 & 2.08 & SS \\
\hline Fair & $6413 E$ & 4 & 1.38 & SS \\
\hline Fair & $6413 E$ & 5 & 1.24 & SS \\
\hline Fair & $6413 E$ & 6 & 1.10 & SS \\
\hline
\end{tabular}


SNF-10293, REV 0

\begin{tabular}{|c|c|c|c|c|}
\hline Fair & $6413 \mathrm{E}$ & 11 & 1.37 & SS \\
\hline Fair & $6413 E$ & 12 & 1.24 & SS \\
\hline Fair & $6143 W$ & 3 & 2.04 & SS \\
\hline Fair & $6143 \mathrm{~W}$ & 4 & 2.04 & SS \\
\hline Fair & $6143 \mathrm{~W}$ & 5 & 1.61 & SS \\
\hline Fair & $6143 \mathrm{~W}$ & 6 & 2.00 & SS \\
\hline Fair & $6143 W$ & 11 & 1.46 & SS \\
\hline Good & $1845 \mathrm{E}$ & 1 & 2.45 & SS \\
\hline Good & $1845 \mathrm{E}$ & 2 & 2.75 & SS \\
\hline Good & $1845 \mathrm{E}$ & 3 & 1.95 & SS \\
\hline Good & $1845 \mathrm{E}$ & 4 & 2.15 & SS \\
\hline Good & $2244 \mathrm{E}$ & 1 & -0.14 & SS \\
\hline Good & $2244 \mathrm{E}$ & 2 & -0.06 & SS \\
\hline Good & $2244 \mathrm{E}$ & 3 & 0.05 & SS \\
\hline Good & $2244 \mathrm{E}$ & 4 & 0.26 & SS \\
\hline Good & $2244 \mathrm{E}$ & 13 & -0.45 & SS \\
\hline Good & $2244 W$ & 1 & -0.33 & SS \\
\hline Good & $2244 W$ & 2 & -0.06 & SS \\
\hline Good & $2244 W$ & 3 & -0.06 & SS \\
\hline Good & $2244 W$ & 4 & 0.05 & SS \\
\hline Good & $2244 W$ & 5 & -0.33 & SS \\
\hline Good & $2244 W$ & 6 & -0.33 & SS \\
\hline Good & $2244 \mathrm{~W}$ & 13 & -0.06 & SS \\
\hline Good & $2248 \mathrm{~W}$ & 1 & -0.14 & SS \\
\hline Good & $2248 \mathrm{~W}$ & 2 & -0.14 & $\mathrm{~S}$ \\
\hline Good & $2248 W$ & 3 & -0.06 & $S S$ \\
\hline Good & $2248 W$ & 4 & 0.05 & SS \\
\hline Good & $2248 W$ & 6 & -0.14 & SS \\
\hline Good & $2248 W$ & 13 & -0.06 & SS \\
\hline Good & $4661 \mathrm{E}$ & 1 & -0.12 & $\mathrm{~S}$ \\
\hline Good & $4661 \mathrm{E}$ & 2 & 0.14 & SS \\
\hline Good & $4661 \mathrm{E}$ & 3 & -0.12 & $\mathrm{~S}$ \\
\hline Good & $4661 \mathrm{E}$ & 4 & -0.25 & SS \\
\hline Good & $4661 \mathrm{E}$ & 8 & 0.23 & SS \\
\hline Done & 9999R & 0 & 0.0 & WW \\
\hline
\end{tabular}




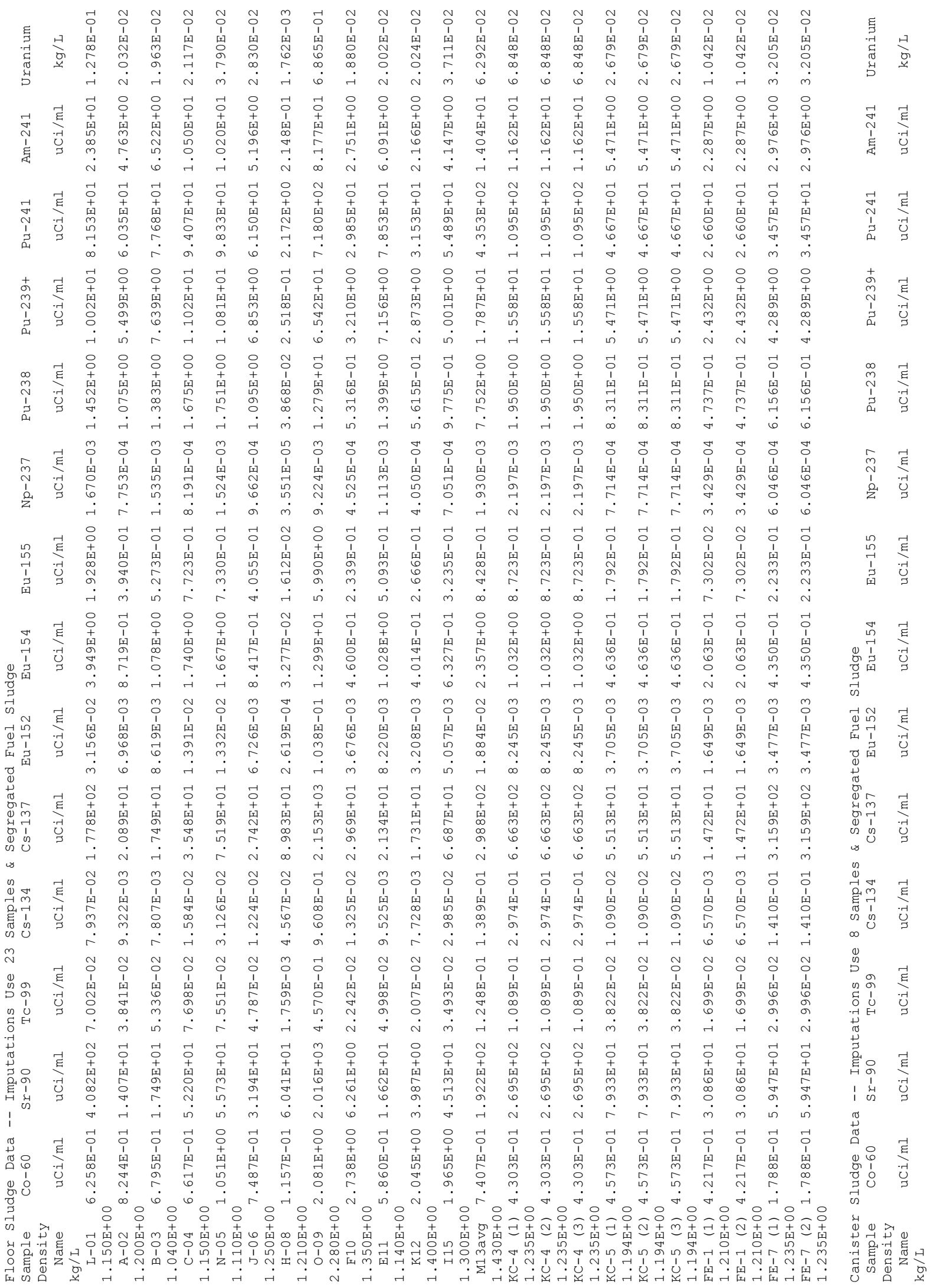




\section{SNF-10293. REV 0}

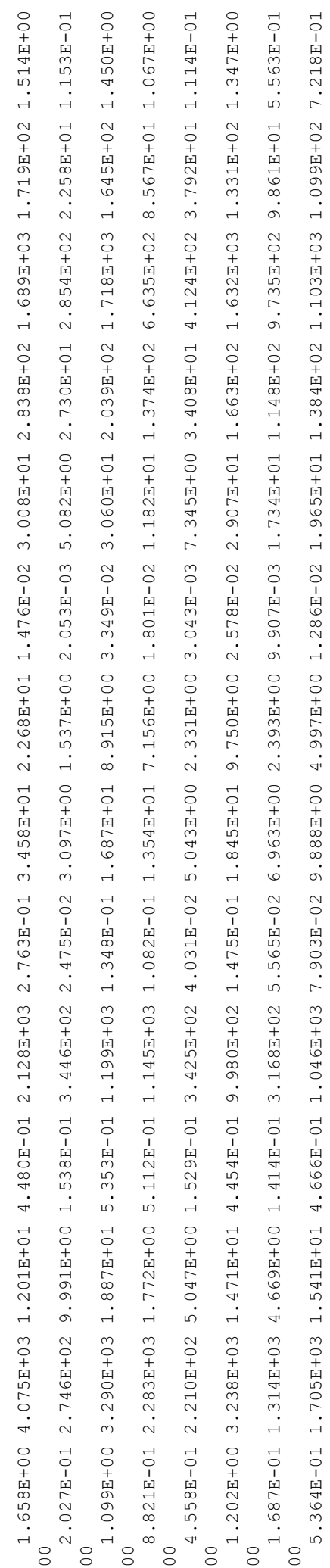

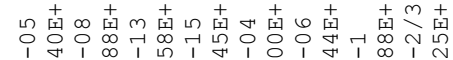

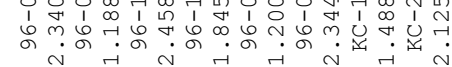

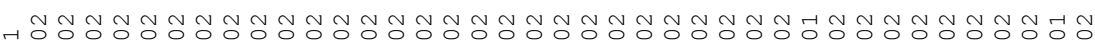

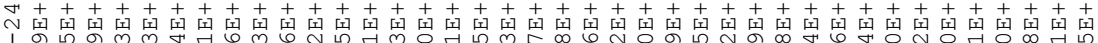

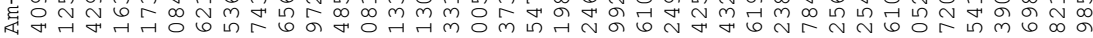

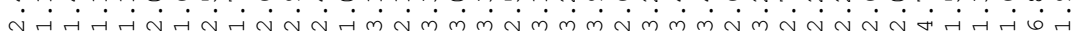

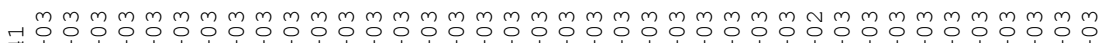

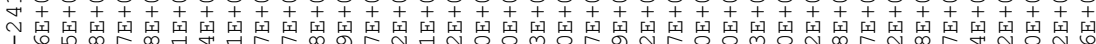

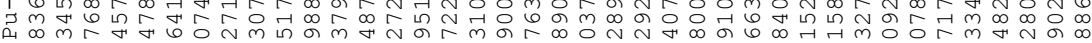

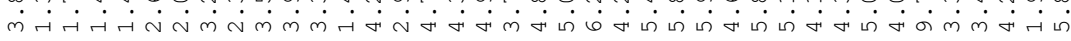

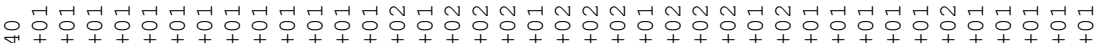

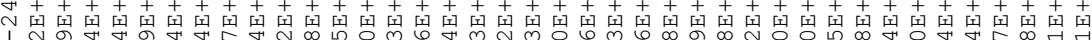

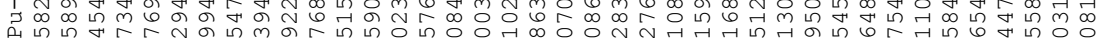

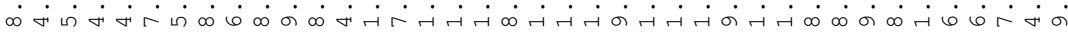
の

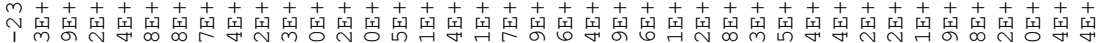

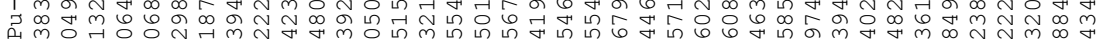

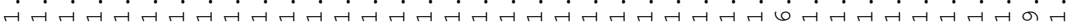

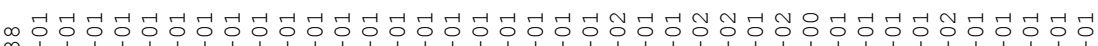

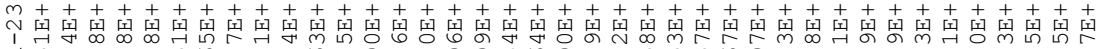

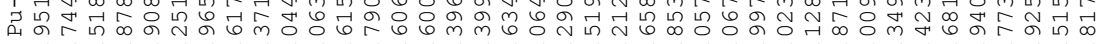

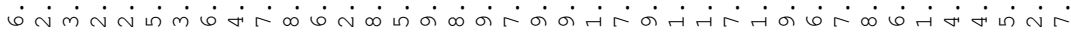
గ N $\mathcal{O}$ O N $\begin{aligned} & 1 \\ & T\end{aligned}$

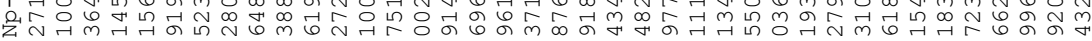

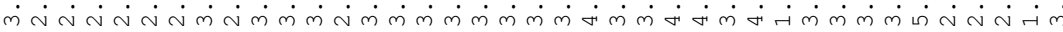

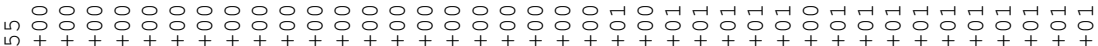

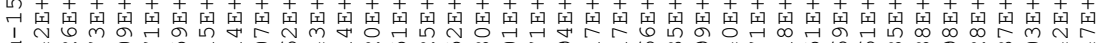

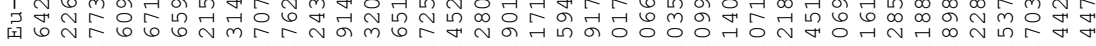

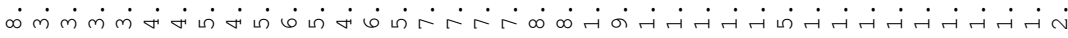

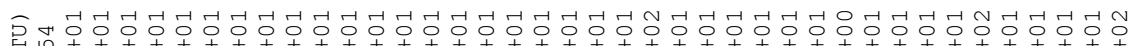

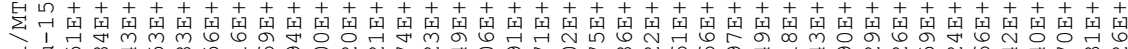

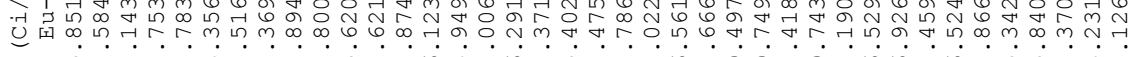

出

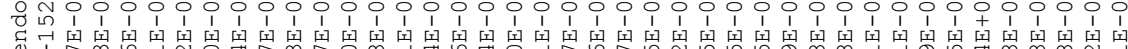

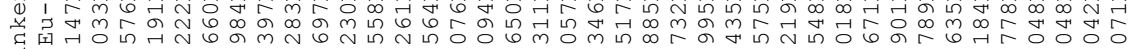

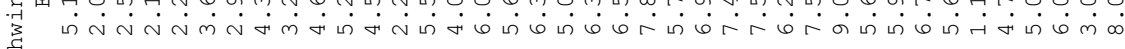

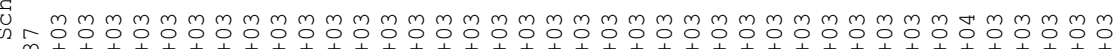

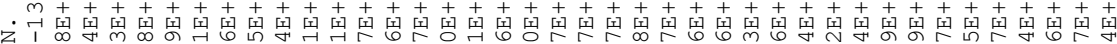

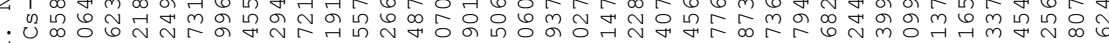

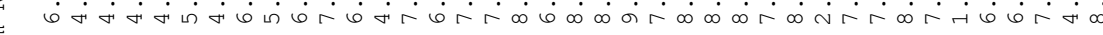

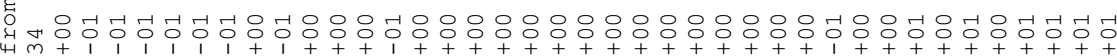

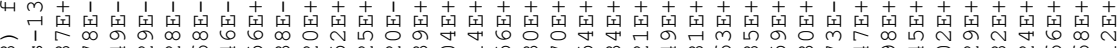

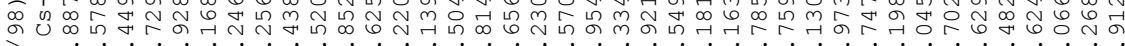

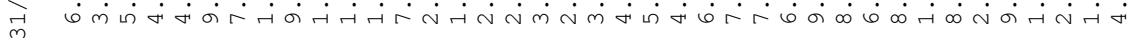

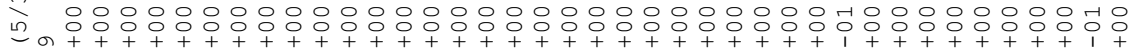

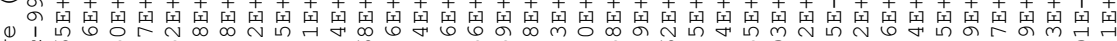

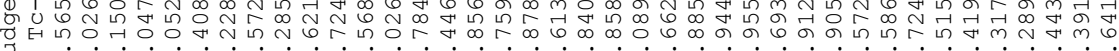

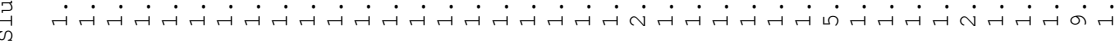

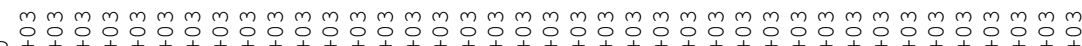

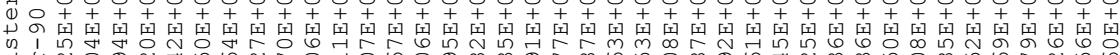
ت

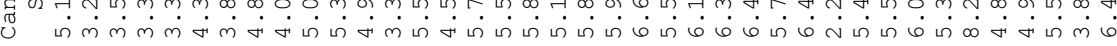

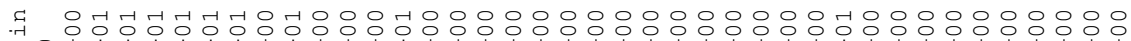

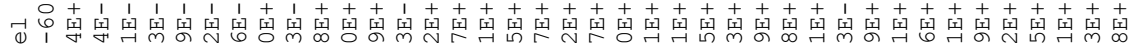

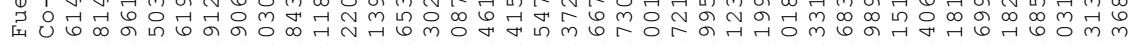
ช 范口

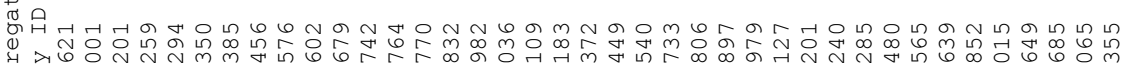

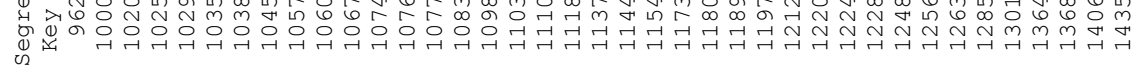


SNF-10293. REV 0

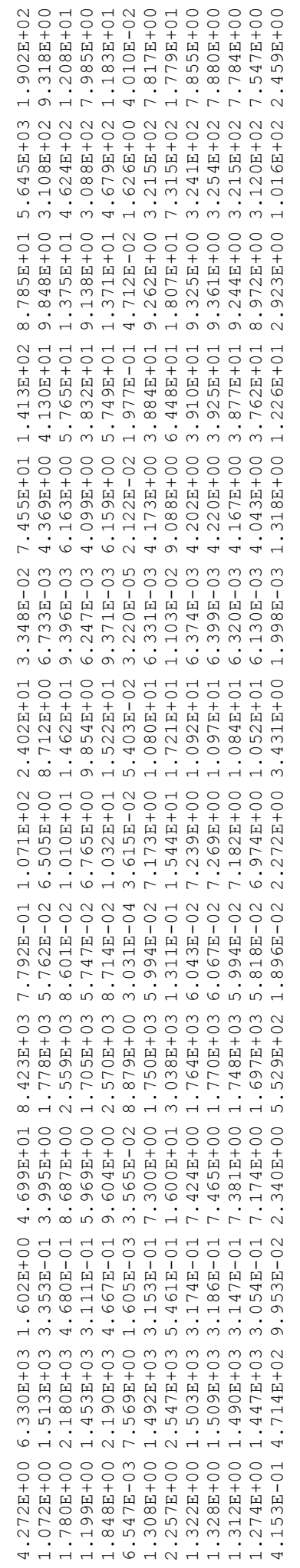

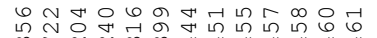

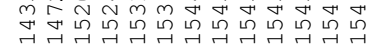




\section{SNF-10293, REV 0}

\section{The Data File PDF-11.HDR}

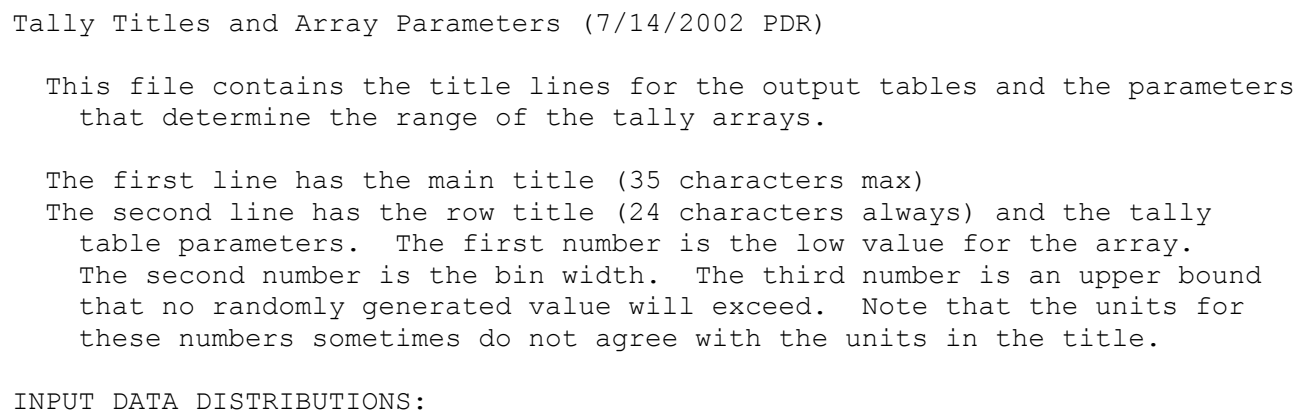




\section{SNF-10293, REV 0}

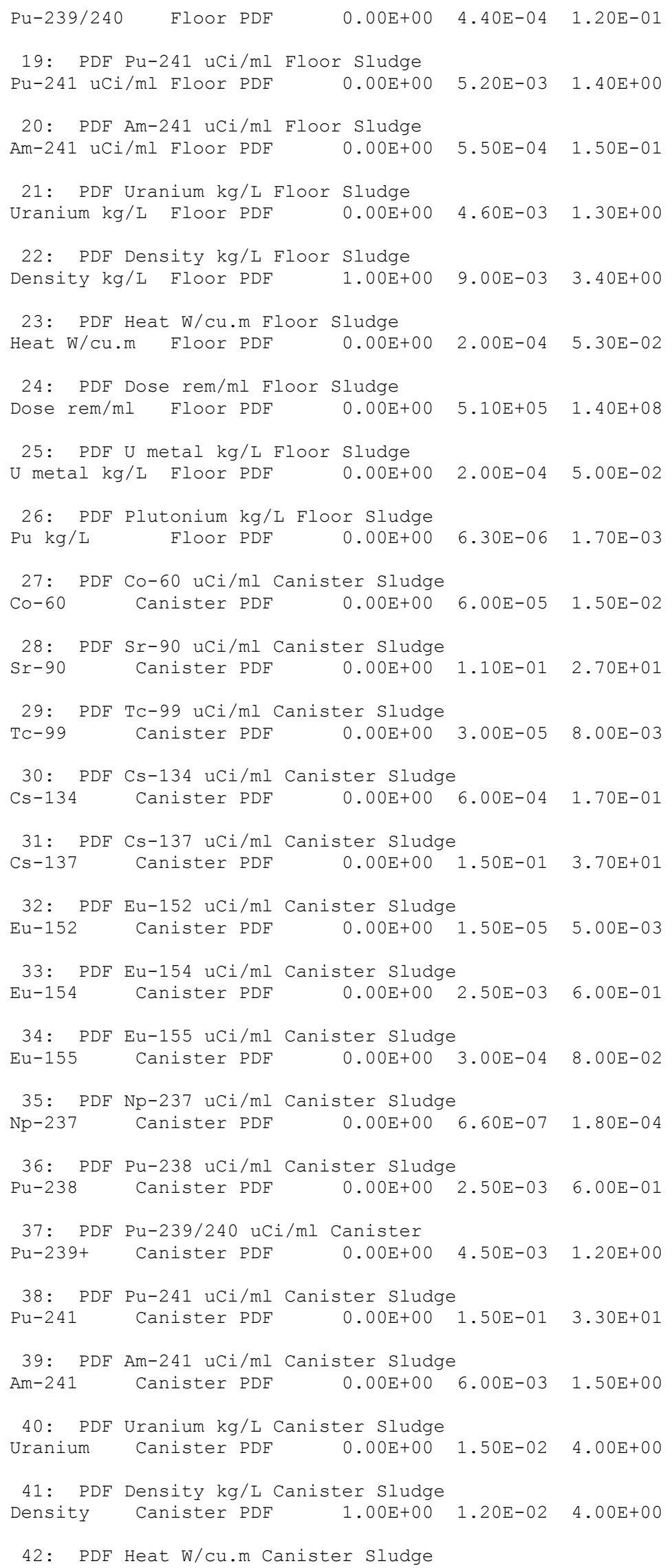


SNF-10293, REV 0

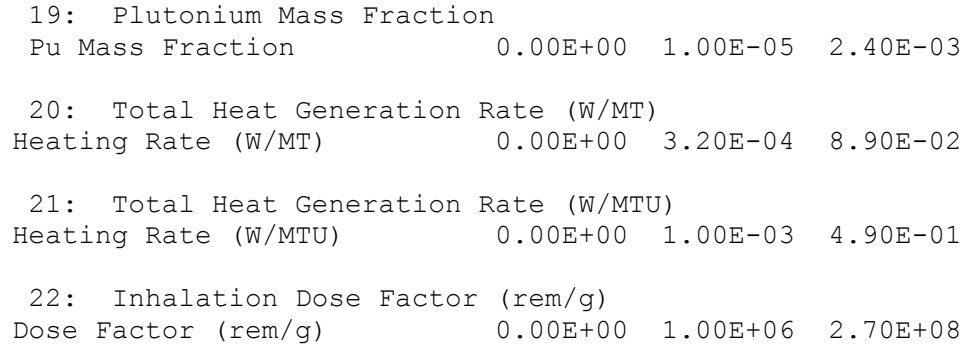


SNF-10293, REV 0

\section{APPENDIX G}

LISTING OF THE PROGRAM, PDF-11.PAS 
SNF-10293, REV 0

This page intentionally left blank.

G-ii 


\section{APPENDIX G \\ LISTING OF THE PROGRAM, PDF-11.PAS}

Program SludgePDF;

\{ Author: Paul D. Rittmann, PhD CHP

Purpose: Compute the distribution of floor \& canister sludge amounts

in the sludge storage container.

Input: Command line repetition constants and container volumes.

Data file with canister counts and floor sludge volumes by key, canister sludge depths, floor sludge concentrations, and fuel compositions. A second file has the tally identifier and tally array parameters.

Output: Output file has three main parts. First is input data.

(1) canister sludge depths (means of various readings)

(2) all key sludge volumes using the mean depths

(3) canister sludge depths sorted for probability distribution

(4) floor sludge compositions sorted for probability distribution

second is stochastic results for floor \& canister sludge

(1) canister sludge depths

(2) all key sludge volumes (floor, canister, total)

(3) concentrations of 13 isotopes (floor \& canister)

(4) U total density and sludge wet density (floor \& canister)

(5) thermal power and inhalation dose (floor \& canister)

(6) U metal density and Pu density (floor \& canister)

(7) summary table

Third is the stochastic results for each sludge container volume

(1) canister-to-total sludge ratio

(2) concentrations of 13 isotopes

(3) U total density and sludge wet density

(4) thermal power and inhalation dose

(5) U metal density and Pu density

(6) summary table

Version History:

PDF-1 calculated canister-to-total ratios using sludge volumes read from the read from the data file. Container filling is the only random variable.

PDF-2 uses floor sludge uniform distribution and canister sludge depth

distributions. Calculates canister-to-total ratio only.

PDF-3 uses floor and canister sludge isotopic information

PDF-4 adds calculation of $U, W / M T, W / M T U$ and Pu fraction

PDF-5 adds an option: the floor sludge composition is computed using one random number for each key applied to distributions of each item of interest.

PDF- 6 refines the calculation of plutonium mass by including a distribution of specific actities for floor sludge Pu-239+ and the actual Pu-239/Pu-240 content of canister sludge

PDF-7 improves the floor sludge mass estimate, simplifies the calculation of retrieval order, and requires the last container to be at least $90 \%$ full, added flags to calculate floor sludge volume for each key, or use a larger canister sludge density

PDF-8 corrects the continuous distributions to preserve the mean. The canister sludge depths can be treated as a discrete distribution with a smearing factor. The larger canister sludge density option was changed to use a discrete distribution with a smearing factor.

PDF-9 adds Pu-241 to the list of nuclides, improves the calculation of the mean values, and shows standard deviations. Lower bound for U metal was changed from the nominal to $0 \%$. Added tallies for heat, dose, isotopics, $\mathrm{U}$, and $\mathrm{Pu}$ input PDFs.

PDF-10 adds tallies for the input floor sludge concentrations and canister sludge densities. The sensitivity options are rearranged. Separate input files are used for segregated and non-segregated canister sludge. Added Geometric mean \& standard deviation. Tally arrays are initialized in the HeaderFile, which is read after allocating dynamic memory. Change the way canister sludge density \& U conc are calculated.

Add option to use canister sludge sample data rather than RADNUC estimates. Add option to use specific retrieval orders rather than any order. Add option to set the fullness criteria for containers and to choose 


\title{
SNF-10293, REV 0
}

\author{
whether to use the container volume or sludge volume to calculate \\ PDF-11 modifies the command line input to control more parameters, \\ restores random key sequence checks, adds a 2-D tally of canister \\ sludge density versus $U$ concentration, and an option to use lognormal \\ lognormal distributions for floor sludge \\ \} \\ Uses Crt, DOS; \\ Const Space $=$ ' '; Author $=$ ' by Paul D. Rittmann PhD CHP'; \\ ProgTitle = 'PDF-11 July 27, 2002'; \\ SludgeFile = 'PDF-11.DAT'; \{file with floor \& canister sludge volumes \} \\ HeaderFile = 'PDF-11.HDR'; \{file with tally titles \& parameters \} \\ DistrFile = 'PDF-11.OUT'; \{ output file with probability density function \} \\ NumKey $=52 ;$ number of keys \\ Num2D $=57 ;$ \{ array size for canister density versus U concentration \} \\ Volmax $=4 ; \quad\{$ maximum number of volumes handled at one time \\ MsgMax $=1000 ;\{$ maximum number of out of range values for tally arrays $\}$ \\ \{ constants for the input PDF records \} \\ BinMaxInp $=300$; \{ number of bins for PDFs of floor \& canister sludge inputs \} \\ BinDelInp $=1.0 / 250.0 ;$ \{ working limit is 250 bins \} \\ NumDpth $=4 ; \quad\{$ number of sludge depth PDFs $\}$ \\ LastVol $=7 ; \quad$ index to last of the all key sludge volumes \\ NumCmp $=19 ;$ \{ number of input PDFs with floor/canister sludge composition \} \\ LastFlrP = LastVol + NumCmp; \{ index to last floor sludge PDF \} \\ MaxRecInp = LastFlrP + NumCmp; \{ index to last canister sludge all key averages \} \\ \{ constants for the container PDFs \} \\ BinMaxOut $=900 ;\{$ number of bins available for container PDFs \\ Binoutput $=400 ;\{$ minimum number of bins to print $\}$ \\ KeycHdis $=16$ '; \{ fuel discharge date retrieval sequence \} \\ KeyMaxCH $=$ '5'; \{ number of stored retrieval sequences \} \\ FullAuto $=0.90 ;\{$ default criteria for discarding partly filled containers \\ VolStp $=23$; \{ separation in PDFout between different volumes \\ MaxRecOut $=$ VolMax*VolStp-1; \{ number of container PDFs $\}$ \\ IndxAm $=13 ; \quad\{$ number of radionuclides, last is Am-241 \} \\ IndxUall $=14 ; \quad\{$ index to uranium masses $\}$ \\ IndxDen $=15 ; \quad\{$ index to sludge density $\}$ \\ IndxWv $=16 ; \quad\{$ index to heat generation rates per unit volume \\ IndxDosV $=17 ; \quad\{$ index to unit dose in $\mathrm{rem} / \mathrm{ml}\}$ \\ IndxUmet $=18 ; \quad\{$ index to uranium metal concentration in $\mathrm{kg} / \mathrm{L}\}$ \\ IndxPu $=19 ; \quad$ index to plutonium mass fraction in kg Pu per kg sludge $\}$ \\ IndxWmt $=20 ; \quad$ \{ index to heat rate in $\mathrm{W} / \mathrm{MT}$ \} \\ IndxWmtu $=21 ; \quad\{$ index to heat rate in $\mathrm{W} / \mathrm{MTU}\}$ \\ IndxDosM $=22 ; \quad\{$ index to unit dose in rem/g \\ $\{$ canister sludge constants \} \\ ThkMax $=35 ;\{$ maximum number of canisters with sludge depth measurements $\}$ \\ FuCoN $=5 ;$ \{ number of canister fuels: 1=good, 2=fair, 3=poor, 4=bad, 5=total \} \\ $\mathrm{FuCON1}=\mathrm{FuCON}-1 ; \quad \mathrm{FuCON} 2=\mathrm{FuCON} 1-1 ;$ \\ FairAL $=1256.0 / 1586.0 ;$ FairSS $=330.0 / 1586.0 ; \quad\{$ stainless \& aluminum weighting factors \} \\ CanDepLoDef $=0.5 ;$ \{ default canister sludge depth variability factor \} \\ CanSmploDef $=0.4 ;$ \{ default canister sludge sample conc variability factor \} \\ CanSIDnLo $=1.05 ;$ CanSlDnHi $=2.70 ;$ range of canister sludge densities, $\mathrm{kg} / \mathrm{L}\}$ \\ CanDense $=1.8735 ;\{\mathrm{kg} / \mathrm{L}$ mean canister sludge density $\}$ \\ UCanDens $=0.8604 ;\{\mathrm{kg} / \mathrm{L}$ actual mean uranium density in canister sludge $\}$ \\ \{ uranium concentration constants \} \\ UslpHi $=3.5 ; \quad\{$ where $\mathrm{U}$ conc $=$ sludge density $\}$ \\ Uslp0x $=1.2 ; \quad\{$ where lower bound reaches zero $\}$ \\ Uslp0y $=2.0 *($ Uslp0x-1.0); $\{$ upper bound when lower bound reaches zero $\}$ \\ UslpDel $=(2.0$-Uslp0y) $/($ UslpHi-Uslp0x $) ;\{$ slope difference upper minus lower $\}$ \\ UslpLo $=($ UslpHi-2.0)/(UslpHi-Uslp0x); \{ slope of lower bound line \} \\ \{floor sludge constants \} \\ FlrVolLoDef $=0.8 ; \quad\{$ default floor sludge volume variability \\ FlrSmploDef $=0.2$; \{ default floor sample concentration variability factor \\ $\{$ indices for the data arrays \} \\ FlrSmp1 = 5; \{first (smallest) item in the sorted floor sludge arrays \\ NumFlrSmp $=23 ;$ \{ number of floor sludge samples analyzed \\ FlrSmpN = FlrSmp1 + NumFlrSmp - 1; \{ last floor sludge sample $\}$ \\ CanSmpl $=$ FlrSmp1 + NumFlrSmp $+4 ;\{$ first item in the canister sludge arrays $\}$ \\ NumCanSmp $=8 ;\{$ number of canister sludge density measurements $\}$ \\ CanSmpN = CanSmp1 + NumCanSmp - 1; \\ \{ uranium metal constants \} \\ UFlrMfHi $=0.015 ; \mathrm{UCanMfHi}=0.05 ;\{\mathrm{U}$ metal fraction upper bounds $\}$
}




\section{SNF-10293, REV 0}

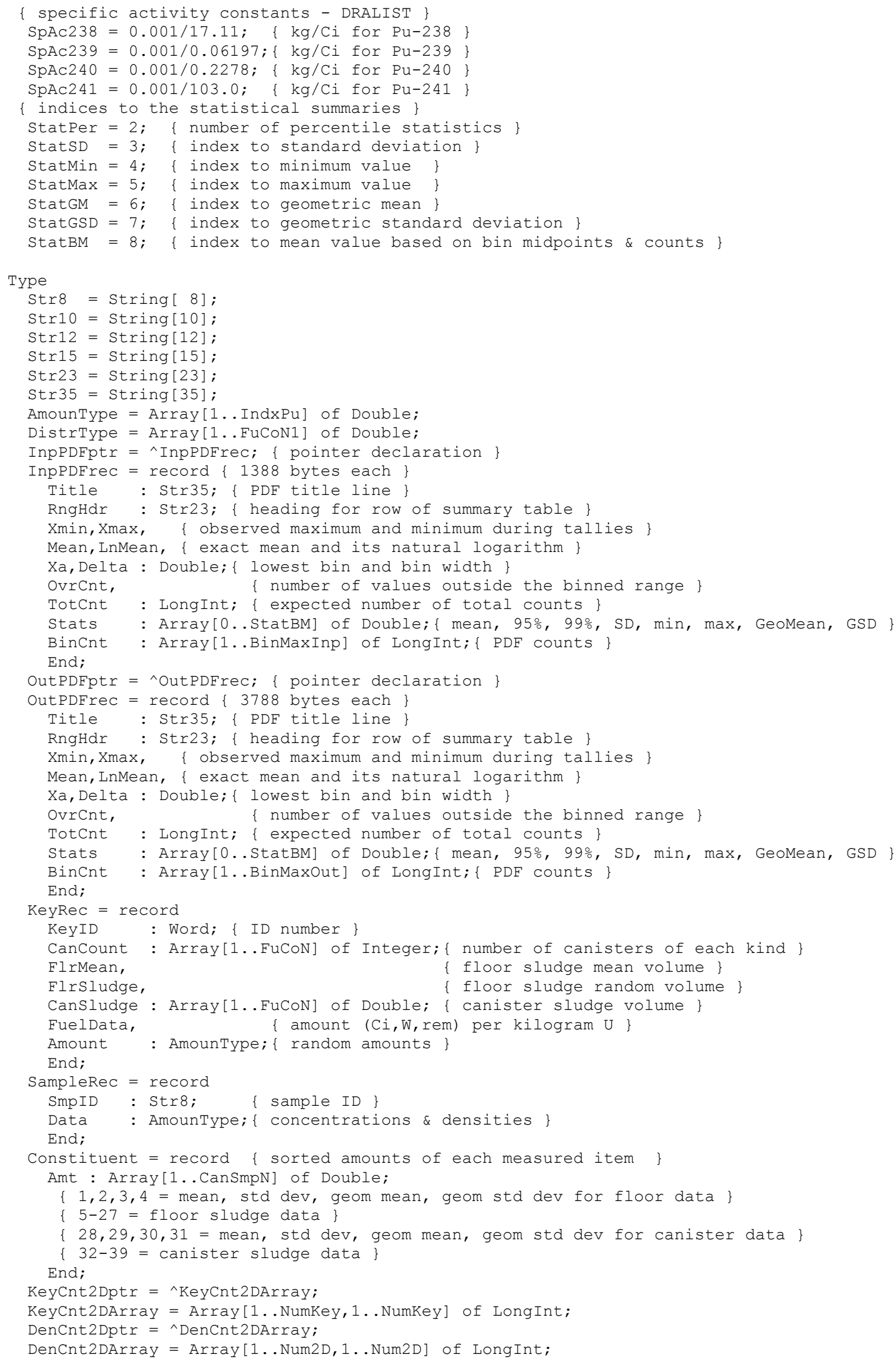




\section{SNF-10293, REV 0}

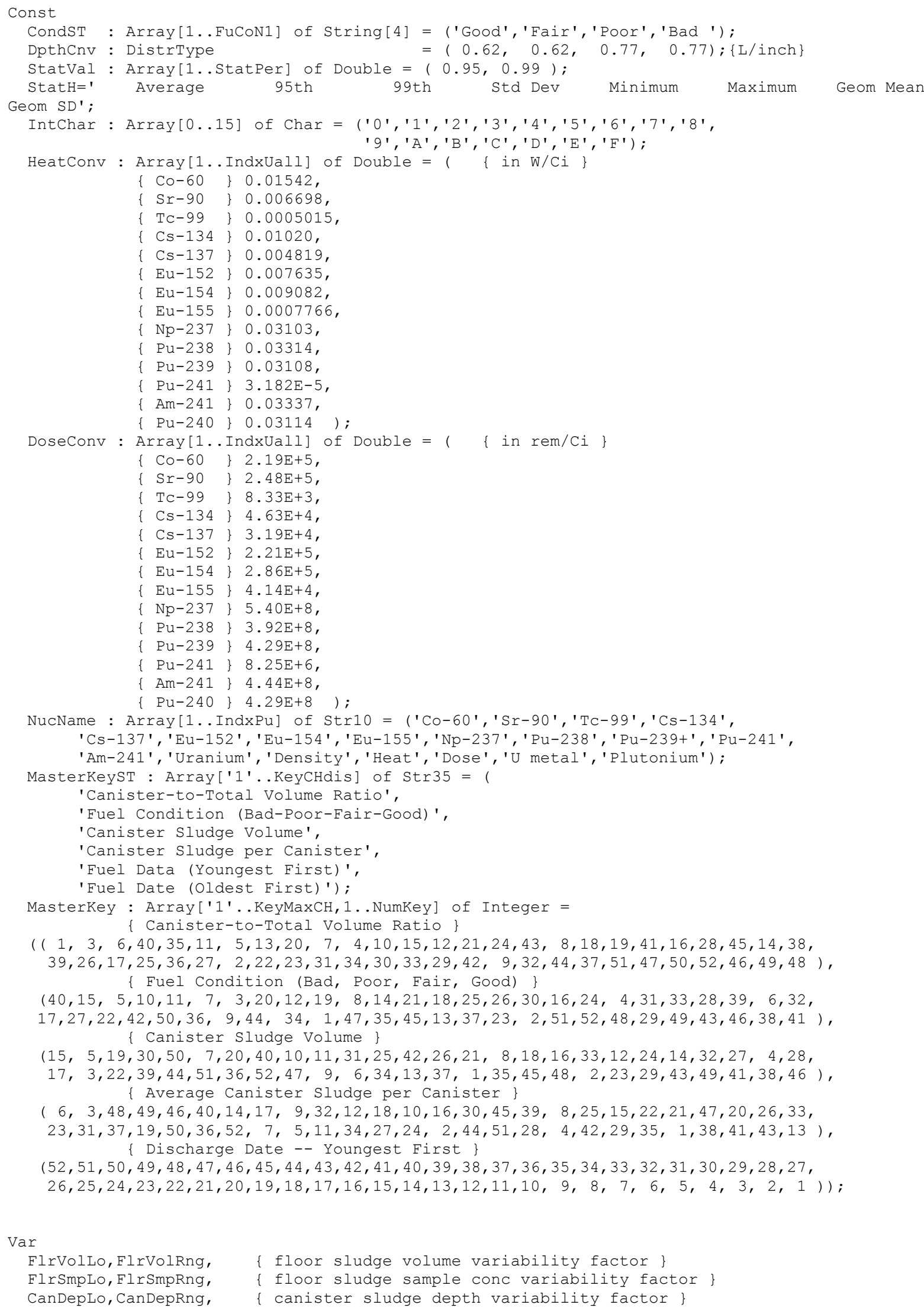




\section{SNF-10293, REV 0}

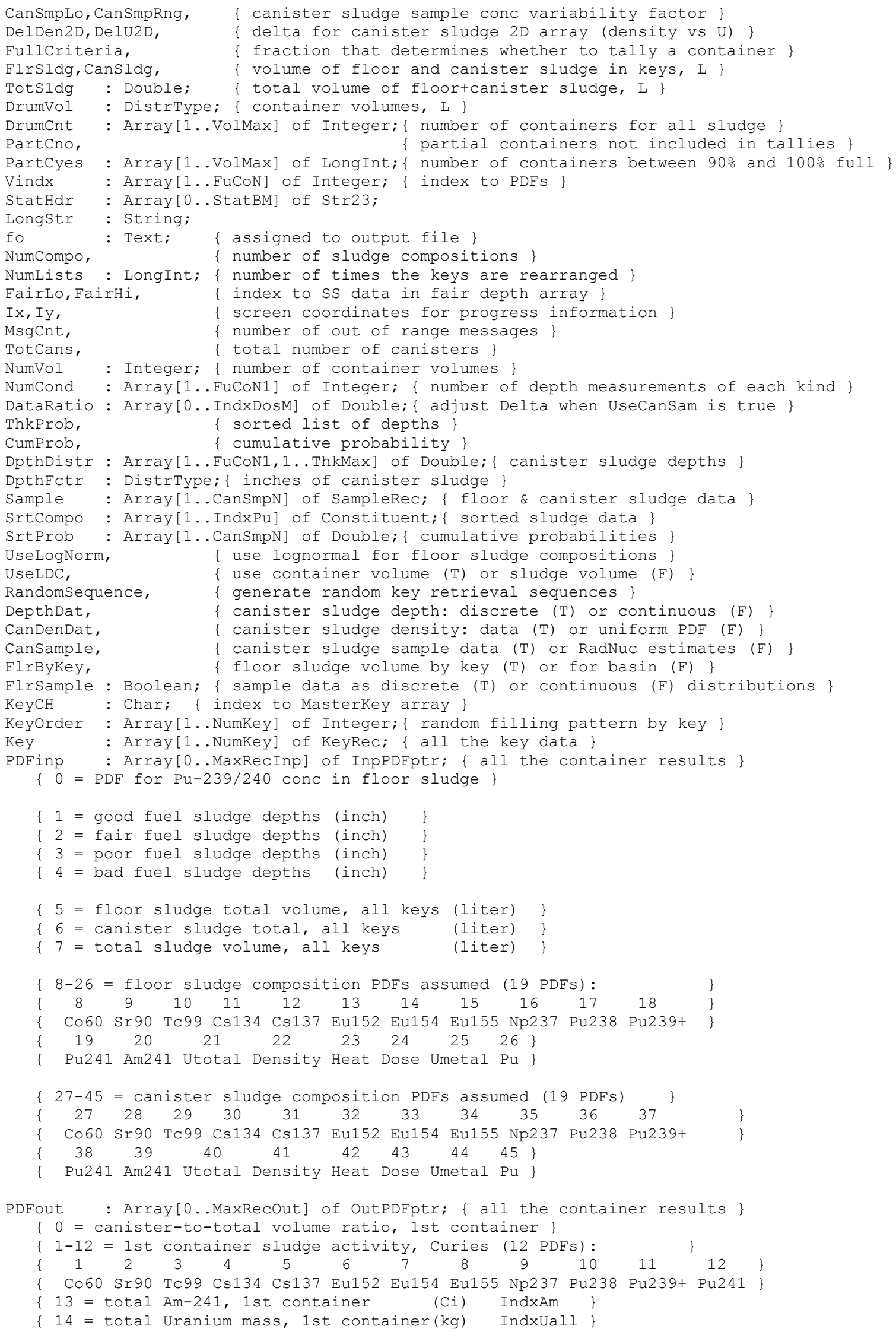




\section{SNF-10293, REV 0}

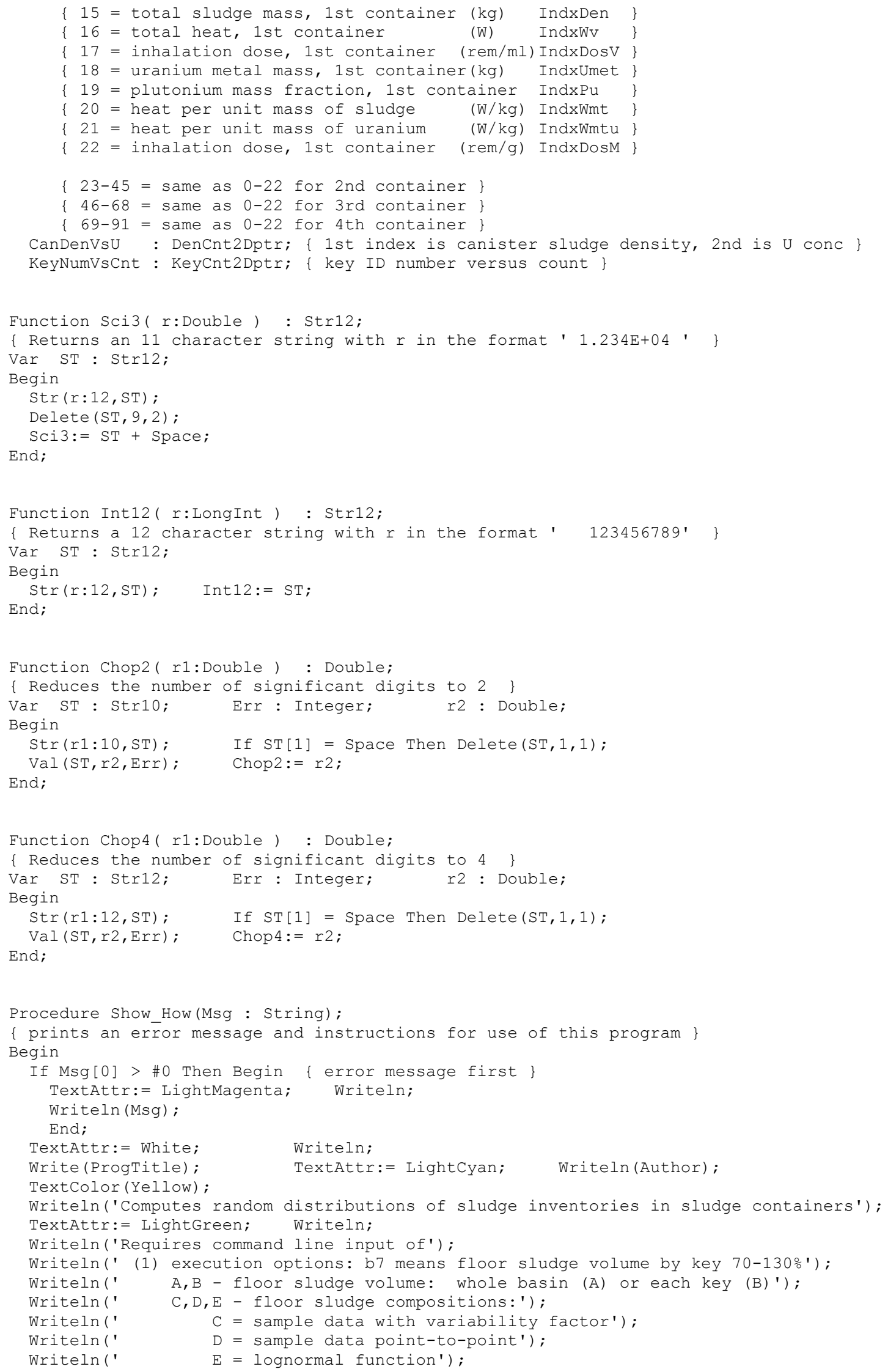




\section{SNF-10293, REV 0}

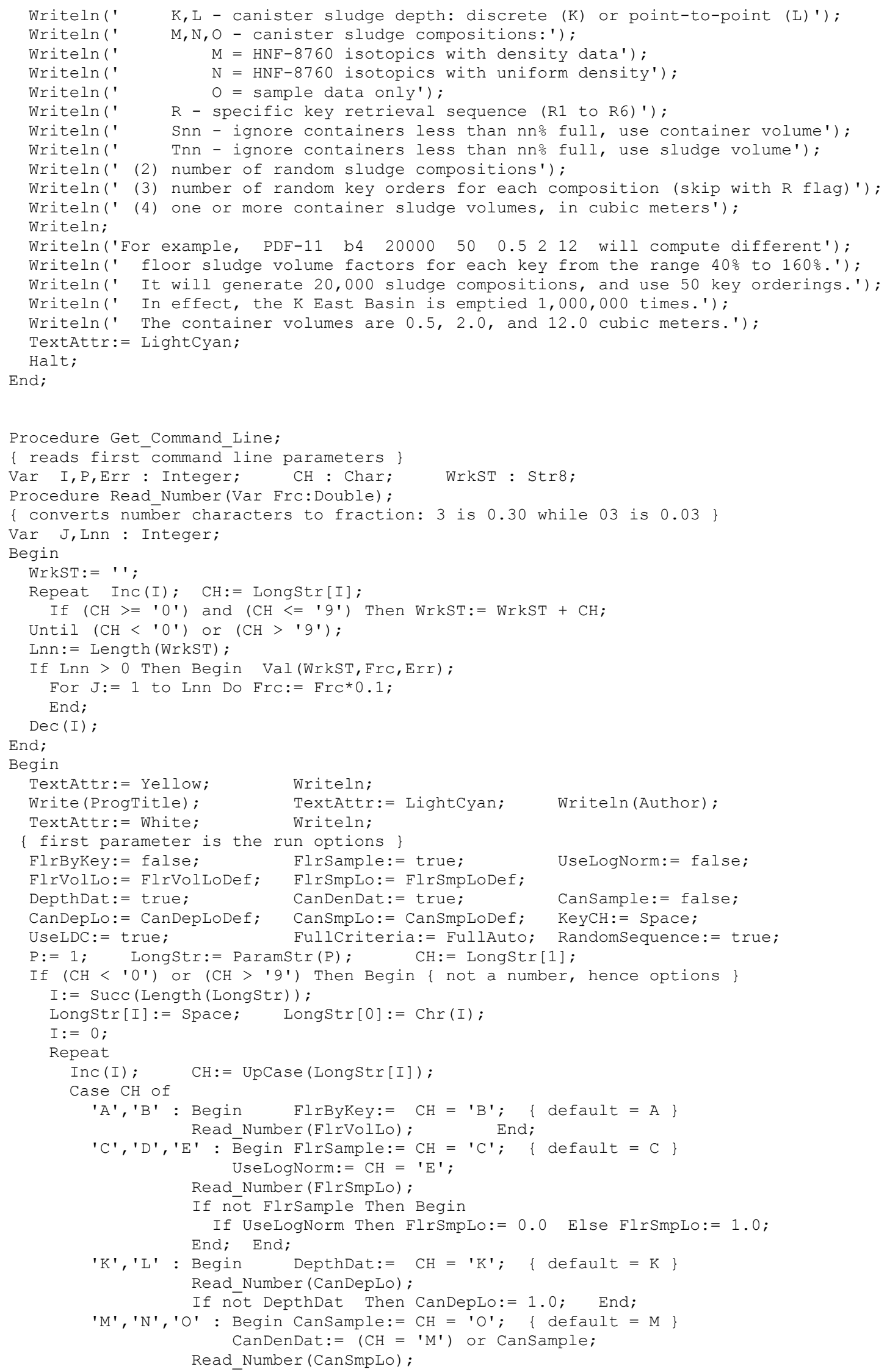




\section{SNF-10293, REV 0}

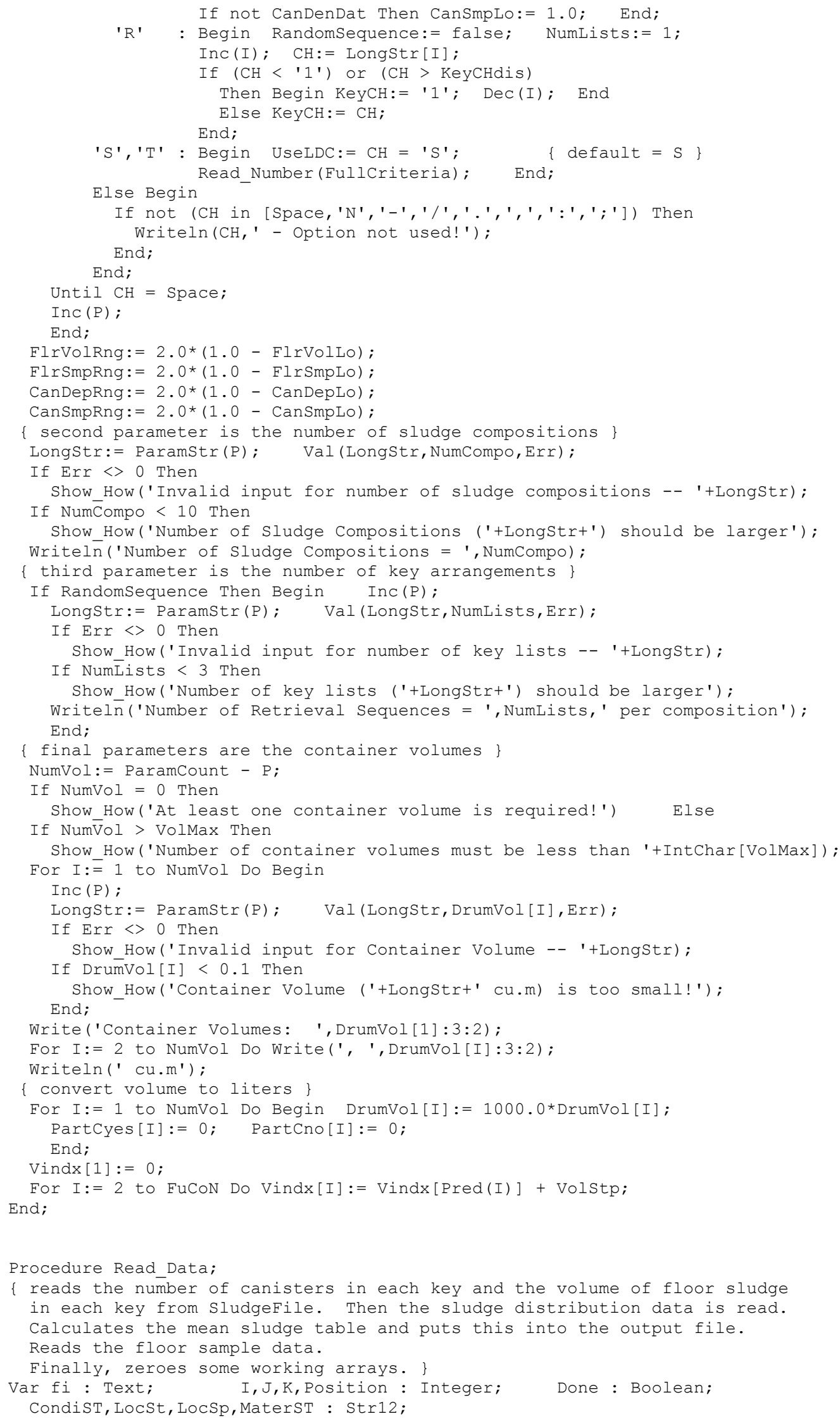




\section{SNF-10293, REV 0}

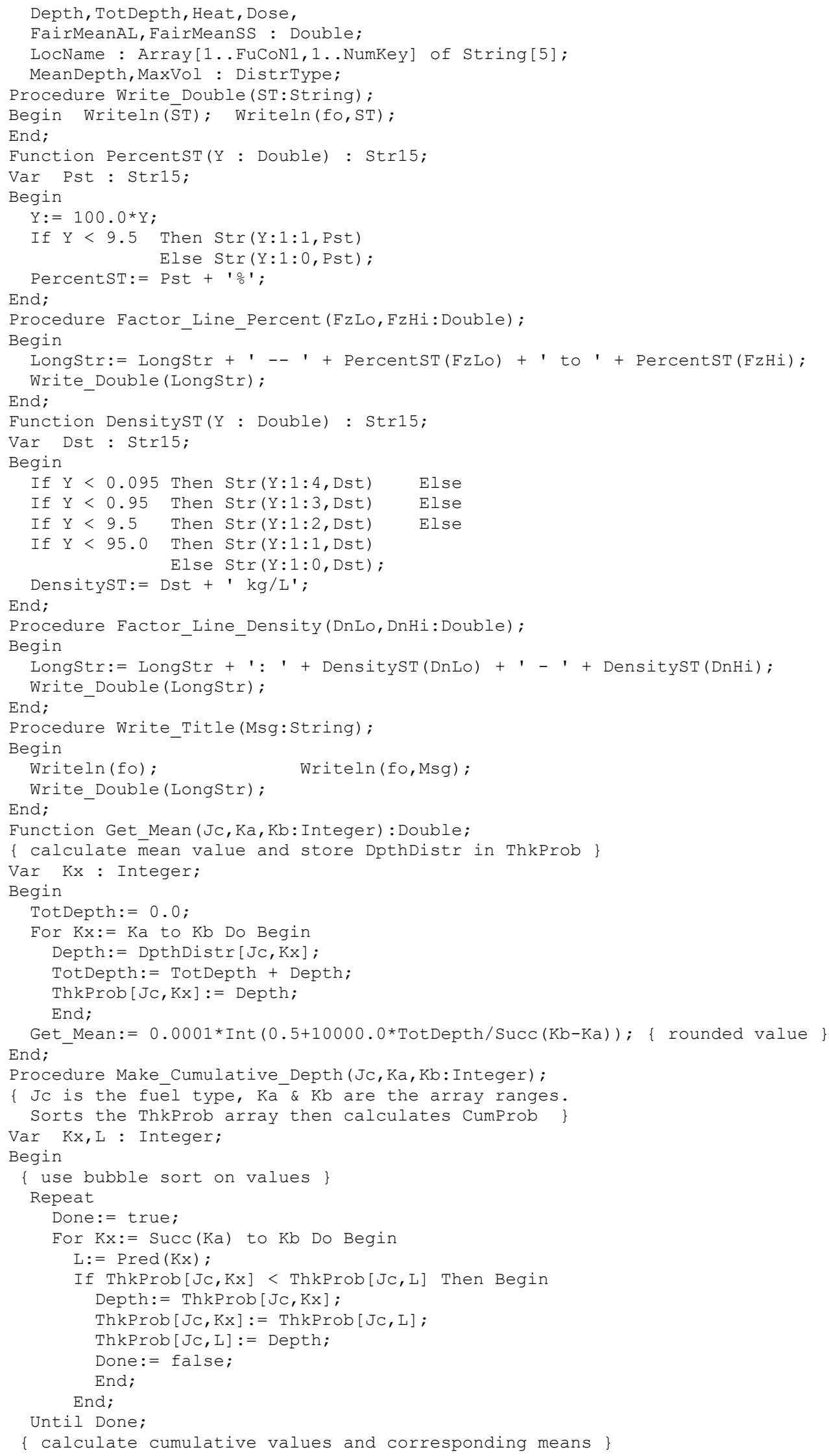




\section{SNF-10293, REV 0}

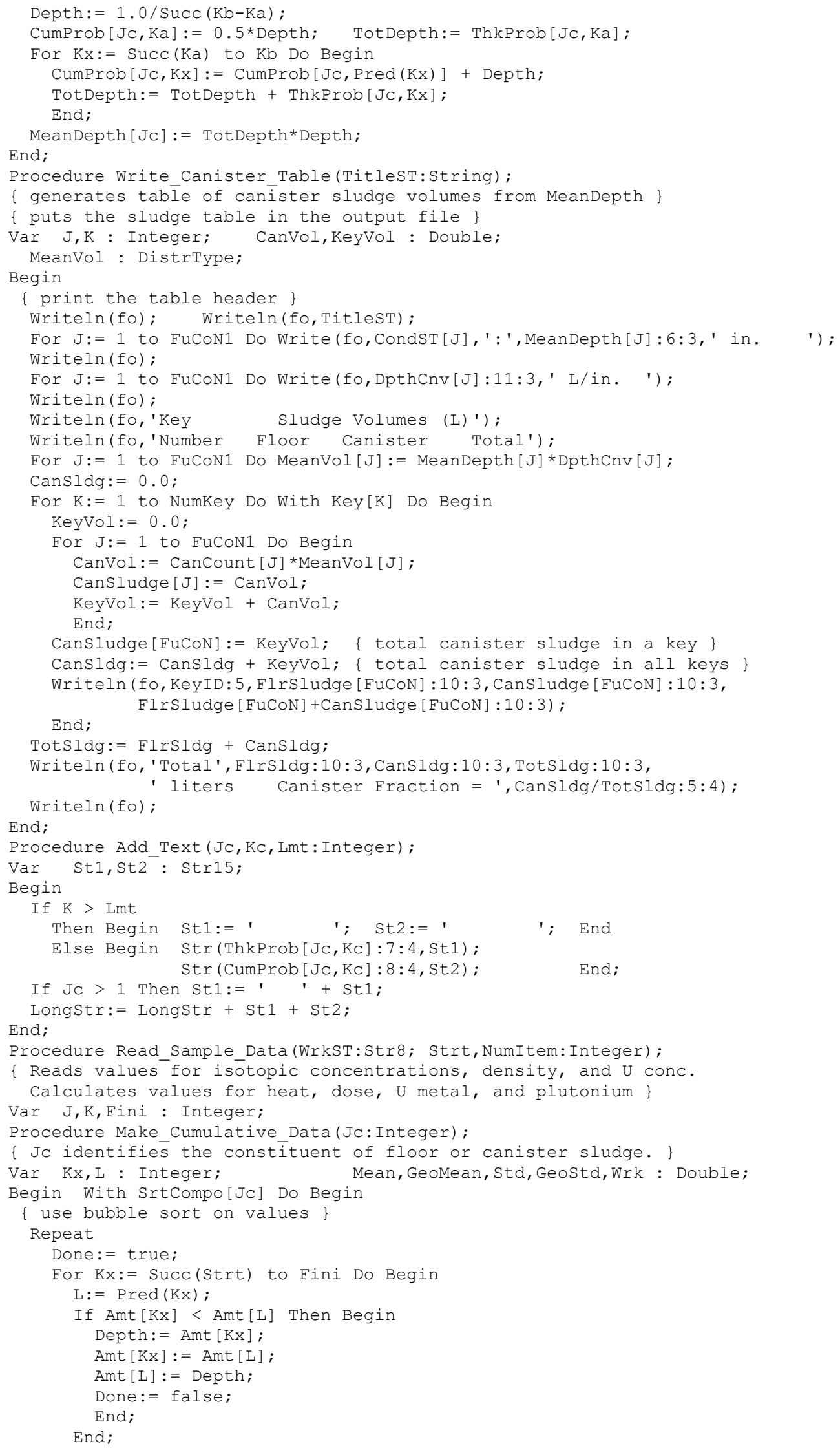




\section{SNF-10293, REV 0}

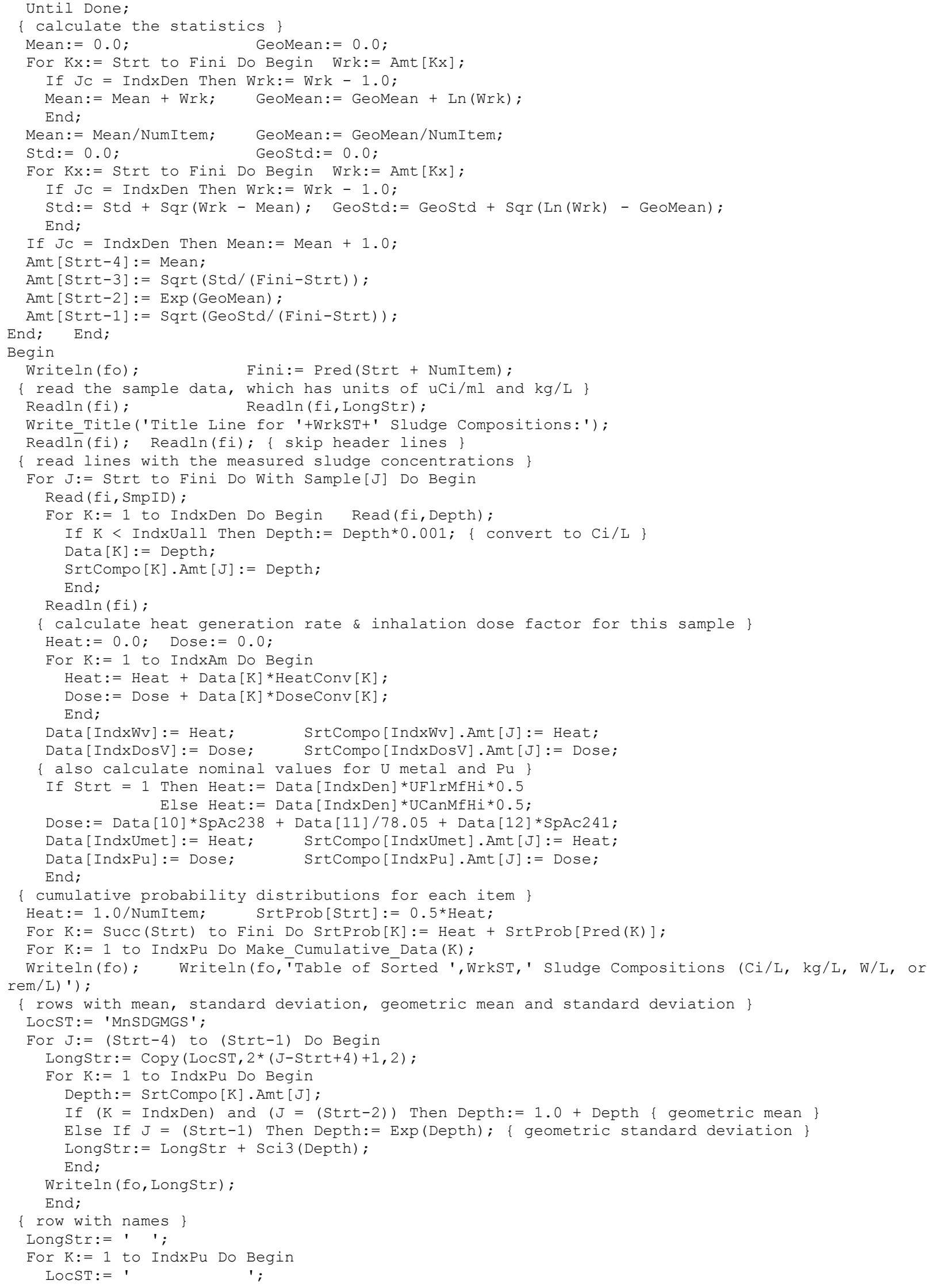




\section{SNF-10293, REV 0}

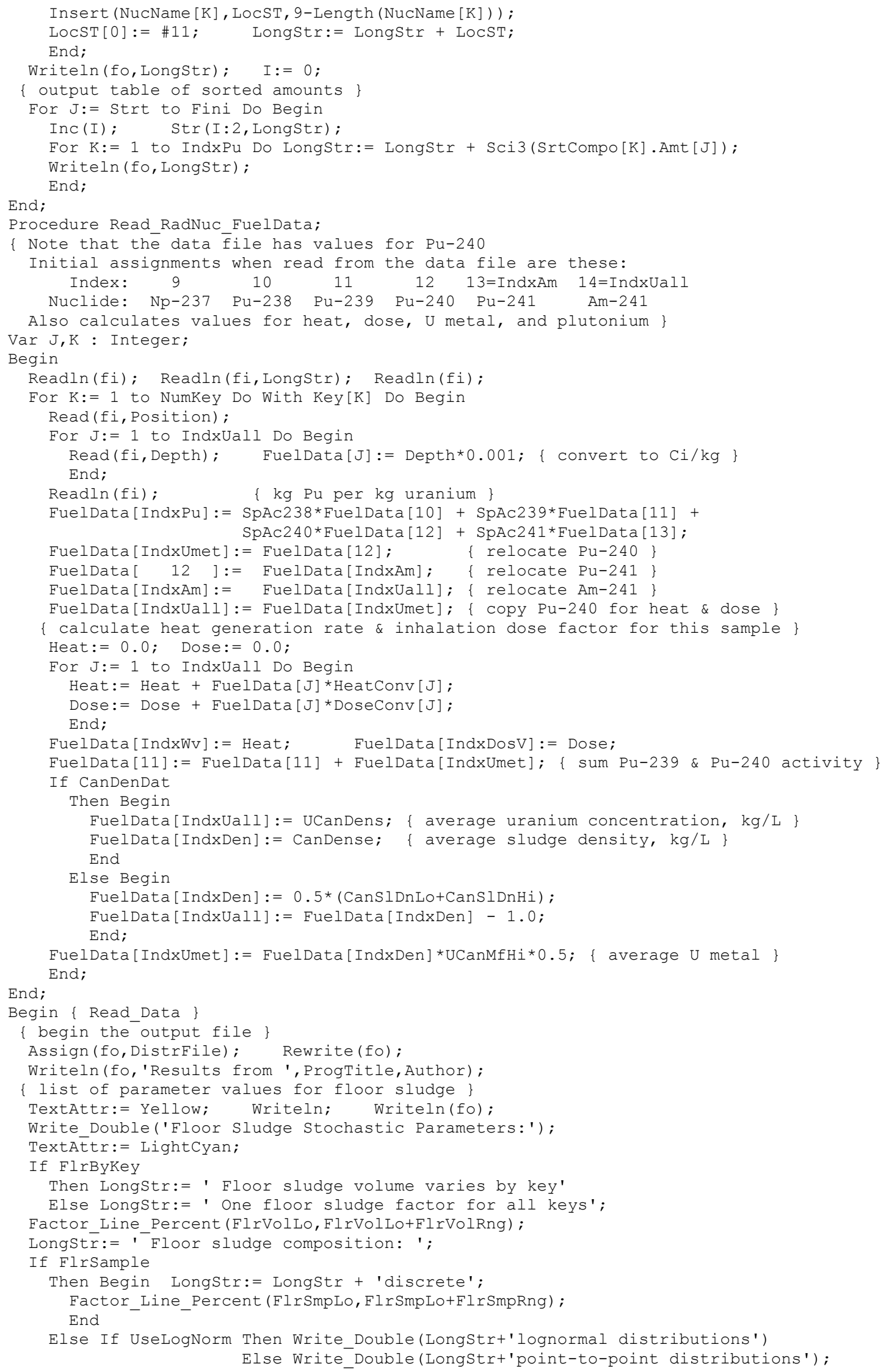




\section{SNF-10293, REV 0}

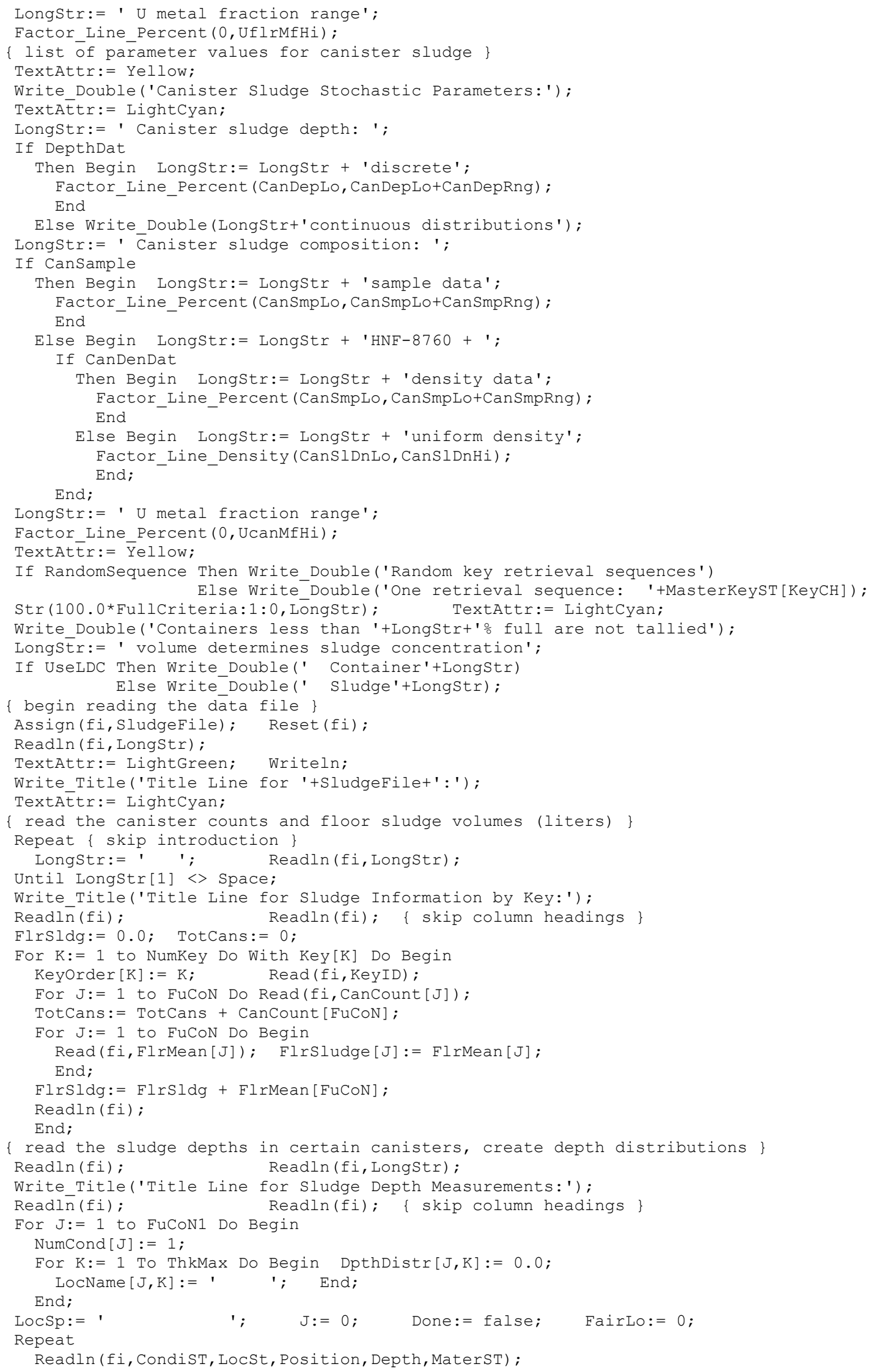




\section{SNF-10293, REV 0}

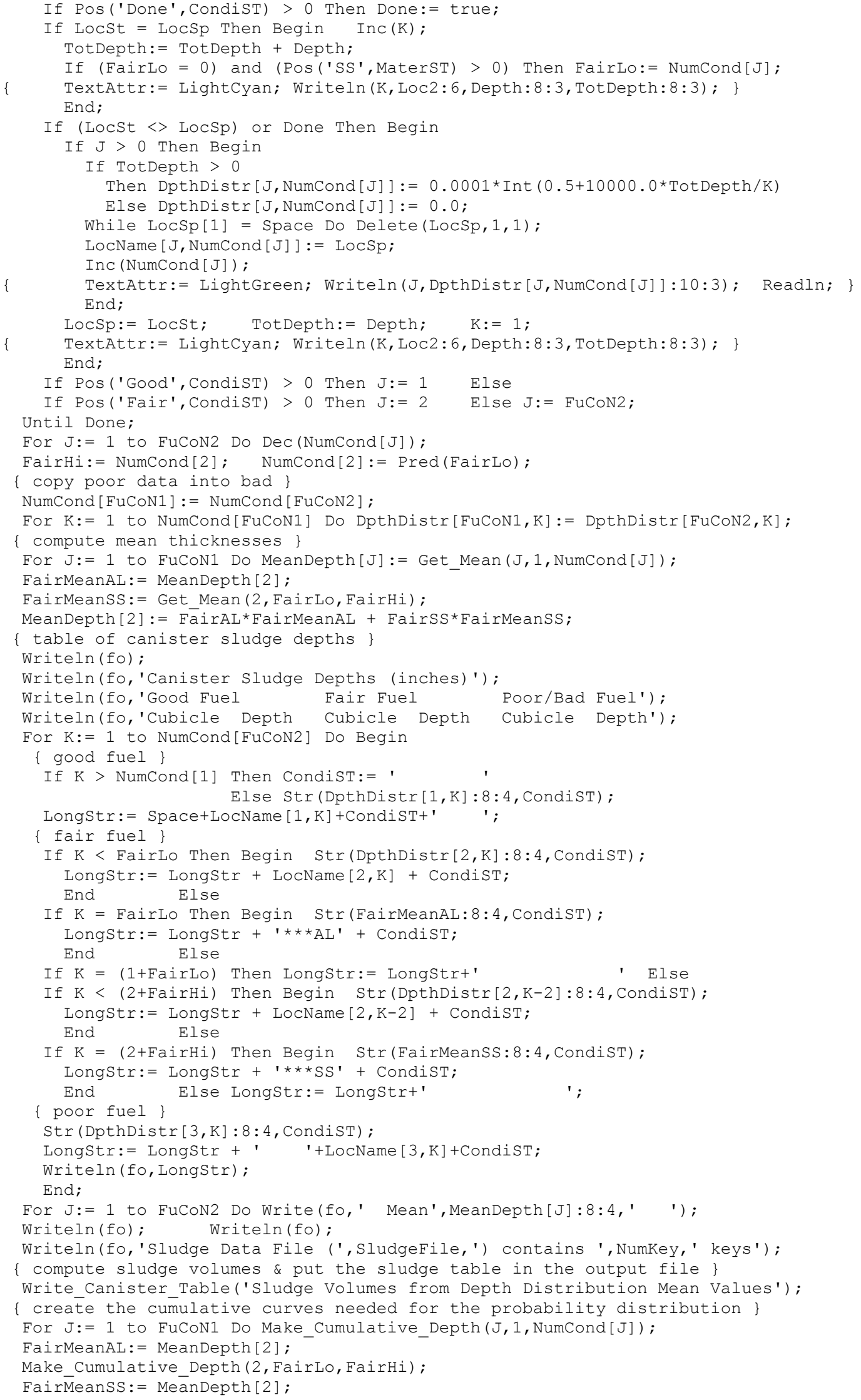




\section{SNF-10293, REV 0}

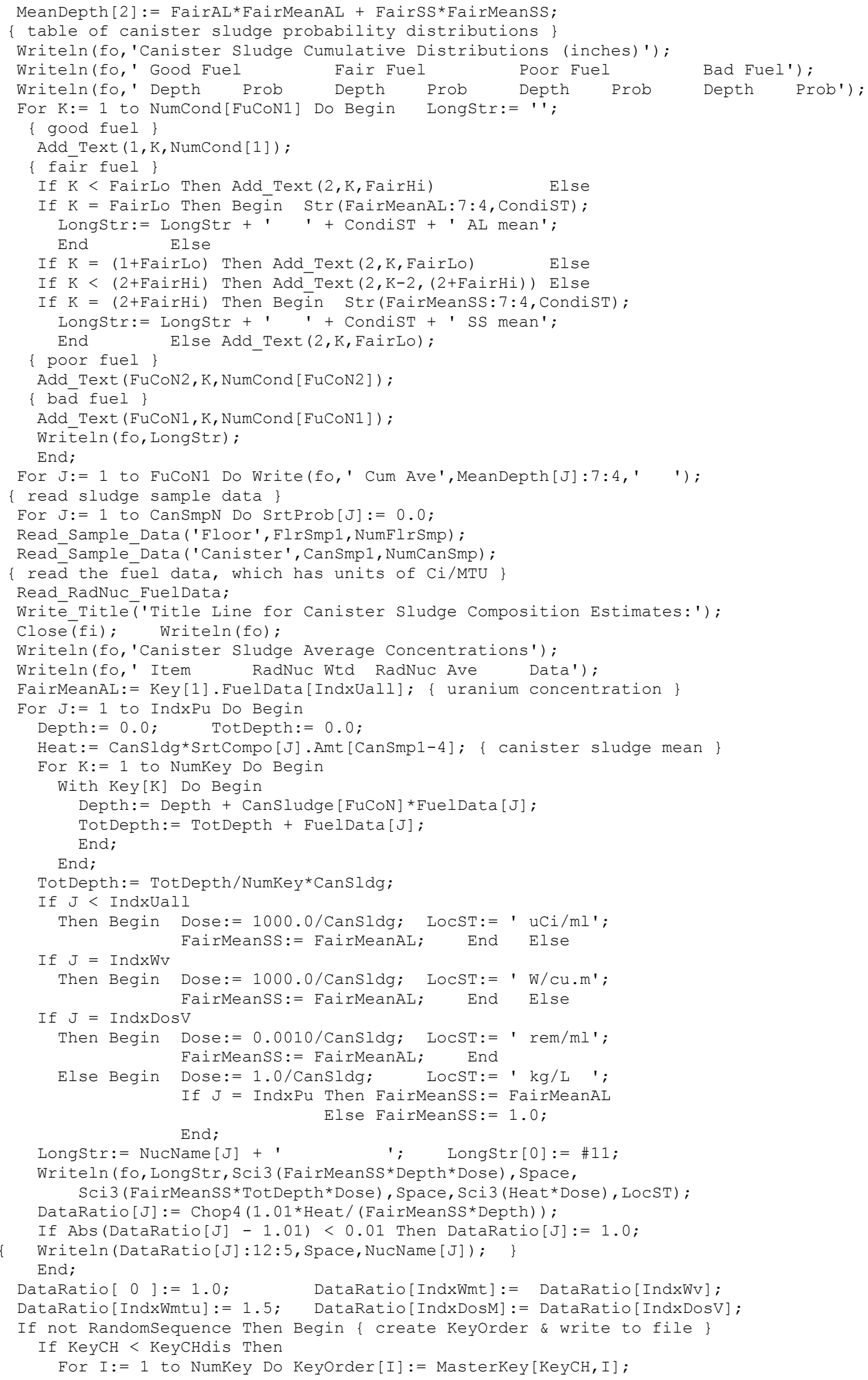




\section{SNF-10293, REV 0}

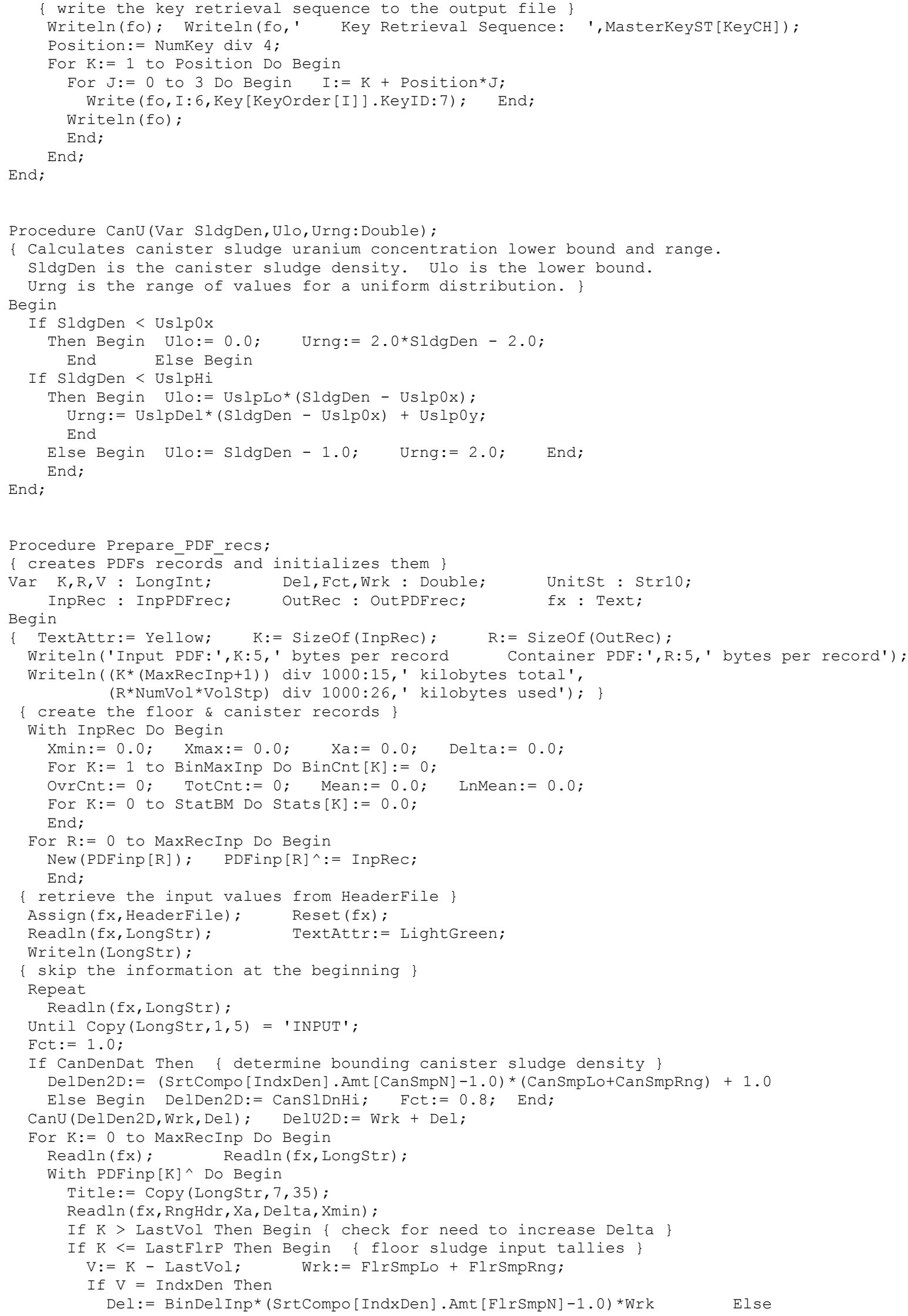




\section{SNF-10293, REV 0}

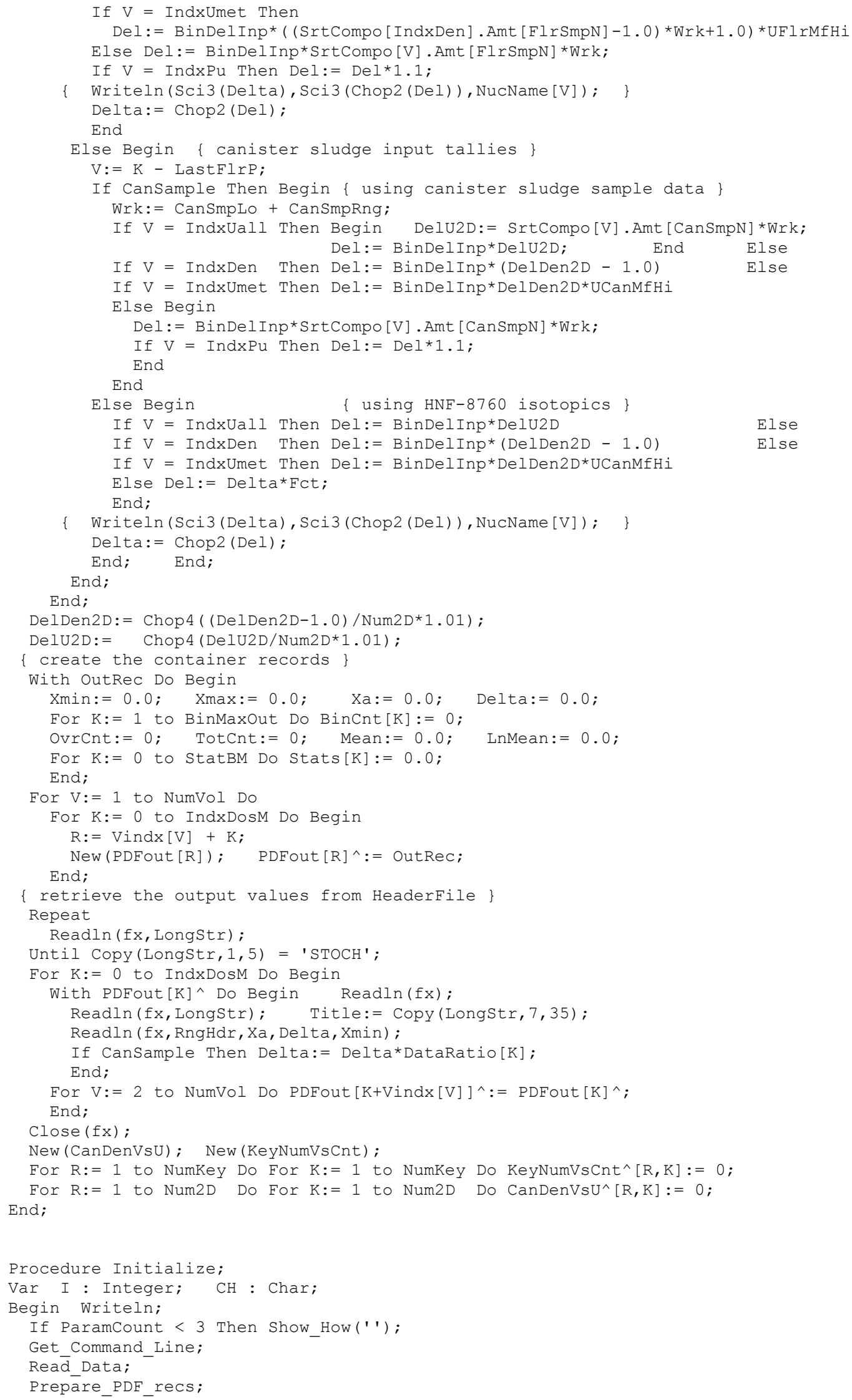




\section{SNF-10293, REV 0}

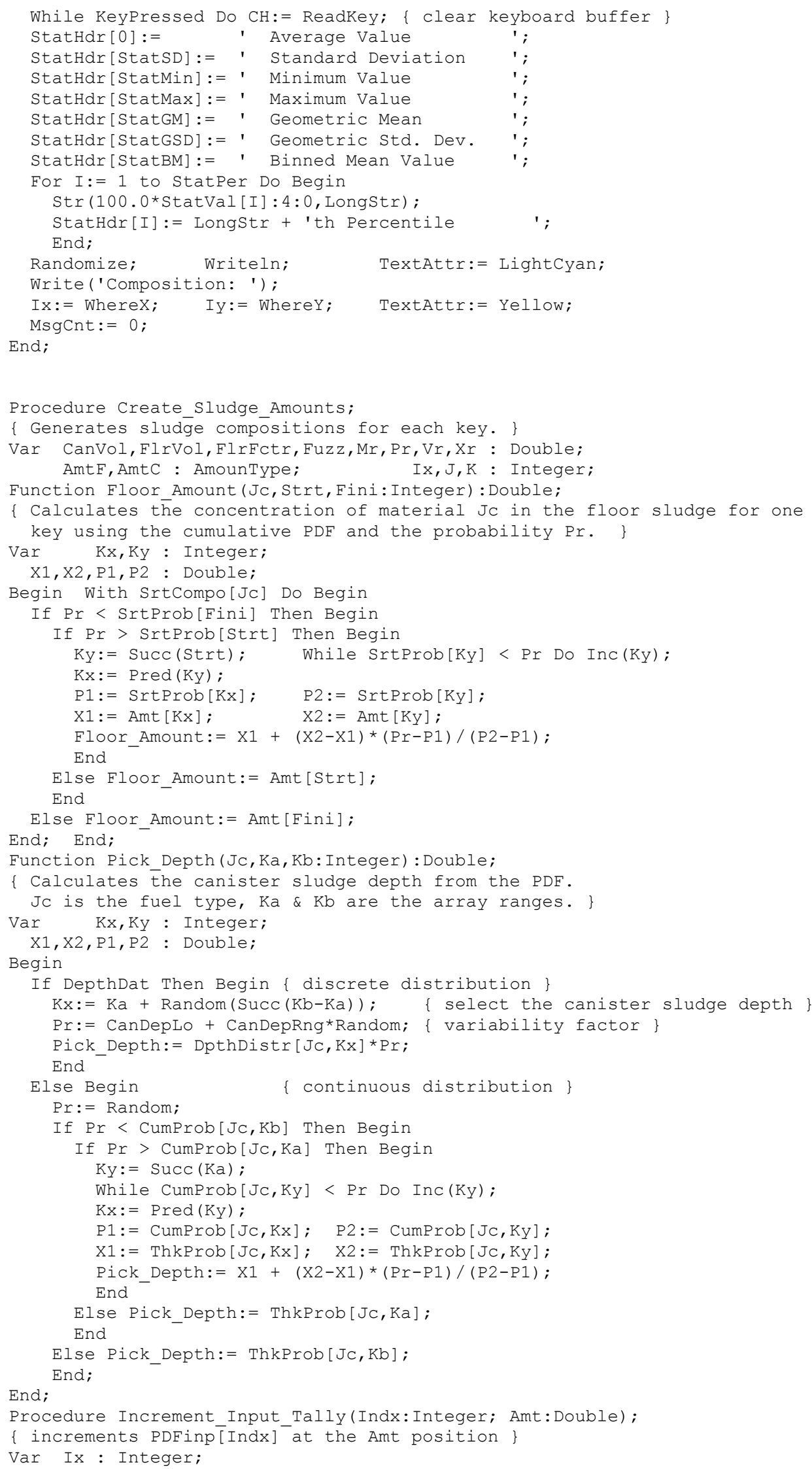




\section{SNF-10293, REV 0}

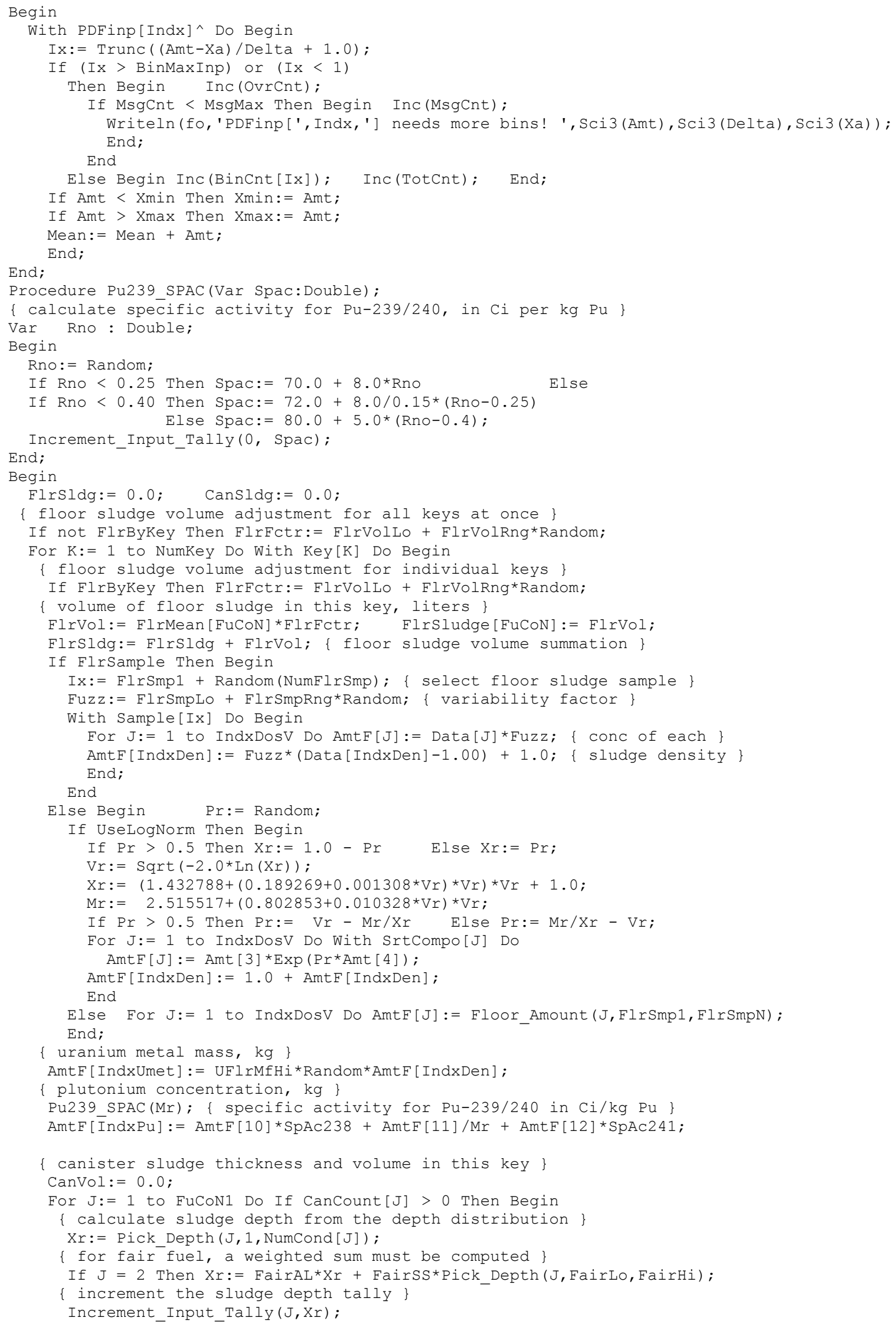




\section{SNF-10293, REV 0}

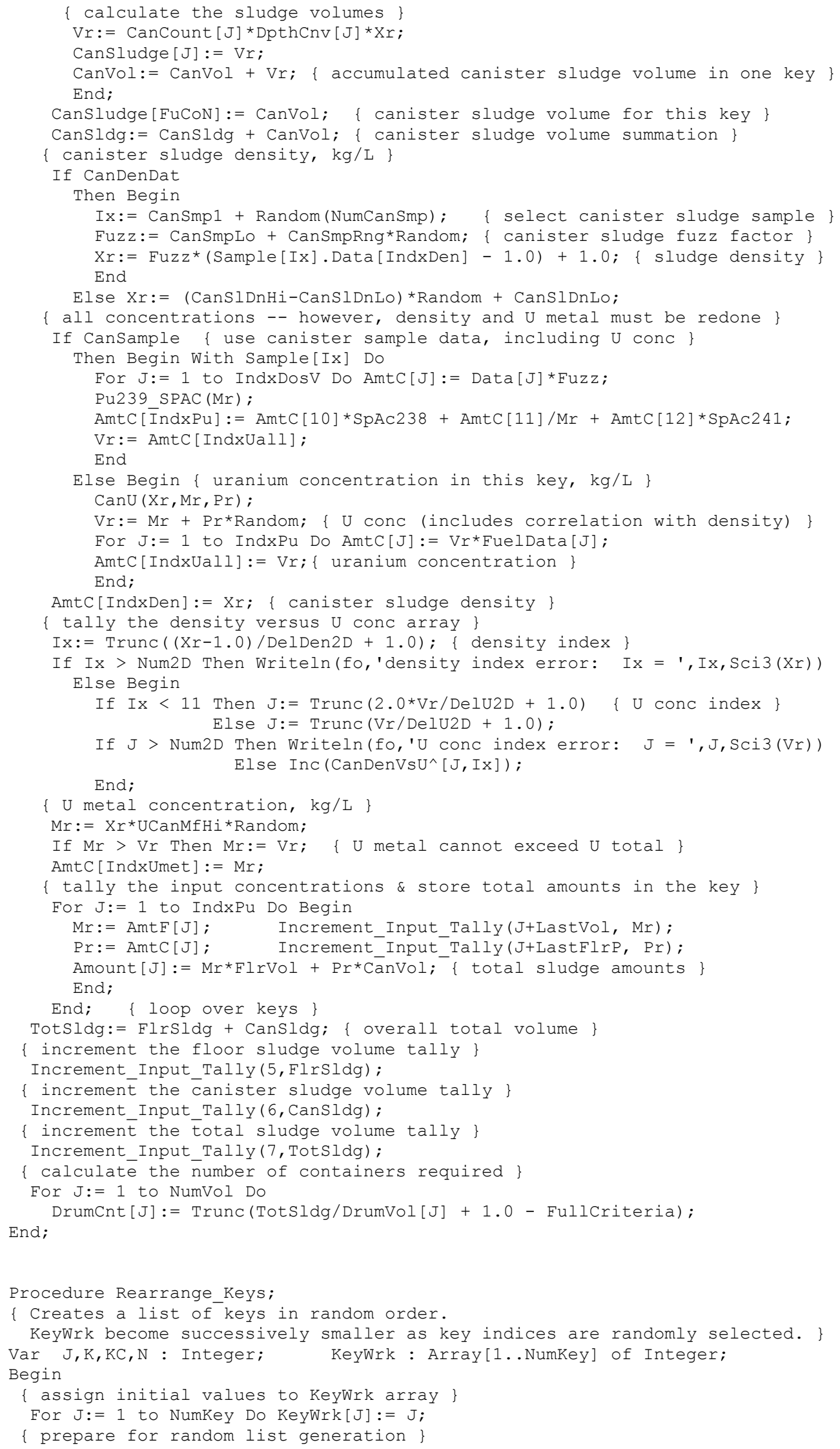




\section{SNF-10293, REV 0}

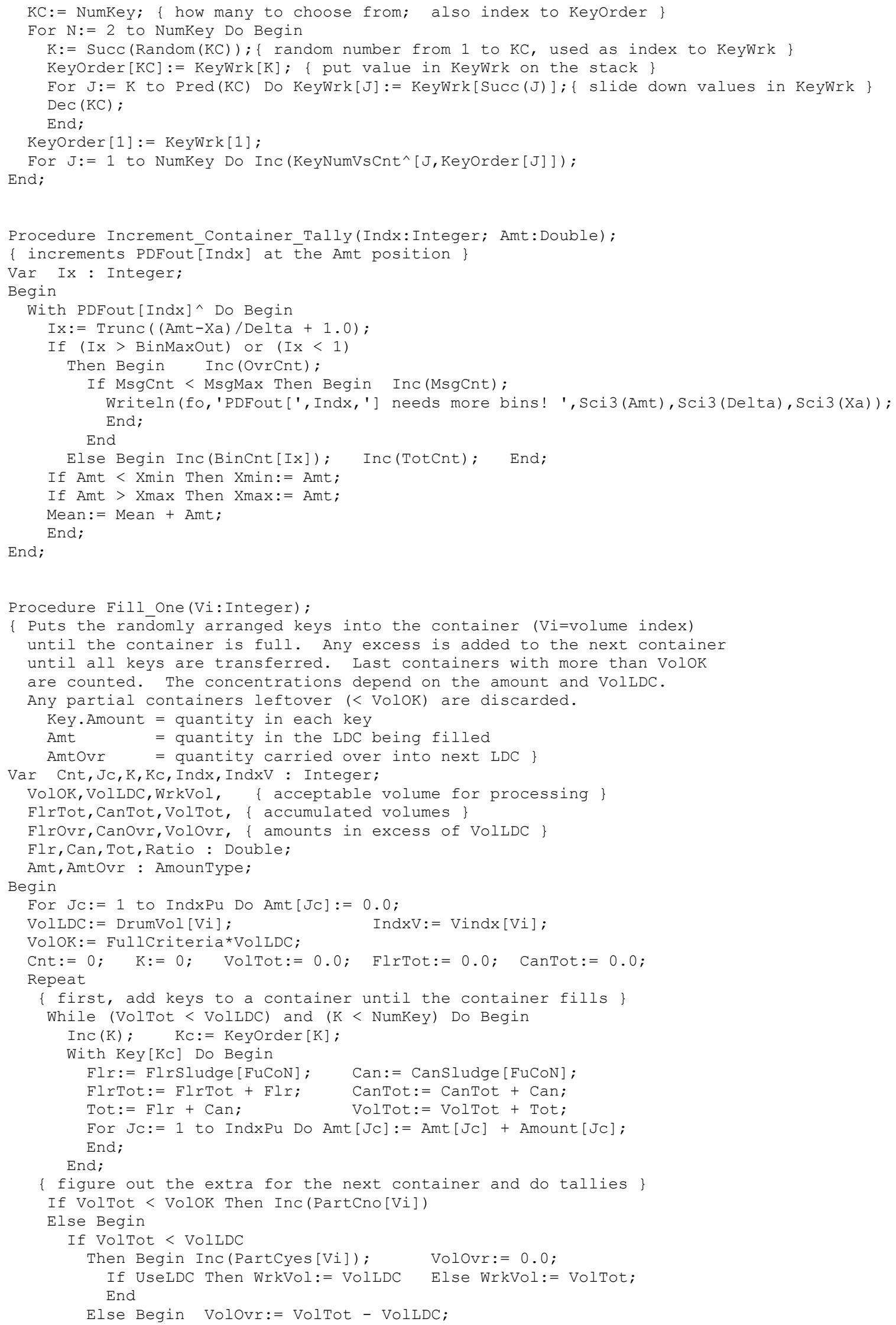




\section{SNF-10293, REV 0}

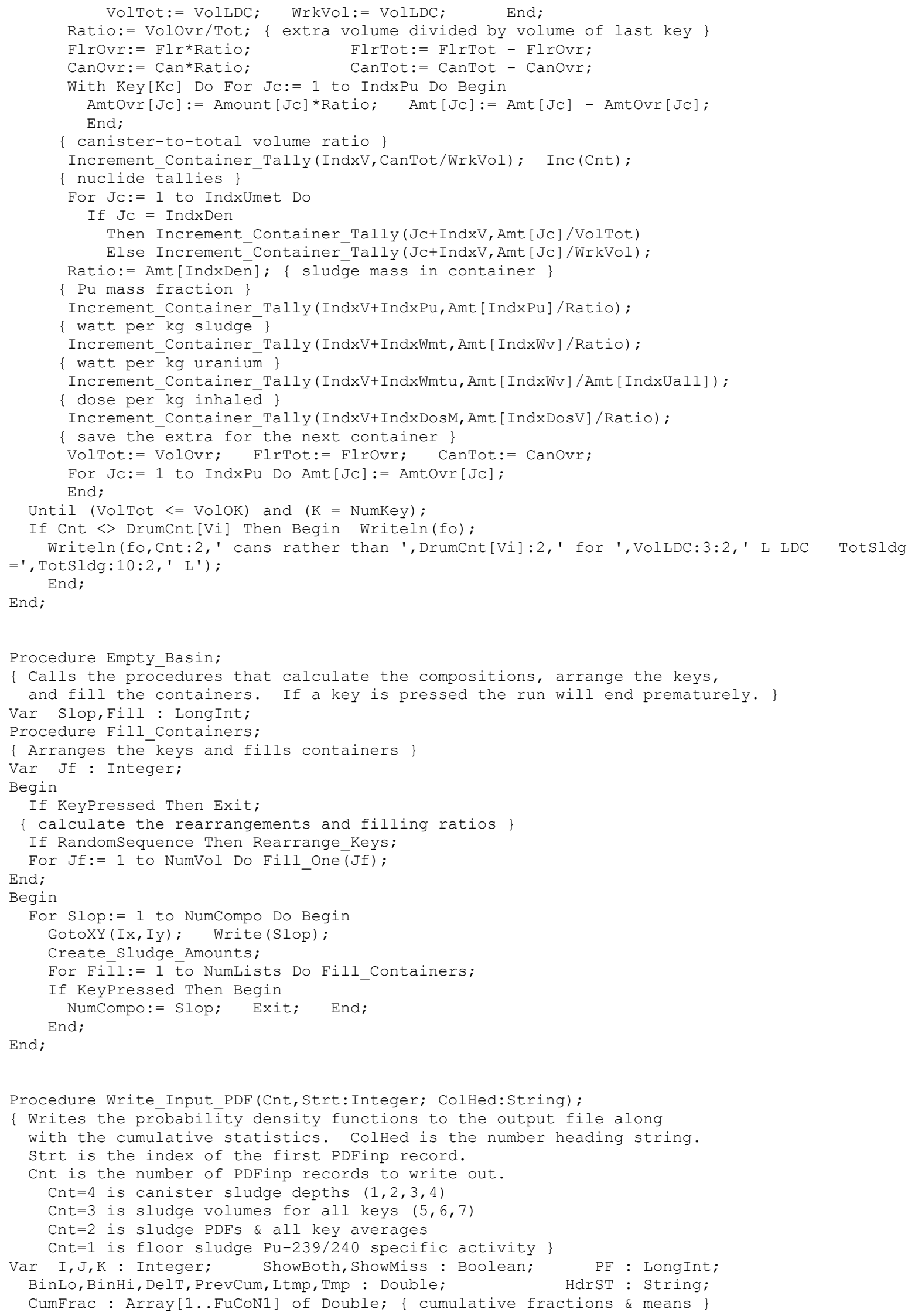




\section{SNF-10293, REV 0}

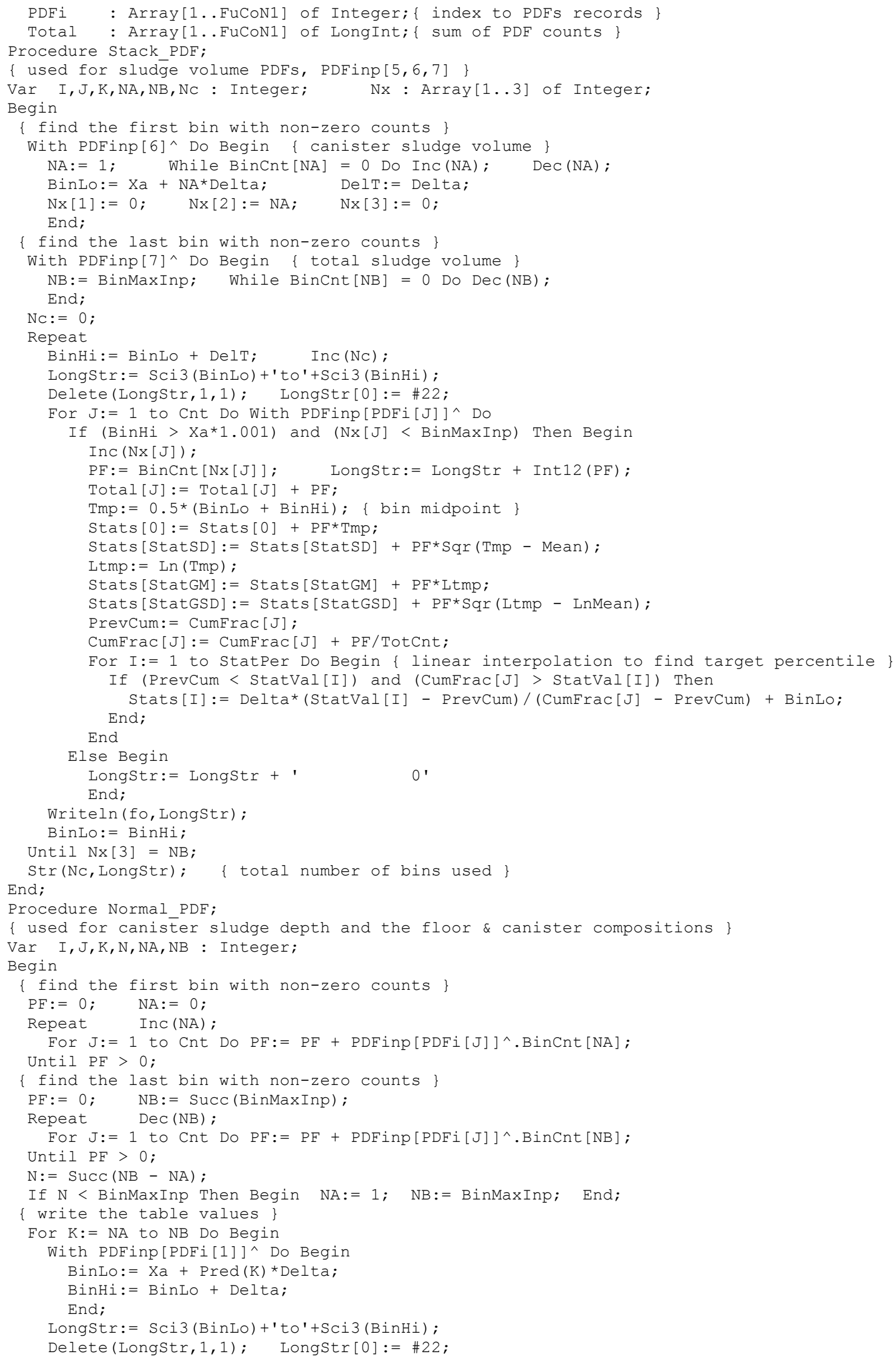




\section{SNF-10293, REV 0}

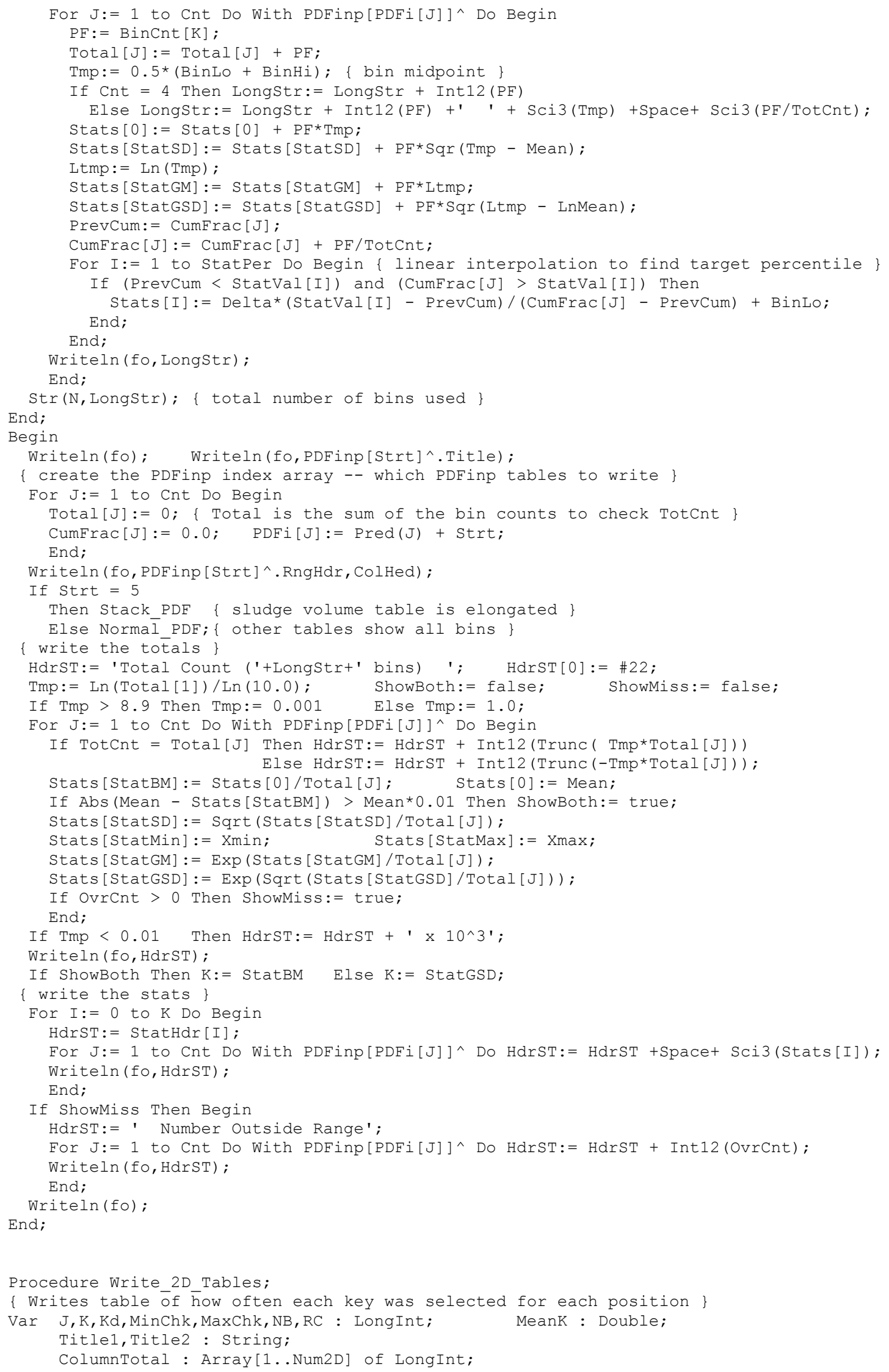




\section{SNF-10293, REV 0}

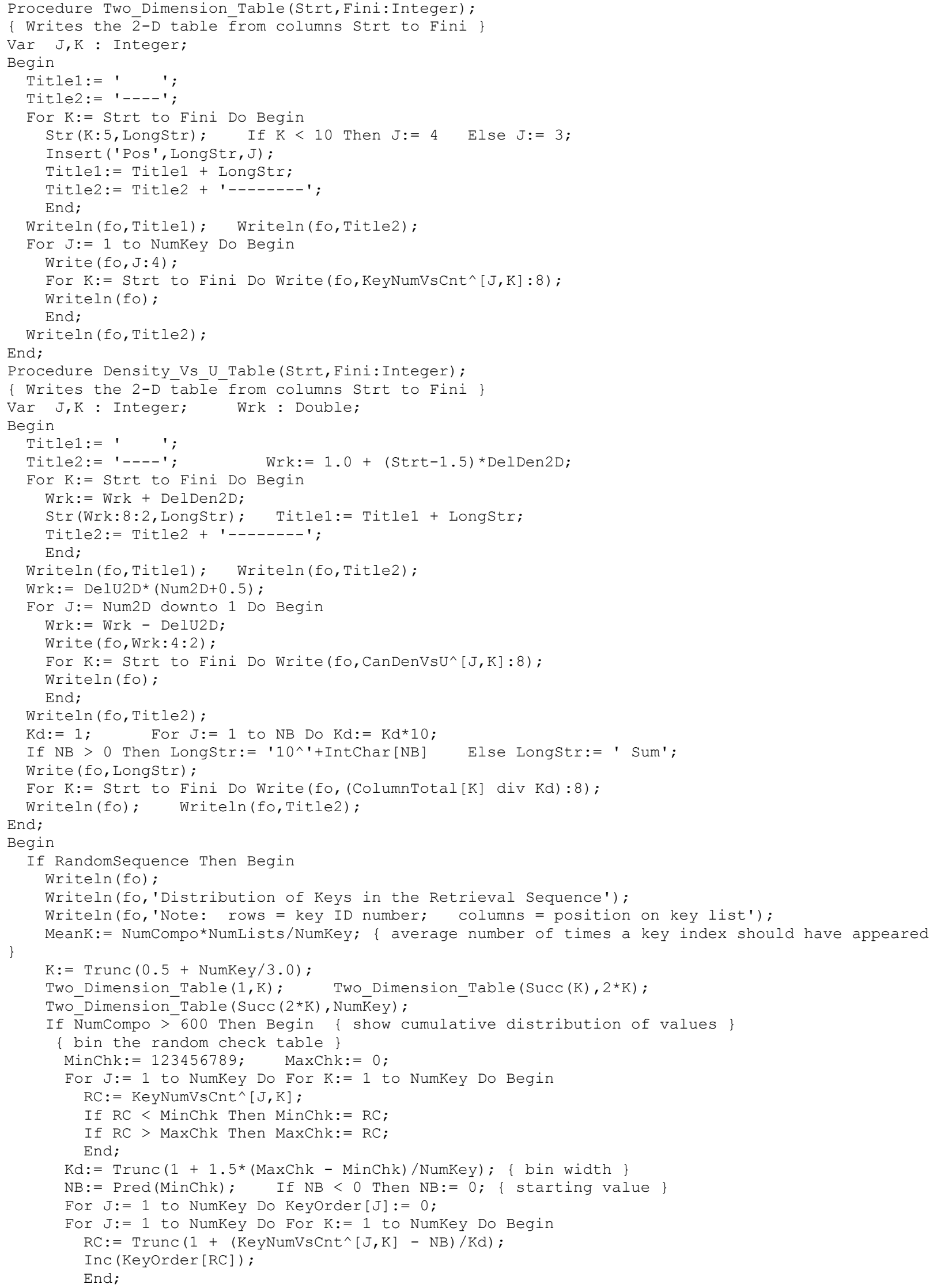




\section{SNF-10293, REV 0}

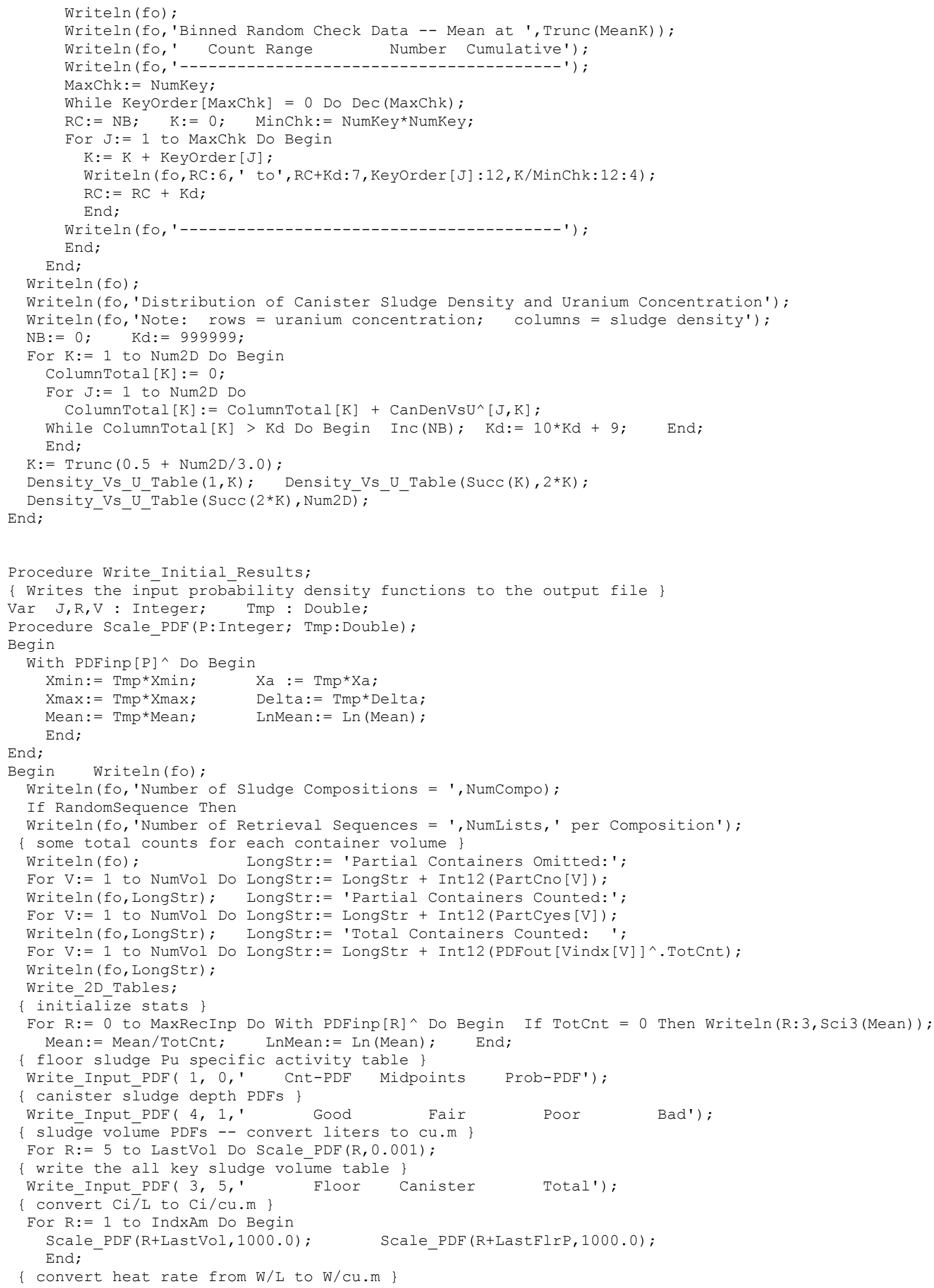




\section{SNF-10293, REV 0}

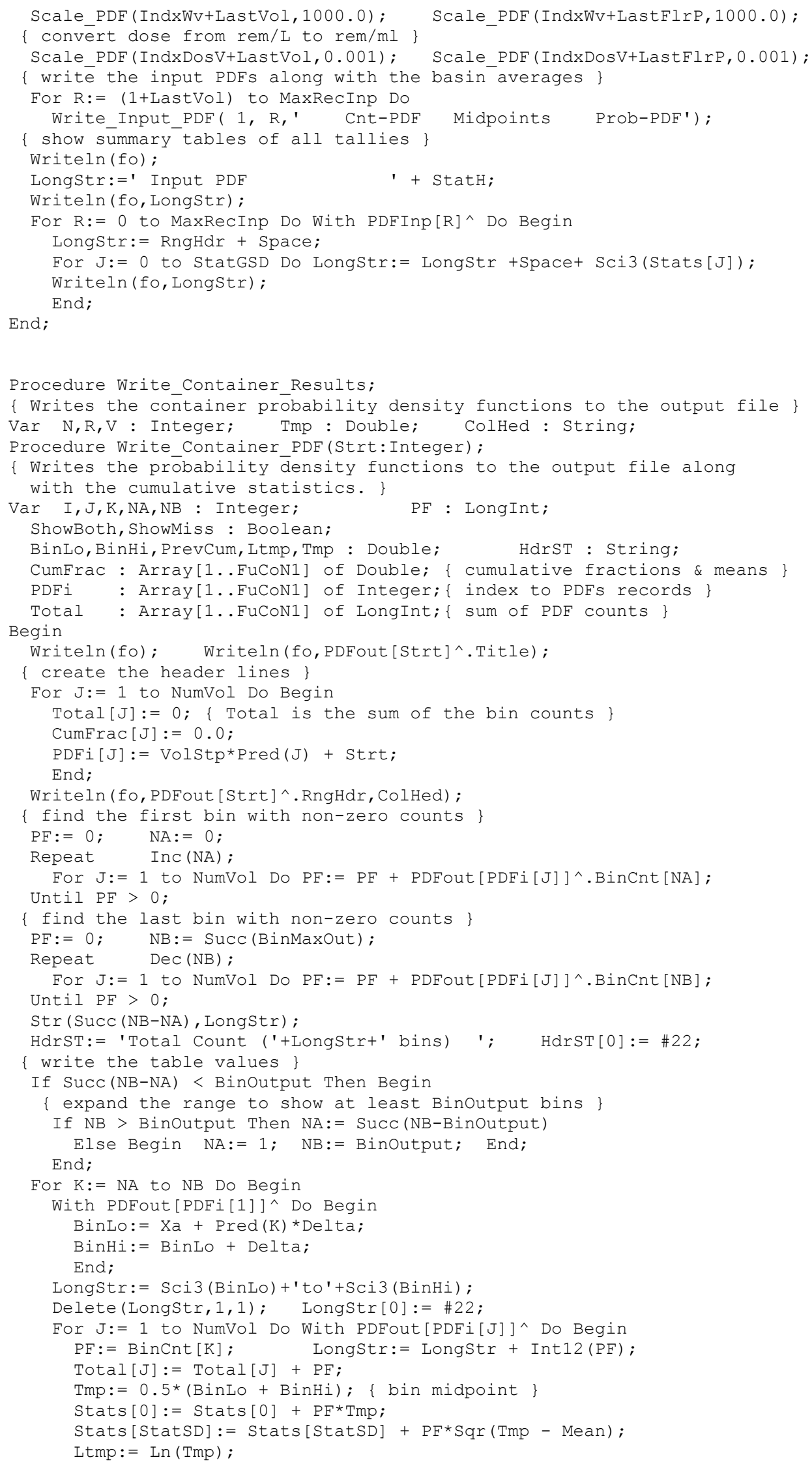




\section{SNF-10293, REV 0}

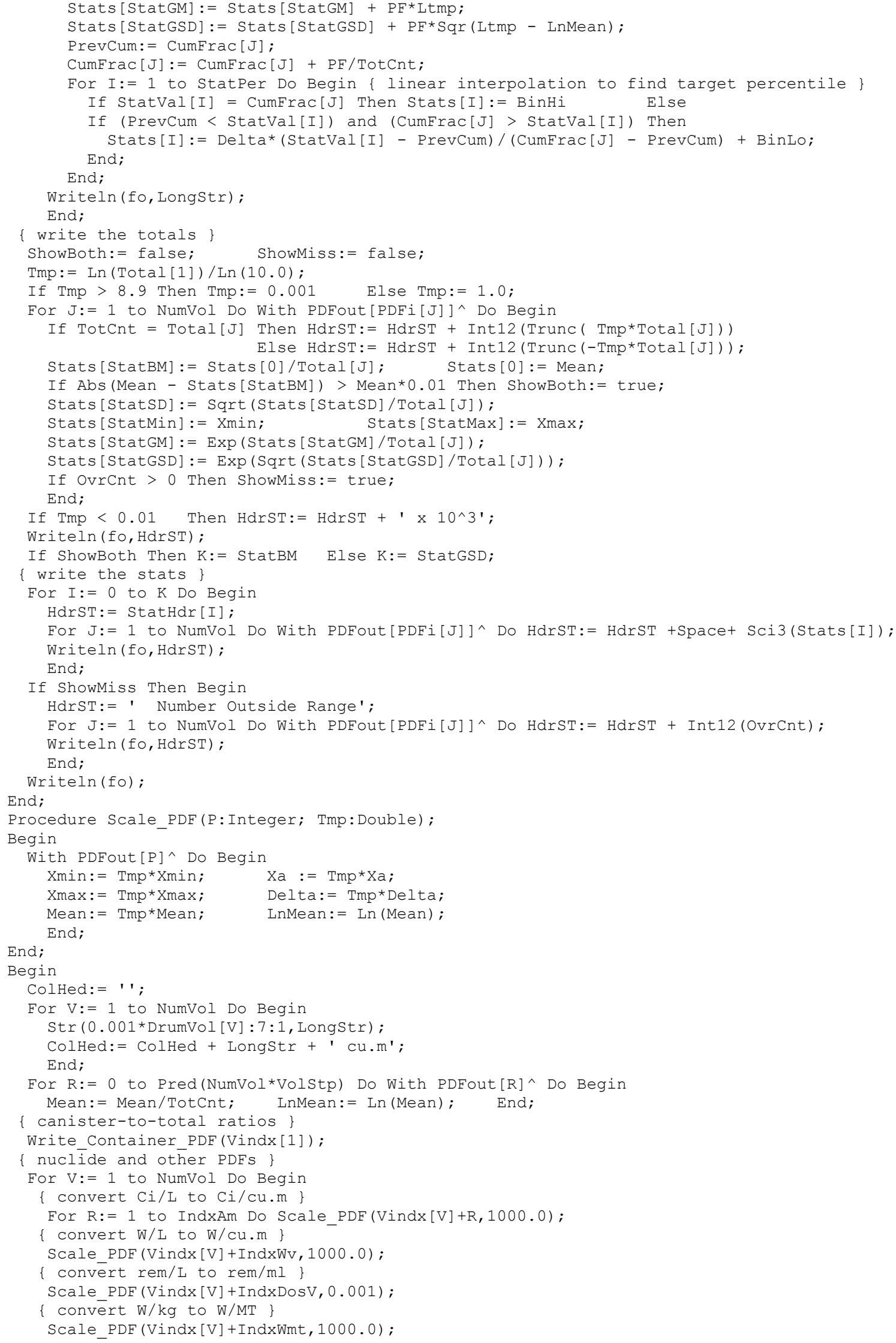


SNF-10293, REV 0

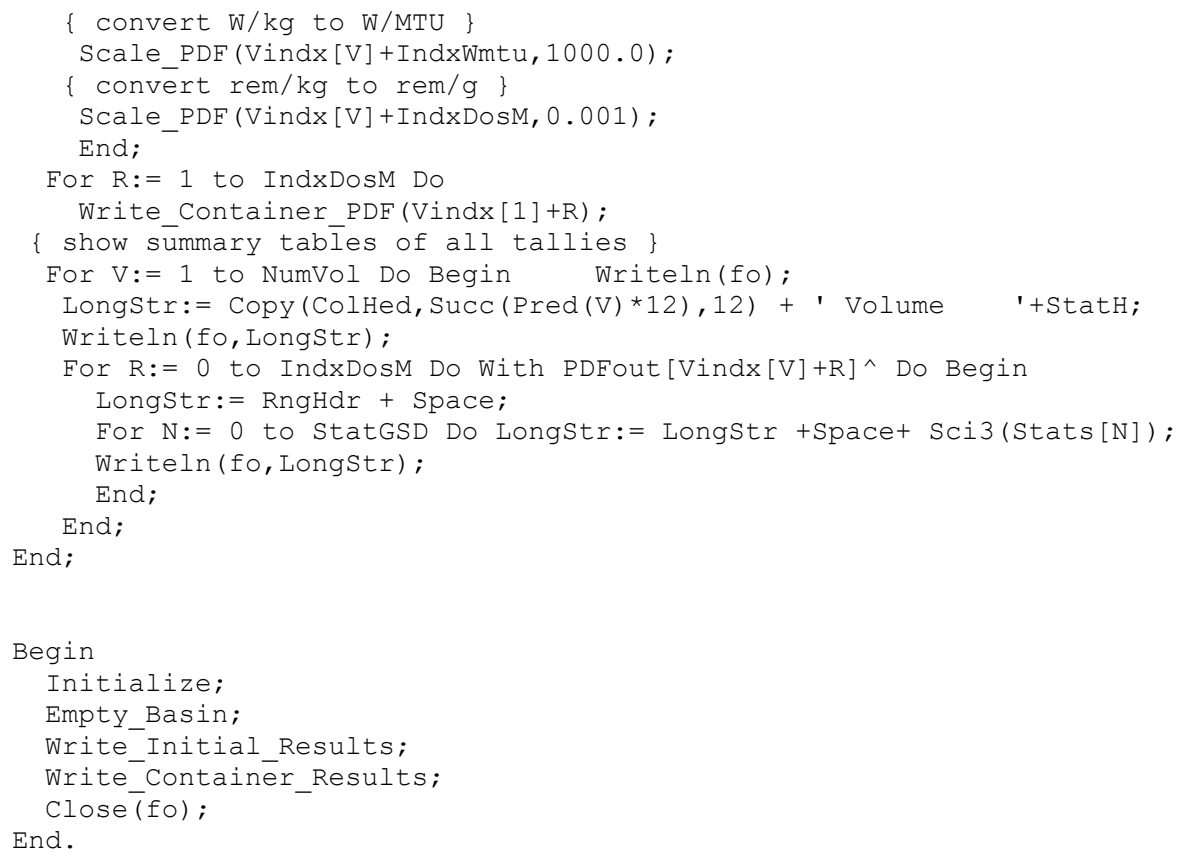


SNF-10293, REV 0

This page intentionally left blank. 
SNF-10293, REV 0

\section{APPENDIX H}

PEER REVIEW AND APPROVAL 
SNF-10293, REV 0

This page intentionally left blank.

H-ii 


\section{La}

Fauske \& Associates, Inc.

Date: $\quad$ August 1, 2002

To: $\quad$ Darrel Duncan and Jim Sloughter, Hanford Spent Nuclear Fuel Project

Cc: $\quad$ Ray Crawford, Brit Hey, Phil Loscoe, Chris Petersen, Ray Puigh, and Joe Swenson, Hanford Spent Nuclear Fuel Project

From: $\quad$ Marty Plys

Reference: D.J. Strom and P.D. Rittman, Evaluation of KE Basin Sludge Radioisotopics and Volumes in Support of the Sludge Water System Safety Analysis, SNF-10293, July. 2002.

Subject: $\quad$ Independent Review of SNF-10293

I have reviewed the reference report and I believe that it provides a reasonable model approach to prediction of expected values and variability in sludge radioisotopics and volumes. The authors have been responsive to comments during the course of the work, and my most recent comments are being satisfactorily considered. I do not require a formal indication of comment disposition.

The present report version clearly describes the methodology and results, and is careful to point out where alternate approaches may be taken, and to evaluate the impact. I was able to quickly understand the calculation process due to clarity in writing and the helpful figures, and I was able to quickly formulate technical questions. These are important points, because there is no single ideal way to perform the kind of stochastic analysis contained in SNF-10293, and the authors' congnizance of these issues and their technical skills are reflected in the product.

Revision of this document into its present state was well worth the time and effort which will pay off during external review and for long-term acceptance of its results. 
SNF-10293, REV 0

\section{REVIEW CHECKLIST}

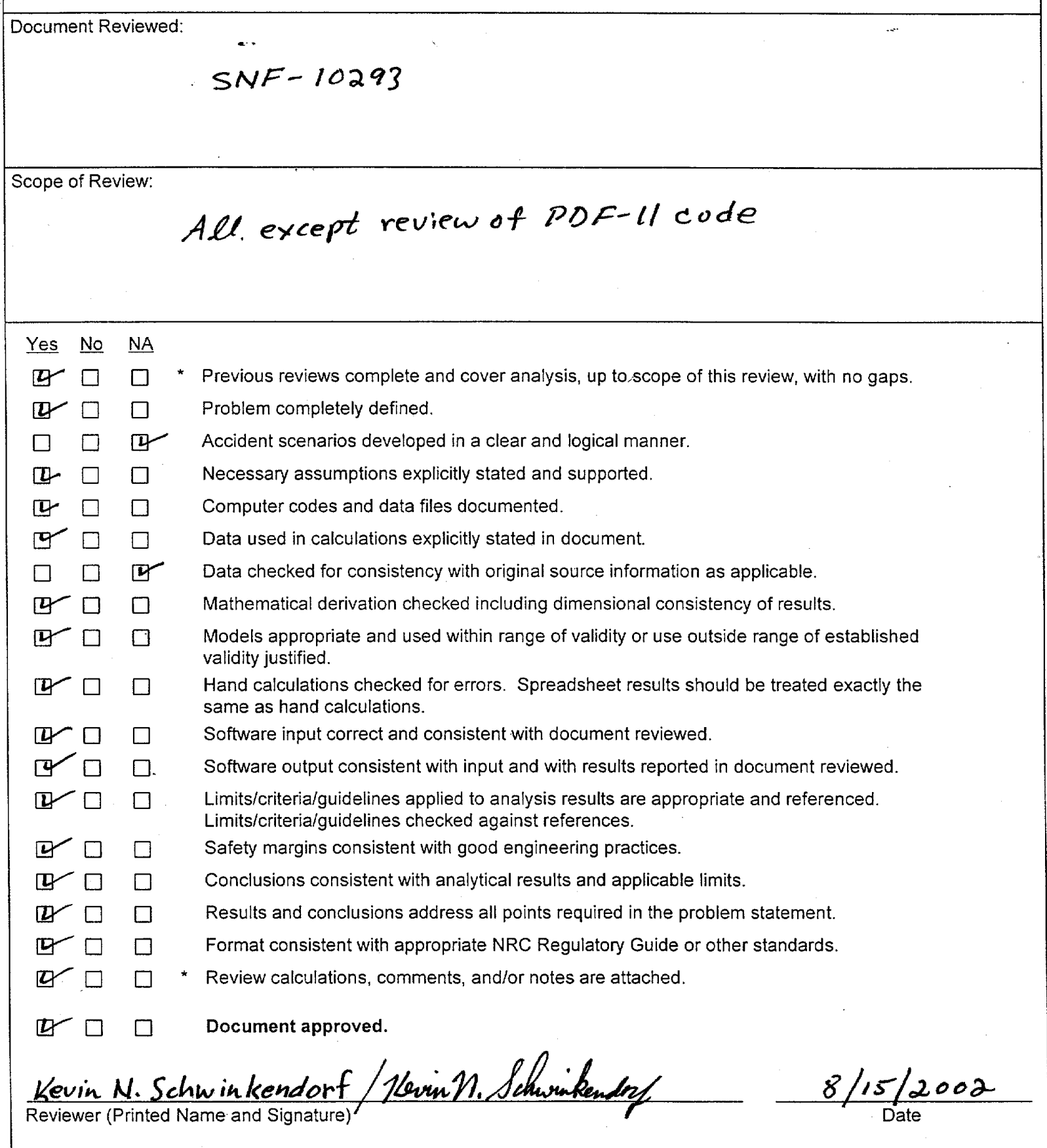

*Any calculations, comments, or notes generated as part of this review should be signed, dated and attached to this checklist. Such material should be labeled and recorded in such a manner as to be intelligible to a technically qualified third party. 
Independent Peer Reviewer Comments: K. N. Schwinkendorf- 08/13/2002

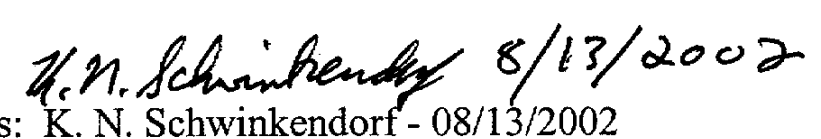

This report was peer reviewed by K. N. Schwinkendorf of the Criticality and Shielding group of Fluor Federal Services. This review covered all aspects of this report, except for PDF-11. The PDF-11 computer code was written in Pascal, and compiled using Borland's compiler, version 7. The implementation of the Monte Carlo simulation logic described in this report was reviewed by S. H. Finfrock of the Environmental and Nuclear Initiatives group of Fluor Federal Services.

This report documents an impressive and thorough analysis of loading $\mathrm{K}$ East Basin sludge into containers of various sizes using Monte Carlo simulation. Distribution functions for heat sources, dose factors, as well as uranium, plutonium, and radionuclide concentrations were carefully generated for three container sizes of interest for K East Basin sludge retrieval. The effects of changing modeling assumptions were addressed, and both measured data and model predictions (HNF-8760) were used as bases for the final results. Editorial comments were suggested, and were incorporated into the final version of this report. This report is approved. 
SNF-10293, REV 0

\section{REVIEW CHECKLIST}

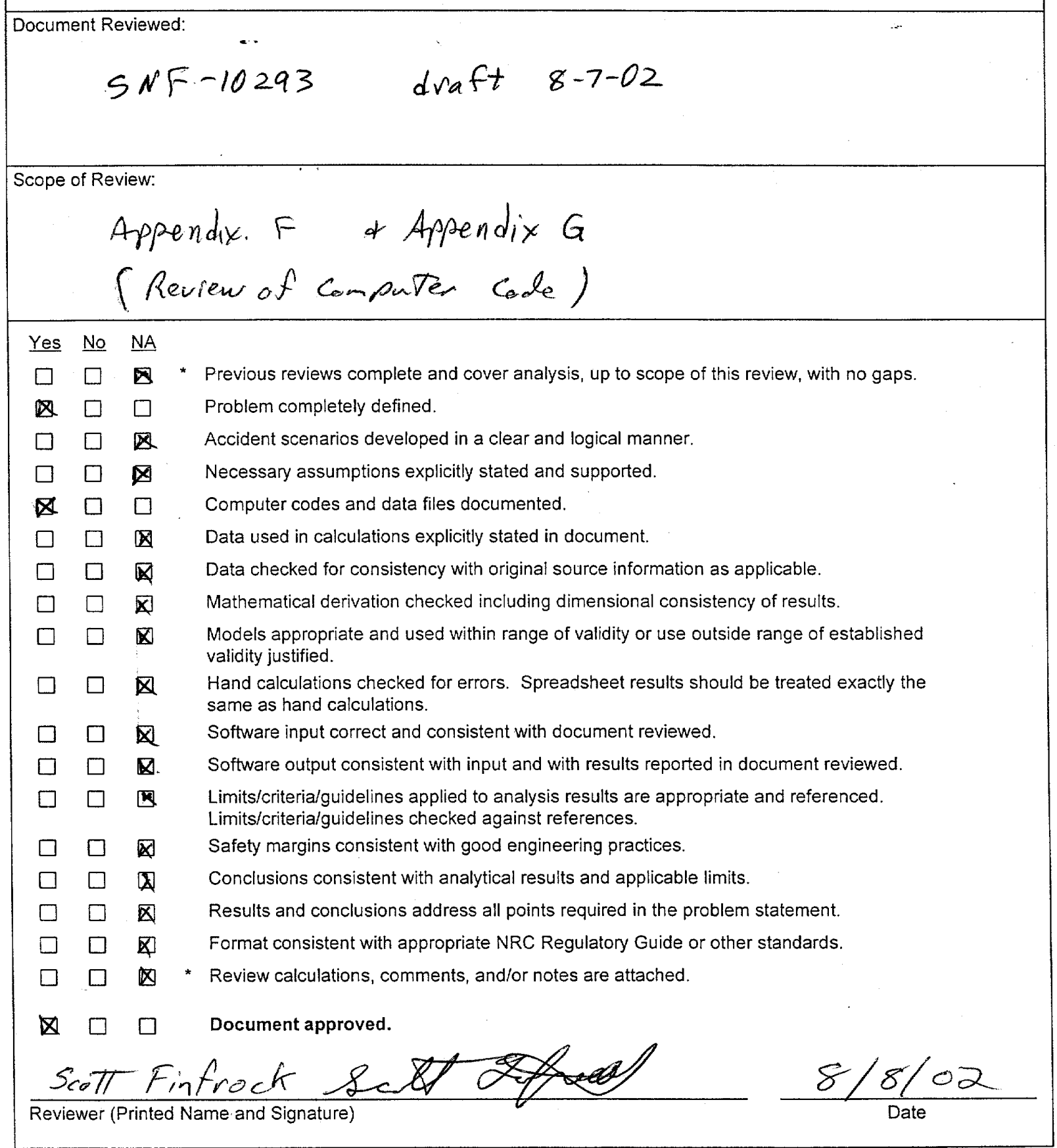

*Any calculations, comments, or notes generated as part of this review should be signed, dated and attached to this checklist. Such material should be labeled and recorded in such a manner as to be intelligible to a technically qualified third party. 